\title{
DETERMINANTS OF SYNAPTIC INFORMATION TRANSFERH FROM CA2+ BINDING PROTEINS TO GA2+ SIGNALING DOMAINS
}

\section{EDITED BY: Philippe Isope, Christian D. Wilms and Hartmut Schmidt}

PUBLISHED IN: Frontiers in Cellular Neuroscience
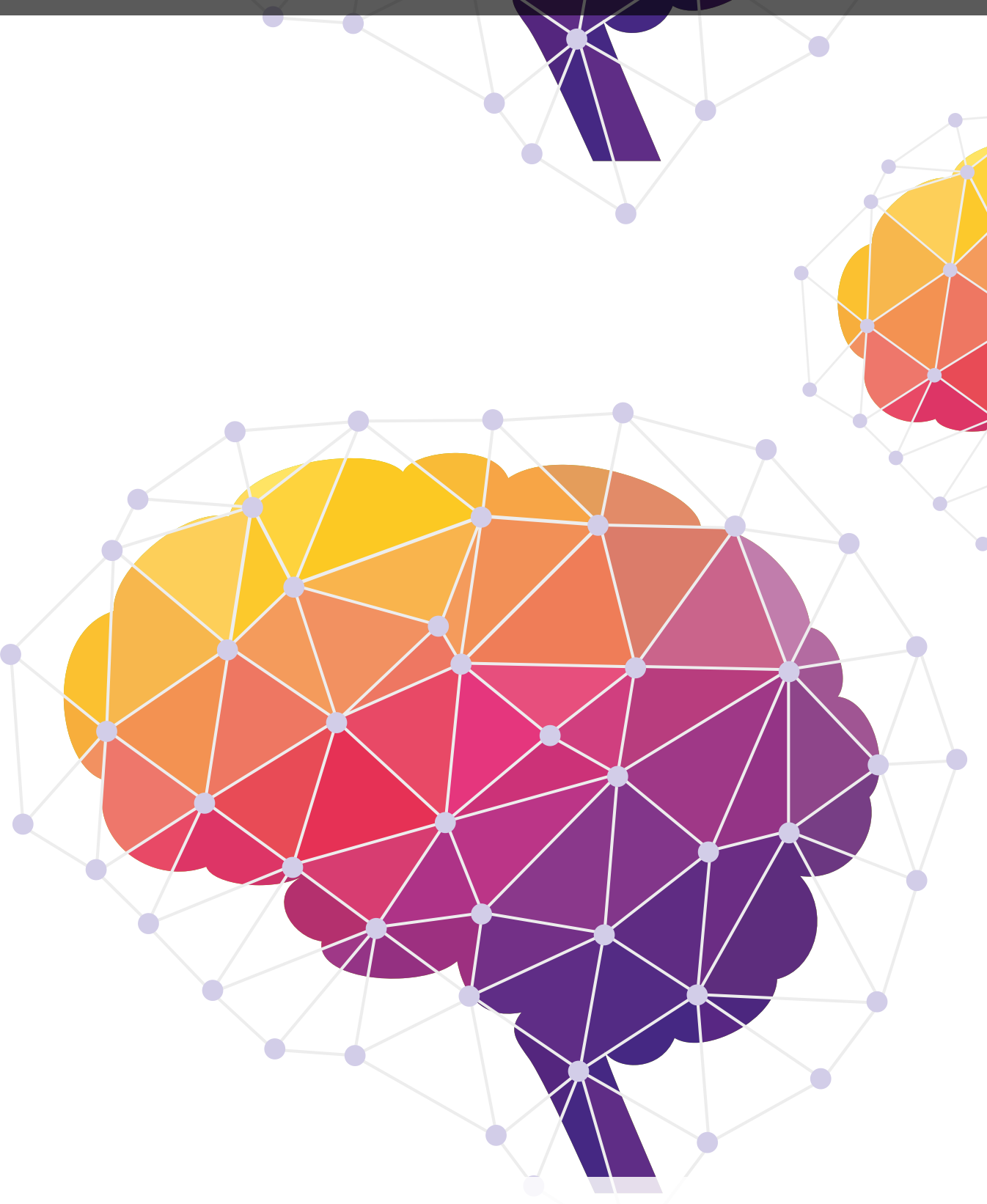


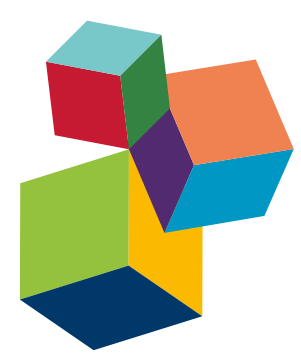

Frontiers Copyright Statement

(c) Copyright 2007-2016 Frontiers Media SA. All rights reserved.

All content included on this site, such as text, graphics, logos, button icons, images, video/audio clips, downloads, data compilations and software, is the property of or is licensed to Frontiers Media SA

("Frontiers") or its licensees and/or subcontractors. The copyright in the text of individual articles is the property of their respective authors, subject to a license granted to Frontiers.

The compilation of articles constituting

this e-book, wherever published,

as well as the compilation of all other content on this site, is the exclusive property of Frontiers. For the conditions for downloading and copying of e-books from Frontiers website, please see the Terms for Website Use. If purchasing Frontiers e-books from other websites or sources, the conditions of the website concerned apply.

Images and graphics not forming part of user-contributed materials may not be downloaded or copied without permission.

Individual articles may be downloaded and reproduced in accordance with the principles of the $C C-B Y$

licence subject to any copyright or other notices. They may not be re-sold as an e-book.

As author or other contributor you grant a CC-BY licence to others to reproduce your articles, including any graphics and third-party materials supplied by you, in accordance with the Conditions for Website Use and subject to any copyright notices which you include in connection with your articles and materials.

All copyright, and all rights therein, are protected by national and international copyright laws.

The above represents a summary only. For the full conditions see the Conditions for Authors and the Conditions for Website Use.

ISSN 1664-8714 ISBN 978-2-88919-834-4

DOI 10.3389/978-2-88919-834-4

\section{About Frontiers}

Frontiers is more than just an open-access publisher of scholarly articles: it is a pioneering approach to the world of academia, radically improving the way scholarly research is managed. The grand vision of Frontiers is a world where all people have an equal opportunity to seek, share and generate knowledge. Frontiers provides immediate and permanent online open access to all its publications, but this alone is not enough to realize our grand goals.

\section{Frontiers Journal Series}

The Frontiers Journal Series is a multi-tier and interdisciplinary set of open-access, online journals, promising a paradigm shift from the current review, selection and dissemination processes in academic publishing. All Frontiers journals are driven by researchers for researchers; therefore, they constitute a service to the scholarly community. At the same time, the Frontiers Journal Series operates on a revolutionary invention, the tiered publishing system, initially addressing specific communities of scholars, and gradually climbing up to broader public understanding, thus serving the interests of the lay society, too.

\section{Dedication to Quality}

Each Frontiers article is a landmark of the highest quality, thanks to genuinely collaborative interactions between authors and review editors, who include some of the world's best academicians. Research must be certified by peers before entering a stream of knowledge that may eventually reach the public - and shape society; therefore, Frontiers only applies the most rigorous and unbiased reviews.

Frontiers revolutionizes research publishing by freely delivering the most outstanding research, evaluated with no bias from both the academic and social point of view. By applying the most advanced information technologies, Frontiers is catapulting scholarly publishing into a new generation.

\section{What are Frontiers Research Topics?}

Frontiers Research Topics are very popular trademarks of the Frontiers Journals Series: they are collections of at least ten articles, all centered on a particular subject. With their unique mix of varied contributions from Original Research to Review Articles, Frontiers Research Topics unify the most influential researchers, the latest key findings and historical advances in a hot research area! Find out more on how to host your own Frontiers Research Topic or contribute to one as an author by contacting the Frontiers Editorial Office: researchtopics@frontiersin.org 


\title{
DETERMINANTS OF SYNAPTIC INFORMATION TRANSFER: FROM $\mathrm{CA}^{2+}$ BINDING PROTEINS TO $\mathrm{CA}^{2+}$ SIGNALING DOMAINS
}

\author{
Topic Editors: \\ Philippe Isope, CNRS, University of Strasbourg, France \\ Christian D. Wilms, University College London, UK; present: Scientifica Ltd. Uckfield, UK \\ Hartmut Schmidt, CLI, University of Leipzig, Germany
}

The cytoplasmic free $\mathrm{Ca}^{2+}$ concentration $\left(\left[\mathrm{Ca}^{2+}\right]_{\mathrm{i}}\right)$ is a key determinant of neuronal information transfer and processing. It controls a plethora of fundamental processes, including transmitter release and the induction of synaptic plasticity. This enigmatic second messenger conveys its wide variety of actions by binding to a subgroup of $\mathrm{Ca}^{2+}$ binding proteins (CaBPs) known as " $\mathrm{Ca}^{2+}$ sensors". Well known examples of $\mathrm{Ca}^{2+}$ sensors are Troponin-C in skeletal muscle, Synaptotagmin in presynaptic terminals, and Calmodulin $(\mathrm{CaM})$ in all eukaryotic cells. Since the levels of $\left[\mathrm{Ca}^{2+}\right]_{i}$ directly influence the potency of $\mathrm{Ca}^{2+}$ sensors, the $\mathrm{Ca}^{2+}$ concentration is tightly controlled by several mechanisms including another type of $\mathrm{Ca}^{2+}$ binding proteins, the $\mathrm{Ca}^{2+}$ buffers. Prominent examples of $\mathrm{Ca}^{2+}$ buffers include Parvalbumin (PV), Calbindin-D28k (CB) and Calretinin (CR), although for the latter two $\mathrm{Ca}^{2+}$ sensor functions were recently also suggested. $\mathrm{Ca}^{2+}$ buffers are distinct from sensors by their purely buffering action, i.e. they influence the spatio-temporal extent of $\mathrm{Ca}^{2+}$ signals, without directly binding downstream target proteins. Details of their action depend on their binding kinetics, mobility, and concentration. Thus, neurons can control the range of action of $\mathrm{Ca}^{2+}$ by the type and concentration of CaBPs expressed.

Since buffering strongly limits the range of action of free $\mathrm{Ca}^{2+}$, the structure of the $\mathrm{Ca}^{2+}$ signaling domain and the topographical relationships between the sites of $\mathrm{Ca}^{2+}$ influx and the location of the $\mathrm{Ca}^{2+}$ sensors are central determinants in neuronal information processing. For example, postsynaptic dendritic spines act to compartmentalize $\mathrm{Ca}^{2+}$ depending on their geometry and expression of $\mathrm{CaBPs}$, thereby influencing dendritic integration. At presynaptic sites it has been shown that tight, so called nanodomain coupling between $\mathrm{Ca}^{2+}$ channels and the sensor for vesicular transmitter release increases speed and reliability of synaptic transmission. Vice versa, the influence of an individual CaBP on information processing depends on the topographical relationships within the signaling domain. If e.g. source and sensor are very close, only buffers with rapid binding kinetics can interfere with signaling. 
This Research Topic contains a collection of work dealing with the relationships between different $\left[\mathrm{Ca}^{2+}\right]_{\mathrm{i}}$ controlling mechanisms in the structural context of synaptic sites and their functional implications for synaptic information processing as detailed in the below Editorial.

Citation: Isope, P., Wilms, C. D., Schmidt, H., eds. (2016). Determinants of Synaptic Information Transfer: From $\mathrm{Ca}^{2+}$ Binding Proteins to $\mathrm{Ca}^{2+}$ Signaling Domains. Lausanne: Frontiers Media. doi: $10.3389 / 978-2-88919-834-4$ 


\section{Table of Contents}

05 Editorial: Determinants of Synaptic Information Transfer: From $\mathrm{Ca}^{2+}$ Binding Proteins to $\mathrm{Ca}^{2+}$ Signaling Domains

Philippe Isope, Christian D. Wilms and Hartmut Schmidt

07 Presynaptic nanodomains: a tale of two synapses

Lu-Yang Wang and George J. Augustine

17 A use-dependent increase in release sites drives facilitation at calretinindeficient cerebellar parallel-fiber synapses

Simone Brachtendorf, Jens Eilers and Hartmut Schmidt

28 Subcellular structural plasticity caused by the absence of the fast $\mathrm{Ca}^{2+}$ buffer calbindin D-28k in recurrent collaterals of cerebellar Purkinje neurons

David Orduz, Alain Boom, David Gall, Jean-Pierre Brion, Serge N. Schiffmann and Beat Schwaller

42 Calmodulin as a major calcium buffer shaping vesicular release and short-term synaptic plasticity: facilitation through buffer dislocation

Yulia Timofeeva and Kirill E. Volynski

55 Buffer mobility and the regulation of neuronal calcium domains

Elizabeth A. Matthews and Dirk Dietrich

66 Translating neuronal activity at the synapse: presynaptic calcium sensors in short-term plasticity

Arthur P. H. de Jong and Diasynou Fioravante

74 Bruchpilot and Synaptotagmin collaborate to drive rapid glutamate release and active zone differentiation

Mila M. Paul, Martin Pauli, Nadine Ehmann, Stefan Hallermann, Markus Sauer,

Robert J. Kittel and Manfred Heckmann

86 Super-resolution microscopy of the synaptic active zone

Nadine Ehmann, Markus Sauer and Robert J. Kittel

94 Spontaneous vesicle recycling in the synaptic bouton

Sven Truckenbrodt and Silvio O. Rizzoli

101 Inhibitory and excitatory axon terminals share a common nano-architecture of their $\mathrm{Ca}_{v} 2.1$ (P/Q-type) $\mathrm{Ca}^{2+}$ channels

Daniel Althof, David Baehrens, Masahiko Watanabe, Noboru Suzuki, Bernd Fakler and Ákos Kulik

112 Dendritic diameters affect the spatial variability of intracellular calcium dynamics in computer models

Haroon Anwar, Christopher J. Roome, Hermina Nedelescu, Weiliang Chen, Bernd Kuhn and Erik De Schutter

126 Dendritic patch-clamp recordings from cerebellar granule cells demonstrate electrotonic compactness

Igor Delvendahl, Isabelle Straub and Stefan Hallermann 


\title{
Editorial: Determinants of Synaptic Information Transfer: From $\mathrm{Ca}^{2+}$ Binding Proteins to $\mathrm{Ca}^{2+}$ Signaling Domains
}

\author{
Philippe Isope ${ }^{1}$, Christian D. Wilms ${ }^{2}$ and Hartmut Schmidt ${ }^{3 *}$ \\ ${ }^{1}$ Institut des Neurosciences Cellulaires et Integratives, Centre National de la Recherche Scientifique UPR 3212, Strasbourg, \\ France, ${ }^{2}$ Wolfson Institute for Biomedical Research and Department of Neuroscience, Physiology and Pharmacology, \\ University College London, London, UK, ${ }^{3}$ Medical Faculty, Carl-Ludwig Institute for Physiology, University of Leipzig, Leipzig, \\ Germany
}

Keywords: synaptic calcium signaling, calcium binding proteins, localization, calcium domains, transmitter release, methods

\section{The Editorial on the Research Topic}

Determinants of Synaptic Information Transfer: From Ca2+ Binding Proteins to Ca2+ Signaling Domains

$\mathrm{Ca}^{2+}$ ions are key regulators of fundamental synaptic processes including transmitter release and the induction of plasticity. They act within complex topographical relationships between the sites of $\mathrm{Ca}^{2+}$ influx and those sites where the $\mathrm{Ca}^{2+}$ controlled effector proteins are located. These topographies are dynamically shaped by protein-complexes and the spatio-temporal extent of $\mathrm{Ca}^{2+}$ elevations within these topographies is controlled by $\mathrm{Ca}^{2+}$ buffers. Ultimately, these spatiotemporal relationships determine the details of $\mathrm{Ca}^{2+}$ induced effects. This e-book deals with the significance of localized synaptic calcium signaling.

Synaptic information transfer begins with presynaptic transmitter release. Wang and Augustine

\section{OPEN ACCESS}

Edited and reviewed by: Egidio D'Angelo,

University of Pavia, Italy

*Correspondence:

Hartmut Schmidt

hartmut.schmidt@

medizin.uni-leipzig.de

Received: 24 February 2016 Accepted: 02 March 2016 Published: 16 March 2016

Citation:

Isope P, Wilms CD and Schmidt H (2016) Editorial: Determinants of Synaptic Information Transfer: From

$\mathrm{Ca}^{2+}$ Binding Proteins to $\mathrm{Ca}^{2+}$ Signaling Domains.

Front. Cell. Neurosci. 10:69.

doi: 10.3389/fncel.2016.00069 review the concept of local presynaptic $\mathrm{Ca}^{2+}$ signaling domains and their functional importance for release, focusing on two giant presynaptic terminals, the squid giant synapse and the calyx of Held. Central concepts that still dominate our view about the significance of presynaptic $\mathrm{Ca}^{2+}$ domains were originally developed using these two synapses. The authors describe the distinction between nano- and micro-domain topographies and the evidence for a developmental regulation of $\mathrm{Ca}^{2+}$ domains at several synapses.

A synapse that operates with nano-domain influx-release coupling is the parallel-fiber (PF) to Purkinje cell (PC) synapse. These synapses show use-dependent facilitation that surprisingly persists in mutant mice lacking calretinin - the major buffer of PF terminals-which leads to increased release probability. Brachtendorf et al. analyzed mechanisms of facilitation at individual PF-PC synapses of calretinin mutant mice using paired patch-clamp recordings. They suggest that a $\mathrm{Ca}^{2+}$-driven process that rapidly replenishes releasable vesicles operates more effectively in the absence of calretinin, thereby explaining the persistence of facilitation.

How critical the maintenance of calcium homeostasis on synaptic function can be is demonstrated by Orduz et al. In their study, knocking out calbindin, a calcium binding protein (CaBP) related to calretinin, had surprisingly little effect on the amplitude of postysynaptic potentials (PSPs) at PC-to-PC synapses. Their detailed study of presynaptic morphology revealed larger boutons and AZs and a higher number of docked vesicles in the mutants. The authors view these changes as a compensatory mechanism to maintain central characteristics of release in the face of a major perturbation. 
For a given topology, CaBPs are key controllers of synaptic efficacy. Whether they simply act as buffers or have an additional sensor function is a critical question. Using constrained modeling, Timofeeva and Volynski shed light onto an oftenoverlooked candidate for the control of neurotransmitter release, Calmodulin (CaM). Acting as a buffer, CaM can influence calcium concentration near the AZ. The authors demonstrate that the fast calcium binding properties of $\mathrm{CaM}$ combined with its mobility inhibit vesicular release and favor short term facilitation during repeated action potential (AP) firing.

Many studies have established how CaBPs shape the calcium wave in the presynaptic bouton or the dendritic spines. Indeed, the mobility of CaBPs will determine how they influence calcium dynamics. Matthews and Dietrich review and discuss the role and the properties of immobile or poorly mobile buffers, demonstrating that they also participate in the control of the apparent diffusion of free calcium and its decay time constant.

The combination of calcium influx, extrusion, and buffering result in the calcium wave that triggers release De Jong and Fioravante review how this wave is translated into actual vesicular release. They described the mechanisms by which the calcium sensors expressed in the presynaptic compartment undergo conformational changes when calcium binds to specific domains and the role of specific candidates in the control of vesicular release.

A calcium sensor central to synaptic release is synaptotagmin. Using a combination of electro-physiological recordings and super-resolution microscopy, Paul et al. reveal an unexpected interaction between the AZ protein Bruchpilot and synaptotagmin. They find that in Drosophila differentiation of neuromuscular junctions require concerted action of both Bruchpilot and synaptotagmin to result in structurally and functionally normal synapses. This study exemplifies how the combination of physiology and high-resolution microscopy can be harnessed to elucidate synaptic function at the nanometer scale.

Estimates of nano-scale topographical relationships at AZs are often derived by combining quantitative physiological techniques with modeling approaches. However, as Ehmann et al. point out in their Perspective, without direct microscopic access to the synaptic fine structure, our picture of the function of the surrounding protein networks will remain incomplete. Due to the diffraction-limit for light investigations of the AZ ultrastructure have been restricted to EM, which however provides only a static snap-shot. The authors explore, how recent advances in super resolution light microscopy like STED and STORM bridge the gap between EM and conventional light microscopy.

Next to AP triggered release, vesicle exocytosis also occurs spontaneously at most synapses. Truckenbrodt and Rizzoli review the controversy about the mechanism of spontaneous exocytosis. It is thought that either spontaneous fluctuations in local calcium trigger fusion of vesicles residing in the same vesicle pool that normally fuses during an AP albeit via a different release sensor, or else that release is driven from a separate pool of synaptic vesicles probably independent of $\mathrm{Ca}^{2+}$. The authors speculate on the origins of this controversy and suggest that a potential solution could be related to developmental effects.
Neurotransmitter release is highly influenced by the structural organization of calcium channels. Notably, the distance and the spatial organization between synaptic vesicles and calcium channels affect the probability of release. Using high-resolution freeze fracture immuno-EM and automatized reconstruction of Cav2.1 channel distribution, Althof et al. demonstrated that inhibitory and excitatory synapses in the CA1 area of the hippocampus share a common spatial organization.

While the papers above focus on the role of calcium topography and dynamics in the presynaptic compartment, the central role of these parameters is of course not limited to the presynapse.

The amplitude and time course of dendritic calcium transients are not only determined by buffering and extrusion, but also by morphology, specifically the dendritic diameter. Anwar et al. explore the expected variance of calcium concentrations due to differences in dendritic diameter using a modeling approach. In addition the authors demonstrate how to implement diameterdependent effects on calcium signaling in the NEURON simulator. Armed with this knowledge researchers will be able to include such strong morphological effects in their models of dendritic function.

To close the cycle of information flow, PSPs generated by synaptic inputs are conducted to the soma. How strongly these PSPs are filtered and attenuated is to a larger part determined by the electrotonic compactness of the dendritic branch. Using whole-cell recordings directly from the dendrites of cerebellar granule cells (GCs), Delvendahl et al. analyze the electrotonic compactness of these minute neurons. They find that GCs are electrotonically extremely compact, making them ideally suited for the efficient transfer of high-frequency information.

This research topic gives a prospect onto how the multitude of synaptic $\mathrm{Ca}^{2+}$ functions arises from the localization of $\mathrm{Ca}^{2+}$ signals to specific sub-compartments by the joined action of topographical relationships with CaBPs and emphasize the importance of technical breakthroughs in promoting our understand compartmentalization.

\section{AUTHOR CONTRIBUTIONS}

All authors listed, have made substantial, direct and intellectual contribution to the work, and approved it for publication.

\section{FUNDING}

This work was supported by an ARN grant to PI (FRM 2014 DEQ20140329514) and a DFG grant to HS (SCHM1838).

\footnotetext{
Conflict of Interest Statement: The authors declare that the research was conducted in the absence of any commercial or financial relationships that could be construed as a potential conflict of interest.

Copyright (C) 2016 Isope, Wilms and Schmidt. This is an open-access article distributed under the terms of the Creative Commons Attribution License (CC BY). The use, distribution or reproduction in other forums is permitted, provided the original author(s) or licensor are credited and that the original publication in this journal is cited, in accordance with accepted academic practice. No use, distribution or reproduction is permitted which does not comply with these terms.
} 


\title{
Presynaptic nanodomains: a tale of two synapses
}

\author{
Lu-Yang Wang ${ }^{1,2}$ and George J. Augustine , $^{3,5,6}$ * \\ 1 Program in Neurosciences and Mental Health, SickKids Research Institute, Toronto, Canada \\ 2 Department of Physiology, University of Toronto, Toronto, Canada \\ ${ }^{3}$ Lee Kong Chian School of Medicine, Nanyang Technological University, Singapore, Singapore \\ ${ }^{4}$ Institute of Molecular and Cell Biology, Singapore, Singapore \\ ${ }^{5}$ Center for Functional Connectomics, Korea Institute of Science and Technology, Seoul, South Korea \\ ${ }^{6}$ Marine Biological Laboratory, Woods Hole, MA, USA
}

\section{Edited by:}

Hartmut Schmidt, University of

Leipzig, Germany

\section{Reviewed by:}

Manfred Heckmann, University of

Wuerzburg, Germany

Samuel Young, Max Planck Florida

Institute for Neuroscience, USA

\section{*Correspondence:}

George J. Augustine, Lee Kong

Chian School of Medicine, Nanyang

Technological University, 61 Biopolis

Drive, Proteos, Singapore 138673,

Singapore

e-mail: george.augustine@

ntu.edu.sg
Here we summarize the evidence from two "giant" presynaptic terminals-the squid giant synapse and the mammalian calyx of Held-supporting the involvement of nanodomain calcium signals in triggering of neurotransmitter release. At the squid synapse, there are three main lines of experimental evidence for nanodomain signaling. First, changing the size of the unitary calcium channel current by altering external calcium concentration causes a non-linear change in transmitter release, while changing the number of open channels by broadening the presynaptic action potential causes a linear change in release. Second, low-affinity calcium indicators, calcium chelators, and uncaging of calcium all suggest that presynaptic calcium concentrations are as high as hundreds of micromolar, which is more compatible with a nanodomain type of calcium signal. Finally, neurotransmitter release is much less affected by the slow calcium chelator, ethylene glycol tetraacetic acid (EGTA), in comparison to the rapid chelator 1,2-bis(oaminophenoxy)ethane- $N, N, N^{\prime}, N^{\prime}$-tetraacetic acid (BAPTA). Similarly, as the calyx of Held synapse matures, EGTA becomes less effective in attenuating transmitter release while the number of calcium channels required to trigger a single fusion event declines. This suggests a developmental transformation of microdomain to nanodomain coupling between calcium channels and transmitter release. Calcium imaging and uncaging experiments, in combination with simulations of calcium diffusion, indicate the peak calcium concentration seen by presynaptic calcium sensors reaches at least tens of micromolar at the calyx of Held. Taken together, data from these provide a compelling argument that nanodomain calcium signaling gates very rapid transmitter release.

Keywords: neurotransmitter release, calcium signaling, calcium channels, presynaptic terminals, synaptic vesicle trafficking

\section{INTRODUCTION TO THE CALCIUM DOMAIN CONCEPT}

The concept of local "domains" of intracellular calcium concentration developed from mathematical models of the diffusion of Ca ions $\left(\mathrm{Ca}^{2+}\right)$ away from the mouth of open calcium channels. The first published model, by Chad and Eckert (1984), laid out several key features of this concept. This paper proposed that opening of individual $\mathrm{Ca}^{2+}$ channels could form discrete spatial domains where $\mathrm{Ca}^{2+}$ concentration is very high locallywithin nanometers of a single open $\mathrm{Ca}^{2+}$ channel-but drops off steeply with distance away from the channel. In addition, increasingly large depolarizations of the membrane potential should have dual effects: increasing the number of such domains, by increasing the number of open $\mathrm{Ca}^{2+}$ channels, yet reducing the magnitude of the local $\mathrm{Ca}^{2+}$ concentration change within a domain because of the reduced unitary current through an open channel.

Simon and Llinás (1985) independently developed the $\mathrm{Ca}^{2+}$ domain concept and considered the possible role of such domains in triggering of neurotransmitter release from presynaptic terminals. In addition to confirming the deductions of Chad and Eckert (1984) regarding the basic properties of $\mathrm{Ca}^{2+}$ domains, they further concluded that these domains usually do not overlap appreciably for the case of squid presynaptic terminals. When many $\mathrm{Ca}^{2+}$ channels are opened by large, prolonged depolarizations, there can be a small degree of spatial overlap due to summation of $\mathrm{Ca}^{2+}$ from adjacent open channels. The modeling studies of Zucker and Fogelson (1986) reached a similar conclusion. Notably, Simon and Llinás (1985) concluded that an endogenous $\mathrm{Ca}^{2+}$ buffer could eliminate summation of $\mathrm{Ca}^{2+}$ signals associated with neighboring domains, but should have minimal effect on the peak $\mathrm{Ca}^{2+}$ concentration change occurring in the immediate vicinity of an open channel.

At its simplest, diffusion of $\mathrm{Ca}^{2+}$ from an open channel can be described by the following equation (Smith and Augustine, 1988):

$$
\left[\mathrm{Ca}^{2+}\right](x, t)=\frac{J_{C a}}{2 \pi D x} \operatorname{erfc} \frac{x}{2 \sqrt{D t}}
$$


Table 1 | "Rule of thumb" relationships for $\mathrm{Ca}^{2+}$ diffusion within domains.

\begin{tabular}{lcr}
\hline Distance & {$\left[\mathbf{C a}^{\mathbf{2 +}}\right](\sim \mathbf{1 / x})$} & Time $\left(\sim \boldsymbol{x}^{\mathbf{2}}\right)$ \\
\hline $10 \mathrm{~nm}$ & $100 \mu \mathrm{M}$ & $1 \mu \mathrm{sec}$ \\
$100 \mathrm{~nm}$ & $10 \mu \mathrm{M}$ & $100 \mu \mathrm{sec}$ \\
$1000 \mathrm{~nm}$ & $1 \mu \mathrm{M}$ & $10000 \mu \mathrm{sec}$ \\
\hline
\end{tabular}

where $x$ is distance from the channel, $t$ is time, $J_{C a}$ is the flux of $\mathrm{Ca}^{2+}$ through a single open channel, $D$ is the diffusion coefficient for $\mathrm{Ca}^{2+}$ and erfc is the complementary error function. This equation predicts that (1) while the channel is open, there will be an inverse relationship between $\mathrm{Ca}^{2+}$ concentration and distance from the channel; and (2) $\mathrm{Ca}^{2+}$ diffusion time will increase with the square of distance. To aid comprehension of these relationships, we provide "rule of thumb" $\mathrm{Ca}^{2+}$ diffusion parameters in Table 1. These parameters are to be taken as orderof-magnitude approximations because their precise values will depend on the value chosen for $D$, as well as the unitary current flowing through the open $\mathrm{Ca}^{2+}$ channel and the properties of cellular $\mathrm{Ca}^{2+}$ buffering. More complete mathematical treatments can be found elsewhere, for example in Smith and Augustine (1988), Naraghi and Neher (1997) and Neher (1998), as well as in the pioneering domain papers described above.

As mentioned above, it is possible that $\mathrm{Ca}^{2+}$ signals from adjacent open $\mathrm{Ca}^{2+}$ channels could overlap under some conditions. The degree of overlap leads to several qualitatively different forms of local $\mathrm{Ca}^{2+}$ signaling that are depicted in Figure 1. If there is no spatial overlap between the local $\mathrm{Ca}^{2+}$ domains, relative to the position of the relevant $\mathrm{Ca}^{2+}$ sensor, then a nanodomain occurs (Figure 1A). This type of signal is called a nanodomain because $\mathrm{Ca}^{2+}$ signaling will occur over dimensions of nanometers (Kasai, 1993; Schweizer et al., 1995; Augustine et al., 2003). If there is some degree of spatial overlap between neighboring domains and the $\mathrm{Ca}^{2+}$ sensor receives contributions from domains summing over a distance of approximately a micrometer, then this is microdomain-style $\mathrm{Ca}^{2+}$ signaling (Figure 1B). Finally, if the sensor receives $\mathrm{Ca}^{2+}$ ions diffusing over distances greater than a micrometer, due to summation of microdomains, then a radial gradient style of $\mathrm{Ca}^{2+}$ signaling is said to occur (Figure 1C). The differences in the spatial range of these different modes of $\mathrm{Ca}^{2+}$ signaling necessarily are associated with differences in the speed of $\mathrm{Ca}^{2+}$ triggering of biological processes, as indicated by the $t$-term in equation (1) and in the right column of Table 1.

From a physical perspective, there is no question that local domains of $\mathrm{Ca}^{2+}$ signaling occur in the immediate vicinity of an open $\mathrm{Ca}^{2+}$ channel. The question addressed in this review is how relevant such domain signaling is for release of neurotransmitters from presynaptic terminals. We will compare $\mathrm{Ca}^{2+}$ signaling at two presynaptic terminals whose large size makes them particularly amenable to experimental analysis: the squid giant synapse and the mammalian calyx of Held. From results obtained at these synapses, as well as selected results from other synapses, we will develop the argument that nanodomain $\mathrm{Ca}^{2+}$ signaling is prevalent at presynaptic terminals that rapidly release neurotransmitters, though microdomain signaling also has been established for some types of presynaptic terminals. Radial gradients are likely to be important only for presynaptic terminals that slowly release neuropeptides or for slower modulatory presynaptic actions of $\mathrm{Ca}^{2+}$ within fastacting synapses, for example $\mathrm{Ca}^{2+}$-dependent forms of synaptic plasticity.

\section{EVIDENCE FOR NANODOMAINS AT THE SOUID GIANT SYNAPSE}

Virtually all of the early experimental tests of the role of $\mathrm{Ca}^{2+}$ domains in triggering neurotransmitter release were performed at the squid giant synapse. As a result, work at this synapse established most of the experimental paradigms that have been used to probe local $\mathrm{Ca}^{2+}$ signaling in presynaptic terminals. In this section we will describe some of these key experiments.

The first clues regarding the possible role of presynaptic domain signaling came from analysis of the kinetics of transmitter release. The minimum synaptic delay between a presynaptic $\mathrm{Ca}^{2+}$ "tail" current (following a depolarization to a
A

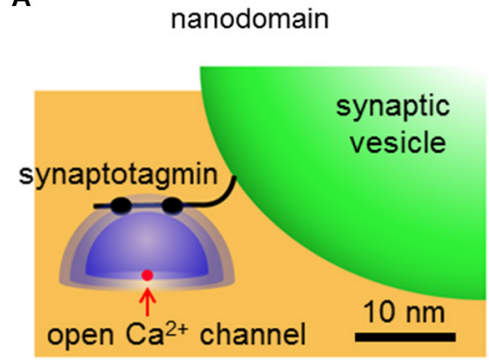

B

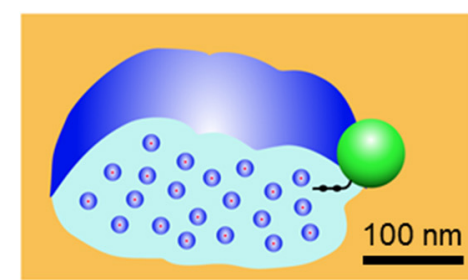

C

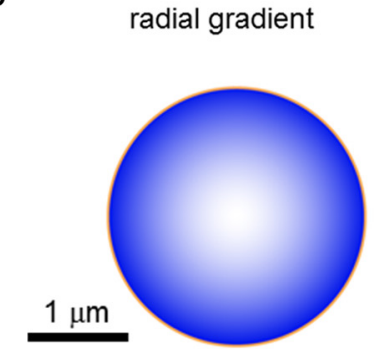

FIGURE 1 | Three types of local $\mathrm{Ca}^{2+}$ signaling. (A) Nanodomains arise from $\mathrm{Ca}^{2+}$ (blue) diffusing away from a single open $\mathrm{Ca}^{2+}$ channel (red) in the presynaptic plasma membrane (orange). The green spherical structure is a synaptic vesicle and a synaptotagmin-like $\mathrm{Ca}^{2+}$ sensor is shown protruding from the surface of the vesicle. (B) Microdomains arise from spatial overlap of local $\mathrm{Ca}^{2+}$ signals contributed by multiple open $\mathrm{Ca}^{2+}$ channels within a micrometer of each other. (C) Terminal-wide radial gradients of $\mathrm{Ca}^{2+}$ arise from long-distance diffusion of $\mathrm{Ca}^{2+}$ from channels at the plasma membrane. Derived from illustrations in Schweizer et al. (1995) and Augustine et al. (2003). 
very positive membrane potential) and the resultant postsynaptic response is very brief, on the order of hundreds of microseconds (Llinás et al., 1981; Augustine et al., 1985). Given the "rule of thumb" relationships for local $\mathrm{Ca}^{2+}$ signaling described above (Table 1), this indicates that $\mathrm{Ca}^{2+}$ must diffuse on the order of $100 \mathrm{~nm}$ or less before binding to the $\mathrm{Ca}^{2+}$ sensor for neurotransmitter release. This is consistent with a nanodomain type of presynaptic $\mathrm{Ca}^{2+}$ signal. Further, Simon and Llinás (1985) indicated that the relationship between presynaptic $\mathrm{Ca}^{2+}$ current and postsynaptic response could be explained by a model where domains do not overlap appreciably, i.e., nanodomain signaling.

While these initial observations provided indirect support for the role of nanodomain $\mathrm{Ca}^{2+}$ signals in triggering transmitter release at the squid giant synapse, they were open to alternative interpretations. However, several additional experiments have greatly bolstered acceptance of this notion. This experimental evidence can be grouped into three categories.

\section{MANIPULATIONS OF DOMAIN SIZE AND NUMBER}

Since the classic work of Dodge and Rahamimoff (1967), it has been known that $\mathrm{Ca}^{2+}$ acts "cooperatively", meaning that there is a high-order relationship between extracellular $\mathrm{Ca}^{2+}$ concentration and the amount of neurotransmitter release evoked by presynaptic action potentials. Cooperative triggering of transmitter release can be used as a tool to probe the spatial organization of presynaptic $\mathrm{Ca}^{2+}$ signaling.

Voltage clamp experiments at the squid giant synapse allowed the first direct measurement of $\mathrm{Ca}^{2+}$ entry into a presynaptic terminal and established that this cooperativity arises from a non-linear relationship between $\mathrm{Ca}^{2+}$ entry and the resultant release of neurotransmitters. Initially $\mathrm{Ca}^{2+}$ entry was varied by depolarizing the presynaptic membrane potential to different levels, thereby varying the number of open $\mathrm{Ca}^{2+}$ channels. While an initial account proposed a linear relationship between $\mathrm{Ca}^{2+}$ entry and transmitter release (Llinás et al., 1981), a follow-up study that optimized spatial control of presynaptic membrane potential indicated a third- or fourth-power relationship (Augustine et al., 1985). Because depolarizing to different membrane potentials changes the number of $\mathrm{Ca}^{2+}$ domains, as well as the $\mathrm{Ca}^{2+}$ concentration within each domain, these results are not easily interpreted in terms of local $\mathrm{Ca}^{2+}$ signals. A follow-up study by Augustine and Charlton (1986) circumvented this problem by depolarizing to a constant presynaptic membrane potential, to open a constant number of $\mathrm{Ca}^{2+}$ channels, while varying $\mathrm{Ca}^{2+}$ entry by changing external $\mathrm{Ca}^{2+}$ concentration. Under such conditions, an approximate fourthpower relationship between $\mathrm{Ca}^{2+}$ entry and transmitter release was observed (Figure 2A). Given that the number of $\mathrm{Ca}^{2+}$ domains will be constant under these conditions (Figure 2A insets), this result reveals that cooperativity occurs within the domains.

A strikingly different result is obtained when $\mathrm{Ca}^{2+}$ entry is varied by altering the duration of the presynaptic action potential. Blockade of potassium channels prolongs the duration of the presynaptic action potential and thereby increases $\mathrm{Ca}^{2+}$ entry into the terminal by increasing the number of
A Vary $\mathrm{Ca}^{2+}$ influx by varying external $\mathrm{Ca}^{2+}$

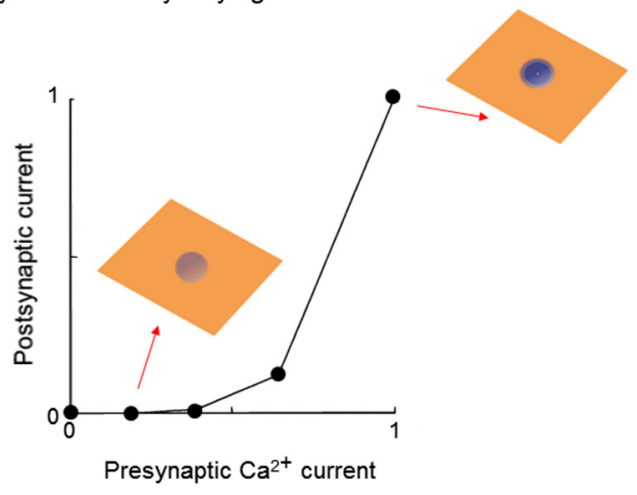

B

Vary $\mathrm{Ca}^{2+}$ influx by broadening action potential

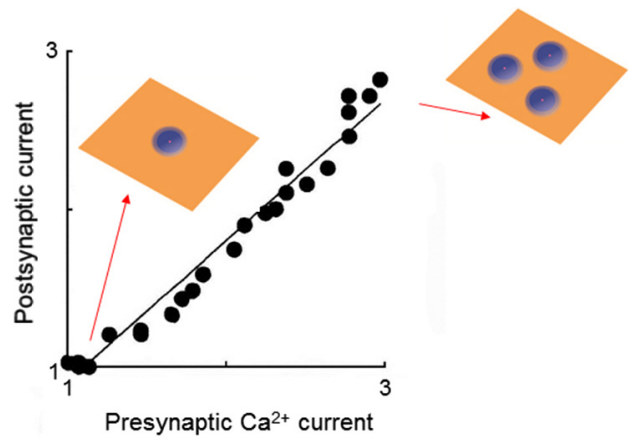

FIGURE 2 | Experimental strategies for probing local $\mathrm{Ca}^{2+}$ signaling during neurotransmitter release. (A) At a voltage-clamped squid giant synapse, varying external $\mathrm{Ca}^{2+}$ concentration changes presynaptic $\mathrm{Ca}^{2+}$ influx by keeping number of $\mathrm{Ca}^{2+}$ domains constant, while changing the amount of $\mathrm{Ca}^{2+}$ within each domain (insets). Under these conditions, the relationship between presynaptic $\mathrm{Ca}^{2+}$ current and amount of transmitter release, assessed by amplitude of the postsynaptic current, is a highly non-linear power function. Both presynaptic and postsynaptic responses are normalized to the maximum values measured at the highest external $\mathrm{Ca}^{2+}$ concentration. Data replotted from Augustine and Charlton (1986). (B) Pharmacologically broadening the presynaptic action potential at the squid giant synapse varies $\mathrm{Ca}^{2+}$ influx by opening additional $\mathrm{Ca}^{2+}$ channels, thereby increasing the number of $\mathrm{Ca}^{2+}$ domains (insets). Under these conditions, the relationship between presynaptic $\mathrm{Ca}^{2+}$ current and amount of transmitter release is a linear function. Both presynaptic and postsynaptic responses are normalized to the values measured under control conditions (narrowest presynaptic action potential). Data replotted from Augustine et al. (1991).

open $\mathrm{Ca}^{2+}$ channels (Klein and Kandel, 1980). Under such conditions, a linear relationship between action potential duration and postsynaptic response was observed (Augustine, 1990). Although $\mathrm{Ca}$ influx was not measured directly, the known gating properties of the squid presynaptic $\mathrm{Ca}^{2+}$ channels allowed calculation of the presynaptic $\mathrm{Ca}^{2+}$ current and indicated a linear relationship between action potential duration and $\mathrm{Ca}^{2+}$ current magnitude (Augustine, 1990). Thus, there is a linear relationship between $\mathrm{Ca}^{2+}$ current and transmitter release when $\mathrm{Ca}^{2+}$ influx is varied by changing the number of open $\mathrm{Ca}^{2+}$ channels (Figure 2B; Augustine et al., 1991). 


\section{A}

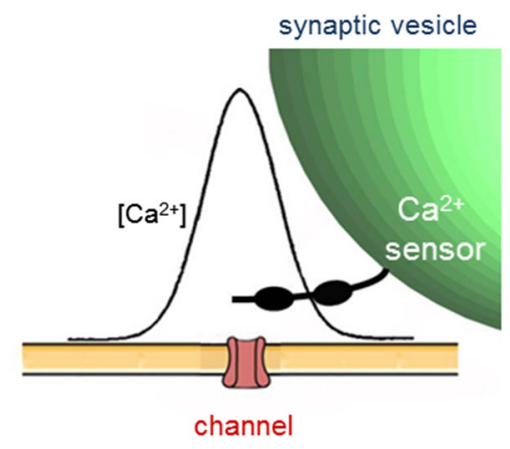

B

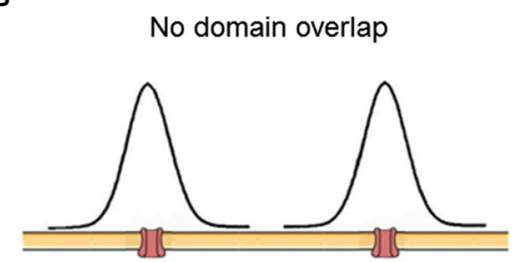

C

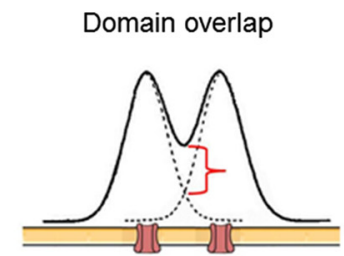

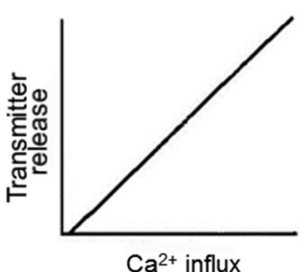

$\mathrm{Ca}^{2+}$ influx

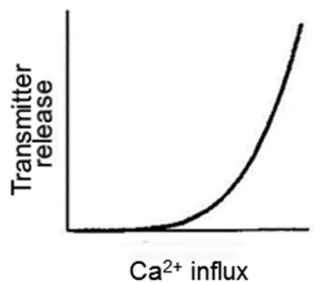

FIGURE 3 | Evaluating local $\mathrm{Ca}^{2+}$ signaling during broadening of presynaptic action potentials. (A) Diagram of spatial distribution of $\mathrm{Ca}^{2+}$ associated with a single open $\mathrm{Ca}^{2+}$ channel. (B) If open $\mathrm{Ca}^{2+}$ channels are sufficiently far apart, then $\mathrm{Ca}^{2+}$ signals associated with each channel will not overlap, creating a nanodomain arrangement (left). In this case, broader presynaptic action potentials will increase the number of discrete domains, leading to a proportional increase in transmitter release and a linear relationship between $\mathrm{Ca}^{2+}$ influx and release (right). (C) If open $\mathrm{Ca}^{2+}$ channels are close together, then $\mathrm{Ca}^{2+}$ signals associated with each channel will overlap, causing an increase in $\mathrm{Ca}^{2+}$ concentration (red bracket) within the local $\mathrm{Ca}^{2+}$ signal associated with each channel (left). This creates a microdomain $\mathrm{Ca}^{2+}$ signal. In this case, broader presynaptic action potentials will increase the $\mathrm{Ca}^{2+}$ concentration within each domain, leading to a supralinear increase in transmitter release due to the cooperative triggering of transmitter release by $\mathrm{Ca}^{2+}$. This spatial summation will yield a supralinear relationship between $\mathrm{Ca}^{2+}$ influx and release (right) when presynaptic action potentials are broadened. After Augustine et al. (1991).
Given that cooperativity occurs within a domain (Figure 2A), the fact that increasing the number of domains causes a linear increase in transmitter release (Figure 2B) leads to the conclusion that domains do not overlap during a presynaptic action potential. The logic behind this deduction is shown in Figure 3. In brief, if domains do not overlap then transmitter release will increase linearly according to the number of $\mathrm{Ca}^{2+}$ channels opened (Figures 3A,B). If domains overlap, then release will increase supralinearly due to summation of $\mathrm{Ca}^{2+}$ from neighboring domains, as well as the cooperative action of $\mathrm{Ca}^{2+}$ within a domain (Figure 3C). The results of Figure 2B are consistent with the expectations if domains do not overlap (Figure 3B, right). Thus, domains do not overlap during a presynaptic action potential at the squid giant synapse. Similar lines of experimentation have been used to probe the spatial dimensions of $\mathrm{Ca}^{2+}$ signaling in other presynaptic terminals (e.g., Wu and Saggau, 1994; Mintz et al., 1995).

\section{MEASUREMENTS OF LOCAL CALCIUM CONCENTRATION}

If the $\mathrm{Ca}^{2+}$ sensor for neurotransmitter release is located within nanometers of an open $\mathrm{Ca}^{2+}$ channel, as postulated by a nanodomain coupling scheme, then the local $\mathrm{Ca}^{2+}$ concentration experienced by this sensor should be in the range of tens to hundreds of micromolar (Table 1). Two types of experimental paradigms have tested this premise at the squid giant synapse.

The first experiment compared the ability of $\mathrm{Ca}^{2+}$ chelators of differing affinities to block transmitter release. For this purpose, analogs of the $\mathrm{Ca}^{2+}$ chelator 1,2-bis(o-aminophenoxy)ethane$\mathrm{N}, \mathrm{N}, \mathrm{N}$ 'N'-tetraacetic acid (BAPTA) were microinjected into the giant presynaptic terminal. A complex relationship between
$\mathrm{Ca}^{2+}$ affinity and chelator efficacy was observed: the most potent inhibitor of transmitter release was dibromoBAPTA, a chelator with intermediate $\mathrm{Ca}^{2+}$ affinity $(4.9 \mu \mathrm{M})$, while chelators with lower or higher affinities were less potent (Adler et al., 1991). A numerical model could predict such results only by assuming that the presynaptic $\mathrm{Ca}^{2+}$ sensor for transmitter release encounters $\mathrm{Ca}^{2+}$ levels on the order of $100 \mu \mathrm{M}$ or higher (Augustine et al., 1991). This prediction is consistent with the results of experiments employing photolysis of a caged $\mathrm{Ca}^{2+}$ chelator; even raising presynaptic $\mathrm{Ca}^{2+}$ concentration to $50 \mu \mathrm{M}$ produced rates of transmitter release lower than those evoked by a presynaptic action potential (Hsu et al., 1996). Together, these results are compatible with the predictions of a nanodomain coupling model.

The second experiment imaged presynaptic $\mathrm{Ca}^{2+}$ concentration during transmitter release. For this purpose, Llinás et al. (1992) used a version of the bioluminescent $\mathrm{Ca}^{2+}$-binding protein, aequorin, that was engineered to have an especially low affinity for $\mathrm{Ca}^{2+}$. This indicator protein, called n-Aequorin-J, only emits light at $\mathrm{Ca}^{2+}$ concentrations in the micromolar range or higher, with the amount of light emission increasing steeply as a function of $\mathrm{Ca}^{2+}$ concentration. Based on the amount of bioluminescence emitted by $n$-Aequorin-J injected into the squid giant presynaptic terminal, Llinás et al. (1992) concluded that presynaptic $\mathrm{Ca}^{2+}$ concentration reached 200-300 $\mu \mathrm{M}$ during transmitter release. Further, their measurements indicated that the regions of high $\mathrm{Ca}^{2+}$ concentration were quite localized, being a fraction of a square micrometer in area. While there are caveats regarding calibration of this indicator, as well as the spatial resolution of the $\mathrm{Ca}^{2+}$ imaging method, the results are compatible with the nanodomain coupling model. 
In sum, estimates provided by two complementary experimental approaches indicate that $\mathrm{Ca}^{2+}$ concentration rises on the order of hundreds of micromolar in the squid giant presynaptic terminal during transmitter release. Such results favor nanodomain coupling of $\mathrm{Ca}^{2+}$ channels to transmitter release at this synapse.

\section{DIFFERENTIAL EFFECTS OF CALCIUM CHELATORS}

The final experimental paradigm — and the one that has proven most useful for probing local $\mathrm{Ca}^{2+}$ signaling in presynaptic terminals - is based on comparison of $\mathrm{Ca}^{2+}$ chelators with different rates of $\mathrm{Ca}^{2+}$ binding (Adler et al., 1991). BAPTA binds $\mathrm{Ca}^{2+}$ very rapidly, with its on rate being diffusion-limited, while ethylene glycol tetraacetic acid (EGTA) binds $\mathrm{Ca}^{2+}$ more slowly because it is protonated at physiological $\mathrm{pH}$ and the bound proton must dissociate before $\mathrm{Ca}^{2+}$ can bind. This greatly slows the binding of $\mathrm{Ca}^{2+}$ to EGTA in comparison to BAPTA, even though the equilibrium affinity of these two chelators is roughly similar (Augustine et al., 1991). The slow binding of $\mathrm{Ca}^{2+}$ allows EGTA to serve as a high-pass temporal (and spatial) filter for $\mathrm{Ca}^{2+}$.

Neurotransmitter release evoked by presynaptic action potentials is completely inhibited when BAPTA is microinjected into the squid giant presynaptic terminal (Figure 4A). In contrast, neurotransmitter release is unaffected when EGTA is injected even at concentrations as high as $80 \mathrm{mM}$ (Figure 4B). Injection of EGTA is capable of buffering slower $\mathrm{Ca}^{2+}$ signaling processes, such as $\mathrm{Ca}^{2+}$ triggering of synaptic augmentation (Swandulla et al., 1991).

The interpretation of these results is that differences in the $\mathrm{Ca}^{2+}$ binding rates of these two chelators are responsible for their differential effects on neurotransmitter release. Thus, BAPTA can block transmitter release because it binds $\mathrm{Ca}^{2+}$ more rapidly than the $\mathrm{Ca}^{2+}$ sensor for transmitter release, while EGTA fails to block transmitter release because it binds $\mathrm{Ca}^{2+}$ slower than this sensor. Under conditions appropriate for the squid giant synapse, $80 \mathrm{mM}$ EGTA will bind $\mathrm{Ca}^{2+}$ with a time constant of approximately $50 \mu \mathrm{sec}$, while the same concentration of BAPTA requires only a fraction of a microsecond (Augustine et al., 1991). Thus, the $\mathrm{Ca}^{2+}$ sensor for transmitter release must bind $\mathrm{Ca}^{2+}$ in more than
$1 \mu \mathrm{sec}$ and in less than $50 \mu \mathrm{sec}$. Knowledge of this temporal constraint has been useful in establishing synaptotagmin as the $\mathrm{Ca}^{2+}$ sensor for transmitter release (Hui et al., 2005). In regard to the spatial dimensions of $\mathrm{Ca}^{2+}$ signaling during transmitter release, a time window of tens of microseconds means that $\mathrm{Ca}^{2+}$ diffuses no more than tens of nanometers prior to triggering transmitter release (Table 1). Thus, a process that is blocked by BAPTA but not by EGTA must be mediated by nanodomain $\mathrm{Ca}^{2+}$ signaling and this is the case for transmitter release at the squid giant synapse.

In summary, a variety of different experimental paradigms all point to the conclusion that transmitter release at the squid giant synapse is caused by nanodomain-style coupling of presynaptic $\mathrm{Ca}^{2+}$ channels to the presynaptic $\mathrm{Ca}^{2+}$ sensor, which presumably is synaptotagmin. In the next section we will see how these and other paradigms have been used to probe the spatial dimensions of presynaptic $\mathrm{Ca}^{2+}$ signaling at a different "giant" synapse, the mammalian calyx of Held.

\section{EVIDENCE FOR NANODOMAINS AT THE CALYX OF HELD SYNAPSE}

The giant calyx of Held synapse has been one of the most prominent preparations developed over the past two decades for analysis of synaptic transmission in the mammalian brain (Forsythe, 1994; Borst et al., 1995). Unfortunately, there is conflicting evidence about whether microdomain or nanodomain $\mathrm{Ca}^{2+}$ signaling mediates transmitter release at this synapse (Borst and Sakmann, 1996; Fedchyshyn and Wang, 2005). In this section we will survey this evidence and suggest that the conflict can be resolved by concluding that the spatial range of presynaptic $\mathrm{Ca}^{2+}$ signaling changes over the course of maturation of this synapse.

The calyx of Held synapse undergoes tremendous morphological remodeling in the course of its development, with its presynaptic terminal transforming from a spoon- or clublike structure with thin filopodia before the onset of hearing (P11-12) to a highly digitated structure with stalks and swellings containing a total of 500-800 active zones at maturity ( $>$ P16; Figure 5). Such structural transformations are

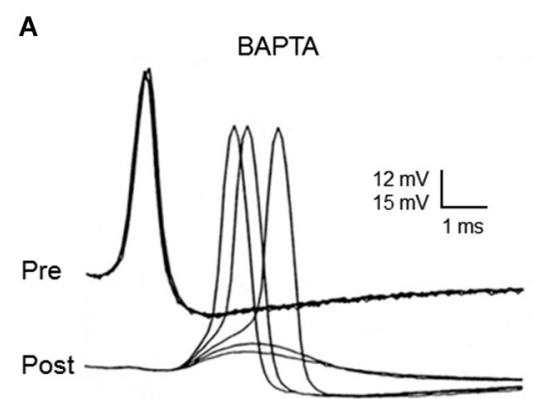

FIGURE 4 | Differential effects of EGTA and BAPTA on transmitter release at the squid giant synapse. While presynaptic microinjection of the rapid $\mathrm{Ca}^{2+}$ chelator, BAPTA, blocks transmitter release $(\mathbf{A})$, microinjection of the slower chelator, EGTA, has no effect. (B) Each family of traces shows five superimposed presynaptic action potentials
B

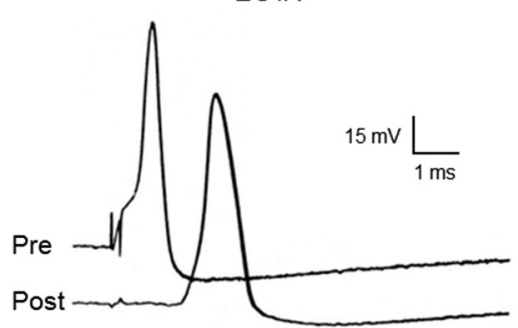

(Pre), as well as the postsynaptic responses (Post) that they evoke, during the course of chelator injection. While BAPTA reduces synaptic transmission to the point that postsynaptic responses are subthreshold, EGTA has no effect on postsynaptic response strength. Traces originally from Adler et al. (1991). 


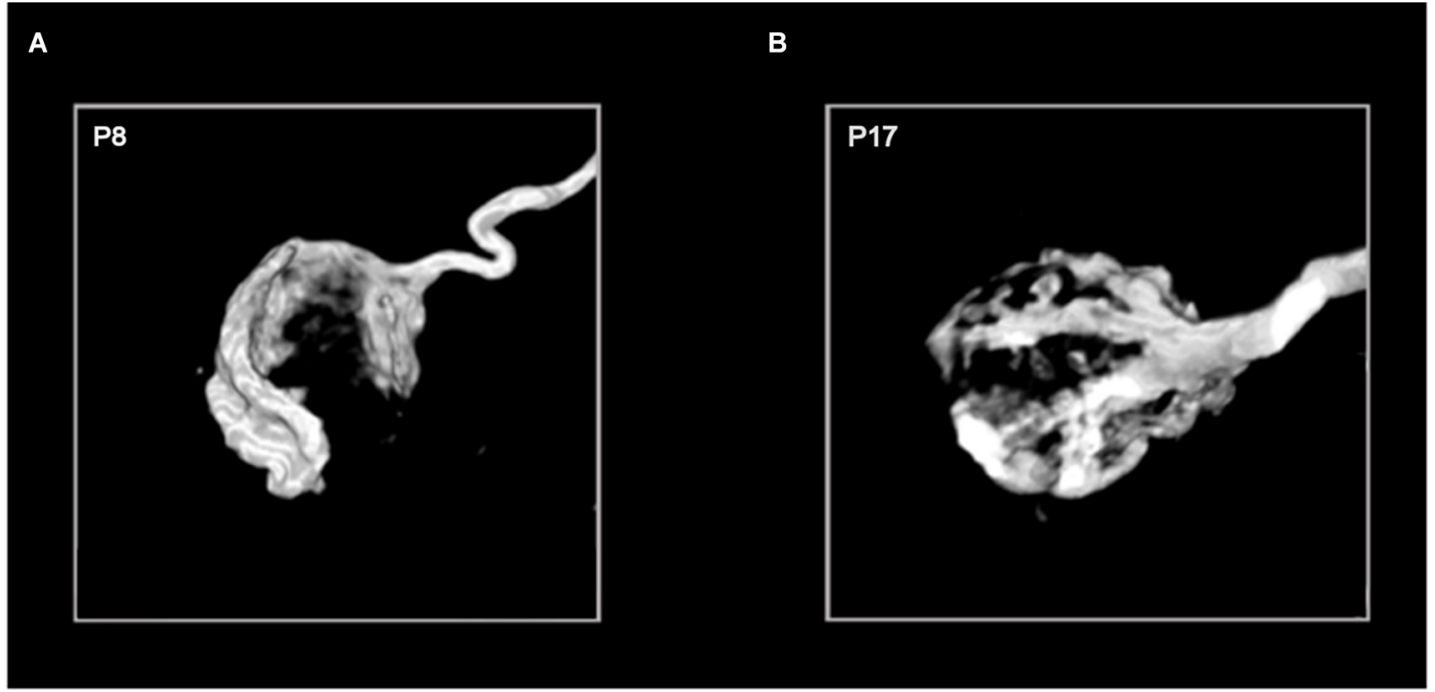

FIGURE 5 | Age-dependent morphological remodeling of the calyx of Held. Images of the 3-dimensional structure of P8 (A) and P17 (B) calyces filled with the fluorescent marker biotinylated dextran amine (courtesy of G. Grande). The diameter of each calyx sphere is approximately 15-20 micrometers.

thought to be important for supporting release and replenishment of synaptic vesicles (SVs), as well as facilitating clearance of neurotransmitter to alleviate postsynaptic receptor desensitization during high-frequency transmission (Trussell, 1999; von Gersdorff and Borst, 2002). In practice, calyces become less visible in brainstem slices for patch-clamping as myelination progresses during maturation, meaning that most early studies on the calyx of Held synapse focused on immature synapses (e.g., P8-11).

\section{EVIDENCE AGAINST NANODOMAIN COUPLING}

Using recordings of presynaptic $\mathrm{Ca}^{2+}$ currents $\left(I_{C a}\right)$ and excitatory postsynaptic currents $\left(I_{E P S C}\right)$ from young (P8-11) calyx of Held synapses, Borst and Sakmann (1996) first demonstrated that the amplitude of excitatory postsynaptic currents (EPSCs) follows the fourth power of presynaptic $\mathrm{Ca}^{2+}$ influx, similar to the squid giant synapse (Figure 2A). However, unlike the squid giant synapse, injection of EGTA at a concentration as low as $1 \mathrm{mM}$ attenuated EPSCs by about 50\%, leading to the conclusion that $\mathrm{Ca}^{2+}$ influx from many open channels (specifically $>60$ ) triggers fusion of a single synaptic vesicle. Furthermore, N-, $\mathrm{P} / \mathrm{Q}$, and R-type $\mathrm{Ca}^{2+}$ channels were found to jointly mediate transmitter release at this synapse, with $\mathrm{P} / \mathrm{Q}$-type $\mathrm{Ca}^{2+}$ channels being the most prominent subtype (Forsythe et al., 1998; Iwasaki and Takahashi, 1998; Wu et al., 1998, 1999). By photolysis of caged $\mathrm{Ca}^{2+}$ and computer simulations with $\mathrm{Ca}^{2+}$ buffer models (Bollmann et al., 2000; Schneggenburger and Neher, 2000), it was estimated that the peak $\mathrm{Ca}^{2+}$ concentration at $\mathrm{Ca}^{2+}$ sensors on SV is as low as $10 \mu \mathrm{M}$, an order of magnitude lower than that deduced for the squid giant synapse. These results are in striking contrast to what would be expected for nanodomain coupling, arguing in favor of a microdomain mode of $\mathrm{Ca}^{2+}$ signaling at the immature calyx of Held synapse (Meinrenken et al., 2002).

\section{EVIDENCE FOR NANODOMAIN COUPLING}

In parallel with morphological remodeling at the developing calyx of Held, presynaptic action potentials undergo a dramatic shortening in their duration (Taschenberger and von Gersdorff, 2000), which would reduce the number of activated $\mathrm{Ca}^{2+}$ channels and thereby attenuate $\mathrm{Ca}^{2+}$ influx. Given that the relationship between $I_{C a}$ and $I_{E P S C}$ is a power function

$$
I_{E P S C} \propto\left[I_{C a}\right]^{n}
$$

where $n$ denotes $\mathrm{Ca}^{2+}$ cooperativity (typically a value of 3-5; Augustine et al., 1985), spike narrowing would lead to a significant reduction in $I_{E P S C}$. In fact, quantal output is increased in older mice (Joshi and Wang, 2002) or maintained in rats (Iwasaki and Takahashi, 2001). This created a paradox: how could the fewer $\mathrm{Ca}^{2+}$ channels recruited by narrower action potentials during development somehow become more effective in triggering transmitter release (Yang and Wang, 2006; Kochubey et al., 2009; Leão and von Gersdorff, 2009)?

Two possible mechanisms have been considered: (1) the spatial coupling between open $\mathrm{Ca}^{2+}$ channels and $\mathrm{Ca}^{2+}$ sensors tightens to yield nanodomain signaling; and/or (2) the $\mathrm{Ca}^{2+}$ sensor (e.g., synaptotagmin) becomes more sensitive to $\mathrm{Ca}^{2+}$ to detect smaller rises in presynaptic $\mathrm{Ca}^{2+}$ concentration. To test the first possibility, Fedchyshyn and Wang (2005) injected EGTA (10 $\mathrm{mM}$ ) into developing calyx of Held synapses and found that while this slow $\mathrm{Ca}^{2+}$ buffer potently attenuates transmitter release in immature terminals ( $\mathrm{P} 8-12$ mice), as previously reported (Borst and Sakmann, 1996), EGTA had surprisingly little effect at mature synapses (P16-18). This indicates that the distance between $\mathrm{Ca}^{2+}$ channels and $\mathrm{Ca}^{2+}$ sensors shortens over the course of development. A similar conclusion was reached by using capacitance measurements of exocytosis under different $\mathrm{Ca}^{2+}$ buffering conditions (Leão and von Gersdorff, 2009). By using a depolarizing paradigm that recruits an increasing number 
of $\mathrm{Ca}^{2+}$ channels without changing the kinetics of $I_{\mathrm{Ca}}$ or the driving force for $\mathrm{Ca}^{2+}$, Fedchyshyn and Wang (2005) further demonstrated that $\mathrm{Ca}^{2+}$ cooperativity values ( $n$ ) were significantly higher in immature synapses than in mature synapses. Based on the logic depicted in Figure 3, this suggests that the number of $\mathrm{Ca}^{2+}$ channels required for triggering release of a synaptic vesicle decreases. It has also been shown that while transmitter release at $\mathrm{P} 8-12$ synapses requires both $\mathrm{N}$ and $\mathrm{P} / \mathrm{Q}$-type $\mathrm{Ca}^{2+}$ channels, only $\mathrm{P} / \mathrm{Q}$-type $\mathrm{Ca}^{2+}$ channels are involved at mature synapses. This suggests that nanodomains contain specific type of $\mathrm{Ca}^{2+}$ channels as part of their molecular architecture.

To test the second possibility, $\mathrm{Ca}^{2+}$ sensor sensitivity was measured in the developing calyx of Held synapse via simultaneous $\mathrm{Ca}^{2+}$ imaging and flash photolysis of caged $\mathrm{Ca}^{2+}$ chelators (Wang et al., 2008; Kochubey et al., 2009). Flash photolysis of caged $\mathrm{Ca}^{2+}$ has the advantage of bypassing $\mathrm{Ca}^{2+}$ entry through $\mathrm{Ca}^{2+}$ channels, instead directly triggering release by increasing intracellular $\mathrm{Ca}^{2+}$ concentration $\left(\left[\mathrm{Ca}^{2+}\right]_{i}\right)$ in a spatially homogeneous manner. Surprisingly, $\mathrm{Ca}^{2+}$ sensor sensitivity decreases over development (e.g., $K_{D}$ values rise from $\sim 80 \mu \mathrm{M}$ for P9-P11 vs. $\sim 120 \mu \mathrm{M}$ for P16-P19 synapses) while cooperativity ( $n$ ) remains the same. Taken together, these studies led to the conclusion that the spatial coupling between $\mathrm{Ca}^{2+}$ channels and $\mathrm{Ca}^{2+}$ sensors switches from microdomain to nanodomain signaling.
Such changes can account for the upregulation of transmitter release over the course of development of the calyx of Held synapse. Computer simulations with a linearized buffered $\mathrm{Ca}^{2+}$ diffusion model and a five-site kinetic model of transmitter release (Naraghi and Neher, 1997; Schneggenburger and Neher, 2000) deduced that during development the peak $\mathrm{Ca}^{2+}$ concentration seen by a $\mathrm{Ca}^{2+}$ sensor during an action potential increases from 35 to $56 \mu \mathrm{M}$ (Figures 6A,B). This is a consequence of tightened spatial coupling between $\mathrm{Ca}^{2+}$ channels and sensors; the modeling results indicate that the microdomain signaling during an action potential in immature synapses results from 12 $\mathrm{Ca}^{2+}$ channels with $50 \%$ open probability and $61 \mathrm{~nm}$ separation, while the nanodomain coupling at mature synapses results from $9 \mathrm{Ca}^{2+}$ channels with $35 \%$ open probability and $23 \mathrm{~nm}$ spacing (Figure 6C; Wang et al., 2008, 2009). This change in the spatial dimensions of presynaptic $\mathrm{Ca}^{2+}$ signaling nearly doubles the release rate and enables the peak of the local $\left[\mathrm{Ca}^{2+}\right]_{\mathrm{i}}$ transient to occur $\sim 410 \mu$ s earlier in mature synapses than in immature synapses (Figures 6A,B). Although direct evidence for the topographic organization of $\mathrm{Ca}^{2+}$ channels at the developing calyx remains elusive, these simulation results provide a theoretical framework for envisaging how a developmental transition from microdomain to nanodomain coupling can recapitulate a number of experimental observations, including an explanation for how narrower action potentials become more effective in
A

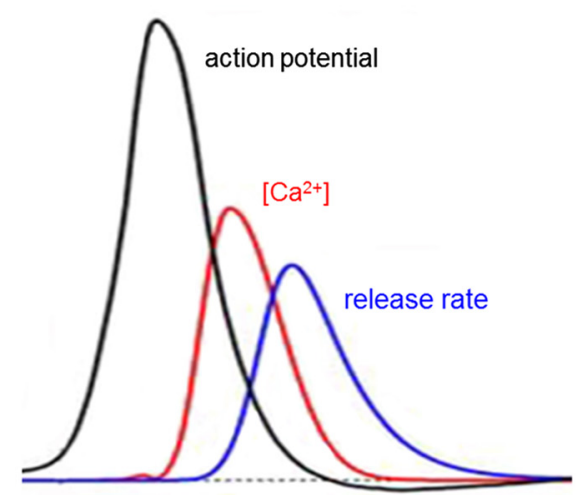

C

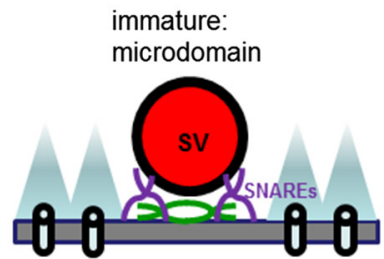

FIGURE 6 | Developmental change in presynaptic $\mathrm{Ca}^{2+}$ signaling at the calyx of Held synapse. (A,B) Developmental tightening of coupling between $\mathrm{Ca}^{2+}$ channels and synaptic vesicles boosts the local $\left[\mathrm{Ca}^{2+}\right]_{i}$ transient seen by the $\mathrm{Ca}^{2+}$ sensor (red traces) and the rate of transmitter release (blue traces) as action potentials (black traces) narrow (from Wang et al., 2008). (C) Transformation from microdomain to nanodomain coupling of $\mathrm{Ca}^{2+}$ channels
B

P16-19
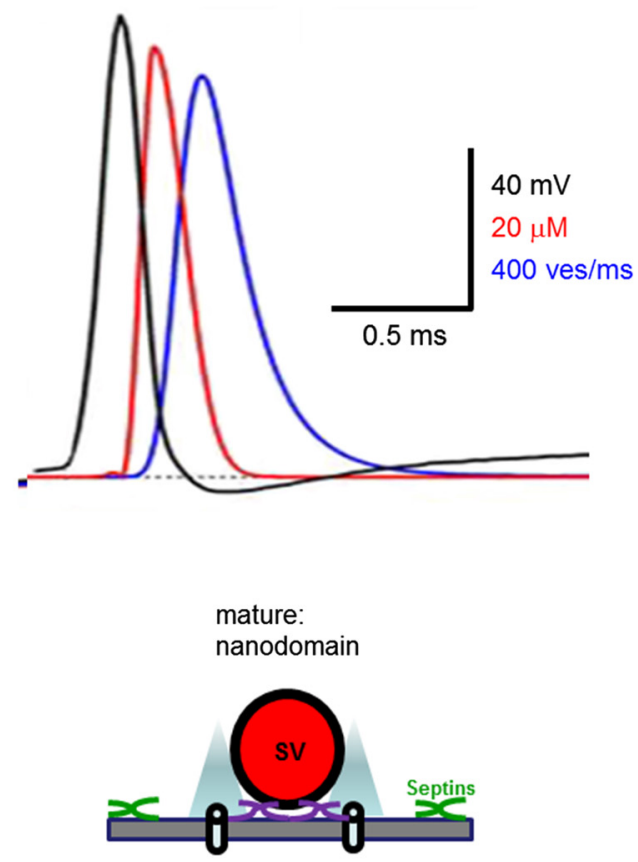

to transmitter release. Note that nanodomains are associated with shorter distance between $\mathrm{Ca}^{2+}$ channels and synaptic vesicles (SV) both in the plane of the presynaptic plasma membrane, as well as orthogonal to this membrane. This spatial reorganization of $\mathrm{Ca}^{2+}$ signaling is associated with translocation of septins (green) away from the active zone during development. 
triggering transmitter release (Taschenberger and von Gersdorff, 2000).

\section{EVIDENCE FOR NANODOMAIN COUPLING AT OTHER SYNAPSES}

The squid giant synapse and calyx of Held synapse are often considered to be specialized synapses because of their extraordinary size, speed and strength of synaptic transmission. Hence nanodomain coupling could be a feature unique to such highfidelity, excitatory "relay" synapses. However, this clearly is not the case because a number of synapses have now been shown to release neurotransmitters via nanodomain coupling.

In line with experiments suggesting nanodomain coupling of $\mathrm{Ca}^{2+}$ influx to transmitter release at the squid giant synapse and calyx of Held, early studies indicated that $\mathrm{Ca}^{2+}$ channels are tightly coupled to $\mathrm{Ca}^{2+}$ sensors to enable highly efficient transmission at neuromuscular synapses (Yoshikami et al., 1989; Delaney et al., 1991). In some cases, opening of a single $\mathrm{Ca}^{2+}$ channel is sufficient for triggering of fusion events at the active zones of chick ciliary ganglion synapses (Stanley, 1993; Gentile and Stanley, 2005).

Initial studies in mammalian synapses appeared to argue in favor of microdomain coupling (Luebke et al., 1993; Takahashi and Momiyama, 1993; Wheeler et al., 1994; Wu and Saggau, 1994; Mintz et al., 1995). These studies combined $\mathrm{Ca}^{2+}$ imaging in presynaptic terminals with patch-clamp recordings from postsynaptic neurons and treatment with subtype-specific $\mathrm{Ca}^{2+}$ channel blockers. The results collectively showed that not only are multiple $\mathrm{Ca}^{2+}$ channels involved in presynaptic $\mathrm{Ca}^{2+}$ signaling but also that different subtypes of $\mathrm{Ca}^{2+}$ channels can mediate transmitter release at a synapse, leading to the idea that opening of many adjacent $\mathrm{Ca}^{2+}$ channels forms microdomains.

It should be noted that the conditions employed in these different experiments differ in many ways. For example, the fraction of $\mathrm{Ca}^{2+}$ channels activated by an action potential differ, ranging from $\sim 10 \%$ at the squid giant synapse to $>70 \%$ at some mammalian central synapses. In addition, extracellular $\mathrm{Ca}^{2+}$ concentrations ranged from $11 \mathrm{mM}$ for the squid synapse to $1-2 \mathrm{mM}$ for mammalian central synapses and experimental temperatures varied from 15 to $37^{\circ} \mathrm{C}$. All of these variables profoundly influence the number of $\mathrm{Ca}^{2+}$ channels opened by an action potential, as well as the driving force on $\mathrm{Ca}^{2+}$ and the kinetics of $\mathrm{Ca}^{2+}$ diffusion within nerve terminals, thereby affecting the coupling of $\mathrm{Ca}^{2+}$ channels to transmitter release.

Under experimental conditions where these variables have been somewhat controlled and are comparable to conditions employed at the developing calyx of Held synapse, emerging evidence suggests that several conventional mammalian central synapses employ nanodomain $\mathrm{Ca}^{2+}$ signaling (Eggermann et al., 2011). For example, nanodomain $\mathrm{Ca}^{2+}$ signaling is found both at hippocampal basket cell-granule cell inhibitory synapses and at cerebellar parallel fiber-Purkinje cell excitatory synapses (Bucurenciu et al., 2008; Eggermann and Jonas, 2011; Schmidt et al., 2013). In addition, transmission at sensory synapses other than the calyx of Held apparently employ nanodomain coupling (Brandt et al., 2005; Jarsky et al., 2010). Together, results from a wide variety of mammalian synapses support the notion that nanodomain coupling is highly conserved and is therefore a general scheme for $\mathrm{Ca}^{2+}$ triggering of rapid neurotransmitter release.

\section{CONCLUSIONS}

We have highlighted key evidence from two giant synapses, the squid giant synapse and the calyx of Held synapse, that firmly establish nanodomains as the predominant mode of $\mathrm{Ca}^{2+}$ signaling that couples $\mathrm{Ca}^{2+}$ influx through presynaptic $\mathrm{Ca}^{2+}$ channels to vesicular fusion and synaptic transmission. There is no doubt that nanodomains exist at many synapses, but it is interesting to note that this mode of $\mathrm{Ca}^{2+}$ signaling appears to be primarily associated with synapses for fast-spiking neurons, where synaptic transmission is particularly important for preserving temporal precision of information flow (Yang et al., 2014). Nanodomains with tight $\mathrm{Ca}^{2+}$ signaling may be particularly suited to trigger highly synchronized fusion events with minimal synaptic delay, temporal jitter, or asynchronous transmitter release. The narrow action potentials typical of fast-spiking neurons are likely to produce brief $\mathrm{Ca}^{2+}$ signals with rapid rise and collapse of local $\left[\mathrm{Ca}^{2+}\right]$. Brief $\mathrm{Ca}^{2+}$ transients will also serve to prevent presynaptic $\mathrm{Ca}^{2+}$ overload and facilitate $\mathrm{Ca}^{2+}$ clearance during prolonged high-frequency transmission. On the other hand, microdomains with loose coupling between $\mathrm{Ca}^{2+}$ channels and $\mathrm{Ca}^{2+}$ sensors may allow a broader dynamic range for tuning quantal output at synapses where activity-dependent forms of synaptic plasticity play a key role in dynamically regulating synaptic efficacy (Vyleta and Jonas, 2014).

Given the dimensions of SV and active zones, it is reasonable to postulate that the nanodomain $\mathrm{Ca}^{2+}$ signaling requires specific molecular specializations to optimize physical interactions between $\mathrm{Ca}^{2+}$ channels and SV (Catterall and Few, 2008; Südhof, 2013). To advance our understanding of local $\mathrm{Ca}^{2+}$ signaling in presynaptic terminals, it will next be important to define the specific molecular specializations underlying both microdomain and nanodomain $\mathrm{Ca}^{2+}$ signaling, and how these two modes of $\mathrm{Ca}^{2+}$ signaling are regulated during development. Emerging evidence indicate that several presynaptic proteins help tether $\mathrm{Ca}^{2+}$ channels to SV; these proteins include RIM (Kiyonaka et al., 2007; Kaeser et al., 2011), Munc 13 (Chen et al., 2013), bassoon (Davydova et al., 2014) and even synaptotagmin (Young and Neher, 2009). Genetic deletion of the cytometrix protein Bruchpilot loosens the coupling of $\mathrm{Ca}^{2+}$ channels and sensors at Drosophila presynaptic terminals (Kittel et al., 2006). In contrast, knockout of septin 5, a filamentous protein that interacts with the SNARE proteins that mediate exocytosis, transforms microdomain coupling typical of immature synapses to nanodomain coupling with functional and morphological phenotypes nearly identical to mature calyces (Figure 6C; Yang et al., 2010). These results suggest that these two modes of local $\mathrm{Ca}^{2+}$ signaling are differentiated by specific molecular substrates and potentially could be inter-convertible to confer distinctive $\mathrm{Ca}^{2+}$ signaling properties to synapses.

\section{ACKNOWLEDGMENTS}

We thank Karen Chung for her help in preparing this paper. This work was supported by a CRP grant from the National Research 
Foundation of Singapore and by the World Class Institute (WCI) Program of the National Research Foundation of Korea (NRF) funded by the Ministry of Education, Science and Technology of Korea (MEST) (NRF Grant Number: WCI 2009-003) (to George J. Augustine), and by Operating Grants from the Canadian Institutes of Health Research (MOP-77610, MOP-81159, MOP-14692, VIH-105441) and Canada Research Chair (to Lu-Yang Wang).

\section{REFERENCES}

Adler, E. M., Augustine, G. J., Duffy, S. N., and Charlton, M. P. (1991). Alien intracellular calcium chelators attenuate neurotransmitter release at the squid giant synapse. J. Neurosci. 11, 1496-1507.

Augustine, G. J. (1990). Regulation of transmitter release at the squid giant synapse by presynaptic delayed rectifier potassium current. J. Physiol. 431, 343-364. doi: 10.1113/jphysiol.1990.sp018333

Augustine, G. J., Adler, E. M., and Charlton, M. P. (1991). The calcium signal for transmitter secretion from presynaptic nerve terminals. Ann. N Y Acad. Sci. 635, 365-381. doi: 10.1111/j.1749-6632.1991.tb36505.x

Augustine, G. J., and Charlton, M. P. (1986). Calcium dependence of presynaptic calcium current and post-synaptic response at the squid giant synapse. J. Physiol. 381, 619-640.

Augustine, G. J., Charlton, M. P., and Smith, S. J. (1985). Calcium entry and transmitter release at voltage-clamped nerve terminals of squid. J. Physiol. 367, $163-181$.

Augustine, G. J., Santamaria, F., and Tanaka, K. (2003). Local calcium signaling in neurons. Neuron 40, 331-346. doi: 10.1016/s0896-6273(03)00639-1

Bollmann, J. H., Sakmann, B., and Borst, J. G. (2000). Calcium sensitivity of glutamate release in a calyx-type terminal. Science 289, 953-957. doi: 10.1126/science. 289.5481 .953

Borst, J. G., Helmchen, F., and Sakmann, B. (1995). Pre- and postsynaptic wholecell recordings in the medial nucleus of the trapezoid body of the rat. J. Physiol. 489, 825-840. doi: 10.1113/jphysiol.1995.sp021095

Borst, J. G., and Sakmann, B. (1996). Calcium influx and transmitter release in a fast CNS synapse. Nature 383, 431-434. doi: 10.1038/383431a0

Brandt, A., Khimich, D., and Moser, T. (2005). Few CaV1.3 channels regulate the exocytosis of a synaptic vesicle at the hair cell ribbon synapse. J. Neurosci. 25, 11577-11585. doi: 10.1523/jneurosci.3411-05.2005

Bucurenciu, I., Kulik, A., Schwaller, B., Frotscher, M., and Jonas, P. (2008). Nanodomain coupling between $\mathrm{Ca}^{2+}$ channels and $\mathrm{Ca}^{2+}$ sensors promotes fast and efficient transmitter release at a cortical GABAergic synapse. Neuron 57, 536545. doi: 10.1016/j.neuron.2007.12.026

Catterall, W. A., and Few, A. P. (2008). Calcium channel regulation and presynaptic plasticity. Neuron 59, 882-901. doi: 10.1016/j.neuron.2008.09.005

Chad, J. E., and Eckert, R. (1984). Calcium domains associated with individual channels can account for anomalous voltage relations of Ca-dependent responses. Biophys. J. 45, 993-999. doi: 10.1016/s0006-3495(84)84244-7

Chen, Z., Cooper, B., Kalla, S., Varoqueaux, F., and Young, S. M. (2013). The Munc13 proteins differentially regulate readily releasable pool dynamics and calcium-dependent recovery at a central synapse. J. Neurosci. 33, 8336-8351. doi: 10.1523/jneurosci.5128-12.2013

Davydova, D., Marini, C., King, C., Klueva, J., Bischof, F., Romorini, S., et al. (2014). Bassoon specifically controls presynaptic P/Q-type $\mathrm{Ca}^{2+}$ channels via RIM-binding protein. Neuron 82, 181-194. doi: 10.1016/j.neuron.2014.02.012

Delaney, K., Tank, D. W., and Zucker, R. S. (1991). Presynaptic calcium and serotonin-mediated enhancement of transmitter release at crayfish neuromuscular junction. J. Neurosci. 11, 2631-2643.

Dodge, F. A. Jr., and Rahamimoff, R. (1967). Co-operative action a calcium ions in transmitter release at the neuromuscular junction. J. Physiol. 193, 419-432.

Eggermann, E., Bucurenciu, I., Goswami, S. P., and Jonas, P. (2011). Nanodomain coupling between $\mathrm{Ca}^{2+}$ channels and sensors of exocytosis at fast mammalian synapses. Nat. Rev. Neurosci. 13, 7-21. doi: 10.1038/nrn3125

Eggermann, E., and Jonas, P. (2011). How the 'slow' $\mathrm{Ca}^{2+}$ buffer parvalbumin affects transmitter release in nanodomain-coupling regimes. Nat. Neurosci. 15, 20-22. doi: 10.1038/nn.3002

Fedchyshyn, M. J., and Wang, L. Y. (2005). Developmental transformation of the release modality at the calyx of Held synapse. J. Neurosci. 25, 4131-4140. doi: 10. 1523/jneurosci.0350-05.2005
Forsythe, I. D. (1994). Direct patch recording from identified presynaptic terminals mediating glutamatergic EPSCs in the rat CNS, in vitro. J. Physiol. 479, 381-387. doi: 10.1113/jphysiol.1994.sp020303

Forsythe, I. D., Tsujimoto, T., Barnes-Davies, M., Cuttle, M. F., and Takahashi, T. (1998). Inactivation of presynaptic calcium current contributes to synaptic depression at a fast central synapse. Neuron 20, 797-807. doi: 10.1016/s08966273(00)81017-x

Gentile, L., and Stanley, E. F. (2005). A unified model of presynaptic release site gating by calcium channel domains. Eur. J. Neurosci. 21, 278-282. doi: 10.1111/j. 1460-9568.2004.03841.x

Hsu, S. F., Augustine, G. J., and Jackson, M. B. (1996). Adaptation of $\mathrm{Ca}^{2+}$-triggered exocytosis in presynaptic terminals. Neuron 17, 501-512. doi: 10.1016/s08966273(00)80182-8

Hui, E., Bai, J., Wang, P., Sugimori, M., Llinas, R. R., and Chapman, E. R. (2005). Three distinct kinetic groupings of the synaptotagmin family: candidate sensors for rapid and delayed exocytosis. Proc. Natl. Acad. Sci. U S A 102, 5210-5214. doi: 10.1073/pnas.0500941102

Iwasaki, S., and Takahashi, T. (1998). Developmental changes in calcium channel types mediating synaptic transmission in rat auditory brainstem. J. Physiol. 509, 419-423. doi: 10.1111/j.1469-7793.1998.419bn.x

Iwasaki, S., and Takahashi, T. (2001). Developmental regulation of transmitter release at the calyx of Held in rat auditory brainstem. J. Physiol. 534, 861-871. doi: 10.1111/j.1469-7793.2001.00861.x

Jarsky, T., Tian, M., and Singer, J. H. (2010). Nanodomain control of exocytosis is responsible for the signaling capability of a retinal ribbon synapse. J. Neurosci. 30, 11885-11895. doi: 10.1523/jneurosci.1415-10.2010

Joshi, I., and Wang, L. Y. (2002). Developmental profiles of glutamate receptors and synaptic transmission at a single synapse in the mouse auditory brainstem. J. Physiol. 540, 861-873. doi: 10.1111/j.1469-7793.2002.00861.x

Kaeser, P. S., Deng, L., Wang, Y., Dulubova, I., Liu, X., Rizo, J., et al. (2011). RIM proteins tether $\mathrm{Ca}^{2+}$ channels to presynaptic active zones via a direct PDZdomain interaction. Cell 144, 282-295. doi: 10.1016/j.cell.2010.12.029

Kasai, H. (1993). Cytosolic $\mathrm{Ca}^{2+}$ gradients, $\mathrm{Ca}^{2+}$ binding proteins and synaptic plasticity. Neurosci. Res. 16, 1-7. doi: 10.1159/000154621

Kittel, R. J., Wichmann, C., Rasse, T. M., Fouquet, W., Schmidt, M., Schmid, A., et al. (2006). Bruchpilot promotes active zone assembly, $\mathrm{Ca}^{2+}$ channel clustering and vesicle release. Science 312, 1051-1054. doi: 10.1126/science.11 26308

Kiyonaka, S., Wakamori, M., Miki, T., Uriu, Y., Nonaka, M., Bito, H., et al. (2007). RIM1 confers sustained activity and neurotransmitter vesicle anchoring to presynaptic $\mathrm{Ca}^{2+}$ channels. Nat. Neurosci. 10, 691-701. doi: 10.1038/ nn 1904

Klein, M., and Kandel, E. R. (1980). Mechanism of calcium current modulation underlying presynaptic facilitation and behavioral sensitization in Aplysia. Proc. Natl. Acad. Sci. U S A 77, 6912-6916. doi: 10.1073/pnas.77.11. 6912

Kochubey, O., Han, Y., and Schneggenburger, R. (2009). Developmental regulation of the intracellular $\mathrm{Ca}^{2+}$ sensitivity of vesicle fusion and $\mathrm{Ca}^{2+}$-secretion coupling at the rat calyx of Held. J. Physiol. 587, 3009-3023. doi: 10.1113/jphysiol. 2009.172387

Leão, R. M., and von Gersdorff, H. (2009). Synaptic vesicle pool size, release probability and synaptic depression are sensitive to $\mathrm{Ca}^{2+}$ buffering capacity in the developing rat calyx of Held. Braz. J. Med. Biol. Res. 42, 94-104. doi: 10. 1590/s0100-879x2009000100014

Llinás, R., Steinberg, I. Z., and Walton, K. (1981). Relationship between presynaptic calcium current and postsynaptic potential in squid giant synapse. Biophys. J. 33, 323-351. doi: 10.1016/s0006-3495(81)84899-0

Llinás, R., Sugimori, M., and Silver, R. B. (1992). Microdomains of high calcium concentration in a presynaptic terminal. Science 256, 677-679. doi: 10. 1126/science. 1350109

Luebke, J. I., Dunlap, K., and Turner, T. J. (1993). Multiple calcium channel types control glutamatergic synaptic transmission in the hippocampus. Neuron 11 , 895-902. doi: 10.1016/0896-6273(93)90119-c

Meinrenken, C. J., Borst, J. G., and Sakmann, B. (2002). Calcium secretion coupling at calyx of held governed by nonuniform channel-vesicle topography. $J$. Neurosci. 22, 1648-1667.

Mintz, I. M., Sabatini, B. L., and Regehr, W. G. (1995). Calcium control of transmitter release at a cerebellar synapse. Neuron 15, 675-688. doi: 10.1016/08966273(95)90155-8 
Naraghi, M., and Neher, E. (1997). Linearized buffered $\mathrm{Ca}^{2+}$ diffusion in microdomains and its implications for calculation of $\left[\mathrm{Ca}^{2+}\right]$ at the mouth of a calcium channel. J. Neurosci. 15, 6961-6973.

Neher, E. (1998). Usefulness and limitations of linear approximations to the understanding of $\mathrm{Ca}^{2+}$ signals. Cell Calcium 24, 345-357. doi: 10.1016/s01434160(98)90058-6

Schmidt, H., Brachtendorf, S., Arendt, O., Hallermann, S., Ishiyama, S., Bornschein, G., et al. (2013). Nanodomain coupling at an excitatory cortical synapse. Curr. Biol. 23, 244-249. doi: 10.1016/j.cub.2012.12.007

Schneggenburger, R., and Neher, E. (2000). Intracellular calcium dependence of transmitter release rates at a fast central synapse. Nature 406, 889-893. doi: 10. $1038 / 35022702$

Schweizer, F. E., Betz, H., and Augustine, G. J. (1995). From vesicle docking to endocytosis: intermediate reactions of exocytosis. Neuron 14, 689-696. doi: 10. 1016/0896-6273(95)90213-9

Simon, S. M., and Llinás, R. R. (1985). Compartmentalization of the submembrane calcium activity during calcium influx and its significance in transmitter release. Biophys. J. 48, 485-498. doi: 10.1016/s0006-3495(85)83804-2

Smith, S. J., and Augustine, G. J. (1988). Calcium ions, active zones and synaptic transmitter release. Trends Neurosci. 11, 458-464. doi: 10.1016/01662236(88)90199-3

Stanley, E. F. (1993). Single calcium channels and acetylcholine release at a presynaptic nerve terminal. Neuron 11, 1007-1011. doi: 10.1016/08966273(93)90214-c

Südhof, T. C. (2013). Neurotransmitter release: the last millisecond in the life of a synaptic vesicle. Neuron 80, 675-690. doi: 10.1016/j.neuron.2013.10.022

Swandulla, D., Hans, M., Zipser, K., and Augustine, G. J. (1991). Role of residual calcium in synaptic depression and posttetanic potentiation: fast and slow calcium signaling in nerve terminals. Neuron 7, 915-926. doi: 10.1016/08966273(91)90337-y

Takahashi, T., and Momiyama, A. (1993). Different types of calcium channels mediate central synaptic transmission. Nature 366, 156-158. doi: 10.1038/36 $6156 \mathrm{a} 0$

Taschenberger, H., and von Gersdorff, H. (2000). Fine-tuning an auditory synapse for speed and fidelity: developmental changes in presynaptic waveform, EPSC kinetics and synaptic plasticity. J. Neurosci. 20, 9162-9173.

Trussell, L. O. (1999). Synaptic mechanisms for coding timing in auditory neurons. Annu. Rev. Physiol. 61, 477-496. doi: 10.1146/annurev.physiol.61.1.477

von Gersdorff, H., and Borst, J. G. (2002). Short-term plasticity at the calyx of held. Nat. Rev. Neurosci. 3, 53-64. doi: 10.1038/nrn705

Vyleta, N. P., and Jonas, P. (2014). Loose coupling between $\mathrm{Ca}^{2+}$ channels and release sensors at a plastic hippocampal synapse. Science 343, 665-670. doi: 10. 1126/science.1244811

Wang, L. Y., Fedchyshyn, M. J., and Yang, Y. M. (2009). Action potential evoked transmitter release in central synapses: insights from the developing calyx of Held. Mol. Brain 2:36. doi: 10.1186/1756-6606-2-36

Wang, L. Y., Neher, E., and Taschenberger, H. (2008). Synaptic vesicles in mature calyx of Held synapses sense higher nanodomain calcium concentrations during action potential-evoked glutamate release. J. Neurosci. 28, 14450-14458. doi: 10. 1523/jneurosci.4245-08.2008
Wheeler, D. B., Randall, A., and Tsien, R. W. (1994). Roles of N-type and Q-type $\mathrm{Ca}^{2+}$ channels in supporting hippocampal synaptic transmission. Science 264, 107-111. doi: 10.1126/science.7832825

Wu, L. G., Borst, J. G., and Sakmann, B. (1998). R-type $\mathrm{Ca}^{2+}$ currents evoke transmitter release at a rat central synapse. Proc. Natl. Acad. Sci. U S A 95, 47204725. doi: 10.1073/pnas.95.8.4720

Wu, L. G., and Saggau, P. (1994). Pharmacological identification of two types of presynaptic voltage-dependent calcium channels at CA3-CA1 synapses of the hippocampus. J. Neurosci. 14, 5613-5622.

Wu, L. G., Westenbroek, R. E., Borst, J. G., Catterall, W. A., and Sakmann, B. (1999). Calcium channel types with distinct presynaptic localization couple differentially to transmitter release in single calyx-type synapses. J. Neurosci. 19, 726-736.

Yang, Y. M., Fedchyshyn, M. J., Grande, G., Aitoubah, J., Tsang, C. W., Xie, H., et al. (2010). Septins regulate developmental switching from microdomain to nanodomain coupling of $\mathrm{Ca}^{2+}$ influx to neurotransmitter release at a central synapse. Neuron 67, 100-115. doi: 10.1016/j.neuron.2010.06.003

Yang, Y. M., and Wang, L. Y. (2006). Amplitude and kinetics of action potentialevoked $\mathrm{Ca}^{2+}$ current and its efficacy in triggering transmitter release at the developing calyx of held synapse. J. Neurosci. 26, 5698-5708. doi: 10. 1523/jneurosci.4889-05.2006

Yang, Y. M., Wang, W., Fedchyshyn, M. J., Zhou, Z., Ding, J., and Wang, L. Y. (2014). Enhancing the fidelity of neurotransmission by activity-dependent facilitation of presynaptic potassium currents. Nat. Commun. 5:4564. doi: 10 . 1038/ncomms5564

Yoshikami, D., Bagabaldo, Z., and Olivera, B. M. (1989). The inhibitory effects of omega-conotoxins on Ca channels and synapses. Ann. N Y Acad. Sci. 560, 230248. doi: 10.1111/j.1749-6632.1989.tb24100.x

Young, S. M., and Neher, E. (2009). Synaptotagmin has an essential function in synaptic vesicle positioning for synchronous release in addition to its role as a calcium sensor. Neuron 63, 482-496. doi: 10.1016/j.neuron.2009.07.028

Zucker, R. S., and Fogelson, A. L. (1986). Relationship between transmitter release and presynaptic calcium influx when calcium enters through discrete channels. Proc. Natl. Acad. Sci. U S A 83, 3032-3036. doi: 10.1073/pnas.83.9.3032

Conflict of Interest Statement: The authors declare that the research was conducted in the absence of any commercial or financial relationships that could be construed as a potential conflict of interest.

Received: 16 October 2014; paper pending published: 18 November 2014; accepted: 16 December 2014; published online: 26 January 2015.

Citation: Wang L-Y and Augustine GJ (2015) Presynaptic nanodomains: a tale of two synapses. Front. Cell. Neurosci. 8:455. doi: 10.3389/fncel.2014.00455

This article was submitted to the journal Frontiers in Cellular Neuroscience.

Copyright (c) 2015 Wang and Augustine. This is an open-access article distributed under the terms of the Creative Commons Attribution License (CC BY). The use, distribution and reproduction in other forums is permitted, provided the original author(s) or licensor are credited and that the original publication in this journal is cited, in accordance with accepted academic practice. No use, distribution or reproduction is permitted which does not comply with these terms. 


\title{
A use-dependent increase in release sites drives facilitation at calretinin-deficient cerebellar parallel-fiber synapses
}

\author{
Simone Brachtendorf, Jens Eilers and Hartmut Schmidt* \\ Medical Faculty, Carl-Ludwig-Institute for Physiology, University of Leipzig, Leipzig, Germany
}

\author{
Edited by: \\ Tycho M. Hoogland, Netherlands \\ Institute for Neuroscience, \\ Netherlands \\ Reviewed by: \\ Stéphane Dieudonné, Institut de \\ Biologie de l'Ecole Normale \\ Supérieure, France \\ Lisa Mapelli, University of Pavia, \\ Italy \\ *Correspondence: \\ Hartmut Schmidt, Medical Faculty, \\ Carl-Ludwig-Institute for Physiology, \\ University of Leipzig, Liebigstr. 27, \\ 04103 Leipzig, Germany \\ e-mail: hartmut.schmidt@ \\ medizin.uni-leipzig.de
}

\begin{abstract}
Endogenous $\mathrm{Ca}^{2+}$-binding proteins affect synaptic transmitter release and short-term plasticity (STP) by buffering presynaptic $\mathrm{Ca}^{2+}$ signals. At parallel-fiber (PF)-to-Purkinje neuron (PN) synapses in the cerebellar cortex loss of calretinin (CR), the major buffer at PF terminals, results in increased presynaptic $\mathrm{Ca}^{2+}$ transients and an almost doubling of the initial vesicular releases probability $\left(p_{r}\right)$. Surprisingly, however, it has been reported that loss of CR from PF synapses does not alter paired-pulse facilitation (PPF), while it affects presynaptic $\mathrm{Ca}^{2+}$ signals as well as $p_{\mathrm{r}}$. Here, we addressed this puzzling observation by analyzing the frequency- and $\mathrm{Ca}^{2+}$-dependence of PPF at unitary PF-to-PN synapses of wild-type (WT) and CR-deficient $\left(\mathrm{CR}^{-1-}\right)$ mice using paired recordings and computer simulations. Our analysis revealed that PPF in $\mathrm{CR}^{-/-}$is indeed smaller than in the WT, to a degree, however, that indicates that rapid vesicle replenishment and recruitment of additional release sites dominate the synaptic efficacy of the second response. These $\mathrm{Ca}^{2+}$-driven processes operate more effectively in the absence of $\mathrm{CR}$, thereby, explaining the preservation of robust PPF in the mutants.
\end{abstract}

Keywords: short-term plasticity, calretinin, paired-pulse facilitation, paired recordings, granule cells, readyreleasable pool

\section{INTRODUCTION}

$\mathrm{Ca}^{2+}$ regulates use-dependent presynaptic short-term plasticity (STP) by controlling the initial vesicular releases probability $\left(p_{\mathrm{r}}\right)$, the facilitation status of the release apparatus, and by regulating the size and restoration of vesicle pools (Zucker and Regehr, 2002; Regehr, 2012). Endogenous $\mathrm{Ca}^{2+}$ buffers (CaBs) are important regulators of presynaptic $\mathrm{Ca}^{2+}$ signals (Eggermann et al., 2012; Schmidt, 2012). Due to their diffusional mobility (Schmidt et al., 2003, 2005; Arendt et al., 2013), they get close to the site of $\mathrm{Ca}^{2+}$ entry and buffer the release triggering $\mathrm{Ca}^{2+}$ signal even if the diffusional distance between $\mathrm{Ca}^{2+}$ channel and release sensor is a few tens of nanometers only (Eggermann and Jonas, 2012; Bornschein et al., 2013; Schmidt et al., 2013). Well-known examples of at least partly mobile neuronal CaBs involved in the regulation of STP include parvalbumin (PV; Caillard et al., 2000), and calbindin-D28k (CB; Blatow et al., 2003).

Calretinin (CR) is an endogenous $\mathrm{CaB}$ closely related to $\mathrm{CB}$. Four of its binding sites bind $\mathrm{Ca}^{2+}$ in a cooperative manner with partly rapid kinetics (Faas et al., 2007). Thus, similar to loss of CB (Bornschein et al., 2013), loss of CR results in significantly increased synaptic $\mathrm{Ca}^{2+}$ transients, reduced initial synaptic failure rate (F1), and increased $p_{r}$ (Schmidt et al., 2013). Surprisingly, however, at the same synapses, the cerebellar parallel-fiber (PF)-to-Purkinje neuron (PN) synapses, which express $\mathrm{CR}$ as their major buffer, loss of $\mathrm{CR}$ has been reported not to be associated with significant alterations in paired-pulse facilitation (PPF; Schiffmann et al., 1999). In order to resolve this discrepancy, we analyzed PPF at unitary PF-to-PN connections in recordings from pairs of connected granule cells (GCs) and PNs in wild-type (WT) and CR deficient $\left(\mathrm{CR}^{-/-}\right)$mice over a broad range of frequencies (5-1000 ms) and extracellular $\mathrm{Ca}^{2+}$ concentrations $(1-10 \mathrm{mM})$. Contrary to the previous report (Schiffmann et al., 1999), we found that loss of CR resulted in a significant reduction in PPF. This reduction, however, was less prominent than would have been expected for the high $p_{\mathrm{r}}$ at $\mathrm{CR}^{-/-}$synapses. The fraction of synaptic failures to the second stimulus (F2) was significantly reduced in the mutants, suggesting that a rapid recovery of the releasable vesicle pool (RP) may compensate for its depletion during the first stimulus. Experimentally constrained computer simulations combined with an analysis of successes and failures, and multiple probability fluctuation analysis (MPFA) suggest the involvement of a $\mathrm{Ca}^{2+}$-driven mechanism in PPF (Millar et al., 2005; Sakaba, 2008; Valera et al., 2012), which restores and overfills the RP more effectively in $\mathrm{CR}^{-/-}$than in $\mathrm{WT}$, thereby, essentially preserving PPF in the mutants.

\section{MATERIALS AND METHODS SLICE PREPARATION}

Horizontal cerebellar slices (300 $\mu \mathrm{m}$ thick) were prepared (HM $650 \mathrm{~V}$; Microm, Walldorf, Germany) from the vermis region of 21-24-day-old C57BL/6 and $\mathrm{CR}^{-1-}$ mice (Schiffmann et al., 1999). The animals were decapitated following anesthesia with isoflurane (Curamed, Karlsruhe, Germany) and the cerebella were rapidly removed and placed in cooled $\left(0-4^{\circ} \mathrm{C}\right)$ artificial cerebrospinal fluid (ACSF) containing (in $\mathrm{mM}$ ): $125 \mathrm{NaCl}$, $2.5 \mathrm{KCl}, 1.25 \mathrm{NaH}_{2} \mathrm{PO}_{4}, 26 \mathrm{NaHCO}_{3}, 1 \mathrm{MgCl}_{2}, 2 \mathrm{CaCl}_{2}$ and 
20 glucose, bubbled with 95\% $\mathrm{O}_{2}$ and 5\% $\mathrm{CO}_{2}(\mathrm{pH} 7.3-7.4$ at $20-22^{\circ} \mathrm{C}$ ). Slices were kept for $30 \mathrm{~min}$. at $35^{\circ} \mathrm{C}$ prior to the experiments. Unless stated otherwise, all chemicals were obtained from Sigma-Aldrich, Seelze, Germany.

\section{ELECTROPHYSIOLOGY}

Patch pipettes were prepared from borosilicate glass (Hilgenberg, Malsfeld, Germany) with a PC-10 puller (Narishige, Tokyo, Japan) and had final resistances of 4-5 $\mathrm{M} \Omega$ or 10-11 $\mathrm{M} \Omega$ when filled with intracellular solution for recordings from PNs or GCs, respectively. The intracellular solution contained (in $\mathrm{mM}$ ): $150 \mathrm{~K}$-gluconate, $10 \mathrm{NaCl}, 3 \mathrm{Mg}$-ATP, $0.3 \mathrm{GTP}$, 10 HEPES and $50 \mu \mathrm{M}$ EGTA dissolved in purified water (SigmaAldrich, Seelze, Germany).The $\mathrm{pH}$ was adjusted to 7.3 with $\mathrm{KOH}$. Slices were transferred to the bath chamber perfused continuously at $3 \mathrm{ml} / \mathrm{min}$. with ACSF containing $10 \mu \mathrm{M}$ of the $\mathrm{GABA}_{\mathrm{a}}$-receptor blocker gabazine (SR-95531). Experiments were carried out at room temperature. Cells were visualized with an upright microscope (BX50WI, Olympus, Lambrecht, Germany) equipped with a $60 \times / 0.9 \mathrm{NA}$ water immersion objective (Olympus). Somatic whole-cell patch-clamp recordings from PNs were obtained using an EPC10-2 amplifier (HEKA, Lambrecht, Germany) and "Patchmaster 2.6" software. The liquid junction potential $(15 \mathrm{mV})$ was corrected for. During the experiments, the series resistance and leak current were monitored continuously and experiments were rejected if either the series resistance exceeded $30 \mathrm{M} \Omega$, deviated by more than $15 \%$ from its initial value, or the leak current fell below $-350 \mathrm{pA}$.

Stimulation of individual GCs was achieved in the loose-cell attached configuration in the voltage-clamp mode by using the current response of the amplifier to positive voltage steps (Perkins, 2006) and borosilicate patch-pipettes (10-11 M $\Omega$ ) filled with intracellular solution. GCs connected to the patched PN were located by briefly puffing the potassium containing intracellular solution to the GC layer at a distance of $>100 \mu \mathrm{m}$ laterally from the PN. If current responses $>20 \mathrm{pA}$ were recorded from the PN during puff-application, GCs in this region were tested sequentially by using electrical stimulation (stimulus duration: $500 \mathrm{~ms} ; 10$ repeats) in the loose-patch configuration with reuse of the pipette. Upon identification of a connected GC, stimulations at different interstimulus intervals (ISI: 5-100 ms) were performed with an inter-sweep interval of 5 s. Excitatory postsynaptic currents (EPSCs) were recorded in PNs at a holding potential of $-70 \mathrm{mV}$ to $-80 \mathrm{mV}$, filtered at $5 \mathrm{kHz}$ and sampled at $10 \mathrm{kHz}$. The stimulus duration was $\leq 2 \mathrm{~ms}$ and the stimulus amplitude was adjusted to induce a single action current only, in order to avoid stimulation of neighboring cells or fibers (Schmidt et al., 2013).

All experiments were carried out in accordance with institutional guidelines for animal experiments, and were approved by the state directorate of Saxony, Germany.

\section{ANALYSIS AND STATISTICS}

EPSCs were analyzed with "Patchmaster 2.6" software and custom written routines in Igor Pro 6.21 (Wavematrics, Lake Oswego, Oregon). paired-pulse ratios (PPRs) were calculated by dividing the average of the second EPSC amplitude of a given ISI by the average of the first EPSC amplitude of all ISIs at a given synapse. Amplitudes were determined by fitting a product of two exponential functions to the baseline-subtracted currents, which allowed for independent adjustment of the time constants of the rising and falling phases. The amplitude of the second EPSC was determined as the difference between its peak and the decay of the first EPSC, thereby eliminating the effects of electrical summation in particular at short ISIs. EPSCs were classified as failures if their amplitude was $<5$ pA i.e., 2-fold root mean square (RMS) noise. It cannot be excluded that some EPSCs with smaller amplitude remained undetected.

Quantal synaptic parameters were determined from EPSC amplitudes recorded at an ISI of $10 \mathrm{~ms}$ at different $\left[\mathrm{Ca}^{2+}\right]_{\mathrm{e}}(1$, 2 and $10 \mathrm{mM}, \geq 50$ repetitions per concentration) and a $5 \mathrm{~s}$ interval between paired stimulations. In indicated experiments $5 \mathrm{mM}$ of the competitive low-affinity AMPA receptor antagonist $\gamma$-D-glutamylglycine $(\gamma$-DGG) was added to the bath to relieve postsynaptic receptor saturation.

For MPFA, which corrected for non-uniform quantal size (Clements and Silver, 2000; Scheuss et al., 2002), noise-corrected variances $\left(\sigma^{2}\right)$ were plotted against mean EPSC amplitudes $(I)$ and fitted by a parabola of the form:

$$
\sigma^{2}=I q-\frac{I^{2}}{N}\left(1+C V_{I I}^{2}\right)+q I C V_{I}^{2}
$$

with $\mathrm{q}$ being the quantal size, $\mathrm{N}$ a binominal parameter indicating the number of release sites or releasable vesicles, and $C V_{I}$ and $C V_{I I}$ the coefficients of intra- and inter-site quantal variability, assumed to be 0.3 (Valera et al., 2012; Schmidt et al., 2013). Non-integer values for $\mathrm{N}$ were allowed to indicate the uncertainty of the fit (for further detail see Hallermann et al., 2010; Schmidt et al., 2013). MPFA is well suited for unitary connections (Clements and Silver, 2000) and has previously been shown to be applicable at the unitary PF to PN synapses (e.g., Sims and Hartell, 2006; Valera et al., 2012; Schmidt et al., 2013).

Unless stated otherwise, data are presented as mean and \pm SE. Parametric statistical tests were performed if data were normally distributed (Shapiro-test) with equal variance and if the number of data points was sufficiently large (as indicated by the power of the test); non-parametric tests were used otherwise or in addition, as indicated. Statistical significance was tested for either with the $t$-test (two groups, normally distributed), the MannWhitney rank sum test (two groups, non-normally distributed) or with parametric (more than two groups, normally distributed) or nonparametric (more than two groups, either non-normally distributed or normally distributed and low n; post hoc testing with Dunn's method) ANOVA designs, using Sigma Plot 11.0 software (Erkrath, Germany).

\section{SIMULATION OF CA $^{2+}$ DYNAMICS AND FACILITATED TRANSMITTER RELEASE}

The reaction schemes of a kinetic model were transformed into the corresponding ordinary differential equations and numerically solved using Mathematica 10.0 (Wolfram Research). The model (Schmidt et al., 2013) included a Gaussian-shaped 
$\mathrm{Ca}^{2+}$ influx, buffering of $\mathrm{Ca}^{2+}$ by ATP, Calmodulin, and cooperative CR (Faas et al., 2007), $\mathrm{Ca}^{2+}$ extrusion, diffusion of all species and $\mathrm{Ca}^{2+}$-dependent vesicle fusion and replenishment (Millar et al., 2005; Sakaba, 2008). Resting $\mathrm{Ca}^{2+}$ was set to $45 \mathrm{nM}$. Release rates were obtained by differentiation of the fused state. PPRs were calculated from the $p_{\mathrm{r}}$ ratios, obtained by integration of the release rates. The model was adjusted to yield an initial $p_{\mathrm{r}}$ of $\sim 0.25$ and $\sim 0.41$ in WT and $\mathrm{CR}^{-/-}$, respectively (Schmidt et al., 2013), and a PPR at $10 \mathrm{~ms}$ ISI consistent with the experiments.

Parameters of the release and replenishment model were similar to previously published values (Millar et al., 2005; Sakaba, 2008). The release sensor (V) was modeled with a binding rate $k_{\text {on }}=1.2^{*} 10^{8} \mathrm{M}^{-1} \mathrm{~s}^{-1}$, unbinding rate $k_{\text {off }}=1000$ or $3500 \mathrm{~s}^{-1}$, cooperativity $b=0.3$, and release rate $g=10000 \mathrm{~s}^{-1}$. The replenishment part $\left(R_{0}, R_{1}\right)$ was simulated with forward and backward rate constants of $\mathrm{Ca}^{2+}$-dependent priming and unpriming of $k_{\text {prim }}=3.5^{*} 10^{8}$ or $1.8^{*} 10^{8} \mathrm{M}^{-1} \mathrm{~s}^{-1}$ and $k_{\text {unprim }}=500 \mathrm{~s}^{-1}$, respectively, followed by $\mathrm{Ca}^{2+}$-independent rates for forward and backward transition into or out of the RP with $k_{\mathrm{t}+}=500 \mathrm{~s}^{-1}$ and $k_{\mathrm{t}-}=50 \mathrm{~s}^{-1}$, respectively. For simplicity, release and replenishment were driven by the same local $\mathrm{Ca}^{2+}$ signal (Millar et al., 2005). Model settings were identical for WT and $\mathrm{CR}^{-/-}$and the mutant was simulated by only removing $\mathrm{CR}$ from the model.

\section{RESULTS}

\section{IDENTIFICATION OF GC-TO-PN PAIRS}

The GC to PN connectivity is rather low in slice preparations (Isope and Barbour, 2002) and we used the following procedure to establish paired recordings (Figure 1): After the wholecell configuration had been established on a PN, potassium containing pipette solution was puffed from a second patchpipette to regions of the GC layer at a distance of $>100 \mu \mathrm{m}$ from the soma of the PN. This distance was chosen to ensure potassium-mediated activation of PF synapses rather than synapses formed by the ascending axon of the GC (Isope and Barbour, 2002). If cells or fibers connecting to the PN were present in this region, EPSCs were apparent in the recording from the PN soma (Figure 1A). Subsequently, individual GCs in this region were activated by electrical stimulation of their somata in the loose-cell configuration (Perkins, 2006) making repeated use of the same patch-pipette (Figure 1B). After identification of a GC-PN pair, tetrodotoxin (TTX) sensitive action currents (ACs) were elicited in the GC by brief current pulse $(<2 \mathrm{~ms})$ at different ISI and evoked EPSCs recorded from the PN (Figure 1C). Assuming reliable propagation of the action potential (AP; Isope and Barbour, 2002), stimulation and release failures could be clearly distinguished in these recordings (cf. Schmidt et al., 2013).

\section{REDUCED FAILURE RATES IN THE SECOND RESPONSE CONTRIBUTES TO PPF}

Following the establishment of a recording from a GCPN pair connected via a PF synapse, we recorded the frequency dependence of PPF at ISIs ranging from 5 to $1000 \mathrm{~ms}$ (Figure 2A). PPF was maximal $(2.8 ; n=12$
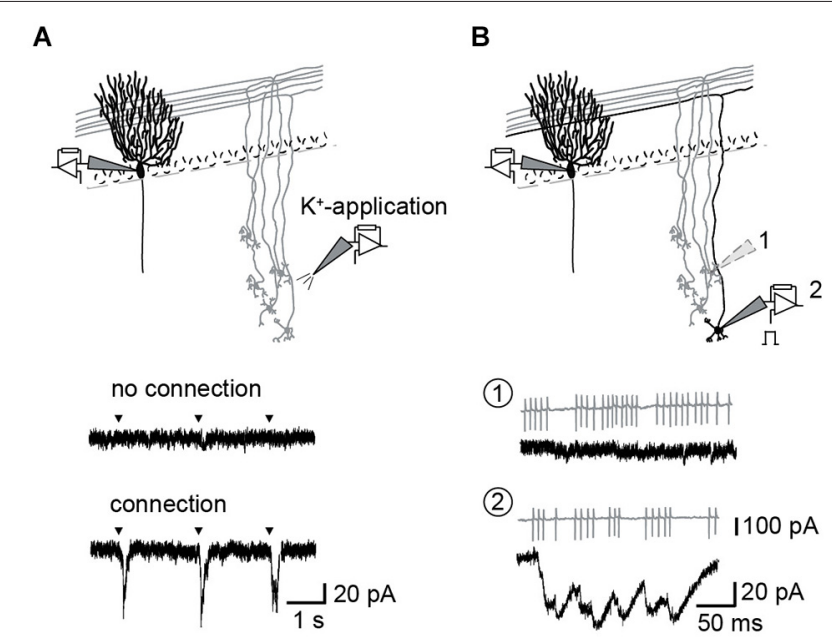

C
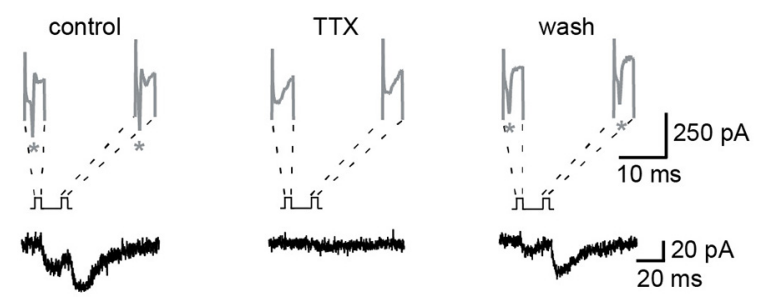

FIGURE 1 | Identification of unitary GC-PN connections. (A,B) Schemes of experimental approaches. (A) Top: PNs were held in the whole-cell configuration. GCs were stimulated via puffs of $\mathrm{K}^{+}$-containing pipette solution applied to the granule cell layer (GCL). Example recordings from a $\mathrm{PN}$ during $\mathrm{K}^{+}$-application to different regions of the $\mathrm{GCL}$ are shown below. Middle: no activation of connected GCs; bottom: inward currents recorded in response to activation of connected GCs. Triangles indicate times of $\mathrm{K}^{+}$-application to the GLC. (B) Top: Single GCs were stimulated in the loose-cell attached configuration within the region that responded to $\mathrm{K}^{+}$-puffs. Bottom: Example recording from a not connected (1) and a connected (2) GC-PN pair. Action currents (ACs) (gray) elicited in the GC and the corresponding postsynaptic response (black). (C) Presynaptic ACs were reversibly inhibited by bath application of $1 \mu \mathrm{M}$ TTX. Currents from GC (gray; stars denote ACs; initial peaks are capacitive currents) and the corresponding current traces (black) recorded from the connected PN.

pairs) at 5-10 ms ISI. It declined to unity with a time constant $(\tau)$ of $96 \mathrm{~ms}$ (Figure 2B). The rate of synaptic failures in the second response (F2) was lowest at short ISIs and increased with the interval between stimuli until it reached the value of the first responses (F1) at $\sim 300 \mathrm{~ms}$ (Figure 2C).

Provided that the quantal size (q) remains unaltered between first and second pulse, the EPSC2/EPSC1 PPR is given by (N2 $\left.p_{r 2}\right) /\left(\mathrm{N} 1 p_{\mathrm{r}}\right)$, with $\mathrm{N} 1$ and $\mathrm{N} 2$ indicating the number of available release sites or releasable vesicles at the first and second activation, respectively. Thus, $\mathrm{F} 2<\mathrm{F} 1$ and PPF result either from increasing $p_{\mathrm{r} 2}$ or N2 or both. N2 is reduced initially by vesicles released during the first pulse but may be replenished by a fraction $\mathrm{R}$ between pulses, i.e., $\mathrm{N} 2=\mathrm{N} 1-\left(\mathrm{N} 1 p_{\mathrm{r}}\right)+\mathrm{R}\left(\mathrm{N} 1 p_{\mathrm{r}}\right)$. The above results have interesting implications for the mechanisms of PPF at PF synapses: The PPR of 2.8 at short ISI is close to the theoretical maximum of 3 in the absence of vesicle replenishment 


\section{A}
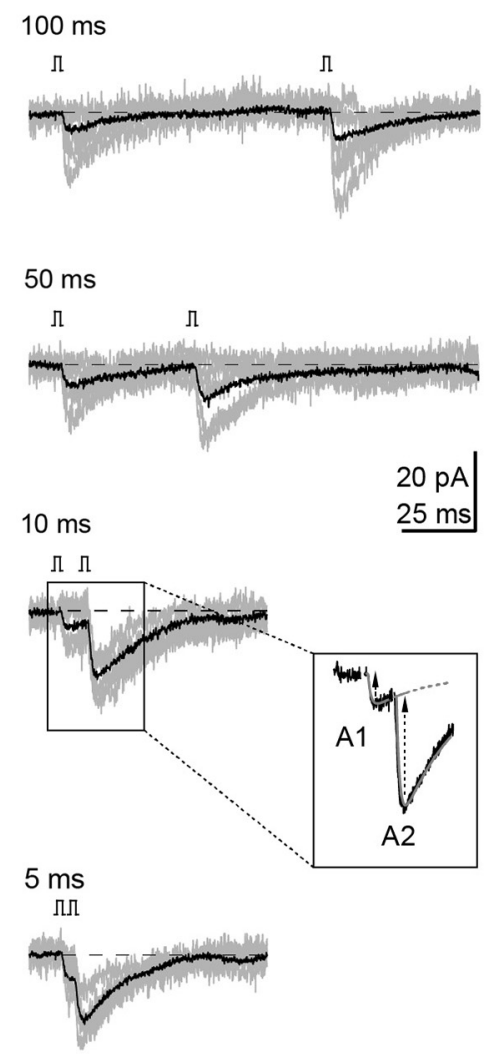

FIGURE 2 | Properties of connected GC-PN pairs in the WT. (A) Example of a paired-pulse experiment at denoted ISI from a unitary GC-PN connection (individual traces in gray; average, including failures, in black). Inset: EPSC amplitudes (black arrows) were determined by fitting products of two exponentials (dashed black lines) to the currents, allowing for subtracting the decay of the first EPSC (A1) from the peak of the second EPSC (A2). (B) PPRs
B

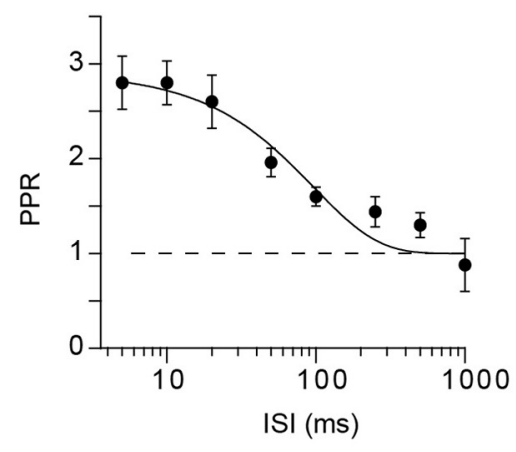

C

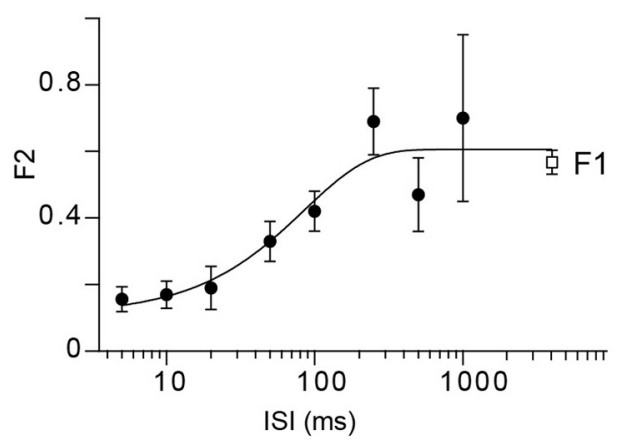

plotted against the indicated ISI (mean $\pm \mathrm{SE}, n=12$ ). The solid line represents an exponential fit to the data $\left(\tau=96 \mathrm{~ms} ; \chi^{2}=1.435\right)$. (C) Fraction of synaptic failures in the second response (F2) plotted vs. the indicated ISI (mean \pm SE, $n=12$ ). The fraction of synaptic failure in the first response ( $F 1=0.57 \pm 0.04$, $n=16)$ is plotted at the interval between successive recordings ( $5 \mathrm{~s})$. Solid line represents exponential fit to the data $\left(\tau=85 \mathrm{~ms} ; \chi^{2}=0.621\right)$. $\left[p_{\mathrm{r} 2}=1 ; \mathrm{PPR}_{\max }=1 / p_{\mathrm{r}}^{*}\left(1-p_{\mathrm{r}}\right)\right]$, using $p_{\mathrm{r}}$ of 0.25 for WT PF synapses (Sims and Hartell, 2005; Valera et al., 2012; Schmidt et al., 2013). However, our failure analysis showed that at ISI of 5 and $10 \mathrm{~ms}$ F2 was $0.16 \pm 0.04$ and $0.17 \pm 0.04$, respectively, demonstrating that $p_{\mathrm{r} 2}$ has not reached a value of one but is $\leq \sim 0.84\left[\mathrm{~F}=\left(1-p_{\mathrm{r}}\right)^{N}\right]$. Thus, rapid replenishment of $\mathrm{N}$ (Crowley et al., 2007) and/or an increase in N (Sakaba, 2008; Valera et al., 2012) may be involved in PPF. Assuming that this process is $\mathrm{Ca}^{2+}$ dependent (Millar et al., 2005; Sakaba, 2008), it could provide a mechanistic explanation for PPF in $\mathrm{CR}^{-/-}$since in the absence of this endogenous $\mathrm{Ca}^{2+}$ buffer from the synapse it could operate more effectively.

\section{PPF AT INDIVIDUAL CR-DEFICIENT PF SYNAPSES}

To probe whether $\mathrm{Ca}^{2+}$-dependent replenishment and/or increase in $\mathrm{N}$ (either by overfilling (Sakaba, 2008) or by recruitment of additional, reluctant release sites (Valera et al., 2012)) could explain the unexpected observation that $\mathrm{PPF}$ in $\mathrm{CR}^{-/-}$is not different from WT synapses (Schiffmann et al., 1999), we analyzed the frequency-dependence of PPF in recordings from pairs of connected GCs and PNs in $\mathrm{CR}^{-/-}$mice ( $n=6$ pairs; Figure $3 \mathrm{~A}$ ), using the above described procedure. In contrast to the previous report of unaltered $\mathrm{PPF}$ in $\mathrm{CR}^{-/-}$obtained with fiber tract stimulations (Schiffmann et al., 1999), in our paired recordings the magnitude of PPF was moderately but significantly reduced in $\mathrm{CR}^{-/-}$compared to WT in particular at ISI $\leq 20 \mathrm{~ms}$ (Figure 3B; $\mathrm{PPR}=2.37 \pm 0.58$ at $5 \mathrm{~ms}$ and $2.14 \pm 0.25$ at $10 \mathrm{~ms} ; P=0.038$ ). PPR showed a frequency dependence almost identical to the WT, dropping with a time constant of $100 \mathrm{~ms}$ to unity. Consistent with a reduced PPF (and an increased $p_{\mathrm{r}}$ ) F1 was significantly reduced in $\mathrm{CR}^{-/-}$compared to the WT $(0.41 \pm 0.06$ and $0.57 \pm 0.04$ for $\mathrm{CR}^{-/-}$and WT, respectively, $\left.P=0.027\right)$.

$\mathrm{PPF}$ in the mutants was still unexpectedly large. Using the above calculations and a $p_{\mathrm{r}}$ of 0.41 (Schmidt et al., 2013) the theoretical $\mathrm{PPR}_{\max }$ (for $p_{\mathrm{r} 2}$ of 1 ) is $\sim 1.4$ in the absence of replenishment. With full replenishment $\left(\mathrm{N} 2=\mathrm{N} 1=>\mathrm{PPR}=p_{\mathrm{r} 2}\right.$ $\left./ p_{\mathrm{r}}\right) \mathrm{PPR}_{\max }$ would be $\sim 2.4$, i.e., the measured PPR substantially exceeds the theoretical $\mathrm{PPR}_{\max }$ without replenishment and at an ISI of $5 \mathrm{~ms}$ is close to $\mathrm{PPR}_{\max }$ with $100 \%$ replenishment. F2 in the mutants was significantly reduced compared to WT (Figure 3C; 
A
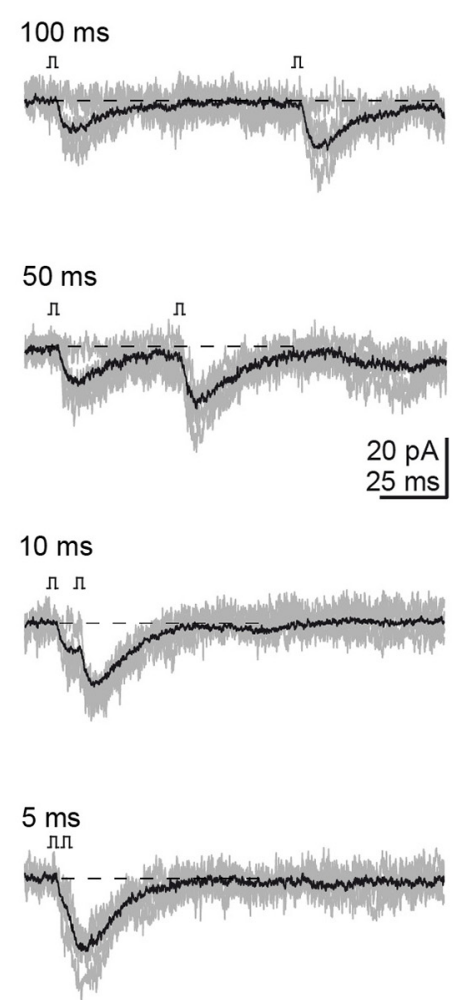

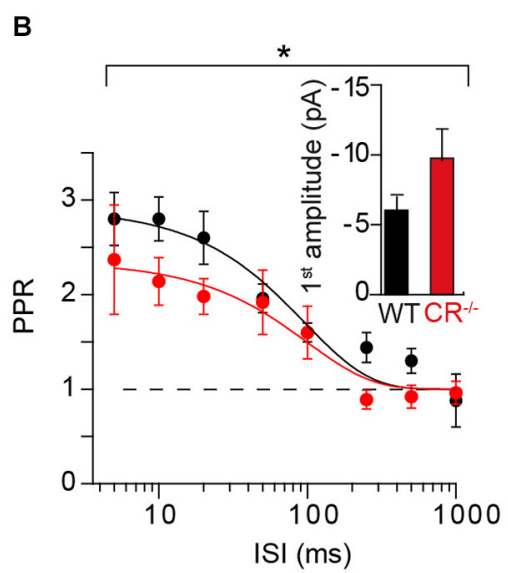

C

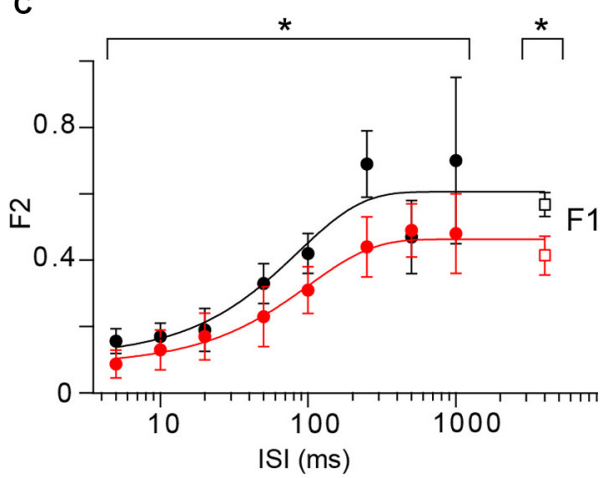

first EPSC amplitude (including failures) in WT and CR KO (mean \pm SE, $n=16$ and 11, respectively; $P=0.059, t$-test). (C) Comparison of the frequency dependence of synaptic failures in the second response (F2) in WT (black; cf. Figure 2C) and $\mathrm{CR}^{-/-}$(red; mean $\pm \mathrm{SE}, n=6$; ${ }^{*} P=0.022$ two-way ANOVA, $P<0.01$ with K-W ANOVA) terminals. The initial failure rate (F1) was determined at the interval between successive recordings $\left(\mathrm{CR}^{-/-}: n=11\right.$, ${ }^{*} P=0.027, t$-test $)$. Solid lines represent exponential fits to the data $\left(\mathrm{CR}^{-1-}\right.$ : $\left.\tau=101 \mathrm{~ms} ; \chi^{2}=0.156\right)$.
$P=0.022)$ and, as in the WT, reached the F1 value at ISI $\sim 300$ $\mathrm{ms}$. Yet, it did not drop to 0 even at an ISI of $5 \mathrm{~ms}(0.09 \pm 0.04)$, i.e., also in the mutants $p_{\mathrm{r} 2}$ did not reach a value of one but rather is $\leq \sim 0.91$. This suggests that even a full replenishment of $\mathrm{N}$ between pulses is not sufficient to account for the measured PPF in $\mathrm{CR}^{-/-}$. In addition, these data suggest that the control of the size of $\mathrm{N}$ is dependent on $\mathrm{Ca}^{2+}$, since it operated more effectively in the absence of CR.

\section{PPF AT HIGH EXTRACELLULAR CA ${ }^{2+}$ CONCENTRATIONS EXCEEDS THEORETICAL MAXIMA}

To further test the hypothesis that a $\mathrm{Ca}^{2+}$-dependent increase in $\mathrm{N}$ contributes to PPF, we measured PPF in paired recordings at WT and $\mathrm{CR}^{-/-}$unitary connections at different extracellular $\mathrm{Ca}^{2+}$ concentrations $\left(\left[\mathrm{Ca}^{2+}\right]_{\mathrm{e}}\right)$ of 1,2 , and $10 \mathrm{mM}$ at an ISI of $10 \mathrm{~ms}$ (Figure 4A). Alterations in $\left[\mathrm{Ca}^{2+}\right]_{\mathrm{e}}$ alter $p_{\mathrm{r}}$ but may also affect the replenishment/recruitment of $\mathrm{N}$, if $\mathrm{Ca}^{2+}$ dependent. We found that for all $\left[\mathrm{Ca}^{2+}\right]_{\mathrm{e}}$ concentrations tested, PPR in the mutant was significantly smaller than in WT (Figure 4B; $P<0.01$ ). PPF was strongest at $1 \mathrm{mM}\left[\mathrm{Ca}^{2+}\right]_{\mathrm{e}}$ with an average PPR of $6.47 \pm 1.36$ in the WT and $3.56 \pm 0.44$ in $\mathrm{CR}^{-/-}$. The PPR dropped with increasing $\left[\mathrm{Ca}^{2+}\right]_{\mathrm{e}}$ being only $1.00 \pm 0.11$ and $0.73 \pm 0.08$ in WT and $\mathrm{CR}^{-1-}$ in $10 \mathrm{mM}\left[\mathrm{Ca}^{2+}\right]_{\mathrm{e}}$, respectively.

It has been shown previously that single EPSC amplitudes at PF synapses are not significantly affected by postsynaptic receptor saturation at high $\left[\mathrm{Ca}^{2+}\right]_{\mathrm{e}}$ (Valera et al., 2012; Schmidt et al., 2013). Yet, the PPR analysis may underestimate the magnitude of presynaptic facilitation due to saturation of postsynaptic receptors in the second response. We therefore recorded PPRs in WT in the presence of the competitive low-affinity AMPA receptor antagonist $\gamma$-DGG $(n=4)$ which relieves receptor saturation (Foster et al., 2005). Under these conditions, PPR at $2 \mathrm{mM}$ and $10 \mathrm{mM}\left[\mathrm{Ca}^{2+}\right]_{\mathrm{e}}$ increased to $4.45 \pm 0.38$ and $1.52 \pm 0.26$, respectively (Figure 4B), indicating that receptor saturation indeed led to an underestimation of the real magnitude of presynaptic facilitation.

Plotting the PPR values for each $\left[\mathrm{Ca}^{2+}\right]_{\mathrm{e}}$ against the previously quantified $p_{\mathrm{r}}$ values (Schmidt et al., 2013) revealed the expected 
A
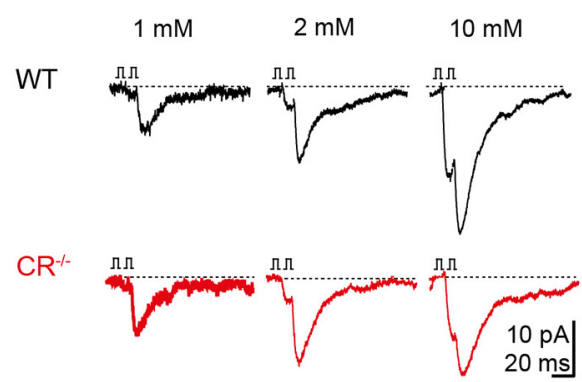

B

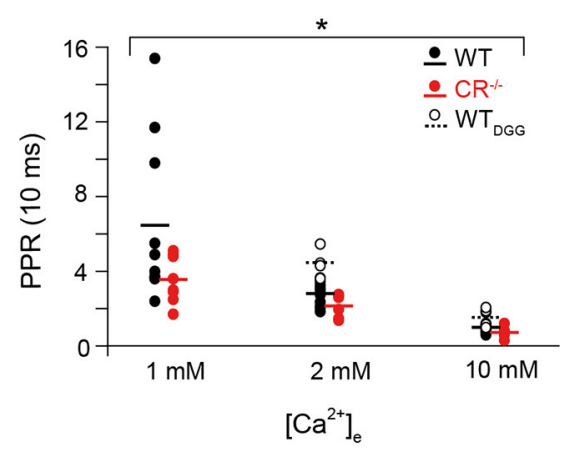

C

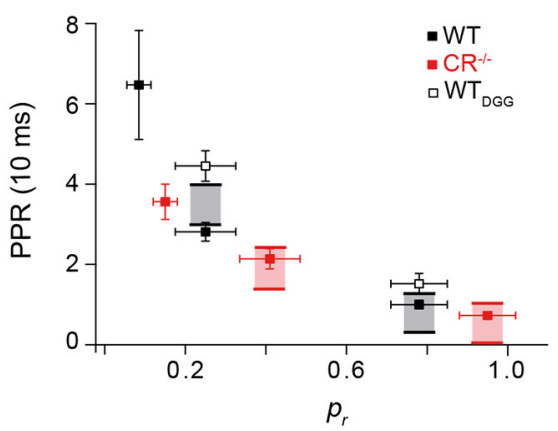

FIGURE 4 | Rapid replenishment and overfilling contribute to PPF. (A) Examples of EPSCs recorded at an ISI of $10 \mathrm{~ms}$ at different extracellular $\mathrm{Ca}^{2+}$-concentrations from a representative WT (black) and $\mathrm{CR}^{-1-}$ (red)

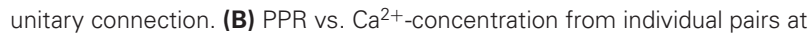
an ISI of $10 \mathrm{~ms}$ for WT (black; solid circles, solid line indicates the average; $n=10), \mathrm{CR}^{-1-}($ red; $n=8)$, and WT in the presence of $\gamma$-DGG $(5 \mathrm{mM}$, $W_{D G G}$ open circles; dashed line indicates average; $\left.n=4\right)$. For all $\left[\mathrm{Ca}^{2+}\right]_{\mathrm{e}}$ concentrations tested, PPR in the mutant was significantly smaller than in WT $\left({ }^{*} P<0.01\right.$, K-W ANOVA). (C) Plot of PPR vs. $p_{r}$ for all $\left[\mathrm{Ca}^{2+}\right]_{e}$ revealing a decline in PPR with increasing $p_{\mathrm{r}}$ in WT and $\mathrm{CR}^{-1-}$ (PPR: mean \pm SE (cf. Panel B), $p_{\mathrm{r}}:$ median $\pm \mathrm{SE}$; WT (black): $n=10 ; \mathrm{CR}^{-/-}($red): $n=8$ ). Shaded areas indicate the maximum theoretical values of PPR $\left(P P R_{\max }\right.$ for $\left.p_{\mathrm{r} 2}=1\right)$ in presence (top line) and in absence (bottom line) of full vesicle replenishment. PPR values at high $p_{r}$ are close to the theoretical PPR $R_{\max }$ with full vesicle replenishment for mutant and WT. PPR values determined in the presence of $\gamma$-DGG (open squares, $W_{D G G}: n=4$ ) exceed the theoretical PPR $\max$ with full vesicle replenishment.

dependency of PPF on $p_{\mathrm{r}}$ in both genotypes (Figure 4C). Inclusion of the theoretical $\mathrm{PPR}_{\max }$ values in the absence of replenishment (lower boundary of the shaded areas in Figure 4C) showed a clear discrepancy to the measured PPR at 2 and $10 \mathrm{mM}$ $\left[\mathrm{Ca}^{2+}\right]_{\mathrm{e}}$ for the mutant and in $10 \mathrm{mM}\left[\mathrm{Ca}^{2+}\right]_{\mathrm{e}}$ also for the WT, with the experimental values being larger than the theoretical
$\mathrm{PPR}_{\max }$ value. These data underline that a rapid vesicle pool restoration or recruitment mechanism is likely to contribute to PPF. Remarkably, PPR values determined in the presence of $\gamma$-DGG substantially exceeded even the theoretical $P R_{\max }$ with full replenishment, demonstrating that replenishment and increased $p_{\mathrm{r} 2}$ alone are not sufficient to explain PPR, i.e., an increase in $\mathrm{N}$ is required to explain the experimentally observed PPF value. In addition, these data further substantiate the above notion that the mechanism controlling the size of $\mathrm{N}$ is $\mathrm{Ca}^{2+}$. dependent since it was influenced by alterations in $\left[\mathrm{Ca}^{2+}\right]_{\mathrm{e}}$ and operated more effectively in the absence of the endogenous $\mathrm{Ca}^{2+}$ buffer CR.

\section{USE DEPENDENT INCREMENT IN N CAN ACCOUNT FOR PPF}

In order to further test the hypothesis that replenishment and increasing $\mathrm{N}$ contribute to the only moderate reduction of PPF in $\mathrm{CR}^{-/-}$, we analyzed a model of release and replenishment that allows for increasing N2 above N1. This model was previously shown to reproduce central characteristics of phasic and tonic synapses (Millar et al., 2005; Sakaba, 2008). In this model, release is triggered via a cooperative $\mathrm{Ca}^{2+}$-driven fivesite sensor from a population of releasable vesicles. These vesicles become replenished in two steps with the first step being $\mathrm{Ca}^{2+}$ dependent.

In our simulations the release-triggering $\mathrm{Ca}^{2+}$ signals in WT and $\mathrm{CR}^{-/-}$were adjusted according to previously measured $\mathrm{Ca}^{2+}$ transients in PF boutons and corresponding estimates of the $\mathrm{Ca}^{2+}$ dynamics at the release sites (Schmidt et al., 2013). For simplicity, $\mathrm{Ca}^{2+}$-dependent replenishment was driven by the same local $\mathrm{Ca}^{2+}$ signal (Millar et al., 2005). The $\mathrm{Ca}^{2+}$ dependent replenishment steps allow for a transient, several hundred ms long increment in N, corresponding to an overfilling or recruitment process (Figures 5A,B; Millar et al., 2005; Sakaba, 2008). The major endogenous $\mathrm{CaB}$ used was $\mathrm{CR}$, and the two genotypes were simulated by either including (WT) or removing $\left(\mathrm{CR}^{-1-}\right) \mathrm{CR}$ from the simulations without changing other parameters.

Pairs of release processes were modeled at ISI of 5-500 ms. PPRs were calculated from the ratio of the time-integrals of the release rates. Plotting the resulting PPRs against the corresponding ISI showed a simulated frequency dependence of facilitation that was in good accordance to the experimental values in both genotypes. In particular, a slightly reduced PPF in $\mathrm{CR}^{-1-}$ compared to the WT was obtained (Figure 5C). When the replenishment steps were excluded from the simulations a residual facilitation remained at short ISI of $\leq 20 \mathrm{~ms}$ (dashed lines in Figure $5 \mathrm{C}$ ). This resulted from the release sensor remaining facilitated for short intervals determined by its $\mathrm{Ca}^{2+}$ unbinding rate ("active $\mathrm{Ca}^{2+}$ ", Katz and Miledi, 1968; Millar et al., 2005; Bornschein et al., 2013). The magnitude of PPF caused by the active $\mathrm{Ca}^{2+}$, however, was too small to account for the experimental values as expected from the uncompensated depletion during the first release process, indicating that the transient increase in $\mathrm{N}$ is a major determinant of PPF (solid lines in Figure 5C).

To experimentally test the hypothesis of an increase in $\mathrm{N}$ between pulses ("overfilling" or "recruitment"), we performed 


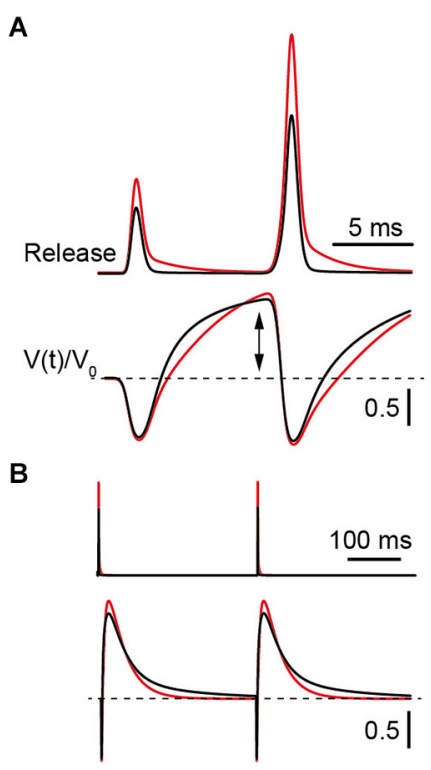

FIGURE 5 | Model of PPF in WT and $\mathbf{C R}^{-/-}$synapses. (A) Upper: Simulated transmitter release rates during a pair of synaptic activations at an ISI of $10 \mathrm{~ms}$ in WT (black) or $\mathrm{CR}^{-/}$- synapses (red) normalized to the first release process in the WT. Lower: Temporal variation of $\mathrm{Ca}^{2+}$-free release sensor sites $(\mathrm{V}(\mathrm{t}))$ normalized to their value prior to the first stimulus $\left(V_{0}\right)$ during $100 \mathrm{~Hz}$ activation in WT (black) or KO synapses (red). Note the increase (arrow) above $V_{0}$ (dashed line) between pulses. (B) Same as in (A) but for two activations with an ISI of $300 \mathrm{~ms}$. (C) Upper: Scheme of the model which includes a release sensor with $5 \mathrm{Ca}^{2+}$ binding sites $(\mathrm{V})$ and a two-step replenishment $\left(R_{0}, R_{1}\right)$ with the first step being $\mathrm{Ca}^{2+}$ dependent and the second step indirectly $\mathrm{Ca}^{2+}$ dependent (Millar et al., 2005; Sakaba, 2008). Lower: Paired

C

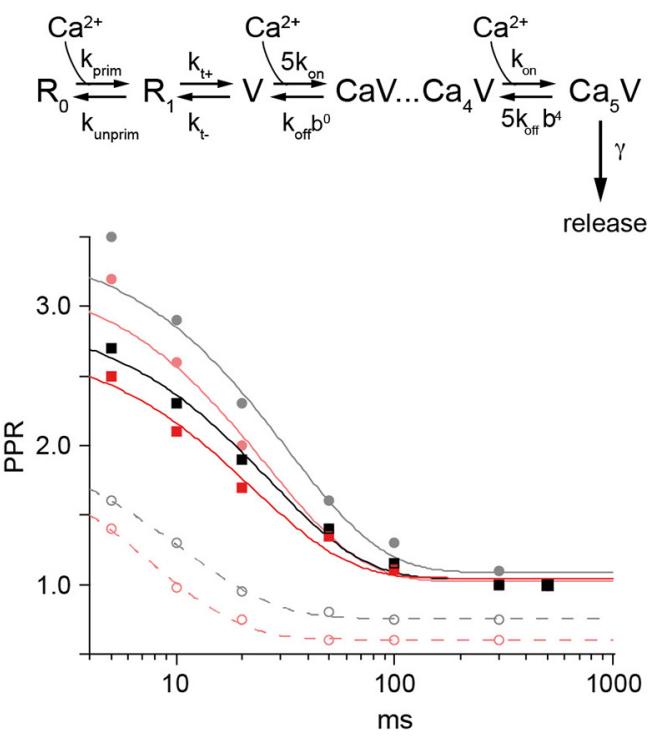

pulse ratios (PPRs) calculated as the ratio of release probabilities between the second and the first pulse plotted against the ISI. Lines represent exponential fits. Curves for the WT are in gray and black and those for the $\mathrm{CR}^{-1-}$ in red. Solid gray and light red curves represent simulations with $k_{\text {off }}$ set to $1000 \mathrm{~s}^{-1}$ (Millar et al., 2005). The corresponding PPRs result from the combined action of $\mathrm{Ca}^{2+}$ remaining bound to the release sensor (active $\mathrm{Ca}^{2+}$ ) between stimuli at small ISI (dashed lines) and overfilling of the pool V (cf. (A)). Increasing $k_{\text {off }}$ to $3500 \mathrm{~s}^{-1}$ eliminated facilitation due to active $\mathrm{Ca}^{2+}$ and resulted in the black and red curves for the frequency dependence of PPR in WT and KO, respectively. Irrespective of model details only slightly decreased facilitation is predicted for the $\mathrm{KO}$ in comparison to the WT.
MPFA of second EPSC amplitudes recorded at an ISI of $10 \mathrm{~ms}$ at $\left[\mathrm{Ca}^{2+}\right]_{\mathrm{e}}$ of 1, 2 and $10 \mathrm{mM}$ (Clements and Silver, 2000). The average initial values of $\mathrm{N}(\mathrm{N} 1)$ in $2 \mathrm{mM}\left[\mathrm{Ca}^{2+}\right]_{\mathrm{e}}$ at unitary PF synapses were previously published to be 2.9 and 2.7 in WT and $\mathrm{CR}^{-/-}$, respectively (Schmidt et al., 2013). The present MPFA of second EPSC amplitudes in WT showed an increase in $\mathrm{N}(\mathrm{N} 2)$ to $3.2 \pm 0.5(n=7)$ and for $\mathrm{CR}^{-/-}$an increase to $3.5 \pm 1(n=7$; Figures 6A,B). Thus, MPFA indicates that $\mathrm{N} 2>\mathrm{N} 1$. Considering that postsynaptic receptor saturation affected the amplitude of second EPSCs (see above), this analysis will even underestimate $\mathrm{N} 2$ as an index of release sites or RP vesicles (Scheuss et al., 2002; Silver, 2003), indicating that the increment in $\mathrm{N} 2$ is even larger. In order to experimentally probe this, we performed MPFA in the presence of $\gamma$-DGG (Figure 6C). EPSC amplitudes recorded from GC-to-PN pairs are small even under control conditions, making MPFA from unitary PF synapses demanding (Valera et al., 2012; Schmidt et al., 2013). On average, these amplitudes are further reduced in the presence of $\gamma$-DGG and we succeeded in performing MPFA at only three GC-PN pairs in the WT. In these experiments, we found that $\mathrm{N} 2$ was indeed prominently raised to a value of $11 \pm 3$ (Figure 6D). It is conceivable that the effects of $\gamma$ DGG deviate from linearity for low glutamate concentrations (Liu et al., 1999), which may lead to an overestimation of $\mathrm{N}$ in its presence, i.e., the value of 11 may be considered an upper limit for N2. Yet, these data in conjunction with the simulations (Figure 5) indicate that increased $\mathrm{N}$ in the second pulse will make a major contribution to PPF. Taken together, our experiments and simulations corroborate the finding that overfilling or apparent recruitment of release sites is a central mechanism of PPF at PF-to-PN synapses (Valera et al., 2012). They extend this result by showing that a transient, $\mathrm{Ca}^{2+}$-dependent overfilling / recruitment mechanisms can account for the preservation of PPF at CR-deficient PF synapses.

\section{COEXISTENCE OF MULTIPLE FORMS OF PLASTICITY MECHANISMS}

So far we focused on the synaptic responses obtained from averaging over several paired-pulse trials at a given synapse. However, individual synaptic responses to a pair of invading APs may be composed of differential sequences of successes and failures. Paired recordings allow obtaining deeper insights into the composition of individual paired-pulse trials and their relative contributions to $\mathrm{PPF}$ in $\mathrm{WT}$ and $\mathrm{CR}^{-/-}$. We performed a binary group analysis of the four possible synaptic responses in a paired pulse experiment, which consist of double successes ("1_1"), a success followed by a failure ("1_0"), a failure followed by a success ("0_1"), or double failures ("0_0"). The percentage of each of these response types in WT and $\mathrm{CR}^{-/-}$ is given in Figure 7A. In the WT 0_1 responses were recorded most frequently $(41 \%)$, while in $\mathrm{CR}^{-/-}$the contribution of 
A
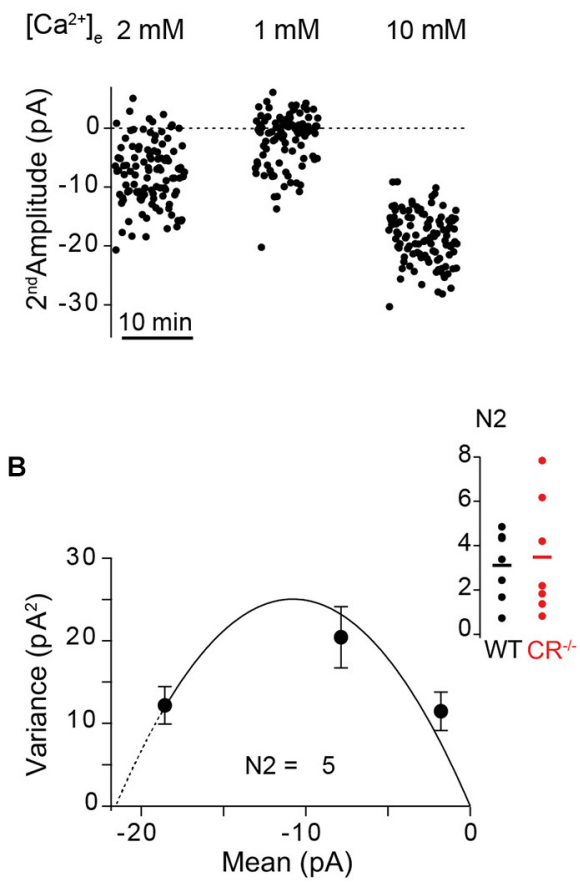
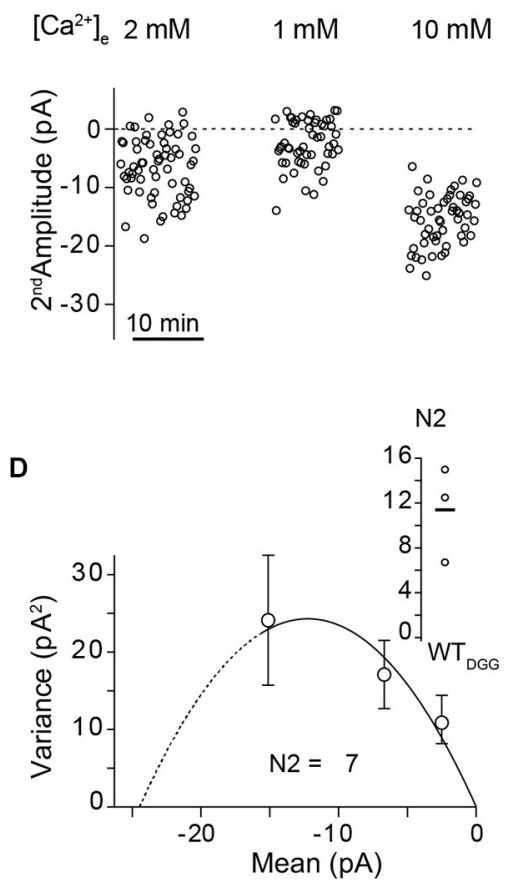

variance. The solid lines represent the parabolic MPFA fit ((B), $\chi^{2}=3.709$; (D), $\left.\chi^{2}=0.665\right)$, which yielded the binominal parameter $\mathrm{N}$ for the second pulse (N2). Line dashing indicates the region over which the fit has been extrapolated. Inset: Summary of the estimated N2 values in absence (B) and presence of $\gamma$-DGG (D). Solid lines: Mean of N2 (WT: $3.2 \pm 0.5, n=7 ; \mathrm{CR}^{-/-}$: $3.5 \pm 1, n=7 ; \mathrm{WT}_{\mathrm{DGG}}: 11 \pm 3, n=3$ ).
$1 \_1$ responses (53\%) was highest. Thus, there is a clear shift towards double successes in the mutants, which is consistent with increased release probability and accelerated re- and overfilling of $\mathrm{N}$.

We next focused on the $1 \_1$ responses and calculated PPRs of individual trials by making the ratio of their second to their first EPSC amplitude. PPRs of these responses result from the combined action of presynaptic depletion and postsynaptic effects on the one hand and presynaptic facilitation mechanisms like active $\mathrm{Ca}^{2+}$ and overfilling on the other hand. Thus, they are an index for the relative dominance of either depression or facilitation mechanisms. At ISIs $\leq 100 \mathrm{~ms} \mathrm{PPR}_{1 \_1 / 1}$ (response types used for calculating the ratio in bold) was $\sim 1.2$ in the WT, dropping to unity at larger ISI (Figure 7B open circles). PPR at short ISI was significantly larger than PPR of a hypothetical distribution of PPRs scattering around 1 with the same SE as the real data $\left(P=0.01\right.$; Kruskal-Wallis $(\mathrm{K}-\mathrm{W})$ ANOVA). In $\mathrm{CR}^{-/-}$ $\mathrm{PPR}_{1 \_1 / 1 \_1}$ was not significantly smaller than in WT $(P=0.64$; $\mathrm{K}-\mathrm{W}$ ANOVA). This shows that in both genotypes PPF does not only result from a higher failure rate in the first response (F1) but that the amplitude of the second response itself is facilitated rather than depressed. Since PF terminals harbor a single AZ only, this finding can only be explained if at a single AZ more than one vesicle can fuse per AP. This corroborates the notion that multivesicular release (MVR) can occur from PF terminals (Foster et al., 2005). It shows that the probability of MVR is increased for the second AP. Thus, second EPSC amplitudes per se are increased compared to the first amplitudes and contribute to PPF, both in WT and $\mathrm{CR}^{-/-}$.

We plotted 2nd EPSC amplitudes of double successes (1_1) against their corresponding first amplitudes (Figure 7C) and made linear fits to these data. The slopes of these fits indicate the interplay between presynaptic depletion plus postsynaptic saturation on the one hand and presynaptic replenishment on the other hand. On average (Figure 7D), the slopes of these fits were close to zero, both in WT $(0.04(-0.16-$ $0.215)$, median and IQR) and in $\mathrm{CR}^{-/-}(-0.14(-0.275-0.17)$; $P=0.442$; Mann-Whitney rank sum test), indicating that median second EPSC amplitudes are "stabilized" at a given, on average slightly facilitated (Figure 7B), level, i.e., they appear essentially independent of the amplitude of the first EPSC, both in WT and $\mathrm{KO}$.

The magnitude of this $1 \_\mathbf{1} / \mathbf{1} \_1$ PPF was much smaller than PPF of average responses (Figures 3B, 7B). This may not only result from $\mathrm{F} 1>\mathrm{F} 2$, but in part also from a combination of presynaptic depletion and postsynaptic effects (cf. above). In order to substantiate this, we calculated $\mathrm{PPR}_{0 \_1 / 1 \_x}$ from the ratio of the second amplitude of $0 \_1$ responses to the average of the first amplitude of $1 \_\mathrm{x}(\mathrm{x}=0$ or 1$)$ responses for each ISI (Saviane and Silver, 2006; Bornschein et al., 2013). The 0_1 responses are 
A

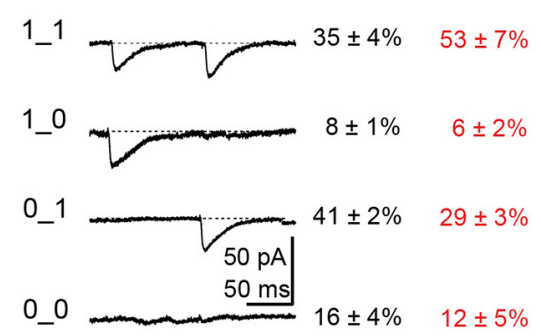

B

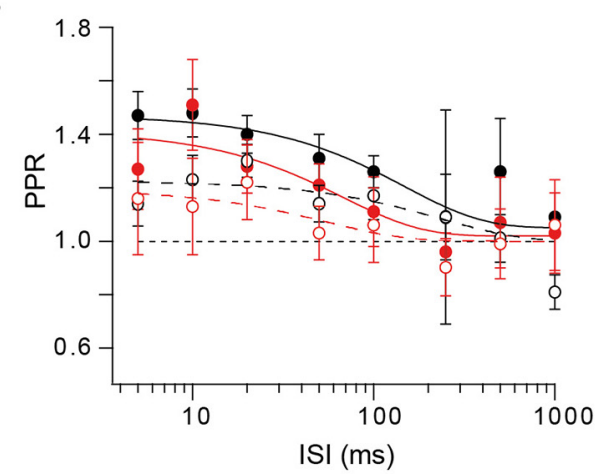

C

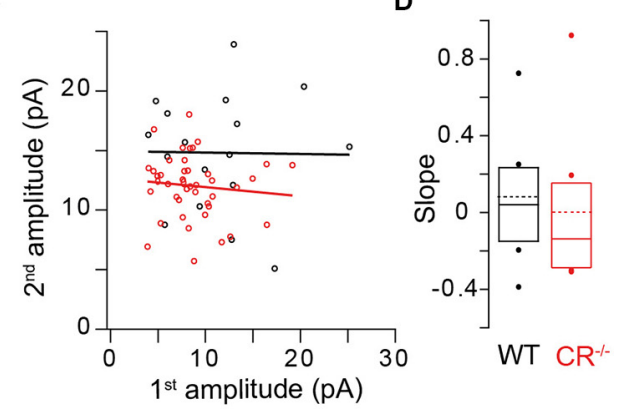

FIGURE 7 | Relationship between release and replenishment. (A) Binary group analysis of the four possible synaptic responses in paired-pulse experiments. Left: Averaged traces from an example cell of successes on both trials (1_1), of failures on both trials $\left(0 \_0\right)$ and of 1_0; $0 \_1$ responses. Right: Percentages (mean $\pm \mathrm{SE}$ ) of the different synaptic responses in WT (black; $n=16$ ) and $\mathrm{CR}^{-/-}$synapses (red; $n=12$ ). (B) ISI dependency of $\mathrm{PPR}_{1 \_1 / 1 \_1}$ (open circles and dashed lines representing exponential fits; mean \pm SE) in WT (black; $n=12 ; \chi^{2}=4.603$ ) and $\mathrm{CR}^{-/}$- synapses (red; $n=12 ; \chi^{2}=0.272$ ). Note that in both genotypes the amplitude of the second EPSC is increased at short ISI rather than depressed. The solid circles and lines show the frequency dependence of PPR __1 1_x $_{\text {f }}$ for WT (black; $\left.\chi^{2}=0.32\right)$ and $\mathrm{CR}^{-1-}\left(\right.$ red; $\left.\chi^{2}=0.378\right)$. Lines represent exponential fits to the data. (C) Second EPSC amplitudes of 1_1 events at ISI $10 \mathrm{~ms}$ plotted against their corresponding first amplitudes for an individual WT (black) or $\mathrm{CR}^{-1-}$ (red) synapse. Lines indicate linear fits to the data (black: slope $=-0.01$, Pearson's $R(\operatorname{Pr})=-0.013, r^{2}=0.0002$; red: slope $=-0.08$, $\left.\operatorname{Pr}=-0.1, r^{2}=0.01\right)$. (D) Summary of all slopes obtained from plots as those shown in (C). Solid lines and boxes indicate median and IQR (see text), dots outlier, dashed lines the mean values for WT (black; $0.08 \pm 0.11$, mean $\pm \mathrm{SE} ; n=8$ ) and $\mathrm{CR}^{-/-}$(red; $0 \pm 0.14 ; n=8 ; P=0.442$;

Mann-Whitney rank sum test).

neither affected by depletion, nor by desensitization since no prior release has occurred. In addition, release machineries of vesicles that were not released during the first pulse may still have been facilitated due to $\mathrm{Ca}^{2+}$ influx during the first AP. Indeed we found that $\mathrm{PPR}_{0 \_1 / 1 \_x}$ was larger $(P=0.019$; K-W ANOVA) than $\mathrm{PPR}_{1 \_1 / 1_{-} 1}$, being $\sim 1.5$ at ISIs $\leq 100 \mathrm{~ms}$ in the WT (Figure $7 \mathrm{~B}$ closed circles). In $\mathrm{CR}^{-/-}$also $\mathrm{PPR}_{0 \_1 / 1 \times x}$ tended to be smaller than in the WT but again the difference was too small to reach the level of significance ( $P=0.3$; K-W ANOVA).

Taken together, our data indicate that multiple forms of plasticity mechanisms, including $\mathrm{Ca}^{2+}$-dependent overfilling/ recruitment, active $\mathrm{Ca}^{2+}$, MVR, and postsynaptic effects coexist at the PF synapse. Consistent with a previous report (Valera et al., 2012), they suggest that overfilling/recruitment is the dominating mechanism of PPF at the PF to PN synapse and explain the persistence of robust PPF at CR-deficient synapses by the presumed $\mathrm{Ca}^{2+}$-dependence of this mechanism.

\section{DISCUSSION}

Using recordings from pairs of GCs and PNs connected via unitary PF synapses, we provide evidence that a $\mathrm{Ca}^{2+}$-driven mechanism that recovers and transiently increases $\mathrm{N}$ between pairs of synaptic activations dominates PPF at PF synapses of mutant mice lacking CR. Thereby, we resolve the apparent discrepancy between high $p_{\mathrm{r}}$ and the preservation of substantial PPF in these mutants. Our findings extend previous results from WT PF synapses (Valera et al., 2012) by estimating $\mathrm{N}$ in the second pulse, by explaining PPF in CR mutants, and by dissecting the contribution of other mechanisms to PPF, including active $\mathrm{Ca}^{2+}$ and MVR (cf. Foster et al., 2005).

Different mechanisms were proposed to generate PPF at distinct synapses (Zucker and Regehr, 2002; Regehr, 2012). Originally, it has been suggested that active $\mathrm{Ca}^{2+}$, i.e., a release machinery facilitated by a residue of bound $\mathrm{Ca}^{2+}$, causes facilitation (Katz and Miledi, 1968). In a simpler form of the "residual $\mathrm{Ca}^{2+}$ hypothesis" a residue of free $\mathrm{Ca}^{2+}\left(\left[\mathrm{Ca}^{2+}\right]_{\text {res }}\right)$ from the first AP summates with free $\mathrm{Ca}^{2+}\left(\left[\mathrm{Ca}^{2+}\right]_{i}\right)$ from the second AP, thereby, causing amplified release. However, it has been recognized early that $\left[\mathrm{Ca}^{2+}\right]_{\text {res }}$ alone cannot account for facilitation (Blundon et al., 1993). In particular due to the large amplitude difference between $\left[\mathrm{Ca}^{2+}\right]_{\text {res }}$ (typically $<1 \mu \mathrm{M}$ ) and nano- or microdomain $\left[\mathrm{Ca}^{2+}\right]_{\mathrm{i}}$ at the release site during the second AP (tens to hundred $\mu \mathrm{M}$ ), simple $\mathrm{Ca}^{2+}$ summation is unlikely to be the major source of facilitation (Zucker and Regehr, 2002). Thus, at different synapses different mechanisms were suggested to underlie PPF. These include slow $\mathrm{Ca}^{2+}$ relaxation of the $\mathrm{Ca}^{2+}$-bound release sensor [i.e., a variant of the original active $\mathrm{Ca}^{2+}$ hypothesis (Yamada and Zucker, 1992; Bertram et al., 1996; Matveev et al., 2002; Bornschein et al., 2013)], separate high-affinity, slow off-rate sites for facilitation (Atluri and Regehr, 1996), elevated release site $\left[\mathrm{Ca}^{2+}\right]_{\mathrm{i}}$ during the second pulse (Geiger and Jonas, 2000; Felmy et al., 2003; Bollmann and Sakmann, 2005), buffer saturation (Neher, 1998a; Rozov et al., 2001), MVR (Foster et al., 2005), or an increase in N, resulting either from transient overfilling of the RP or recruitment of additional (reluctant) release sites (Millar et al., 2005; Sakaba, 2008; Valera et al., 2012).

The size of the release triggering $\mathrm{Ca}^{2+}\left(\left[\mathrm{Ca}^{2+}\right]_{\text {local }}\right)$ has been estimated to be $\sim 20 \mu \mathrm{M}$ at PF synapses, decaying from peak to $\left[\mathrm{Ca}^{2+}\right]_{\text {res }}$ of $\sim 0.5 \mu \mathrm{M}$ within $\sim 2 \mathrm{~ms}$ (Schmidt et al., 2013). 
Assuming a fourth power relationship between $\mathrm{Ca}^{2+}$ and EPSC amplitude, the linear summation of $\left[\mathrm{Ca}^{2+}\right]_{\text {res }}$ and $\left[\mathrm{Ca}^{2+}\right]_{\text {local }}$ can only account for $\leq 10 \%$ facilitation. In addition, it has been estimated that $\left[\mathrm{Ca}^{2+}\right]_{\text {res }}$ decays with an average $\tau$ of $42 \mathrm{~ms}$ under unperturbed conditions (Brenowitz and Regehr, 2007). Thus, PPF, which decayed with $\tau$ of $100 \mathrm{~ms}$ lasted much longer than the elevation in $\left[\mathrm{Ca}^{2+}\right]_{\text {res }}$ (cf. Atluri and Regehr, 1996).

The signature of saturation of endogenous $\mathrm{CaBs}$ is a nonlinear increase in $\left[\mathrm{Ca}^{2+}\right]_{i}$ during repeated synaptic activations (Neher, 1998b). Similarly, non-linear summation of $\left[\mathrm{Ca}^{2+}\right]_{\mathrm{i}}$ would be expected if $\mathrm{Ca}^{2+}$ current facilitation is involved in the generation of PPF (Bollmann and Sakmann, 2005). Yet, at PF synapses $\mathrm{Ca}^{2+}$ sums linearly during repeated activations, both in WT and in $\mathrm{CR}^{-/-}$, making saturation of $\mathrm{CR}$ or $\mathrm{Ca}^{2+}$ influx facilitation unlikely to be involved in facilitation of release from PF terminals (Brenowitz and Regehr, 2007; Schmidt et al., 2013). Consequently, we show here that, consistent with the previous report (Schiffmann et al., 1999), robust PPF persisted in CR mutants.

Specifically for facilitation of release from PF terminals a facilitation sensor (Atluri and Regehr, 1996) or recruitment of additional release sites (Valera et al., 2012) were suggested. Present release sensor models are based on cooperative binding of several $\mathrm{Ca}^{2+}$ ions (Lou et al., 2005; Millar et al., 2005; Sun et al., 2007; Sakaba, 2008). Depending on the rate constants for $\mathrm{Ca}^{2+}$ binding and unbinding, conditioning pulses facilitate these release sensors in the absence of separate facilitation sensors. Thus, the facilitation sensor could be a variant of the facilitated release sensor and not necessarily distinct from the latter one. Considering this, our simulations explain PPF at PF synapses by a synthesis of the two previous suggestions, with the increase in $\mathrm{N}$ making the dominating contribution.

The magnitude of facilitation caused either by facilitation of the release machinery of non-released vesicles or by $\mathrm{Ca}^{2+}$-driven increase in $\mathrm{N}$ may change during postnatal synapse maturation since the active zone and the $\mathrm{Ca}^{2+}$ influx-release coupling undergoes substantial postnatal rearrangement at PF terminals (Baur et al., in press). This may explain why from experiments in younger rats the former process has been suggested (Atluri and Regehr, 1996) and in older mice the latter one (Valera et al., 2012).

STP is a central factor in neuronal information processing (Abbott and Regehr, 2004) with $\mathrm{Ca}^{2+}$-binding proteins being considered important regulators of PPF. Consistent with one previous report (Schiffmann et al., 1999), we found that lack of CR does not dramatically alter PPF of release from PF terminals. Since the mossy-fiber-GC-PF-pathway appears to be specialized for transmitting information in short, facilitating broad-bandwidths bursts of up to $1 \mathrm{kHz}$ (Gall et al., 2003; Chadderton et al., 2004; Rancz et al., 2007; Valera et al., 2012; Ritzau-Jost et al., 2014; Rössert et al., 2014), this is a puzzling result. What might be the functional significance-if anyof CR for synaptic facilitation? Since lack of CR leads to a significant increase in $p_{\mathrm{r}}$ (Schmidt et al., 2013) the preservation of PPF is balanced by an increased vesicle replenishment and an increase in N. Given the enormous number of PF synapses in the mammalian brain and the energy costs of transmitter release and vesicle recycling, this preservation might be rather energy demanding. This suggests that one synaptic function of CR could be optimization of cerebellar energy consumption.

\section{ACKNOWLEDGMENTS}

We thank Gudrun Bethge for technical assistance. This work was supported a DFG grant to HS (SCHM1838/1-1).

\section{REFERENCES}

Abbott, L. F., and Regehr, W. G. (2004). Synaptic computation. Nature 431, 796803. doi: $10.1038 /$ nature 03010

Arendt, O., Schwaller, B., Brown, E. B., Eilers, J., and Schmidt, H. (2013). Restricted diffusion of calretinin in cerebellar granule cell dendrites implies $\mathrm{Ca}^{2+}$-dependent interactions via its EF-hand 5 domain. J. Physiol. 591, 38873899. doi: 10.1113/jphysiol.2013.256628

Atluri, P. P., and Regehr, W. G. (1996). Determinants of the time course of facilitation at the granule cell to Purkinje cell synapse. J. Neurosci. 16, 56615671.

Baur, D., Bornschein, G., Althof, D., Watanabe, M., Kulik, A., Eilers, J., et al. (in press). Developmental tightening of cerebellar cortical synaptic influx-release coupling. J. Neurosci.

Bertram, R., Sherman, A., and Stanley, E. F. (1996). Single-domain/bound calcium hypothesis of transmitter release and facilitation. J. Neurophysiol. 75, 1919-1931.

Blatow, M., Caputi, A., Burnashev, N., Monyer, H., and Rozov, A. (2003). $\mathrm{Ca}^{2+}$ buffer saturation underlies paired pulse facilitation in calbindind28k-containing terminals. Neuron 38, 79-88. doi: 10.1016/s0896-6273(03) 00196-x

Blundon, J. A., Wright, S. N., Brodwick, M. S., and Bittner, G. D. (1993). Residual free calcium is not responsible for facilitation of neurotransmitter release. Proc. Natl. Acad. Sci. U S A 90, 9388-9392. doi: 10.1073/pnas.90.20.9388

Bollmann, J. H., and Sakmann, B. (2005). Control of synaptic strength and timing by the release-site $\mathrm{Ca}^{2+}$ signal. Nat. Neurosci. 8, 426-434. doi: 10.1038/nn1417

Bornschein, G., Arendt, O., Hallermann, S., Brachtendorf, S., Eilers, J., and Schmidt, H. (2013). Paired-pulse facilitation at recurrent Purkinje neuron synapses is independent of calbindin and parvalbumin during high-frequency activation. J. Physiol. 591, 3355-3370. doi: 10.1113/jphysiol.2013.254128

Brenowitz, S. D., and Regehr, W. G. (2007). Reliability and heterogeneity of calcium signaling at single presynaptic boutons of cerebellar granule cells. J. Neurosci. 27, 7888-7898. doi: 10.1523/jneurosci.1064-07.2007

Caillard, O., Moreno, H., Schwaller, B., Llano, I., Celio, M. R., and Marty, A. (2000). Role of the calcium-binding protein parvalbumin in short-term synaptic plasticity. Proc. Natl. Acad. Sci. U S A 97, 13372-13377. doi: 10.1073/pnas. 230362997

Chadderton, P., Margrie, T. W., and Häusser, M. (2004). Integration of quanta in cerebellar granule cells during sensory processing. Nature 428, 856-860. doi: 10 . 1038/nature02442

Clements, J. D., and Silver, R. A. (2000). Unveiling synaptic plasticity: a new graphical and analytical approach. Trends Neurosci. 23, 105-113. doi: 10 . 1016/s0166-2236(99)01520-9

Crowley, J. J., Carter, A. G., and Regehr, W. G. (2007). Fast vesicle replenishment and rapid recovery from desensitization at a single synaptic release site. $J$. Neurosci. 27, 5448-5460. doi: 10.1523/jneurosci.1186-07.2007

Eggermann, E., Bucurenciu, I., Goswami, S. P., and Jonas, P. (2012). Nanodomain coupling between $\mathrm{Ca}^{2+}$ channels and sensors of exocytosis at fast mammalian synapses. Nat. Rev. Neurosci. 13, 7-21. doi: 10.1038/nrn3125

Eggermann, E., and Jonas, P. (2012). How the 'slow' $\mathrm{Ca}^{2+}$ buffer parvalbumin affects transmitter release in nanodomain-coupling regimes. Nat. Neurosci. 15, 20-22. doi: 10.1038/nn.3002

Faas, G. C., Schwaller, B., Vergara, J. L., and Mody, I. (2007). Resolving the fast kinetics of cooperative binding: $\mathrm{Ca}^{2+}$ buffering by calretinin. PLoS Biol. 5:e311. doi: 10.1371/journal.pbio.0050311

Felmy, F., Neher, E., and Schneggenburger, R. (2003). Probing the intracellular calcium sensitivity of transmitter release during synaptic facilitation. Neuron 37 801-811. doi: 10.1016/s0896-6273(03)00085-0 
Foster, K. A., Crowley, J. J., and Regehr, W. G. (2005). The influence of multivesicular release and postsynaptic receptor saturation on transmission at granule cell to Purkinje cell synapses. J. Neurosci. 25, 11655-11665. doi: 10. 1523/jneurosci.4029-05.2005

Gall, D., Roussel, C., Susa, I., D’Angelo, E., Rossi, P., Bearzatto, B., et al. (2003). Altered neuronal excitability in cerebellar granule cells of mice lacking calretinin. J. Neurosci. 23, 9320-9327.

Geiger, J. R., and Jonas, P. (2000). Dynamic control of presynaptic $\mathrm{Ca}^{2+}$ inflow by fast-inactivating $\mathrm{K}^{+}$channels in hippocampal mossy fiber boutons. Neuron 28 , 927-939. doi: 10.1016/s0896-6273(00)00164-1

Hallermann, S., Fejtova, A., Schmidt, H., Weyhersmüller, A., Silver, R. A., Gundelfinger, E. D., et al. (2010). Bassoon speeds vesicle reloading at a central excitatory synapse. Neuron 68, 710-723. doi: 10.1016/j.neuron.2010.10.026

Isope, P., and Barbour, B. (2002). Properties of unitary granule cell->Purkinje cell synapses in adult rat cerebellar slices. J. Neurosci. 22, 9668-9678.

Katz, B., and Miledi, R. (1968). The role of calcium in neuromuscular facilitation. J. Physiol. 195, 481-492. doi: 10.1113/jphysiol.1968.sp008469

Liu, G., Choi, S., and Tsien, R. W. (1999). Variability of neurotransmitter concentration and nonsaturation of postsynaptic AMPA receptors at synapses in hippocampal cultures and slices. Neuron 22, 395-409. doi: 10.1016/s08966273(00)81099-5

Lou, X., Scheuss, V., and Schneggenburger, R. (2005). Allosteric modulation of the presynaptic $\mathrm{Ca}^{2+}$ sensor for vesicle fusion. Nature 435, 497-501. doi: 10 . 1038/nature03568

Matveev, V., Sherman, A., and Zucker, R. S. (2002). New and corrected simulations of synaptic facilitation. Biophys. J. 83, 1368-1373. doi: 10 . 1016/s0006-3495(02)73907-6

Millar, A. G., Zucker, R. S., Ellis-Davies, G. C., Charlton, M. P., and Atwood, H. L. (2005). Calcium sensitivity of neurotransmitter release differs at phasic and tonic synapses. J. Neurosci. 25, 3113-3125. doi: 10.1523/jneurosci.4717-04.2005

Neher, E. (1998a). Usefulness and limitations of linear approximations to the understanding of $\mathrm{Ca}^{++}$signals. Cell Calcium 24, 345-357. doi: 10.1016/s01434160(98)90058-6

Neher, E. (1998b). Vesicle pools and $\mathrm{Ca}^{2+}$ microdomains: new tools for understanding their roles in neurotransmitter release. Neuron 20, 389-399. doi: 10.1016/s0896-6273(00)80983-6

Perkins, K. L. (2006). Cell-attached voltage-clamp and current-clamp recording and stimulation techniques in brain slices. J. Neurosci. Methods 154, 1-18. doi: 10.1016/j.jneumeth.2006.02.010

Rancz, E. A., Ishikawa, T., Duguid, I., Chadderton, P., Mahon, S., and Häusser, M. (2007). High-fidelity transmission of sensory information by single cerebellar mossy fibre boutons. Nature 450, 1245-1248. doi: 10.1038/nature05995

Regehr, W. G. (2012). Short-term presynaptic plasticity. Cold Spring Harb. Perspect. Biol. 4:a005702. doi: 10.1101/cshperspect.a005702

Ritzau-Jost, A., Delvendahl, I., Rings, A., Byczkowicz, N., Harada, H., Shigemoto, R., et al. (2014). Ultrafast action potentials mediate kilohertz signaling at a central synapse. Neuron 84, 152-163. doi: 10.1016/j.neuron.2014.08.036

Rössert, C., Solinas, S., D'Angelo, E., Dean, P., and Porrill, J. (2014). Model cerebellar granule cells can faithfully transmit modulated firing rate signals. Front. Cell. Neurosci. 8:304. doi: 10.3389/fncel.2014.00304

Rozov, A., Burnashev, N., Sakmann, B., and Neher, E. (2001). Transmitter release modulation by intracellular $\mathrm{Ca}^{2+}$ buffers in facilitating and depressing nerve terminals of pyramidal cells in layer $2 / 3$ of the rat neocortex indicates a target cell-specific difference in presynaptic calcium dynamics. J. Physiol. 531, 807826. doi: 10.1111/j.1469-7793.2001.0807h.x

Sakaba, T. (2008). Two $\mathrm{Ca}^{2+}$-dependent steps controlling synaptic vesicle fusion and replenishment at the cerebellar basket cell terminal. Neuron 57, 406-419. doi: 10.1016/j.neuron.2007.11.029

Saviane, C., and Silver, R. A. (2006). Fast vesicle reloading and a large pool sustain high bandwidth transmission at a central synapse. Nature 439, 983-987. doi: 10 . 1038/nature04509
Scheuss, V., Schneggenburger, R., and Neher, E. (2002). Separation of presynaptic and postsynaptic contributions to depression by covariance analysis of successive EPSCs at the calyx of held synapse. J. Neurosci. 22, $728-739$.

Schiffmann, S. N., Cheron, G., Lohof, A., D’Alcantara, P., Meyer, M., Parmentier, M., et al. (1999). Impaired motor coordination and Purkinje cell excitability in mice lacking calretinin. Proc. Natl. Acad. Sci. U S A 96, 5257-5262. doi: 10. 1073/pnas.96.9.5257

Schmidt, H. (2012). Three functional facets of calbindin D-28k. Front. Mol. Neurosci. 5:25. doi: 10.3389/fnmol.2012.00025

Schmidt, H., Brachtendorf, S., Arendt, O., Hallermann, S., Ishiyama, S., Bornschein, G., et al. (2013). Nanodomain coupling at an excitatory cortical synapse. Curr. Biol. 23, 244-249. doi: 10.1016/j.cub.2012.12.007

Schmidt, H., Brown, E. B., Schwaller, B., and Eilers, J. (2003). Diffusional mobility of parvalbumin in spiny dendrites of cerebellar Purkinje neurons quantified by fluorescence recovery after photobleaching. Biophys. J. 84, 2599-2608. doi: 10. 1016/s0006-3495(03)75065-6

Schmidt, H., Schwaller, B., and Eilers, J. (2005). Calbindin D28k targets myoinositol monophosphatase in spines and dendrites of cerebellar Purkinje neurons. Proc. Natl. Acad. Sci. U S A 102, 5850-5855. doi: 10.1073/pnas. 0407855102

Silver, R. A. (2003). Estimation of nonuniform quantal parameters with multipleprobability fluctuation analysis: theory, application and limitations. J. Neurosci. Methods 130, 127-141. doi: 10.1016/j.jneumeth.2003.09.030

Sims, R. E., and Hartell, N. A. (2005). Differences in transmission properties and susceptibility to long-term depression reveal functional specialization of ascending axon and parallel fiber synapses to Purkinje cells. J. Neurosci. 25, 3246-3257. doi: 10.1523/jneurosci.0073-05.2005

Sims, R. E., and Hartell, N. A. (2006). Differential susceptibility to synaptic plasticity reveals a functional specialization of ascending axon and parallel fiber synapses to cerebellar Purkinje cells. J. Neurosci. 26, 5153-5159. doi: 10. 1523/jneurosci.4121-05.2006

Sun, J., Pang, Z. P., Qin, D., Fahim, A. T., Adachi, R., and Südhof, T. C. (2007). A dual- $\mathrm{Ca}^{2+}$-sensor model for neurotransmitter release in a central synapse. Nature 450, 676-682. doi: 10.1038/nature06308

Valera, A. M., Doussau, F., Poulain, B., Barbour, B., and Isope, P. (2012). Adaptation of granule cell to Purkinje cell synapses to high-frequency transmission. J. Neurosci. 32, 3267-3280. doi: 10.1523/JNEUROSCI.3175-11. 2012

Yamada, W. M., and Zucker, R. S. (1992). Time course of transmitter release calculated from simulations of a calcium diffusion model. Biophys. J. 61, 671682. doi: 10.1016/s0006-3495(92)81872-6

Zucker, R. S., and Regehr, W. G. (2002). Short-term synaptic plasticity. Annu. Rev. Physiol. 64, 355-405. doi: 10.1146/annurev.physiol.64.092501.114547

Conflict of Interest Statement: The authors declare that the research was conducted in the absence of any commercial or financial relationships that could be construed as a potential conflict of interest.

Received: 02 December 2014; accepted: 15 January 2015; published online: 03 February 2015.

Citation: Brachtendorf S, Eilers J and Schmidt H (2015) A use-dependent increase in release sites drives facilitation at calretinin-deficient cerebellar parallel-fiber synapses. Front. Cell. Neurosci. 9:27. doi: 10.3389/fncel.2015.00027

This article was submitted to the journal Frontiers in Cellular Neuroscience.

Copyright (c) 2015 Brachtendorf, Eilers and Schmidt. This is an open-access article distributed under the terms of the Creative Commons Attribution License (CC BY). The use, distribution and reproduction in other forums is permitted, provided the original author(s) or licensor are credited and that the original publication in this journal is cited, in accordance with accepted academic practice. No use, distribution or reproduction is permitted which does not comply with these terms. 


\section{Subcellular structural plasticity caused by the absence of the fast $\mathrm{Ca}^{2+}$ buffer calbindin D-28k in recurrent collaterals of cerebellar Purkinje neurons}

\section{David Orduz $^{1}{ }^{*}$, Alain Boom ${ }^{2}$, David Gall ${ }^{1}$, Jean-Pierre Brion ${ }^{2}$, Serge N. Schiffmann ${ }^{1}$ and Beat Schwaller ${ }^{3} *$}

' Laboratory of Neurophysiology, UNI, Université Libre de Bruxelles (ULB), Bruxelles, Belgium

${ }^{2}$ Laboratory of Histology, Neuroanatomy and Neuropathology, UNI, Université Libre de Bruxelles (ULB), Bruxelles, Belgium

${ }^{3}$ Anatomy, Department of Medicine, University of Fribourg, Fribourg, Switzerland

\section{Edited by:}

Christian D. Wilms, University College

London, UK

\section{Reviewed by:}

Laurens Bosman, Erasmus Medical

Center, Netherlands

Davide Pozzi, Humanitas Research

Hospital, Italy

\section{${ }^{*}$ Correspondence}

David Orduz, Neurophysiology and New Microscopies, Laboratory, INSERM U1128, Paris Descartes University, 45 Rue des Saints-Pères, 75006, Paris, France

e-mail: david.orduz-perez@ parisdescartes.fr;

Beat Schwaller, Anatomy, Department of Medicine, University of Fribourg, Route Albert-Gockel 1, CH-1700 Fribourg, Switzerland e-mail: beat.schwaller@unifr.ch
Purkinje cells (PC) control spike timing of neighboring PC by their recurrent axon collaterals. These synapses underlie fast cerebellar oscillations and are characterized by a strong facilitation within a time window of $<20 \mathrm{~ms}$ during paired-pulse protocols. $\mathrm{PC}$ express high levels of the fast $\mathrm{Ca}^{2+}$ buffer protein calbindin D-28K (CB). As expected from the absence of a fast $\mathrm{Ca}^{2+}$ buffer, presynaptic action potential-evoked $\left[\mathrm{Ca}^{2+}\right]_{i}$ transients were previously shown to be bigger in PC boutons of young (second postnatal week) CB-/- mice, yet IPSC mean amplitudes remained unaltered in connected CB-/- PC. Since PC spine morphology is altered in adult CB-/- mice (longer necks, larger spine head volume), we summoned that morphological compensation/adaptation mechanisms might also be induced in $\mathrm{CB}-/-$ PC axon collaterals including boutons. In these mice, biocytin-filled PC reconstructions revealed that the number of axonal varicosities per PC axon collateral was augmented, mostly confined to the granule cell layer. Additionally, the volume of individual boutons was increased, evidenced from z-stacks of confocal images. EM analysis of PC-PC synapses revealed an enhancement in active zone (AZ) length by approximately $23 \%$, paralleled by a higher number of docked vesicles per AZ in CB-/- boutons. Moreover, synaptic cleft width was larger in CB-/- $(23.8 \pm 0.43 \mathrm{~nm})$ compared to wild type $(21.17 \pm 0.39 \mathrm{~nm})$ synapses. We propose that the morphological changes, i.e., the larger bouton volume, the enhanced AZ length and the higher number of docked vesicles, in combination with the increase in synaptic cleft width likely modifies the GABA release properties at this synapse in CB-/- mice. We view these changes as adaptation/homeostatic mechanisms to likely maintain characteristics of synaptic transmission in the absence of the fast $\mathrm{Ca}^{2+}$ buffer $\mathrm{CB}$. Our study provides further evidence on the functioning of the $\mathrm{Ca}^{2+}$ homeostasome.

Keywords: calcium buffer, calbindin D-28k, Cerebellum, axonal recurrent collateral, synaptic cleft, synaptic bouton

\section{INTRODUCTION}

Activity-dependent synaptic plasticity, i.e., modulation of the synaptic strength of two neurons coupled by chemical synapses is the hallmark of most synapses (Kim and Linden, 2007). Induction of plasticity occurs at highly variable time scales. Such changes may be $(i)$ very brief and restricted to the time period from the start to the end of trains of APs and are named STP, (ii) longer lasting as in the case of Hebbian-type plasticity including LTP or depression (LTP and LTD, respectively), or (iii) operating at

\footnotetext{
Abbreviations: AP, action potential; AZ, active zone; $\mathrm{CB}$, calbindin D-28k; CB-/-, calbindin knock-out mice; $\mathrm{CF}$, climbing fiber; $\mathrm{CV}$, coefficient of variation; $\mathrm{DCN}$, deep cerebellar nuclei; EM, electron microscopy; GABA, gamma amino butyric acid; GCL, granular cell layer; HSP, homeostatic synaptic plasticity; IPSC, inhibitory postsynaptic current; LTD, long-term depression; LTP, long-term potentiation; ML, molecular layer; MLI, molecular layer interneuron; $p$, release probability; PC, Purkinje cell; PCL, Purkinje cell layer; PF, parallel fiber; PN, postnatal day; PSD, postsynaptic density; $q$, quantal size; STP, short-term plasticity.
}

time scales of hours to days and the process is referred to as HSP (Turrigiano, 2012). The latter is thought to maintain network stability by fine-tuning global synaptic strength under conditions when its activity diverges from tolerant (stable) physiological levels, e.g., as the consequence of an insult, chronic suppression of activity or mutations in genes implicated in synaptic transmission. Many different processes occurring in the pre- and/or postsynaptic compartment have been described for the various types of plasticity (Zucker and Regehr, 2002; Blitz et al., 2004; Vitureira and Goda, 2013). Since transmitter release at presynaptic terminals is a $\mathrm{Ca}^{2+}$-dependent process, the precise shape of $\mathrm{Ca}^{2+}$ signals within presynaptic terminals is a critical determinant (Cavazzini et al., 2005). Among other components linked to $\mathrm{Ca}^{2+}$ entry and extrusion, $\mathrm{Ca}^{2+}$ buffers are considered as relevant modulators of these presynaptic $\mathrm{Ca}^{2+}$ signals. Examples of such buffers characterized by either slow or fast $\mathrm{Ca}^{2+}$-binding kinetics include parvalbumin (PV) and $\mathrm{CB}$, respectively (Schwaller, 
2010). Both of these proteins were previously shown to modulate STP (Blatow et al., 2003; Collin etal., 2005; Orduz et al., 2013). The quantitative aspects of a presynaptic $\mathrm{Ca}^{2+}$ signal then determine the time course and amount of neurotransmitter released into the synaptic cleft. This, in turn, leads to an appropriate response in the postsynaptic neuron, in the form of an inhibitory or excitatory postsynaptic response, depending on the type of neurotransmitter and on the type(s) of receptors. Also at the postsynaptic side, several mechanisms leading to modulation of synaptic transmission have been described and include receptor saturation, receptor desensitization, receptor distribution/clustering and/or phosphorylation (von Gersdorff and Borst, 2002), but also morphological changes (e.g., spine shape; Alvarez and Sabatini, 2007). Thus, while pre- and postsynaptic compartments show a large degree of plasticity, the architecture of the synaptic cleft, most notably cleft width is considered as relatively resistant to plasticity. The cleft width is assumed to be essentially determined by interactions of proteins anchored in the pre- and postsynaptic membrane including neurexin family members and neuroligins, respectively. Consequently, the cleft width for a particular synapse shows extremely little variation, most often less than $5 \%$, i.e., $0.4 \mathrm{~nm}$ variation for a "typical" synapse cleft width of approximately $20 \mathrm{~nm}$, as modeled based on Monte Carlo simulations (Savtchenko and Rusakov, 2007) or measured from EM images. Structural changes with respect to synaptic cleft width, dimension of the AZ/PSD, curvature and presynaptic bouton volume have been reported. These changes are often the result of short- or long-term pathological insults such as oxygen/glucose deprivation (OGD; Lushnikova et al., 2011), long-term exposure to lead (Han et al., 2014), aluminum (Jing et al., 2004) or biphenyl-A, a presumed endocrine disruptor (Xu et al., 2013a,b). But also exposure of ovarectomized (OVX) rats to estradiol benzoate increases the synaptic cleft width of CA1 hippocampal synapses in the pyramidal cell layer (Xu and Zhang, 2006). Most recently, a change in the synaptic cleft architecture was reported in mice deficient for the neurexin family member contactin associated protein-like 4 (CNTNAP4), a protein implicated in autism spectrum disorders (ASD; Karayannis etal., 2014). Based on the observation that the absence of the fast $\mathrm{Ca}^{2+}$ buffer $\mathrm{CB}$ in presynaptic boutons of PC recurrent axon collaterals of PN7-PN12 mice had no effect on either basic synaptic transmission (mean IPSC amplitude) or on paired-pulse facilitation (PPF; Bornschein et al., 2013), we summoned adaptive/homeostatic mechanisms to be present at this synapse and focused on changes in the synapse morphology. We observed an increase in presynaptic bouton volume, paralleled by an increase in the AZ/PSD length, in the number of docked vesicles and moreover, an increase in synaptic cleft width. This is in line with the proposition of Loebel et al. (2013) that "ongoing synaptic plasticity results in matched presynaptic and postsynaptic modifications, in which elementary modules that span the synaptic cleft are added or removed as a function of experience."

\section{MATERIALS AND METHODS ANIMALS}

The experiments and procedures conformed to the regulations of the Institutional Ethical Committee of the School of Medicine of the Université Libre de Bruxelles, Belgium and the University of Fribourg, Switzerland. In this study we used C57Bl/6J mice as control animals and null-mutant mice for (CB-/- mice; Airaksinen et al., 1997).

\section{CB IMMUNOHISTOCHEMISTRY AND WESTERN BLOT ANALYSES}

To assess developmental $\mathrm{CB}$ expression in the cerebellum, immunohistochemistry with $\mathrm{CB}$ antibodies was performed with sections from mice of the same litters (PN5-PN25). Animals were perfused intracardially with phosphate-buffered saline (PBS), followed by paraformaldehyde $(4 \%)$ and rinsed with PBS $(0.1 \mathrm{M})$. Cerebella were removed and placed overnight in a 4\% PFA solution. Sagittal slices of the cerebellar vermis $(80 \mu \mathrm{m})$ were prepared in ice-cold PBS solution $\left(4^{\circ} \mathrm{C}\right)$. Slices from animals belonging to the same litter were processed simultaneously. Primary antibody incubations were performed at $4^{\circ} \mathrm{C}$ for $24 \mathrm{~h}$, using rabbit anti-CB 38a antiserum (1:500, Swant, Marly, Switzerland) and secondary antibody incubations for $1 \mathrm{~h}$ at room temperature with Alexa Fluor-labeled donkey anti-rabbit 488 (1:500, Invitrogen).

For Western blot analyses, mice from similar age groups (PN6-PN25; $n=3$ animals for each age group/series, 2 series for all but one for the PN25 group) were deeply anesthetized by $\mathrm{CO}_{2}$ inhalation and perfused transcardially with ice-cold PBS solution $\left(4^{\circ} \mathrm{C}\right)$. Dissected cerebellum were homogenized in $10 \mathrm{mM}$ Tris- $\mathrm{HCl} / 1 \mathrm{mM}$ EDTA, $\mathrm{pH}$ 7.4. Soluble protein fractions (supernatant) were obtained by centrifugation of homogenates at $15,000 \times \mathrm{g}$ for $30 \mathrm{~min}$. Proteins $(1 \mu \mathrm{g})$ were separated by SDS-PAGE (12\%) and transferred on nitrocellulose membranes. Membranes were incubated with a blocking solution (LI-COR Biosciences GmbH, Bad Homburg, Germany) for $1 \mathrm{~h}$. Next, they were incubated overnight with primary antibodies against CB (CB 38a; dilution, 1:1000; Swant) or against $\alpha$-actin (mouse monoclonal, dilution 1:1000; Sigma). As secondary antibodies we used either anti-rabbit labeled with IRDye 800CW or anti-mouse labeled with IRDye 680RD (dilution 1:10,000, incubation for $1 \mathrm{~h}$ ). The bands corresponding to $\mathrm{CB}$ and $\alpha$ actin were visualized and quantified by the Odyssey ${ }^{\circledR}$ Infrared Imaging System (LI-COR) and the corresponding software, respectively.

\section{BIOCYTIN-FILLING OF PC AND SYNAPTOPHYSIN LABELING}

Sagittal cerebellar slices $(180 \mu \mathrm{m})$ were prepared from WT and $\mathrm{CB}-/-$ mice (PN18-PN25). Animals were anesthetized with halothane before decapitation. After rapid removal of the cerebellum, slices were cut with a Leica VT1000S vibratome (Leica Microsystems), in ice-cold bicarbonate-buffered saline (BBS) at $4^{\circ} \mathrm{C}$, containing (in $\mathrm{mM}$ ): $125 \mathrm{NaCl}, 2.5 \mathrm{KCl}, 1.25 \mathrm{NaH}_{2} \mathrm{PO}_{4}, 26$ $\mathrm{NaHCO}_{3}, 2 \mathrm{CaCl}_{2}, 1 \mathrm{MgCl}_{2}, 10$ glucose and equilibrated with a $95 \% \mathrm{O}_{2}-5 \% \mathrm{CO}_{2}$ mixture $(\mathrm{pH} 7.3)$. Before experiments, slices were incubated at $34^{\circ} \mathrm{C}$ for $45 \mathrm{~min}$ in the same saline. After this period, a single slice was transferred to a recording chamber and submerged in continuously flowing BBS at $22-24^{\circ} \mathrm{C}$ with a flow rate of $1.5 \mathrm{ml} / \mathrm{min}$.

To increase the probability of finding PC with intact axons and recurrent collateral arbors, we targeted PC located (i) in the second/third somata below the surface of the slice and (ii) 
in flat regions between the apex and base of a lobule. These regions were clearly less damaged during the slicing procedure. PC were visualized with a $63 \mathrm{x}$ water immersion objective placed in a Zeiss upright microscope (Axioskop 2FS Plus, Zeiss) and recorded using the whole-cell configuration of the patch-clamp technique (WCR) with a double EPC-10 operational amplifier (Heka Elektronik). Patch pipettes were made from borosilicate glass capillaries (Hilgenberg $\mathrm{GmbH}$ ) with a two-stage vertical puller (PIP 5, Heka Elektronik) with resistances between 3.5 and $5 \mathrm{M} \Omega$. The intracellular solution contained the following (in $\mathrm{mM}$ ): $150 \mathrm{~K}$-gluconate, $4.6 \mathrm{MgCl}_{2}, 10 \mathrm{~K}$-Hepes, $0.1 \mathrm{~K}$ EGTA, $0.4 \mathrm{Na}-\mathrm{GTP}, 4 \mathrm{Na}-\mathrm{ATP}$, and $0.1 \mathrm{CaCl}_{2}$ (pH 7.2) and $0.4 \%$ biocytin.

WCR was maintained for at least $20 \mathrm{~min}$ after break-in to improve labeling up to distal regions of recurrent collaterals. Once this time period had elapsed, high-resistance outside-out patches were obtained during pipette withdrawal. An extra time period of 15 min was allotted for biocytin diffusion, then slices were fixed overnight in $4 \%$ PFA $\left(4^{\circ} \mathrm{C}\right)$ and rinsed with PBS $(0.1 \mathrm{M})$. For double immunostainings we first incubated slices with a primary rabbit anti-synaptophysin 1 antibody (1:200, Synaptic systems) for $24 \mathrm{~h}$, followed by secondary anti-rabbit 633 antibodies (1:500, Invitrogen) and streptavidin-conjugated NL557 (1:5000, R\&D systems) at room temperature during $1 \mathrm{~h}$.

\section{CONFOCAL MICROSCOPY AND IMAGE ANALYSIS}

Confocal acquisitions were obtained by using an Axiovert 200MLSM 510 META microscope (Zeiss) equipped with a PlanNeofluar $10 \mathrm{x} / 0.3 \mathrm{~W}$ or a C-Apochromat $40 \mathrm{x} / 1.2 \mathrm{~W}$ objective. We used three laser beams (a $488 \mathrm{~nm}$ argon and 543 and $633 \mathrm{~nm}$ helium-neon laser lines) with filters to selectively detect emitted fluorescence from CB-positive regions (BP 500-550 nm), biocytin-filled cells (BP 565-615 nm) and synaptophysin-positive zones (BP 650-710).

For the $\mathrm{CB}$ quantification during $\mathrm{PN}$ development by immunofluorescence acquisition, parameters were optimized for non-saturated CB signals at PN20 on $60 \mu \mathrm{m}$-thick z-stacks composed of $115 \times 115 \mu \mathrm{m}$ images with a z-step of $3 \mu \mathrm{m}$. Subsequently similar stacks for sections from younger mice from the same litter were acquired using the identical parameters. Because fluorescence can be artificially reduced on optical slices as the result of incomplete antibody penetration, we used ImageJ software (http://imagej.nih.gov/ij/) to identify the five images within each stack with the strongest fluorescence intensities. A maximal intensity z-projection was made with these five selected optical slices and we calculated the mean fluorescence values of the PC layer as percentage of fluorescence normalized to PN20. For colocalization studies, the fluorescence intensity profiles were obtained from single optical sections of a PC after biocytin-filling (green) and synaptophysin labeling (red).

For the localization of PC boutons in the different PC layers, biocytin-loaded PC were reconstructed from an area of $350 \times 350 \mu \mathrm{m}$ and consisting of $80 \mathrm{z}$-sections $(0.8 \mu \mathrm{m}$ each $)$. To visualize recurrent collateral boutons on PC somata, z-stacks containing 30 images (thickness of $0.4 \mu \mathrm{m}$ per section) and covering a region of $14 \times 14 \mu \mathrm{m}$ were selected. We applied a median filter to each z-projection from the stacks to reduce noise and to perform morphology measurements (e.g., bouton volume) with ImageJ tools. To assess the distribution pattern of boutons from several PC, all PC somata were superimposed and axonal arbors were oriented in such a way as to respect their position within cerebellar layers and to position the main axon path toward the white matter. We then determined $\mathrm{x}$ and $\mathrm{y}$ positions for each bouton. These coordinates were used to build a $2 \mathrm{D}$-matrix in MatLab environment permitting to quantify the number of boutons within $20-\mu \mathrm{m}$ side length squares. This size was chosen, since it corresponds approximately to the diameter of a PC soma. We then calculated the bouton density as the number of boutons per $\mu \mathrm{m}^{2}$ and used a color gradient scale (heat map) to visualize regions with high and low bouton densities. For bouton volume measurements, we first defined the "boundaries of a bouton" along the axon recurrent collateral as the points at which collaterals dilate to twice its axonal diameter. Next we counted the number of fluorescent voxels between these points (or in the case of terminal boutons only from the starting point). Because a single voxel corresponded to a cube of $0.00004 \mu \mathrm{m}^{3}$ $(0.01 \times 0.01 \times 0.4 \mu \mathrm{m}$ for $\mathrm{x}, \mathrm{y}$, and $\mathrm{z}$ dimensions $)$, we were able to calculate the approximated value of bouton volumes. Changes in volume were more evident when we performed $3 \mathrm{D}$-surface reconstructions of each bouton with Osirix software, as it is shown in Figure 3D.

\section{ELECTRON MICROSCOPY AND ULTRASTRUCTURAL MEASUREMENTS}

Mice (PN20) were anaesthetized and were perfused transcardially with a heparinized saline solution followed by fixative containing $4 \%$ PFA and glutaraldehyde $(0.25 \%)$ in $0.1 \mathrm{M}$ phosphate buffer ( $\mathrm{pH}$ 7.3). Brains were removed, cerebella were dissected and further fixed for $4 \mathrm{~h}$ in the same fixative. For pre-embedding immunolabeling, cerebella were sagittally sectioned on a vibrating blade microtome (Ted Pella, Inc., USA) at a thickness of 35$40 \mu \mathrm{m}$. The sections were processed for immunolabeling with the streptavidin-biotin-peroxidase complex (ABC, Vector, UK). In brief, the tissue sections were pre-incubated for $1 \mathrm{~h}$ in $5 \%$ normal goat serum with $0.5 \%$ Triton X-100 and then incubated for $24 \mathrm{~h}$ at $4^{\circ} \mathrm{C}$ with TBS containing anti-L7 polyclonal rabbit antibody (1:500, Santa Cruz). After washing in TBS, sections were incubated for $4 \mathrm{~h}$ with the goat anti-rabbit antibody conjugated to biotin (1:100, Vector, UK) followed by the streptavidin-biotinperoxidase complex (Vector). The peroxidase activity was revealed using diaminobenzidine as chromogen (DAB; Dako, Belgium). 35-40 $\mu \mathrm{m}$-thick cerebellar slices were then observed with transmitted light on an inverted microscope (Zeiss, Axiovert $200 \mathrm{M}$ ), which corroborated L7-labeling on PC somata and PC collateral boutons from both WT and $\mathrm{CB}-/-$ mice. After washing in Millonig's buffer with $0.5 \%(\mathrm{w} / \mathrm{v})$ sucrose for $24 \mathrm{~h}$, sections were post-fixed in $2 \%(\mathrm{w} / \mathrm{v}) \mathrm{OsO}_{4}$ for $30 \mathrm{~min}$, dehydrated in a graded series of ethanol and embedded in Epon-resin LX112 (Ladd Research Industries, Inc., USA). Semi-thin sections were stained with toluidine blue. Ultrathin sections $(30 \mathrm{~nm})$ collected on nickel grids were stained in $2 \%$ aqueous uranyl acetate and lead citrate and observed with a Zeiss EM 809 microscope at $80 \mathrm{kV}$ coupled to a digital camera (Jenoptik, Prog Res C14, Germany).

PC - PC contacts were found by careful inspection of PC somata. PC slightly labeled by L7 antibodies presented a better 
ultrastructure, which facilitated the extraction of morphological parameters. In contrast, strong labeling hid the most important details by obscuring the synapses. Thus, we focused our analyses on morphologically well preserved, lightly stained PC e.g., shown in Figure 4B.

Morphological measurements were made with ImageJ tools on EM images at x 30,000 magnifications. To analyze PC-PC synapses, we first identified PC soma based on their characteristic soma shape and size. The PC soma was surveyed under high magnification and PC-PC synapses were recognized by the presence of $(i)$ L7-labeling, (ii) symmetrical membrane appositions, (iii) pleomorphic synaptic vesicles, and (iv) eventually its myelinated axon, when the slicing procedure preserved it. We counted the number of AZ per bouton and their individual lengths. Only synapses that had a clear synaptic cleft separating the pre- and postsynaptic elements were taken for width measurements. These measurements required a more standardized method, because the synaptic cleft boundaries are not really well defined when measuring it on EM images. A true consensus is missing on how to reduce the observer's bias in measurements of "the real cleft width." It is often defined as the brightest region between pre and postsynaptic membranes and usually measured as the distance between pre- and postsynaptic electro-dense (black) peaks. However, this procedure can lead to mistakes, because those peaks can vary significantly from one synapse to the other.

To solve this problem we adapted the following method consisting of three steps: (1) on digital-zoomed images, we traced five $100 \mathrm{~nm}$-length straight lines from the pre- to the postsynaptic side, perpendicular to the membranes. These lines were separated from each other by 10 -nm intervals; (2) we extracted the intensity profiles for each line and averaged them. This reduced the noise to better resolve the valley between peaks that is considered to be the real synaptic width; and (3) we identified the point at which the intensity of the signal decreased by $10 \%$ from the presynaptic peak to the minimal signal value into the valley (as it is shown by the gray dotted line in Figure 4C) and similarly for the postsynaptic peak with respect to the minimal value into the valley. The distance between both were assumed to represent the real synaptic width. We found that our method is more robust and less biased compared to measurements by experimenter's eyeinspection as demonstrated by manual-blind measurements $v s$. the standardized method (see Figure S1).

\section{DATA ANALYSIS AND STATISTICS}

Data analyses were performed using Neuromatic software package and custom routines within the IgorPro (Wavemetrics, Lake Oswego, OR, USA) or Matlab environment. All values are expressed as mean \pm SEM. Student's $t$-test were performed within the Excel software package (Microsoft). The significance level was established at $P<0.05\left(^{*}\right)$. Cumulative distributions were compared using the Kolmogorov-Smirnov test. A homoscedasticity test was used to determine whether measurements of synaptic cleft width within a group have equal variances or not (separately for either WT or $\mathrm{CB}-/-$ ). This was important to justify that the homeostatic rearrangement in synaptic cleft values are indeed genotypically stable changes (low variability for each genotype, independent of the number of samples). For this the test initially consists of calculating the ratio of the largest to the smallest of several sample variances for a genotype $\left(\mathrm{F}_{\max }\right)$. If this ratio is equal to unit, then the null hypothesis is accepted (variances are not different), but this was not the case for both genotypes. In that case, one needs to know how high $\mathrm{F}_{\max }$ may be for each genotype before to accept the null hypothesis. This is given by a statistical value called critical- $\mathrm{F}_{\max }=\mathrm{F}_{\max } \alpha[\mathrm{k}, \mathrm{n}-1]$, where $\alpha=0.05 ; k=$ number of mice; $n=$ number of observations per mouse. The critical$\mathrm{F}_{\max }$ for both genotypes, obtained from statistic tables (David, 1952), presented higher values compared to $F_{\max }$ for each genotype, demonstrating that variances are equal within each genotype group. Coefficients of variation were calculated as the square root of the ratio of SD to mean. The box-and-whiskers graphs were performed using Prism 4.0 (GraphPad software) and show the median (the line in the middle) and the 25 th -75 th percentile (the box extension).

\section{RESULTS}

\section{DEVELOPMENTAL INCREASE IN CB EXPRESSION IN MOUSE CEREBELLAR PC REACHING A PLATEAU AT PC MATURITY}

The first three postnatal (PN) weeks are critical for the establishment of a mature cerebellar circuit in rodents (van Welie et al., 2011). Only at the end of the second PN week PC axon collateral arbors stabilize their length, spatial distribution and number of axonal boutons, a process probably achieved by cerebellar myelination that limits the expansion of axon collaterals and conserves final and stable synapses (Gianola et al., 2003). CB has been commonly used as "the prototypical PC marker," but little attention has been paid to the temporal aspect of CB expression during this critical period of cerebellar development. With this aim, we quantified CB expression levels in the cerebellum of WT mice by immunofluorescence and Western blot analyses from PN5 to PN20 (Figure 1). Cerebellar slices at different ages were processed simultaneously and values for the two types of measurements were normalized to PN20 levels. Slices obtained from younger animals (PN5-PN14) were imaged with the same optical settings (see Materials and Methods). CB immunofluorescence was detected at the first PN week (PN5) and the staining intensity increased progressively until PN20 (Figure 1A). Semiquantification of $\mathrm{CB}$ expression measured by the intensity of fluorescence in the PC layer indicated a developmental upregulation of $\mathrm{CB}$ (Figures 1A,B). As a second method to quantify the developmental increase in CB expression levels, we performed Western blot analyses with two additional time points that confirmed the increase in $\mathrm{CB}$ expression during that period; a plateau was reached during the third PN week (Figures 1C,D). The combined results from immunofluorescence and Western blot analyses indicated an approximately twofold increase in CB expression levels taking place between the end of the first and the end of the third PN week. This strongly indicates that morphological stabilization of PC axon collaterals and CB-regulated intracellular $\mathrm{Ca}^{2+}$-signaling processes in PC recurrent collaterals, as well as in the soma and dendrites are likely to be temporally coordinated. We then focused our further investigation on mice from PN18-PN25, time points when CB expression levels had attained steady-state levels, thus all further experiments were carried out with animals of this age range. 

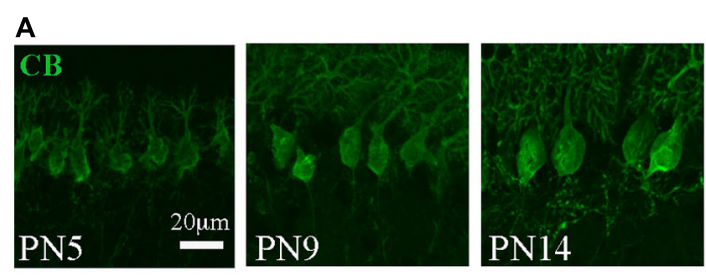

C

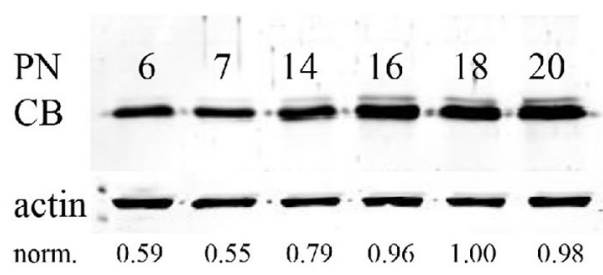

B

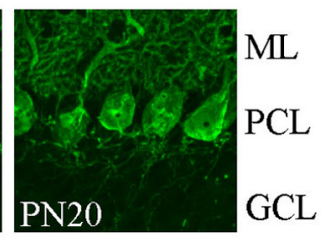

D
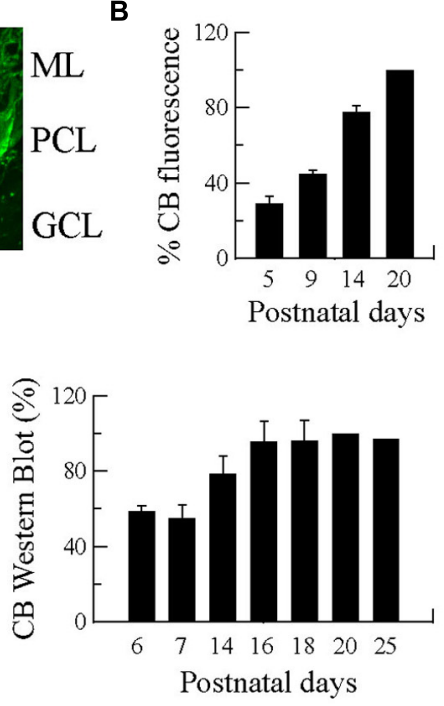

FIGURE 1 | Developmental regulation of calbindin-D28k (CB) expression in mouse cerebellar PC. (A) Confocal images of the cerebellar cortex at different ages from PN5-PN20 on sagittal slices treated with anti-CB antibodies. The expression of $\mathrm{CB}$ is detected early during the first PN week in dendrites, somata and axons of PC. The intensity of IHC signals increases progressively until PN20. (B) Mean CB fluorescence values of the PC layer at different ages $(n=3$ mice per age). Data are normalized to PN20. (C) Age-dependent increase in the total CB protein expression levels from PN6-PN20 determined by quantitative Western blots (average from 2 independent experiments, $n=3$ mice per time point). The actin signal was used for normalization (norm.). In each of the 2 experiments, the normalized signal at PN20 was set as 1.00. (D) Pooled data for Western-blots of cerebella from PN6-PN25 ( $n=3$ mice per age) reached a plateau in CB expression at the end of the third week (ML, molecular layer; PCL, Purkinje cell layer, $\mathrm{GCL}$, granule cell layer).

\section{TERMINAL BOUTONS OF PC COLLATERALS ON NEIGHBOR PC ARE SYNAPSES AND CO-LOCALIZE WITH CB}

The trilaminar architecture of the cerebellar cortex is easily distinguished by $\mathrm{CB}$ immunolabeling of a cerebellar lobule (Figure 2A). PC dendrites, somata, and axons determine the boundaries of the molecular, PC and granule cell layers, respectively. This labeling also distinguishes recurrent collateral arbors and their terminal boutons on neighbor PC (Figure 2B). The strong $\mathrm{CB}$ immunofluorescence of PC somata makes it difficult to resolve presynaptic boutons, a requirement for detailed morphological analyses of PC-PC connections. To surmount this difficulty we loaded PC with biocytin via the patch pipette during whole cell recordings in PN18-25 mice. This approach permitted us to identify from a single PC the following structures: the main axon, recurrent collaterals terminating on the PC layer and terminal boutons on neighbor PC somata (Figure 2C). We confirmed that these boutons made synapses, since they co-localized with the synaptic vesicle glycoprotein, synaptophysin (Figure 2D). With this tool at hand we proceeded to analyze numerous $\mathrm{PC}$ boutons from both WT and CB-/- mice in order to determine their spatial distribution patterns in the presence/absence of $\mathrm{CB}$.

\section{ABSENCE OF CB INDUCES A CHANGE IN NUMBER AND VOLUME OF PRESYNAPTIC TERMINALS OF PC COLLATERALS}

Dendrites and the main axon of PC are aligned in an almost perfect sagittal plane with minimal transversal deviations (Chan-Palay, 1971; King and Bishop, 1982). This is also the case for the recurrent collateral arbors that are entirely confined to very thin optical stacks from the confocal reconstructions of single biocytin-loaded PC, being the thickness of this stack generally $<50 \mu \mathrm{m}$. This planar disposition of recurrent collaterals enabled us to examine confocal projections of PC in order to test whether morphological differences existed with respect to axon collateral synapses between WT and $\mathrm{CB}-/-$ mice.

We compared the distance to the first branch point, the angle that gave rise to the collateral and the total length of the collateral arbor; no statistically significant differences existed between genotypes (Table 1). Second, we evaluated the orientation/distribution of recurrent collateral arbors and boutons. For this we superimposed somata of biocytin-loaded PC and oriented their main axons toward the white matter, in order to align cortical layers (black traces in Figure 3A, $n=10$ cells per genotype). Independent of the genotype, the main axons pointed in the direction of the DCN (to the base of the lobule) with their recurrent collaterals also pointing toward that direction. None to very few of recurrent collaterals returned in the opposite direction, i.e., in the direction toward the apex of the lobule. This indicates that $\mathrm{CB}$ deletion in $\mathrm{CB}-/-$ mice did not affect the spatial orientation either of the principal PC axon or their recurrent collaterals. This is in line with a previous report demonstrating that in connected PC pairs a majority of PC collaterals $(>80 \%)$ projected from the apex to the base of the lobule in both genotypes (Bornschein et al., 2013).

Next, we calculated the bouton density on the superimposed PC per area represented as heat color maps shown in Figure 3A (for details, see Materials and Methods). In both genotypes the highest bouton densities (warm colors in the heat map) were seen 
A

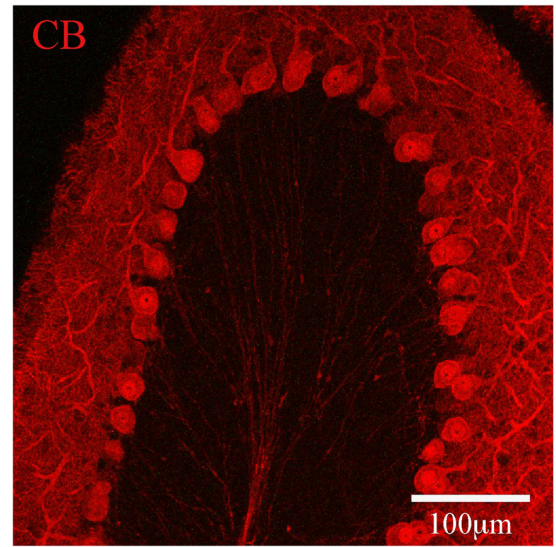

B

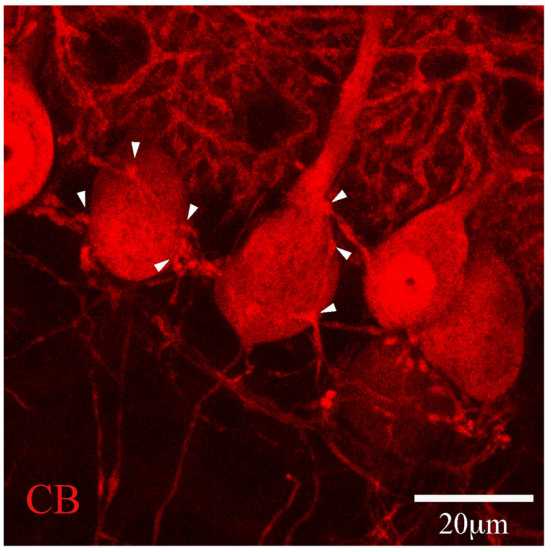

D

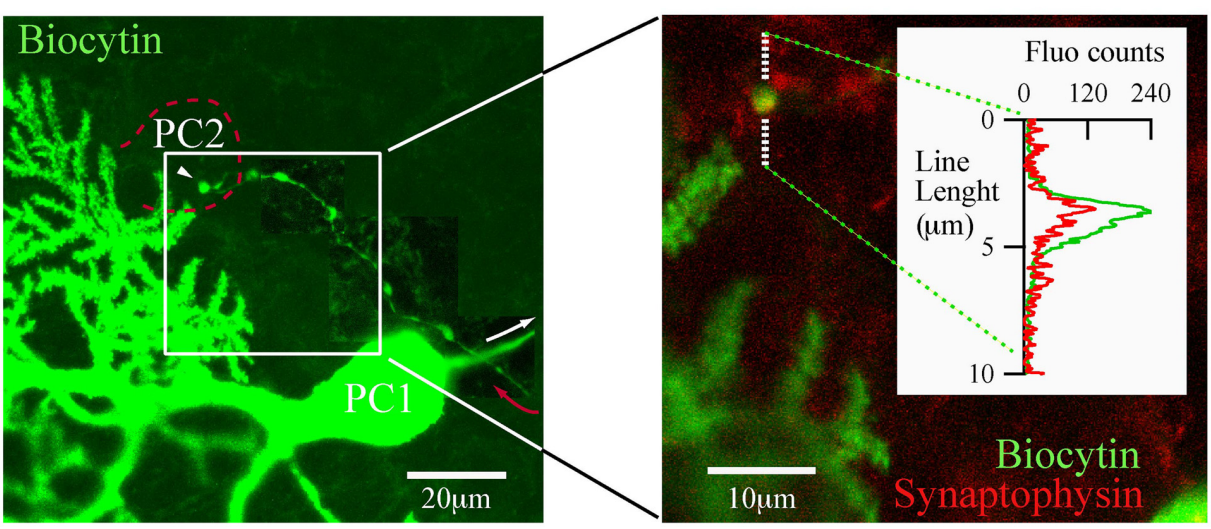

FIGURE 2 | calbindin D-28k labels presynaptic PC collateral boutons onto neighbor PC. (A) Confocal image of a cerebellar lobule showing that in the cerebellar cortex, CB expression is restricted to PC (PN18, sagittal slice). (B) At high resolution, $\mathrm{CB}$ labels the recurrent axon collateral plexus and their boutons on PC somata (white arrowheads). (C) Confocal image of one biocytin-filled PC (PC1; green)) in which the main axon (white arrow) gives rise to a recurrent collateral that returns to the $\mathrm{PC}$ layer (red arrow) and terminates on a neighbor PC soma (PC2, dotted red line). To reveal the axon collateral, the contrast was adapted for three adjacent areas of the final stack. (D) Zoom of the white square in (C) shows a biocytin-loaded bouton (green) on the PC layer (white square) co-localizing with synaptophysin (red). Line analysis (inset) confirmed that boutons on PC layer are bona fide PC-PC synapses. to the right of the parental somata, i.e., in the direction to the base of the lobule. A high prevalence of boutons within the PC layer was evident in comparison to the other layers. This indicates that the main postsynaptic targets of PC collaterals in either genotype were neighbor PC somata located approximately $20-80 \mu \mathrm{m}$ away from the parental soma. However, the total number of boutons per collateral was increased in $\mathrm{CB}-/-$ mice compared to WT animals (Figure 3B, left panel). This difference was mostly due to an increase in GCL boutons $(P<0.05)$ that was accompanied by an increase in the number of branching points in the GCL of the cerebellar cortex (Figure 3B, right panel).

Finally, we focused on the morphology of boutons located on neighbor PC somata to explore subtle presynaptic changes in $\mathrm{PC}-\mathrm{PC}$ synapses resulting from $\mathrm{CB}$ deletion. On images of

Table 1 | Morphological measurements of axonal recurrent collaterals from WT and CB-/- mice.

\begin{tabular}{|c|c|c|c|}
\hline$n$ & WT 10 & CB-/- 10 & $P$ \\
\hline Distance until first branch ( $\mu \mathrm{m})$ & $134.68 \pm 14.61$ & $134.02 \pm 25.6$ & NS \\
\hline Angle deviation of axon collateral $\left(^{\circ}\right)$ & $145.4 \pm 3.51$ & $141.2 \pm 6.26$ & NS \\
\hline Total length collateral arbor ( $\mu \mathrm{m})$ & $807.8 \pm 81.15$ & $950.19 \pm 112.71$ & NS \\
\hline
\end{tabular}

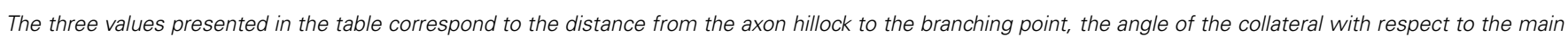

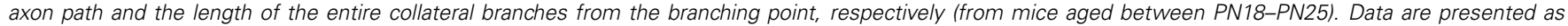
mean \pm SEM. No significant differences (NS) were observed between the two genotypes (Student's t tests). 

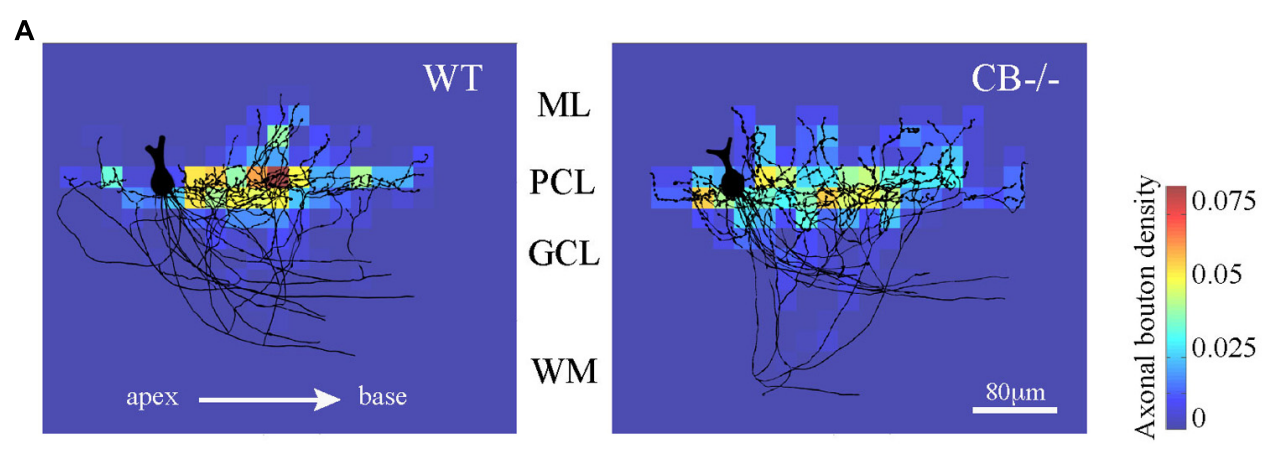

B
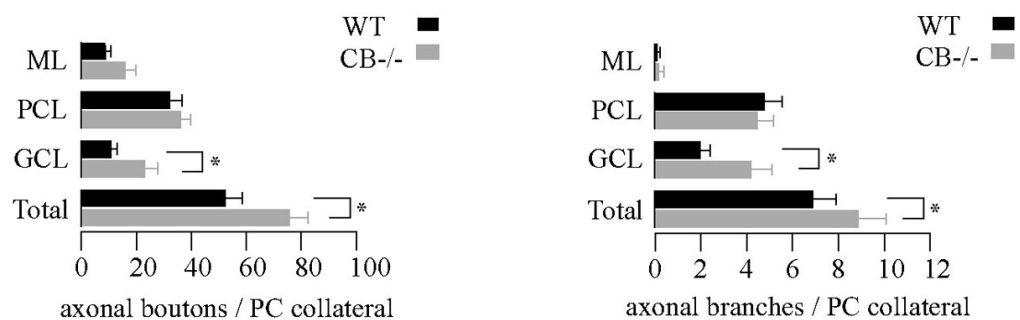

C

D
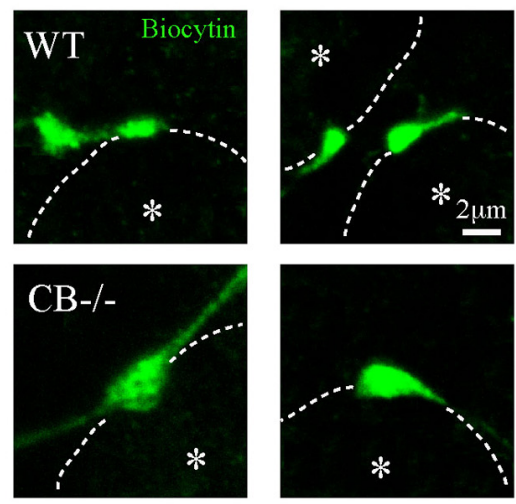
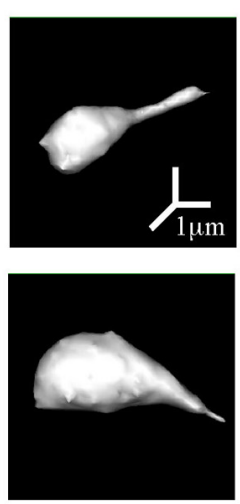

E
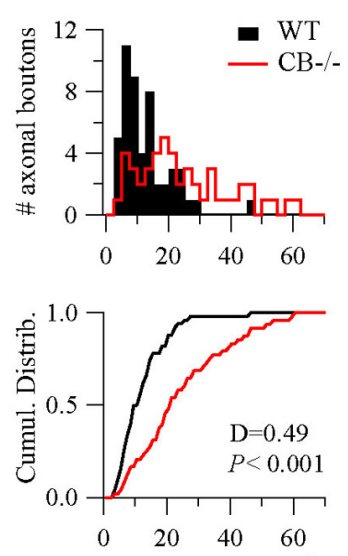

Bouton volume $\left(\mu \mathrm{m}^{3}\right)$
FIGURE 3 | Homeostatic changes in presynaptic boutons of PC recurrent collaterals of $\mathbf{C B}-/-$ mice. (A) Axonal bouton density of PC collaterals in the cerebellar cortex. Biocytin-loaded PC were confocally imaged and superimposed for analysis of bouton distribution $(n=10$ per genotype, PN18-25, see Materials and Methods). Somata were aligned (only one soma is shown, left) and axonal plexuses were oriented in the direction of the main PC axon pointing toward the DCN (right). As indicated by the white arrow, axonal plexuses ramified from the apex to the base of the cerebellar lobule. Heat maps were built and the number of boutons per area was calculated as bouton densities (boutons $/ \mu \mathrm{m}^{2}$ ). Red and blue colors indicate maximal and minimal density of boutons, respectively. Axonal boutons from WT and CB-/- PC showed a similar spatial distribution by targeting more the PC layer and by respecting the apex-to-base orientation. (B) Pooled data for boutons and branches per PC collateral in the three layers of the cerebellar cortex from PC in A. CB-/- PC showed an increase in total number of boutons and branches compared to WT PC. This difference was essentially due to an increase in GCL boutons and branches without significant changes either in PCL or in ML. (C) Confocal projections of biocytin-loaded boutons terminating on neighbor PC somata (dotted white line). White asterisks show the half-radius of the targeted PC soma center. Note the size increase of $\mathrm{CB}-/$ - terminal boutons when compared to WT ones. (D) 3D-projections of the examples in C (right panels) corroborated the increase in volume. (E) Histogram for bouton volume values and the cumulative distributions showed a statistically significant difference between CB-/- compared to the WTs PC boutons. ${ }^{*} P<0.05$, Student's $t$-test z-stack projections of these boutons an increase in the size of CB-/- boutons compared to WT ones was observed (Figure 3C). A 3D-reconstruction pointed toward an increase in volume (Figure 3D), which was confirmed by counting the number of voxels per bouton. The volume distribution histograms showed a right-shift indicative of bigger values for CB-/- boutons compared to the WT boutons, also evidenced in the cumulative distribution curve shown in Figure 3E. In summary, these results imply that a likely homeostatic program of morphological axonal remodeling is induced in the absence of CB. This strongly hints toward changes at the subcellular level possibly in order to cope with the altered $\mathrm{Ca}^{2+}$ transients in $\mathrm{CB}-/-$ presynaptic PC terminals reported before (Bornschein et al., 2013). 


\section{SUBCELLULAR ADAPTATIONS OF PC-PC SYNAPSES IN CB-I- MICE}

To further explore putative changes at the subcellular level of PCPC synapses from both WT and CB-/- mice (PN20), we used EM to visualize presynaptic terminals onto PC somata and to determine their ultra-structural profile. Evidently when analyzing $\mathrm{CB}-/-$ mice, $\mathrm{CB}$ immunohistochemistry could not be used as a means to identify presynaptic terminals. To circumvent this problem we used the L7 protein (also named PCP-2), a marker exclusively localized within PC, expressed in all neuronal compartments and detected from early stages of neuronal maturation (Oberdick et al., 1990; Berrebi and Mugnaini, 1992). Moreover the L7 gene promoter has been widely used to efficiently direct targeted gene expression to PC (Smeyne et al., 1995) and L7 protein expression is observed throughout the PC cytosol. At the light and electron microscopic levels, L7 was visualized on somata and axonal terminals of PC from both genotypes (Figure 4A). Of note, $\mathrm{L}^{+}$boutons surrounding the $\mathrm{PC}$ somata were thinner in WT mice compared to CB-/- animals, which confirmed our previous observations obtained in single biocytin-loaded PC (Figure 3C).

As shown in Figure 4B, we identified PC-PC synapses by a detailed inspection of the somatic PC plasma membrane in order to localize $\mathrm{L}^{+}$boutons with symmetrical synaptic densities and pleomorphic synaptic vesicles as previously reported (Larramendi and Victor, 1967; Chan-Palay, 1971). The number of AZ per bouton was rather small (in the order of $1-3$ ) and similar when comparing WT $(1.19 \pm 0.08 ; n=32$ from 2 mice) vs. $\mathrm{CB}-/-$ synapses $(1.38 \pm 0.1, n=35$ from 2 mice, $P=\mathrm{NS})$. However, the length of individual AZ was 23\% larger at CB-/synapses $(292.08 \pm 15.89 \mathrm{~nm}, n=35)$ compared to AZ of WT synapses $(237.01 \pm 14.43 \mathrm{~nm}, n=32, P<0.05)$. We also measured the synaptic cleft width between pre-post synaptic membranes of those synapses by using a newly developed method aimed to standardize the measurements and to maximally reduce experimenter bias (Figure 4C; also see Materials and Methods and Figure S1). Interestingly, we found that $\mathrm{CB}-/-$ cleft widths were bigger than WT ones $(23.8 \pm 0.43 \mathrm{~nm}$ vs. $21.17 \pm 0.39 \mathrm{~nm}$, respectively; $P<0.001$; Figure 4D). To determine whether variances are equal or not within each group we performed homoscedasticity tests. In this test one calculates first the ratio of the largest to the smallest of the sample variances within one group $\left(\mathrm{F}_{\max }\right)$. If this ratio is bigger than unit, then one calculates what values $F_{\max }$ can attain (critical$\mathrm{F}_{\max }$ ) before one needs to reject the null hypotheses, which is that "variances are different" (see Materials and Methods). Neither for WT nor for $\mathrm{CB}-/-$ mice, $\mathrm{F}_{\max }$ exceeded the critical- $\mathrm{F}_{\max }$ values (1.19 vs. 2.46 for WT and 1.47 vs. 3.72, for CB-/-). This corroborated that synaptic cleft width values were homogeneous within each genotype and are in support that $\mathrm{CB}$ deletion in $\mathrm{CB}-/-$ mice induced a steady and constant change in the pre-post synaptic distance, which is assumed to have an impact on released GABA reaching the postsynaptic PC somata and thus on PC-PC IPSC characteristics (Bornschein et al., 2013).

Based on the observed changes in AZ length in CB-/- mice, we determined the vesicle number within this region, since it constitutes the site of synaptic vesicle clustering, docking and transmitter release (Rizzoli and Betz, 2005). Vesicles were counted in (i) the vicinity of the AZ, a region defined as a half circle with a diameter of the AZ length and (ii) in proximity of the AZ membrane, the latter representing the docked vesicle population (Figure 5). In both compartments, the number of vesicles was higher in $\mathrm{CB}-/$ - presynaptic terminals compared to WT, however, the overall vesicle density was similar in both genotypes: $8.97 \pm 0.82$ vs. $7.21 \pm 0.58$ vesicles per $100 \mathrm{~nm}^{2}$ for WT and CB-/- synapses, respectively ( $P=\mathrm{NS}$ ). This indicates that the number of vesicles was proportional to the AZ length, irrespective of the genotype and the vesicle/AZ length relationship is shown in Figure 5C. The vesicle number depended linearly on the AZ length, as it has been previously reported also for glutamatergic synapses (Holderith et al., 2012). Thus, the higher number of docked vesicles in PC-PC CB-/-synapses is in line with the observation of bigger $q$ quanta values previously recorded in $\mathrm{PC}-\mathrm{PC}$ CB-/- synapses (Bornschein et al., 2013). Of note the coefficient of determination ( $\mathrm{R}$ ) was smaller in $\mathrm{CB}-/$ - synapses compared to WT synapses, for both, the docked vesicles and the ones in the AZ vicinity. This might be an indication of a less tight organization of vesicles in the CB-/- presynaptic compartment and that potentially more vesicles were available for GABA release. This in turn, could underlie the reported increase in $p$. Thus, our results indicate that the absence of $\mathrm{CB}$ also affects the distribution/organization of vesicles at this synapse, likely also affecting the exocytotic machinery and consequently GABA release properties. Interestingly, in line with our results, an increase in the amount of $\mathrm{Ca}^{2+}$ entering the presynaptic terminal, e.g., via $\mathrm{Ca}_{V} 2$-type $\mathrm{Ca}^{2+}$ channels is often paralleled by an increase in the AZ size and the size of the ready-releasable pool of vesicles (Frank, 2014); other reported presynaptic HSP mechanisms include changes in $p$ (Murthy et al., 2001).

\section{DISCUSSION}

The brain is characterized by an extraordinary degree of plasticity, however, controlled by the presence of homeostatic signaling systems to keep the excitatory and inhibitory activity (E/I balance) within a "physiological" range thus allowing for a stable communication between neurons. From the viewpoint of information transfer at synapses, it is evident that synapses are under a bidirectional homeostatic control (Vitureira et al., 2012). By the process of HSP, neurons may modulate their excitability, firing properties and short- and/or long-term synaptic modulation in response to changes in activity in either direction, i.e., as the result of increased or decreased net activity (Lee et al., 2014). Given the importance of $\mathrm{Ca}^{2+}$ ions in the process of synaptic transmission, both preand postsynaptically, an involvement of many components of the $\mathrm{Ca}^{2+}$-signaling toolkit (Berridge et al., 2003) in essentially all forms of synaptic plasticity has been reported (Cavazzini et al., 2005). Examples include presynaptic high-voltage activated $\mathrm{Ca}_{V} 2$ type $\mathrm{Ca}^{2+}$ channels that gate forms of HSP. Weakened synapse activity leads to increased influx through $\mathrm{Ca}_{V} 2$ channels, while enhanced influx via Cav1-type channels elicits homeostatic adaptation by removal of postsynaptic excitatory receptors (for details, see Frank, 2014). The entity of all molecules that build the network of $\mathrm{Ca}^{2+}$ signaling components, and that are involved in their own regulation as to maintain physiological $\mathrm{Ca}^{2+}$ homeostasis resulting in phenotypic stability is named the $\mathrm{Ca}^{2+}$ homeostasome (Schwaller, 2009, 2012a). 
A

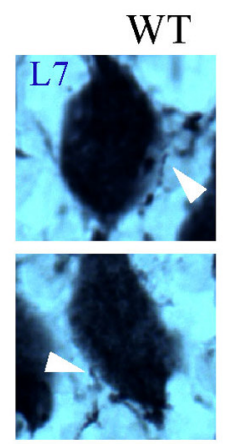

CB-/-
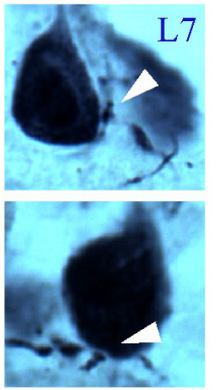

C
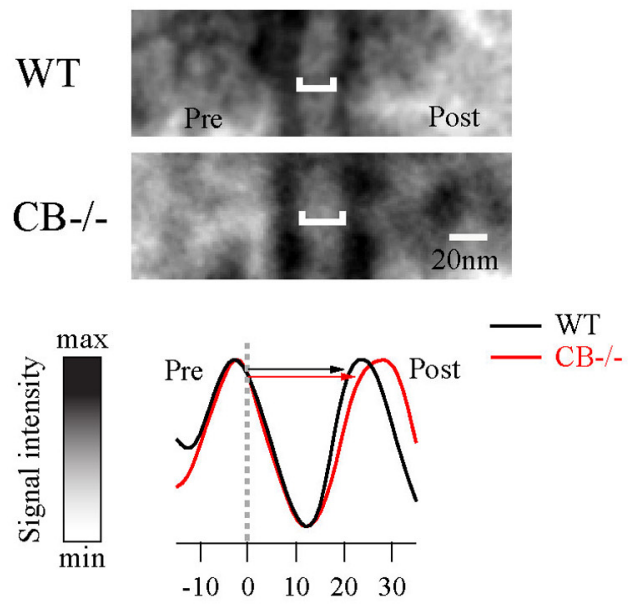

Synaptic Cleft Width $(\mathrm{nm})$

B

CB-/-
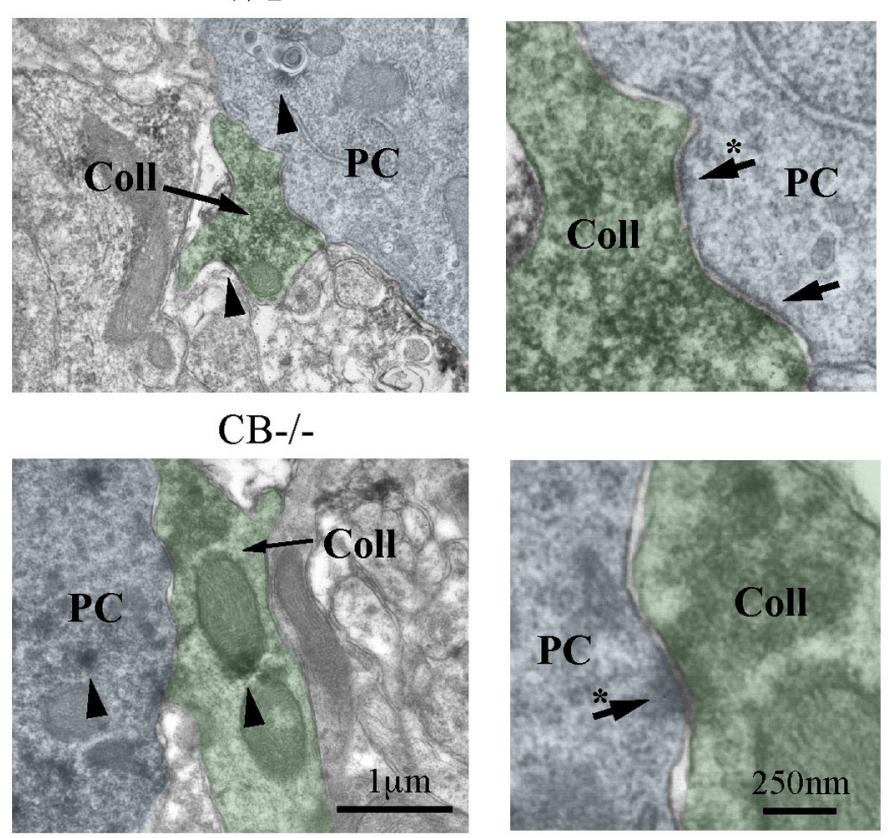

D
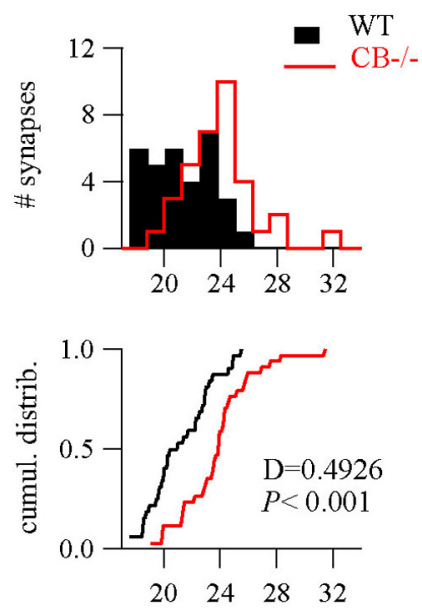

Synaptic Cleft Width (nm)
FIGURE 4 | Ultrastructural changes of PC-PC synapses in CB-/- mice.

(A) L7-immunolabelings were used to visualize axonal boutons on PC somata (white arrowheads) in both WT and CB-/- mice (PN20). WT boutons exhibited a smaller size than those from $\mathrm{CB}-/-$ mice. (B) Electronmicrographs of PC somata (blue background) and their presynaptic PC collateral boutons (green background) from both genotypes were recognized by their $\mathrm{L}^{+}$labeling (arrowheads) at $X 30,000$ magnification. Right panels are zoomed regions to better distinguish AZs (arrows). (C) Zoomed region of AZs from (B) (indicated by arrows with an asterisk on the right panels) showed an enlargement of synaptic cleft width in the CB-/- synapses compared to WT synapses (top). The line analysis method to properly measure synaptic cleft widths (bottom). Averages of five lines including pre/postsynaptic densities per synapse were normalized to peak/valley (see Materials and Methods). (D) Histograms and cumulative distributions showed that in CB-/- PC-PC synapses the right shift in the histogram (upper part) and cumulative distribution plot (lower part) indicate larger synaptic cleft values at CB-/- PC-PC synapses.
The cerebellum represents a model system to investigate synaptic plasticity and the involved $\mathrm{Ca}^{2+}$-dependent processes, both accessible to experimental (reviewed in Lamont and Weber, 2012) and modeling approaches (Achard and Schutter, 2008). This highly repetitive structure consisting of relatively few distinct elements and a rather stereotyped wiring pattern, has allowed to investigate the role of proteins implicated in $\mathrm{Ca}^{2+}$ signaling in the various forms of plasticity. While rather much attention was given to systems implicated in $\mathrm{Ca}^{2+}$ entry from the extracellular side and to release mechanisms from internal stores, as well as systems leading to a decrease in $\left[\mathrm{Ca}^{2+}\right]_{\mathrm{i}}$, studies on the role of intracellular $\mathrm{Ca}^{2+}$-binding proteins in synaptic plasticity in the cerebellum are rather underrepresented. The "fast" buffer CB and the "slow buffer" PV are expressed in PC and PV additionally 
A

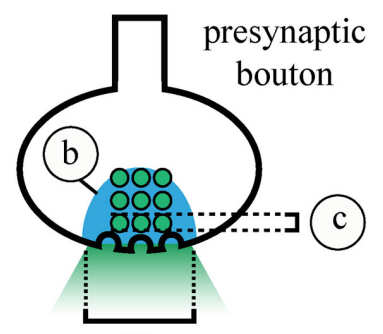

(a)

C

(a) AZ length

(b) Vesicles in AZ-vecinity

(c) Docked vesicles
B

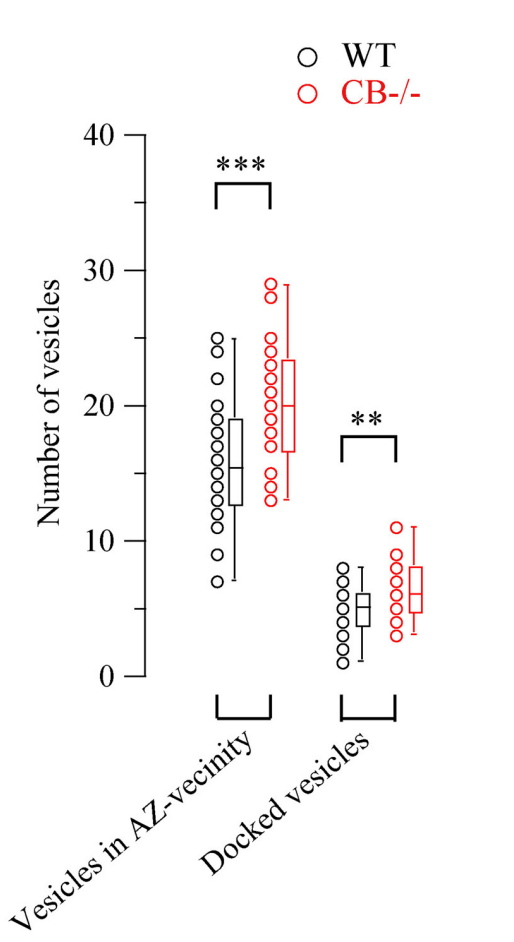

FIGURE 5 | The number of synaptic vesicles scales linearly with AZ length in both WT and CB-I- presynaptic boutons. (A) The scheme shows the two approaches to count vesicles within a bouton in respect to the $A Z$. Vesicles in the "AZ vicinity" are considered as those located in an area covered by a half-circle with the diameter of the $A Z$ length (blue region). Vesicles localized at a distance $<10 \mathrm{~nm}$ from the plasma membrane of the AZ were counted as "docked vesicles." (B) Pooled data from the two defined regions. CB-/- boutons contained more vesicles in the zone defined as "AZ vicinity" as well as docked vesicles per AZ.
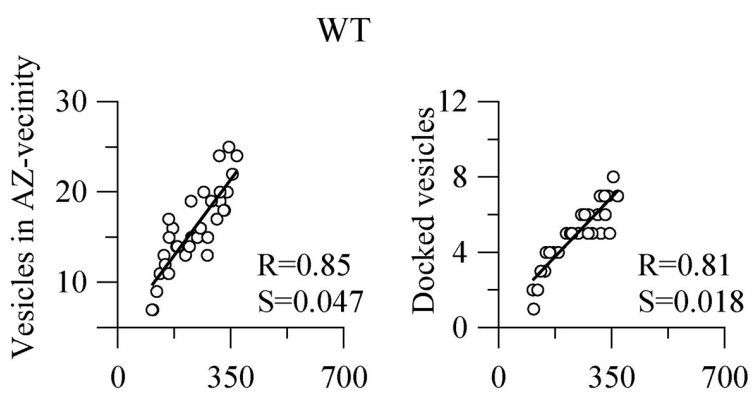

CB-/-

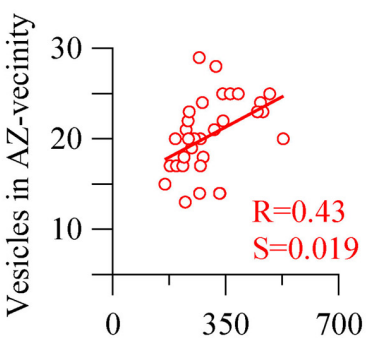

AZ length (nm)

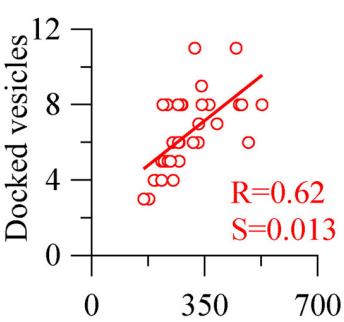

AZ length (nm)

(C) Comparison between vesicle numbers and AZ length for each bouton. A strong linear relationship between $A Z$ length and either docked or associated vesicles strongly indicated that the $A Z$ length dictates the number of vesicles immediately ready to be released as well as those belonging to the pool for slower release. Statistical differences were calculated by Student's $t$ tests ( $\left.{ }^{* *} P<0.01 ;{ }^{* *} P<0.001\right)$. A 2-tailed test of significance was used for linear correlations (Pearson correlation) in (C) showing $P$ values $<0.001$ with the exception of $P<0.01$ for the right bottom panel. $R$, coefficient of determination; $S$, slope.

in stellate and basket cells. The effects that the absence of these proteins entail in PC and MLIs have been described in detail, both at the functional level (Schmidt et al., 2003; Collin et al., 2005; Franconville et al., 2011; Bornschein et al., 2013; reviewed in Schwaller, 2012b), but also at the level of PC morphology (Vecellio et al., 2000; Chen et al., 2006). The increased spine head volume and the longer spine shafts on $\mathrm{CB}-/-\mathrm{PC}$ dendrites had been discussed as a HSP mechanism possibly contributing to unaltered LTD in $\mathrm{CB}-/-$ mice, although dendritic and spine $\left[\mathrm{Ca}^{2+}\right]_{\mathrm{i}}$ dynamics were altered in the absence of CB (Barski et al., 2003).
With respect to the presynaptic function of $\mathrm{CB}$, previous studies on recurrent PC axon collaterals forming synapses on neighboring PC (Orduz and Llano, 2007) have lead to several unexpected findings in CB-deficient mice (Bornschein et al., 2013). Although $\left[\mathrm{Ca}^{2+}\right]_{\mathrm{i}}$ amplitudes evoked by $10 \mathrm{APs}$ delivered at $200 \mathrm{~Hz}$ were clearly larger in PC boutons of CB-/- PC, the evoked IPSC in the postsynaptic PC was unaltered and also PPF characteristics was unchanged in PN7-PN12 mice. This is at an when CB expression levels have not yet attained adult levels and based on our results were estimated to be in the order of 50\% compared 
to values in PN18-PN25 mice (Figure 1). This relates to a CB concentration in the range of $50-180 \mu \mathrm{M}$, since $\mathrm{CB}$ levels in $\mathrm{PC}$ of adult mice were reported to be in the range of $100-$ $360 \mu \mathrm{M}$ (for details, see Schmidt, 2012). Based on a modeling approach, the authors proposed that slow $\mathrm{Ca}^{2+}$ unbinding from the sensor for transmitter release was the main determinant for PPF dynamics and thus independent from CB (Bornschein et al., 2013). This is in contrast to the synapse between cortical CBexpressing multipolar bursting (MB) cells and pyramidal cells, where rapid $\mathrm{Ca}^{2+}$ buffer $(\mathrm{CB})$ saturation resulted in a decreased IPSC amplitude of the first response and increased PPF by this buffer effect, a process termed "facilitation by $\mathrm{Ca}^{2+}$ buffer $(\mathrm{CB})$ saturation (Maeda et al., 1999; Blatow et al., 2003) or "pseudofacilitation" (Neher, 1998; Rozov et al., 2001; Zucker and Regehr, 2002). These experiments had been carried out in acute slices after washout of $\mathrm{CB}$ via the patch pipette (representing the $\mathrm{CB}-/-$ situation) and reverted by loading of terminals with CB or BAPTA, in both cases not allowing for homeostatic plasticity mechanisms to come into play. Additional experiments showed that PPF at $\mathrm{MB}$ cell terminals depend on $\mathrm{Ca}^{2+}$ influx rather than on the initial $p$.

In PC-PC connections in CB-/- mice, both $p$ and $q$ are increased, findings that hinted toward an induction of likely homeostatic mechanisms possibly aimed at restoring IPSC amplitudes and STP as seen in WT terminals. Both parameters, $p$ and $q$, strongly correlate with bouton volume (Schikorski and Stevens, 1997; Murthy etal., 2001) and peak amplitude of presynaptic $\left[\mathrm{Ca}^{2+}\right]$ transients has been seen positively correlated with the AZ area (Holderith et al., 2012). Such a volume increase was also evident in PC boutons from CB-/- mice (Figure 3); moreover an increase in bouton volume is also associated with a larger AZ, paralleled by an almost identical increase in the size of the PSD (Murthy et al., 2001). While a larger AZ encompasses more docked vesicles (Holderith et al., 2012), which is in line with an increase in the magnitude of $q$, such a straightforward correlation appears not to hold true for the $p$. Several possibilities were considered to explain differences in $p$ at CF and PF synapses, despite the number of docked vesicles being similar (Xu-Friedman et al., 2001). Alterations in the priming process of docked vesicles or in the phosphorylation state of proteins implicated in the exocytotic machinery, but also changes in the $\mathrm{Ca}^{2+}$ signal resulting from changes in $\mathrm{Ca}^{2+}$ influx or $\mathrm{Ca}^{2+}$ buffering were discussed to affect $p$. An example of the former was observed in boutons of hippocampal neurons, where an increase in $\mathrm{Ca}^{2+}$ influx by TTX pretreatment increased $p$, while the number of readily releasable vesicles was only marginally reduced (Zhao et al., 2011).

Although EM 3D-reconstructions from serial ultrathin sections could permit a most accurate view of the ultrastructure (Pierce and Mendell, 1993), we considered the AZ/PSD length of recurrent PC axon collaterals as a proxy measure for the area of these appositions, i.e., we assumed these contacts to be circular in shape. Accepting these shortcomings, the area of a PC-PC contact was calculated to be approximately 50\% larger in CB-/- terminals. Studies that reported on the width of the synaptic cleft found the cleft width to show extremely little variation (SD in the order of $<5 \%$ ) for a given synapse type (Jing et al., 2004; Xu and Zhang, 2006; Xu et al., 2013a,b; Han et al., 2014). Changes in cleft width are most often the result of experimental manipulations including OGD, "metal-poisoning" by lead and aluminum (Jing et al., 2004; Lushnikova et al., 2011; Han et al., 2014), but were also observed in genetic models, e.g., CNTNAP4-KO mice (Karayannis et al., 2014). Of note cleft width changes appear to be independent from changes in the length/area of the AZ. After 60 min OGD, AZ area increased by 58\% (Lushnikova et al., 2011) and also the volume of the presynaptic terminal was augmented. In the other models, where cleft width was increased, the length of the AZ was either unaltered, as in the case of estradiol benzoate treatment (Xu et al., 2006) or even reduced as in the cases of bisphenol-A-mediated inhibition of synaptogenesis (Xu et al., 2013a,b) and exposure to either lead (Han et al., 2014) or aluminum (Jing et al., 2004).

One of the problems with measuring cleft width on EM images is the procedure used to accurately measure this parameter. Different approaches have been chosen. Here we have compared a manual and a standardized procedure for measuring cleft width. Although absolute values are different when applying the 2 methods (Figure S1), synaptic cleft width was clearly larger in CB-/PC-PC synapses. The volume of the synaptic cleft was then estimated to be a cylinder defined by the surface area of the AZ/PSD and the height of the synaptic cleft; this volume was increased by $70 \%$ in CB-/- PC-PC synapses. Thus, it is foreseeable that the increased volume would suffice to diminish the GABA concentration at the surface of the postsynaptic side and thus IPSC amplitude, as previously modeled for excitatory synaptic transmission (Wahl et al., 1996). This, in turn may lead to a postsynaptic response (IPSC) of similar magnitude as observed in WT PC (Figure 6).

Besides the changes in the architecture of PC-PC contacts in $\mathrm{CB}-1-$ mice, the number of boutons per PC collateral was increased. Studies on cerebellar synapse formation during postnatal development have mostly focused on the formation of PF-PC synapses and CF-PC synapses. In the mouse the process of CF-PC synapse formation/elimination is a multistep process consisting of initially multiple innervation of a PC soma by several CF (PN3) followed by functional differentiation (PN7). At this time point also PF synapses, as well as MLI synapses start to form. The later stages consist of CF synapse translocation essentially to PC dendrites (PN9) and an early (PN8-PN11) and late (PN12-PN17) phase of CF elimination, resulting in a single CF innervating each PC (reviewed in Hashimoto and Kano, 2013). What is currently known about the temporal development of PC-PC synapses? The PC intracortical plexus starts to form during the first postnatal week characterized by sprouting with a maximal branching reached around PN6 (Gianola et al., 2003). PC collateral start to form synapses around PN7, just at the time when functional differentiation of CF synapses is finished. CF synapse formation is considered to be the first step during cerebellar synaptogenesis (Larramendi, 1969). The second postnatal week is then exemplified by augmented structural plasticity consisting of trimming of collateral branches to less than half compared to PN6 and in remodeling of terminal arbors. Excess fibers that make up this tangled plexus disappear largely around PN20, but stable long-term connections are preserved from PN15 onward in rats (Gianola et al., 2003). Paired PC-PC recordings in young rodents revealed a decrease in the probability of connections between the first and 


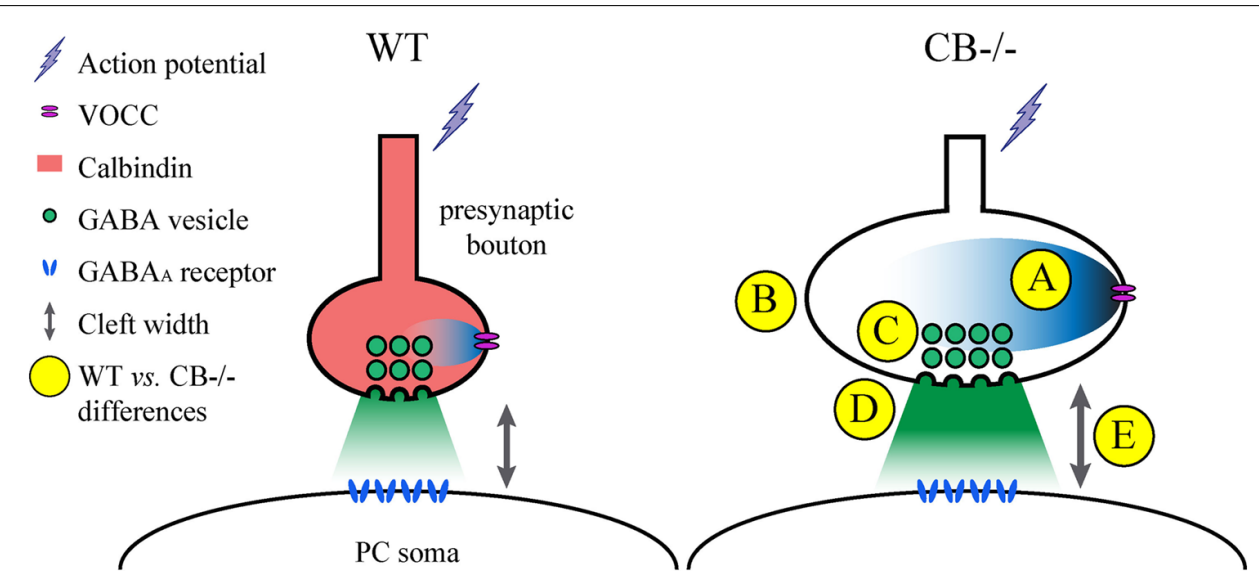

FIGURE 6 | Summary of the observed and proposed alterations at PC-PC synapses due to $\mathbf{C B}$ deletion. (A) An AP arrives at the presynaptic terminal and causes opening of voltage-operated $\mathrm{Ca}^{2+}$ channels (VOCC). The resulting intracellular rise in $\left[\mathrm{Ca}^{2+}\right]_{i}$ is shaped by the presence of CB (Bornschein etal., 2013), which determines the efficacy of synaptic transmission. In the absence of $\mathrm{CB}$, the maximal amplitude of $\mathrm{Ca}^{2+}$ transients is increased in $\mathrm{CB}-/-$ presynaptic boutons compared to WT controls. (B) To possibly reduce the impact of the $\mathrm{Ca}^{2+}$ transients on the amount of GABA released into the synaptic cleft, the volume (and surface) of presynaptic boutons of CB-/- mice is increased. (C) This is also reflected by a stretching of the $A Z$ length, which is accompanied by a proportional increase in the number of ready releasable vesicles. (D) This might explain the enhancement in synaptic efficacy characterized by increased $p$ and $q$ previously observed at CB-/- PC-PC synapses (Bornschein etal., 2013). Yet postsynaptic PC responses with respect to IPSC mean amplitudes or STP are indistinguishable between PC pairs from WT or CB-/- mice (Bornschein et al., 2013). (E) An enlargement of synaptic cleft width and $A Z$ length resulting in an approximately $70 \%$ larger volume of the synaptic cleft might reduce the GABA concentration reaching the postsynaptic GABA receptors finally resulting in unaltered IPSC amplitudes and PPF characteristics at CB-/- PC-PC synapses. It can't be excluded that other mechanisms, e.g., subtle alterations in the subunit composition of $\mathrm{GABA}_{A}$ receptors might also contribute to this likely homeostatic mechanism of plasticity. second PN weeks compared to the third PN week: 26\% at PN4-14 (Watt et al., 2009) and 10\% at PN15-19 (Orduz and Llano, 2007). This decrease may be viewed as an advantage of CF-PC somatic innervation $v s$. PC-PC connections during postnatal development (Larramendi, 1969). Of interest, a mathematical model recapitulating in vivo recordings of cerebellar oscillations in adult rats, in which PC-PC connections are fully operational, requires a connection probability of $20 \%$ between PC in order to reproduce this oscillatory behavior (de Solages et al., 2008). Thus, the higher number of axonal branches and the increased number of boutons in the GCL of CB-/- mice might, in part, explain the strong $160-\mathrm{Hz}$ oscillations observed in adult $\mathrm{CB}-/-$ mice that are essentially absent in WT mice (Servais et al., 2005). Thus, one might speculate that the situation of $\mathrm{PC}$ collaterals in $\mathrm{CB}-/-$ mice resembles the situation of early development, when collaterals are more numerous, inhibition via MLI has not taken place yet, resulting in synchronization in the sagittal plane in the form of traveling waves (Watt et al., 2009).

We reasoned that the time window of the formation of PCPC synapses would be the same as for spine development, i.e., in conjunction with the functional maturation of the cerebellum. In developing hippocampal neurons during the period of rapid synaptogenesis in vitro, the blocking of spike activity by tetrodotoxin (TTX) reduces the density of inhibitory synapses, both onto glutamatergic and GABAergic target neurons, without affecting density of glutamatergic synapses (Hartman et al., 2006). Yet in other experimental settings using cortical or hippocampal cultures, also the density of excitatory synapses was found to be increased after TTX treatment (Wierenga et al., 2006) and decreasing neuronal circuit activity in hippocampal cultures resulted in an increase in the number of connected neuron pairs (Nakayama et al., 2005). Thus, both excitatory as well as inhibitory synapses are receptive for activity-dependent modulation by modifying synapse numbers. Here we propose that the increase in CB-/- PC synaptic boutons might be viewed as a means to increase inhibition onto close neighbor PC. The most prominent increase in axonal boutons was observed in the GCL and less in the PCL indicating that possibly axo-axonic and not axo-somatic inhibition might be increased. Thus, these morphological findings indicate that the output of an individual PC to the DCN is possibly more strongly controlled by neighbor PC. Another interesting point concerns the fact that myelin formation shapes cerebellar connections by removing excess collateral branches of Purkinje neurons (Gianola et al., 2003). In the case of CB-/- mice, the increased branching in the GCL, the layer in which more myelin is wrapped around the PC axons might be indicative of a permissive signal generating branching and possibly more synaptic contacts onto postsynaptic targets of PC axon collaterals, e.g., on other PC axons, on interneurons or even on NG2 ${ }^{+}$progenitors (Boda and Buffo, 2014).

What are the consequences of the absence of $\mathrm{CB}$ with respect to cerebellar function and do the reported HSP mechanisms suffice to "prevent" a CB-/- motor phenotype? As mentioned above, LTD is not affected in CB-/- mice, while motor coordination and motor learning are impaired (Barski et al., 2003; Farré-Castany et al., 2007), as shown in the runway assay, and by measuring the optokinetic reflex OKR (Barski et al., 2003). This has, in part, been attributed to the presence of $160 \mathrm{~Hz}$ cerebellar oscillations recorded from alert $\mathrm{CB}-/-$ mice that are essentially absent in WT mice (Servais et al., 2005). These oscillations are the result of synchronous activity along the PF beam with a likely contribution 
of the recurrent PC axon collaterals (Maex and Schutter, 2005). Whether higher cognitive functions are impaired in $\mathrm{CB}-/-$ mice is currently unknown. A decrease in CB expression levels and/or $\mathrm{CB}$-ir neurons has been reported in several neurological diseases including schizophrenia, bipolar disorder and autism spectrum disorders (Wondolowski and Dickman, 2013). It remains to be investigated whether $\mathrm{CB}-/-$ mice also show behavioral changes reminiscent of these pathologies.

\section{AUTHOR CONTRIBUTIONS}

David Orduz, David Gall, Serge N. Schiffmann, and Beat Schwaller conceived and designed the experiments; David Orduz, Alain Boom, and Beat Schwaller performed research; David Orduz and Beat Schwaller analyzed the data; all authors interpreted the data; David Orduz and Beat Schwaller drafted the manuscript, all authors critically revised the manuscript and approved the final version of the manuscript.

\section{ACKNOWLEDGMENTS}

David Orduz was supported by a postdoctoral fellowship from ULB. We thank Michele Authelet, Brussels, for her advices and expertise in EM that was fundamental for this study. Also we thank the imaging platform LiMif (Belgium) for help with confocal imaging, Patrick Bischop, Brussels, for advices on Igor programming and Andrea Helo, Paris, for help with image analysis on Matlab environment. The help of Martine Steinauer, Fribourg, for the Western blot analyses is highly appreciated. This study was supported by FMRE-Belgium, FRS-FNRS (Belgium) and Interuniversity Attraction Pole (IUAP - P7/10) from Belgian Federal Scientific Affairs.

\section{SUPPLEMENTARY MATERIAL}

The Supplementary Material for this article can be found online at: http://www.frontiersin.org/journal/10.3389/fncel.2014.00364/ abstract

\section{REFERENCES}

Achard, P., and Schutter, E. D. (2008). Calcium, synaptic plasticity and intrinsic homeostasis in purkinje neuron models. Front. Comput. Neurosci. 2:8. doi: 10.3389/neuro.10.008.2008

Airaksinen, M. S., Eilers, J., Garaschuk, O., Thoenen, H., Konnerth, A., and Meyer, M. (1997). Ataxia and altered dendritic calcium signaling in mice carrying a targeted null mutation of the calbindin D28k gene. Proc. Natl. Acad. Sci. U.S.A. 94, 1488-1493. doi: 10.1073/pnas.94.4.1488

Alvarez, V. A., and Sabatini, B. L. (2007). Anatomical and physiological plasticity of dendritic spines. Annu. Rev. Neurosci. 30, 79-97. doi 10.1146/annurev.neuro.30.051606.094222

Barski, J. J., Hartmann, J., Rose, C. R., Hoebeek, F., Mörl, K., Noll-Hussong, M., et al. (2003). Calbindin in cerebellar Purkinje cells is a critical determinant of the precision of motor coordination. J. Neurosci. 23, 3469-3477.

Berrebi, A. S., and Mugnaini, E. (1992). Characteristics of labeling of the cerebellar Purkinje neuron by L7 antiserum. J. Chem. Neuroanat. 5, 235-243. doi: 10.1016/0891-0618(92)90048-U

Berridge, M. J., Bootman, M. D., and Roderick, H. L. (2003). Calcium signalling: dynamics, homeostasis and remodelling. Nat. Rev. Mol. Cell Biol. 4, 517-529. doi: $10.1038 / \mathrm{nrm} 1155$

Blatow, M., Caputi, A., Burnashev, N., Monyer, H., and Rozov, A. (2003). Ca ${ }^{2+}$ buffer saturation underlies paired pulse facilitation in calbindin-D28k-containing terminals. Neuron 38, 79-88. doi: 10.1016/S0896-6273(03)00196-X

Blitz, D. M., Foster, K. A., and Regehr, W. G. (2004). Short-term synaptic plasticity: a comparison of two synapses. Nat. Rev. Neurosci. 5, 630-640. doi: 10.1038/nrn1475
Boda, E., and Buffo, A. (2014). Beyond cell replacement: unresolved roles of NG2expressing progenitors. Front. Neurosci. 8:122. doi: 10.3389/fnins.2014.00122

Bornschein, G., Arendt, O., Hallermann, S., Brachtendorf, S., Eilers, J., and Schmidt, H. (2013). Paired-pulse facilitation at recurrent Purkinje neuron synapses is independent of calbindin and parvalbumin during high-frequency activation. J. Physiol. 591, 3355-3370.

Cavazzini, M., Bliss, T., and Emptage, N. (2005). $\mathrm{Ca}^{2+}$ and synaptic plasticity. Cell Calcium 38, 355-367. doi: 10.1016/j.ceca.2005.06.013

Chan-Palay, V. (1971). The recurrent collaterals of Purkinje cell axons: a correlated study of the rat's cerebellar cortex with electron microscopy and the Golgi method. Z. Anat. Entwicklungsgesch. 134, 200-234. doi: 10.1007/BF00519300

Chen, G., Racay, P., Bichet, S., Celio, M. R., Eggli, P., and Schwaller, B. (2006). Deficiency in parvalbumin, but not in calbindin D-28k upregulates mitochondrial volume and decreases smooth endoplasmic reticulum surface selectively in a peripheral, subplasmalemmal region in the soma of Purkinje cells. Neuroscience 142, 97-105. doi: 10.1016/j.neuroscience.2006.06.008

Collin, T., Chat, M., Lucas, M. G., Moreno, H., Racay, P., Schwaller, B., et al. (2005). Developmental changes in parvalbumin regulate presynaptic $\mathrm{Ca}^{2+}$ signaling. J. Neurosci. 25, 96-107. doi: 10.1523/JNEUROSCI.3748-04.2005

David, H. A. (1952). Upper 5 and 1\% points of the maximum F-ratio. Biometrika 39, 422-424. doi: 10.1093/biomet/39.3-4.422

de Solages, C., Szapiro, G., Brunel, N., Hakim, V., Isope, P., Buisseret, P., et al. (2008). High-frequency organization and synchrony of activity in the purkinje cell layer of the cerebellum. Neuron 58, 775-788. doi: 10.1016/j.neuron.2008.05.008

Farré-Castany, M. A., Schwaller, B., Gregory, P., Barski, J., Mariethoz, C., Eriksson, J. L., et al. (2007). Differences in locomotor behavior revealed in mice deficient for the calcium-binding proteins parvalbumin, calbindin D-28k or both. Behav. Brain Res. 178, 250-261. doi: 10.1016/j.bbr.2007.01.002

Franconville, R., Revet, G., Astorga, G., Schwaller, B., and Llano, I. (2011). Somatic calcium level reports integrated spiking activity of cerebellar interneurons in vitro and in vivo. J. Neurophysiol. 106, 1793-1805. doi: 10.1152/jn.00133.2011

Frank, C. A. (2014). How voltage-gated calcium channels gate forms of homeostatic synaptic plasticity. Front. Cell. Neurosci. 8:40. doi: 10.3389/fncel.2014.00040

Gianola, S., Savio, T., Schwab, M. E., and Rossi, F. (2003). Cell-autonomous mechanisms and myelin-associated factors contribute to the development of Purkinje axon intracortical plexus in the rat cerebellum. J. Neurosci. 23, 4613-4624.

Han, X., Xiao, Y., Ai, B., Hu, X., Wei, Q., and Hu, Q. (2014). Effects of organic selenium on lead-induced impairments of spatial learning and memory as well as synaptic structural plasticity in rats. Biol. Pharm. Bull. 37, 466-474. doi: 10.1248/bpb.b13-00892

Hartman, K. N., Pal, S. K., Burrone, J., and Murthy, V. N. (2006). Activity-dependent regulation of inhibitory synaptic transmission in hippocampal neurons. Nat. Neurosci. 9, 642-649. doi: 10.1038/nn1677

Hashimoto, K., and Kano, M. (2013). Synapse elimination in the developing cerebellum. Cell Mol. Life Sci. 70, 4667-4680. doi: 10.1007/s00018-013-1405-2

Holderith, N., Lorincz, A., Katona, G., Rózsa, B., Kulik, A., Watanabe, M., et al. (2012). Release probability of hippocampal glutamatergic terminals scales with the size of the active zone. Nat. Neurosci. 15, 988-997. doi: 10.1038/nn.3137

Jing, Y., Wang, Z., and Song, Y. (2004). Quantitative study of aluminuminduced changes in synaptic ultrastructure in rats. Synapse 52, 292-298. doi: 10.1002/syn.20025

Karayannis, T., Au, E., Patel, J. C., Kruglikov, I., Markx, S., Delorme, R., et al. (2014). Cntnap4 differentially contributes to GABAergic and dopaminergic synaptic transmission. Nature 511, 236-240. doi: 10.1038/nature13248

Kim, S. J., and Linden, D. J. (2007). Ubiquitous plasticity and memory storage. Neuron 56, 582-592. doi: 10.1016/j.neuron.2007.10.030

King, J. S., and Bishop, G. A. (1982). The synaptic features of horseradish peroxidaselabelled recurrent collaterals in the ganglionic plexus of the cat cerebellar cortex. J. Neurocytol. 11, 867-880. doi: 10.1007/BF01148305

Lamont, M. G., and Weber, J. T. (2012). The role of calcium in synaptic plasticity and motor learning in the cerebellar cortex. Neurosci. Biobehav. Rev. 36, 1153-1162. doi: 10.1016/j.neubiorev.2012.01.005

Larramendi, E. M., and Victor, T. (1967). Synapses on the purkinje cell spines in the mouse. An electronmicroscopic study. Brain Res. 5, 15-30. doi: 10.1016/00068993(67)90216-8

Larramendi, L. M. (1969). “Analysis of synaptogenesis in the cerebellum of mice," in Neurobiology of Cerebellar Evolution and Development, ed. R. Llinas (Chicago: American Medical Assoc), 803-843. 
Lee, K. F. H., Soares, C., and Béïque, J.-C. (2014). Tuning into diversity of homeostatic synaptic plasticity. Neuropharmacology 78, 31-37. doi: 10.1016/j.neuropharm.2013.03.016

Loebel, A., Bé, J.-V. L., Richardson, M. J. E., Markram, H., and Herz, A. V. M. (2013). Matched pre- and post-synaptic changes underlie synaptic plasticity over long time scales. J. Neurosci. 33, 6257-6266. doi: 10.1523/JNEUROSCI.374012.2013

Lushnikova, I., Skibo, G., Muller, D., and Nikonenko, I. (2011). Excitatory synaptic activity is associated with a rapid structural plasticity of inhibitory synapses on hippocampal CA1 pyramidal cells. Neuropharmacology 60, 757-764. doi: 10.1016/j.neuropharm.2010.12.014

Maeda, H., Ellis-Davies, G. C., Ito, K., Miyashita, Y., and Kasai, H. (1999). Supralinear $\mathrm{Ca}^{2+}$ signaling by cooperative and mobile $\mathrm{Ca}^{2+}$ buffering in Purkinje neurons. Neuron 24, 989-1002. doi: 10.1016/S0896-6273(00)81045-4

Maex, R., and Schutter, E. D. (2005). Oscillations in the cerebellar cortex: a prediction of their frequency bands. Prog. Brain Res. 148, 181-188. doi: 10.1016/S0079-6123(04)48015-7

Murthy, V. N., Schikorski, T., Stevens, C. F., and Zhu, Y. (2001). Inactivity produces increases in neurotransmitter release and synapse size. Neuron 32, 673-682. doi: 10.1016/S0896-6273(01)00500-1

Nakayama, K., Kiyosue, K., and Taguchi, T. (2005). Diminished neuronal activity increases neuron-neuron connectivity underlying silent synapse formation and the rapid conversion of silent to functional synapses. J. Neurosci. 25, 4040-4051. doi: 10.1523/JNEUROSCI.4115-04.2005

Neher, E. (1998). Usefulness and limitations of linear approximations to the understanding of Ca++ signals. Cell Calcium 24, 345-357. doi: 10.1016/S01434160(98)90058-6

Oberdick, J., Smeyne, R. J., Mann, J. R., Zackson, S., and Morgan, J. I. (1990). A promoter that drives transgene expression in cerebellar Purkinje and retinal bipolar neurons. Science 248, 223-226. doi: 10.1126/science. 2109351

Orduz, D., Bischop, D. P., Schwaller, B., Schiffmann, S. N., and Gall, D. (2013). Parvalbumin tunes spike-timing and efferent short-term plasticity in striatal fast spiking interneurons. J. Physiol. 591, 3215-3232.

Orduz, D., and Llano, I. (2007). Recurrent axon collaterals underlie facilitating synapses between cerebellar Purkinje cells. Proc. Natl. Acad. Sci. U.S.A. 104, 17831-17836. doi: 10.1073/pnas.0707489104

Pierce, J. P., and Mendell, L. M. (1993). Quantitative ultrastructure of Ia boutons in the ventral horn: scaling and positional relationships. J. Neurosci. 13, 4748-4763.

Rizzoli, S. O., and Betz, W. J. (2005). Synaptic vesicle pools. Nat. Rev. Neurosci. 6, 57-69. doi: 10.1038/nrn1583

Rozov, A., Burnashev, N., Sakmann, B., and Neher, E. (2001). Transmitter release modulation by intracellular $\mathrm{Ca}^{2+}$ buffers in facilitating and depressing nerve terminals of pyramidal cells in layer $2 / 3$ of the rat neocortex indicates a target cell-specific difference in presynaptic calcium dynamics. J. Physiol. 531, 807-826. doi: 10.1111/j.1469-7793.2001.0807h.x

Savtchenko, L. P., and Rusakov, D. A. (2007). The optimal height of the synaptic cleft. Proc. Natl. Acad. Sci. U.S.A. 104, 1823-1828. doi: 10.1073/pnas.0606636104

Schikorski, T., and Stevens, C. F. (1997). Quantitative ultrastructural analysis of hippocampal excitatory synapses. J. Neurosci. 17, 5858-5867.

Schmidt, H. (2012). Three functional facets of calbindin D-28k. Front. Mol. Neurosci. 5:25. doi: 10.3389/fnmol.2012.00025

Schmidt, H., Stiefel, K. M., Racay, P., Schwaller, B., and Eilers, J. (2003). Mutational analysis of dendritic $\mathrm{Ca}^{2+}$ kinetics in rodent Purkinje cells: role of parvalbumin and calbindin $\mathrm{D}_{28 k}$. J. Physiol. 551, 13-32. doi: 10.1113/jphysiol.2002.035824

Schwaller, B. (2009). The continuing disappearance of "pure" $\mathrm{Ca}^{2+}$ buffers. Cell. Mol. Life Sci. 66, 275-300. doi: 10.1007/s00018-008-8564-6

Schwaller, B. (2010). Cytosolic $\mathrm{Ca}^{2+}$ buffers. Cold Spring Harb. Perspect. Biol. 2, a004051. doi: 10.1101/cshperspect.a004051

Schwaller, B. (2012a). The regulation of a cell's $\mathrm{Ca}^{2+}$ signaling toolkit: the $\mathrm{Ca}^{2+}$ homeostasome. Adv. Exp. Med. Biol. 740, 1-25. doi: 10.1007/978-94-0072888-2_1

Schwaller, B. (2012b). The use of transgenic mouse models to reveal the functions of $\mathrm{Ca}^{2+}$ buffer proteins in excitable cells. Biochim. Biophys. Acta 1820, 1294-1303. doi: 10.1016/j.bbagen.2011.11.008

Servais, L., Bearzatto, B., Schwaller, B., Dumont, M., Saedeleer, C. D., Dan, B. et al. (2005). Mono- and dual-frequency fast cerebellar oscillation in mice lacking parvalbumin and/or calbindin D-28k. Eur. J. Neurosci. 22, 861-870. doi: 10.1111/j.1460-9568.2005.04275.x
Smeyne, R. J., Chu, T., Lewin, A., Bian, F., Sanlioglu, S., S-Crisman, S., et al. (1995) Local control of granule cell generation by cerebellar Purkinje cells. Mol. Cell. Neurosci. 6, 230-251. doi: 10.1006/mcne.1995.1019

Turrigiano, G. (2012). Homeostatic synaptic plasticity: local and global mechanisms for stabilizing neuronal function. Cold Spring Harb. Perspect. Biol. 4:a005736. doi: 10.1101/cshperspect.a005736

van Welie, I., Smith, I. T., and Watt, A. J. (2011). The metamorphosis of the developing cerebellar microcircuit. Curr. Opin. Neurobiol. 21, 245-253. doi: 10.1016/j.conb.2011.01.009

Vecellio, M., Schwaller, B., Meyer, M., Hunziker, W., and Celio, M. R. (2000). Alterations in Purkinje cell spines of calbindin D-28 k and parvalbumin knockout mice. Eur. J. Neurosci. 12, 945-954. doi: 10.1046/j.1460-9568.2000.00986.x

Vitureira, N., and Goda, Y. (2013). Cell biology in neuroscience: the interplay between Hebbian and homeostatic synaptic plasticity. J. Cell Biol. 203, 175-186. doi: $10.1083 /$ jcb. 201306030

Vitureira, N., Letellier, M., and Goda, Y. (2012). Homeostatic synaptic plasticity: from single synapses to neural circuits. Curr. Opin. Neurobiol. 22, 516-521. doi: 10.1016/j.conb.2011.09.006

von Gersdorff, H., and Borst, J. G. G. (2002). Short-term plasticity at the calyx of held. Nat. Rev. Neurosci. 3, 53-64. doi: 10.1038/nrn705

Wahl, L. M., Pouzat, C., and Stratford, K. J. (1996). Monte Carlo simulation of fast excitatory synaptic transmission at a hippocampal synapse. J. Neurophysiol. 75, 597-608.

Watt, A. J., Cuntz, H., Mori, M., Nusser, Z., Sjöström, P. J., and Häusser, M. (2009). Traveling waves in developing cerebellar cortex mediated by asymmetrical Purkinje cell connectivity. Nat. Neurosci. 12, 463-473. doi: 10.1038/nn.2285

Wierenga, C. J., Walsh, M. F., and Turrigiano, G. G. (2006). Temporal regulation of the expression locus of homeostatic plasticity. J. Neurophysiol. 96, 2127-2133. doi: 10.1152/jn.00107.2006

Wondolowski, J., and Dickman, D. (2013). Emerging links between homeostatic synaptic plasticity and neurological disease. Front. Cell. Neurosci. 7:223. doi: 10.3389 /fncel.2013.00223

Xu, X., Liu, X., Zhang, Q., Zhang, G., Lu, Y., Ruan, Q., et al. (2013a). Sex-specific effects of bisphenol-A on memory and synaptic structural modification in hippocampus of adult mice. Horm. Behav. 63, 766-775. doi: 10.1016/j.yhbeh.2013.03.004

Xu, X., Xie, L., Hong, X., Ruan, Q., Lu, H., Zhang, Q., et al. (2013b). Perinatal exposure to bisphenol-A inhibits synaptogenesis and affects the synaptic morphological development in offspring male mice. Chemosphere 91, 1073-1081. doi: 10.1016/j.chemosphere.2012.12.065

$\mathrm{Xu}, \mathrm{X}$., and Zhang, Z. (2006). Effects of estradiol benzoate on learning-memory behavior and synaptic structure in ovariectomized mice. Life Sci. 79, 1553-1560. doi: 10.1016/j.lfs.2006.04.020

Xu-Friedman, M. A., Harris, K. M., and Regehr, W. G. (2001). Three-dimensional comparison of ultrastructural characteristics at depressing and facilitating synapses onto cerebellar Purkinje cells. J. Neurosci. 21, 6666-6672.

Zhao, C., Dreosti, E., and Lagnado, L. (2011). Homeostatic synaptic plasticity through changes in presynaptic calcium influx. J. Neurosci. 31, 7492-7496. doi: 10.1523/JNEUROSCI.6636-10.2011

Zucker, R. S., and Regehr, W. G. (2002). Short-term synaptic plasticity. Annu. Rev. Physiol. 64, 355-405. doi: 10.1146/annurev.physiol.64.092501.114547

Conflict of Interest Statement: The authors declare that the research was conducted in the absence of any commercial or financial relationships that could be construed as a potential conflict of interest.

Received: 26 August 2014; accepted: 14 October 2014; published online: 05 November 2014.

Citation: Orduz D, Boom A, Gall D, Brion J-P, Schiffmann SN and Schwaller B (2014) Subcellular structural plasticity caused by the absence of the fast $\mathrm{Ca}^{2+}$ buffer calbindin D-28k in recurrent collaterals of cerebellar Purkinje neurons. Front. Cell. Neurosci. 8:364. doi: 10.3389/fncel.2014.00364

This article was submitted to the journal Frontiers in Cellular Neuroscience. Copyright (C) 2014 Orduz, Boom, Gall, Brion, Schiffmann and Schwaller. This is an open-access article distributed under the terms of the Creative Commons Attribution License (CC BY). The use, distribution or reproduction in other forums is permitted, provided the original author(s) or licensor are credited and that the original publication in this journal is cited, in accordance with accepted academic practice. No use, distribution or reproduction is permitted which does not comply with these terms. 


\section{OPEN ACCESS}

Edited by:

Hartmut Schmidt,

University of Leipzig, Germany

Reviewed by:

David Gall,

University Libre de Bruxelles, Belgium

Guido C. Faas,

University of California, Los Angeles,

USA

*Correspondence:

Kirill E. Volynski,

University College London Institute of Neurology, University College London,

Queen Square, London WC1N 3BG,

UK

k.volynski@ucl.ac.uk

Received: 27 February 2015 Accepted: 12 June 2015

Published: 01 July 2015

Citation:

Timofeeva Y and Volynski KE (2015) Calmodulin as a major calcium buffer shaping vesicular release and short-term synaptic plasticity:

facilitation through buffer dislocation.

Front. Cell. Neurosci. 9:239.

doi: 10.3389/fncel.2015.00239

\section{Calmodulin as a major calcium buffer shaping vesicular release and short-term synaptic plasticity: facilitation through buffer dislocation}

\author{
Yulia Timofeeva ${ }^{1,2}$ and Kirill E. Volynski ${ }^{3 *}$ \\ ${ }^{1}$ Department of Computer Science, University of Warwick, Coventry, UK, ${ }^{2}$ Centre for Complexity Science, University of \\ Warwick, Coventry, UK, ${ }^{3}$ University College London Institute of Neurology, University College London, London, UK
}

Action potential-dependent release of synaptic vesicles and short-term synaptic plasticity are dynamically regulated by the endogenous $\mathrm{Ca}^{2+}$ buffers that shape $\left[\mathrm{Ca}^{2+}\right]$ profiles within a presynaptic bouton. Calmodulin is one of the most abundant presynaptic proteins and it binds $\mathrm{Ca}^{2+}$ faster than any other characterized endogenous neuronal $\mathrm{Ca}^{2+}$ buffer. Direct effects of calmodulin on fast presynaptic $\mathrm{Ca}^{2+}$ dynamics and vesicular release however have not been studied in detail. Using experimentally constrained three-dimensional diffusion modeling of $\mathrm{Ca}^{2+}$ influx-exocytosis coupling at small excitatory synapses we show that, at physiologically relevant concentrations, $\mathrm{Ca}^{2+}$ buffering by calmodulin plays a dominant role in inhibiting vesicular release and in modulating short-term synaptic plasticity. We also propose a novel and potentially powerful mechanism for short-term facilitation based on $\mathrm{Ca}^{2+}$-dependent dynamic dislocation of calmodulin molecules from the plasma membrane within the active zone.

Keywords: synaptic transmission, synaptic vesicles, short-term plasticity, calcium channels, modeling biological systems

\section{Introduction}

Calmodulin (CaM) is a major neuronal protein that acts as a key mediator of multiple $\mathrm{Ca}^{2+}$ dependent intracellular signaling cascades in the brain. CaM regulates synaptic transmission and synaptic plasticity via $\mathrm{Ca}^{2+}$-dependent binding to its target proteins in both the pre- and the postsynaptic compartments. These include protein kinases, adenylyl cyclases, calcineurin, Munc13s, and voltage-gated $\mathrm{Ca}^{2+}$ channels (VGCCs) (Xia and Storm, 2005; Pang et al., 2010; Sun et al., 2010; Lipstein et al., 2013; Ben-Johny and Yue, 2014). It has been recently demonstrated that $\mathrm{CaM}$ binds $\mathrm{Ca}^{2+}$ ions with much faster kinetics than other major neuronal $\mathrm{Ca}^{2+}$ buffers such as calbindin-D28k (CB), parvalbumin, and calretinin (Faas et al., 2011). However, in comparison to the other buffers direct effects of CaM-dependent $\mathrm{Ca}^{2+}$ buffering on action potential (AP)-evoked presynaptic $\mathrm{Ca}^{2+}$ dynamics and vesicular release have not been systematically studied.

In this work we performed realistic, experimentally constrained model simulations of AP-evoked presynaptic $\mathrm{Ca}^{2+}$ dynamics and synaptic vesicle fusion in small excitatory synapses. We compared the relative contributions of $\mathrm{Ca}^{2+}$ buffering by $\mathrm{CB}$ and $\mathrm{CaM}$ to modulation of vesicular release probability and short-term synaptic plasticity. Our simulations demonstrate that, at physiologically relevant concentrations, fast $\mathrm{Ca}^{2+}$ binding to the $\mathrm{N}$ lobe of CaM has a dominant effect in inhibiting AP-evoked vesicular release. We also show 
that the predicted effect of $\mathrm{CaM} \mathrm{Ca}{ }^{2+}$ buffering on short-term synaptic plasticity strongly depends on the location and mobility of CaM molecules.

Finally, we propose a novel mechanism for a dynamic regulation of presynaptic strength based on $\mathrm{Ca}^{2+}$-dependent interaction of $\mathrm{CaM}$ with membrane-associated proteins that contain the isoleucine-glutamine (IQ) binding motif (e.g., neuromodulin and VGCCs) (Alexander et al., 1988; Xia and Storm, 2005; Ben-Johny and Yue, 2014). Our simulations demonstrate that $\mathrm{Ca}^{2+}$-induced dislocation of $\mathrm{CaM}$ molecules from the plasma membrane could lead to a significant reduction of $\mathrm{Ca}^{2+}$ buffering capacity within the active zone (AZ). This in turn, leads to an increase of vesicular release probability during repeated APs. Thus, AP-evoked dislocation of CaM may provide a powerful mechanism for short-term facilitation of synaptic transmission.

\section{Materials and Methods}

\section{Modeling of Presynaptic $\mathrm{Ca}^{2+}$ Dynamics}

Three-dimensional modeling of dynamic AP-evoked presynaptic $\mathrm{Ca}^{2+}$ influx, buffering, and diffusion, on a millisecond timescale, was performed in the Virtual Cell (VCell) simulation environment (http://vcell.org) using the fully implicit finite volume regular grid solver and a $10 \mathrm{~nm}$ mesh. In contrast to the simplified steady-state and/or non-stationary single compartment models that are normally used to approximate presynaptic $\mathrm{Ca}^{2+}$ dynamics on tens to hundreds of milliseconds timescale (Neher, 1998; Sabatini and Regehr, 1998; Scott and Rusakov, 2006; Ermolyuk et al., 2012), no assumptions regarding $\mathrm{Ca}^{2+}$ buffer binding and/or diffusional equilibration were made in the VCell model used here. VCell simulations using a $10 \mathrm{~nm}$ mesh produced solutions for presynaptic $\mathrm{Ca}^{2+}$ dynamics at vesicular release sensors similar to those obtained in our previous work with a $5 \mathrm{~nm}$ mesh (Ermolyuk et al., 2013). The use of the larger mesh substantially increased the computation speed and allowed us to simulate $\mathrm{Ca}^{2+}$ dynamics in the whole presynaptic bouton on the longer time scale.

The presynaptic bouton was considered as a truncated sphere (Figure 1A) of radius $R_{\text {bout }}=0.3 \mu \mathrm{m}$ (described by the equation $\left[x^{2}+y^{2}+z^{2} \leq 0.09\right] \cdot[z \leq 0.25]$, all distances are in $\left.\mu \mathrm{m}\right)$. The AZ containing VGCCs was modeled as a circle of radius $R_{A Z}=0.16 \mu \mathrm{m}$ situated in the center of the truncated plane $z=$ $0.25 \mu \mathrm{m}$. VGCCs were evenly distributed within a rectangular cluster ( 40 by $80 \mathrm{~nm}$ ) which was placed in the center of the AZ. The cluster contained 7 P/Q-type, $8 \mathrm{~N}$-type, and 1 R-type VGCCs (Ermolyuk et al., 2013).

The model assumed $\mathrm{Ca}^{2+}$ binding to the three endogenous buffers present in the presynaptic bouton: $\mathrm{CaM}, \mathrm{CB}$, and ATP. $\mathrm{Ca}^{2+}$ interaction with free CaM was simulated using a two-step cooperative binding model to the $\mathrm{N}$ - and the C-lobes of CaM molecule (Faas et al., 2011):

$N_{T} N_{T}+C a^{2+} \underset{k_{o f f}^{(T), N}}{\stackrel{2 \cdot k_{o n}^{(T), N}}{\rightleftarrows}} C_{a N} N_{R}+C a^{2+} \underset{2 \cdot k_{o f f}^{(R), N}}{\stackrel{k_{o n}^{(R), N}}{\rightleftarrows}} C_{a N} \operatorname{CaN}_{R}$,
$C_{T} C_{T}+C a^{2+} \underset{k_{o f f}^{(T), C}}{\stackrel{2 \cdot k_{o n}^{(T), C}}{\rightleftarrows}} C a C_{T} C_{R}+C a^{2+} \underset{2 \cdot k_{o f f}^{(R), C}}{\stackrel{k_{o n}^{(R), C}}{\rightleftarrows}} C a C_{R} C a C_{R}$ $k_{o n}^{(T), N}=770 \mu \mathrm{M}^{-1} \mathrm{~s}^{-1}, k_{o f f}^{(T), N}=1.6 \times 10^{5} \mathrm{~s}^{-1}, k_{o n}^{(R), N}=$ $3.2 \times 10^{4} \mu \mathrm{M}^{-1} \mathrm{~s}^{-1}, k_{\text {off }}^{(R), N}=2.2 \times 10^{4} \mathrm{~s}^{-1}, k_{o n}^{(T), C}=84 \mu \mathrm{M}^{-1}$ $\mathrm{s}^{-1}, k_{\text {off }}^{(T), C}=2.6 \times 10^{3} \mathrm{~s}^{-1}, k_{o n}^{(R), C}=25 \mu \mathrm{M}^{-1} \mathrm{~s}^{-1}, k_{o f f}^{(R), C}=$ $6.5 \mathrm{~s}^{-1}$. The total average $\mathrm{CaM}$ concentration was $[\mathrm{CaM}]_{t o t}=$ $100 \mu \mathrm{M}$ (Faas et al., 2011). Depending on the type of simulation (as indicated in the text) CaM was considered either as a mobile buffer with diffusion coefficient $D_{C a M}=20 \mu \mathrm{m}^{2} \mathrm{~s}^{-1}$, or as an immobile buffer which was either evenly distributed throughout the bouton volume or bound to the plasma membrane (within a $10 \mathrm{~nm}$ single layer adjacent to the bouton membrane in VCell simulations). In the case of CaM associated with neuromodulin we assumed that $k_{\text {off }}^{(R), C}$ was increased 50-fold (Gaertner et al., 2004; Hoffman et al., 2014) $\left(k_{\text {off }}^{(R), C}=325 \mathrm{~s}^{-1}\right)$.

Each CB molecule contained four independent $\mathrm{Ca}^{2+}$ binding sites (two fast and two slow) (Nagerl et al., 2000):

$$
\begin{aligned}
& C B_{\text {fast }}+C a^{2+} \underset{k_{\text {off }}^{C B \_a s t}}{\stackrel{k_{\text {on }}^{C B_{f} \text { fast }}}{\rightleftarrows}} C a C B_{\text {fast }}, \\
& C B_{\text {slow }}+C a^{2+} \underset{k_{\text {off }}^{C B \_s l o w}}{\stackrel{k_{\text {on }}^{\text {CBslow }}}{\rightleftarrows}} C a C B_{\text {slow }} \text {, }
\end{aligned}
$$

$k_{\text {on }}^{C B \_f a s t}=87 \mu \mathrm{M}^{-1} \mathrm{~s}^{-1}, k_{\text {off }}^{C B \_f a s t}=35.8 \mathrm{~s}^{-1}, k_{\text {on }}^{C B \_s l o w}=$ $11 \mu \mathrm{M}^{-1} \mathrm{~s}^{-1}, k_{o f f}^{C B \_s l o w}=2.6 \mathrm{~s}^{-1}$. The diffusion coefficient for both $\mathrm{Ca}^{2+}$-free and $\mathrm{Ca}^{2+}$-bound $\mathrm{CB}$ molecules was $D_{C B}=$ $20 \mu \mathrm{m}^{2} \mathrm{~s}^{-1}$ and the total $\mathrm{CB}$ concentration was $[C B]_{t o t}=$ 47.5 $\mu \mathrm{M}$ (Muller et al., 2005).

$\mathrm{Ca}^{2+}$ binding to ATP was modeled as a second order reaction:

$$
A T P+C a^{2+} \underset{k_{o f f}^{A T P}}{\stackrel{k_{o n}^{A T P}}{\rightleftarrows}} C a A T P
$$

$k_{\text {on }}^{A T P}=500 \mu \mathrm{M}^{-1} \mathrm{~s}^{-1}, k_{\text {off }}^{A T P}=1.0 \times 10^{5} \mathrm{~s}^{-1}$. The diffusion coefficient of free and $\mathrm{Ca}^{2+}$ bound ATP was $D_{\text {ATP }}=220 \mu \mathrm{m}^{2}$ $s^{-1}$ (Meinrenken et al., 2002). The total ATP concentration was $[A T P]_{\text {tot }}=0.9 \mathrm{mM}$, corresponding to $58 \mu \mathrm{M}[A T P]_{\text {free }}$ at resting physiological conditions (assuming $1 \mathrm{mM}$ intracellular $\left[\mathrm{Mg}^{2+}\right]$ ) (Faas et al., 2011).

$\mathrm{Ca}^{2+}$ extrusion by the bouton surface pumps (excluding the AZ) was approximated by a first-order reaction: $j_{\text {extr }}=-k_{\text {extr }}$. $\left(\left[\mathrm{Ca}^{2+}\right]-\left[\mathrm{Ca}^{2+}\right]_{\text {rest }}\right)$ (Matveev et al., 2006; Ermolyuk et al., 2013), with $k_{\text {extr }}=125 \mu \mathrm{m} \mathrm{s}^{-1}$ and $\left[\mathrm{Ca}^{2+}\right]_{\text {rest }}=50 \mathrm{nM}$.

AP-evoked $\mathrm{Ca}^{2+}$ currents through P/Q-, N-, and R-type VGCCs were modeled in the NEURON simulation environment (Hines and Carnevale, 1997) using a six-state channel gating kinetic model of P/Q-, N-, and R-type VGCCs in hippocampal mossy fiber boutons (Li et al., 2007) as described in detail previously (Ermolyuk et al., 2013). The mean AP-evoked $\mathrm{Ca}^{2+}$ current at the VGCC cluster was approximated by averaging 500 
A

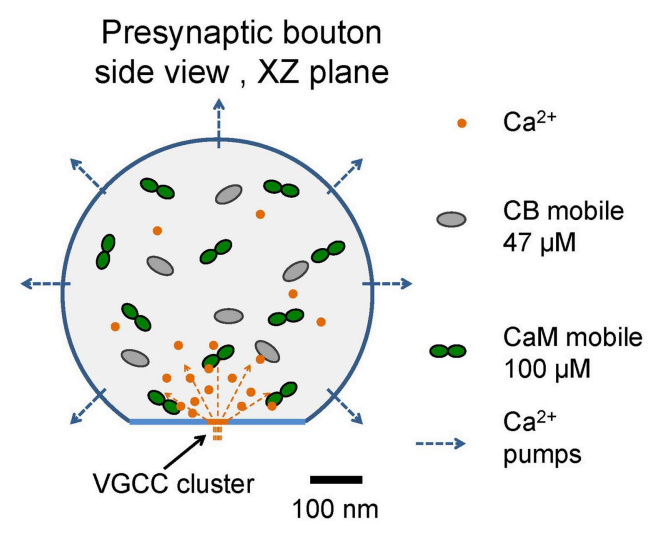

Active zone $(A Z)$, $\mathrm{XY}$ plane

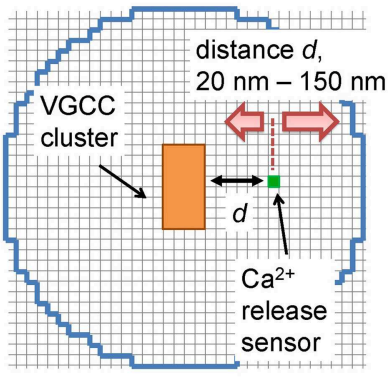

$$
\begin{aligned}
& \text { Time, ms } \\
& \begin{array}{llllll}
\hline & 1 & 2 & 3 & 4 & 5
\end{array}
\end{aligned}
$$

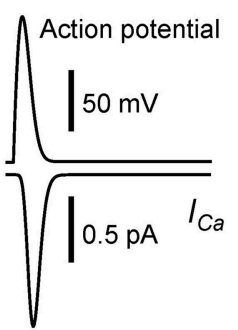

C

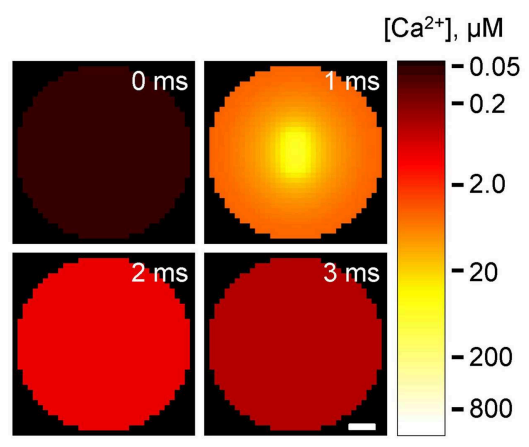

D

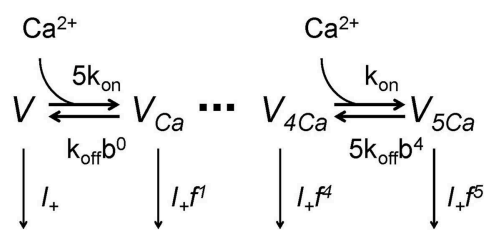

FIGURE 1 | Modeling of AP-evoked synaptic vesicle exocytosis in a small presynaptic bouton. (A) Presynaptic bouton geometry. Left, side view of a bouton modeled as a truncated sphere of $R_{\text {bout }}=0.3 \mu \mathrm{m}$. Right, the AZ plane containing the VGCC cluster modeled as a $40 \times 80 \mathrm{~nm}$ rectangle (orange); red arrows depict the range of tested coupling distances $d(20-150 \mathrm{~nm})$ between the VGCC cluster and the vesicular $\mathrm{Ca}^{2+}$ release sensor (green dot). Grid $10 \mathrm{~nm}$. (B) AP waveform (top trace) and corresponding average $\mathrm{Ca}^{2+}$ current $I_{\mathrm{Ca}}$ (bottom trace) through 7 P/Q-type, $8 \mathrm{~N}$-type, and 1 R-type VGCCs. (C) Snapshots of spatial AP-evoked $\left[\mathrm{Ca}^{2+}\right]$ within a $10 \mathrm{~nm}$ thick plane immediately above
E

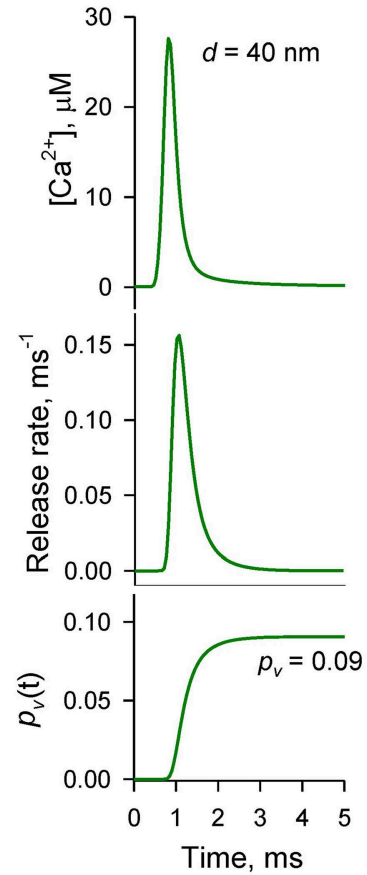

$\mathbf{F}$

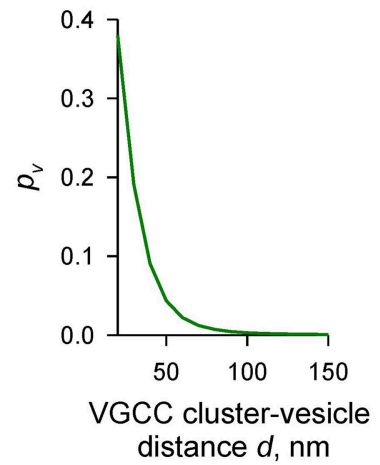

independent NEURON simulations of AP-evoked $\mathrm{Ca}^{2+}$ currents for each channel sub-type, followed by fitting the sum of average $\mathrm{Ca}^{2+}$ currents corresponding to $7 \mathrm{P} / \mathrm{Q}$-type, $8 \mathrm{~N}$-type, and $1 \mathrm{R}$ type VGCCs with the function $I_{C a}(t)=\frac{A}{t} \exp \left[-B \cdot\left[\ln \left(t / t_{0}\right)\right]^{2}\right]$, where $A=9.2246 \times 10^{-4} \mathrm{pA} \mathrm{s}, B=15.78, t_{0}=8.036 \times$ $10^{-4} \mathrm{~s}$ (Figure 1B). We did not consider any possible effects of AP waveform changes during repeated AP stimulations and assumed that the magnitude of $\mathrm{Ca}^{2+}$ influx was the same at the $A Z$, scale bar $50 \mathrm{~nm}$. Logarithmic color coding bar is shown on the right. (D) Allosteric model of $\mathrm{Ca}^{2+}$ activation of vesicle fusion (Lou et al., 2005) (E) Results of simulations for a representative VGCC $-\mathrm{Ca}^{2+}$ sensor coupling distance $d=40 \mathrm{~nm}:\left[\mathrm{Ca}^{2+}\right]$ time course (top trace), corresponding vesicular release rate time course (middle trace), and time dependency of cumulative vesicular release probability $p_{v}(t)$ (bottom trace). Final AP-evoked vesicular release probability $p_{V}$ is shown in the insert. This was determined as the horizontal asymptote of a cumulative probability after the AP (in practice we defined it as a value of $p_{V}(t)$ at $\left.5 \mathrm{~ms}\right)(\mathbf{F})$ Dependency of $p_{V}$ on distance $d$. each AP. Access to the VCell simulations is available upon request.

\section{Modeling of $\mathrm{Ca}^{2+}$-triggered Synaptic Vesicle Fusion}

Vesicular release rates were calculated using a previously published six-state allosteric model of $\mathrm{Ca}^{2+}$ activation of vesicle fusion in the calyx of Held (Lou et al., 2005) (Figure 1D). The 
model parameters were: $k_{\text {on }}=1 \times 10^{8} \mathrm{M}^{-1} \mathrm{~s}^{-1}, k_{\text {off }}=4 \times 10^{3}$ $\mathrm{s}^{-1}, b=0.5, f=31.3$, and $I_{+}=2 \times 10^{-4} \mathrm{~s}^{-1}$. The model was solved using a variable-order stiff multistep method based on the numerical differentiation formulas (function ode15s in MATLAB, MathWorks USA) for AP-evoked $\mathrm{Ca}^{2+}$ concentration profiles obtained in VCell simulations at each of the $10 \times 10 \times 10 \mathrm{~nm}$ voxels located immediately above the AZ plane (Figure 1C). MATLAB computer code is enclosed (Supplementary MATLAB code).

The time-dependent vesicular release probability at each voxel in the AZ was calculated as $p_{v}(t)=1-\sum_{i} V_{i}(t)$, where $\sum_{i} V_{i}(t)$ is the sum of occupancies of all six model states $V_{i}$ (Figure 1D). The release rate was then calculated as $R_{r e l}=d p_{v}(t) / d t$. In this work we were specifically interested in dissecting the relative effects of $\mathrm{CaM}$ and $\mathrm{CB}$ on vesicular release and short-term facilitation. Therefore, we did not take into account any changes in the number of release-ready vesicles that occur during pairedpulse stimulation due to vesicle depletion and replenishment. We thus considered that at the onsets of both the first and second APs the vesicular release sensor was in $\mathrm{Ca}^{2+}$ unbound state $V_{t=0 m s}=V_{t=20 m s}=(1,0,0,0,0,0)$. To account for sensitivity of AP-evoked release observed in small excitatory hippocampal and neocortical synapses to the slow endogenous buffer EGTA (e.g., Rozov et al., 2001; Ermolyuk et al., 2013), voxels located closer than $20 \mathrm{~nm}$ to the VGCC clusters were excluded from the analysis.

\section{Results}

\section{Experimentally Constrained Model of AP-evoked Synaptic Vesicle Exocytosis in Small Central Synapses}

To compare the effects of $\mathrm{CB}$ and $\mathrm{CaM} \mathrm{Ca}{ }^{2+}$ buffering on APevoked vesicular release and short-term synaptic plasticity we used a realistic experimentally constrained three-dimensional model of AP-evoked VGCC-mediated $\mathrm{Ca}^{2+}$ influx, $\mathrm{Ca}^{2+}$ buffering and diffusion, and $\mathrm{Ca}^{2+}$-dependent activation of vesicular release sensors. The modeling framework consisted of two steps: simulation of buffered $\mathrm{Ca}^{2+}$ diffusion in the presynaptic bouton using VCell environment, and calculation of vesicular release rates and fusion probabilities $p_{v}$ using an allosteric model of the $\mathrm{Ca}^{2+}$ activation of vesicle fusion developed in the calyx of Held (Lou et al., 2005) (Materials and Methods).

The presynaptic bouton was considered as a truncated sphere $\left(R_{\text {bout }}=0.3 \mu \mathrm{m}\right)$ with the $\mathrm{AZ}$ located at the truncated plane (Figure 1A). Immunogold electron microscopy has shown that P/Q-type VGCCs in small excitatory CA3 hippocampal synapses are mainly situated in small oval-shaped clusters with typical dimensions of 50-100 nm, and that the number of such clusters linearly scales with the size of the AZ (Holderith et al., 2012). To simplify our model we assumed that the AZ had only a single VGCC cluster of rectangular shape: $40 \times 80 \mathrm{~nm}$ (Figure 1A). Indeed, several studies have argued that for a given release-ready vesicle docked at the AZ its AP-evoked release probability $p_{v}$ is mainly determined by the closest VGCC cluster (Meinrenken et al., 2002; Ermolyuk et al., 2013; Nakamura et al., 2015).
AP-evoked release in small central excitatory synapses is triggered by mixed populations of P/Q-, N-, and R-type VGCCs (Wu and Saggau, 1994; Reid et al., 1998; Li et al., 2007; Holderith et al., 2012; Sheng et al., 2012). Based on experimental data for the relative numbers of P/Q-, N-, and R-type VGCCs in small hippocampal boutons (Ermolyuk et al., 2013) and for the average channel density within VGCC clusters (Holderith et al., 2012) we considered that the VGCC cluster contains $7 \mathrm{P} / \mathrm{Q}$-type, $8 \mathrm{~N}$-type, and 1 R-type VGCCs. In this simplified model we did not take into account the stochastic behavior of individual VGCCs during an $\mathrm{AP}$ and assumed that all channels are evenly distributed within the VGCC cluster. Thus, total AP-evoked $\mathrm{Ca}^{2+}$ influx at the VGCC cluster was approximated as the sum of average $\mathrm{Ca}^{2+}$ currents corresponding to $7 \mathrm{P} / \mathrm{Q}$-type, $8 \mathrm{~N}$-type, and 1 R-type VGCCs (Figure 1B and Materials and Methods).

We considered that in addition to ATP the presynaptic bouton contains two major presynaptic $\mathrm{Ca}^{2+}$ buffers found in central excitatory synapses: $\mathrm{CB}$ [physiological $[C B]_{\text {tot }} \sim 47.5 \mu \mathrm{M}$, total concentration of $\mathrm{Ca}^{2+}$ binding sites $190 \mu \mathrm{M}$; (Berggard et al., 2002; Jackson and Redman, 2003; Muller et al., 2005; Scott and Rusakov, 2006)] and CaM (physiological $[\mathrm{CaM}]_{t o t} \sim 100 \mu \mathrm{M}$, total concentration of $\mathrm{Ca}^{2+}$ binding sites $400 \mu \mathrm{M}$; Faas et al., 2011). In the first set of simulations we assumed that CaM molecules are mobile and have the same coefficient of diffusion in $\mathrm{Ca}^{2+}$-free and $\mathrm{Ca}^{2+}$-bound states equal to that of $\mathrm{CB}\left(D_{\mathrm{CaM}}=\right.$ $\left.D_{C B}=20 \mu \mathrm{m}^{2} \mathrm{~s}^{-1}\right)$.

To calculate the AP-evoked synaptic vesicle release probability $p_{v}$ as a function of distance between the VGCC cluster and the vesicular release sensor (coupling distance $d$, Figure 1A) we extracted from the three-dimensional VCell model $\mathrm{Ca}^{2+}$ dynamics at the AZ (Figure 1C) and then calculated $p_{v}$ at different $d$ using the allosteric model of $\mathrm{Ca}^{2+}$-triggered synaptic vesicle fusion (Figure 1D). Consistent with experimental data (Murthy et al., 2001; Ariel and Ryan, 2010; Ermolyuk et al., 2012) the model predicted that physiologically relevant $p_{v}$-values $(0.05-$ 0.15 range) should correspond to an average coupling distance $d$ within a $30-50 \mathrm{~nm}$ range (Figures 1E,F).

\section{Dominant Effect of CaM Ca ${ }^{2+}$ Buffering on AP-evoked Vesicular Release}

To compare the relative contributions of $\mathrm{CB}$ and $\mathrm{CaM}$ to buffering of AP-evoked $\left[\mathrm{Ca}^{2+}\right]$ transients at the $\mathrm{AZ}$ (and, as a consequence, to inhibition of vesicular release) we performed simulations using different combinations of $\mathrm{CB}$ and $\mathrm{CaM}$ either absent or present at physiological concentrations (Figures 2A,B). The model predicted that each buffer on its own efficiently inhibited AP-evoked $\mathrm{AZ}\left[\mathrm{Ca}^{2+}\right]$ transients and $p_{v}$. At a typical coupling distance $d=40 \mathrm{~nm} \mathrm{CB}$ caused $\sim 50 \%$ reduction of $p_{v}$ (from 0.58 to 0.31 ) relative to control simulations without $\mathrm{CB}$ and CaM. CaM had even stronger inhibitory effect: $\sim 80 \%$ reduction of $p_{v}$ at $d=40 \mathrm{~nm}$ (from 0.58 to 0.12 ). Consistent with the steep power relationship between vesicular release rate and $\left[\mathrm{Ca}^{2+}\right]$ at the release sensors (Mintz et al., 1995; Lou et al., 2005) $\mathrm{Ca}^{2+}$ buffering by $\mathrm{CB}$ and $\mathrm{CaM}$ caused a non-additive supralinear reduction of $p_{v}$. Notably, addition of CB on top of CaM caused only a minor further decrease of $p_{v}$ (e.g., from 80 to $85 \%$ at $d=40 \mathrm{~nm}$ ). 


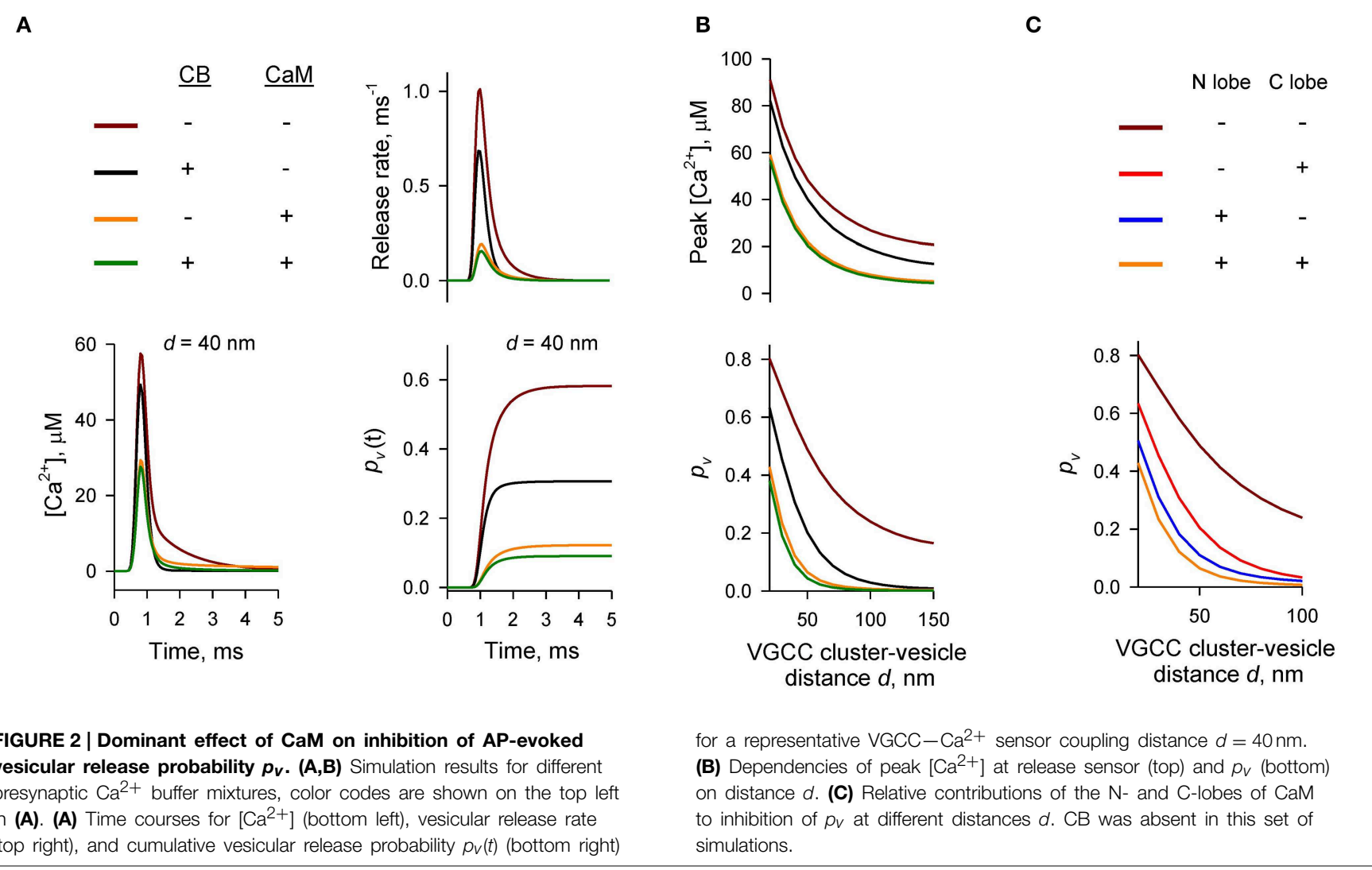

We next compared the relative contributions of the fast $\mathrm{Ca}^{2+}$ binding to the CaM N-lobe (limiting rate constant $k_{o n}^{(T), N}=$ $770 \mu \mathrm{M}^{-1} \mathrm{~s}^{-1}$ ) and the slower $\mathrm{Ca}^{2+}$ binding to the CaM Clobe (limiting rate constant $k_{o n}^{(T), C}=84 \mu \mathrm{M}^{-1} \mathrm{~s}^{-1}$ ) to inhibition of $p_{v}$. Consistent with its $\sim$ ten-fold higher $\mathrm{Ca}^{2+}$ binding rate the N-lobe had a dominant effect in reducing AP-evoked $\left[\mathrm{Ca}^{2+}\right]$ transients at the $\mathrm{AZ}$ and $p_{v}$ (Figure 2C). The C-lobe on its own produced an inhibitory effect similar to that of $\mathrm{CB}$.

Thus, our simulations show that fast synchronous AP-evoked vesicular release at synapses that contain both $\mathrm{CB}$ and $\mathrm{CaM}$ is mainly inhibited by fast $\mathrm{Ca}^{2+}$ binding to the $\mathrm{N}$-lobe of $\mathrm{CaM}$ and that the CaM C-lobe and CB play only secondary roles.

\section{Effect of Mobile CaM on Paired-pulse Facilitation}

At certain types of central synapses $\mathrm{CB}$ has been shown to contribute to short-term facilitation of AP-evoked vesicular release through $\mathrm{Ca}^{2+}$ buffer saturation (e.g., Blatow et al., 2003; Jackson and Redman, 2003). Given the predicted dominant effect of CaM on AP-evoked release we asked how $\mathrm{Ca}^{2+}$ buffering by CaM affects short-term synaptic plasticity in presynaptic boutons that contain both $\mathrm{CB}$ and CaM. Facilitation through buffer saturation strongly depends on the mobility of the endogenous $\mathrm{Ca}^{2+}$ buffers (e.g., Matveev et al., 2004). CaM binds to multiple soluble and membrane-bound proteins (Xia and Storm, 2005; Villarroel et al., 2014). However, the precise distribution of presynaptic $\mathrm{CaM}$ molecules between the mobile and immobile states is not known. Therefore, we explored several limiting cases with respect to the diffusional properties and spatial distribution of presynaptic CaM.

We first considered the case of mobile CaM (Figure 3, see also Figures 1, 2). We modeled $\mathrm{Ca}^{2+}$ dynamics and vesicular release during $50 \mathrm{~Hz}$ paired-pulse AP stimulation (inter-spike interval $\Delta t_{A P}=20 \mathrm{~ms}$ ) and calculated the dependencies of paired-pulse ratios (PPRs) on the coupling distance $d$ both for peak $\left[\mathrm{Ca}^{2+}\right]$ $\left(P P R\left[\mathrm{Ca}^{2+}\right]_{\text {peak }}=\left[\mathrm{Ca}^{2+}\right]_{\text {peak }}^{A P 2} /\left[\mathrm{Ca}^{2+}\right]_{\text {peak }}^{A P 1}\right)$ and for the vesicular release probability $\left(P P R_{p_{v}}=p_{v}^{A P 2} / p_{v}^{A P 1}\right)$ (Figures $3 \mathbf{A}-\mathbf{C}$ ). It should be noted that because we were specifically interested in the effects of $\mathrm{CaM}$ and $\mathrm{CB}$ on shaping the vesicular release, when calculating $P P R_{p_{v}}$ we did not consider any changes in the number of release-ready vesicles that may occur as a result of vesicle depletion and replenishment during repetitive stimulation (Materials and Methods).

In comparison to the control simulations where only $\mathrm{CB}$ was present, inclusion of mobile CaM led to a noticeable decrease of both $P P R\left[\mathrm{Ca}^{2+}\right]_{\text {peak }}$ and $P P R_{p_{v}}$ (Figure 3C). CB has a relatively high affinity to $\mathrm{Ca}^{2+}\left(K_{D}^{\text {eff }}{ }_{C B}=0.31 \mu \mathrm{M}\right.$, Supplementary Figure 1) and binds $\mathrm{Ca}^{2+}$ ions that enter the bouton during the first $\mathrm{AP}$ both within the transient $\mathrm{Ca}^{2+}$-nano/microdomain (local $\left[\mathrm{Ca}^{2+}\right]$ up to $10-100 \mu \mathrm{M}$ within $20-150 \mathrm{~nm}$ from the VGCC cluster) and in the rest of the bouton volume (global $\left[\mathrm{Ca}^{2+}\right] \sim 1.0-$ $1.5 \mu \mathrm{M}$ ) (Figures 3D,E). Thus, at the onset of the second AP the concentration of free $\mathrm{CB}$ binding sites was noticeably reduced in 


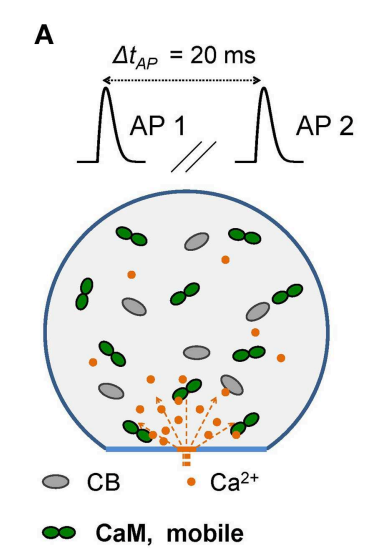

B

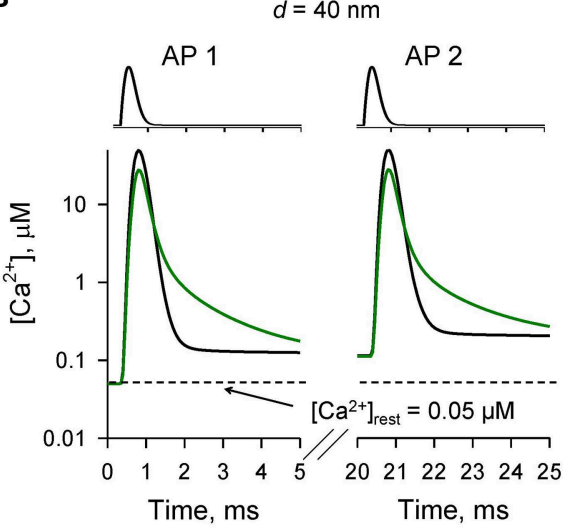

C

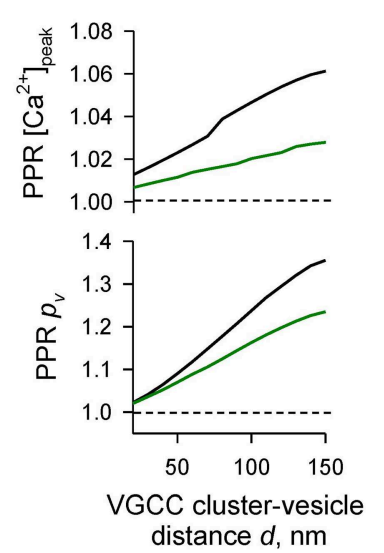
distance $d, \mathrm{~nm}$
D

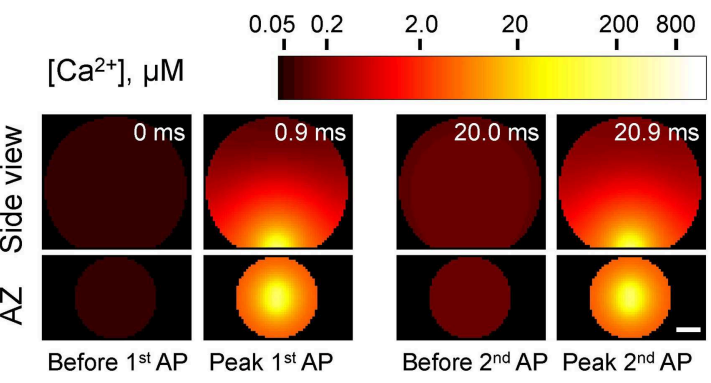

$\mathbf{F}$

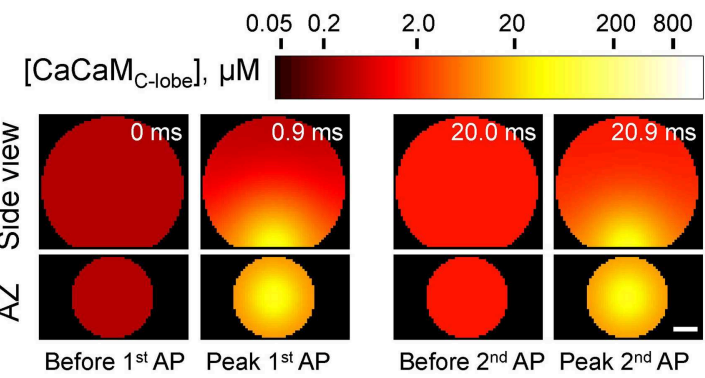

FIGURE 3 | Effect of mobile CaM on short-term synaptic plasticity. (A) Schematic representation of the paired-pulse simulation experiment $\left(\Delta t_{A P}=20 \mathrm{~ms}\right)$ with mobile CaM, see text for details. (B) Comparison of $\left[\mathrm{Ca}^{2+}\right]$ time courses at the vesicular release $\mathrm{Ca}^{2+}$ sensor for a representative coupling distance $d=40 \mathrm{~nm}$ during paired-pulse stimulation (AP 1 and AP 2) with and without mobile CaM in the presynaptic bouton. Left, $\left[\mathrm{Ca}^{2+}\right]$ transients; right, net increase of $\left[\mathrm{Ca}^{2+}\right]$ at the second AP $\left(\Delta\left[\mathrm{Ca}^{2+}\right]_{A P 2}=\left[\mathrm{Ca}^{2+}\right]_{A P 2}-\left[\mathrm{Ca}^{2+}\right]_{A P 1}\right)$. (C) Dependencies of $P P R\left[\mathrm{Ca}^{2+}\right]_{\text {peak }}=\left[\mathrm{Ca}^{2+}\right]_{\text {peak }}^{A P 2} /\left[\mathrm{Ca}^{2+}\right]_{\text {peak }}^{A P 1}$ and $P P R p_{V}=p_{V}{ }^{A P 2} / p_{V}{ }^{A P 1}$ on

comparison to the first AP (by $\sim 10 \%$, from 163.0 to $148.5 \mu \mathrm{M}$, Supplementary Figure 2). In contrast both the C- and the Nlobes of CaM have low $\mathrm{Ca}^{2+}$ affinities $\left(K_{D}^{e f f}{ }_{C-l o b e}=2.84 \mu \mathrm{M}\right.$ and $K_{D N-\text { lobe }}^{\text {eff }}=12.0 \mu \mathrm{M}$, Supplementary Figure 1) and bind $\mathrm{Ca}^{2+}$ ions mainly within the $\mathrm{Ca}^{2+}$-nano/microdomain (Figures 3F,G). Therefore, because of the diffusional equilibration at the onset of the second AP over $99 \%$ of $\mathrm{CaM} \mathrm{Ca}{ }^{2+}$ binding sites at the $\mathrm{AZ}$ remained in the unbound state (Supplementary Figure 2). Thus,
E

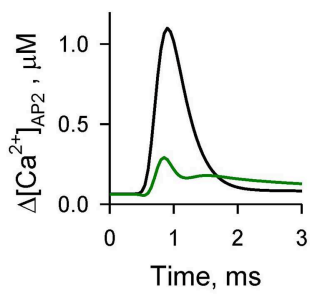

$\mathrm{CB}$ only $\mathrm{CB}$ and CaM

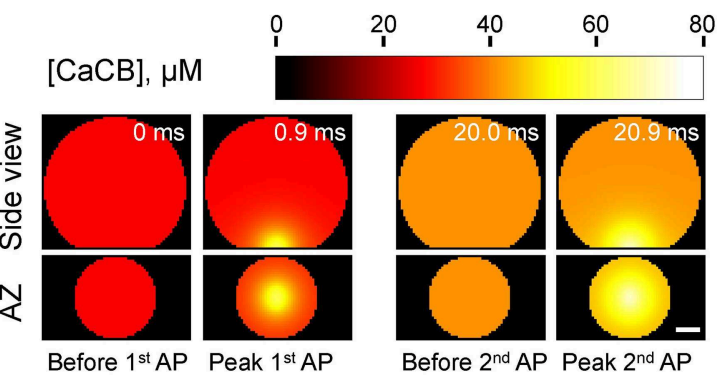

G
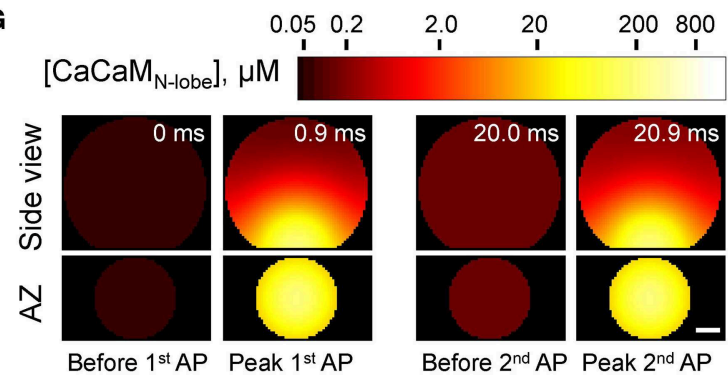

the coupling distance $d . p_{V}{ }^{A P 1}$ and $p_{V}{ }^{A P 2}$ were determined as the horizontal asymptotes of $p_{V}{ }^{A P 1}(t)$ and $p_{V}{ }^{A P 2}(t)$ after the first and the second APs respectively. (D-G) Snapshots of spatial distribution of $\mathrm{Ca}^{2+}\left(D,\left[\mathrm{Ca}^{2+}\right]\right)$, $\mathrm{Ca}^{2+}$ bound to $\mathrm{CB}\left(\mathrm{E},[\mathrm{CaCB}]=\left[\mathrm{CaCB}_{\text {fast }}\right]+\left[\mathrm{CaCB}_{\text {slow }}\right]\right)$, and $\mathrm{Ca}^{2+}$ bound to the C-lobe $\left(\mathrm{F},\left[\mathrm{CaCaM}_{\mathrm{C}-\text { lobe }}\right]=\left[\mathrm{CaC}_{\mathrm{T}} \mathrm{C}_{\mathrm{R}}\right]+2 \cdot\left[\mathrm{CaC}_{\mathrm{R}} \mathrm{CaC}_{\mathrm{R}}\right]\right)$ and the $\mathrm{N}$-lobe $\left(\mathrm{G},\left[\mathrm{CaCaM}_{\mathrm{N} \text {-lobe }}\right]=\left[\mathrm{CaN}_{T} \mathrm{~N}_{R}\right]+2 \cdot\left[\mathrm{CaN}_{\mathrm{R}} \mathrm{CaN}_{\mathrm{R}}\right]\right)$ of $\mathrm{CaM}$ during paired-pulse stimulation. Side view, $X Z$ plane through the center of the bouton (as in A); AZ, $10 \mathrm{~nm}$ thick plane immediately above the AZ. Scale bar $100 \mathrm{~nm}$.

the presence of mobile CaM, which directly competes with $\mathrm{CB}$ for $\mathrm{Ca}^{2+}$ in the $\mathrm{AZ}$, occludes the short-term facilitation caused by saturation of $\mathrm{CB}$.

\section{Effect of Immobile CaM on Paired-pulse Facilitation}

In the next set of simulations (Figure 4) we considered another limiting case and assumed that all CaM molecules were immobile (e.g., bound to immobile target proteins) and were evenly 


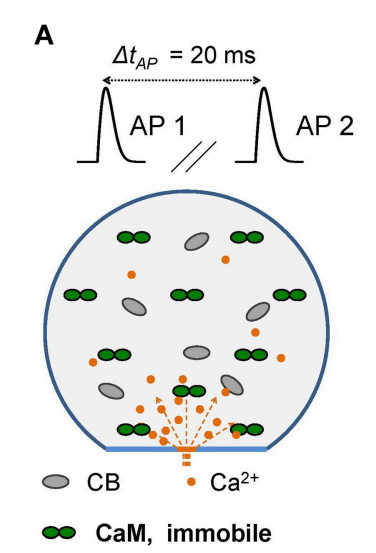

B

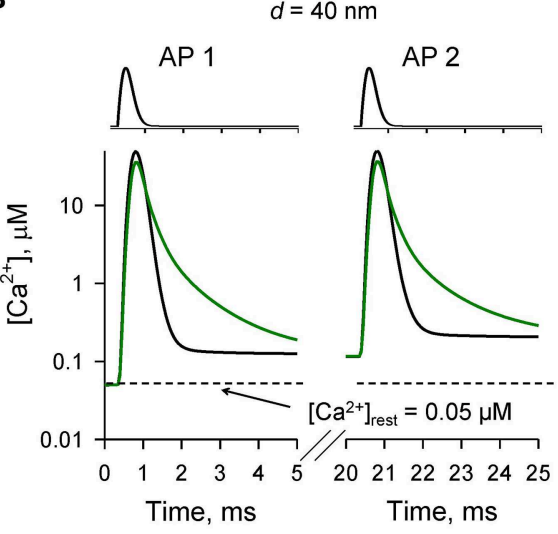

C

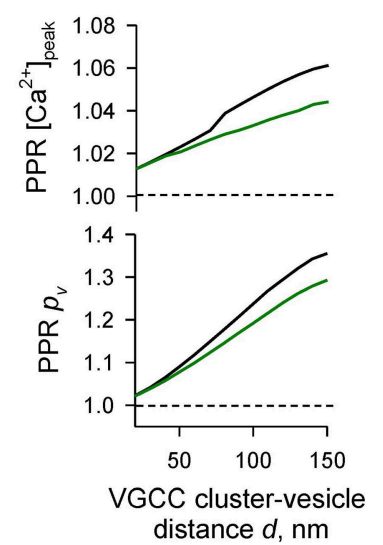
distance $d, \mathrm{~nm}$
D

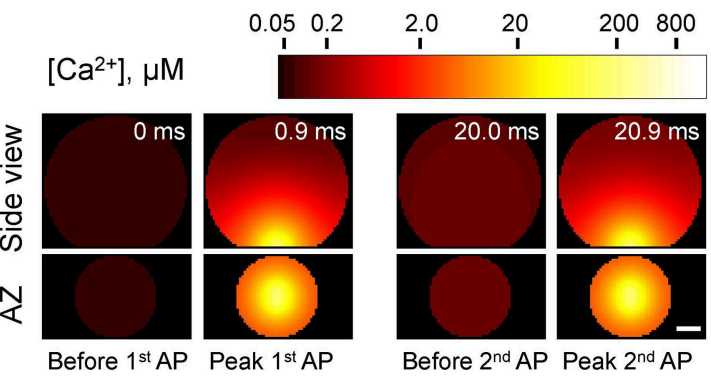

$\mathbf{F}$

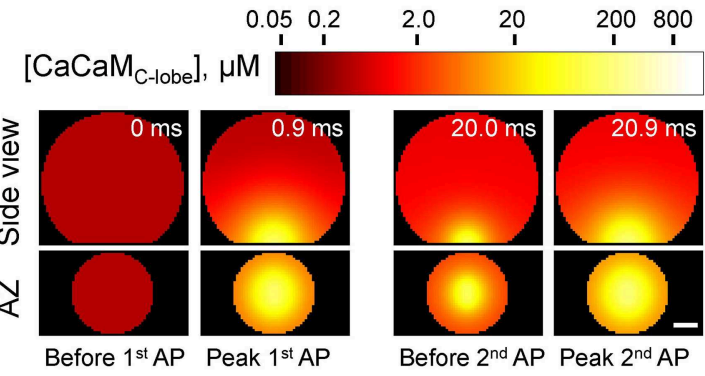

FIGURE 4 | Effect of immobile CaM on short-term synaptic plasticity. (A) Schematic representation of the paired-pulse simulation experiment $\left(\Delta t_{A P}=20 \mathrm{~ms}\right)$ with immobile CaM, see text for details. (B) Comparison of $\left[\mathrm{Ca}^{2+}\right]$ time courses at the vesicular release $\mathrm{Ca}^{2+}$ sensor for a representative coupling distance $d=40 \mathrm{~nm}$ during paired-pulse stimulation (AP 1 and AP 2) with and without immobile CaM in the presynaptic bouton. Left, $\left[\mathrm{Ca}^{2+}\right]$
E

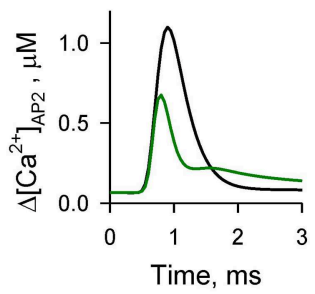

$\mathrm{CB}$ only $\mathrm{CB}$ and CaM

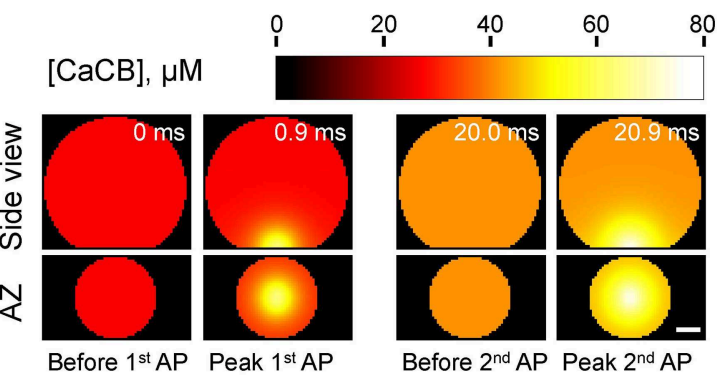

G
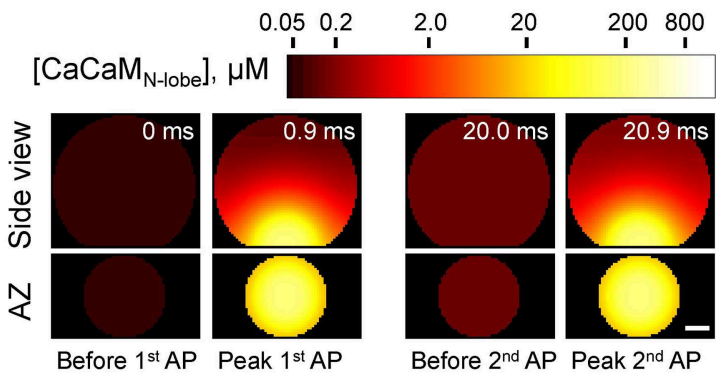

transients; right, net increase of $\left[\mathrm{Ca}^{2+}\right]$ at the second AP. (C) Dependencies of PPR $\left[\mathrm{Ca}^{2+}\right]_{\text {peak }}$ and $P P R_{p_{V}}$ on the coupling distance $d$. (D-G) Snapshots of spatial distribution of $\mathrm{Ca}^{2+}(\mathbf{D}), \mathrm{Ca}^{2+}$ bound to $\mathrm{CB}(\mathbf{E})$, and $\mathrm{Ca}^{2+}$ bound to the $\mathrm{C}$-lobe $(\mathbf{F})$ and the $\mathrm{N}$-lobe $(\mathbf{G})$ of CaM during paired-pulse stimulation. Side view, $X Z$ plane through the center of the bouton (as in $\mathbf{A}$ ); $A Z, 10 \mathrm{~nm}$ thick plane immediately above the AZ. Scale bar $100 \mathrm{~nm}$.

distributed throughout the bouton volume. The presence of immobile CaM still led to a reduction of paired-pulse facilitation mediated by buffer saturation, although on a smaller scale than in the case of mobile CaM (Figures $4 \mathbf{A}-\mathbf{C}$ ). This was due to the contribution of partial saturation of the immobile CaM Clobe within the $\mathrm{Ca}^{2+}$-nano/microdomain (Figure 4F, snapshot "Before $2^{\text {nd }} \mathrm{AP}^{\text {") }} . \mathrm{Ca}^{2+}$ unbinding from the fully occupied Clobe occurs on a longer timescale $\left(\mathrm{Ca}^{2+}\right.$ dwell time $\sim 150 \mathrm{~ms}$, $\left.k_{o f f}^{(R), C}=6.5 \mathrm{~s}^{-1}\right)$ than the $20 \mathrm{~ms}$ inter-spike interval. Therefore, at a typical coupling distance $d=40 \mathrm{~nm}$ only $80 \%$ of $\mathrm{Ca}^{2+}$ binding sites on the C-lobe were free at the onset of the second
AP (Supplementary Figure 3). In contrast $\mathrm{Ca}^{2+}$ unbinding from the $\mathrm{N}$-lobe occurs on a much faster timescale $\left(\mathrm{Ca}^{2+}\right.$ dwell time $\left.\sim 0.05 \mathrm{~ms}, k_{\text {off }}^{(R), N}=2.2 \times 10^{4} \mathrm{~s}^{-1}\right)$. Therefore, concentrations of the available $\mathrm{N}$-lobe $\mathrm{Ca}^{2+}$ binding sites were similar at the onsets of the first and the second APs, which led to occlusion of the paired-pulse facilitation caused by saturation of $\mathrm{CB}$ and the C-lobe of CaM. In this set of simulations we used $\mathrm{Ca}^{2+}$ binding kinetics determined for free CaM (Faas et al., 2011). However, $\mathrm{CaM} \mathrm{Ca}^{2+}$ binding properties are affected by binding of CaM to its target proteins. These can either increase (e.g., CaM kinase II) or decrease (e.g., neuromodulin) $\mathrm{Ca}^{2+}$ affinity of CaM (Gaertner 
et al., 2004; Xia and Storm, 2005). Therefore, the effects of the immobile CaM on vesicular release probability $p_{v}$ and short-term plasticity are expected to be also influenced by the distribution of bound CaM among different target proteins.

\section{The Case of Membrane-bound CaM}

Many CaM binding partners are located on the presynaptic plasma membrane. In particular, neuromodulin is an abundant presynaptic protein which is found in the brain at similar levels to CaM (Alexander et al., 1988; Xia and Storm, 2005; Kumar et al., 2013). Neuromodulin is a member of the IQ motif family of CaM-binding proteins which also includes neurogranin and PEP-19 (Putkey et al., 2003; Xia and Storm, 2005). CaM binds to the IQ motif via the C-lobe at low $\left[\mathrm{Ca}^{2+}\right]$, and dissociates when $\mathrm{Ca}^{2+}$ levels increase (Alexander et al., 1988; Xia and Storm, 2005; Kumar et al., 2013). It was proposed that at resting $\left[\mathrm{Ca}^{2+}\right]_{\text {rest }}$ most of presynaptic CaM is bound to the membrane anchored neuromodulin (Xia and Storm, 2005). Indeed, our model predicts that at $\left[\mathrm{Ca}^{2+}\right]_{\text {rest }}=50 \mathrm{nM}$, over $99.8 \%$ of CaM C-lobes should be in the $\mathrm{Ca}^{2+}$-free apo-state which has high affinity of binding to neuromodulin.

We first considered a limiting case where all CaM molecules were irreversibly bound to neuromodulin molecules located in the bouton plasma membrane. In the VCell simulations we assumed that all CaM molecules were located within a single $10 \mathrm{~nm}$ layer adjacent to the plasma membrane (Figure 5). This led to $\sim$ a ten-fold increase of $[\mathrm{CaM}]_{\text {tot }}$ near the plasma membrane $(1023 \mu \mathrm{M})$ in comparison to the case with evenly distributed CaM $(100 \mu \mathrm{M})$. The detailed $\mathrm{Ca}^{2+}$ binding kinetics to $\mathrm{CaM}$ associated with neuromodulin remains unknown. However, binding of CaM to the post-synaptically expressed neurogranin (which contains a similar CaM-binding IQ motif) has been shown to decrease $\mathrm{Ca}^{2+}$ affinity of the CaM C-lobe because of $\sim$ a fifty-fold acceleration of $\mathrm{Ca}^{2+}$ dissociation rate $k_{\text {off }}^{(R), C}$ (Gaertner et al., 2004; Hoffman et al., 2014). Therefore, in this set of simulations we also increased $k_{\text {off }}^{(R), C} 50$-fold (from 6.5 to $\left.325 \mathrm{~s}^{-1}\right)$.

The simulations revealed that in the case of irreversible binding of CaM to membrane associated neuromodulin, the presence of CaM still partially occludes the short-term facilitation caused by saturation of CB (Figure 5 and Supplementary Figure 4) to the degree similar to that observed in the case of evenly distributed immobile CaM (Figure 4 and Supplementary Figure 3).

\section{Short-term Facilitation through $\mathrm{Ca}^{2+}$-induced Dislocation of CaM from the Plasma Membrane}

We next considered a more realistic case of dynamic $\mathrm{Ca}^{2+}$. dependent interaction between $\mathrm{CaM}$ and neuromodulin. $\mathrm{Ca}^{2+}$ binding by the C-lobe of CaM reduces its affinity to neuromodulin several fold which leads to dissociation of $\mathrm{CaM}-$ neuromodulin complex (Alexander et al., 1988; Kumar et al., 2013; Hoffman et al., 2014). This prompts the hypothesis that $\mathrm{Ca}^{2+}$-induced dislocation of CaM molecules from the membrane bound neuromodulin may decrease the $\mathrm{Ca}^{2+}$ buffering capacity at the $\mathrm{AZ}$ during repetitive $\mathrm{AP}$ stimulation, which, in turn, should lead to a use-dependent increase in the vesicular release probability $p_{v}$.

To test the feasibility of this hypothesis we modeled how $\mathrm{Ca}^{2+}$-dependent dislocation of CaM molecules from the plasma membrane to the cytosol affects presynaptic $\mathrm{Ca}^{2+}$ dynamics and vesicular release during paired-pulse stimulation (Figure 6). As in Section The Case of Membrane-bound CaM we considered that at the beginning of each simulation $\left(\left[\mathrm{Ca}^{2+}\right]_{\text {rest }}=50 \mathrm{nM}\right)$ all CaM molecules were bound to the plasma membrane via the interaction with neuromodulin. We assumed that upon binding of two $\mathrm{Ca}^{2+}$ ions by the C-lobe (independently of the $\mathrm{Ca}^{2+}$ occupancy of the N-lobe), a CaM molecule can irreversibly dissociate from the plasma membrane and freely diffuse in the cytosol (with $D_{C a M}=20 \mu \mathrm{m}^{2} \mathrm{~s}^{-1}$ ) (Figure 6A). The dissociation rate of the $\mathrm{Ca}^{2+}$ bound $\mathrm{C}$-lobe from neuromodulin $\left(k_{o f f}^{C a M}\right)$ is unknown, but based on thermodynamics principles it is likely to be comparable to the effective $\mathrm{Ca}^{2+}$ dissociation rate. Therefore, we assumed that upon $\mathrm{Ca}^{2+}$ binding by the $\mathrm{C}$-lobe there is a $50 \%$ chance of CaM dissociation from neuromodulin (i.e., $k_{o f f}^{C a M}=$ $\left.2 \cdot k_{\text {off }}^{(R), C}=650 \mathrm{~s}^{-1}\right)$.

Simulations revealed a reduction of $[\mathrm{CaM}]_{\text {tot }}$ in the $\mathrm{AZ}$ caused by $\mathrm{Ca}^{2+}$ influx during the first AP (Figures 6F, G and Supplementary Figure 5). In comparison to the simulations where paired-pulse facilitation was mediated only by the buffer saturation mechanism (Figures 3-5) CaM dislocation led to a noticeably stronger increase in peak $\left[\mathrm{Ca}^{2+}\right]$ and $p_{v}$ at the second AP (Figures 6B,C). Indeed, in the case of buffer dislocation the decrease of $\mathrm{Ca}^{2+}$ buffering at the second AP was not only due to saturation of the relatively slow $\mathrm{CB}$ and CaM C-lobe $\mathrm{Ca}^{2+}$ binding sites, but also due to a direct reduction in fast $\mathrm{Ca}^{2+}$ binding to the N-lobe of CaM, which dominates regulation of fast AP-evoked $\mathrm{Ca}^{2+}$-nano/microdomain dynamics and $p_{v}$ (Figure 2).

Finally we considered the effect of CaM membrane dislocation on AP-evoked release during physiological firing patterns typical for CA1 hippocampal pyramidal cells. These are characterized by short high-frequency bursts of APs that are interleaved by single APs (O'Keefe and Dostrovsky, 1971; Dobrunz and Stevens, 1999). We modeled AP-evoked presynaptic $\mathrm{Ca}^{2+}$ dynamics and vesicular release during a $50 \mathrm{~Hz}$ burst of six APs which was followed by a single AP $300 \mathrm{~ms}$ after the burst (Figure 7A). The results of our simulations show that cumulative dislocation of CaM from the AZ plasma membrane during the AP burst leads to a prominent and lasting longer facilitation of vesicular release, as evidently from the comparison with the control simulations where all CaM molecules were irreversibly bound to the plasma membrane (Figure 7).

\section{Discussion}

This modeling study investigates the effects of $\mathrm{Ca}^{2+}$ buffering by $\mathrm{CaM}$ on AP-evoked synaptic vesicle release and short-term synaptic plasticity. The multiple roles of $\mathrm{CaM}$ in modulating synaptic transmission, which it exerts via interactions with its target proteins, have been extensively characterized (Xia and Storm, 2005; Pang et al., 2010; Sun et al., 2010; Lipstein et al., 

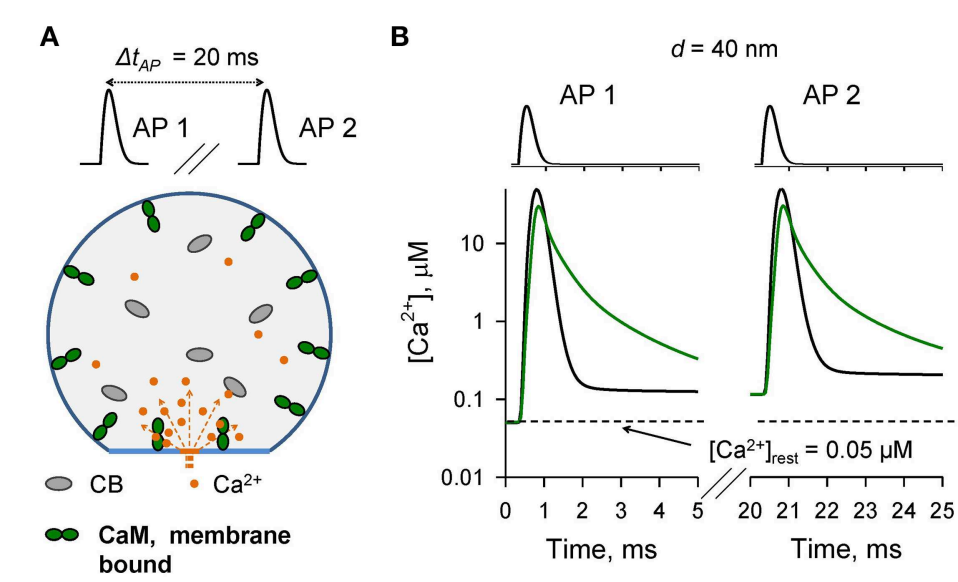

C

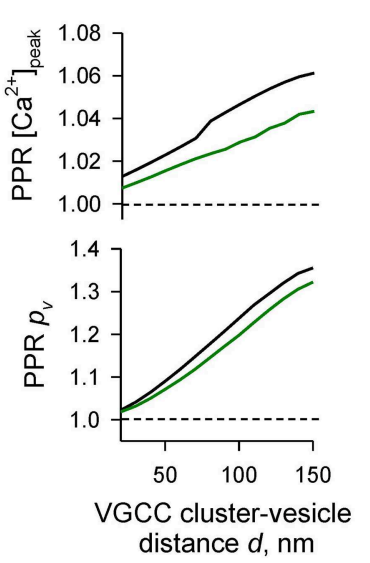

D

$\left[\mathrm{Ca}^{2+}\right], \mu \mathrm{M}$

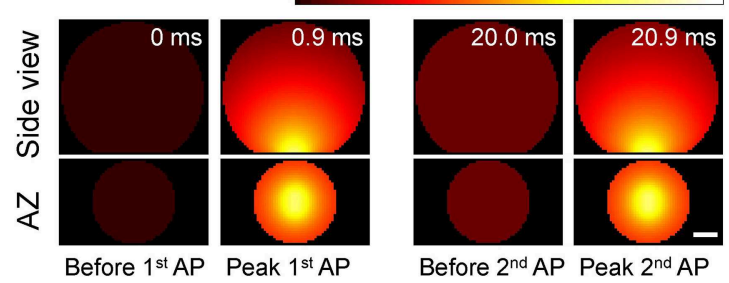

$\mathbf{F}$

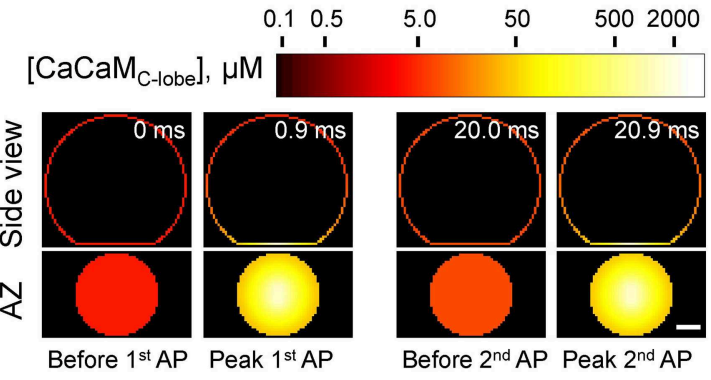

FIGURE 5 | Effect of membrane-bound CaM on short-term synaptic plasticity. (A) Schematic representation of the paired-pulse simulation experiment $\left(\Delta t_{A P}=20 \mathrm{~ms}\right)$ with membrane-bound CaM, see text for details. (B) Comparison of $\left[\mathrm{Ca}^{2+}\right]$ time courses at the vesicular release $\mathrm{Ca}^{2+}$ sensor for a representative coupling distance $d=40 \mathrm{~nm}$ during paired-pulse stimulation (AP 1 and $A P$ 2) with and without membrane-bound CaM in the presynaptic bouton. Left, $\left[\mathrm{Ca}^{2+}\right]$
E

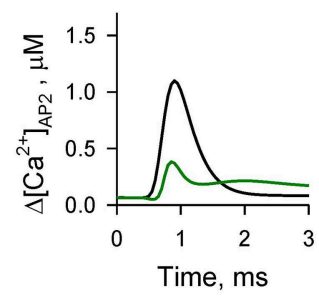

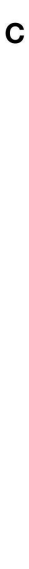

CB only $\mathrm{CB}$ and CaM distance $d, \mathrm{~nm}$

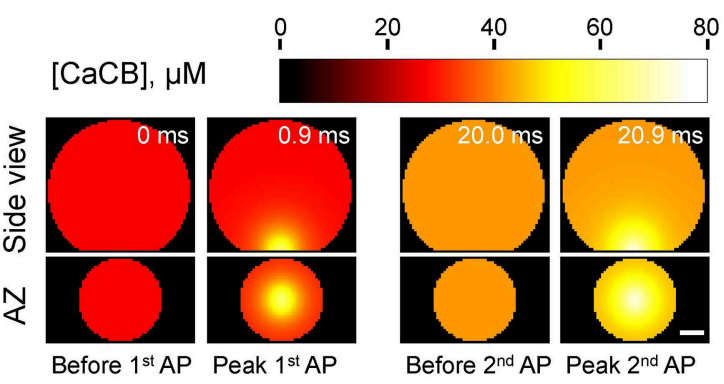

G

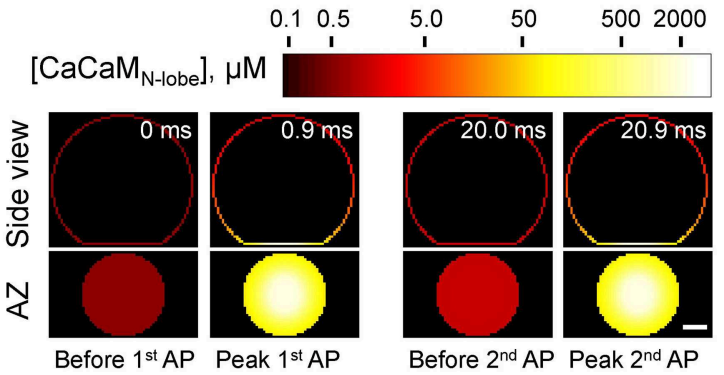

transients; right, net increase of $\left[\mathrm{Ca}^{2+}\right]$ at the second AP. (C) Dependencies of $P P R\left[\mathrm{Ca}^{2+}\right]_{\text {peak }}$ and $P P R_{p_{V}}$ on the coupling distance d. (D-G) Snapshots of spatial distribution of $\mathrm{Ca}^{2+}$ (D), $\mathrm{Ca}^{2+}$ bound to $\mathrm{CB}(\mathbf{E})$, and $\mathrm{Ca}^{2+}$ bound to the C-lobe $(\mathbf{F})$ and the $\mathrm{N}$-lobe $(\mathbf{G})$ of $\mathrm{CaM}$ during paired-pulse stimulation. Side view, $X Z$ plane through the center of the bouton (as in $\mathbf{A}$ ); $A \mathbf{Z}, 10 \mathrm{~nm}$ thick plane immediately above the AZ. Scale bar $100 \mathrm{~nm}$.

2013; Ben-Johny and Yue, 2014). Hitherto however, the direct effects of $\mathrm{Ca}^{2+}$ buffering by CaM on AP-evoked presynaptic $\mathrm{Ca}^{2+}$ dynamics and vesicular release have not been systematically investigated.

We used a realistic three-dimensional computational model of AP-evoked presynaptic $\left[\mathrm{Ca}^{2+}\right]$ dynamics and $\mathrm{Ca}^{2+}$-triggered vesicular fusion in small excitatory synapses (Ermolyuk et al., 2013). We systematically compared the effects of physiologically relevant concentrations of $\mathrm{CaM}$ and $\mathrm{CB}$ (the two major $\mathrm{Ca}^{2+}$ buffers found in central excitatory synapses) on vesicular release probability and short-term synaptic plasticity. To constrain the model parameters we used recently published detailed kinetics of $\mathrm{Ca}^{2+}$ binding to CaM (Faas et al., 2011), which reveal that the Nlobe of $\mathrm{CaM}$ binds $\mathrm{Ca}^{2+}$ much faster than any other characterized presynaptic $\mathrm{Ca}^{2+}$ buffer, whilst the CaM C-lobe binds $\mathrm{Ca}^{2+}$ with a rate comparable to that of $\mathrm{CB}$. Consistently with this, our modeling shows that fast $\mathrm{Ca}^{2+}$ binding by the N-lobe of CaM plays a dominant role in shaping $\left[\mathrm{Ca}^{2+}\right]$ within the transient AP-evoked $\mathrm{Ca}^{2+}$-nano/microdomains and as a consequence in inhibition of vesicular release probability $p_{v}$. In contrast, slower $\mathrm{Ca}^{2+}$ binding by the CaM C-lobe and by $\mathrm{CB}$ plays only a secondary role. 
A

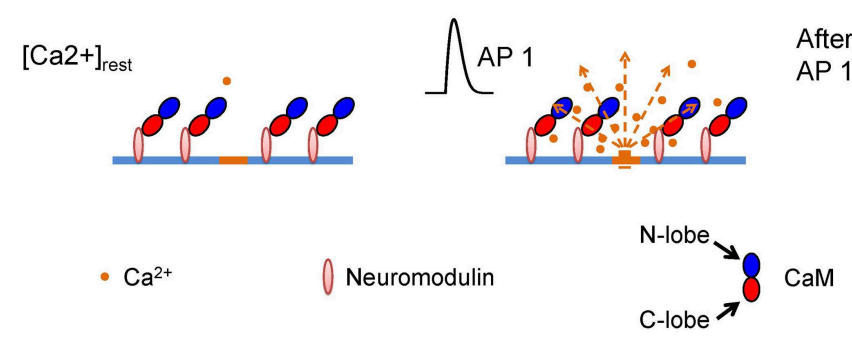

B

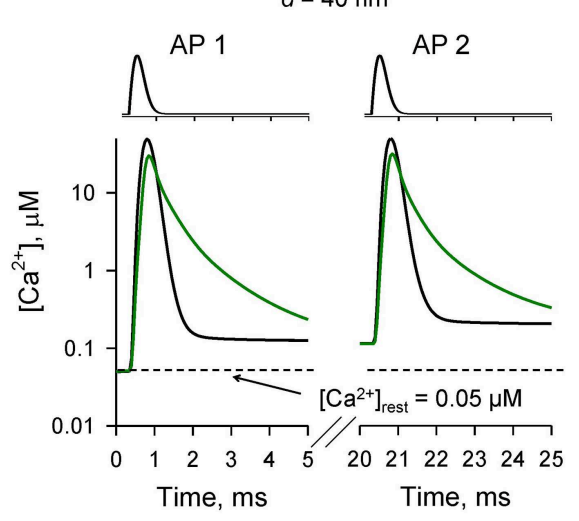

C
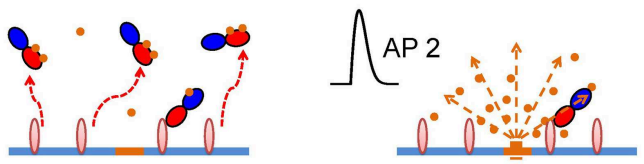
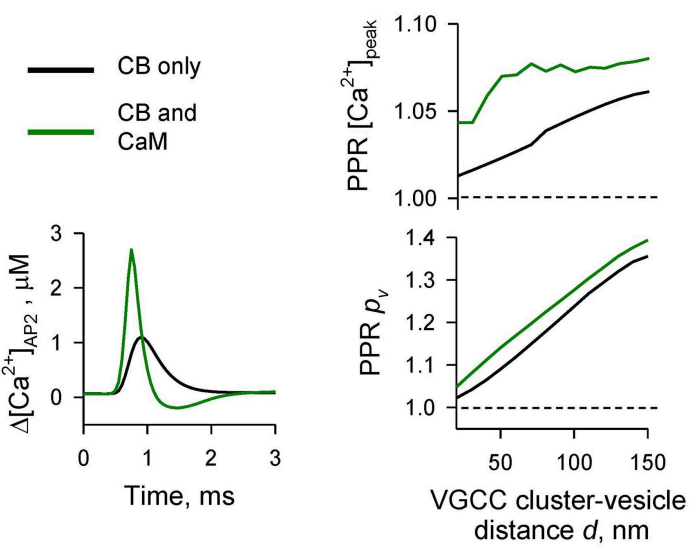

E

[CaCB], $\mu \mathrm{M}$
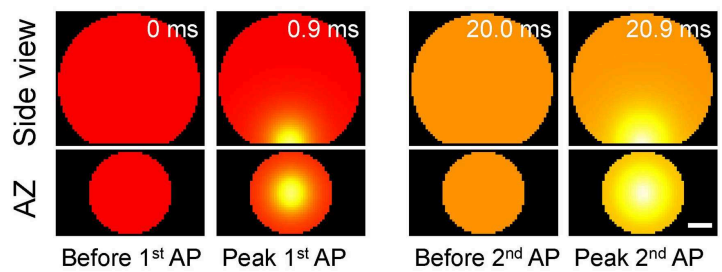

G
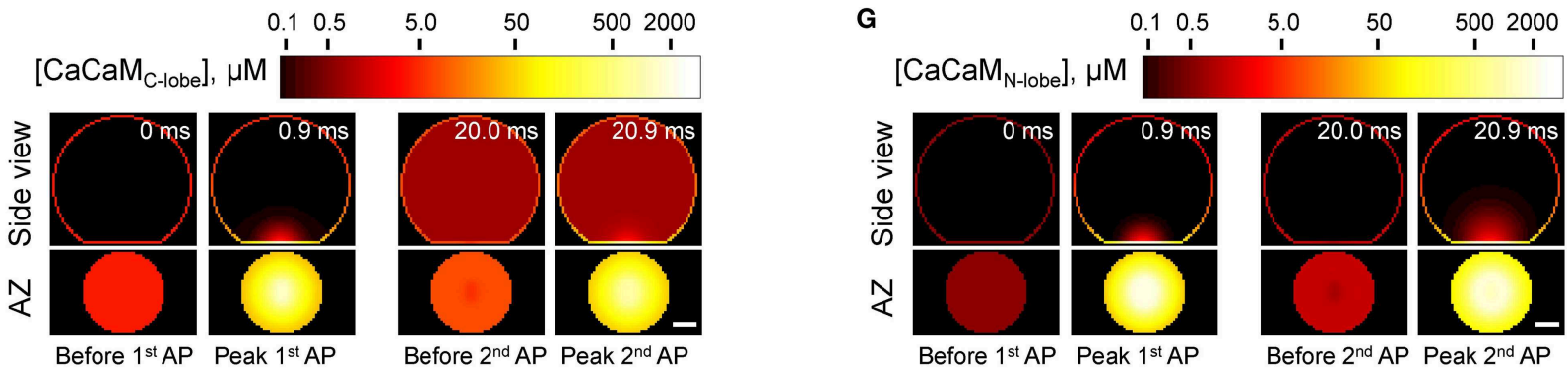

FIGURE 6 | $\mathrm{Ca}^{2+}$-dependent CaM dislocation from the $\mathrm{AZ}$ as a mechanism of short-term facilitation. (A) Schematics depicting the model of $\mathrm{Ca}^{2+}$-dependent $\mathrm{CaM}$ dislocation from the membrane during paired-pulse simulation experiment. We assumed that at resting $\left[\mathrm{Ca}^{2+}\right]_{\text {rest }}$ all CaM molecules were in the $\mathrm{Ca}^{2+}$-free apo state and bound via the $\mathrm{C}$-lobes to the membrane-associated neuromodulin molecules. Binding of two $\mathrm{Ca}^{2+}$ ions by the C-lobe during the first AP leads to its dissociation from neuromodulin and to reduction of $\mathrm{Ca}^{2+}$ buffering at the $\mathrm{AZ}$ during the second AP. (B) Comparison of $\left[\mathrm{Ca}^{2+}\right]$ time courses at the vesicular release

$\mathrm{Ca}^{2+}$ sensor for a representative coupling distance $d=40 \mathrm{~nm}$ during paired-pulse stimulation (AP 1 and AP 2) with and without CaM in the presynaptic bouton. Left, $\left[\mathrm{Ca}^{2+}\right]$ transients; right, net increase of $\left[\mathrm{Ca}^{2+}\right]$ at the second AP. (C) Dependencies of $P P R\left[\mathrm{Ca}^{2+}\right]_{\text {peak }}$ and $P P R_{p_{V}}$ on the coupling distance $d$. (D-G) Snapshots of spatial distribution of $\mathrm{Ca}^{2+}$ (D), $\mathrm{Ca}^{2+}$ bound to $\mathrm{CB}(\mathbf{E})$, and $\mathrm{Ca}^{2+}$ bound to the $\mathrm{C}$-lobe $(\mathbf{F})$ and the N-lobe (G) of CaM during paired-pulse stimulation. Side view, XZ plane through the center of the bouton (as in $\mathbf{A}$ ); $A Z, 10 \mathrm{~nm}$ thick plane immediately above the AZ. Scale bar $100 \mathrm{~nm}$. 
A

\section{CaM dislocation}
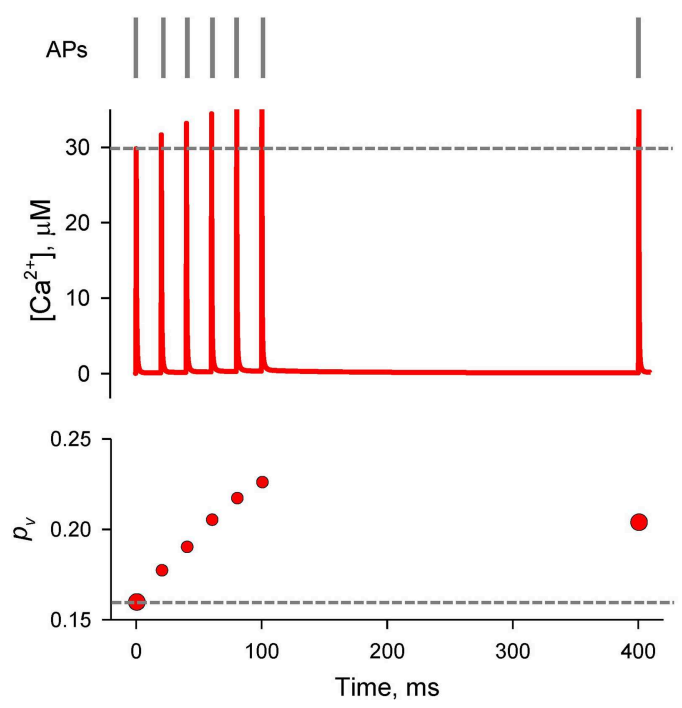

B

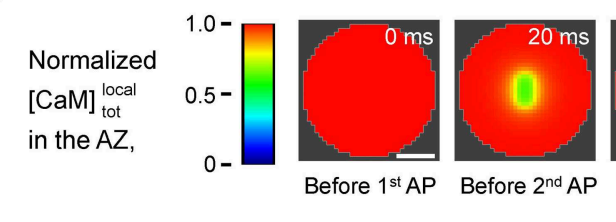

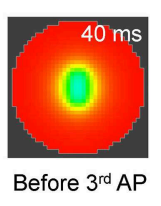
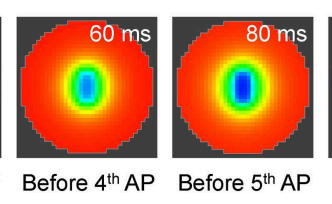

Before $6^{\text {th }}$ AP

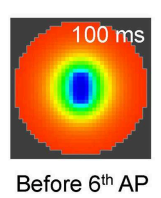

Control
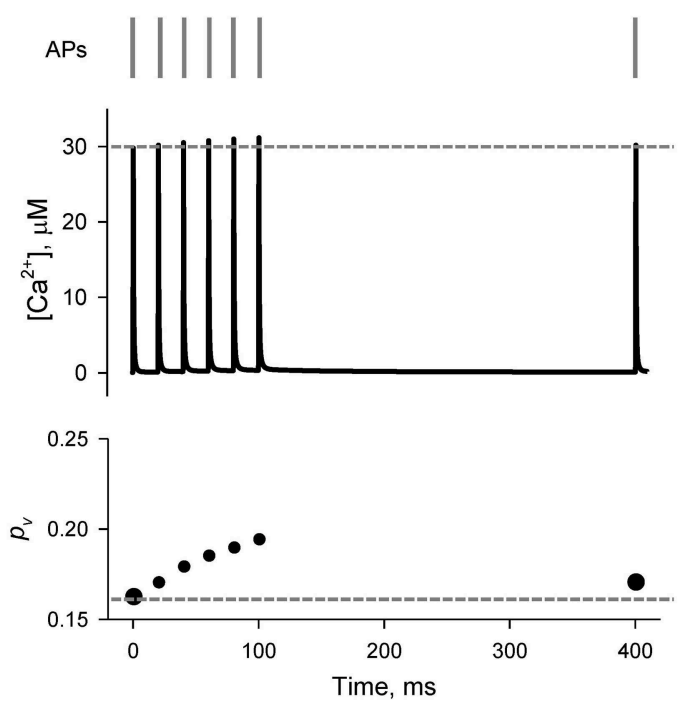

C

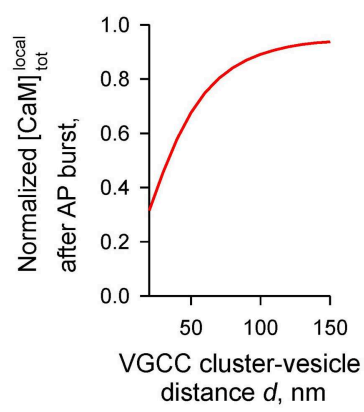

FIGURE 7 | Effect of CaM dislocation on short-term facilitation during physiological patterns of AP firing. (A) Comparison of $\mathrm{Ca}^{2+}$ dynamics and vesicular release probability $p_{V}$ at a representative coupling distance $d=40 \mathrm{~nm}$ for the case of $\mathrm{Ca}^{2+}$-dependent CaM dislocation (left) and for the control case of irreversible membrane bound CaM (right). Top, physiological AP stimulus pattern; middle, $\left[\mathrm{Ca}^{2+}\right]$ time courses; bottom, $p_{v}$ plots. (B) Snapshots of spatial distribution of normalized local total [CaM] tot (which accounts for all $\mathrm{CaM}$ molecules in a given voxel, irrespective of their $\mathrm{Ca}^{2+}$
Before $5^{\text {th }} \mathrm{AP}$
D

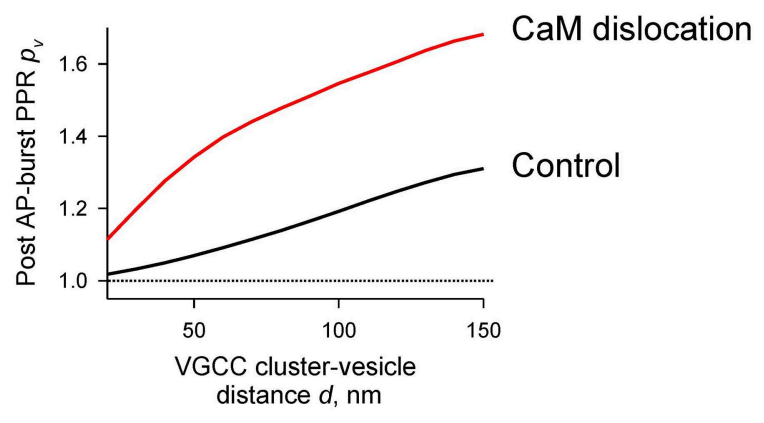

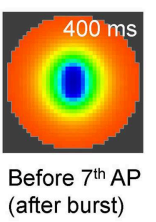

(after burst) binding state) in the AZ, illustrating progressive dislocation of CaM from the AZ during the burst of APs [the same stimulation pattern as in (A)]. Scale bar $100 \mathrm{~nm}$. (C) Dependency of normalized $[\mathrm{CaM}]_{\text {tot }}^{\text {local }}$ after the burst of APs on the coupling distance $d$. Normalized $[\mathrm{CaM}]_{\text {tot }}^{\text {local }}$ in (B) and (C) were calculated by dividing the spatially dependent [CaM] tocal by the initial total $[\mathrm{CaM}]_{\text {tot }}=1023 \mu \mathrm{M}$. (D) Dependences of post-AP burst $P P R_{p_{V}}$ on the coupling distance $d$ calculated for the single AP at $t=400 \mathrm{~ms}$ and for the first AP in the burst.
Our simulations also demonstrate that, depending on its mobility and location, CaM may exert opposite effects on short-term facilitation of synaptic responses. First, the fast $\mathrm{Ca}^{2+}$ binding/unbinding by the CaM N-lobe generally occludes paired-pulse facilitation of vesicular release caused by partial saturation of $\mathrm{CB}$ and the CaM C-lobe (which release $\mathrm{Ca}^{2+}$ on a slow time scale). Such an occlusion mechanism, and possible differences in concentration, location and mobility of CaM may explain why $\mathrm{Ca}^{2+}$ saturation of $\mathrm{CB}$ contributes to short-term facilitation only in certain types of synapses (e.g., Blatow et al., 2003; Muller et al., 2005; Bornschein et al., 2013).

Second, we propose a novel mechanism of short-term facilitation through $\mathrm{Ca}^{2+}$-induced dislocation of $\mathrm{CaM}$ from the plasma membrane. It is thought that at resting conditions 
most of the presynaptic CaM is bound to the membraneassociated protein neuromodulin (Alexander et al., 1988; Xia and Storm, 2005). The binding occurs at low $\left[\mathrm{Ca}^{2+}\right]$ via interaction between the apoCaM C-lobe and the IQ-motif of neuromodulin. Upon $\mathrm{Ca}^{2+}$ binding by the C-lobe when $\left[\mathrm{Ca}^{2+}\right]$ increases this interaction becomes weaker and $\mathrm{CaM}$ dissociates from neuromodulin (Xia and Storm, 2005; Kumar et al., 2013). Thus, we hypothesize that transient increase of $\left[\mathrm{Ca}^{2+}\right]$ within $\mathrm{Ca}^{2+}$. nano/microdomains may lead to a dislocation of CaM molecules from the plasma membrane at the $\mathrm{AZ}$ into the cytosol.

Indeed, our simulations show that even a single AP would lead to a reduction in $[\mathrm{CaM}]_{t o t}$ in the AZ. Such a stimulationdependent reduction of $\mathrm{Ca}^{2+}$ buffering capacity within the $\mathrm{AZ}$ results in a noticeable increase in the paired-pulse ratio when compared to the control simulation with irreversible membranebound CaM. The effect of $\mathrm{Ca}^{2+}$-dependent CaM dislocation was even more prominent during the physiological burst-like AP firing of pyramidal cells.

When modeling the effect of $\mathrm{Ca}^{2+}$-dependent $\mathrm{CaM}$ dislocation we assumed that the effective concentration of $\mathrm{CaM}$ at the membrane was $\sim 1000 \mu \mathrm{M}$ (to maintain the experimentally estimated $[\mathrm{CaM}]_{\text {tot }}$ in the entire bouton at $100 \mu \mathrm{M})$. This corresponds to $\sim 25 \mathrm{CaM}$ molecules located at an average sized $\mathrm{AZ}$ with an area $S_{A Z}=0.04 \mu \mathrm{m}^{2}$ (Schikorski and Stevens, 1997; Holderith et al., 2012). In reality it is likely that the density of CaM molecules bound at the $\mathrm{AZ}$ is even higher than that because at $\left[\mathrm{Ca}^{2+}\right]_{\text {rest }}$ apoCaM molecules are also bound to the presynaptic VGCCs via a similar IQ-motif interaction (Ben-Johny and Yue, 2014).

In this work we used a simplified model that did not take into account the mobility of VGCCs in the presynaptic membrane (Schneider et al., 2015) and also assumed irreversible dissociation of CaM from neuromodulin when both binding

\section{References}

Alexander, K. A., Wakim, B. T., Doyle, G. S., Walsh, K. A., and Storm, D. R. (1988). Identification and characterization of the calmodulin-binding domain of neuromodulin, a neurospecific calmodulin-binding protein. J. Biol. Chem. 263, 7544-7549.

Ariel, P., and Ryan, T. A. (2010). Optical mapping of release properties in synapses. Front. Neural Circuits 4:18. doi: 10.3389/fncir.2010.00018

Ben-Johny, M., and Yue, D. T. (2014). Calmodulin regulation (calmodulation) of voltage-gated calcium channels. J. Gen. Physiol. 143, 679-692. doi: 10.1085/jgp.201311153

Berggard, T., Miron, S., Onnerfjord, P., Thulin, E., Akerfeldt, K. S., Enghild, J. J., et al. (2002). Calbindin D28k exhibits properties characteristic of a Ca2+ sensor. J. Biol. Chem. 277, 16662-16672. doi: 10.1074/jbc.M200 415200

Blatow, M., Caputi, A., Burnashev, N., Monyer, H., and Rozov, A. (2003). $\mathrm{Ca} 2+$ buffer saturation underlies paired pulse facilitation in calbindin-D28kcontaining terminals. Neuron 38, 79-88. doi: 10.1016/S0896-6273(03)00196-X

Bornschein, G., Arendt, O., Hallermann, S., Brachtendorf, S., Eilers, J., and Schmidt, H. (2013). Paired-pulse facilitation at recurrent Purkinje neuron synapses is independent of calbindin and parvalbumin during high-frequency activation. J. Physiol. 591, 3355-3370. doi: 10.1113/jphysiol.2013.254128

Dobrunz, L. E., and Stevens, C. F. (1999). Response of hippocampal synapses to natural stimulation patterns. Neuron 22, 157-166. doi: 10.1016/S08966273(00)80687-X sites on the CaM C-lobe were occupied by $\mathrm{Ca}^{2+}$ ions. Yet, the detailed kinetics of $\mathrm{CaM}$ and neuromodulin interaction in the presence and in the absence of $\mathrm{Ca}^{2+}$ remains largely unknown. Thus, further experimental and modeling work is required to obtain more realistic models of the complex kinetics of $\mathrm{Ca}^{2+}$-dependent interaction of $\mathrm{CaM}$ with its binding partners at the AZ. Furthermore, activity-dependent phosphorylation of neuromodulin and other IQ-motif containing proteins prevents their interaction with CaM (Xia and Storm, 2005; Kumar et al., 2013). This should lead to long-lasting changes in the distribution of CaM molecules between the membrane-bound and mobile states, thus regulating $\mathrm{Ca}^{2+}$ buffering capacity at the $\mathrm{AZ}$ and $p_{v}$ on a longer timescale. Our theoretical modeling study thus argues that $\mathrm{Ca}^{2+}$-dependent $\mathrm{CaM}$ dislocation from the plasma membrane could provide a powerful mechanism for dynamic modulation of vesicular release during physiological patterns of activity, and calls for direct experimental testing of this hypothesis.

\section{Acknowledgments}

This study was supported by the Welcome Trust. The Virtual Cell simulation environment is supported by NIH Grant Number P41 GM103313 from the National Institute for General Medical Sciences. We are grateful to J. Jepson, S. Krishnakumar, D. M. Kullmann, I. Pavlov, and S. Schorge for critical reading of the manuscript.

\section{Supplementary Material}

The Supplementary Material for this article can be found online at: http://journal.frontiersin.org/article/10.3389/fncel. 2015.00239
Ermolyuk, Y. S., Alder, F. G., Surges, R., Pavlov, I. Y., Timofeeva, Y., Kullmann, D. M., et al. (2013). Differential triggering of spontaneous glutamate release by P/Q-, N- and R-type $\mathrm{Ca}(2+)$ channels. Nat. Neurosci. 16, 1754-1763. doi: 10.1038/nn.3563

Ermolyuk, Y. S., Alder, F. G., Henneberger, C., Rusakov, D. A., Kullmann, D. M., and Volynski, K. E. (2012). Independent regulation of basal neurotransmitter release efficacy by variable $\mathrm{Ca} 2+$ influx and bouton size at small central synapses. PLoS Biol. 10:e1001396. doi: 10.1371/journal.pbio.1001396

Faas, G. C., Raghavachari, S., Lisman, J. E., and Mody, I. (2011). Calmodulin as a direct detector of $\mathrm{Ca} 2+$ signals. Nat. Neurosci. 14, 301-304. doi: 10.1038/nn.2746

Gaertner, T. R., Putkey, J. A., and Waxham, M. N. (2004). RC3/Neurogranin and $\mathrm{Ca} 2+/$ calmodulin-dependent protein kinase II produce opposing effects on the affinity of calmodulin for calcium. J. Biol. Chem. 279, 39374-39382. doi: 10.1074/jbc.M405352200

Hines, M. L., and Carnevale, N. T. (1997). The NEURON simulation environment. Neural Comput. 9, 1179-1209. doi: 10.1162/neco.1997.9.6.1179

Hoffman, L., Chandrasekar, A., Wang, X., Putkey, J. A., and Waxham, M. N. (2014). Neurogranin alters the structure and calcium binding properties of calmodulin. J. Biol. Chem. 289, 14644-14655. doi: 10.1074/jbc.M114. 560656

Holderith, N., Lorincz, A., Katona, G., Rozsa, B., Kulik, A., Watanabe, M., et al. (2012). Release probability of hippocampal glutamatergic terminals scales with the size of the active zone. Nat. Neurosci. 15, 988-997. doi: 10.1038/ nn.3137 
Jackson, M. B., and Redman, S. J. (2003). Calcium dynamics, buffering, and buffer saturation in the boutons of dentate granule-cell axons in the hilus. J. Neurosci. 23, 1612-1621.

Kumar, V., Chichili, V. P., Zhong, L., Tang, X., Velazquez-Campoy, A., Sheu, F. S., et al. (2013). Structural basis for the interaction of unstructured neuron specific substrates neuromodulin and neurogranin with Calmodulin. Sci. Rep. 3:1392. doi: $10.1038 /$ srep 01392

Li, L., Bischofberger, J., and Jonas, P. (2007). Differential gating and recruitment of P/Q-, N-, and R-type Ca2+ channels in hippocampal mossy fiber boutons. J. Neurosci. 27, 13420-13429. doi: 10.1523/JNEUROSCI.1709-07.2007

Lipstein, N., Sakaba, T., Cooper, B. H., Lin, K. H., Strenzke, N., Ashery, U., et al. (2013). Dynamic control of synaptic vesicle replenishment and short-term plasticity by $\mathrm{Ca}(2+)$-calmodulin-Munc13-1 signaling. Neuron $79,82-96$. doi: 10.1016/j.neuron.2013.05.011

Lou, X., Scheuss, V., and Schneggenburger, R. (2005). Allosteric modulation of the presynaptic Ca2+ sensor for vesicle fusion. Nature 435, 497-501. doi: 10.1038/nature03568

Matveev, V., Bertram, R., and Sherman, A. (2006). Residual bound Ca2+ can account for the effects of $\mathrm{Ca} 2+$ buffers on synaptic facilitation. J. Neurophysiol. 96, 3389-3397. doi: 10.1152/jn.00101.2006

Matveev, V., Zucker, R. S., and Sherman, A. (2004). Facilitation through buffer saturation: constraints on endogenous buffering properties. Biophys. J. 86, 2691-2709. doi: 10.1016/S0006-3495(04)74324-6

Meinrenken, C. J., Borst, J. G., and Sakmann, B. (2002). Calcium secretion coupling at calyx of held governed by nonuniform channel-vesicle topography. J. Neurosci. 22, 1648-1667.

Mintz, I. M., Sabatini, B. L., and Regehr, W. G. (1995). Calcium control of transmitter release at a cerebellar synapse. Neuron 15, 675-688. doi: 10.1016/0896-6273(95)90155-8

Muller, A., Kukley, M., Stausberg, P., Beck, H., Muller, W., and Dietrich, D. (2005). Endogenous Ca2+ buffer concentration and $\mathrm{Ca} 2+$ microdomains in hippocampal neurons. J. Neurosci. 25, 558-565. doi: 10.1523/JNEUROSCI.3799-04.2005

Murthy, V. N., Schikorski, T., Stevens, C. F., and Zhu, Y. (2001). Inactivity produces increases in neurotransmitter release and synapse size. Neuron 32, 673-682. doi: 10.1016/S0896-6273(01)00500-1

Nagerl, U. V., Novo, D., Mody, I., and Vergara, J. L. (2000). Binding kinetics of calbindin- $\mathrm{D}(28 \mathrm{k})$ determined by flash photolysis of caged $\mathrm{Ca}(2+)$. Biophys. J. 79, 3009-3018. doi: 10.1016/S0006-3495(00)76537-4

Nakamura, Y., Harada, H., Kamasawa, N., Matsui, K., Rothman, J. S., Shigemoto, R., et al. (2015). Nanoscale distribution of presynaptic $\mathrm{Ca}(2+)$ channels and its impact on vesicular release during development. Neuron $85,145-158$. doi: 10.1016/j.neuron.2014.11.019

Neher, E. (1998). Usefulness and limitations of linear approximations to the understanding of Ca2+ signals. Cell Calcium 24, 345-357. doi: 10.1016/S01434160(98)90058-6

O'Keefe, J., and Dostrovsky, J. (1971). The hippocampus as a spatial map. Preliminary evidence from unit activity in the freely-moving rat. Brain Res. 34, 171-175. doi: 10.1016/0006-8993(71)90358-1

Pang, Z. P., Cao, P., Xu, W., and Sudhof, T. C. (2010). Calmodulin controls synaptic strength via presynaptic activation of calmodulin kinase II. J. Neurosci. 30, 4132-4142. doi: 10.1523/JNEUROSCI.3129-09.2010
Putkey, J. A., Kleerekoper, Q., Gaertner, T. R., and Waxham, M. N. (2003). A new role for IQ motif proteins in regulating calmodulin function. J. Biol. Chem. 278, 49667-49670. doi: 10.1074/jbc.C300372200

Reid, C. A., Bekkers, J. M., and Clements, J. D. (1998). N- and P/Q-type $\mathrm{Ca} 2+$ channels mediate transmitter release with a similar cooperativity at rat hippocampal autapses. J. Neurosci. 18, 2849-2855.

Rozov, A., Burnashev, N., Sakmann, B., and Neher, E. (2001). Transmitter release modulation by intracellular $\mathrm{Ca} 2+$ buffers in facilitating and depressing nerve terminals of pyramidal cells in layer $2 / 3$ of the rat neocortex indicates a target cell-specific difference in presynaptic calcium dynamics. J. Physiol. 531, 807-826. doi: 10.1111/j.1469-7793.2001.0807h.x

Sabatini, B. L., and Regehr, W. G. (1998). Optical measurement of presynaptic calcium currents. Biophys. J. 74, 1549-1563. doi: 10.1016/S0006-3495(98)77867-1

Schikorski, T., and Stevens, C. F. (1997). Quantitative ultrastructural analysis of hippocampal excitatory synapses. J. Neurosci. 17, 5858-5867.

Schneider, R., Hosy, E., Kohl, J., Klueva, J., Choquet, D., Thomas, U., et al. (2015). Mobility of calcium channels in the presynaptic membrane. Neuron 86 , 672-679. doi: 10.1016/j.neuron.2015.03.050

Scott, R., and Rusakov, D. A. (2006). Main determinants of presynaptic $\mathrm{Ca} 2+$ dynamics at individual mossy fiber-CA3 pyramidal cell synapses. J. Neurosci. 26, 7071-7081. doi: 10.1523/JNEUROSCI.094606.2006

Sheng, J., He, L., Zheng, H., Xue, L., Luo, F., Shin, W., et al. (2012). Calcium-channel number critically influences synaptic strength and plasticity at the active zone. Nat. Neurosci. 15, 998-1006. doi: 10.1038/ nn.3129

Sun, T., Wu, X. S., Xu, J., McNeil, B. D., Pang, Z. P., Yang, W., et al. (2010). The role of calcium/calmodulin-activated calcineurin in rapid and slow endocytosis at central synapses. J. Neurosci. 30, 11838-11847. doi: 10.1523/JNEUROSCI.148110.2010

Villarroel, A., Taglialatela, M., Bernardo-Seisdedos, G., Alaimo, A., Agirre, J., Alberdi, A., et al. (2014). The ever changing moods of calmodulin: how structural plasticity entails transductional adaptability. J. Mol. Biol. 426, 2717-2735. doi: 10.1016/j.jmb.2014.05.016

Wu, L. G., and Saggau, P. (1994). Pharmacological identification of two types of presynaptic voltage-dependent calcium channels at CA3-CA1 synapses of the hippocampus. J. Neurosci. 14, 5613-5622.

Xia, Z., and Storm, D. R. (2005). The role of calmodulin as a signal integrator for synaptic plasticity. Nat. Rev. Neurosci. 6, 267-276. doi: 10.1038/ nrn1647

Conflict of Interest Statement: The authors declare that the research was conducted in the absence of any commercial or financial relationships that could be construed as a potential conflict of interest.

Copyright (c) 2015 Timofeeva and Volynski. This is an open-access article distributed under the terms of the Creative Commons Attribution License (CC BY). The use, distribution or reproduction in other forums is permitted, provided the original author(s) or licensor are credited and that the original publication in this journal is cited, in accordance with accepted academic practice. No use, distribution or reproduction is permitted which does not comply with these terms. 


\section{Buffer mobility and the regulation of neuronal calcium domains}

\section{Elizabeth A. Matthews* and Dirk Dietrich}

Experimental Neurophysiology, Department of Neurosurgery, University Clinic Bonn, Bonn, Germany

\section{Edited by:}

Philippe Isope, Centre National pour la

Recherche Scientifique, France

\section{Reviewed by:}

Heinz Beck, University of Bonn Medical Center, Germany

Guido C. Faas, University of California, Los Angeles, USA

\section{${ }^{*}$ Correspondence:}

Elizabeth A. Matthews, Experimental Neurophysiology, Department of Neurosurgery, University Clinic Bonn, Sigmund-Freud Straße 25D, 53105 Bonn, Germany

e-mail: emat@uni-bonn.de
The diffusion of calcium inside neurons is determined in part by the intracellular calcium binding species that rapidly bind to free calcium ions upon entry. It has long been known that some portion of a neuron's intracellular calcium binding capacity must be fixed or poorly mobile, as calcium diffusion is strongly slowed in the intracellular environment relative to diffusion in cytosolic extract. The working assumption was that these immobile calcium binding sites are provided by structural proteins bound to the cytoskeleton or intracellular membranes and may thereby be relatively similar in composition and capacity across different cell types. However, recent evidence suggests that the immobile buffering capacity can vary greatly between cell types and that some mobile calcium binding proteins may alter their mobility upon binding calcium, thus blurring the line between mobile and immobile. The ways in which immobile buffering capacity might be relevant to different calcium domains within neurons has been explored primarily through modeling. In certain regimes, the presence of immobile buffers and the interaction between mobile and immobile buffers have been shown to result in complex spatiotemporal patterns of free calcium. In total, these experimental and modeling findings call for a more nuanced consideration of the local intracellular calcium microenvironment. In this review we focus on the different amounts, affinities, and mobilities of immobile calcium binding species; propose a new conceptual category of physically diffusible but functionally immobile buffers; and discuss how these buffers might interact with mobile calcium binding partners to generate characteristic calcium domains.

Keywords: calcium buffer, mobile, immobile, calcium domains, diffusion coefficient
Calcium is a broadly active signaling molecule in neurons and can initiate a diverse set of actions, from neurotransmitter release, to induction of synaptic plasticity, to gene transcription. But intracellular calcium is not indiscriminately active, indicating that systems for controlling and directing the signaling cascade must exist. This regulation results from the combined effects of localized calcium entry, binding by mobile and immobile endogenous buffering species, distribution within intracellular compartments, and finally removal from the intracellular space. In combination, these regulatory actions produce different spatial and temporal domains of calcium: microdomains around single channels, calcium signals limited to spines or small dendritic segments, and global calcium elevations involving the entire cell (Augustine et al., 2003). Calcium entry, binding, diffusion, and removal have been topics of intense focus in order to better predict the types of calcium domains that could arise during physiological neuronal activity and to better understand the functional consequences of each calcium domain on information transfer and the pattern of pre- and post-synaptic activity. In the period of time after calcium has entered a cell, but before it has been removed or sequestered into internal stores, the distribution and availability of calcium is dictated by the presence of endogenous buffering molecules.

\section{CONSIDERING BUFFER MOBILITY}

Initially, endogenous calcium buffers were assumed to be "unsaturable, infinitely fast, and immobile" (Sala and Hernandez Cruz, 1990). However, the development of fast calcium dyes that allowed for direct measures of the intracellular free calcium concentration in real time made it clear that there must be fast buffers present in cells that could be washed out relatively rapidly by the patch pipette (Thayer and Miller, 1990; Zhou and Neher, 1993; Mueller et al., 2005), and were thus mobile. The impact of mobile buffers on the time course and spatial range of calcium signals was first shown by modeling (Sala and Hernandez Cruz, 1990), and later experimentally. Since then, the molecular identities of many of these mobile binding partners have been uncovered, and their binding properties have been intensely studied. The properties and functional role of mobile calcium binding proteins have been well reviewed (Baimbridge et al., 1992; Schwaller, 2010), and so this review will instead focus attention on the somewhat neglected topic of immobile calcium buffers in neurons. Immobile buffers, although their molecular identities remain unknown, deserve separate consideration because of their unique role in shaping the spatial domain of intracellular free calcium. Immobile buffers alone greatly slow the spatial spread of calcium, while at the same time prolonging the temporal duration of the signal. In the presence of mobile buffers, immobile 
buffers increase the complexity of the spatio-temporal signaling repertoires available to the neuron, based on the relative affinities, kinetics, and concentrations of the different buffers.

At the outset, we should define "immobile." Simplistically, the physical definition is a buffer that has a diffusion coefficient of $0 \mu \mathrm{m}^{2} / \mathrm{s}$, while a mobile buffer has a diffusion coefficient $>0 \mu \mathrm{m}^{2} / \mathrm{s}$. Of more interest though, is the functional mobility, i.e., how does the buffer affect the amount and distribution of free calcium. Under certain physiological conditions, even mobile buffers can function like an immobile buffer by slowing and restricting the increase and spread of free calcium concentration. Even a highly mobile buffer retards calcium diffusion relative to the diffusion of unbound calcium $\left(\sim 220 \mu \mathrm{m}^{2} / \mathrm{s}\right.$ for free calcium in cytosol (Allbritton et al., 1992), implying that mobile buffers will also slow calcium diffusion and can act analogously to immobile buffers. In contrast, small exogenously applied calcium chelators such as BAPTA, Oregon Green-BAPTA, or EGTA that are commonly used in experiments will virtually always function as mobile buffers, as their estimated diffusion coefficients are nearly the same as for free calcium (Naraghi and Neher, 1997). So in what context is a mobile buffer functionally immobile? Using Equation 1, presented later in this paper, we have calculated buffer diffusion coefficient threshold values to predict, whether the effect of a generic diffusible buffer on free calcium will be that of a functionally mobile or immobile buffer within three spatial domains representing microdomains around a channel, in a spine-like compartment, or in a dendritic segment. Buffer capacity is quantified by the unit-less measure $\kappa$ (see following paragraphs). With a background of high immobile buffer capacity $\left(\kappa_{\text {immobile }}=150\right)$, the mobile buffer must have a diffusion coefficient greater than $2 \mu \mathrm{m}^{2} / \mathrm{s}$ to increase the apparent diffusion coefficient of calcium $\left(\mathrm{D}_{\mathrm{app}}\right)$ by $\sim 10 \%$. However, in the context of low immobile buffering $\left(\kappa_{\text {immobile }}=15\right)$, even a mobile protein with a diffusion coefficient of $\sim 14 \mu \mathrm{m}^{2} / \mathrm{s}$ would act as a functionally immobile buffer, slowing the apparent diffusion of calcium. Many well-known calcium binding proteins (parvalbumin, calbindin, etc) are mobile, with diffusion coefficients that have been measured in cells and in vitro. The diffusion of mobile proteins varies between sub-cellular compartments (Schmidt et al., 2003, 2007), and in the case of calretinin, following neuronal activity (Arendt et al., 2013); these measured diffusion coefficients cover a large range [calretinin: $2.2 \mu \mathrm{m}^{2} / \mathrm{s}$ (Arendt et al., 2013), calbindin: $20 \mu \mathrm{m}^{2} / \mathrm{s}$ (Schmidt et al., 2005), parvalbumin: $43 \mu \mathrm{m}^{2} / \mathrm{s}$ (Schmidt et al., 2007)]. For the mobile proteins at the slower end of the range, whether they function as mobile or immobile at the microdomain level depends entirely on the background of physically immobile buffer that is concurrently available.

\section{THEORETICAL DEFINITION AND EXPERIMENTAL QUANTIFICATION OF BUFFERING}

Buffering capacity $(\kappa)$ describes how much calcium will be bound and how much will remain free following an increase in free calcium inside the neuron. It is typically quantified at resting calcium concentration of the cell (Box 1). A high buffering capacity means that very little calcium will remain free following an action potential or other calcium-generating event. Numerous studies have examined the total endogenous buffer capacity present in various cell types, although because of the different experimental approaches, comparisons between the reported binding capacities are complicated (see Table 1). Further confusing the issue, the relative contributions of the mobile and immobile buffering fractions have not always been directly or independently measured in these different studies. In some cases, the authors disregarded the question of buffer mobility entirely.

\section{Box 1}

\section{Some helpful relationships}

Buffering Reaction

$$
\left[\mathrm{Ca}^{2+}\right]+[B] \underset{k_{\text {off }}}{\stackrel{k_{\text {on }}}{\rightleftarrows}}\left[B C \mathrm{Ca}^{2+}\right]
$$

Dissociation Constant $\left(K_{d}\right)$

$$
K_{d}=\frac{k_{o f f}}{k_{o n}}
$$

Buffering Capacity $(\kappa)$

$$
\kappa=\frac{K_{d}[B]}{\left(K_{d}+\left[C a^{2+}\right]_{i}\right)^{2}}=\frac{\mathrm{d}\left[B C a^{2+}\right]}{\mathrm{d}\left[C a^{2+}\right]} \approx \frac{[B]}{K_{d}}
$$

The "added buffer" method is typically used to measure the endogenous buffer capacity. This method involves the addition of exogenous calcium buffer (dye or chelator) via a patch pipette. The endogenous $\kappa$ value is then extrapolated from the linear relationship found by plotting amplitude or decay of a calcium transient against the added buffer. The experiment can be done in either a population of cells or in a single cell. In population experiments, different cells are loaded with different, known amounts of exogenous buffer. If the measures of endogenous buffering are repeated at fixed time points during the recording, then changes in endogenous buffering (due to washout), run-down or run-up of extrusion, and changes in calcium entry are easily identified by comparing the fitted data from early in the experiment to later time points. The second approach is to load dye into a single cell and use the increasing exogenous $\kappa$ during the initial loading period to extract the endogenous buffer capacity. Neither of these methods can inherently differentiate between the contributions of mobile and immobile buffers to the total endogenous $\kappa$. If the recording durations, the distance of the imaging location from the patch pipette, and the expression of mobile calcium binding proteins are reported, an educated guess can be made as to the extent of washout of the mobile fraction (Pusch and Neher, 1988; Scott and Rusakov, 2006). In the absence of such specific information or an explicit check of the mobility of the endogenous buffering species, the total endogenous $\kappa$ reported by the added buffer method sets an upper limit for the amount of immobile $\kappa$ present - either the reported buffer capacity is entirely immobile, or the reported value represents a mixture of mobile and immobile components, and the immobile buffering capacity is less than the measured value.

Most studies using the dye loading method report loading times between 3 and 10 min for proximal dendrites to achieve a plateau fluorescence; even with these short loading times, mobile buffers may be lost to the recording pipette, especially from proximal 


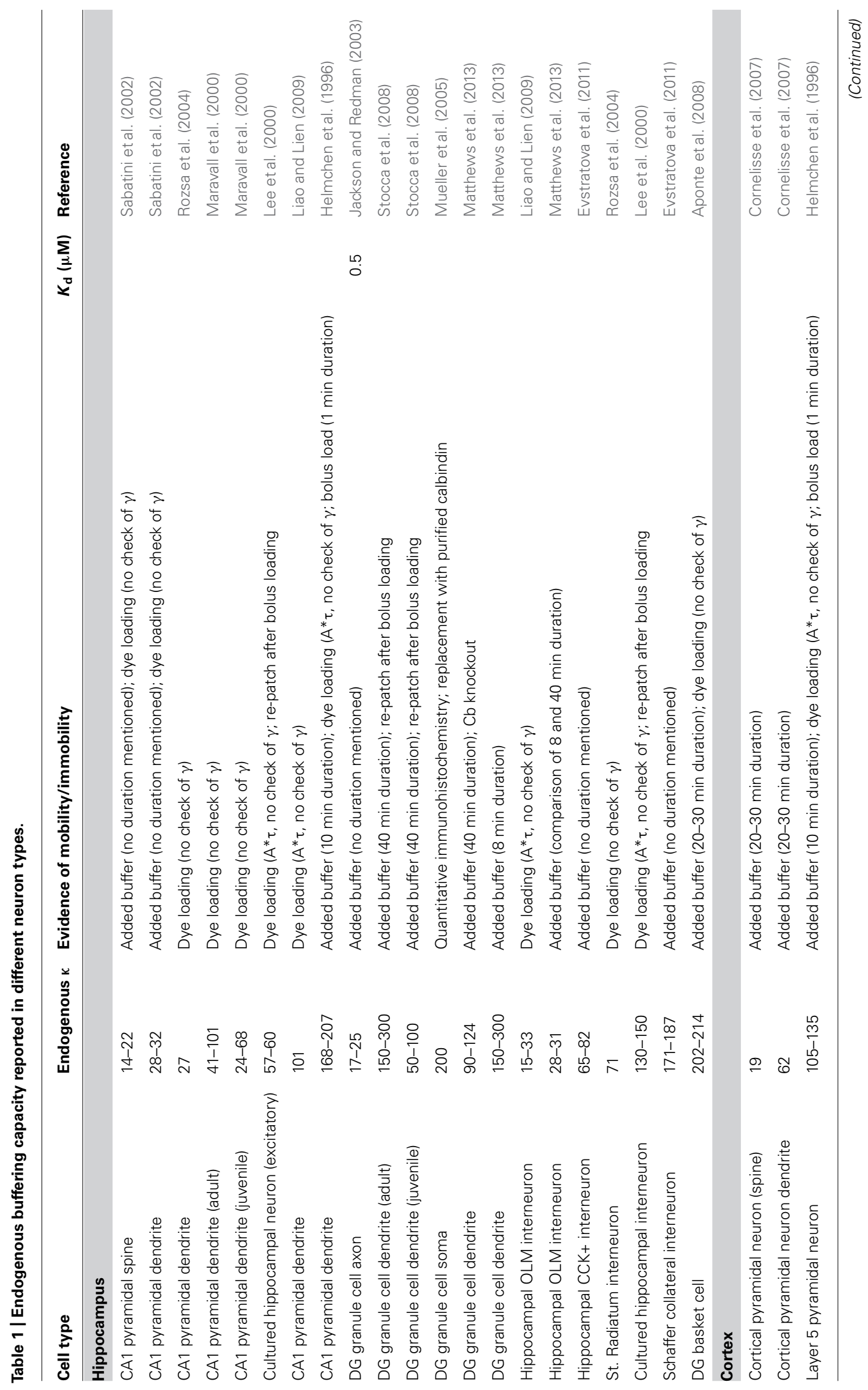




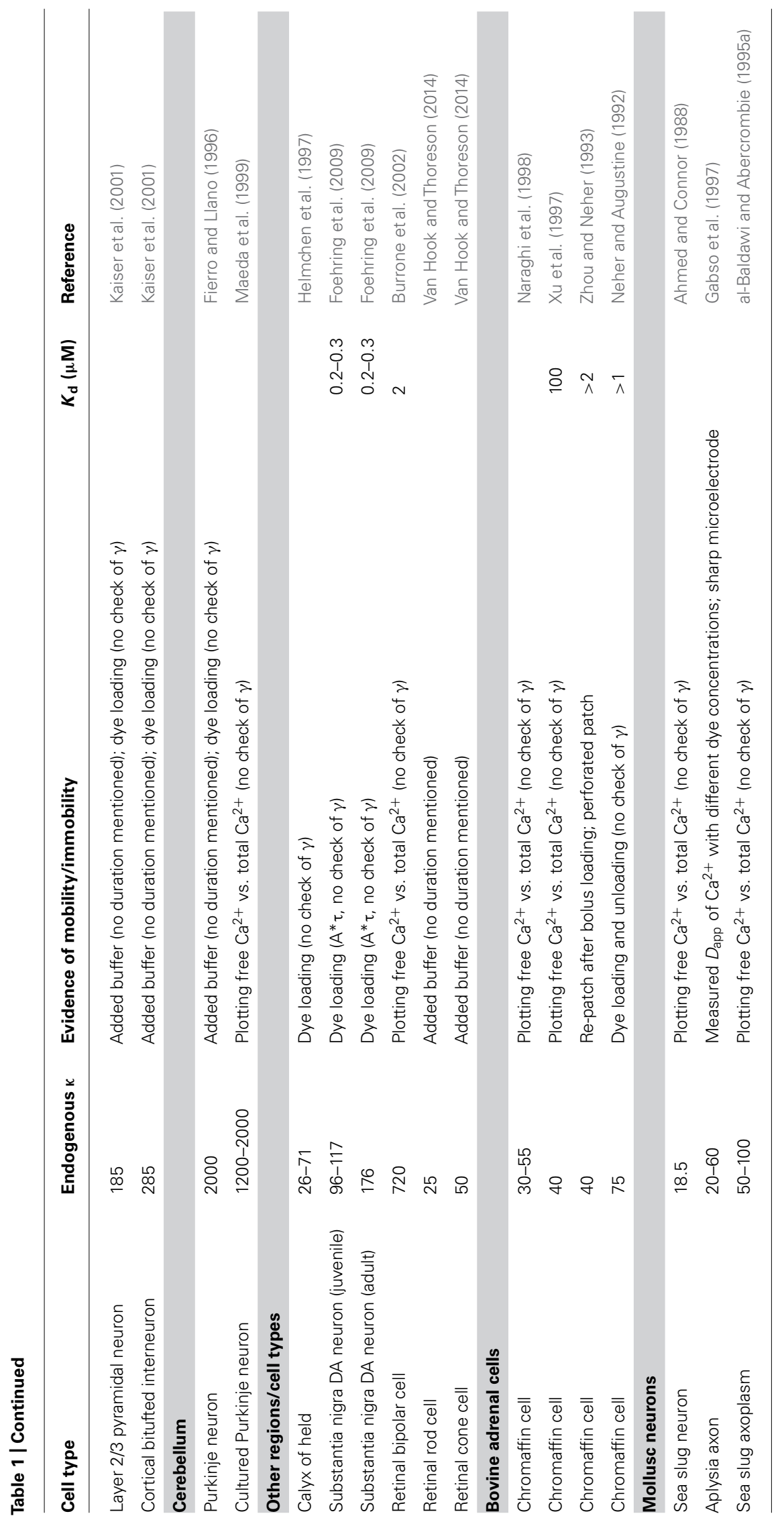


sites (Mueller et al., 2005). Some of these studies have used the product of the transient amplitude and decay time constant $\left(A^{*} \tau\right)$, which has been assumed to be constant throughout the experiment (Helmchen et al., 1996; Lee et al., 2000), to ensure that there are not alterations in calcium entry or extrusion $(\gamma)$ during the dye loading period and that the decay time constant or the amplitude truthfully reflect the total buffer concentration. However, if both entry and extrusion change in tandem as has been observed (Matthews et al., 2013; see Discussion), the product $A^{*} \tau$ may remain constant but neither $\tau$ nor A would truthfully reflect the total buffer concentration and may mask loss of endogenous buffer. The population approach, by virtue of allowing measurements to be taken across the cell population at identical experimental time points, ensures that calcium extrusion and entry are the same for all "added" calcium dye values, and thus is an uncontaminated measure of the endogenous buffering capacity, and thus $\tau$ and A can be used to assess the total endogenous buffer capacity. Note that, long recording times do not guarantee that only immobile buffering species are left; it can take over $45 \mathrm{~min}$ to fill the fine distal dendrites or axons with dye, and a large protein is unlikely to be washed out more rapidly than a small dye molecule can be loaded (Scott and Rusakov, 2006).

In hippocampal pyramidal neurons, values for the total endogenous $\kappa$ range from 15 to 20 (Sabatini et al., 2002; Rozsa et al., 2004) to 150-200 (Helmchen et al., 1996). For cortical and hippocampal interneurons the reported $\kappa$ values range from 15 to 30 (Liao and Lien, 2009; Matthews et al., 2013) to 285 (Kaiser et al., 2001). The highest reported value is for Purkinje neurons, with a measured calcium buffering capacity of $\sim 2000$ (Fierro and Llano, 1996; Maeda et al., 1999). It is therefore clear that $\kappa$ varies strongly between cell types and even between different sub-compartments (spines, axon boutons) within the same cell (Sabatini et al., 2002; Jackson and Redman, 2003). All of these factors make it difficult to ascertain the source of the large variances between cell types - are these differences due to different measurement techniques, or the whole or partial removal of mobile buffering species, or are they integral to the spatio-temporal calcium profiles of the different neurons? Clearly, the expression of calcium binding proteins will vary from cell type to cell type, and may even vary at different developmental states of the neurons, as calbindin does for dentate granule cells (Yoon et al., 2000); it has not been as obvious whether reported differences in the immobile buffering components are similarly cell type specific.

The most reliable measures of immobile buffering capacity come from studies that have explicitly separated the mobile and immobile fraction, by either long duration recordings that monitor for a change in buffering capacity over time, or use of perforated patch recordings to prevent washout followed by whole cell recording. Applying this strict criteria to the list in Table 1 results in an estimate of endogenous immobile buffering capacity of 90-124 in dentate gyrus granule cells (Matthews et al., 2013); 28-31 in hippocampal OLM interneurons (Matthews et al., 2013); and 40-75 in bovine chromaffin cells (Zhou and Neher, 1993) Other studies have made concerted efforts to detect a change in buffering capacity over the course of the recording by comparing the endogenous buffering after very short recordings to measures made after the longer dye-loading process. Including these measurements suggests that the immobile fraction of the total buffer capacity for CA1 pyramidal neurons is 168-207 (Helmchen et al., 1996); 105-135 in Layer V pyramidal neurons (Helmchen et al., 1996); and 285-288 in cortical bi-tufted interneurons (Kaiser et al., 2001). These measures provide evidence that endogenous immobile buffering capacity is not a general property of cells, but is tailored to the cell type and must play a role in setting different calcium signaling regimes from cell type to cell type.

\section{ADDITIONAL PROPERTIES OF THE IMMOBILE BUFFER}

The effect of any buffer on the calcium distribution does not solely depend on $\kappa$. The affinity, concentration, and on-rate of the buffer are also important, especially if several buffering species compete for the available free calcium (Markram et al., 1998; Helmchen and Tank, 2005). For instance, a buffer with a slow on-rate will have difficulty impacting the local calcium concentration, even if it is present in high concentration, because the calcium cloud can disperse through diffusion before the buffer has time to bind many molecules (Eggermann and Jonas, 2012). In addition to ascertaining the immobile endogenous buffering capacity, it is also important to consider the probable affinity, concentration, and speed of the calcium binding species that contribute to the immobile fraction. Unfortunately, the literature on affinity, concentration and on-rate of the endogenous immobile buffer is sparse. The most comprehensive studies were done by the group of Neher using bovine adrenal chromaffin cells. They reported an immobile buffering capacity of $\sim 30$ and a dissociation constant $\left(K_{\mathrm{d}}\right)$ greater than $2 \mu \mathrm{M}$ in these cells (Neher and Augustine, 1992; Zhou and Neher, 1993). In a later study, they refined the $K_{\mathrm{d}}$ measurement and report a dissociation constant of $100 \mu \mathrm{M}$ and a concentration of $4 \mathrm{mM}$ for the immobile buffer (Xu et al., 1997). This is in good agreement with other indicators that the immobile buffer would be low affinity based on the equivalent saturation of BAPTA (Tillotson and Gorman, 1980; Lumpkin and Hudspeth, 1998) and the constancy of the buffering capacity with large elevations in calcium (Thayer and Miller, 1990; Allbritton et al., 1992; al-Baldawi and Abercrombie, 1995b). If the resting calcium level is much smaller than the $K_{d}$ for a given buffer, then $\kappa$ can be approximated as the concentration of the buffer divided by the $\mathrm{K}_{\mathrm{d}}$ (Box 1). The resting calcium concentration in neurons is reported to be $\sim 20-100 \mathrm{nM}$ (Schwaller, 2010), which is much less than even the smallest estimated value for the $K_{d}$ of the endogenous immobile buffer. We can therefore estimate a range for the concentration of this buffer as between $90 \mu \mathrm{M}$ $\left(\kappa=30, K_{\mathrm{d}}=3 \mu \mathrm{M}\right)$ and $12 \mathrm{mM}\left(\kappa=120, K_{\mathrm{d}}=100 \mu \mathrm{M}\right)$. The kinetics of calcium binding by the immobile buffer are also unclear; the on-rate has only been measured directly in bovine chromaffin cells and was reported to be $1.07 \times 10^{8} \mathrm{M}^{-1} \mathrm{~s}^{-1}$ (Xu etal., 1997). The experimental data from other neurons suggests that the on-rate of the endogenous buffers must be fast enough to compete with typical calcium indicators. EGTA has a relatively slow on-rate: $2.5 \times 10^{6} \mathrm{M}^{-1} \mathrm{~s}^{-1}$, while BAPTA and Fura have faster on-rates in the range of $5 \times 10^{8} \mathrm{M}^{-1} \mathrm{~s}^{-1}$ (Naraghi etal., 1998). A conservative estimate of the on-rate for the endogenous immobile buffer would be in the range of $10^{7}-10^{8} \mathrm{M}^{-1} \mathrm{~s}^{-1}$. 


\section{POTENTIAL CANDIDATES CONTRIBUTING AN IMMOBILE BUFFERING CAPACITY}

What molecular species might contribute this immobile buffering? Because of indications that the buffering in the middle of cells was lower than near the cell membrane (Tillotson and Gorman, 1983; Klingauf and Neher, 1997; Naraghi et al., 1998), it has been suggested that the immobile buffer might be a combination of negatively charged phospholipid groups on the intracellular face of the membrane (McLaughlin et al., 1981), in particular, phosphatidylserine, which is enriched in neurons (Kim et al., 2014). The affinity of these groups is indeed quite low: the estimated $K_{d}$ of phospholipids for calcium is $10-80 \mathrm{mM}$ (McDaniel and McLaughlin, 1985). However, there is no evidence that the membranes of different cell types would contain different enough amounts of phospholipids to create such a marked variation in buffering capacity as has been observed, and the concentration would have to be quite high for such a low affinity buffer to achieve a buffering capacity in the observed range (1500-10,000 mM). Another possibility is that the immobile calcium buffering capacity is contributed by a heterogeneous mix of proteins which sense or bind calcium such as the calcium sensing domains of calcium dependent ion channels, cytoskeletal molecules and transport motors, or membrane-associated calcium binding kinases (Smith et al., 1996; Schwaller, 2010). This suggestion would fit the observation that immobile buffering capacity varies markedly between cell types, and can even vary between different regions in the same cell (spines vs. dendrites). To date, direct experimental evidence to either support or contradict the idea that the immobile buffer is composed of an assortment of phospholipids and calcium sensing membrane proteins is lacking.

Mitochondria are sometimes suggested as a potential candidate for the immobile calcium binding species. It is true that the majority of mitochondria are immobile (50-85\%), and the fraction of mobile mitochondria decreases to approximately $5 \%$ when internal calcium is elevated above $400 \mathrm{nM}$ (Wang and Schwarz, 2009). It would therefore be expected that all mitochondria in the vicinity of calcium channels or calcium permeable receptors will become immobile during neuronal firing. In addition to their role in energy production, mitochondria remove calcium from the intracellular space via their uniporters (Gunter and Pfeiffer, 1990). The uniporter has a low affinity for calcium, estimated at $\sim 10 \mu \mathrm{M}$ (Patterson et al., 2007; Santo-Domingo and Demaurex, 2010). The rate of removal from the cytosol depends on the intracellular free calcium concentration, but the maximal rate has been estimated in the range of 4-225 $\mu \mathrm{M} / \mathrm{s}$ when intracellular calcium is in the micromolar range (Babcock et al., 1997; Montero etal., 2000). When intracellular free calcium is strongly elevated, mitochondria may account for the majority of calcium binding and removal (Herrington et al., 1996). However, at calcium levels more commonly occurring in neurons, calcium uptake from the cytosol by mitochondria is considerably slower (Kann and Kovacs, 2007), and would not strongly compete with most endogenous buffers. Calcium is also extruded from mitochondria, primarily via a $\mathrm{Na}^{+} / \mathrm{Ca}^{2+}$ exchanger in the inner mitochondrial membrane (White and Reynolds, 1995; Kann and Kovacs, 2007), which can prolong the cytosolic elevation of calcium (Herrington et al., 1996; Babcock et al., 1997). Although mitochondria bind and release calcium in a calcium-dependent fashion, the mechanisms responsible for each action are independent of each other; the uniporter responds to the cytosolic calcium concentration, while the $\mathrm{Na}^{+} / \mathrm{Ca}^{2+}$ exchange responds to mitochondrial calcium concentration and cytosolic $\mathrm{Na}^{+}$concentration. Because the calcium uptake and release are realized through distinct protein actors, and are coupled via intramitochondrial calcium, the application of classical biochemical treatments of buffers is not warranted. Mitochondria do play a role in supporting and shaping neuronal calcium signals, especially under conditions of strongly elevated cytosolic free calcium, but are not fast enough to remove a substantial portion of calcium within a physiologically relevant time window of a couple hundred milliseconds and thus are not a good candidate for the immobile buffer shaping calcium signals in the context discussed here.

\section{MODELING THE ROLE OF IMMOBILE BUFFERS IN THREE SCENARIOS}

Thus far, the accumulated experimental evidence suggests that the endogenous immobile buffer varies by cell type, but in general has a buffering capacity in the range of $\sim 30-120$, a low affinity with a dissociation constant in the range of 3-100 $\mu \mathrm{M}$, and an on-rate similar to BAPTA. In the next section, we present an estimate of the impact of functionally immobile buffers on free calcium, exclusively. Whether and how the mobility or immobility of a buffer is important for its effect on free calcium depends on the spatial domain under consideration. We differentiate three scenarios: calcium signals spreading along dendrites, calcium signals with no relevant spatial concentration gradients, and submicroscopic calcium signals (microdomains). For our calculations, we assume that every cell has at least a minimal immobile buffering capacity of 15 . This is justified by our previous work (Matthews et al., 2013) and also by the fact that none of the other previous studies found a lower total $\kappa$ value in any type of neuron (Table 1). Only recently it was highlighted that the immobile buffer content may be significantly higher in certain types of neurons, which strongly affects the competition for calcium between mobile and immobile buffers (Matthews et al., 2013). We therefore also consider a condition with high immobile buffer capacity of 150 . The other parameter values were: $k_{\mathrm{on}}=1 \times 10^{8} \mathrm{M}^{-1} \mathrm{~s}^{-1}$ and $K_{\mathrm{d}}=5 \mu \mathrm{M}$. The immobile buffering capacity was assumed to be evenly distributed in the space. For simplicity, when mobile buffers were simulated with immobile buffers, the $k_{\text {on }}$ and $K_{\mathrm{d}}$ were the same for both buffers.

In our first scenario, calcium diffusing along a dendrite, the spatial domains are sufficiently large, so that diffusional equilibrium takes longer than local chemical equilibrium between calcium and its binding partners. Such domains typically arise from a local calcium source in a dendrite with ensuing spread of the calcium ions along the dendrite. The diffusion of calcium within three dimensional space in the presence of multiple buffers of mixed mobility is a complex phenomenon, described by a set of partial differential equations, which can be linearized to describe individual interactions of calcium ions with buffers at the nano- and microdomain level (Zador and Koch, 1994; Naraghi and Neher, 1997). An additional simplification assumes that interactions between 
calcium and buffers are instantaneous (Rapid Buffer Approximation) and that the spatio-temporal localization of calcium depends only on the diffusion coefficients and affinities of the various buffers (Wagner and Keizer, 1994; Smith et al., 1996; Neher, 1998). This yields a very useful analytical expression, which describes calcium diffusion in the presence of multiple buffers using a new, smaller diffusion coefficient of calcium, depending on the number, amount and mobility of the calcium buffers present:

$$
D_{a p p}=D_{C a^{2+}} \frac{\left(1+\frac{D_{\text {mobile }}}{D_{C a^{2}}} \kappa_{\text {mobile }}\right)}{\left(1+\kappa_{\text {mobile }}+\kappa_{\text {immobile }}\right)}
$$

where, $D_{\mathrm{Ca}^{2+}}$ is the diffusion coefficient of free calcium in the cytosol, $D_{\text {mobile }}$ is the diffusion coefficient of mobile buffers, and $\kappa_{\text {mobile }}$ and $\kappa_{i \text { mmobile }}$ are the calcium buffering capacities of mobile and immobile buffers, respectively (Wagner and Keizer, 1994; Zador and Koch, 1994).

Both mobile and immobile buffers will increase the endogenous $к$. By slowing down calcium diffusion, immobile buffers delay the spread of the calcium cloud, prolong the local availability of calcium ions, and decrease the apparent calcium diffusion coefficient. The two immobile calcium buffer backgrounds considered here, $\kappa_{\text {immobile }} 15$ or 150 , both hinder calcium diffusion and reduce $D_{\text {app }}$ from 220 to $13.8 \mu \mathrm{m}^{2} / \mathrm{s}$ ("low immobile buffer background") and $1.5 \mu \mathrm{m}^{2} / \mathrm{s}$ ("high immobile buffer background"), respectively (Equation 1).

Mobile buffers can either increase or decrease the $D_{\text {app }}$. Consider a system with only immobile buffer and a $D_{\text {app }}$ of $13.8 \mu \mathrm{m}^{2} / \mathrm{s}$. Now we add a mobile buffer with a diffusion coefficient of $5 \mu \mathrm{m}^{2} / \mathrm{s}$. The new $D_{\text {app }}$ will depend on the buffering capacity of the mobile species $\left(\kappa_{\text {mobile }}\right)$, but will always be less than the original $D_{\text {app }}$ of the system, $13.8 \mu \mathrm{m}^{2} / \mathrm{s}$. Even increasing $\kappa_{\text {mobile }}$ to very large values will not alter the slowing effect of this mobile buffer and will decrease $D_{\text {app }}$ of the resultant system to the low diffusion coefficient of the mobile buffer. Eq. 1 dictates that adding a mobile calcium buffer to a system can accelerate calcium diffusion (increase $D_{\text {app }}$ ), only if the mobile buffer's diffusion coefficient is larger than the $D_{a p p}$ of the system in the absence of the mobile buffer. Further, if the mobile buffer's diffusion coefficient is smaller than the original $D_{\text {app }}$, it will slow calcium diffusion in the system. This means that in the presence of a low immobile buffer background even mobile calcium binding proteins with a diffusion coefficient up to $\sim 14 \mu \mathrm{m}^{2} / \mathrm{s}$ will influence calcium diffusion as if they were immobile; conversely, in the presence of a large amount of immobile buffer only poorly mobile buffers with a diffusion coefficient $<1.5 \mu \mathrm{m}^{2} / \mathrm{s}$ act as immobile buffers. For a scenario of calcium diffusion in a dendrite, the general limit below which a physically mobile buffer will functionally impact the diffusion of calcium in the system in the same manner as an immobile buffer is given by $D_{\mathrm{m}}<D_{\mathrm{Ca}^{2+}} /\left(1+\kappa_{\text {immobile }}\right)$. One should note that equating the impact of mobile and immobile buffers on the apparent diffusion coefficient neglects other consequences of adding a buffer, such as the total buffering capacity. For example, addition of a small amount of immobile buffer or a larger amount of poorly mobile buffer may have the same effect on $D_{\text {app }}$, but each addition will lead to concomitantly small or large increases in the total endogenous buffering capacity.

The second scenario we consider is a calcium signal with no relevant concentration gradient. Relevant calcium concentration gradients are absent if either the compartment of calcium diffusion is so small that diffusional equilibration is very rapidly achieved ( $<\sim 5 \mathrm{~ms}$, e.g., in spines) or if calcium entry is spatially homogenous across a larger compartment (global calcium signal). As there are no gradients, diffusion does not play a role and therefore immobile and mobile buffers affect calcium transients similarly, according to their $\kappa$ values and the predictions of the single compartment model (Helmchen et al., 1997; Neher, 1998).

Our final scenario examines the function of immobile buffers within microdomains. Calcium microdomains are characterized by a very spatially restricted $(<500 \mathrm{~nm})$ standing free calcium concentration gradient rapidly building up around one or several calcium channels after their opening. These gradients persist as long as the channels are open based on a local, steady state equilibrium between calcium binding and calcium and buffer diffusion. Although a steady state is established, there is no chemical equilibrium between the local calcium concentration and mobile buffers because on this small spatial scale diffusion is as rapid as binding kinetics. This is not the case for immobile buffers, which by definition cannot diffuse. Therefore, depletion of calcium-free immobile buffer within the microdomain cannot be replaced by diffusion of calcium-free buffer from remote sites. For this reason immobile buffers do reach chemical equilibrium once the standing gradient of free calcium has fully developed. Further, chemical equilibrium means that at every point in space immobile buffers bind the same number of calcium ions per time as they release and do not affect the standing gradient of free calcium. Simply stated, once calcium microdomains achieve steady state they are not affected by the presence of immobile buffers. But how long does it take to reach steady state? The seminal work by Naraghi and Neher (1997) evaluated the temporal evolution of calcium microdomains. They used millimolar amounts of exogenous calcium chelators, such as EGTA and BAPTA, to probe the spatial extent of microdomain signaling and showed that steady state is reached in only a fraction of a millisecond. They concluded that it is reasonable and accurate to only consider the steady state of a microdomain and neglect immobile buffers. However, as shown below, under physiological conditions, i.e., in the absence of exogenous chelators, it may take 10s of milliseconds to achieve a full steady-state situation even very near the calcium source (Figure 1A). In fact, immobile buffers exacerbate the delay to steady state because of the time required before calcium can occupy the local immobile binding sites. Calcium sources such as voltage-gated calcium channels would typically open for less than $1 \mathrm{~ms}$ during an action potential (Lee and Elmslie, 1999; Mueller et al., 2005), or $<10 \%$ of the time it takes to reach steady state, so it can be expected that microdomain steady states are rarely achieved near calcium channels and, accordingly immobile buffers do play a role in shaping calcium microdomains near voltage-gated channels.

When simulating the microdomain with either a low or a high immobile $\kappa$, free calcium progresses to steady state substantially more slowly than in the absence of immobile buffering. Figure 1A 

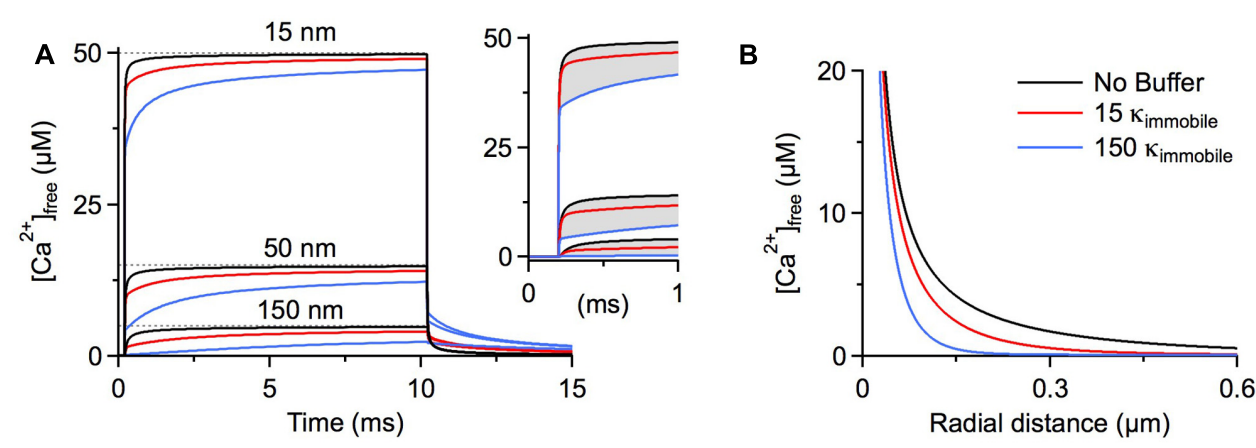

C
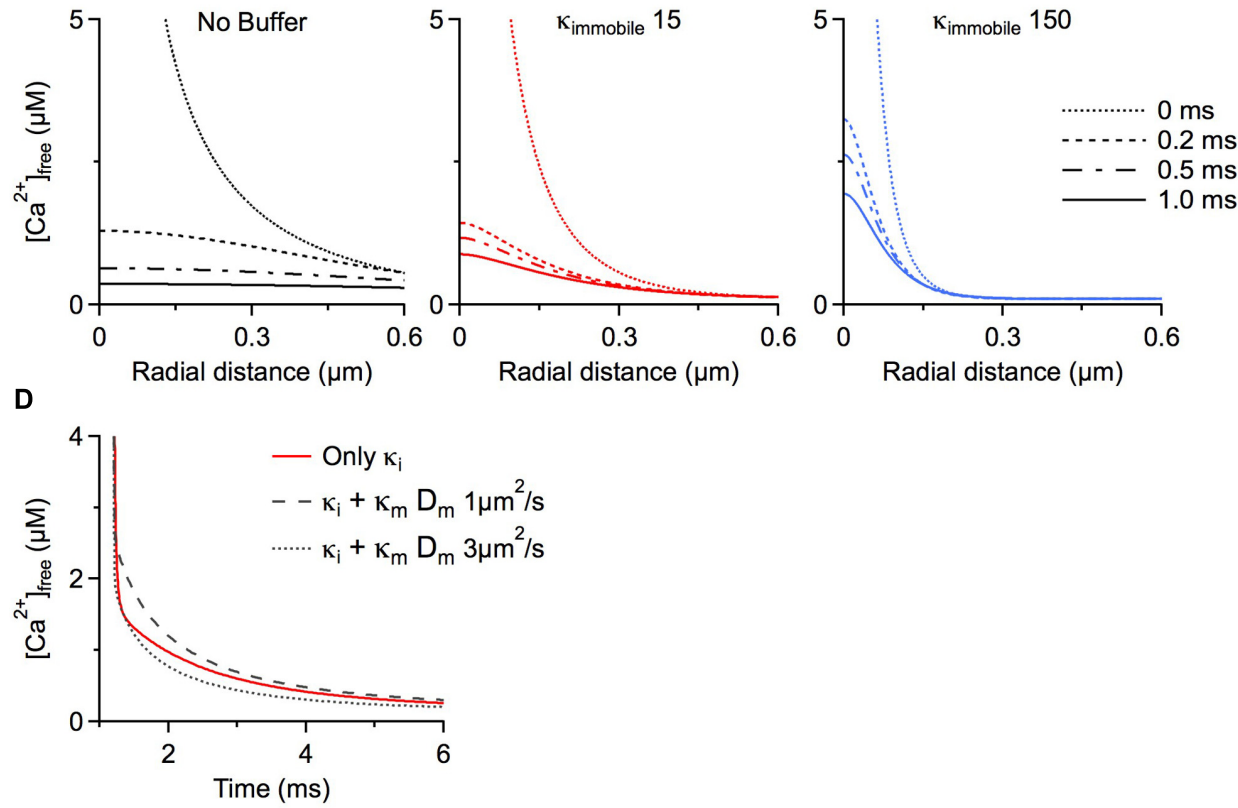

FIGURE 1 | Simulations were run using CalC (Matveev et al., 2004; http://web.njit.edu/ matveev; Version 6.7.4). Resting calcium

concentration was set at $100 \mathrm{nM}$. We simulate a calcium current of $0.2 \mathrm{pA}$ through a generic calcium channel. Parameters for the immobile buffer were taken from the experimental results summarized in Table 1. (A) The presence of immobile buffer slows the arrival at steady state free calcium concentrations at all points in space surrounding a calcium source. The time of channel opening was increased to a non-physiological $10 \mathrm{~ms}$ so that the prolonged time course could be better illustrated. However, in the case of high immobile buffering capacity, steady state concentrations were clearly not reached even after $10 \mathrm{~ms}$. In the case of a more physiologically relevant open time of $0.8 \mathrm{~ms}$, the free calcium concentration is even further from reaching a steady state level and the slowing effect of immobile buffers is even more prominent (inset). A similar effect is seen after the channel closes. Calcium is gradually released from immobile buffers, prolonging the return to resting calcium concentrations. (B) The localizing effect of immobile buffers on microdomains during calcium entry was plotted for a $1 \mathrm{~ms}$ calcium channel opening. At all illustrated points in space, the immobile buffer reduces free calcium concentration; this localizing effect on peak free calcium concentration is known to be similar for a mobile buffer. (C) The collapse of a microdomain after the closing of a calcium channel is illustrated for conditions with no buffering (left), a low immobile buffer capacity (center) and a high immobile buffer capacity (right). The spatial calcium gradient is shown immediately before closing $(0 \mathrm{~ms})$, and at $0.2,0.5$, and $1.0 \mathrm{~ms}$ following channel closure. The strong prolongation of the free calcium domain by the immobile buffer is apparent. With a high immobile buffer capacity, the domain remains above $1 \mu \mathrm{M}$ free calcium even $1 \mathrm{~ms}$ after the channel has closed. This effect is unique to immobile buffers; a mobile buffer will disperse the spatial gradient and speed the local return to resting levels. (D) To explore the diffusion limits at which a mobile buffer behaves as a functionally immobile buffer, the prolongation of a calcium microdomain was examined. A calcium source was opened for $1 \mathrm{~ms}$, then the decay of the microdomain at $15 \mathrm{~nm}$ from the channel was plotted over time. The initial condition had an immobile buffer with a capacity of 15 ; under these conditions, the free calcium concentration remains elevated for several milliseconds. When the diffusion coefficient was $\leq 1 \mu \mathrm{m}^{2} / \mathrm{s}$, the lifetime was further increased by the mobile buffer. This indicates that the poorly mobile buffer functions as though it were physically immobile. depicts the time course of free calcium around a single calcium channel at three distances, with the dashed line representing the eventual steady state concentration of free calcium. Purely for better illustration of the time course of microdomain calcium we assume a non-physiological single channel open time of $10 \mathrm{~ms}$. Even in the absence of any buffers, the free calcium concentration remains $\sim 5 \%$ below the steady state concentration after $1 \mathrm{~ms}$ at distances $50 \mathrm{~nm}$ from the channel (physiological open times would be even shorter). Evaluating the free calcium concentration in the presence of immobile buffers at the same time point shows a strong reduction in free calcium concentration because of the slower rise to steady state (Figure 1A inset). In fact, immobile buffers 
reduce the amplitude and restrict the spatial extent of calcium microdomains just as mobile buffers do (Figures 1A,B; B shows radial profiles after $1 \mathrm{~ms}$ ).

After closure of the channel the calcium microdomain rapidly collapses (Figure 1A). Mobile buffers accelerate the collapse of the microdomain by shuttling calcium away from the source, thereby strongly reducing the local concentration. This is in contrast to immobile buffers, which not only delay the build-up of the microdomain but also increase microdomain lifetimes after closure of the calcium source because the calcium bound to immobile buffers is locally released, prolonging the return to a resting calcium concentration (Nowycky and Pinter, 1993; see Figure 1C, note the localization and prolongation of the microdomain with $\kappa_{\text {immobile }} 15$ and 150). The outcome of a microdomain on the whole neuron depends on the calcium signaling cascade which is initiated by the microdomain. The question of which calcium signaling cascades might be optimally activated by microdomains with different lifetimes hinges crucially on the on-rates of the different sensor molecules that initiate the cascades, on the different affinities of the sensors, and on the location of the sensor relative to the calcium source. In general, the presence of longer lasting, localized calcium microdomains would allow for the recruitment of calcium signaling cascades initiated by slower acting calcium binding proteins such as parvalbumin. The above discussion of action potential-associated calcium microdomains is also generally applicable to synaptically activated calcium sources. NMDA receptors, for example, are highly permeable to calcium, and are the primary ligand-gated source for postsynaptic calcium. These receptors have longer open times than voltage gated channels, in the range of 2-5 ms (Rosenmund et al., 1995; Lieberman and Mody, 1999). Still, as can be seen in Figure 1, adding $150 \kappa_{\text {immobile }}$ reduces free calcium at $50 \mathrm{~nm}$ from the source by $\sim 25 \%$. Considering that many postsynaptic densities are $>100 \mathrm{~nm}$ the effect of fixed calcium buffers may be even more pronounced.

Increasing the microdomain lifetime is a function unique to immobile buffers. To define the family of functionally immobile buffers we therefore have to ask under which conditions buffers with a diffusion coefficient $>0$ will mimic this behavior and prolong the persistence of calcium microdomains in addition to slowing the apparent diffusion coefficient. Poorly mobile buffers increase the lifetime of a microdomain after channel closure by releasing the calcium they had bound during calcium entry while still very close to the channel, due to low buffer mobility. We initially simulated the collapse of a microdomain with a low amount of immobile buffer $(\kappa=15)$, and then added a mobile buffer with identical kinetics and affinity, but with a $\kappa_{\text {mobile }}$ of 100 . The diffusion coefficient of the mobile buffer was then changed to find a value that would mimic the slow collapse of the microdomain. It turns out that mobile buffers with diffusion coefficients less than $1 \mu \mathrm{m}^{2} / \mathrm{s}$ will act as functionally immobile buffers, further prolonging microdomain lifetime (Figure 1D). This estimate holds true for a large range of calcium association rates of the mobile buffer. In fact, increasing the on-rate strongly enhances the prolongation effect on the microdomain. However, when the on-rate of a poorly mobile buffer drops below $5 \times 10^{7} \mathrm{M}^{-1} \mathrm{~s}^{-1}$, the buffer will cease to mimic the microdomain effect of a physically immobile buffer (in our model). If diffusing freely, only very large mobile calcium binding proteins, $>100 \mathrm{kDa}$, will display diffusion coefficients $\leq 1 \mu \mathrm{m}^{2} / \mathrm{s}$. Using the higher immobile buffer capacity $(\kappa=150)$, the lower limit of the diffusion coefficient that allows a protein to behave as a functionally mobile buffer further drops to $0.2 \mu \mathrm{m}^{2} / \mathrm{s}$ (not shown).

It is unlikely that any of the known calcium binding proteins would have such a low diffusion coefficient, so in cells with a high background of immobilized buffer, all known mobile calcium binding proteins would act as both physically and functionally mobile buffers. The degree of microdomain suppression by immobile buffers depends on their concentration, binding kinetics, and channel open time, and therefore cannot be generally predicted. Nevertheless, since the assumed properties of immobile buffers used for these simulations represent probable mean values, our conclusions about the impact of immobile buffers on microdomain signaling at least serve as a good first approximation.

\section{CONCLUSION}

The interplay between mobile and immobile buffers provides neurons with a broad range of options for specifying calcium domains. Immobile buffers play a unique role in shaping calcium signals if relevant concentration gradients are present in dendrites, where they dramatically slow the spread of a calcium cloud. In contrast to common assumptions, immobile buffers are also important regulators of calcium microdomains under physiological conditions when steady state concentration gradients are not expected to occur. Furthermore, depending on the background of immobile buffering capacity, even physically mobile calcium-binding proteins with non-zero diffusion coefficients can function as immobile buffers insofar as they influence the apparent diffusion of calcium and the lifetime of the microdomain. Our simulation is by no means an exhaustive description of the possible parameters that are known to influence free calcium concentration at all spatial and temporal scales. Rather we sought to illustrate a narrower set of circumstances, and in particular the effect of immobile and slowly diffusing buffers on the apparent diffusion of free calcium. The interdependent relationship between the amount of immobile buffer present and the functional behavior of co-expressed, physically mobile buffers highlights the importance of careful experimentation to separate mobile from immobile buffering capacity and highlights the importance of gaining a clearer picture of the kinetics, molecular identity, and prevalence of immobile buffers in neurons.

\section{ACKNOWLEDGMENTS}

This review was supported by Deutsche Forschungsgemeinschaft (DFG SFB1089 B02, B04, PP 1757), and University Clinic Bonn grants (BONFOR).

\section{REFERENCES}

Ahmed, Z., and Connor, J. A. (1988). Calcium regulation by and buffer capacity of molluscan neurons during calcium transients. Cell calcium 9, 57-69. doi: 10.1016/0143-4160(88)90025-5

al-Baldawi, N. F., and Abercrombie, R. F. (1995a). Calcium diffusion coefficient in Myxicola axoplasm. Cell calcium 17, 422-430. doi: 10.1016/01434160(95)90088-8 
al-Baldawi, N. F., and Abercrombie, R. F. (1995b). Cytoplasmic calcium buffer capacity determined with Nitr-5 and DM-nitrophen. Cell calcium 17, 409-421. doi: 10.1016/0143-4160(95)90087-X

Allbritton, N. L., Meyer, T., and Stryer, L. (1992). Range of messenger action of calcium ion and inositol 1,4,5-trisphosphate. Science 258, 1812-1815. doi: 10.1126/science. 1465619

Aponte, Y., Bischofberger, J., and Jonas, P. (2008). Efficient $\mathrm{Ca}^{2+}$ buffering in fast-spiking basket cells of rat hippocampus. J. Physiol. 586, 2061-2075. doi: 10.1113/jphysiol.2007.147298

Arendt, O., Schwaller, B., Brown, E. B., Eilers, J., and Schmidt, H. (2013) Restricted diffusion of calretinin in cerebellar granule cell dendrites implies $\mathrm{Ca}^{+}{ }^{+}$-dependent interactions via its EF-hand 5 domain. J. Physiol. 591(Pt 16), 3887-3899. doi: 10.1113/jphysiol.2013.256628

Augustine, G. J., Santamaria, F., and Tanaka, K. (2003). Local calcium signaling in neurons. Neuron 40, 331-346. doi: 10.1016/S0896-6273(03)00639-1

Babcock, D. F., Herrington, J., Goodwin, P. C., Park, Y. B., and Hille, B. (1997) Mitochondrial participation in the intracellular $\mathrm{Ca}^{2+}$ network. J. Cell. Biol. 136 , 833-844. doi: 10.1083/jcb.136.4.833

Baimbridge, K. G., Celio, M. R., and Rogers, J. H. (1992). Calcium-binding proteins in the nervous system. Trends Neurosci. 15, 303-308. doi: 10.1016/01662236(92)90081-I

Burrone, J., Neves, G., Gomis, A., Cooke, A., and Lagnado, L. (2002). Endogenous calcium buffers regulate fast exocytosis in the synaptic terminal of retinal bipolar cells. Neuron 33, 101-112. doi: 10.1016/S0896-6273(01)00565-7

Cornelisse, L. N., van Elburg, R. A., Meredith, R. M., Yuste, R., and Mansvelder, H. D. (2007). High speed two-photon imaging of calcium dynamics in dendritic spines: consequences for spine calcium kinetics and buffer capacity. PLoS ONE 2:e1073. doi: 10.1371/journal.pone.0001073

Eggermann, E., and Jonas, P. (2012). How the 'slow' $\mathrm{Ca}^{2+}$ buffer parvalbumin affects transmitter release in nanodomain-coupling regimes. Nat. Neurosci. 15, 20-22. doi: $10.1038 / \mathrm{nn} .3002$

Evstratova, A., Chamberland, S., and Topolnik, L. (2011). Cell type-specific and activity-dependent dynamics of action potential-evoked $\mathrm{Ca}^{2+}$ signals in dendrites of hippocampal inhibitory interneurons. J. Physiol. 589(Pt 8), 1957-1977. doi: 10.1113/jphysiol.2010.204255

Fierro, L., and Llano, I. (1996). High endogenous calcium buffering in Purkinje cells from rat cerebellar slices. J. Physiol. 496(Pt 3), 617-625. doi: 10.1113/jphysiol.1996.sp021713

Foehring, R. C., Zhang, X. F., Lee, J. C., and Callaway, J. C. (2009). Endogenous calcium buffering capacity of substantia nigral dopamine neurons. J. Neurophysiol. 102, 2326-2333. doi: 10.1152/jn.00038.2009

Gabso, M., Neher, E., and Spira, M. E. (1997). Low mobility of the $\mathrm{Ca}^{2+}$ buffers in axons of cultured Aplysia neurons. Neuron 18, 473-481. doi: 10.1016/S08966273(00)81247-7

Gunter, T. E., and Pfeiffer, D. R. (1990). Mechanisms by which mitochondria transport calcium. Am. J. Physiol. 258(5 Pt 1), C755-C786.

Helmchen, F., Borst, J. G., and Sakmann, B. (1997). Calcium dynamics associated with a single action potential in a CNS presynaptic terminal. Biophys. J. 72, 1458-1471. doi: 10.1016/S0006-3495(97)78792-7

Helmchen, F., Imoto, K., and Sakmann, B. (1996). $\mathrm{Ca}^{2+}$ buffering and action potential-evoked $\mathrm{Ca}^{2+}$ signaling in dendrites of pyramidal neurons. Biophys. J. 70, 1069-1081. doi: 10.1016/S0006-3495(96)79653-4

Helmchen, F., and Tank, D. W. (2005). "A single-compartment model of calcium dynamics in nerve terminals and dendrites," in Imaging in Neuroscience: A Laboratory Manual, eds F. Helmchen and A. Konnerth (New York, NY: Cold Spring Harbor Laboratory Press).

Herrington, J., Park, Y. B., Babcock, D. F., and Hille, B., (1996). Dominant role of mitochondria in clearance of large $\mathrm{Ca}^{2+}$ loads from rat adrenal chromaffin cells. Neuron 16, 219-228. doi: 10.1016/S0896-6273(00)80038-0

Jackson, M. B., and Redman, S. J. (2003). Calcium dynamics, buffering, and buffer saturation in the boutons of dentate granule-cell axons in the hilus. J. Neurosci. $23,1612-21$

Kaiser, K. M., Zilberter, Y., and Sakmann, B. (2001). Back-propagating action potentials mediate calcium signalling in dendrites of bitufted interneurons in layer $2 / 3$ of rat somatosensory cortex. J. Physiol. 535(Pt 1), 17-31. doi: 10.1111/j.1469-7793.2001.t01-1-00017.x

Kann, O., and Kovacs, R. (2007). Mitochondria and neuronal activity. Am. J. Physiol. Cell Physiol. 292, C641-C657. doi: 10.1152/ajpcell.00222.2006
Kim, H. Y., Huang, B. X., and Spector, A. A. (2014). Phosphatidylserine in the brain: metabolism and function. Prog. Lipid. Res. 56, 1-18. doi: 10.1016/j.plipres.2014.06.002

Klingauf, J., and Neher, E. (1997). Modeling buffered $\mathrm{Ca}^{2+}$ diffusion near the membrane: implications for secretion in neuroendocrine cells. Biophys. J. 72(2 Pt 1), 674-690. doi: 10.1016/S0006-3495(97)78704-6

Lee, H. K., and Elmslie, K. S. (1999). Gating of single N-type calcium channels recorded from bullfrog sympathetic neurons. J. Gen. Physiol. 113, 111-124. doi: 10.1085/jgp.113.1.111

Lee, S. H., Rosenmund, C., Schwaller, B., and Neher, E. (2000). Differences in $\mathrm{Ca}^{2+}$ buffering properties between excitatory and inhibitory hippocampal neurons from the rat. J. Physiol. 525(Pt 2), 405-418. doi: 10.1111/j.1469-7793.2000.t01-300405.x

Liao, C. W., and Lien, C. C. (2009). Estimating intracellular $\mathrm{Ca}^{2+}$ concentrations and buffering in a dendritic inhibitory hippocampal interneuron. Neuroscience 164, 1701-1711. doi: 10.1016/j.neuroscience.2009.09.052

Lieberman, D. N., and Mody, I. (1999). Properties of single NMDA receptor channels in human dentate gyrus granule cells. J. Physiol. 518(Pt 1), 55-70. doi: 10.1111/j.1469-7793.1999.0055r.x

Lumpkin, E. A., and Hudspeth, A. J. (1998). Regulation of free $\mathrm{Ca}^{2+}$ concentration in hair-cell stereocilia. J. Neurosci 18, 6300-6318.

Maeda, H., Ellis-Davies, G. C., Ito, K., Miyashita, Y., and Kasai, H. (1999). Supralinear $\mathrm{Ca}^{2+}$ signaling by cooperative and mobile $\mathrm{Ca}^{2+}$ buffering in Purkinje neurons. Neuron 24, 989-1002. doi: 10.1016/S0896-6273(00)81045-4

Maravall, M., Mainen, Z. F., Sabatini, B. L., and Svoboda, K. (2000). Estimating intracellular calcium concentrations and buffering without wavelength ratioing. Biophys. J. 78, 2655-2667. doi: 10.1016/S0006-3495(00)76809-3

Markram, H., Roth, A., and Helmchen, F. (1998). Competitive calcium binding: implications for dendritic calcium signaling. J. Comput. Neurosci. 5, 331-348. doi: 10.1023/A:1008891229546

Matthews, E. A., Schoch, S., and Dietrich, D. (2013). Tuning local calcium availability: cell-type-specific immobile calcium buffer capacity in hippocampal neurons. J Neurosci. 33, 14431-14445. doi: 10.1523/jneurosci.4118-12.2013

Matveev, V., Zucker, R. S., and Sherman, A. (2004). Facilitation through buffer saturation: constraints on endogenous buffering properties. Biophys. J. 86, 26912709. doi: 10.1016/S0006-3495(04)74324-6

McDaniel, R., and McLaughlin, S. (1985). The interaction of calcium with gangliosides in bilayer membranes. Biochim. Biophys. Acta 819, 153-160. doi: 10.1016/0005-2736(85)90169-5

McLaughlin, S., Mulrine, N., Gresalfi, T., Vaio, G., and McLaughlin, A. (1981). Adsorption of divalent cations to bilayer membranes containing phosphatidylserine. J. Gen. Physiol. 77, 445-473. doi: 10.1085/jgp.77.4.445

Montero, M., Alonso, M. T., Carnicero, E., Cuchillo-Ibanez, I., Albillos, A., Garcia, A. G., et al. (2000). Chromaffin-cell stimulation triggers fast millimolar mitochondrial $\mathrm{Ca}^{2+}$ transients that modulate secretion. Nat. Cell Biol. 2, 57-61. doi: $10.1038 / 35000001$

Mueller, A., Kukley, M., Stausberg, P., Beck, H., Muller, W., and Dietrich, D. (2005). Endogenous $\mathrm{Ca}^{2+}$ buffer concentration and $\mathrm{Ca}^{2+}$ microdomains in hippocampal neurons. J. Neurosci. 25, 558-565. doi: 10.1523/JNEUROSCI.3799-04.2005

Naraghi, M., Muller, T. H., and Neher, E. (1998). Two-dimensional determination of the cellular $\mathrm{Ca}^{2+}$ binding in bovine chromaffin cells. Biophys. J. 75, 1635-1647. doi: 10.1016/S0006-3495(98)77606-4

Naraghi, M., and Neher, E. (1997). Linearized buffered $\mathrm{Ca}^{2+}$ diffusion in microdomains and its implications for calculation of $\left[\mathrm{Ca}^{2+}\right]$ at the mouth of a calcium channel. J. Neurosci. 17, 6961-6973.

Neher, E. (1998). Usefulness and limitations of linear approximations to the understanding of $\mathrm{Ca}^{++}$signals. Cell calcium 24, 345-357. doi: 10.1016/S01434160(98)90058-6

Neher, E., and Augustine, G. J. (1992). Calcium gradients and buffers in bovine chromaffin cells. J. Physiol. 450, 273-301. doi: 10.1113/jphysiol.1992. sp019127

Nowycky, M. C., and Pinter, M. J. (1993). Time courses of calcium and calciumbound buffers following calcium influx in a model cell. Biophys. J. 64, 77-91. doi: 10.1016/S0006-3495(93)81342-0

Patterson, M., Sneyd, J., and Friel, D. D. (2007). Depolarization-induced calcium responses in sympathetic neurons: relative contributions from $\mathrm{Ca}^{2+}$ entry, extrusion, ER/mitochondrial $\mathrm{Ca}^{2+}$ uptake and release, and $\mathrm{Ca}^{2+}$ buffering. J. Gen. Physiol. 129, 29-56. doi: 10.1085/jgp.200609660 
Pusch, M., and Neher, E. (1988). Rates of diffusional exchange between small cells and a measuring patch pipette. Pflugers Arch. 411, 204-211. doi: 10.1007/BF00582316

Rosenmund, C., Feltz, A., and Westbrook, G. L. (1995). Synaptic NMDA receptor channels have a low open probability. J. Neurosci. 15, 2788-2895.

Rozsa, B., Zelles, T., Vizi, E. S., and Lendvai, B. (2004). Distance-dependent scaling of calcium transients evoked by backpropagating spikes and synaptic activity in dendrites of hippocampal interneurons. J. Neurosci. 24, 661-670. doi: 10.1523/JNEUROSCI.3906-03.2004

Sabatini, B. L., Oertner, T. G., and Svoboda, K. (2002). The life cycle of $\mathrm{Ca}^{(2+)}$ ions in dendritic spines. Neuron 33, 439-452. doi: 10.1016/S0896-6273(02)00573-1

Sala, F., and Hernandez Cruz, A. (1990). Calcium diffusion modeling in a spherical neuron. Relevance of buffering properties. Biophys. J. 57, 313-324. doi: 10.1016/S0006-3495(90)82533-9

Santo-Domingo, J., and Demaurex, N. (2010). Calcium uptake mechanisms of mitochondria. Biochim. Biophys. Acta 1797, 907-912. doi: 10.1016/j.bbabio.2010.01.005

Schmidt, H., Arendt, O., Brown, E. B., Schwaller, B., and Eilers, J. (2007). Parvalbumin is freely mobile in axons, somata and nuclei of cerebellar Purkinje neurones. J. Neurochem. 100, 727-735. doi: 10.1111/j.1471-4159.2006.04231.x

Schmidt, H., Brown, E. B., Schwaller, B., and Eilers, J. (2003). Diffusional mobility of parvalbumin in spiny dendrites of cerebellar Purkinje neurons quantified by fluorescence recovery after photobleaching. Biophys. J. 84, 2599-2608. doi: 10.1016/S0006-3495(03)75065-6

Schmidt, H., Schwaller, B., and Eilers, J. (2005). Calbindin D28k targets myo-inosito monophosphatase in spines and dendrites of cerebellar Purkinje neurons. Proc Natl. Acad. Sci. U.S.A. 102, 5850-5855. doi: 10.1073/pnas.0407855102

Schwaller, B. (2010). Cytosolic $\mathrm{Ca}^{2+}$ buffers. Cold Spring Harb. Perspect. Biol. 2:a004051. doi: 10.1101/cshperspect.a004051

Scott, R., and Rusakov, D. A. (2006). Main determinants of presynaptic $\mathrm{Ca}^{2+}$ dynamics at individual mossy fiber-CA3 pyramidal cell synapses. J. Neurosci. 26, 7071-7081. doi: 10.1523/JNEUROSCI.0946-06.2006

Smith, G. D., Wagner, J., and Keizer, J. (1996). Validity of the rapid buffering approximation near a point source of calcium ions. Biophys. J. 70, 2527-2539. doi: 10.1016/S0006-3495(96)79824-7

Stocca, G., Schmidt-Hieber, C., and Bischofberger, J. (2008). Differential dendritic $\mathrm{Ca}^{2+}$ signalling in young and mature hippocampal granule cells. J. Physiol. 586 3795-3811. doi: 10.1113/jphysiol.2008.155739

Thayer, S. A., and Miller, R. J. (1990). Regulation of the intracellular free calcium concentration in single rat dorsal root ganglion neurones in vitro. J. Physiol. 425, 85-115. doi: 10.1113/jphysiol.1990.sp018094

Tillotson, D., and Gorman, A. L. (1980). Non-uniform $\mathrm{Ca}^{2+}$ buffer distribution in a nerve cell body. Nature 286, 816-817. doi: 10.1038/286816a0
Tillotson, D. L., and Gorman, A. L. (1983). Localization of neuronal $\mathrm{Ca}^{2+}$ buffering near plasma membrane studied with different divalent cations. Cell. Mol. Neurobiol. 3, 297-310. doi: 10.1007/BF00734712

Van Hook, M. J., and Thoreson, W. B. (2014). Endogenous calcium buffering at photoreceptor synaptic terminals in salamander retina. Synapse 68, 518-528. doi: 10.1002/syn. 21768

Wagner, J., and Keizer, J. (1994). Effects of rapid buffers on $\mathrm{Ca}^{2+}$ diffusion and $\mathrm{Ca}^{2+}$ oscillations. Biophys. J. 67, 447-56. doi: 10.1016/S0006-3495(94) 80500-4

Wang, X., and Schwarz, T. L. (2009). The mechanism of $\mathrm{Ca}^{2+}$-dependent regulation of kinesin-mediated mitochondrial motility. Cell 136, 163-174. doi: 10.1016/j.cell.2008.11.046

White, R. J., and Reynolds, I. J. (1995). Mitochondria and $\mathrm{Na}^{+} / \mathrm{Ca}^{2+}$ exchange buffer glutamate-induced calcium loads in cultured cortical neurons. J. Neurosci. 15, 1318-1328.

Xu, T., Naraghi, M., Kang, H., and Neher, E. (1997). Kinetic studies of $\mathrm{Ca}^{2+}$ binding and $\mathrm{Ca}^{2+}$ clearance in the cytosol of adrenal chromaffin cells. Biophys. J. 73, 532-545. doi: 10.1016/S0006-3495(97)78091-3

Yoon, S. P., Chung, Y. Y., Chang, I. Y., Kim, J. J., Moon, J. S., and Kim, H. S. (2000). Postnatal development of parvalbumin and calbindin D-28k immunoreactivities in the canine hippocampus. J. Chem. Neuroanat. 19, 143-154. doi: 10.1016/S0891-0618(00)00059-4

Zador, A., and Koch, C. (1994). Linearized models of calcium dynamics: formal equivalence to the cable equation. J. Neurosci. 14, 4705-4715.

Zhou, Z., and Neher, E. (1993). Mobile and immobile calcium buffers in bovine adrenal chromaffin cells. J. Physiol. 469, 245-273. doi: 10.1113/jphysiol.1993.sp019813

Conflict of Interest Statement: The authors declare that the research was conducted in the absence of any commercial or financial relationships that could be construed as a potential conflict of interest.

Received: 31 October 2014; accepted: 31 January 2015; published online: 20 February 2015.

Citation: Matthews EA and Dietrich D (2015) Buffer mobility and the regulation of neuronal calcium domains. Front. Cell. Neurosci. 9:48. doi: 10.3389/fncel.2015.00048 This article was submitted to the journal Frontiers in Cellular Neuroscience. Copyright (C) 2015 Matthews and Dietrich. This is an open-access article distributed under the terms of the Creative Commons Attribution License (CC BY). The use, distribution or reproduction in other forums is permitted, provided the original author(s) or licensor are credited and that the original publication in this journal is cited, in accordance with accepted academic practice. No use, distribution or reproduction is permitted which does not comply with these terms. 


\title{
Translating neuronal activity at the synapse: presynaptic calcium sensors in short-term plasticity
}

\author{
Arthur P. H. de Jong ${ }^{1 *}$ and Diasynou Fioravante ${ }^{2 *}$ \\ ' Department of Neurobiology, Harvard Medical School, Boston, MA, USA \\ ${ }^{2}$ Department of Neurobiology, Physiology and Behavior, Center for Neuroscience, University of California Davis, Davis, CA, USA
}

\author{
Edited by: \\ Philippe Isope, Centre National pour \\ la Recherche Scientifique, France \\ Reviewed by: \\ Marco Canepari, Institut National de \\ la Santé et de la Recherche \\ Médicale, France \\ Erwin Neher, Max Planck Institute \\ for Biophysical Chemistry, Germany \\ ${ }^{*}$ Correspondence: \\ Arthur P. H. de Jong, Department of \\ Neurobiology, Harvard Medical \\ School, 220 Longwood Ave, Boston, \\ MA 02115, USA \\ e-mail: arthur_de_jong@hms. \\ harvard.edu; \\ Diasynou Fioravante, Department of \\ Neurobiology, Physiology and \\ Behavior, Center for Neuroscience, \\ University of California Davis, 1544 \\ Newton Court, Davis, CA 95618, \\ USA \\ e-mail:dfioravante@ucdavis.edu
}

The complex manner in which patterns of presynaptic neural activity are translated into short-term plasticity (STP) suggests the existence of multiple presynaptic calcium $\left(\mathrm{Ca}^{2+}\right)$ sensors, which regulate the amplitude and time-course of STP and are the focus of this review. We describe two canonical $\mathrm{Ca}^{2+}$-binding protein domains (C2 domains and EF-hands) and define criteria that need to be met for a protein to qualify as a $\mathrm{Ca}^{2+}$ sensor mediating STP. With these criteria in mind, we discuss various forms of STP and identify established and putative $\mathrm{Ca}^{2+}$ sensors. We find that despite the multitude of proposed sensors, only three are well established in STP: Munc13, protein kinase C (PKC) and synaptotagmin-7. For putative sensors, we pinpoint open questions and potential pitfalls. Finally, we discuss how the molecular properties and modes of action of $\mathrm{Ca}^{2+}$ sensors can explain their differential involvement in STP and shape net synaptic output.

\author{
Keywords: C2 domain, protein kinase C, Munc13, synaptotagmin, calmodulin, post-tetanic potentiation, residual \\ calcium, short-term plasticity
}

\section{INTRODUCTION}

Synaptic transmission is initiated by action potential-evoked influx of calcium $\left(\mathrm{Ca}^{2+}\right)$ into the presynaptic terminal, which triggers fusion of vesicles by binding to a specialized $\mathrm{Ca}^{2+}$ sensor. Bursts of action potentials lead to the buildup of residual $\mathrm{Ca}^{2+}$ $\left(\left[\mathrm{Ca}^{2+}\right]_{\text {residual }}\right)$ in the terminal, which outlives neuronal activity, and induce multiple forms of short-term presynaptic plasticity (STP), including facilitation, depression, augmentation and posttetanic potentiation (PTP) (reviewed in Fioravante and Regehr, 2011). STP plays a crucial role in synaptic computations and shapes the properties of microcircuits (reviewed in Abbott and Regehr, 2004; Regehr, 2012).

The dynamics of some forms of STP are dictated by the

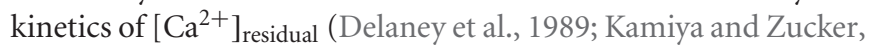
1994) and can be explained by changes in vesicular release probability (Katz and Miledi, 1968; Zucker and Stockbridge, 1983) or by depletion of the readily releasable pool (RRP) of vesicles (Bailey and Chen, 1988; Liu and Tsien, 1995; von Gersdorff and Matthews, 1997). However, at several synapses the magnitude of facilitation is higher than can be explained by $\left[\mathrm{Ca}^{2+}\right]_{\text {residual }}$ alone, and both facilitation and PTP decay slower than the $\left[\mathrm{Ca}^{2+}\right]_{\text {residual }}$ signal (Regehr et al., 1994; Atluri and Regehr, 1996; Brager et al., 2003; Felmy et al., 2003; Fioravante et al., 2011; Figure 1). Furthermore, many types of STP rely on the regulation of steps upstream of vesicle fusion (Dittman and Regehr, 1998;
Wang and Kaczmarek, 1998), including RRP refilling and $\mathrm{Ca}^{2+}$ influx through voltage-gated $\mathrm{Ca}^{2+}$ channels (VGCCs; Stevens and Wesseling, 1998; Xu and Wu, 2005; Mochida et al., 2008; Müller et al., 2008; Leal et al., 2012). These events are strongly $\mathrm{Ca}^{2+}$. dependent, and thus $\mathrm{Ca}^{2+}$ sensors must be activated to induce and sustain STP. The $\mathrm{Ca}^{2+}$ sensors that mediate STP are the topic of this mini-review. First, we will discuss the molecular structure and function of two $\mathrm{Ca}^{2+}$-binding domains employed by $\mathrm{Ca}^{2+}$ sensors: C2 domains and EF-hands. Subsequently, we will define the criteria for establishing $\mathrm{Ca}^{2+}$ sensors for STP, and, guided by these criteria, discuss a body of recent literature on well accepted and putative sensors that regulate STP.

\section{$\mathrm{Ca}^{2+}$-BINDING MOTIFS C2 DOMAINS}

The best described $\mathrm{Ca}^{2+}$ sensors in the context of synapses are C2 domains, which are found in many signal transduction and membrane trafficking proteins (Rizo and Südhof, 1998). C2 domains consist of $\sim 130$ amino acids that form a compact $\beta$ sandwich of two 4 -stranded $\beta$-sheets. Three loops connecting the $\beta$-sheets at the top of the domain contain 4-5 highly conserved aspartates that coordinate the binding of 2 to $3 \mathrm{Ca}^{2+}$ ions (Shao et al., 1996; Ubach et al., 1998; Fernandez et al., 2001). The $\mathrm{Ca}^{2+}$-binding properties of $\mathrm{C} 2$ domains have been described in detail in synaptotagmin (syt), which acts as the $\mathrm{Ca}^{2+}$ sensor for 

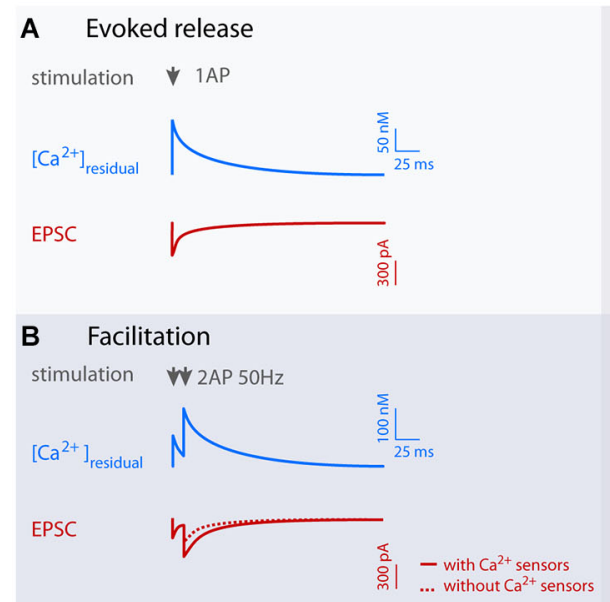

C Depression and augmentation

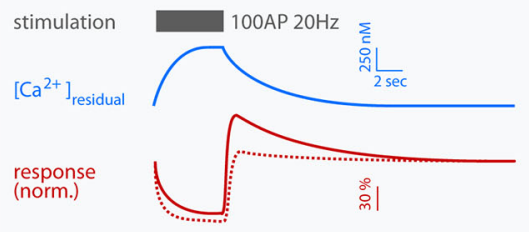

\section{PTP}

stimulation

$\left[\mathrm{Ca}^{2+}\right]_{\text {residual }}$

- $400 \mathrm{AP} 100 \mathrm{~Hz}$

response (norm.)

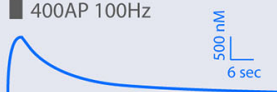

\&̊ำ

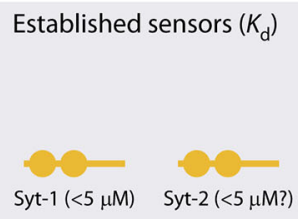

Putative sensors $\left(K_{\mathrm{d}}\right)$

Syt-1 $(<5 \mu \mathrm{M}) \quad$ Syt-2 $(<5 \mu \mathrm{M}$ ?)

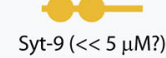

Syt-9 $(<<5 \mu \mathrm{M}$ ?)
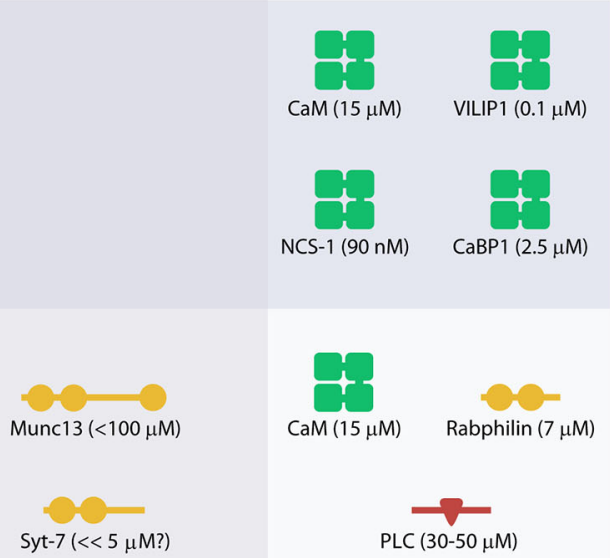

Syt-7 $(<<5 \mu \mathrm{M} ?)$

$\operatorname{PLC}(30-50 \mu \mathrm{M})$

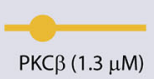

$\operatorname{PKC} \beta(1.3 \mu \mathrm{M})$

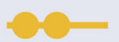

Syt-2 $(<5 \mu M$ ?)

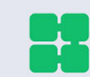

CaM $(15 \mu \mathrm{M})$

$\operatorname{PKC} \alpha(0.9 \mu \mathrm{M})$

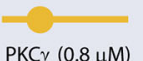

$a^{2+}$ sensing domain:
FIGURE 1 | Overview of established and putative presynaptic $\mathrm{Ca}^{2+}$ sensors in evoked release and short-term plasticity (STP). Left panel displays idealized traces of $\left[\mathrm{Ca}^{2+}\right]_{\text {residual }}$ and excitatory postsynaptic currents (EPSCs; $\mathbf{A}$ and $\mathbf{B}$ ) or baseline-normalized responses (C and $\mathbf{D}$ ) during synaptic plasticity based on experiments at parallel fiber synapses, mossy fiber-CA3 synapses, the crayfish neuromuscular junction and the calyx of Held. Typical stimulation paradigms used to elicit various forms of STP are indicated in gray. Scale bars are approximate, but note that the amplitude and kinetics of the $\mathrm{Ca}^{2+}$ signal and STP vary significantly between preparations. Right panels show established and putative $\mathrm{Ca}^{2+}$ sensors for evoked release (A) and each form of STP (B-D), and their $\mathrm{Ca}^{2+}$ dissociation constant $\left(K_{d}\right)$. $K_{d}$ values were obtained from: syt-1 C2AB (with PIP2) (van den Bogaart et al., 2012), free calmodulin (CaM; Xia and Storm, 2005), visin-like protein (VILIP-1) (myristroylated) (Li et al., 2011), neuronal calcium sensor 1 (NCS-1) (myristroylated) and CaBP1 (Aravind et al., 2008), Munc13 C2B (Shin et al., 2010), Rabphilin C2B (Ubach et al., 1999), PLC 81 (Grobler and Hurley, 1998) PKC $\alpha,-\beta$ and $\gamma$ (Torrecillas et al., 2004). The $K_{d}$ values of syt-2, -7 and -9 have not been measured directly, but indirect measurements suggest that syt-2 is similar to syt-1, whereas syt-7 and 9 may have lower $K_{d}$ (Sugita et al., 2002). synchronous vesicle fusion at most synapses (Pang and Südhof, 2010). Mutations that interfere with $\mathrm{Ca}^{2+}$ binding on syt-1 alter the $\mathrm{Ca}^{2+}$-sensitivity of vesicle fusion (Nishiki and Augustine, 2004; Shin et al., 2009; Kochubey and Schneggenburger, 2011; Kochubey et al., 2011). Analogous mutation analyses of other $\mathrm{C} 2$ domains showed similar effects on $\mathrm{Ca}^{2+}$ binding (Shin et al., 2010; Fioravante et al., 2014; Liu et al., 2014). Some C2 domains naturally lack these aspartate residues and cannot bind $\mathrm{Ca}^{2+}$ (e.g., Pappa et al., 1998). $\mathrm{Ca}^{2+}$ binding increases the affinity of C2 domains for phospholipids (Brose et al., 1992; Fernandez et al., 2001), thus recruiting the domain to the plasma membrane. In addition, it may trigger a conformational change that increases association with effector proteins (for instance syt-1 binding to SNAREs (Bai et al., 2004)) or exposes a domain within the protein (e.g., the MUN domain of Munc13 (Shin et al., 2010)). Many C2 domains display a steep increase in $\mathrm{Ca}^{2+}$ affinity in the presence of phosphatidylinositol 4,5-biphosphate (PIP2; van den Bogaart et al., 2012), which helps localize the domain to the PIP2-enriched active zone (Rohrbough and Broadie, 2005).

\section{EF-HANDS}

The EF-hand is the most common $\mathrm{Ca}^{2+}$-binding motif, with diverse cellular functions including cytoplasmic $\mathrm{Ca}^{2+}$ buffering and signal transduction (Skelton et al., 1994; Schaub and Heizmann, 2008; Schwaller, 2009). The motif consists of two 
$\alpha$-helices connected by a linker of 12 amino acids (Lewit-Bentley and Réty, 2000). Six residues within this linker coordinate binding to a single $\mathrm{Ca}^{2+}$ ion, and their mutation abolishes $\mathrm{Ca}^{2+}$ binding (Maune et al., 1992). Examples of EF-hand-containing proteins with proposed $\mathrm{Ca}^{2+}$-sensing roles in STP include calmodulin (CaM), neuronal calcium sensor 1 (NCS-1) and visin-like proteins (VILIPs).

$\mathrm{CaM}$ is the prototypical EF-hand protein that interacts with numerous effector proteins in a $\mathrm{Ca}^{2+}$-dependent manner (Xia and Storm, 2005). Important presynaptic effectors are CaM-dependent kinase II (CaMKII), myosin light chain kinase (MLCK), adenylyl cyclase, the protein phosphatase calcineurin, Munc13, VGCCs and $\mathrm{Ca}^{2+}$-activated potassium channels, all of which regulate presynaptic function (de Jong and Verhage, 2009; Adelman et al., 2012). Because the $\mathrm{Ca}^{2+}$ affinity of CaM is differentially regulated by its binding partners, different CaM-protein complexes vary in their $\mathrm{Ca}^{2+}$ sensitivity (Olwin and Storm, 1985; Xia and Storm, 2005) and could therefore be differentially engaged during various forms of STP. Direct assessment of the role of CaM as a $\mathrm{Ca}^{2+}$ sensor for STP has proven difficult because manipulations of CaM levels alter expression of $>200$ genes (Pang et al., 2010) and rescue experiments in neuronal preparations with $\mathrm{Ca}^{2+}$-binding mutants of CaM have not been conducted thus far.

\section{DEFINITION OF A Ca ${ }^{2+}$ SENSOR FOR STP}

With a plethora of $\mathrm{C} 2$ - and EF-hand-containing proteins in the presynaptic terminal, there are numerous candidate $\mathrm{Ca}^{2+}$ sensors for STP. We propose that in order to qualify as a sensor for STP, a protein must fulfill the following three criteria:

1. $\mathrm{Ca}^{2+}$ must bind directly to the protein. An obvious requirement for a $\mathrm{Ca}^{2+}$ sensor is that it must bind $\mathrm{Ca}^{2+}$. Some EF-hands and $\mathrm{C} 2$ domains lack the $\mathrm{Ca}^{2+}$-coordinating residues and cannot bind $\mathrm{Ca}^{2+}$. Therefore, $\mathrm{Ca}^{2+}$ binding must be experimentally established for each protein.

2. Protein must be part of, or directly modulate, vesicle availability or the vesicle release machinery. Changes in availability and/or fusogenicity of synaptic vesicles and in presynaptic $\mathrm{Ca}^{2+}$ influx shape STP (Dutta Roy et al., 2014). A Ca ${ }^{2+}$ sensor for STP must therefore directly affect vesicle availability (recruitment, docking, priming) and/or the vesicle fusion machinery, including VGCCs and SM proteins (for a discussion of release machinery, see Südhof, 2013). This definition includes enzymes like kinases, which directly regulate the properties of these components. For the purpose of this review, we do not consider $\mathrm{Ca}^{2+}$ buffers (e.g., parvalbumin) and pumps, which indirectly affect STP by changing the spatiotemporal distribution of free $\mathrm{Ca}^{2+}$ through binding or extrusion (Müller et al., 2007; Scullin and Partridge, 2010), or components of the endocytotic machinery, which can affect vesicle or release site availability after prolonged episodes of exocytosis (Wilkinson and Lin, 2004; Hosoi et al., 2009).

3. Mutations that interfere with $\mathrm{Ca}^{2+}$ binding affect STP. Even if a protein satisfies criteria 1 and 2 , it is not a $\mathrm{Ca}^{2+}$ sensor for STP unless $\mathrm{Ca}^{2+}$ binding is required for the protein's function in STP. For instance, whether $\mathrm{Ca}^{2+}$ binding to Doc2 is required for spontaneous release is debated and the role of Doc2 as a
$\mathrm{Ca}^{2+}$ sensor for spontaneous release remains unclear (Groffen et al., 2010; Pang et al., 2011). Therefore, it is necessary to show that mutation of the $\mathrm{Ca}^{2+}$ binding site abolishes function (for example using a knockout/rescue or knockin approach) in order to conclude that a protein is a $\mathrm{Ca}^{2+}$ sensor mediating STP. It could even be argued that a requirement for $\mathrm{Ca}^{2+}$ binding during plasticity must be demonstrated in order to establish a protein as a $\mathrm{Ca}^{2+}$ sensor, but the technology for this type of experiments is currently lacking.

\section{$\mathrm{Ca}^{2+}$ SENSORS IN STP FACILITATION}

At synapses with low initial release probability, brief bursts of activity can induce transient facilitation of release, which relies on increased release probability due to elevated $\left[\mathrm{Ca}^{2+}\right]_{\text {residual }}$ (Katz and Miledi, 1968; Kamiya and Zucker, 1994; Regehr et al., 1994). However, this mechanism alone cannot fully explain the magnitude of facilitation at all synapses (Atluri and Regehr, 1996; Felmy et al., 2003), and additional $\mathrm{Ca}^{2+}$-dependent processes have been suggested (Zucker and Regehr, 2002), including the existence of a yet unidentified presynaptic $\mathrm{Ca}^{2+}$ sensor distinct from syt-1 (Bain and Quastel, 1992; Saraswati et al., 2007). Enhancement of $\mathrm{Ca}^{2+}$ currents is an attractive mechanism to mediate facilitation, and the capability of $\mathrm{Ca}^{2+} / \mathrm{CaM}$ to modulate overexpressed VGCCs during strong depolarization has been studied extensively (Catterall et al., 2013). $\mathrm{Ca}^{2+} / \mathrm{CaM}$ binds to a regulatory domain of $\mathrm{Ca}_{\mathrm{v}} 2.1$, the VGCC that mediates the P/Q type $\mathrm{Ca}^{2+}$ current driving synaptic transmission in most synapses. In heterologous cell lines, this interaction leads to enhancement of $\mathrm{Ca}^{2+}$ currents, which depends on $\mathrm{Ca}^{2+}$ binding to CaM (Lee et al., 1999; DeMaria et al., 2001). Several EF-hand-containing proteins including VILIPs, CaBPs and NCS-1 (collectively named neuronal $\mathrm{Ca}^{2+}$ sensors, or $\left.\mathrm{nCaS}\right)$ also modulate $\mathrm{Ca}^{2+}$ influx through VGCCs (Few et al., 2005; Lautermilch et al., 2005; Burgoyne, 2007; Dason et al., 2012; Catterall et al., 2013) and may affect facilitation in a manner that depends on the nCaS binding domain of VGCCs (Tsujimoto et al., 2002; Sippy et al., 2003; Mochida et al., 2008; Leal et al., 2012). For none of these protein functions, however, has a $\mathrm{Ca}^{2+}$ binding requirement been established, and some of them may actually be independent of $\mathrm{Ca}^{2+}$ (Few et al., 2005). In addition, due to the lack of suitable genetic models, most experiments rely on overexpression of exogenous proteins (Mochida et al., 2003). Whether $\mathrm{nCaS}$ are specifically involved in the regulation of STP, or the altered STP is a consequence of altered basal synaptic properties, remains controversial (Dason et al., 2012).

\section{DEPRESSION AND RECOVERY FROM DEPRESSION}

Prolonged high-frequency stimulation leads to transient decrease in presynaptic strength, which can be due to depletion of the RRP (Elmqvist and Quastel, 1965; Liu and Tsien, 1995; Schneggenburger et al., 2002) and activity-dependent decrease in $\mathrm{Ca}^{2+}$ influx (Forsythe et al., 1998; $\mathrm{Xu}$ and $\mathrm{Wu}, 2005$ ) (for a complete review of known mechanisms of depression, see Regehr, 2012). CaM, CaBP1 and NCS-1 have been proposed as putative $\mathrm{Ca}^{2+}$ sensors to mediate the latter effect (Xu and $\mathrm{Wu}$, 2005; Catterall and Few, 2008; Mochida et al., 2008). Depression can be slowed by $\mathrm{Ca}^{2+}$-dependent replenishment of the RRP 
(Stevens and Wesseling, 1998; Wang and Kaczmarek, 1998). The vesicle priming factor Munc13 acts as a $\mathrm{Ca}^{2+}$ sensor to determine the rate of depression, via its $\mathrm{C} 2 \mathrm{~B}$ and CaM-binding domains. $\mathrm{Ca}^{2+}$ binding to the $\mathrm{C} 2 \mathrm{~B}$ domain of Munc13 activates its MUN domain that promotes assembly of the machinery responsible for vesicle fusion, thereby increasing refilling of the RRP (Shin et al., 2010; Ma et al., 2011). Indeed, Munc13 knockout neurons expressing a variant of the protein with mutated $\mathrm{Ca}^{2+}$-coordinating aspartates display increased synaptic depression without affecting initial release probability (Shin et al., 2010). In addition, Munc13 binds $\mathrm{Ca}^{2+} / \mathrm{CaM}$, and this interaction also accelerates RRP refilling (Junge et al., 2004; Lipstein et al., 2012, 2013). In line with this observation, CaM inhibitors slow the RRP refilling rate (Sakaba and Neher, 2001; Hosoi et al., 2007). Although a $\mathrm{Ca}^{2+}$ binding CaM mutant has not been studied in this context, the CaM/Munc13 interaction is strongly $\mathrm{Ca}^{2+}$-dependent (Junge et al., 2004; Dimova et al., 2006; Lipstein et al., 2012), thus making the $\mathrm{Ca}^{2+} / \mathrm{CaM}-\mathrm{Munc} 13$ complex a likely $\mathrm{Ca}^{2+}$-sensor for STP.

Synaptotagmin-7 has also been identified as a sensor that regulates depression, operating via its two $\mathrm{Ca}^{2+}$-binding $\mathrm{C} 2$ domains (Liu et al., 2014). At the zebrafish neuromuscular junction, syt7 regulates desynchronized release (Wen et al., 2010), but its function in mammalian neurons has been debated (Maximov et al., 2008; Bacaj et al., 2013; Liu et al., 2014). A recent study showed that in syt-7 knockout mice, initial release probability is unaffected but the rate of vesicle replenishment during and after bursts of activity is significantly reduced (Liu et al., 2014). This phenotype is rescued by wild-type syt-7 but not by syt7 carrying mutations of the $\mathrm{Ca}^{2+}$ binding sites, demonstrating that syt-7 is a $\mathrm{Ca}^{2+}$ sensor that mediates RRP refilling. Syt7 also probably interacts with $\mathrm{Ca}^{2+} / \mathrm{CaM}$ (Liu et al., 2014), but the functional significance of this complex remains to be identified.

In contrast to the proteins discussed above that promote recovery from depression, rabphilin is thought to slow down recovery from depression (Deák et al., 2006). Rabphilin is a synaptic vesicle protein with two $\mathrm{Ca}^{2+}$-sensing C2 domains (Yamaguchi et al., 1993; Ubach et al., 1999; Coudevylle et al., 2008), but whether $\mathrm{Ca}^{2+}$ binding is required for its role in STP has not been determined.

\section{AUGMENTATION AND PTP}

Augmentation and PTP are two closely related forms of STP that require prolonged high-frequency stimulation (Magleby, 1973; Magleby and Zengel, 1976a; Stevens and Wesseling, 1999; Habets and Borst, 2005; Korogod et al., 2005). For augmentation, varying stimulus duration increases the peak amplitude of the enhancement without significantly affecting the time course of decay (Magleby, 1979). The mechanisms underlying augmentation are not well understood and changes in both release probability and $\mathrm{Ca}^{2+}$-dependent replenishment of the RRP have been proposed (Magleby and Zengel, 1976b; Stevens and Wesseling, 1999; Rosenmund et al., 2002; Kalkstein and Magleby, 2004). Munc13 and syt-7 have been suggested as $\mathrm{Ca}^{2+}$ sensors for augmentation (Shin et al., 2010; Lipstein et al., 2013; Liu et al., 2014), but since both sensors affect depression as well, dissociation of their roles in synaptic depression vs. augmentation has not been possible. Various phospholipase C (PLC) isoforms could also act as $\mathrm{Ca}^{2+}$ sensors because they require binding of a $\mathrm{Ca}^{2+}$ ion for activation of their catalytic domain (Grobler and Hurley, 1998; Rebecchi and Pentyala, 2000). Pharmacological studies suggest that PLC activation is required for augmentation (Rosenmund et al., 2002) but not PTP (Genc et al., 2014). PLC hydrolyses PIP2 to diacylglycerol, which could lead to potentiation of synaptic transmission via Munc13 and protein kinase C (PKC; de Jong and Verhage, 2009).

PTP typically lasts longer than augmentation and shows a progressive increase in the time course of decay with increased duration and frequency of stimulation (Magleby, 1979; Korogod et al., 2005). Pharmacological (e.g., Alle et al., 2001; Brager et al., 2002; Beierlein et al., 2007; Korogod et al., 2007) and genetic (Fioravante et al., 2011, 2012, 2014; Chu et al., 2014) studies at several synapses have firmly established the requirement for $\mathrm{PKC}$ in PTP. Three PKC isoforms $(\alpha, \beta$ and $\gamma)$ possess a $\mathrm{C} 2$ domain and bind $\mathrm{Ca}^{2+}$ with low micromolar affinity (Torrecillas et al., 2004; Newton, 2010; Figure 1). PKCs enhance release through phosphorylation of effectors, including components of the vesicular release machinery such as Munc18 (Wierda et al., 2007; de Jong and Verhage, 2009; Genc et al., 2014). Mutations of the $\mathrm{Ca}^{2+}$-coordinating aspartates in the $\mathrm{C} 2$ domain of PKC $\beta$ abolish its ability to support PTP, without affecting basal synaptic function (Fioravante et al., 2014).

PKC $\beta$ is probably not the only $\mathrm{Ca}^{2+}$ sensor for PTP. At the immature calyx of Held, PTP depends on PKC $\gamma$ (Chu et al., 2014). Moreover, at the parallel fiber-Purkinje cell synapse in the cerebellum, PKC $\alpha$ can readily support PTP in the absence of PKC $\beta$ and $\gamma$ (Fioravante et al., 2012). It remains to be tested whether $\mathrm{Ca}^{2+}$ binding to PKC $\alpha$ and $\gamma$ is necessary for PTP and whether all PKC isoforms act through Munc18 phosphorylation. Finally, pharmacological studies suggest that $\mathrm{Ca}^{2+} / \mathrm{CaM}$, acting via MLCK, makes a small contribution to PTP at immature, but not functionally mature, synapses (Lee et al., 2008; Fioravante et al., 2011).

Tetanic stimulation enhances not only evoked responses (i.e., PTP) but also spontaneous events in a $\mathrm{Ca}^{2+}$-dependent manner. The frequency (Zengel and Magleby, 1981; Zucker and LaraEstrella, 1983; Eliot et al., 1994; Habets and Borst, 2005), and at some synapses also the amplitude (He et al., 2009), of spontaneous events increase after tetanization. Because of similarities in the time course of these effects with PTP, a common mechanism has been speculated (Zengel and Magleby, 1981). However, the effects of $\left[\mathrm{Ca}^{2+}\right]_{\text {residual }}$ on spontaneous transmission were recently shown to be independent of PKC (Xue and Wu, 2010; Fioravante et al., 2011; but see Brager et al., 2003) and the increase in amplitude requires syt-2 (He et al., 2009). The $\mathrm{Ca}^{2+}$ sensors remain unknown.

\section{DIFFERENTIAL ENGAGEMENT OF $\mathrm{Ca}^{2+}$ SENSORS AND IMPLICATIONS FOR STP}

Different patterns of neuronal activity result in variable $\mathrm{Ca}^{2+}$ signals stretching over an order of magnitude (Figure 1). Diverse 
sensors are therefore needed to translate the $\mathrm{Ca}^{2+}$ signals into distinct forms of STP. Factors such as $\mathrm{Ca}^{2+}$ affinity, specific (sub-)cellular expression and mechanisms of action contribute to the specialization of sensors for different forms of STP. For example, NCS-1 has high affinity for $\mathrm{Ca}^{2+}$ and localizes at the plasma membrane (O'Callaghan et al., 2002; Burgoyne, 2007) where it could rapidly respond to local $\mathrm{Ca}^{2+}$ signals. PKC $\beta$, on the other hand, has lower $\mathrm{Ca}^{2+}$ affinity, is cytoplasmic at rest (Newton, 2010) and likely has to phosphorylate more than one substrates to induce plasticity; therefore, sustained, global $\mathrm{Ca}^{2+}$ increases are likely required for its activation, in agreement with the prolonged stimulation requirement for PTP (Habets and Borst, 2005; Korogod et al., 2005). Even for the same sensor, $\mathrm{Ca}^{2+}$ affinity can vary as a result of effector binding, phospholipid binding, and post-translational modifications (Xia and Storm, 2005; Li et al., 2011; van den Bogaart et al., 2012). Finally, specific expression patterns of $\mathrm{Ca}^{2+}$ sensors could help explain why identical activation regimes do not always lead to the same STP across synapses or during development (Rosenmund et al., 2002; Chu et al., 2014).

Most synapses exhibit multiple forms of STP and the net synaptic output reflects the interaction between these different forms (de Jong and Verhage, 2009). It is therefore likely that different $\mathrm{Ca}^{2+}$ sensors interact, and might even compete (Chu et al., 2014), during STP. The dynamics of these interactions should be considered when building computational models of STP. Traditionally, such models combine use-dependent depletion and $\mathrm{Ca}^{2+}$-dependent facilitation to explain synaptic output (Tsodyks et al., 1998; Fuhrmann et al., 2002; Pfister et al., 2010). Introduction of additional components such as vesicle replenishment, which are engaged under conditions that activate the corresponding $\mathrm{Ca}^{2+}$ sensors, more accurately reflects our understanding of the underlying biology and allows better prediction of synaptic and network behavior (Hennig, 2013).

\section{ACKNOWLEDGMENTS}

We would like to thank Drs. P.S. Kaeser, M. Verhage, M. Thanawala, W. Regehr and E. Antzoulatos for critically reading the manuscript. This work was funded by the Netherlands Organization for Scientific Research (NWO, 825.12.028) to Arthur P. H. de Jong and UC Davis College of Biological Sciences Dean's start-up award to Diasynou Fioravante.

\section{REFERENCES}

Abbott, L. F., and Regehr, W. G. (2004). Synaptic computation. Nature 431, 796-803. doi: 10.10.1038/nature 03010

Adelman, J. P., Maylie, J., and Sah, P. (2012). Small-Conductance $\mathrm{Ca}^{2+}$-Activated $\mathrm{K}^{+}$channels: form and function. Annu. Rev. Physiol. 74, 245-269. doi: 10. 1146/annurev-physiol-020911-153336

Alle, H., Jonas, P., and Geiger, J. R. (2001). PTP and LTP at a hippocampal mossy fiber-interneuron synapse. Proc. Natl. Acad. Sci. U S A 98, 14708-14713. doi: 10. 1073/pnas.251610898

Aravind, P., Chandra, K., Reddy, P. P., Jeromin, A., Chary, K. V. R., and Sharma, Y. (2008). Regulatory and structural EF-hand motifs of neuronal calcium sensor1: $\mathrm{Mg}^{2+}$ modulates $\mathrm{Ca}^{2+}$ binding, $\mathrm{Ca}^{2+}$-induced conformational changes and equilibrium unfolding transitions. J. Mol. Biol. 376, 1100-1115. doi: 10.1016/j. jmb.2007.12.033

Atluri, P. P., and Regehr, W. G. (1996). Determinants of the time course of facilitation at the granule cell to Purkinje cell synapse. J. Neurosci. 16, 56615671.
Bacaj, T., Wu, D., Yang, X., Morishita, W., Zhou, P., Xu, W., et al. (2013). Synaptotagmin-1 and synaptotagmin-7 trigger synchronous and asynchronous phases of neurotransmitter release. Neuron 80, 947-959. doi: 10.1016/j.neuron. 2013.10.026

Bai, J., Wang, C.-T., Richards, D. A., Jackson, M. B., and Chapman, E. R. (2004). Fusion Pore dynamics are regulated by Synaptotagmin $\bullet$-SNARE interactions. Neuron 41, 929-942. doi: 10.1016/s0896-6273(04)00117-5

Bailey, C. H., and Chen, M. (1988). Morphological basis of short-term habituation in Aplysia. J. Neurosci. 8, 2452-2459.

Bain, A. I., and Quastel, D. M. (1992). Multiplicative and additiva $\mathrm{Ca}(2+)$ dependent components of facilitation at mouse endplates. J. Physiol. 455, 383405.

Beierlein, M., Fioravante, D., and Regehr, W. G. (2007). Differential expression of posttetanic potentiation and retrograde signaling mediate target-dependent short-term synaptic plasticity. Neuron 54, 949-959. doi: 10.1016/j.neuron.2007. 06.002

Brager, D. H., Cai, X., and Thompson, S. M. (2003). Activity-dependent activation of presynaptic protein kinase $\mathrm{C}$ mediates post-tetanic potentiation. Nat. Neurosci. 6, 551-552. doi: 10.1038/nn1067

Brager, D. H., Capogna, M., and Thompson, S. M. (2002). Short-term synaptic plasticity, simulation of nerve terminal dynamics, and the effects of protein kinase $\mathrm{C}$ activation in rat hippocampus. J. Physiol. 541, 545-559. doi: 10.1113/ jphysiol.2001.015842

Brose, N., Petrenko, A. G., Südhof, T. C., and Jahn, R. (1992). Synaptotagmin: a calcium sensor on the synaptic vesicle surface. Science 256, 1021-1025. doi: 10. $1126 /$ science. 1589771

Burgoyne, R. D. (2007). Neuronal calcium sensor proteins: generating diversity in neuronal $\mathrm{Ca}^{2+}$ signalling. Nat. Rev. Neurosci. 8, 182-193. doi: 10.1038/nrn2093

Catterall, W. A., and Few, A. P. (2008). Calcium channel regulation and presynaptic plasticity. Neuron 59, 882-901. doi: 10.1016/j.neuron.2008.09.005

Catterall, W. A., Leal, K., and Nanou, E. (2013). Calcium channels and shortterm synaptic plasticity. J. Biol. Chem. 288, 10742-10749. doi: 10.1074/jbc.R112. 411645

Chu, Y., Fioravante, D., Leitges, M., and Regehr, W. G. (2014). Calcium-dependent PKC isoforms have specialized roles in short-term synaptic plasticity. Neuron 82 , 859-871. doi: 10.1016/j.neuron.2014.04.003

Coudevylle, N., Montaville, P., Leonov, A., Zweckstetter, M., and Becker, S. (2008). Structural determinants for $\mathrm{Ca}^{2+}$ and phosphatidylinositol 4,5-bisphosphate binding by the C2A domain of rabphilin-3A. J. Biol. Chem. 283, 35918-35928. doi: 10.1074/jbc.M804094200

Dason, J. S., Romero-Pozuelo, J., Atwood, H. L., and Ferrús, A. (2012). Multiple roles for Frequenin/NCS-1 in synaptic function and development. Mol. Neurobiol. 45, 388-402. doi: 10.1007/s12035-012-8250-4

Deák, F., Shin, O.-H., Tang, J., Hanson, P., Ubach, J., Jahn, R., et al. (2006). Rabphilin regulates SNARE-dependent re-priming of synaptic vesicles for fusion. EMBO J. 25, 2856-2866. doi: 10.1038/sj.emboj.7601165

de Jong, A. P. H., and Verhage, M. (2009). Presynaptic signal transduction pathways that modulate synaptic transmission. Curr. Opin. Neurobiol. 19, 245-253. doi: 10.1016/j.conb.2009.06.005

Delaney, K. R., Zucker, R. S., and Tank, D. W. (1989). Calcium in motor nerve terminals associated with posttetanic potentiation. J. Neurosci. 9, 3558-3567.

DeMaria, C. D., Soong, T. W., Alseikhan, B. A., Alvania, R. S., and Yue, D. T. (2001). Calmodulin bifurcates the local $\mathrm{Ca}^{2+}$ signal that modulates P/Q-type $\mathrm{Ca}^{2+}$ channels. Nature 411, 484-489. doi: 10.1038/35078091

Dimova, K., Kawabe, H., Betz, A., Brose, N., and Jahn, O. (2006). Characterization of the Munc13-calmodulin interaction by photoaffinity labeling. Biochim. Biophys. Acta 1763, 1256-1265. doi: 10.1016/j.bbamcr.2006.09.017

Dittman, J. S., and Regehr, W. G. (1998). Calcium dependence and recovery kinetics of presynaptic depression at the climbing fiber to Purkinje cell synapse. J. Neurosci. 18, 6147-6162.

Dutta Roy, R., Stefan, M. I., and Rosenmund, C. (2014). Biophysical properties of presynaptic short-term plasticity in hippocampal neurons: insights from electrophysiology, imaging and mechanistic models. Front. Cell. Neurosci. 8:141. doi: $10.3389 /$ fncel.2014.00141

Eliot, L. S., Kandel, E. R., and Hawkins, R. D. (1994). Modulation of spontaneous transmitter release during depression and posttetanic potentiation of Aplysia sensory-motor neuron synapses isolated in culture. J. Neurosci. 14, 3280-3292.

Elmqvist, D., and Quastel, D. M. (1965). A quantitative study of end-plate potentials in isolated human muscle. J. Physiol. 178, 505-529. 
Felmy, F., Neher, E., and Schneggenburger, R. (2003). Probing the intracellular calcium sensitivity of transmitter release during synaptic facilitation. Neuron 37 , 801-811. doi: 10.1016/s0896-6273(03)00085-0

Fernandez, I., Araç, D., Ubach, J., Gerber, S. H., Shin, O., Gao, Y., et al. (2001). Three-dimensional structure of the synaptotagmin 1 C2B-domain: synaptotagmin 1 as a phospholipid binding machine. Neuron 32, 1057-1069. doi: 10. 1016/s0896-6273(01)00548-7

Few, A. P., Lautermilch, N. J., Westenbroek, R. E., Scheuer, T., and Catterall, W. A. (2005). Differential regulation of CaV2.1 channels by calcium-binding protein 1 and visinin-like protein-2 requires N-terminal myristoylation. J. Neurosci. 25, 7071-7080. doi: 10.1523/jneurosci.0452-05.2005

Fioravante, D., Chu, Y., de Jong, A. P., Leitges, M., Kaeser, P. S., and Regehr, W. G. (2014). Protein kinase $\mathrm{C}$ is a calcium sensor for presynaptic short-term plasticity. Elife 3:e03011. doi: 10.7554/elife.03011

Fioravante, D., Chu, Y., Myoga, M. H., Leitges, M., and Regehr, W. G. (2011). Calcium-dependent isoforms of protein kinase $\mathrm{C}$ mediate posttetanic potentiation at the calyx of held. Neuron 70, 1005-1019. doi: 10.1016/j.neuron.2011. 04.019

Fioravante, D., Myoga, M. H., Leitges, M., and Regehr, W. G. (2012). Adaptive regulation maintains posttetanic potentiation at cerebellar granule cell synapses in the absence of calcium-dependent PKC. J. Neurosci. 32, 13004-13009. doi: 10. 1523/jneurosci.0683-12.2012

Fioravante, D., and Regehr, W. G. (2011). Short-term forms of presynaptic plasticity. Curr. Opin. Neurobiol. 21, 269-274. doi: 10.1016/j.conb.2011. 02.003

Forsythe, I. D., Tsujimoto, T., Barnes-Davies, M., Cuttle, M. F., and Takahashi, T. (1998). Inactivation of presynaptic calcium current contributes to synaptic depression at a fast central synapse. Neuron 20, 797-807. doi: 10.1016/s08966273(00)81017-x

Fuhrmann, G., Segev, I., Markram, H., and Tsodyks, M. (2002). Coding of temporal information by activity-dependent synapses. J. Neurophysiol. 87, 140-148.

Genc, O., Kochubey, O., Toonen, R. F., Verhage, M., and Schneggenburger, R. (2014). Munc18-1 is a dynamically regulated PKC target during short-term enhancement of transmitter release. Elife 3:e01715. doi: 10.7554/eLife.01715

Grobler, J. A., and Hurley, J. H. (1998). Catalysis by phospholipase C delta1 requires that $\mathrm{Ca}^{2+}$ bind to the catalytic domain, but not the $\mathrm{C} 2$ domain. Biochemistry 37 , 5020-5028. doi: 10.1021/bi972952w

Groffen, A. J., Martens, S., Díez Arazola, R., Cornelisse, L. N., Lozovaya, N., de Jong, A. P. H., et al. (2010). Doc2b is a high-affinity $\mathrm{Ca}^{2+}$ sensor for spontaneous neurotransmitter release. Science 327, 1614-1618. doi: 10.1126/science.11 83765

Habets, R. L. P., and Borst, J. G. G. (2005). Post-tetanic potentiation in the rat calyx of Held synapse. J. Physiol. 564, 173-187. doi: 10.1113/jphysiol.2004.079160

He, L., Xue, L., Xu, J., McNeil, B. D., Bai, L., Melicoff, E., et al. (2009). Compound vesicle fusion increases quantal size and potentiates synaptic transmission. Nature 459, 93-97. doi: 10.1038/nature07860

Hennig, M. H. (2013). Theoretical models of synaptic short term plasticity. Front. Comput. Neurosci. 7:45. doi: 10.3389/fncom.2013.00154

Hosoi, N., Holt, M., and Sakaba, T. (2009). Calcium dependence of exo- and endocytotic coupling at a glutamatergic synapse. Neuron 63, 216-229. doi: 10. 1016/j.neuron.2009.06.010

Hosoi, N., Sakaba, T., and Neher, E. (2007). Quantitative analysis of calciumdependent vesicle recruitment and its functional role at the calyx of Held synapse. J. Neurosci. 27, 14286-14298. doi: 10.1523/jneurosci.4122-07.2007

Junge, H. J., Rhee, J.-S., Jahn, O., Varoqueaux, F., Spiess, J., Waxham, M. N., et al. (2004). Calmodulin and Munc13 form a $\mathrm{Ca}^{2+}$ sensor/effector complex that controls short-term synaptic plasticity. Cell 118, 389-401. doi: 10.1016/j.cell. 2004.06.029

Kalkstein, J. M., and Magleby, K. L. (2004). Augmentation increases vesicular release probability in the presence of masking depression at the Frog neuromuscular junction. J. Neurosci. 24, 11391-11403. doi: 10.1523/jneurosci.275604.2004

Kamiya, H., and Zucker, R. S. (1994). Residual Ca2 + and short-term synaptic plasticity. Nature 371, 603-606. doi: 10.1038/371603a0

Katz, B., and Miledi, R. (1968). The role of calcium in neuromuscular facilitation. J. Physiol. 195, 481-492.

Kochubey, O., Lou, X., and Schneggenburger, R. (2011). Regulation of transmitter release by $\mathrm{Ca}^{2+}$ and synaptotagmin: insights from a large CNS synapse. Trends Neurosci. 34, 237-246. doi: 10.1016/j.tins.2011.02.006
Kochubey, O., and Schneggenburger, R. (2011). Synaptotagmin increases the dynamic range of synapses by driving $\mathrm{Ca}^{2+}$-evoked release and by clamping a near-linear remaining $\mathrm{Ca}^{2+}$ sensor. Neuron 69, 736-748. doi: 10.1016/j.neuron. 2011.01.013

Korogod, N., Lou, X., and Schneggenburger, R. (2005). Presynaptic $\mathrm{Ca}^{2+}$ requirements and developmental regulation of posttetanic potentiation at the calyx of held. J. Neurosci. 25, 5127-5137. doi: 10.1523/jneurosci.1295-05.2005

Korogod, N., Lou, X., and Schneggenburger, R. (2007). Posttetanic potentiation critically depends on an enhanced $\mathrm{Ca}^{2+}$ sensitivity of vesicle fusion mediated by presynaptic PKC. Proc. Natl. Acad. Sci. U S A 104, 15923-15928. doi: 10. 1073/pnas.0704603104

Lautermilch, N. J., Few, A. P., Scheuer, T., and Catterall, W. A. (2005). Modulation of CaV2.1 channels by the neuronal calcium-binding protein visinin-like protein-2. J. Neurosci. 25, 7062-7070. doi: 10.1523/jneurosci.0447-05.2005

Leal, K., Mochida, S., Scheuer, T., and Catterall, W. A. (2012). Fine-tuning synaptic plasticity by modulation of $\mathrm{Ca}(\mathrm{V}) 2.1$ channels with $\mathrm{Ca}^{2+}$ sensor proteins. Proc. Natl. Acad. Sci. U S A 109, 17069-17074. doi: 10.1073/pnas.1215172109

Lee, J. S., Kim, M.-H., Ho, W.-K., and Lee, S.-H. (2008). Presynaptic release probability and readily releasable pool size are regulated by two independent mechanisms during posttetanic potentiation at the calyx of held synapse. $J$. Neurosci. 28, 7945-7953. doi: 10.1523/JNEUROSCI.2165-08.2008

Lee, A., Wong, S., Gallagher, D., Li, B., Storm, D., Scheuer, T., et al. (1999). $\mathrm{Ca}^{2+} /$ calmodulin binds to and modulates P/Q-type calcium channels. Nature 399, 155-159. doi: 10.1038/20194

Lewit-Bentley, A., and Réty, S. (2000). EF-hand calcium-binding proteins. Curr. Opin. Struct. Biol. 10, 637-643. doi: 10.1016/S0959-440X(00)00142-1

Li, C., Pan, W., Braunewell, K. H., and Ames, J. B. (2011). Structural analysis of $\mathrm{Mg}^{2+}$ and $\mathrm{Ca}^{2+}$ binding, myristoylation and dimerization of the neuronal calcium sensor and visinin-like protein 1 (VILIP-1). J. Biol. Chem. 286, 63546366. doi: 10.1074/jbc.M110.173724

Lipstein, N., Sakaba, T., Cooper, B. H., Lin, K.-H., Strenzke, N., Ashery, U., et al. (2013). Dynamic control of synaptic vesicle replenishment and short-term plasticity by $\mathrm{Ca}(2+)$-calmodulin-Munc13-1 signaling. Neuron 79, 82-96. doi: 10.1016/j.neuron.2013.05.011

Lipstein, N., Schaks, S., Dimova, K., Kalkhof, S., Ihling, C., Kölbel, K., et al. (2012). Nonconserved $\mathrm{Ca}(2+) /$ calmodulin binding sites in Munc13s differentially control synaptic short-term plasticity. Mol. Cell. Biol. 32, 4628-4641. doi: 10. 1128/MCB.00933-12

Liu, H., Bai, H., Hui, E., Yang, L., Evans, C. S., Wang, Z., et al. (2014). Synaptotagmin 7 functions as a $\mathrm{Ca}^{2+}$-sensor for synaptic vesicle replenishment. Elife 3:e01524. doi: 10.7554/elife.01524

Liu, G., and Tsien, R. W. (1995). Properties of synaptic transmission at single hippocampal synaptic boutons. Nature 375, 404-408. doi: 10.1038/375404a0

Ma, C., Li, W., Xu, Y., and Rizo, J. (2011). Munc13 mediates the transition from the closed syntaxin-Munc18 complex to the SNARE complex. Nat. Struct. Mol. Biol. 18, 542-549. doi: 10.1038/nsmb.2047

Magleby, K. L. (1973). The effect of repetitive stimulation on facilitation of transmitter release at the frog neuromuscular junction. J. Physiol. 234, 327-352.

Magleby, K. L. (1979). Facilitation, augmentation and potentiation of transmitter release. Prog. Brain Res. 49, 175-182. doi: 10.1016/s0079-6123(08)64631-2

Magleby, K. L., and Zengel, J. E. (1976a). Augmentation: a process that acts to increase transmitter release at the frog neuromuscular junction. J. Physiol. 257, 449-470.

Magleby, K. L., and Zengel, J. E. (1976b). Long term changes in augmentation, potentiation and depression of transmitter release as a function of repeated synaptic activity at the frog neuromuscular junction. J. Physiol. 257, 471-494.

Maune, J. F., Klee, C. B., and Beckingham, K. (1992). $\mathrm{Ca}^{2+}$ binding and conformational change in two series of point mutations to the individual $\mathrm{Ca}(2+)$-binding sites of calmodulin. J. Biol. Chem. 267, 5286-5295.

Maximov, A., Lao, Y., Li, H., Chen, X., Rizo, J., Sorensen, J. B., et al. (2008). Genetic analysis of synaptotagmin-7 function in synaptic vesicle exocytosis. Proc. Natl. Acad. Sci. U S A 105, 3986-3991. doi: 10.1073/pnas.07123 72105

Mochida, S., Few, A. P., Scheuer, T., and Catterall, W. A. (2008). Regulation of Presynaptic CaV2.1 Channels by $\mathrm{Ca}^{2+}$ sensor proteins mediates short-term synaptic plasticity. Neuron 57, 210-216. doi: 10.1016/j.neuron.2007.11.036

Mochida, S., Westenbroek, R. E., Yokoyama, C. T., Itoh, K., and Catterall, W. A. (2003). Subtype-selective reconstitution of synaptic transmission in sympathetic 
ganglion neurons by expression of exogenous calcium channels. Proc. Natl. Acad. Sci. US A 100, 2813-2818. doi: 10.1073/pnas.262787299

Müller, M., Felmy, F., and Schneggenburger, R. (2008). A limited contribution of $\mathrm{Ca}^{2+}$ current facilitation to paired-pulse facilitation of transmitter release at the rat calyx of held. J. Physiol. 586, 5503-5520. doi: 10.1113/jphysiol.2008. 155838

Müller, M., Felmy, F., Schwaller, B., and Schneggenburger, R. (2007). Parvalbumin is a mobile presynaptic $\mathrm{Ca}^{2+}$ buffer in the calyx of held that accelerates the decay of $\mathrm{Ca}^{2+}$ and short-term facilitation. J. Neurosci. 27, 2261-2271. doi: 10. 1523/jneurosci.5582-06.2007

Newton, A. C. (2010). Protein kinase C: poised to signal. Am. J. Physiol. Endocrinol. Metab. 298, E395-E402. doi: 10.1152/ajpendo.00477.2009

Nishiki, T., and Augustine, G. J. (2004). Dual roles of the C2B domain of synaptotagmin I in synchronizing $\mathrm{Ca}^{2+}$-dependent neurotransmitter release. J. Neurosci. 24, 8542-8550. doi: 10.1523/jneurosci.2545-04.2004

O'Callaghan, D. W., Ivings, L., Weiss, J. L., Ashby, M. C., Tepikin, A. V., and Burgoyne, R. D. (2002). Differential use of Myristoyl groups on neuronal calcium sensor proteins as a determinant of spatio-temporal aspects of $\mathrm{Ca}^{2+}$ signal transduction. J. Biol. Chem. 277, 14227-14237. doi: 10.1074/jbc.m1117 50200

Olwin, B. B., and Storm, D. R. (1985). Calcium binding to complexes of calmodulin and calmodulin binding proteins. Biochemistry 24, 8081-8086. doi: 10. 1021/bi00348a037

Pang, Z. P., Bacaj, T., Yang, X., Zhou, P., Xu, W., and Südhof, T. C. (2011). Doc2 supports spontaneous synaptic transmission by a $\mathrm{Ca}(2+)$-independent mechanism. Neuron 70, 244-251. doi: 10.1016/j.neuron.2011.03.011

Pang, Z. P., and Südhof, T. C. (2010). Cell biology of $\mathrm{Ca}^{2+}$-triggered exocytosis. Curr. Opin. Cell Biol. 22, 496-505. doi: 10.1016/j.ceb.2010.05.001

Pang, Z. P., Xu, W., Cao, P., and Südhof, T. C. (2010). Calmodulin suppresses Synaptotagmin-2 transcription in cortical neurons. J. Biol. Chem. 285, 3393033939. doi: 10.1074/jbc.M110.150151

Pappa, H., Murray-Rust, J., Dekker, L. V., Parker, P. J., and McDonald, N. Q. (1998). Crystal structure of the C2 domain from protein kinase C-delta. Structure 6, 885-894. doi: 10.1016/s0969-2126(98)00090-2

Pfister, J.-P., Dayan, P., and Lengyel, M. (2010). Synapses with short-term plasticity are optimal estimators of presynaptic membrane potentials. Nat. Neurosci. 13, 1271-1275. doi: 10.1038/nn.2640

Rebecchi, M. J., and Pentyala, S. N. (2000). Structure, function and control of phosphoinositide-specific Phospholipase C. Physiol. Rev. 80, 1291-1335.

Regehr, W. G. (2012). Short-term presynaptic plasticity. Cold Spring Harb. Perspect. Biol. 4:a005702. doi: 10.1101/cshperspect.a005702

Regehr, W. G., Delaney, K. R., and Tank, D. W. (1994). The role of presynaptic calcium in short-term enhancement at the hippocampal mossy fiber synapse. J. Neurosci. 14, 523-537.

Rizo, J., and Südhof, T. C. (1998). C2-domains, structure and function of a universal $\mathrm{Ca}^{2+}$-binding domain. J. Biol. Chem. 273, 15879-15882. doi: 10. 1074/jbc.273.26.15879

Rohrbough, J., and Broadie, K. (2005). Lipid regulation of the synaptic vesicle cycle. Nat. Rev. Neurosci. 6, 139-150. doi: 10.1038/nrn1608

Rosenmund, C., Sigler, A., Augustin, I., Reim, K., Brose, N., and Rhee, J.S. (2002). Differential control of vesicle priming and short-term plasticity by Munc13 isoforms. Neuron 33, 411-424. doi: 10.1016/s0896-6273(02) 00568-8

Sakaba, T., and Neher, E. (2001). Calmodulin mediates rapid recruitment of fast-releasing synaptic vesicles at a calyx-type synapse. Neuron 32, 1119-1131. doi: 10.1016/s0896-6273(01)00543-8

Saraswati, S., Adolfsen, B., and Littleton, J. T. (2007). Characterization of the role of the Synaptotagmin family as calcium sensors in facilitation and asynchronous neurotransmitter release. Proc. Natl. Acad. Sci. U S A 104, 14122-14127. doi: 10. 1073/pnas.0706711104

Schaub, M. C., and Heizmann, C. W. (2008). Calcium, troponin, calmodulin, S100 proteins: from myocardial basics to new therapeutic strategies. Biochem. Biophys. Res. Commun. 369, 247-264. doi: 10.1016/j.bbrc.2007. 10.082

Schneggenburger, R., Sakaba, T., and Neher, E. (2002). Vesicle pools and short-term synaptic depression: lessons from a large synapse. Trends Neurosci. 25, 206-212. doi: 10.1016/s0166-2236(02)02139-2

Schwaller, B. (2009). The continuing disappearance of "pure" $\mathrm{Ca}^{2+}$ buffers. Cell. Mol. Life Sci. 66, 275-300. doi: 10.1007/s00018-008-8564-6
Scullin, C. S., and Partridge, L. D. (2010). Contributions of SERCA pump and ryanodine-sensitive stores to presynaptic residual $\mathrm{Ca}^{2+}$. Cell Calcium 47 , 326-338. doi: 10.1016/j.ceca.2010.01.004

Shao, X., Davletov, B. A., Sutton, R. B., Südhof, T. C., and Rizo, J. (1996). Bipartite $\mathrm{Ca}^{2+}$-binding motif in $\mathrm{C} 2$ domains of synaptotagmin and protein kinase $\mathrm{C}$. Science 273, 248-251. doi: 10.1126/science.273.5272.248

Shin, O.-H., Lu, J., Rhee, J.-S., Tomchick, D. R., Pang, Z. P., Wojcik, S. M., et al. (2010). Munc13 C2B domain is an activity-dependent $\mathrm{Ca}^{2+}$ regulator of synaptic exocytosis. Nat. Struct. Mol. Biol. 17, 280-288. doi: 10.1038/nsmb.1758

Shin, O.-H., Xu, J., Rizo, J., and Sudhof, T. C. (2009). Differential but convergent functions of $\mathrm{Ca}^{2+}$ binding to synaptotagmin-1 C2 domains mediate neurotransmitter release. Proc. Natl. Acad. Sci. U S A 106, 16469-16474. doi: 10. 1073/pnas.0908798106

Sippy, T., Cruz-Martín, A., Jeromin, A., and Schweizer, F. E. (2003). Acute changes in short-term plasticity at synapses with elevated levels of neuronal calcium sensor-1. Nat. Neurosci. 6, 1031-1038. doi: 10.1038/nn1117

Skelton, N. J., Kördel, J., Akke, M., Forsén, S., and Chazin, W. J. (1994). Signal transduction versus buffering activity in $\mathrm{Ca}(2+)$-binding proteins. Nat. Struct. Biol. 1, 239-245. doi: 10.1038/nsb0494-239

Stevens, C. F., and Wesseling, J. F. (1998). Activity-dependent modulation of the rate at which synaptic vesicles become available to undergo exocytosis. Neuron 21, 415-424. doi: 10.1016/s0896-6273(00)80550-4

Stevens, C. F., and Wesseling, J. F. (1999). Augmentation is a potentiation of the Exocytotic process. Neuron 22, 139-146. doi: 10.1016/s0896-6273(00) 80685-6

Südhof, T. C. (2013). Neurotransmitter release: the last millisecond in the life of a synaptic vesicle. Neuron 80, 675-690. doi: 10.1016/j.neuron.2013.10.022

Sugita, S., Shin, O.-H., Han, W., Lao, Y., and Südhof, T. C. (2002). Synaptotagmins form a hierarchy of exocytotic $\mathrm{Ca}^{2+}$ sensors with distinct $\mathrm{Ca}^{2+}$ affinities. $E M B O$ J. 21, 270-280. doi: 10.1093/emboj/21.3.270

Torrecillas, A., Laynez, J., Menéndez, M., Corbalán-García, S., and GómezFernández, J. C. (2004). Calorimetric study of the interaction of the C2 domains of classical protein kinase $\mathrm{C}$ isoenzymes with $\mathrm{Ca}^{2+}$ and phospholipids. Biochemistry 43, 11727-11739. doi: 10.1021/bi0489659

Tsodyks, M., Pawelzik, K., and Markram, H. (1998). Neural networks with dynamic synapses. Neural Comput. 10, 821-835. doi: 10.1162/089976698300017502

Tsujimoto, T., Jeromin, A., Saitoh, N., Roder, J. C., and Takahashi, T. (2002). Neuronal calcium sensor 1 and activity-dependent facilitation of P/Q-type calcium currents at presynaptic nerve terminals. Science 295, 2276-2279. doi: 10. $1126 /$ science. 1068278

Ubach, J., García, J., Nittler, M. P., Südhof, T. C., and Rizo, J. (1999). Structure of the Janus-faced C2B domain of rabphilin. Nat. Cell Biol. 1, 106-112. doi: 10. 1038/10076

Ubach, J., Zhang, X., Shao, X., Südhof, T. C., and Rizo, J. (1998). $\mathrm{Ca}^{2+}$ binding to synaptotagmin: how many $\mathrm{Ca}^{2+}$ ions bind to the tip of a C2-domain? EMBO J. 17, 3921-3930. doi: 10.1093/emboj/17.14.3921

van den Bogaart, G., Meyenberg, K., Diederichsen, U., and Jahn, R. (2012). Phosphatidylinositol 4,5-bisphosphate increases $\mathrm{Ca}^{2+}$ affinity of synaptotagmin1 by 40 -fold. J. Biol. Chem. 287, 16447-16453. doi: 10.1074/jbc.M112. 343418

von Gersdorff, H., and Matthews, G. (1997). Depletion and Replenishment of vesicle pools at a ribbon-type synaptic terminal. J. Neurosci. 17, 1919-1927.

Wang, L. Y., and Kaczmarek, L. K. (1998). High-frequency firing helps replenish the readily releasable pool of synaptic vesicles. Nature 394, 384-388. doi: 10. $1038 / 28645$

Wen, H., Linhoff, M. W., McGinley, M. J., Li, G.-L., Corson, G. M., Mandel, G., et al. (2010). Distinct roles for two synaptotagmin isoforms in synchronous and asynchronous transmitter release at zebrafish neuromuscular junction. Proc. Natl. Acad. Sci. U S A 107, 13906-13911. doi: 10.1073/pnas.10085 98107

Wierda, K. D. B., Toonen, R. F. G., de Wit, H., Brussaard, A. B., and Verhage, M. (2007). Interdependence of PKC-dependent and PKC-independent pathways for presynaptic plasticity. Neuron 54, 275-290. doi: 10.1016/j.neuron.2007. 04.001

Wilkinson, R. S., and Lin, M. Y. (2004). Endocytosis and synaptic plasticity: might the tail wag the dog? Trends Neurosci. 27, 171-174. doi: 10.1016/j.tins.2004. 01.011

Xia, Z., and Storm, D. R. (2005). The role of calmodulin as a signal integrator for synaptic plasticity. Nat. Rev. Neurosci. 6, 267-276. doi: 10.1038/nrn1647 
$\mathrm{Xu}$, J., and Wu, L.-G. (2005). The decrease in the presynaptic calcium current is a major cause of short-term depression at a calyx-type synapse. Neuron 46, 633-645. doi: 10.1016/j.neuron.2005.03.024

Xue, L., and Wu, L.-G. (2010). Post-tetanic potentiation is caused by two signalling mechanisms affecting quantal size and quantal content. J. Physiol. 588, 49874994. doi: 10.1113/jphysiol.2010.196964

Yamaguchi, T., Shirataki, H., Kishida, S., Miyazaki, M., Nishikawa, J., Wada, K., et al. (1993). Two functionally different domains of rabphilin-3A, Rab3A p25/smg p25A-binding and phospholipid- and $\mathrm{Ca}(2+)$-binding domains. J. Biol. Chem. 268, 27164-27170.

Zengel, J. E., and Magleby, K. L. (1981). Changes in miniature endplate potential frequency during repetitive nerve stimulation in the presence of $\mathrm{Ca}^{2+}, \mathrm{Ba}^{2+}$ and $\mathrm{Sr}^{2+}$ at the frog neuromuscular junction. J. Gen. Physiol. 77, 503-529. doi: 10. 1085/jgp.77.5.503

Zucker, R. S., and Lara-Estrella, L. O. (1983). Post-tetanic decay of evoked and spontaneous transmitter release and a residual-calcium model of synaptic facilitation at crayfish neuromuscular junctions. J. Gen. Physiol. 81, 355-372. doi: 10.1085/jgp.81.3.355

Zucker, R. S., and Regehr, W. G. (2002). Short-term synaptic plasticity. Annu. Rev. Physiol. 64, 355-405. doi: 10.1146/annurev.physiol.64.092501.114547
Zucker, R. S., and Stockbridge, N. (1983). Presynaptic calcium diffusion and the time courses of transmitter release and synaptic facilitation at the squid giant synapse. J. Neurosci. 3, 1263-1269.

Conflict of Interest Statement: The authors declare that the research was conducted in the absence of any commercial or financial relationships that could be construed as a potential conflict of interest.

Received: 18 September 2014; accepted: 09 October 2014; published online: 29 October 2014.

Citation: de Jong APH and Fioravante D (2014) Translating neuronal activity at the synapse: presynaptic calcium sensors in short-term plasticity. Front. Cell. Neurosci. 8:356. doi: $10.3389 /$ fncel.2014.00356

This article was submitted to the journal Frontiers in Cellular Neuroscience.

Copyright (c) 2014 de Jong and Fioravante. This is an open-access article distributed under the terms of the Creative Commons Attribution License (CC BY). The use, distribution and reproduction in other forums is permitted, provided the original author(s) or licensor are credited and that the original publication in this journal is cited, in accordance with accepted academic practice. No use, distribution or reproduction is permitted which does not comply with these terms. 


\section{Bruchpilot and Synaptotagmin collaborate to drive rapid glutamate release and active zone differentiation}

\section{Mila M. Paul ${ }^{1}$, Martin Pauli ${ }^{1}$, Nadine Ehmann ${ }^{1}$, Stefan Hallermann ${ }^{2}$, Markus Sauer ${ }^{3}$, Robert J. Kittel $^{1}$ * and Manfred Heckmann ${ }^{1}$ *}

${ }^{1}$ Department of Neurophysiology, Institute of Physiology, Julius-Maximilians-University Würzburg, Würzburg, Germany

${ }^{2}$ Carl-Ludwig-Institute for Physiology, University of Leipzig, Leipzig, Germany

${ }^{3}$ Department of Biotechnology and Biophysics, Julius-Maximilians-University Würzburg, Würzburg, Germany

Edited by:

Christian D. Wilms, University College London, UK

\section{Reviewed by:}

Annalisa Scimemi, State University of New York Albany, USA

Dion Dickman, University of Southern California, USA

\section{${ }^{*}$ Correspondence:}

Manfred Heckmann and Robert J.

Kittel, Department of

Neurophysiology, Institute of

Physiology, Julius-Maximilians-

University Würzburg, Röntgenring 9,

97070 Würzburg, Germany

e-mail: heckmann@uni-wuerzburg.de;

robert.kitte@@uni-wuerzburg.de
The active zone (AZ) protein Bruchpilot (Brp) is essential for rapid glutamate release at Drosophila melanogaster neuromuscular junctions (NMJs). Quantal time course and measurements of action potential-waveform suggest that presynaptic fusion mechanisms are altered in brp null mutants $\left(b r p^{69}\right)$. This could account for their increased evoked excitatory postsynaptic current (EPSC) delay and rise time (by about $1 \mathrm{~ms}$ ). To test the mechanism of release protraction at $b_{r p} 69 \mathrm{AZs}$, we performed knock-down of Synaptotagmin-1 (Syt) via RNAi $\left(s y t^{K D}\right.$ ) in wildtype (wt), brp ${ }^{69}$ and rab3 null mutants (rab3 $3^{\text {rup }}$ ), where Brp is concentrated at a small number of AZs. At wt and rab3 ${ }^{\text {rup }}$ synapses, syt ${ }^{K D}$ lowered EPSC amplitude while increasing rise time and delay, consistent with the role of Syt as a release sensor. In contrast, syt ${ }^{K D}$ did not alter EPSC amplitude at brp ${ }^{69}$ synapses, but shortened delay and rise time. In fact, following $s y t^{K D}$, these kinetic properties were strikingly similar in wt and brp 69 , which supports the notion that Syt protracts release at $b r p^{69}$ synapses. To gain insight into this surprising role of Syt at brp ${ }^{69} \mathrm{AZs}$, we analyzed the structural and functional differentiation of synaptic boutons at the NMJ. At 'tonic' type Ib motor neurons, distal boutons contain more AZs, more Brp proteins per AZ and show elevated and accelerated glutamate release compared to proximal boutons. The functional differentiation between proximal and distal boutons is Brp-dependent and reduced after $s y t^{K D}$. Notably, syt ${ }^{K D}$ boutons are smaller, contain fewer Brp positive AZs and these are of similar number in proximal and distal boutons. In addition, super-resolution imaging via $d S T O R M$ revealed that $s y t^{K D}$ increases the number and alters the spatial distribution of Brp molecules at AZs, while the gradient of Brp proteins per AZ is diminished. In summary, these data demonstrate that normal structural and functional differentiation of Drosophila AZs requires concerted action of Brp and Syt.

Keywords: Bruchpilot, active zone, neurotransmitter release, synaptic delay, presynaptic differentiation, synaptotagmin, $d$ STORM

\section{INTRODUCTION}

Active zones (AZs) allow exquisite spatial and temporal control of vesicle fusion. Large multidomain proteins rich in coiled-coil sequences such as Bassoon, Piccolo and the CAST/ERC family member Brp are major structural and functional organizers of AZs (Südhof, 2012). Their abundance appears to correlate positively with neurotransmitter release (Graf et al., 2009; Matz etal., 2010; Weyhersmüller etal., 2011; Ehmann etal., 2014; Peled et al., 2014).

At Drosophila melanogaster NMJs, Brp is crucial for synchronous glutamate release and the clustering of calcium channels at AZs (Kittel etal., 2006; Wagh etal., 2006). Linking the amount of Brp or Bassoon per AZ to the number and spatial arrangement of calcium channels may account for the correlation with release probability, e.g., in the context of synaptic homeostasis (Matz et al., 2010; Weyhersmüller et al., 2011; Ehmann etal., 2014). Slight increases in coupling distance in the $20-40 \mathrm{~nm}$ range reduce release probability dramatically while changing kinetic release parameters to a lesser extent (Neher, 1998; Eggermann etal., 2011; Schmidt etal., 2013; Vyleta and Jonas, 2014). Differences in coupling distance are therefore ideal for scaling the amount of release, whereas controlling its time course appears to require additional molecular mechanisms.

The main kinetic transmitter release parameters are synaptic delay, rise, and decay times. In the present study, we focus on synaptic delay and EPSC rise time. Notably, the latter is increased by more than $1 \mathrm{~ms}$ at synapses lacking Brp, while release probability drops by comparison only moderately (Kittel et al., 2006; Eggermann et al., 2011). This marked kinetic change appears disproportional to the reduction in release probability. While synaptic delay has not yet been analyzed at $b r p^{69}$ synapses, it is usually fairly constant for a wide range of release probabilities (Barrett and Stevens, 1972; Datyner and Gage, 1980).

While the molecular mechanisms controlling release kinetics are complex and not well understood (Neher, 2010), it is 
clear that the vesicle protein Syt plays an important role (Brose et al., 2002; Young and Neher, 2009). As initially suggested more than 20 years ago (DiAntonio et al., 1993; Littleton et al., 1993; Geppert etal., 1994), Syt is crucial for triggering release and may act both as a calcium sensor and a vesicle fusion clamp. In fact, its role may change from clamp to sensor upon calcium influx into the presynaptic terminal (DeBello et al., 1993; Walter et al., 2011).

To clarify the molecular mechanisms that shape the time course of release we analyzed the interaction between Brp and Syt. We find that in addition to prolonged EPSC rise time, synaptic delay is strongly increased at $b r p^{69}$ synapses. Interestingly, whereas Syt is necessary for the increase in both kinetic parameters, it has little effect on the amount of transmitter released from $b r p^{69}$ AZs. Following up on the functional interaction of Brp and Syt, our data suggest central roles of these two proteins in the spatial differentiation of AZs and reveal that the number of AZs per bouton, as well as the number and distribution of Brp molecules per AZ is Syt-dependent.

\section{MATERIALS AND METHODS FLY STOCKS}

Drosophila larvae were raised in vials on standard corn meal (Ashburner, 1989) at $25^{\circ} \mathrm{C}$ for focal recordings in Figure $\mathbf{1}$ (wt and $b r p^{69}$ ) or at $29^{\circ} \mathrm{C}$ for reliable RNAi expression in all other experiments (mutant and control groups). For Syt RNAi, we expressed syt1_RNAi ${ }^{8875}$ Vienna Drosophila Resource Center (VDRC) specifically in motor neurons (ok6-GAL4/+; UAS-syt1_RNAi $i^{8875} /+$ ) or panneuronally (elav-GAL4/UAS-syt1_RNAi ${ }^{8875}$ ) using the binary UAS-GAL4 expression system (Brand and Perrimon, 1993). $b r p^{69}$ and rab3 ${ }^{r u p}$ were used as previously described (Kittel etal., 2006; Graf etal., 2009). To combine $b r p^{69}$ and rab3 $3^{\text {rup }}$ with syt1_RNAi $i^{8875}$ the following lines were generated: brp ${ }^{69}$ ok6-GAL4/ $d f(2 R) B S C 29 ; \quad U A S$-syt1_RNAi $i^{8875} /+$ and $b r p^{69} / d f(2 R) B S C 29 ;$ elav-GAL4/UAS-syt1_RNAi ${ }^{8875}$ (brp ${ }^{69}$, syt $\left.{ }^{K D}\right) ; r a b 3^{\text {rup }} / d f(2 R) E D 2076$ ok6-GAL4; UAS-syt1_RNAi ${ }^{8875} /+$ and rab3 ${ }^{\text {rup }} / d f(2 R) E D 2076$; elav-GAL4/UAS-syt1_RNAi ${ }^{8875}$ (rab3 $^{\text {rup }}$, syt $\left.{ }^{K D}\right)$; ok6-GAL4/+ and elav-GAL4/+ served as wt controls. For focal recordings, GFP was expressed in presynaptic terminals: ok6-GAL4/+; UAS-CD8::GFP/+ (wt), brp ${ }^{69}$ ok6GAL4/df(2R)BSC29;UAS-CD8::GFP/+ (brp $\left.{ }^{69}\right)$ and ok6-GAL4/+; UAS-CD8::GFP/UAS-Syt1_RNAi $i^{8875}\left(s y t^{K D}\right)$. For non-allelic noncomplementation the following genotypes were used: $w^{1118}(\mathrm{wt})$, $s y t 1^{A D 4} /+$ (DiAntonio et al., 1993), brp ${ }^{69} /+$ (Kittel et al., 2006) and $s y t 1^{A D 4} / b r p^{69}\left(s y t^{A D 4}+/+b r p^{69}\right)$.

\section{PHYSIOLOGICAL SOLUTION AND PREPARATION}

The composition of the extracellular, physiological, hemolymphlike saline (HL-3, Stewart et al., 1994) was (in mM): $\mathrm{NaCl} 70, \mathrm{KCl}$, $\mathrm{MgCl}_{2}$ 20, $\mathrm{NaHCO}_{3}$ 10, trehalose 5, sucrose 115, HEPES 5, $\mathrm{CaCl}_{2}$ as indicated, $\mathrm{pH}$ adjusted to 7.2. Wandering male third instar larvae were dissected in $\mathrm{HL}-3$ without $\mathrm{CaCl}_{2}$. All experiments were carried out at NMJs formed on ventral abdominal muscles 6/7 in segments A2 and A3.

\section{FOCAL RECORDINGS}

Macropatch recordings in Figure 1 were performed in HL-3 saline containing $1 \mathrm{mM}\left[\mathrm{Ca}^{2+}\right]_{\mathrm{Ex}}$ essentially as reported previously (Pawlu etal., 2004). Bath temperature was kept constant at $18 \pm 0.5^{\circ} \mathrm{C}$ using a Peltier element (27 W, Conrad Electronic) glued to the bath inflow with heat-conductive paste (Fischer Elektronik). EPSCs were elicited using a $0.2 \mathrm{~Hz}$ nerve-stimulation protocol with $0.2 \mathrm{~ms}$ pulse duration and amplitudes slightly above the threshold for eliciting an action potential via a suction electrode (filled with extracellular solution). Recording electrodes with openings of about 5-10 $\mu \mathrm{m}$ diameter below the tip had resistances of $250 \mathrm{k} \Omega$ when filled with HL-3. About 20 EPSCs were recorded per site and analyzed with IgorPro 5.04 (Wavemetrics). The data were digitally filtered at $3 \mathrm{kHz}$ (Gaussian filter), baseline subtracted and the average of all failures was
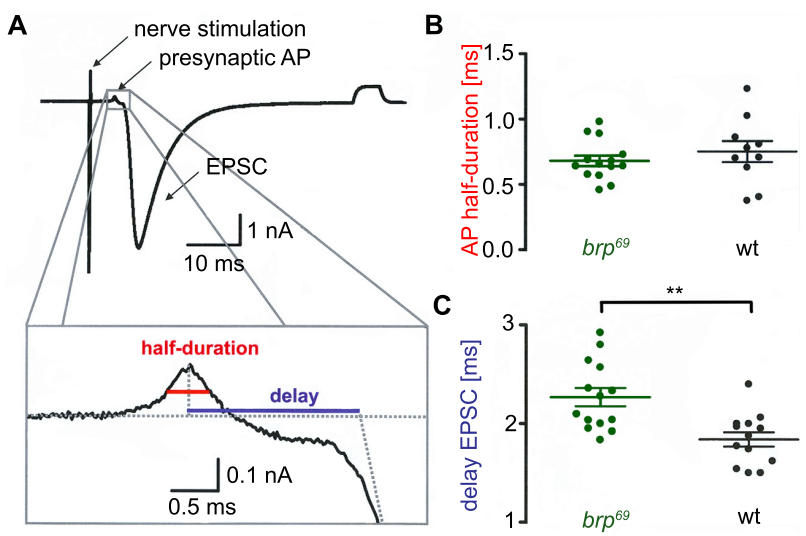

FIGURE 1 | Presynaptic action potential and synaptic delay in $b_{r p}^{69}$. (A) Focal recording from a Drosophila wt NMJ. Nerve stimulation (left arrow) elicited an action potential in the suction electrode which traveled to the presynaptic bouton under the focal electrode and lead to deflections (presynaptic AP, second arrow) prior to the large compound EPSC (third arrow). A subthreshold pulse through the focal electrode elicited the final upward deflection (right side of the trace and allowed to measure electrode and seal resistance). The lower panel shows the enlarged presynaptic AP and illustrates how half-duration of the positive AP deflection (red) and synaptic delay (blue) were determined. (B) While AP half-duration is unchanged between $b_{r p}{ }^{69}$ (green) and wt (black), (C) synaptic delay is prolonged in brp 69 . Shown are single values (dots) and mean \pm SD. 
subtracted from the currents. AP durations were measured at half amplitude of the positive deflection (Dudel, 1965) and synaptic delay was measured from the peak of the AP to the point at which the back extrapolation of the EPSC current rising phase crossed the baseline (Figure 1). Focal recordings in Figure 6 were performed in HL-3 saline containing $0.5 \mathrm{mM}\left[\mathrm{Ca}^{2+}\right]_{\mathrm{Ex}}$. Bath temperature was kept constant at $20 \pm 1^{\circ} \mathrm{C}$. Focal electrodes (resistances $600 \pm 50 \mathrm{k} \Omega$ when filled with HL-3 solution) were positioned on proximal or distal type Ib boutons of muscles 6/7. EPSCs were elicited using a $0.2 \mathrm{~Hz}$ nerve-stimulation protocol with $0.2 \mathrm{~ms}$ pulse duration and $7 \mathrm{~V}$ amplitude. Traces were low-pass filtered at $20 \mathrm{kHz}$, and recorded and stored with Patchmaster using an EPC10 double patch clamp amplifier (HEKA electronics). About 60 EPSCs were averaged per site and analyzed with Igor Pro 6.05 (Wavemetrics). 10 mg EGTA-AM (membrane permeable tetraacetoxymethyl ester of ehtyleneglycol-bis(Baminoethyl)-N,N,N',N'-tetraacetic acid, Calbiochem Germany) was dissolved in DMSO with 20\% Pluronic (Invitrogen) to obtain a stock solution of $10 \mathrm{mM}$ EGTA. This stock solution was diluted 1:100 with calcium-free HL-3 and applied to the dissected preparation for $10 \mathrm{~min}$. After incubation preparations were washed for 5 minutes with HL-3 (Müller et al., 2012) and recordings were performed in HL-3 containing $1.0 \mathrm{mM}\left[\mathrm{Ca}^{2+}\right]_{\mathrm{Ex}}$ as described above.

\section{TWO-ELECTRODE VOLTAGE CLAMP RECORDINGS (TEVC)}

Two-electrode voltage clamp-recordings (Figure 3) were performed essentially as previously described (Kittel et al., 2006) using an Axo Clamp 2B amplifier (Axon Instruments, Molecular Devices). All measurements were made from muscle 6 at $21 \pm 1^{\circ} \mathrm{C}$ bath temperature. Intracellular electrodes were filled with $3 \mathrm{M} \mathrm{KCl}$ and had resistances of $12-15 \mathrm{M} \Omega$. $\mathrm{V}_{\text {holding }}$ was $-60 \mathrm{mV}$ for evoked EPSCs. Only cells with an initial membrane potential of at least $-50 \mathrm{mV}$ and $\geq 4 \mathrm{M} \Omega$ input resistance were analyzed. Synaptic responses were generated by pulses of $0.3 \mathrm{~ms}$ length and $5-10 \mathrm{~V}$ amplitude, applied via a suction electrode (filled with extracellular solution) and low-pass filtered at $10 \mathrm{kHz}$. We applied a $0.2 \mathrm{~Hz}$ stimulation protocol, averaged 20 EPSCs per muscle cell and analyzed the data with Clampfit (Axon Instruments, Molecular Devices).

\section{IMAGING}

Larvae were dissected in ice-cold HL-3 standard saline without $\mathrm{CaCl}_{2}$, fixed with $4 \%$ paraformaldehyde in $0.1 \mathrm{M}$ phosphate buffered saline (PBS) for $10 \mathrm{~min}$ and blocked with PBT (PBS containing $0.05 \%$ Triton X-100, Sigma) including 5\% natural goat serum (Dianova) for $30 \mathrm{~min}$. Primary antibodies were added for overnight staining at $4^{\circ} \mathrm{C}$. After three washing steps with PBS (20 min each), preparations were incubated with secondary antibodies for $2-4 \mathrm{~h}$ at room temperature followed by three washing steps with PBS. Filets were mounted using Vectashield (Vector Laboratories) and images were aquired using an Apotome System (Zeiss, Axiovert 200M Zeiss, objective $63 \mathrm{x}$, NA 1.4, oil). Antibodies were used at the following concentrations: mouse monoclonal antibody $(\mathrm{mAb}) \mathrm{Brp}^{\mathrm{Nc} 82}$ (1:250), Alexa Fluor 488-conjugated goat $\alpha$-mouse (Invitrogen) and Cy3-conjugated goat $\alpha$-horseradish peroxidase (HRP,
Jackson Immuno Research) antibodies (1:250), rabbit $\alpha$-DsytCL1 (Mackler etal., 2002) and Cy3-conjugated goat $\alpha$-rabbit (Jackson Immuno Research, 1:250) antibodies. Z-stacks of 1520 single images taken every $250 \mathrm{~nm}$ were maximum projected and analyzed in Image $(1.440, \mathrm{NIH})$. Brp puncta per NMJ and per bouton were quantified manually. Using the three terminal boutons of type $\mathrm{Ib}$ and Is branches the respective structural gradient was analyzed. Distal boutons were located at the end of bouton chains, while proximal boutons were closer to the entry site of the motor neuron. Bouton area, length (along chain axis) and width ( $90^{\circ}$ to length) were measured using $\alpha$-HRP stainings.

\section{dSTORM (DIRECT STOCHASTIC OPTICAL RECONSTRUCTION MICROSCOPY)}

Larvae were dissected, fixed and washed as described above and super-resolution imaging was performed essentially as previously reported (Ehmann et al., 2014). Preparations were incubated with $\mathrm{mAb} \operatorname{Brp}^{N c 82}$ (1:2000) and secondary antibody goat $\alpha$-mouse $\mathrm{F}(\mathrm{ab})_{2}$ fragments (A10534, Invitrogen) labeled with Cy5-NHS (PA15101, GE Healthcare) at a concentration of $5.2 \times 10^{-8} \mathrm{M}$ or with rabbit $\alpha$-Dsyt-CL1 (1:10000, Mackler et al., 2002) and secondary antibody goat $\alpha$-rabbit $\mathrm{F}(\mathrm{ab})_{2}$ fragments labeled with Alexa Fluor 647 (1:500, Invitrogen). Boutons were visualized with Alexa Fluor 488 conjugated goat $\alpha$-horseradish-peroxidase antibody ( $\alpha$-HRP, 1:250, Jackson Immuno Research). After staining, larval preparations were incubated in $100 \mathrm{mM}$ Mercaptoethylamin (MEA, Sigma-Aldrich) buffer in PBS, pH 7.8-7.9 to allow reversible switching of single fluorophores during data acquisiton (van de Linde etal., 2008). Images were acquired using an inverted microscope (Olympus IX-71, 60x, NA 1.45, oil immersion) equiped with a nosepiece-stage (IX2-NPS, Olympus). $644 \mathrm{~nm}$ (iBEAM-SMART-640-S, Toptica), and $488 \mathrm{~nm}$ (iBEAM-SMART-488-S, Toptica) lasers were used for excitation of Cy5 and Alexa Fluor488, respectively. Laser beams were passed through a clean-up filter (Brightline HC 642/10, Semrock, and ZET 488/10, Chroma, respectively) and two dichroic mirrors (Laser-MUX BS 514-543 and HC-quadband BP, Semrock) onto the probe. The emmitted fluorescence was filtered with a quadband-filter (HC-quadband 446/523/600/677, Semrock) and divided onto two cameras (iXon Ultra DU-897-U, Andor) using a dichroic mirror (HC-BS 640 imaging, Semrock). In addition, fluorescence was filtered using a longpass(Edge Basic 635, Semrock) or bandpass-filter (Brightline HC 525/50, Semrock) for red and green channels, respectively. Pixel sizes were $126 \mathrm{~nm}$ (red) and $128 \mathrm{~nm}$ (green). Single fluorophores were localized and high resolution-images were reconstructed with rapidSTORM (Heilemann et al., 2008; Wolter etal., 2010; van de Linde etal., 2011; Wolter et al., 2012; www.super-resolution.de). Only fluorescence spots with more than 1000 photons were analyzed (10 nm /pixel sub-pixel binning). Data were processed with ImageJ (1.440, NIH), AZ area and Brp localizations per AZ were analyzed as previously described Ehmann etal. (2014). AZs were assigned to single boutons using the $\alpha$-HRP signal. For Syt1 quantification (Figure 2) we determined localization counts in single type Ib boutons that were defined according to the $\alpha$-HRP signal. 


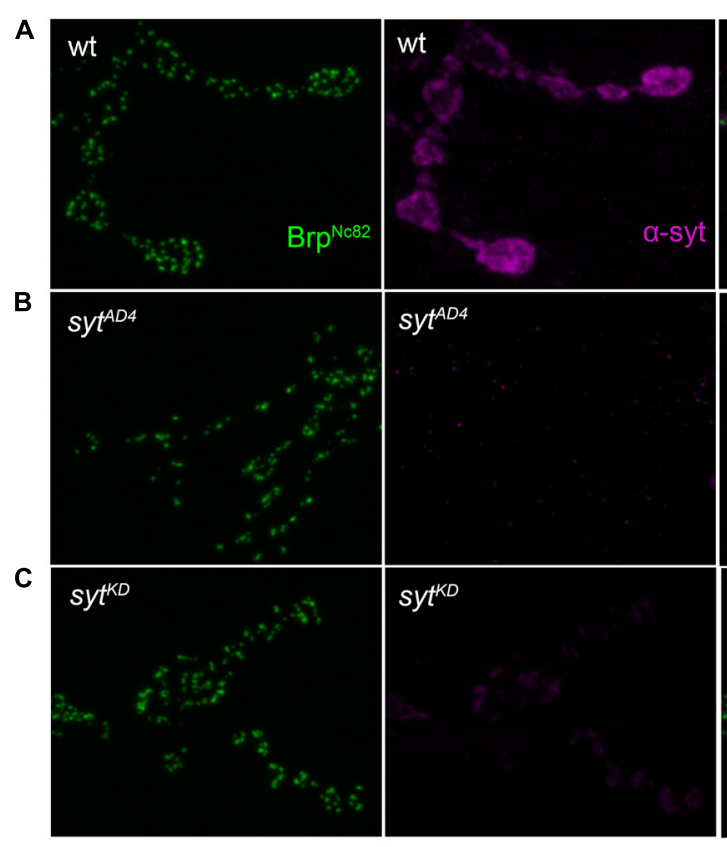

D
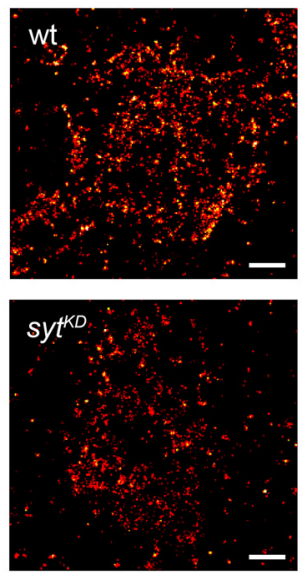

E

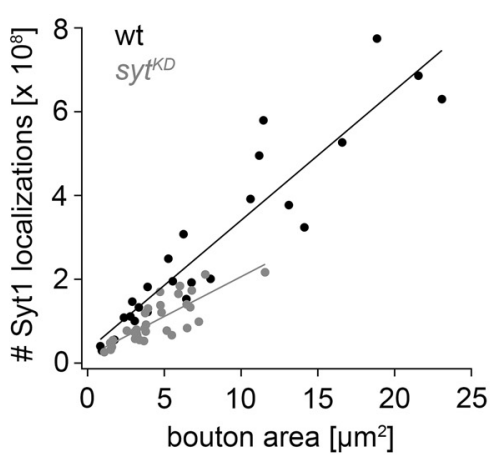

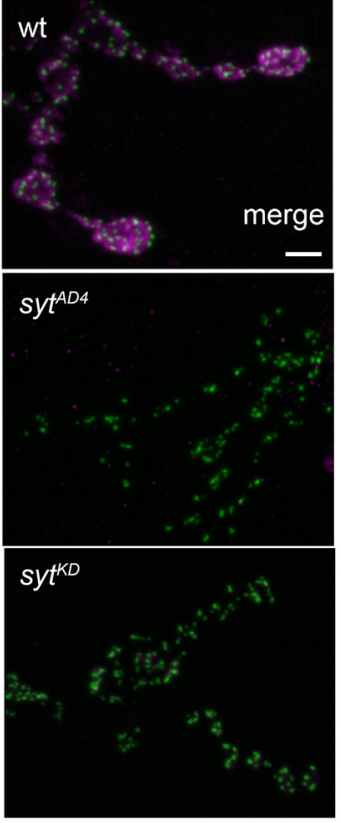

$\mathbf{F}$

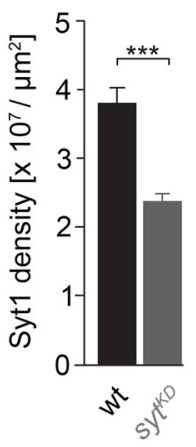

FIGURE 2 | Ribonucleic acid interference decreases presynaptic Syt protein levels. (A) Drosophila wt NMJ on larval abdominal muscles 6/7 stained with antibodies against $\operatorname{Brp}\left(\operatorname{Brp}^{N c 82}\right.$, green) and Synaptotagmin ( $\alpha$-Syt, magenta). Brp demarks individual AZs, whereas Syt labels synaptic vesicles predominately distributed around the bouton circumference. (B) The $\alpha$-Syt signal is absent in Syt null mutans $\left(\right.$ syt $\left.^{A D 4}\right)$ and $(\mathbf{C})$ strongly reduced after Syt knock-down (syt ${ }^{K D}$ ) through presynaptically (ok6-GAL4) driven RNA interference (UAS-syt1-RNA;8875). (D) dSTORM images of single presynaptic boutons stained against Syt1. (E) Number of single-fluorophore localization events by $d$ STORM. Syt1 localizations plotted against bouton area for wt (black) and syt ${ }^{K D}$ (gray) with respective regression lines. Dots represent values for single boutons. (F) Summary bar graph indicates the decrease of Syt1 density in boutons of similar size (below $10 \mu \mathrm{m}^{2}$ ) to $62.38 \%$ in syt ${ }^{K D}$ compared to wt $(p<0.001)$. Scale bar $=2 \mu \mathrm{m}(\mathbf{A}-\mathbf{C})$ and $300 \mathrm{~nm}$ (D).
Localization densities were analyzed only in boutons with areas $<10 \mu \mathrm{m}$.

\section{STATISTICAL ANALYSIS}

Statistical analyses were performed with Sigma Plot 12 (Systat Software) using the non-parametric Mann-Whitney rank sum test. Linear fits for mean \pm SEM Brp localizations per AZ (Figure 9) were made in Igor Pro (Wavemetrics) and statistical analysis was performed using the non-parametric Spearman correlation coefficient. Asterisks indicate the significance level ${ }^{*} p<0.05$, $\left.{ }^{* *} p<0.01,{ }^{* * *} p<0.001\right)$. Data are reported as mean \pm SEM unless indicated otherwise and $n$ denotes sample number.

\section{RESULTS}

\section{SYNAPTIC DELAY IS INCREASED IN $\boldsymbol{b r p}^{69}$}

We performed focal recordings using macropatch electrodes which allow simultaneous measurements of presynaptic action potentials (AP) and synaptic release (Dudel, 1965). EPSCs evoked by $0.2 \mathrm{~Hz}$ nerve stimulation were recorded at $b r p^{69}$ and wt larval NMJs on muscles $6 / 7$ to measure half-duration of the positive AP deflection and synaptic delay (Figure 1A). Whereas AP wave form was unchanged in $b r p^{69}$ compared to wt, synaptic delay was significantly increased $(2.27 \pm 0.3 \mathrm{~ms}$ and $1.8 \pm 0.3 \mathrm{~ms}$ mean $\pm \mathrm{SD}$, $p=0.0014, n=14$, and 13 for $b r p^{69}$ and wt, Figures 1B,C). As quantal time course is normal in $b r p^{69}$ (Kittel etal., 2006), 
the increase in release kinetics at $b r p^{69}$ synapses is likely due to alterations in presynaptic fusion mechanisms.

\section{IMPACT OF SYT ON DIFFERENT AZ STATES}

We used wt, $b r p^{69}$ and $r a b 3^{r u p}$ to define explicit AZ conditions: (i) normal organization, (ii) disorganized lacking Brp (Kittel et al., 2006), and (iii) large accumulation of Brp proteins (Graf et al., 2009; Ehmann et al., 2014), respectively. To determine the impact of the putative calcium sensor Syt on synchronous transmitter release in the context of different AZ states we combined these genotypes with $s y t^{K D}$. Protein levels of endogeneous Syt were decreased via RNAi (syt1-RNAi ${ }^{8875}$, see experimental procedures). By engaging the binary UAS-Gal4 expression system (Brand and Perrimon, 1993), syt1-RNAi was driven in larval glutamatergic motor neurons or panneuronally. To confirm that presynaptic Syt expression was reduced by this strategy, immunostainings of larval NMJs were performed using an antiserum against Syt1 (Mackler et al., 2002; Figure 2). Whereas presynaptic terminals of $s y t^{A D 4}$ were completely devoid of Syt, there was residual though heavily reduced protein expression in $s y t^{K D}$ compared to wt (Figure 2C). Furthermore, we quantified the protein reduction following Syt knock-down with dSTORM (Ehmann et al., 2014). Comparison of Syt1 localization numbers in boutons of similar size in wt and $s y t^{K D}$ revealed a reduction to $62.38 \%$ in $s y t^{K D}$ (Figures 2D-F). To address the functional consequences of $s y t^{K D}$ at wt, $b r p^{69}$, and $r a b 3^{r u p}$ synapses, postsynaptic currents in response to low-frequency nerve stimulation were recorded in two-electrode voltage clamp mode (TEVC) from larval ventral abdominal muscles 6/7 (Figure 3). Both panneuronal (Figure 3) and motoneuronal (data not shown) RNAi expression gave essentially comparable results. At wt synapses, $s y t^{K D}$ decreased EPSC amplitude and lengthened rise time (amplitude: $30.0 \pm 4.5 \mathrm{nA}$ and $53.6 \pm 4.7 \mathrm{nA}, p=0.002$; rt: $1.3 \pm 0.1 \mathrm{~ms}$ and $1.0 \pm 0.03 \mathrm{~ms}$, $p<0.001 ; n=11$ and 17 for $s y t^{K D}$ and wt, Figure 3B) consistent with the role of Syt as a sensor for fast release (DiAntonio et al., 1993; Littleton et al., 1993). Similarly, at rab3 ${ }^{\text {rup }}$ synapses, syt ${ }^{K D}$ reduced the amplitude and increased the delay of postsynaptic responses (amplitude: $19.5 \pm 2.1 \mathrm{nA}$ and $50.3 \pm 5.3 \mathrm{nA}, p<0.001$; delay: $1.7 \pm 0.1 \mathrm{~ms}$ and $1.4 \pm 0.04 \mathrm{~ms}, p=0.013 ; n=14$ and 10 for $r a b 3^{r u p}, s y t^{K D}$, and $\left.r a b 3^{\text {rup }}\right)$. Strikingly, syt $t^{K D}$ at $b r p^{69}$ synapses left current amplitudes unchanged $(35.1 \pm 6.7 \mathrm{nA}$ and $24.8 \pm 3.3 \mathrm{nA}$, $p>0.05)$ and in fact accelerated EPSC rise time and delay (rt: $1.4 \pm 0.1 \mathrm{~ms}$ and $2.2 \pm 0.2 \mathrm{~ms}, p=0.008$; delay: $1.6 \pm 0.03 \mathrm{~ms}$ and $1.9 \pm 0.1 \mathrm{~ms}, p<0.001 ; n=12$ and 11 for $b r p^{69}$, syt $t^{K D}$, and $\left.b r p^{69}\right)$. These results illustrate that Syt is necessary for efficient and rapid vesicle fusion at AZs with normal or increased Brp levels. In contrast, vesicle release from $b r p^{69}$ AZs appears less dependent on Syt. We did not find any changes in size or kinetics of quantal events in $b r p^{69}$ and $b r p^{69}$, syt $t^{K D}$ that could explain these effects (amplitude: $0.89 \pm 0.04 \mathrm{nA}$ and $0.90 \pm 0.04 \mathrm{nA}$; $\mathrm{rt}: 1.0 \pm 0.06 \mathrm{~ms}$ and $1.0 \pm 0.04 \mathrm{~ms}$; tau: $6.02 \pm 0.6 \mathrm{~ms}$ and $7.2 \pm 0.4 \mathrm{~ms}$; all $p>0.05 ; n=10$ and 14 for $b r p^{69}$ and $b r p^{69}$, syt $t^{K D}$, respectively). Thus, the changes in release kinetics following $s y t^{K D}$ suggest that Syt protracts release at $b r p^{69}$ AZs.

\section{REDUCED EGTA SENSITIVITY IN $b r p^{69}$, syt $^{K D}$}

To further clarify how $s y t^{K D}$ affects release we tested the influence of EGTA-AM in $s y t^{K D}$ and $b r p^{69}, s y t^{K D}$ in focal recordings

\section{A}
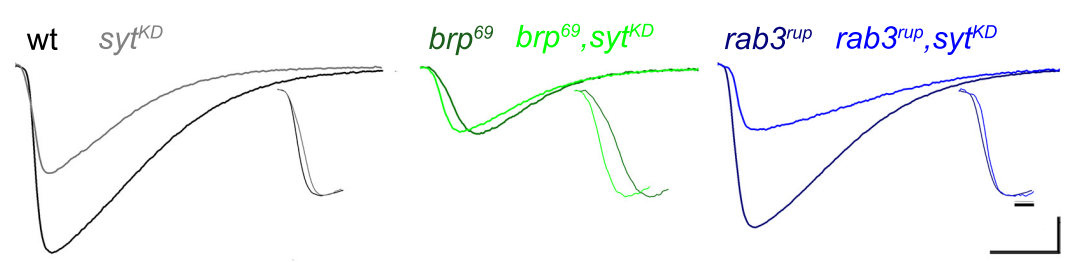

B
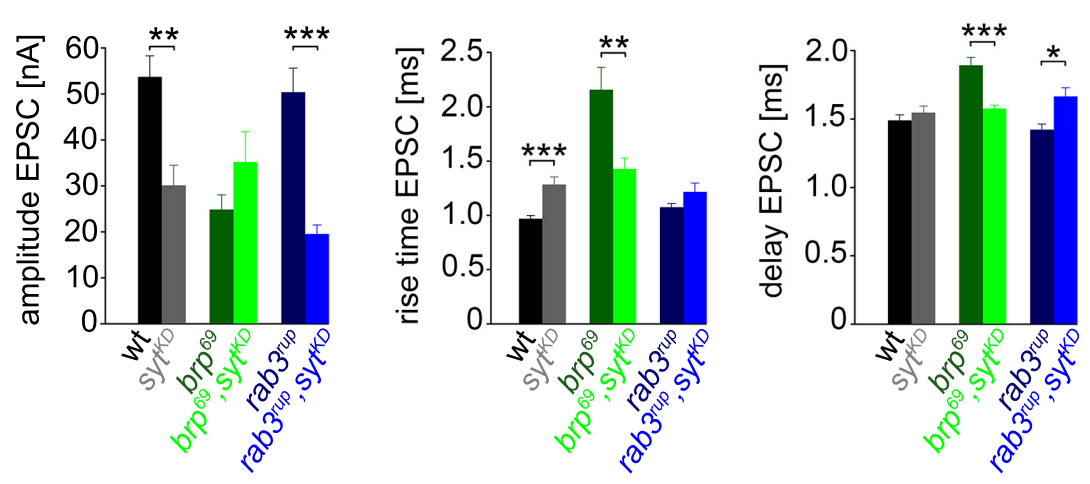

FIGURE 3 | Effect of $s y t^{K D}$ on amplitude and kinetics of the postsynaptic response. (A) Two-electrode voltage clamp recordings in physiological solution with $1.0 \mathrm{mM}\left[\mathrm{Ca}^{2+}\right]_{\text {Ex }}$ and $0.2 \mathrm{~Hz}$ nerve stimulation. Traces from wt (black), brp 69 (dark green), and rab3 $3^{\text {rup }}$ (dark blue) are shown superimposed with recordings of the respective genotypes after syt ${ }^{K D}$ (brighter shades). Insets show normalized events to highlight the differences in synaptic delay and postsynaptic current rise time. Scale bars $=5 \mathrm{~ms}, 10 \mathrm{nA}$ (main traces), and $1 \mathrm{~ms}$ (insets). (B) Summary bar graphs for mean \pm SEM amplitude, current rise time and synaptic delay for all six genotypes, color coded as in (A). 
(Figure 4, Kittel et al., 2006). Consistent with earlier work (Maximov and Südhof, 2005) release in $s y t^{K D}$ was significantly reduced $(0.37 \pm 0.03 \mathrm{nA}$ and $0.22 \pm 0.03 \mathrm{nA}, p=0.002, n=16$ each), whereas the reduction was not significant in $b r p^{69}, s y t^{K D}$ $(0.28 \pm 0.05 \mathrm{nA}$ and $0.21 \pm 0.03 \mathrm{nA}, p>0.05, n=17$, and 10 without and with EGTA). This is in contrast to the findings described in Kittel et al. (2006) for $b r p^{69}$ and suggests that Syt knock-down reduces coupling distance in $b r p^{69}$ and that Syt's role in positional priming (Young and Neher, 2009) requires Brp.

\section{BRP IS DISTRIBUTED UNEVENLY AT WT AND rab3 ${ }^{\text {rup }}$ NMJs}

The innervation of ventral abdominal muscles $6 / 7$ is shared between two functionally distinct motoneurons in Drosophila larvae (Karunanithi et al., 2002): the MN6/7b-Ib (RP3) neuron gives rise to large type Ib boutons (Atwood et al., 1993), whereas the MNSNb/d-Is neuron forms smaller type Is boutons (Kurdyak et al., 1994; Lnenicka and Keshishian, 2000; Hoang and Chiba, 2001). We performed immunostainings and counted the number of Brp puncta per bouton as an estimate of the number of AZs. In addition, staining against horseradish-peroxidase (HRP) was used to measure dimensions of presynaptic arborizations (Jan and Jan, 1982; Figure 5). We found an uneven Brp distribution in wt type Ib motoneurons with more Brp positive AZs in distal than

A

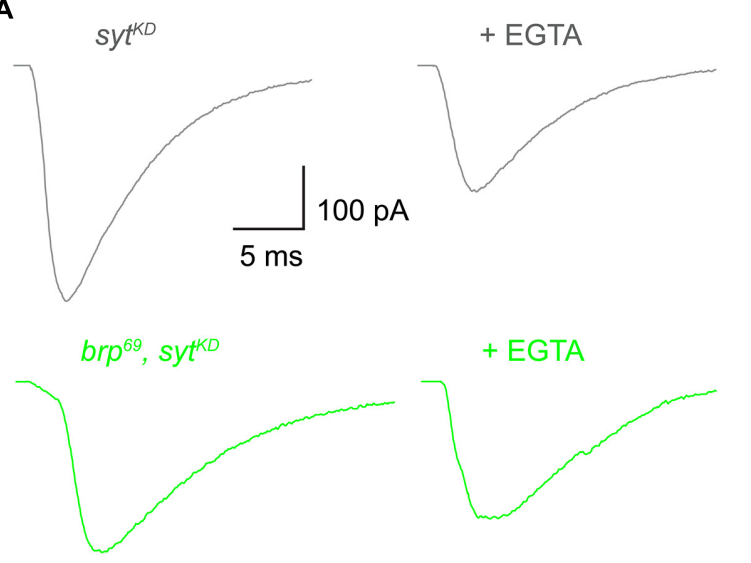

B

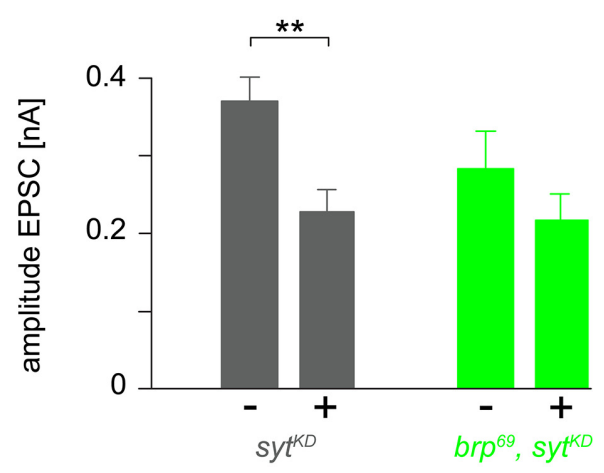

FIGURE 4 | Reduced EGTA sensitivity in brp $^{69}$, syt $^{K D}$. (A) Focally recorded, averaged EPSCs from syt ${ }^{K D}$ (gray) and brp ${ }^{69}$, syt $^{K D}$ (light green) NMJs under control conditions and after incubation with $100 \mu \mathrm{M}$ EGTA-AM for 10 min (+EGTA). (B) Summary bar graph of EPSC amplitudes without (-) or with (+) EGTA-AM in both genotypes. in proximal boutons $(16.5 \pm 0.8,9.8 \pm 0.7,9.6 \pm 0.8, p<0.001$, $n=115$ (1), 70 (2), 70 (3), Figure 5B). In addition, distal type Ib boutons were largest along the bouton chain $\left(10.6 \pm 0.5 \mu \mathrm{m}^{2}\right.$, $\left.6.9 \pm 0.5 \mu \mathrm{m}^{2}, 6.5 \pm 0.5 \mu \mathrm{m}^{2}, p<0.001\right)$. Interestingly, this gradient was not found in type Is boutons regarding both AZ number $[5.5 \pm 0.4,5.5 \pm 0.3,4.6 \pm 0.3, p>0.05, n=73$ (1), 73 (2), 73 (3)] and bouton area $\left(3.0 \pm 0.2 \mu \mathrm{m}^{2}, 3.1 \pm 0.2 \mu \mathrm{m}^{2}, 3.3 \pm 0.2 \mu \mathrm{m}^{2}\right.$, $p>0.05$, Figure 5C). Our data are in line with previous work analyzing functional (Guerrero et al., 2005; Peled and Isacoff, 2011) and structural properties (Ehmann et al., 2014) of the NMJ. Furthermore, the number of AZs per bouton was decreased at rab3 $3^{r u p}$ NMJs compared to wt (Figures 5D-F). This matches earlier work, showing that Rab3 controls the distribution of Brp at the NMJ with decreased $A Z$ numbers per NMJ and increased Brp levels per $\mathrm{AZ}$ in rab3 ${ }^{\text {rup }}$ (Graf et al., 2009). However, the structural gradient regarding AZ number per bouton and bouton size was still present in rab3 $3^{\text {rup }}$ type $\mathrm{Ib}$ axons [Brp: $7.0 \pm 0.3,4.1 \pm 0.3,4.2 \pm 0.3$; area: $10.5 \pm 0.5 \mu \mathrm{m}^{2}, 6.9 \pm 0.5 \mu \mathrm{m}^{2}, 7.5 \pm 0.5 \mu \mathrm{m}^{2}, p<0.001$, respectively, $n=117$ (1), 57 (2), 57 (3), Figure 5E]. Thus, a structural gradient is present along the MN6/7b-Ib motor neuron with larger distal than proximal type Ib boutons. In addition, Brp is unevenly distributed in type Ib boutons of wt and rab3 ${ }^{\text {rup }}$ NMJs.

\section{SYT AND BRP ARE ESSENTIAL FOR FUNCTIONAL PRESYNAPTIC DIFFERENTIATION}

We used focal electrodes as these can be selectively placed on a subset of presynaptic boutons to improve spatial resolution of synaptic measurements. Boutons were visualized by GFP-expression (Pawlu et al., 2004) and postsynaptic currents of proximal and distal type $\mathrm{Ib}$ boutons were measured in response to low-frequency nerve stimulation in $0.5 \mathrm{mM}\left[\mathrm{Ca}^{2+}\right]_{\mathrm{Ex}}$ (Figure 6). Distal boutons of wt NMJs showed larger EPSC amplitudes and shorter rise times than proximal boutons (amplitude: $1.4 \pm 0.1 \mathrm{nA}$ and $1.0 \pm 0.1 \mathrm{nA}$, $p=0.005$; rt: $1.1 \pm 0.07 \mathrm{~ms}$ and $1.3 \pm 0.07 \mathrm{~ms}, p=0.032, n=24$, and 33, Figure 6B). In contrast, at both $b r p^{69}$ and $s y t^{K D}$ NMJs amplitude and kinetics of postsynaptic currents were comparable in distal and proximal boutons $\left(n=10\right.$ and 11 for $b r p^{69}$ and 11 and 13 for $s y t^{K D}$, Figure 6B). These results reveal that Brp and Syt are both essential for the functional differentiation of the NMJ.

\section{SYT AND BRP INTERACT GENETICALLY}

To test for a genetic interaction between Brp and Syt we analyzed non-allelic non-complementation (Yook et al., 2001). This genetic strategy tests for the ability of two recessive mutations to complement one another for a specific phenotype. We studied heterozygous animals carrying either one copy of the syt null allele $S y t^{A D 4}$ (DiAntonio et al., 1993) or the $\operatorname{brp}$ null allele $b r p^{69}$ (Kittel et al., 2006) and trans-heterozygous animals carrying both. We analyzed the structural gradient along the MN6/7b-Ib motor axon regarding AZ number and bouton dimensions (Figure 7). Whereas in heterozygous animals distal boutons contained more Brp puncta than proximal boutons $(p<0.001, n=95$ (1), 72 (2), 72 (3) for $s y t^{A D 4} ; p<0.001, n=51$ (1), 40 (2), 40 (3) for $b r p^{69}$ ), this gradient was lost in trans-heterozygous animals $(9.8 \pm 0.5$, $10.0 \pm 0.4,11.0 \pm 0.5, p>0.05, n=81$ (1), 81 (2), 81 (3), Figure 7A). Interestingly, distal boutons of trans-heterozygous animals were still largest along the bouton chain $\left(7.3 \pm 0.4 \mu \mathrm{m}^{2}\right.$, 
A

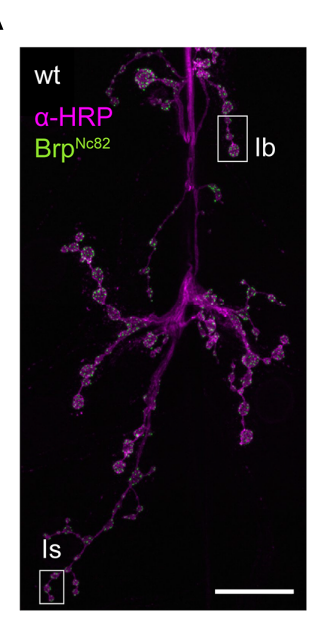

D

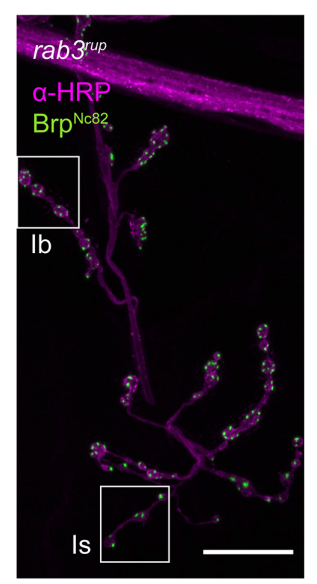

B

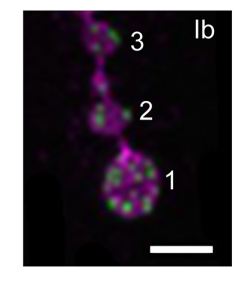

C

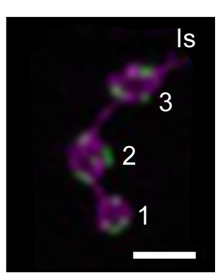

E

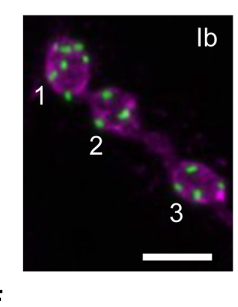

$\mathbf{F}$

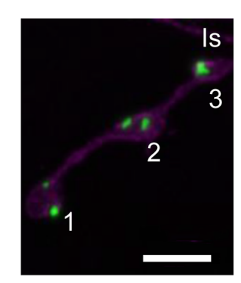

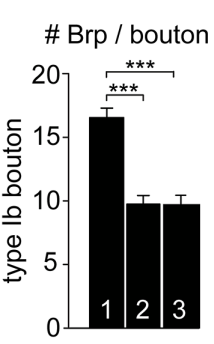
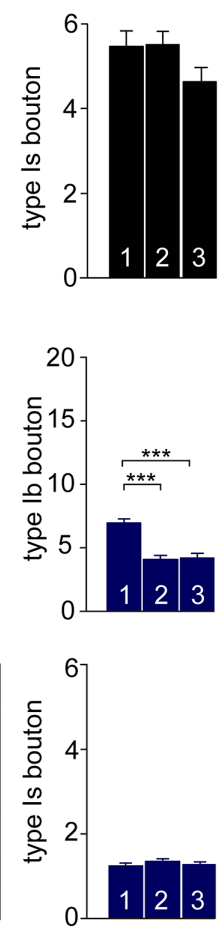
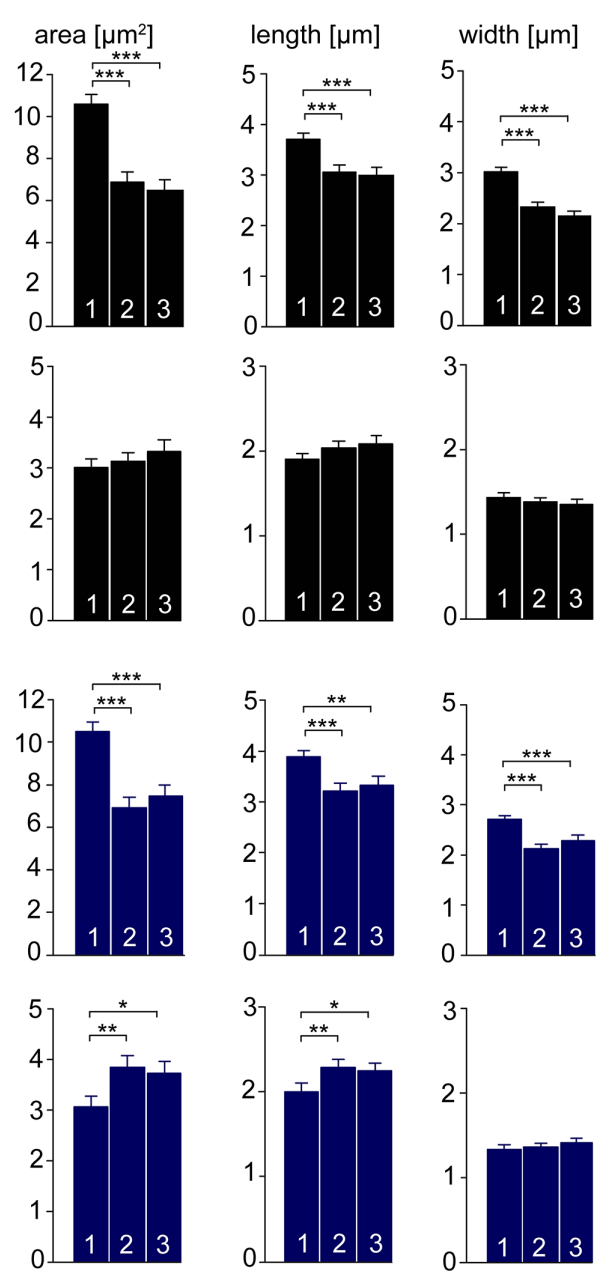

No. of bouton from end

FIGURE 5 | Type lb and type Is bouton morphometry in wt and rab3 ${ }^{\text {rup }}$. (A) Drosophila wt NMJ stained with $\alpha$-HRP against neuronal membranes (magenta) and $\mathrm{mAb} \mathrm{Brp}^{N c 82}$ against Brp (green). White boxes indicate terminal three boutons of type $\mathrm{lb}$ and type Is branches. (B,C) Magnification of boxed regions in (A). Summary bar graphs for the number of Brp puncta per bouton and bouton area, length and width of the terminal three boutons. (D) Drosophila rab3 rup NMJ as in (A). Neurite passing by the NMJ at the top. (E,F) Magnification of boxed regions in (D) show the terminal three boutons of respective type $\mathrm{Ib}$ and type Is endings. Summary bar graphs for the number of Brp puncta per bouton and area, length and width of the terminal three boutons. Scale bars $=20 \mu \mathrm{m}(\mathbf{A}, \mathbf{D})$ and $5 \mu \mathrm{m}$ $(B, C, E, F)$.
$5.5 \pm 0.3 \mu \mathrm{m}^{2}, 5.8 \pm 0.3 \mu \mathrm{m}^{2}, p=0.003$ and 0.014 for (2) and (3), Figure 7B). We conclude that regarding AZ distribution both mutations fail to complement one another, suggesting a direct interaction of Brp and Syt or an action of the two proteins in the same functional pathway.

\section{SYT GUIDES AZ DISTRIBUTION}

To further investigate the impact of Brp and Syt on maintaining the structural gradient we again performed immunostainings (Figure 8). At $b r p^{69} \mathrm{NMJs}$, bouton area, length, and width were larger for distal than for proximal type Ib boutons (data not shown). Analysis of $s y t^{K D}$ NMJs showed profound alterations of synaptic morphology regarding Brp distribution and bouton size. Whereas the number of Brp positive AZs per NMJ was slightly decreased compared to wt $(724 \pm 38$ and $861 \pm 41, p=0.019$, $n=16$ NMJs each), AZ numbers per Ib bouton were reduced to about a quarter. Furthermore, Brp was distributed homogeneously along the MN6/7b-Ib motor neuron and spatial dimensions of type Ib boutons were similar for all locations along the motoneuron $[p>0.05, n=125$ (1), 109 (2), 109 (3), Figures 8B,C]. Compared to wt, bouton size was reduced dramatically. In addition, analysis of the structural gradient in combined rab3 $3^{r u p}, s y t^{K D}$ animals revealed that Syt knock-down also decreases the structural differentation at rab3 ${ }^{\text {rup }}$ NMJs (data not shown). These data demonstrate that Syt is essential for the structural differentation of the NMJ both in wt and rab3 $3^{r u p}$.

\section{SYT INFLUENCES ORGANIZATION AND NUMBER OF BRP PROTEINS AT INDIVIDUAL AZs}

In a final set of experiments we employed $d S T O R M$ to image glutamatergic boutons. This localization microscopy technique substantially increases spatial resolution compared to conventional 

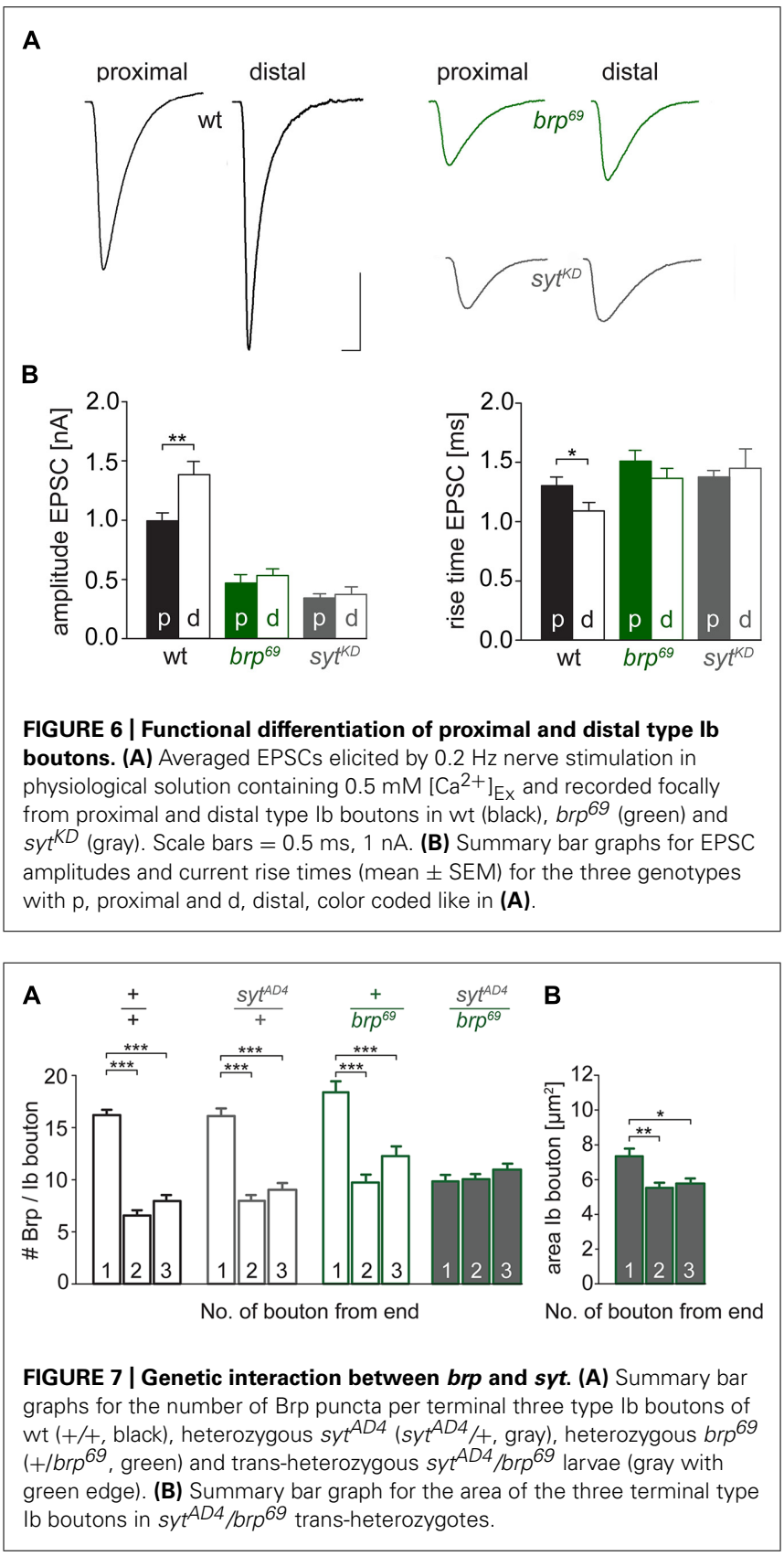

fluorescence light microscopy (Heilemann et al., 2008; van de Linde etal., 2011) and can provide quantitative insight into the nanoscopic organization of presynaptic AZs (Sauer, 2013; Ehmann et al., 2014, 2015). dSTORM resolved the substructural arrangement of indiviudal Brp localizations into multiple clusters within single AZs, which correspond to diffraction-limited Brp puncta in confocal images. We analyzed AZs in the distal six type $\mathrm{Ib}$ boutons of MN6/7b-Ib motor neurons in wt and $s y t^{K D}$ (Figure 9). Interestingly, syt ${ }^{K D} \mathrm{AZs}$ were larger than their wt counterparts $\left(0.079 \pm 0.003 \mu \mathrm{m}^{2}\right.$ and $0.069 \pm 0.002 \mu \mathrm{m}^{2}$, $p=0.003, n=300$, and $468 \mathrm{AZs}$, Figure 9D) and contained more localizations $(710 \pm 30$ and $590 \pm 19, p=0.003$, Figure 9E), which reflects an increased number of Brp protein copies (Ehmann etal., 2014). Furthermore, we observed that syt ${ }^{K D}$ AZs contained a similar number of Brp localizations irrespective of bouton order (Figure 9F, Spearman correlation coefficient $r=-0.169, p<0.001$ for wt and $r=0.014$, $p>0.05$ for $s y t^{K D}$ indicates a moderate negative correlation for wt and no correlation for $s y t^{K D}$ ). Thus, Syt influences the arrangement of Brp at the AZ. Previous work showed that the number of Brp localizations per wt AZ is higher in distal than in proximal type Ib boutons (Ehmann et al., 2014). This is consistent with the electrophysiological and structural data presented here.

\section{DISCUSSION}

While differentiation of presynaptic terminals was initially described more than 50 years ago and has since been studied extensively in various organisms, its mechanisms remain poorly understood (Katz, 1936; Hoyle and Wiersma, 1958; Reyes et al., 1998; reviewed in Atwood and Karunanithi, 2002). In view of the enormous complexity of the relevant molecular mechanisms (Südhof, 2012), the genetically and experimentally accessible NMJ of Drosophila melanogaster provides advantageous features for studying a glutamatergic synaptic system. Type Is- and Ib-boutons of the NMJ exhibit distinct functional properties (Kurdyak et al., 1994; Pawlu et al., 2004), show differences in vesicle size (Karunanithi et al., 2002) and in the amount of Brp molecules per AZ (Ehmann et al., 2014).

Here we show branch-specific differentiation in the MN6/7b-Ib motoneuron regarding structure and function. Distal type Ib boutons are larger than proximal ones, have more Brp positive AZs and show larger and faster postsynaptic responses (Figures $\mathbf{5}$ and $\mathbf{6}$ ). Consistent with these findings, AZs of distal type Ib boutons are larger and possess more Brp molecules per AZ (Ehmann et al., 2014). Presynaptic differentiation is impaired by disrupting either Brp or Syt function (Figures 6, 8, and 9). Postsynaptic responses of proximal and distal boutons in $b r p^{69}$ and $s y t^{K D}$ are comparable and the structural gradient in bouton size, AZs per bouton and AZ size is absent in $s y t^{K D}$. Moreover, genetic evidence suggests that $\mathrm{Brp}$ and Syt act in the same functional pathway to mediate structural heterogeneity (Figure 7). Structural and functional presynaptic differentiation thus clearly requires the concerted action of Brp and Syt.

Interestingly, we obtained consistently lower values for $\mathrm{AZ}$ size and Brp counts per AZ than a recent previous investigation using dSTORM (Ehmann etal., 2014). In the present study, we raised both mutant and control animals at $29^{\circ} \mathrm{C}$ to ensure efficient RNA-mediated $s y t^{K D}$ (expression via the GAL4UAS system is temperature-dependent), whereas Ehmann etal. (2014) raised larvae at $25^{\circ} \mathrm{C}$. Since higher temperature accelerates the development of Drosophila, enlarges presynaptic arborizations and increases the number of AZs per NMJ (Ashburner, 1989; Sigrist etal., 2003), it is conceivable that temperaturedependent plasticity also affects molecular organization at the level of individual AZs.

The molecular mechanisms controlling size, structure and distribution of AZs are complex. Several years ago, the vesicle protein Rab3 was identified as an important regulatory factor of AZ size and distribution (Graf etal., 2009). Rab-proteins 
A

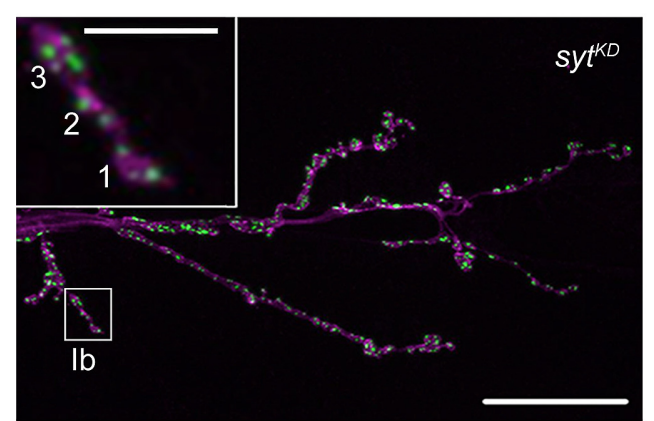

C

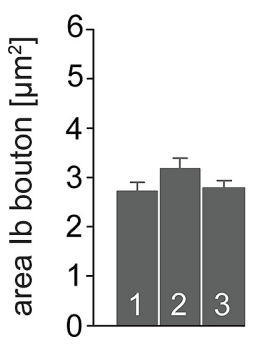

B
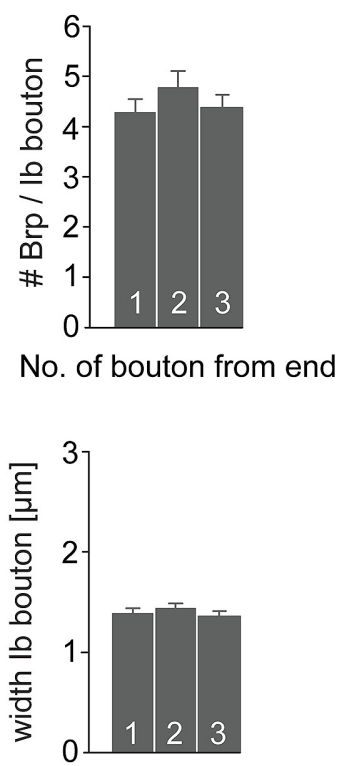

No. of bouton from end

FIGURE 8 |Type lb bouton morphometry in syt ${ }^{K \boldsymbol{D}}$. (A) syt ${ }^{K D} \mathrm{NMJ}$ stained with $\alpha$-HRP against neuronal membranes (magenta) and mAb Brp ${ }^{N c 82}$ (green). Higher magnification of type lb ending (white box) showing the three terminal boutons. Scale bars $=20 \mu \mathrm{m}, 2 \mu \mathrm{m}$ (inset). (B) Summary bar graph for the number of Brp puncta per terminal three type lb boutons in $s y t^{K D}$ and (C) bouton area, length, and width. are key organizers of vesicle trafficking (Harris and Littleton, 2011). Measurements with genetically encoded postsynaptic calcium sensor showed comparable calcium signals in proximal and distal type Ib boutons at rab3 ${ }^{\text {rup }}$ NMJs (Peled and Isacoff, 2011). Here, we found a gradient in rab3 ${ }^{\text {rup }}$ animals in the number of Brp positive AZs along the MN6/7b-Ib motoneuron (Figure 5), unlike in $s y t^{K D}$ (Figure 8). Whereas at rab3 ${ }^{r u p}$ NMJs the overall number of AZs is reduced dramatically (Graf et al., 2009), this reduction is moderate at $s y t^{K D}$ NMJs $(724 \pm 38$ and $861 \pm 41$, see Results). However, at both rab3 ${ }^{r u p}$ and $s y t^{K D}$ type Ib branches, the number of Brp proteins per AZ is increased strongly and moderately, respectively (Ehmann et al., 2014; Figure 9). In contrast to rab3 ${ }^{\text {rup }}$ synapses, $s y t^{K D}$ decreases bouton size. Smaller boutons were reported for $s y t^{A D 4}$ and linked to defects in endocytosis (Dickman etal., 2006). We found that brp and syt interact genetically regarding $\mathrm{AZ}$ number per bouton, but not area of boutons (Figure 7), which suggests that the effects of Syt on bouton size and AZ-differentiation are not strictly linked.

The pronounced presynaptic structural alterations after $s y t^{K D}$ are puzzling. Syt is one of the best-studied synaptic proteins. However, its function has mainly been discussed without considering AZ-differentiation. After $s y t^{K D}$, evoked release was reduced by a factor of 4-5 compared to wt in our focal recordings (Figure 6). However, the number of Brp spots in terminal boutons was also reduced by a factor of 4-5 after $s y t^{K D}$ (Figure 8). Is release probability per $\mathrm{AZ}$ in distal boutons following $s y t^{K D}$ therefore similar to wt? More work perhaps combining optical release sensors, focal recordings and subsequent immunostainings will be necessary to clarify this issue. The present study highlights how interpretations of synaptic function and differentiation profit from electrophysiological recording techniques with improved spatial resolution (focal vs. TEVC). Functional sampling of synaptic subsets appears absolutely necessary when considering the significant differentiation at the structural level. Either way, linking structure and function at one and the same AZ is fundamentally important for a comprehensive mechanistic interpretation (Bailey and Chen, 1983; Wojtowicz et al., 1994).

Our electrophysiological data suggest that Syt protracts release at AZs lacking Brp. In this study, we used $s y t^{K D}$ to reduce the protein level in presynpatic terminals (Figure 2). While we assume there are normally more than 10 Syt molecules on each vesicle in our preparation (Takamori et al., 2006), it is unclear whether $s y t^{K D}$ leads to a reduction in the average number of Syt proteins per vesicle or a reduction in the number of Syt positive vesicles with those remaining possessing a full complement of Syt copies. This is relevant in the context of the molecular interpretation of our results. For example, ring-like oligomerization of Syt's cytosolic C2-domains, which prevents release in the absence of calcium, requires a certain copy number (Wang etal., 2014). Furthermore, quantitative information on Syt's partner molecules, such as Complexin and SNAREproteins, will be required for a mechanistic interpretation down to the level of stochiometric interactions (Mohrmann et al., 2010; Cho et al., 2014). Imaging techniques such as dSTORM can be used to quantify the molecular organization of AZs (Sauer, 2013; Ehmann etal., 2015) and will be necessary to 
A
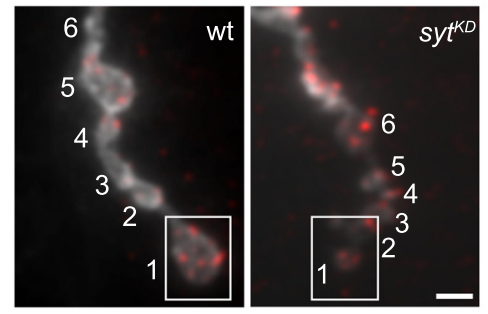

B

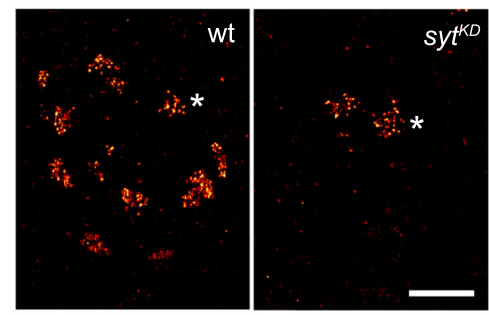

C

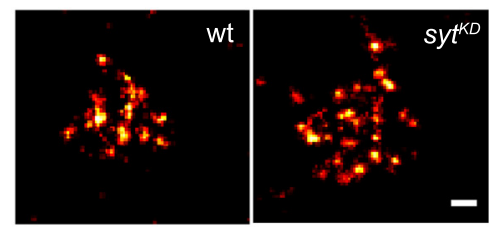

D

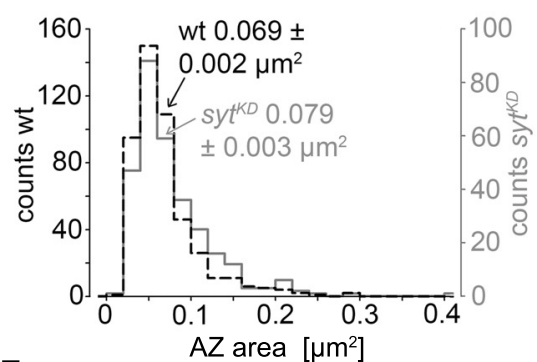

E

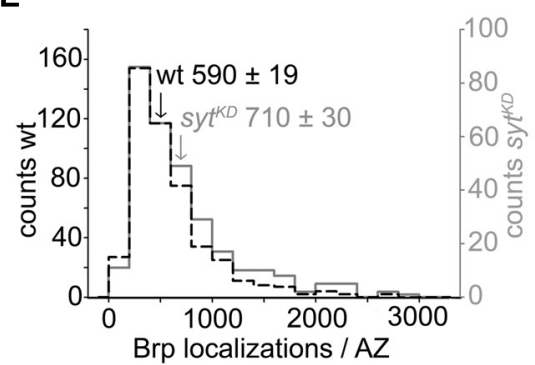

F

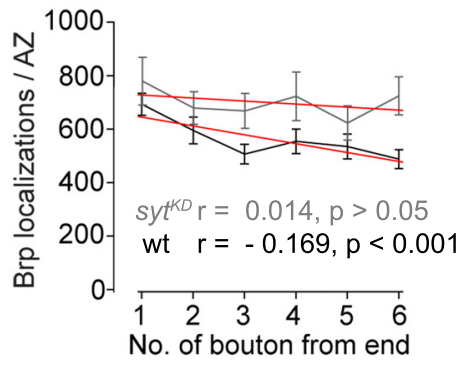

$A Z$ areas and (E) distribution of Brp localizations per $A Z$ are shifted to larger values in $s y t^{K D}$ (gray). Arrows indicate respective mean $\pm S E M$ values. (F) Linear fits (red) of mean \pm SEM Brp localizations per AZ in the terminal six type $\mathrm{lb}$ boutons had slopes of -33.2 (wt) and -11.2 (syt ${ }^{K D}$ ) (non-parametric Spearman correlation coefficient wt: $r=-0.169, p<0.001$; syt ${ }^{K D}: r=0.014$, $p>0.05)$. Scale bars $=2 \mu \mathrm{m}$ (A), $1 \mu \mathrm{m}$ (B), $100 \mathrm{~nm}$ (C).

clarify the so far insufficiently understood kinetic release parameters. In this context, interpretations may well have to take into account the existence of alternative sensors (Walter et al., 2011).

Syt contributes to vesicle docking at the AZ, vesicle positioning within the AZ, clamping and triggering release from the AZ (Walter et al., 2011). While specific amino acids of Syt have been put in connection with certain subsets of these features (e.g., Young and Neher, 2009), it remains unclear which specific functional roles and molecular domains of Syt are responsible for interactions with Brp and structural synaptic specialization. Intriguingly, both Syt and Rab3 are involved in vesicle trafficking and participate in the structural differentiation of AZs. Our work supports the notion that organization of the synaptic vesicle cycle and AZ structure are causally linked.

\section{AUTHOR CONTRIBUTIONS}

Mila M. Paul, Martin Pauli, Nadine Ehmann, Stefan Hallermann, Markus Sauer, and Manfred Heckmann performed experiments.
Mila M. Paul, Stefan Hallermann, Robert J. Kittel, and Manfred Heckmann analyzed the data. Robert J. Kittel and Manfred Heckmann conceived the project and coordinated the study. Mila M. Paul, Robert J. Kittel, and Manfred Heckmann wrote the manuscript with assistance from all co-authors.

\section{ACKNOWLEDGMENTS}

This work was supported by grants from the German Research Foundation (DFG) to Robert J. Kittel (KI 1460/1-1 and SFB 1047/A05), from the IZKF Würzburg to Manfred Heckmann (N229), a BMBF grant to Markus Sauer (133N12781) and a GSLS-fellowship from the University of Würzburg to Nadine Ehmann. The authors thank N. Reist for the $\alpha$-Dsyt-CL1 antibody, D. Ljaschenko for support in the intitial phase of the study and F. Köhler, T. Martini, M. Oppmann, and C. Wirth for technical assistance.

\section{REFERENCES}

Ashburner, M. (1989). Drosophila: A Laboratory Handbook. Cold Spring Harbor, NY: Cold Spring Harbor Laboratory Press. 
Atwood, H. L., Govind, C. K., and Wu, C. F. (1993). Differential ultrastructure of synaptic terminals on ventral longitudinal abdominal muscles in Drosophila larvae. J. Neurobiol. 24, 1008-1024. doi: 10.1002/neu.480240803

Atwood, H. L., and Karunanithi, S. (2002). Diversification of synaptic strength: presynaptic elements. Nat. Rev. Neurosci. 3, 497-516. doi: 10.1038/nrn876

Bailey, C. H., and Chen, M. (1983). Morphological basis of long-term habituation and sensitization in Aplysia. Science 220, 91-93. doi: 10.1126/science.6828885

Barrett, E. F., and Stevens, C. F. (1972). The kinetics of transmitter release at the frog neuromuscular junction. J. Physiol. 227, 691-708. doi: 10.1113/jphysiol.1972.sp010054

Brand, A. H., and Perrimon, N. (1993). Targeted gene expression as a means of altering cell fates and generating dominant phenotypes. Development 118 , 401-415.

Brose, N., Petrenko, A. G., Südhof, T. C., and Jahn, R. (2002). Synaptotagmin: a calcium sensor on the synaptic vesicle surface. Science 256, 1021-1025. doi: $10.1126 /$ science. 1589771

Cho, R. W., Kümmel, D., Li, F., Baguley, S. W., Coleman, J., and Rothman, J. E. et al. (2014). Genetic analysis of the Complexin trans-clamping model for crosslinking SNARE complexes in vivo. Proc. Natl. Acad. Sci. U.S.A. 111, 10317-10322. doi: 10.1073/pnas.1409311111

Datyner, N. B., and Gage, P. W. (1980). Phasic secretion of acetylcholine at a mammalian neuromuscular junction. J. Physiol. 303, 299-314. doi: 10.1113/jphysiol.1980.sp013286

DeBello, W. M., Betz, H., and Augustine, G. J. (1993). Synaptotagmin and neurotransmitter release. Cell 74, 947-950. doi: 10.1016/0092-8674(93)90716-4

DiAntonio, A., Parfitt, K. D., and Schwarz, T. L. (1993). Synaptic transmission persists in synaptotagmin mutants of Drosophila. Cell 73, 1281-1290. doi: 10.1016/0092-8674(93)90356-U

Dickman, D. K., Lu, Z., Meinertzhagen, I. A., and Schwarz, T. L. (2006). Altered synaptic development and active zone spacing in endocytosis mutants. Curr. Biol. 16, 591-598. doi: 10.1016/j.cub.2006.02.058

Dudel, J. (1965). Potential changes in the crayfish motor nerve terminal during repetitive stimulation. Pflügers Arch. Gesamte Physiol. Menschen Tiere 282, 323 337. doi: 10.1007/BF00412507

Eggermann, E., Bucurenciu, I., Goswami, S. P., and Jonas, P. (2011). Nanodomain coupling between $\mathrm{Ca} 2+$ channels and sensors of exocytosis at fast mammalian synapses. Nat. Rev. Neurosci. 13, 7-21. doi: 10.1038/nrn3125

Ehmann, N., Sauer, M., and Kittel, R. J. (2015). Super-resolution microscopy of the synaptic active zone. Front. Cell. Neurosci. 9:7. doi: 10.3389/fncel.2015. 00007

Ehmann, N., van de Linde, S., Alon, A., Ljaschenko, D., Keung, X. Z., Holm, T., et al. (2014). Quantitative super-resolution imaging of Bruchpilot distinguishes active zone states. Nat. Commun. 5, 4650. doi: 10.1038/ncomms5650

Geppert, M., Goda, Y., Hammer, R. E., Li, C., Rosahl, T. W., Stevens, C. F., et al. (1994). Synaptotagmin I: a major Ca2+ sensor for transmitter release at a central synapse. Cell 79, 717-727. doi: 10.1016/0092-8674(94)90556-8

Graf, E. R., Daniels, R. W., Burgess, R. W., Schwarz, T. L., and DiAntonio, A. (2009). Rab3 dynamically controls protein composition at active zones. Neuron 64, 663-677. doi: 10.1016/j.neuron.2009.11.002

Guerrero, G., Reiff, D. F., Agarwal, G., Ball, R. W., Borst, A., Goodman, C. S., et al. (2005). Heterogeneity in synaptic transmission along a Drosophila larval motor axon. Nat. Neurosci. 8, 1188-1196. doi: 10.1038/nn1526

Harris, K. P., and Littleton, J. T. (2011). Vesicle trafficking: a Rab family profile. Curr. Biol. 21, 841-843. doi: 10.1016/j.cub.2011.08.061

Heilemann, M., van de Linde, S., Schüttpelz, M., Kasper, R., Seefeldt, B. Mukherjee, A., et al. (2008). Subdiffraction-resolution fluorescence imaging with conventional fluorescent probes. Angew. Chem. Int. Ed. Engl. 47, 6172-6176. doi: 10.1002/anie. 200802376

Hoang, B., and Chiba, A. (2001). Single-cell analysis of Drosophila larval neuromuscular synapses. Dev. Biol. 229, 55-70. doi: 10.1006/dbio.2000.9983

Hoyle, G., and Wiersma, C. A. (1958). Excitation at neuromuscular junctions in Crustacea. J. Physiol. 143, 403-425. doi: 10.1113/jphysiol.1958.sp006068

Jan, L. Y., and Jan, Y. N. (1982). Antibodies to horseradish peroxidase as specific neuronal markers in Drosophila and in grasshopper embryos. Proc. Natl. Acad. Sci. U.S.A. 79, 2700-2704. doi: 10.1073/pnas.79.8.2700

Karunanithi, S., Marin, L., Wong, K., and Atwood, H. L. (2002). Quantal size and variation determined by vesicle size in normal and mutant Drosophila glutamatergic synapses. J. Neurosci. 22, 10267-10276.
Katz, B. (1936). Neuro-muscular transmission in crabs. J. Physiol. 87, 199-221. doi: 10.1113/jphysiol.1936.sp003401

Kittel, R. J., Wichmann, C., Rasse, T. M., Fouquet, W., Schmidt, M., Schmid, A., et al. (2006). Bruchpilot promotes active zone assembly, Ca2+ channel clustering, and vesicle release. Science 312, 1051-1054. doi: 10.1126/science. 1126308

Kurdyak, P., Atwood, H. L., Stewart, B. A., and Wu, C. F. (1994). Differential physiology and morphology of motor axons to ventral longitudinal muscles in larval Drosophila. J. Comp. Neurol. 350, 463-472. doi: 10.1002/cne. 903500310

Littleton, J. T., Stern, M., Schulze, K., Perin, M., and Bellen, H. J. (1993). Mutational analysis of Drosophila synaptotagmin demonstrates its essential role in Ca2+-activated neurotransmitter release. Cell 74, 1125-1134. doi: 10.1016/0092-8674(93)90733-7

Lnenicka, G. A., and Keshishian, H. (2000). Identified motor terminals in Drosophila larvae show distinct differences in morphology and physiology. J. Neurobiol. 43, 186-197. doi: 10.1002/(SICI) 1097-4695(200005)43:2<186:AIDNEU8>3.0.CO;2-N

Mackler, J. M., Drummond, J. A., Loewen, C. A., Robinson, I. M., and Reist, N. E. (2002). The C2B Ca2+-binding motif of synaptotagmin is required for synaptic transmission in vivo. Nature 418, 340-344. doi: 10.1038/nature00846

Matz, J., Gilyan, A., Kolar, A., McCarvill, T., and Krueger, S. R. (2010). Rapid structural alterations of the active zone lead to sustained changes in neurotransmitter release. Proc. Natl. Acad. Sci. U.S.A. 107, 8836-8841. doi: 10.1073/pnas.0906087107

Maximov, A., and Südhof, T. C. (2005). Autonomous function of synaptotagmin 1 in triggering synchronous release independent of asynchronous release. Neuron 48, 547-554. doi: 10.1016/j.neuron.2005.09.006

Mohrmann, R., de Wit, H., Verhage, M., Neher, E., and Sørensen, J. B. (2010). Fast vesicle fusion in living cells requires at least three SNARE complexes. Science 330, 502-505. doi: 10.1126/science.1193134

Müller, M., Liu, K. S., Sigrist, S. J., and Davis, G. W. (2012). RIM controls homeostatic plasticity through modulation of the readily-releasable vesicle pool. J. Neurosci. 32, 16574-16585. doi: 10.1523/JNEUROSCI.0981-12.2012

Neher, E. (1998). Vesicle pools and Ca2+ microdomains: new tools for understanding their roles in neurotransmitter release. Neuron 20, 389-399. doi: 10.1016/S0896-6273(00)80983-6

Neher, E. (2010). What is rate-limiting during sustained synaptic activity: vesicle supply or the availability of release sites. Front. Synaptic Neurosci. 2:144. doi: 10.3389/fnsyn.2010.00144

Pawlu, C., DiAntonio, A., and Heckmann, M. (2004). Postfusional control of quantal current shape. Neuron 42, 607-618. doi: 10.1016/S0896-6273(04)00269-7

Peled, E. S., and Isacoff, E. Y. (2011). Optical quantal analysis of synaptic transmission in wild-type and rab3-mutant Drosophila motor axons. Nat. Neurosci. 14, 519-526. doi: 10.1038/nn.2767

Peled, E. S., Newman, Z. L., and Isacoff, E. Y. (2014). Evoked and spontaneous transmission favored by distinct sets of synapses. Curr. Biol. 24, 484-493. doi: 10.1016/j.cub.2014.01.022

Reyes, A., Lujan, R., Rozov, A., Burnashev, N., Somogyi, P., and Sakmann, B. (1998). Target-cell-specific facilitation and depression in neocortical circuits. Nat. Neurosci. 1, 279-285. doi: 10.1038/1092

Sauer, M. (2013). Localization microscopy coming of age: from concepts to biological impact. J. Cell Sci. 126, 3505-3513. doi: 10.1242/jcs.123612

Schmidt, H., Brachtendorf, S., Arendt, O., Hallermann, S., Ishiyama, S., Bornschein, G., et al. (2013). Nanodomain coupling at an excitatory cortical synapse. Curr. Biol. 23, 244-249. doi: 10.1016/j.cub.2012.12.007

Sigrist, S. J., Reiff, D. F., Thiel, P. R., Steinert, J. R., and Schuster, C. M. (2003). Experience-dependent strengthening of Drosophila neuromuscular junctions. J. Neurosci. 23, 6546-6556.

Stewart, B. A., Atwood, H. L., Renger, J. J., Wang, J., and Wu, C. F. (1994). Improved stability of Drosophila larval neuromuscular preparations in haemolymph-like physiological solutions. J. Comp. Physiol. A 175, 179-191. doi: 10.1007/BF00215114

Südhof, T. C. (2012). The presynaptic active zone. Neuron 75, 11-25. doi: 10.1016/j.neuron.2012.06.012

Takamori, S., Holt, M., Stenius, K., Lemke, E. A., Grønborg, M., and Riedel, D. et al. (2006). Molecular anatomy of a trafficking organelle. Cell 127, 831-846. doi: 10.1016/j.cell.2006.10.030 
van de Linde, S., Kasper, R., Heilemann, M., and Sauer, M. (2008). Photoswitching microscopy with standard fluorophores. Appl. Phys. B 93, 725-731. doi: 10.1007/s00340-008-3250-9

van de Linde, S., Löschberger, A., Klein, T., Heidbreder, M., Wolter, S., Heilemann, M., etal. (2011). Direct stochastic optical reconstruction microscopy with standard fluorescent probes. Nat. Protoc. 6, 991-1009. doi: 10.1038/nprot.2011.336

Vyleta, N. P., and Jonas, P. (2014). Loose coupling between Ca2+ channels and release sensors at a plastic hippocampal synapse. Science 343, 665-670. doi: $10.1126 /$ science. 1244811

Wagh, D. A., Rasse, T. M., Asan, E., Hofbauer, A., Schwenkert, I., Dürrbeck, H., et al. (2006). Bruchpilot, a Protein with homology to ELKS/CAST, is required for structural integrity and function of synaptic active zones in Drosophila. Neuron 49, 833-844. doi: 10.1016/j.neuron.2006.02.008

Walter, A. M., Groffen, A. J., Sørensen, J. B., and Verhage, M. (2011). Multiple Ca2+ sensors in secretion: teammates, competitors or autocrats? Trends Neurosci. 34, 487-497. doi: 10.1016/j.tins.2011.07.003

Wang, J., Bello, O., Auclair, S. M., Wang, J., Coleman, J., Pincet, F., et al. (2014). Calcium sensitive ring-like oligomers formed by synaptotagmin. Proc. Natl. Acad. Sci. U.S.A. 111, 13966-13971. doi: 10.1073/pnas.1415849111

Weyhersmüller, A., Hallermann, S., Wagner, N., and Eilers, J. (2011). Rapid active zone remodeling during synaptic plasticity. J. Neurosci. 31, 6041-6052. doi: 10.1523/JNEUROSCI.6698-10.2011

Wojtowicz, J. M., Marin, L., and Atwood, H. L. (1994). Activity-induced changes in synaptic release sites at the crayfish neuromuscular junction. J. Neurosci. 14, 3688-3703.

Wolter, S., Löschberger, A., Holm, T., Aufmkolk, S., Dabauvalle, M. C., van de Linde, S., et al. (2012). rapidSTORM: accurate, fast and open-source software for localization microscopy. Nat. Methods 9, 1040-1041. doi: 10.1038/nmeth.2224
Wolter, S., Schüttpelz, M., Tscherepanow, M., van de Linde, S., Heilemann, M., and Sauer, M. (2010). Real-time computation of subdiffraction-resolution fluorescence images. J. Microsc. 237, 12-22. doi: 10.1111/j.1365-2818.2009. 03287.x

Yook, K. J., Proulx, S. R., and Jorgensen, E. M. (2001). Rules of nonallelic noncomplementation at the synapse in Caenorhabditis elegans. Genetics 158, 209-220.

Young, S. M. Jr., and Neher, E. (2009). Synaptotagmin has an essential function in synaptic vesicle positioning for synchronous release in addition to its role as a calcium sensor. Neuron 63, 482-496. doi: 10.1016/j.neuron.2009. 07.028

Conflict of Interest Statement: The authors declare that the research was conducted in the absence of any commercial or financial relationships that could be construed as a potential conflict of interest.

Received: 28 October 2014; accepted: 16 January 2015; published online: 05 February 2015.

Citation: Paul MM, Pauli M, Ehmann N, Hallermann S, Sauer M, Kittel RJ and Heckmann $M$ (2015) Bruchpilot and Synaptotagmin collaborate to drive rapid glutamate release and active zone differentiation. Front. Cell. Neurosci. 9:29. doi: 10.3389/fncel.2015.00029

This article was submitted to the journal Frontiers in Cellular Neuroscience. Copyright (C) 2015 Paul, Pauli, Ehmann, Hallermann, Sauer, Kittel and Heckmann. This is an open-access article distributed under the terms of the Creative Commons Attribution License (CC BY). The use, distribution or reproduction in other forums is permitted, provided the original author(s) or licensor are credited and that the original publication in this journal is cited, in accordance with accepted academic practice. No use, distribution or reproduction is permitted which does not comply with these terms. 


\title{
Super-resolution microscopy of the synaptic active zone
}

\author{
Nadine Ehmann ${ }^{1}$, Markus Sauer ${ }^{2}$ and Robert J. Kittel ${ }^{1 *}$ \\ ${ }^{1}$ Department of Neurophysiology, Institute of Physiology, University of Würzburg, Würzburg, Germany \\ ${ }^{2}$ Department of Biotechnology and Biophysics, University of Würzburg, Würzburg, Germany
}

\author{
Edited by: \\ Hartmut Schmidt, University of \\ Leipzig, Germany \\ Reviewed by: \\ Ania K. Majewska, University of \\ Rochester, USA \\ Valentin Nägerl, University of \\ Bordeaux, France \\ ${ }^{*}$ Correspondence: \\ Markus Sauer, Department of \\ Biotechnology and Biophysics, \\ University of Würzburg, Am \\ Hubland, 97074 Würzburg, \\ Germany \\ e-mail:m.sauer@uni-wuerzburg.de \\ Robert J. Kittel, Department of \\ Neurophysiology, Institute of \\ Physiology, University of Würzburg, \\ Röntgenring 9, 97070 Würzburg, \\ Germany \\ email: robert.kittel@ \\ uni-wuerzburg.de
}

Brain function relies on accurate information transfer at chemical synapses. At the presynaptic active zone (AZ) a variety of specialized proteins are assembled to complex architectures, which set the basis for speed, precision and plasticity of synaptic transmission. Calcium channels are pivotal for the initiation of excitationsecretion coupling and, correspondingly, capture a central position at the AZ. Combining quantitative functional studies with modeling approaches has provided predictions of channel properties, numbers and even positions on the nanometer scale. However, elucidating the nanoscopic organization of the surrounding protein network requires direct ultrastructural access. Without this information, knowledge of molecular synaptic structure-function relationships remains incomplete. Recently, super-resolution microscopy (SRM) techniques have begun to enter the neurosciences. These approaches combine high spatial resolution with the molecular specificity of fluorescence microscopy. Here, we discuss how SRM can be used to obtain information on the organization of AZ proteins.

Keywords: active zone, super-resolution microscopy, excitation-secretion coupling, structure-function relationships, $\mathrm{Ca}^{2+}$ channels

\section{INTRODUCTION}

At chemical synapses, neurotransmitter release takes place at presynaptic active zones (AZs). Morphologically, AZs can be identified via their electron-dense cytomatrix-an intricate network of specialized proteins precisely organized to execute and modulate exocytosis (Zhai and Bellen, 2004; Jahn and Fasshauer, 2012; Südhof, 2012). Structure and function of AZs display varying degrees of diversity between different neuron types, between synapses of the same neuron innervating different follower cells and even between individual synapses formed by the same partner cells (Rozov et al., 2001; Atwood and Karunanithi, 2002; Zhai and Bellen, 2004; Peled and Isacoff, 2011; Ehmann et al., 2014; Paul et al., submitted). Moreover, the functional properties and the molecular composition of AZs are dynamic and can be modified in an activity-dependent manner (e.g., Wojtowicz et al., 1994; Castillo et al., 2002; Matz et al., 2010; Weyhersmüller et al., 2011). Moving from correlation to causality to clarify how different molecular architectures of AZs give rise to specific physiological properties remains a major challenge.

As an AZ contains a multitude of densely packed proteins in a small sub-cellular compartment (around 200-400 nm diameter at a central synapse; Siksou et al., 2007) diffraction-limited light microscopy delivers only very coarse structural information. Hence, morphological investigations of the fine structure and the molecular organization of AZs have mainly been restricted to electron microscopy (EM). Recently, the development of superresolution microscopy (SRM) techniques has provided means to bypass the diffraction barrier of $\sim 300 \mathrm{~nm}$ in lateral dimensions
(Abbe, 1873) and to bridge the gap between conventional light microscopy and EM (for detailed recent overviews, see Hell, 2009; Patterson et al., 2010; Schermelleh et al., 2010; Galbraith and Galbraith, 2011; Sauer, 2013). These emerging technologies offer promising new options for studying nanoscopic sub-cellular structures.

Recent work has reviewed the molecular composition of AZs (Owald and Sigrist, 2009; Jahn and Fasshauer, 2012; Südhof, 2012). This perspective will focus on excitation-secretion coupling, i.e., the transduction of an electrical signal into Calcium $\left(\mathrm{Ca}^{2+}\right)$-dependent neurotransmitter release (Schneggenburger and Neher, 2005; Wojcik and Brose, 2007). We will summarize current information on functional determinants of the $\mathrm{AZ}$ and explore how the search for structural correlates can be supported by SRM to improve our mechanistic understanding of neurotransmission.

\section{MICROSCOPY}

Fluorescence microscopy is the method of choice for visualizing biomolecules in fixed and living cells as it enables their selective and specific detection with a high signal-to-background ratio (Lichtman and Conchello, 2005). However, while light microscopy is ideally suited to investigate macroscopic arrangements, it fails to uncover organizational principles at the molecular scale due to its limited spatial resolution.

EM provides substantially increased resolution, though its application is restricted to lifeless, fixed and embedded biological samples. EM studies have been instrumental in recognizing the 
large morphological diversity of AZs (Zhai and Bellen, 2004) and in identifying repetitive structural elements within individual, chemically fixed AZs (Pfenninger et al., 1972; Phillips et al., 2001). Moreover, alternative tissue preparation and fixation techniques have enabled analyses of filamentous AZ structures and their associated synaptic vesicles in various organisms (Landis et al., 1988; Siksou et al., 2007; Jiao et al., 2010; Wichmann and Sigrist, 2010; Fernández-Busnadiego et al., 2013). The resolving power of EM is exemplified by a classical tomographic study at the frog neuromuscular junction (NMJ). The results revealed an intricate fine structure of the AZ, which establishes a regular and precisely organized arrangement of synaptic vesicles relative to $\mathrm{Ca}^{2+}$ channels at release sites (Harlow et al., 2001). As more substructural details are uncovered (Szule et al., 2012), knowledge of the underlying protein species becomes increasingly desirable. Immunogold labeling provides a means to locate specific proteins in electron micrographs with nanometer resolution and has been used to examine the topology of AZs (e.g., Limbach et al., 2011). However, specific labeling with antibody-coupled gold particles is inefficient and a compromise must be made between optimal tissue preservation and structural resolution. Consequently, the ideal microscope should combine the minimal invasiveness and efficient specific labeling possibilities of optical microscopy with the high spatial resolution of EM. Technologies that merge these features, at least to a certain extent, are collectively termed SRM. These include structured illumination microscopy (SIM), stimulated emission depletion (STED) and single-molecule based localization microscopy methods, such as photo-activated localization microscopy (PALM) and direct stochastic optical reconstruction microscopy (dSTORM). The techniques can be subdivided based on their principle of bypassing the diffraction barrier: deterministic approaches, such as STED, use a phase mask to define the coordinates of fluorescence emission predefined in space by the zero-node, whereas PALM and dSTORM use stochastic activation of individual fluorophores and precise position determination (localization).

SIM relies on patterned illumination of the specimen with a high spatial frequency in various orientations providing a lateral resolution of approximately $100 \mathrm{~nm}$ (Heintzmann and Cremer, 1999; Gustafsson, 2000). Fortunately, SIM does not depend on any specific fluorophore properties, such as high photostability or particular transitions between orthogonal states, and can therefore be generally applied. A further modification of SIM, known as SSIM (saturated-SIM) exhibits higher spatial resolution but requires photostable samples (Gustafsson, 2005). As SIM enables multicolor 3D-imaging with standard fluorescent dyes, it has attracted considerable interest among biologists (Maglione and Sigrist, 2013).

In STED microscopy, the lateral resolution is improved by decreasing the size of the excitation point-spread-function (PSF) by stimulated emission of fluorophores at the rim of the PSF (Hell and Wichmann, 1994). Since the resolution enhancement in STED microscopy scales with the intensity of the depletion beam (Hell, 2007), only very photostable fluorophores allow spatial resolutions in the 30-50 nm range (Hell, 2007; Meyer et al., 2008). Nevertheless, STED has also been used for live-cell super-resolution imaging albeit at lower resolution (Nägerl et al., 2008; Tønnesen et al., 2014).

Single-molecule based localization microscopy techniques such as PALM, STORM and dSTORM rely on stochastic photoactivation, photoconversion, or photoswitching of fluorophores, such that only a small subset emits photons at any given time. By fitting a $2 \mathrm{D}$ Gaussian function to the PSF of individual, spatially isolated emitters, their positions can be precisely localized and used to reconstruct a superresolved image, as long as all fluorophores determining the structure of interest have been detected and localized at least once during acquisition (Betzig et al., 2006; Hess et al., 2006; Rust et al., 2006; Heilemann et al., 2008). Localization microscopy methods differ in their use of fluorescent probes: PALM is conducted with genetically expressed photoactivatable fluorescent proteins (Betzig et al., 2006; Hess et al., 2006), STORM requires photoswitchable dye pairs (Rust et al., 2006) and dSTORM takes advantage of the reversible photoswitching of standard organic fluorophores in thiol-containing aqueous buffer (Heilemann et al., 2008; van de Linde et al., 2011). Since localization microscopy exhibits explicit single-molecule sensitivity, all approaches can deliver quantitative information on molecular distributions and even have the potential to report absolute numbers of proteins present in sub-cellular compartments (Sauer, 2013). These features provide insight into biological systems at a molecular level and can yield direct experimental feedback for modeling the complexity of biological interactions.

\section{FUNCTIONAL PARAMETERS OF THE AZ}

Derived from the quantal hypothesis (Del Castillo and Katz, 1954) it is understood that synaptic strength, i.e., the amplitude of an excitatory postsynaptic current (EPSC), can be described by the product of three basic parameters: $N$, the number of fusion competent synaptic vesicles also termed readily-releasable vesicles (RRVs), $p$, their probability of exocytosis and $q$, usually taken to reflect postsynaptic sensitivity (Equation 1). This conceptual framework plays an important role in explaining synaptic function and plasticity (Zucker and Regehr, 2002), and identifies $N$ and $p$ as major functional determinants of the presynapse.

$$
\mathrm{EPSC}=N p q
$$

The parameter $N$ can be estimated by electrophysiological means, such as high-frequency electrical stimulation or fluctuation analysis of synaptic responses (Clements and Silver, 2000). Results obtained by either approach must, however, be interpreted carefully, as additional factors complicate the analysis (Sakaba et al., 2002; Hallermann et al., 2010a). For example, asynchronous release, the kinetics of vesicle pool refilling (Hosoi et al., 2007) and postsynaptic contributions, such as receptor desensitization and saturation (Scheuss et al., 2002), can influence approximations of $N$. Hypertonic sucrose stimulation can be used as another technique to approximate $N$ (Fatt and Katz, 1952; Rosenmund and Stevens, 1996). However, being independent of $\mathrm{Ca}^{2+}$ triggered fusion, it remains uncertain whether hypertonically 
released vesicles are generally also readily released under physiological conditions (Moulder and Mennerick, 2005).

Alternatively, $N$ can be defined as the number of release sites, in which case $p$ denotes the probability that a vesicle will fuse at a given release site (Schneggenburger et al., 2002). Nerve terminals vary greatly in size and correspondingly contain between one (e.g., at certain cortical synapses; Xu-Friedman et al., 2001) and many hundreds of AZs (e.g., at the Calyx of Held; Sätzler et al., 2002). It is therefore helpful to view the AZ as a fundamental unit of presynaptic function (Alabi and Tsien, 2012). That said, morphology and function of AZs are highly heterogeneous (Zhai and Bellen, 2004), also varying within one and the same neuron (Atwood and Karunanithi, 2002; Peled and Isacoff, 2011; Ehmann et al., 2014). Correspondingly, functional estimates of $p$ at central mammalian synapses have reported both AZs operating with uniquantal release and AZs capable of multivesicular release (Tong and Jahr, 1994; Auger et al., 1998; Silver et al., 2003). To date, this next level of $A Z$ organization has been difficult to study as specific molecular markers or structural correlates of release sites remain uncertain.

Functional estimates of $p$ can be obtained with several methods that provide relative or absolute values. These include electrophysiology-based approaches such as paired-pulse stimulation or fluctuation analysis (Clements and Silver, 2000; Sakaba et al., 2002; Zucker and Regehr, 2002) and dynamic optical readouts of exocytosis or postsynaptic activation (Branco and Staras, 2009; Zhang et al., 2009; Peled and Isacoff, 2011; Marvin et al., 2013). Since $p$ is highly $\mathrm{Ca}^{2+}$-dependent, its value for a given synaptic vesicle will be strongly influenced by the vesicle's position relative to voltage-gated $\mathrm{Ca}^{2+}$ channels at the AZ (Neher, 1998; Eggermann et al., 2012).

$\mathrm{Ca}^{2+}$ channels are essential components of the macromolecular exocytosis machinery. Their opening elicits $\mathrm{Ca}^{2+}$ influx, which serves as the fusion trigger for nearby vesicles. Early computational and functional studies introduced the concept of "microdomains" to describe transient, local regions of high $\mathrm{Ca}^{2+}$ concentration (Chad and Eckert, 1984; Llinás et al., 1992). Such microdomains possess complex spatial distributions of $\mathrm{Ca}^{2+}$ elevation, which are controlled by $\mathrm{Ca}^{2+}$ diffusion, $\mathrm{Ca}^{2+}$ buffering and the geometric arrangement of $\mathrm{Ca}^{2+}$ channels in the AZ membrane (Neher, 1998). Due to their major functional significance for synaptic transmission, detailed understanding of $\mathrm{Ca}^{2+}$ signals and the arrangement of synaptic vesicles relative to local domains is important. Using electrophysiology, modeling, $\mathrm{Ca}^{2+}$ imaging and $\mathrm{Ca}^{2+}$ uncaging, considerable quantitative information on excitationsecretion coupling has been obtained at the Calyx of Held, a large glutamatergic synapse in the mammalian auditory brainstem (Bollmann et al., 2000; Schneggenburger and Neher, 2005; Sun et al., 2007). At calyceal terminals, electrophysiology has even delivered direct functional readouts (Stanley, 1993) and estimates of $\mathrm{AZ} \mathrm{Ca}{ }^{2+}$ channel numbers (Sheng et al., 2012). Application of synthetic $\mathrm{Ca}^{2+}$ chelators with different binding rates [BAPTA (1,2-bis(2-aminophenoxy)ethane$\mathrm{N}, \mathrm{N}, \mathrm{N}^{\prime}, \mathrm{N}^{\prime}$-tetraacetic acid) and EGTA (ethylene glycol-bis(2aminoethylether)-N,N,N',N'-tetraacetic acid)] can differentiate between very tight ("nanodomain", $<100 \mathrm{~nm}$ ) and larger distance ("microdomain", >100 $\mathrm{nm}$ ) coupling regimes of synaptic vesicles and $\mathrm{Ca}^{2+}$ channels (Eggermann et al., 2012). By combining data from such investigations, the vesicle- $\mathrm{Ca}^{2+}$ channel topography has now been modeled at several mammalian central AZs (Meinrenken et al., 2002; Schmidt et al., 2013; Vyleta and Jonas, 2014). While it would be desirable to study the ultrastructural organization underlying coupling modes directly, information on the exact arrangement of $\mathrm{Ca}^{2+}$ channels derived from EM is sparse (Feeney et al., 1998; Holderith et al., 2012; Indriati et al., 2013). Conventional light microscopy, in turn, cannot measure the physical distance between channels and vesicles or resolve whether the $\mathrm{Ca}^{2+}$ signal is shaped by a single channel (Augustine et al., 1991; Stanley, 1993) or the superposition of multiple channels (Borst and Sakmann, 1996).

There appears to be no general map of synaptic vesicle and $\mathrm{Ca}^{2+}$ channel arrangements at the AZ. In fact, vesiclechannel coupling may differ significantly at AZs belonging to the same neuron (Rozov et al., 2001) and at single presynaptic terminals over time (Fedchyshyn and Wang, 2005; Erazo-Fischer et al., 2007; Wong et al., 2013). Before a synaptic vesicle becomes fusion competent, the release machinery must build up a primed state (Wojcik and Brose, 2007). In addition to such "molecular priming", evidence also suggests that "positional priming", i.e., moving primed vesicles closer to $\mathrm{Ca}^{2+}$ channels, can contribute to a heterogeneous $p$ of RRVs (Neher and Sakaba, 2008). However, information on spatial relationships of AZ molecules in these distinct states has not yet been collected. Importantly, proteins which influence AZ function and plasticity by tightening vesicle- $\mathrm{Ca}^{2+}$ channel coupling have been identified in fly and mouse (Kittel et al., 2006; Yang et al., 2010). Investigating the organization of such key components relative to other AZ constituents should help to improve our mechanistic understanding of $A Z$ structure-function relationships.

\section{SRM OF THE AZ}

Quantitative information on functional determinants of the AZ has mainly been derived from large, electrophysiologically accessible presynaptic terminals, such as the Calyx of Held (Forsythe, 1994; Meinrenken et al., 2002; Neher and Sakaba, 2008). While sophisticated electrophysiology has extended direct studies of transmitter release to smaller terminals (see e.g., Hallermann et al., 2003; Rancz et al., 2007; Bucurenciu et al., 2008), there remains an obvious demand for correlative structural information.

Here, SRM techniques can be expected to make a significant contribution. Several SRM studies, mostly conducted in cell culture, have provided indirect information on AZ function by analyzing the vesicle cycle. In one of its first biological applications, STED microscopy showed that the vesicular $\mathrm{Ca}^{2+}$ sensor Synaptotagmin remains clustered in isolated patches following exocytosis in cultured neurons (Willig et al., 2006). Subsequent work introduced live cell STED imaging to visualize synaptic vesicle movement between and within presynaptic boutons (Westphal et al., 2008), while multicolor imaging has been used to differentiate molecularly-defined synaptic vesicle 


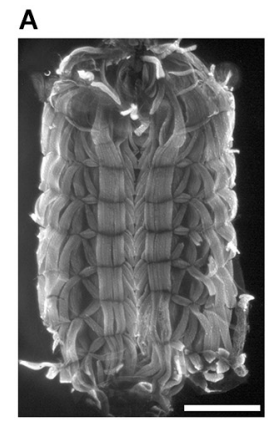

B

Diffraction-limited fluorescence Microscopy

Epifluorescence

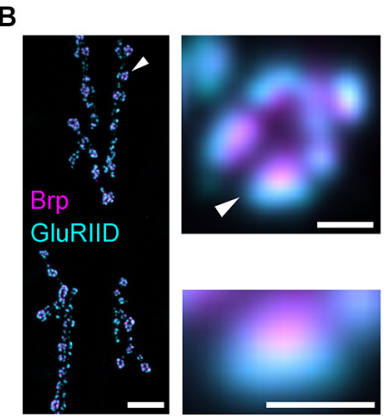

Confocal
C

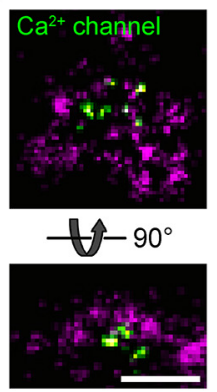

Localization

Microscopy

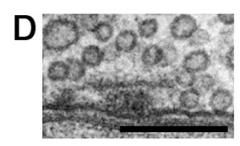

Electron

Microscopy
FIGURE 1 | Imaging Drosophila neuromuscular AZs. Gradual increases in spatial resolution show (A) a Drosophila larval preparation imaged with epifluorescence microscopy (phalloidin staining); (B) a confocal image of the glutamatergic neuromuscular junction (left panel), a single bouton (upper panel) and an individual synapse (lower panel) stained against the AZ protein Brp (magenta) and the postsynaptic glutamate receptor subunit GluRIID (cyan; arrowheads indicate enlarged regions); (C) dSTORM images of AZs stained against Brp (C-terminal epitope, magenta) and $\mathrm{Ca}^{2+}$ channels (nanobody recognizing a GFP-tagged a1-subunit, Cac ${ }^{\text {GFP }}$; Kawasaki et al., 2004) viewed en face (optical axis perpendicular to $A Z$ membrane, upper panel) and from the side (optical axis parallel to AZ membrane, lower panel; cf. D); (D) an electron micrograph of the AZ cytomatrix and opposed pre- and postsynaptic membranes. Electron micrograph kindly provided by C. Wichmann and S.J. Sigrist. Scale bars: (A) 1 mm; (B) $10 \mu \mathrm{m}$ (NMJ), $1 \mu \mathrm{m}$ (bouton), $500 \mathrm{~nm}$ (synapse); (C,D) $200 \mathrm{~nm}$. pools at calyceal synapses in rat brain tissue (Kempf et al., 2013). Focussing on Syntaxin as a component of the vesicle fusion machinery, two independently conducted investigations using STED and dSTORM provided detailed information on its arrangement in clusters at the plasma membrane of PC12 cells (Sieber et al., 2007; Bar-On et al., 2012). Moreover, 3-D applications of STORM and PALM have been utilized to investigate vesicle endocytosis by Clathrin nanostructures in cultured cell lines (Jones et al., 2011; Sochacki et al., 2012).

Analysis of the AZ nanoarchitecture in tissue was first carried out with SRM by using STED at the Drosophila NMJ. Beginning with the identification of Bruchpilot (Brp) as a major component of the AZ cytomatrix (Kittel et al., 2006; Wagh et al., 2006), subsequent work described the polarized, elongated orientation of this large filamentous protein and resolved the organization of further AZ components, such as $\mathrm{Ca}^{2+}$ channels, Syd-1, Liprin- $\alpha$ and RIM binding protein (RBP) relative to the Brp hub (Fouquet et al., 2009; Owald et al., 2010; Liu et al., 2011). This has generated an increasingly detailed picture of the protein scaffold at Drosophila AZs (Maglione and Sigrist, 2013), which is currently being extended by photobleaching microscopy techniques (PiMP, photo-bleaching microscopy with nonlinear processing; Khuong et al., 2013) and SRM via dSTORM (Figure 1; Ehmann et al., 2014, Paul et al., submitted). In a separate effort, STORM was used to measure the axial positions of the AZ-specific proteins RIM1, Piccolo and Bassoon at synapses in mouse brain tissue (Dani et al., 2010). It is of obvious interest to compare such AZ topographies from different synapses, to identify conserved and specialized principles of organization and to test whether these are causally linked to functional diversity.

Extending beyond descriptive ultrastructural studies, microscopy can contribute to identifying structural correlates of synaptic function (Wojtowicz et al., 1994). Considering their fundamental impact on neurotransmission there has thus been a long standing motivation to resolve the nanoscopic organization of $\mathrm{Ca}^{2+}$ channels at the AZ. However, to date little direct information has been collected on their ultrastructural distribution (Haydon et al., 1994; Feeney et al., 1998; Holderith et al., 2012; Indriati et al., 2013). Notably, a recent study at hippocampal neurons elegantly combined $\mathrm{Ca}^{2+}$ imaging with EM to estimate the number of $\mathrm{Ca}^{2+}$ channels contributing to one microdomain and to identify a close correlation between the number of docked vesicles, AZ area and $p$ (Holderith et al., 2012). Combining STED with molecular manipulations and electrophysiology has identified functional roles of the AZ proteins Brp and RBP in the recruitment and spatial arrangement of $\mathrm{Ca}^{2+}$ channels to promote $p$ at the Drosophila AZ (Kittel et al., 2006; Hallermann et al., 2010b; Liu et al., 2011). Moreover, dynamic reorganizations of Brp accompany rapid AZ strengthening and increase the number of release sites during homeostatic synaptic plasticity (Weyhersmüller et al., 2011). Similarly, studies at mammalian hair cell synapses have demonstrated a role of the AZ protein Bassoon, functionally related to Brp (Hallermann and Silver, 2013), in shaping $\mathrm{Ca}^{2+}$ channel arrangement and establishing release sites (Frank et al., 2010).

Despite the high spatial resolution provided by SRM, estimates of protein abundance are mainly obtained from fluorescence intensity measurements and therefore deliver only relative values. However, quantitative information on endogenous protein copies, in addition to their spatial organization, is required for a comprehensive mechanistic understanding of AZ structurefunction relationships. While stepwise photobleaching can be used to count low protein numbers (Ulbrich and Isacoff, 2007) the densely packed protein assembly at the AZ requires alternative methods. Several recent reports have addressed this issue. 
Wilhelm et al. combined quantitative biochemistry with EM and STED to estimate average protein copies and to localize these to specific sub-cellular regions of biochemically isolated presynaptic terminals (Wilhelm et al., 2014). This approach has delivered a wealth of quantitative information on presynaptic proteins. However, it does not connect structural features with functional properties at the single synapse level.

Since localization microscopy is an explicit single-molecule imaging technique, it can be used to obtain quantitative information on both the spatial distribution and the copy number of labeled proteins in situ, as long as antibody binding features (e.g., in dSTORM) or fluorescent protein expression and folding properties (as e.g., in PALM) are taken into account. By engaging dSTORM, this principle was recently utilized to study the nanoscopic arrangement of endogenous Brp proteins at AZs in tissue (Ehmann et al., 2014). The results provided an estimate of the number of Brp copies per AZ and were correlated with electrophysiological features to offer an interpretation of how the protein's organization is linked to AZ function.

These current developments open up new perspectives for clarifying how functional properties are encoded in the protein architecture of AZs. Logical next steps could include searching for molecular determinants of vesicle release sites and quantitative ultrastructural studies of $\mathrm{Ca}^{2+}$ channel-vesicle topographies.

\section{OUTLOOK}

Despite a gradually emerging comprehensive protein catalog, we still lack basic information describing how the nanoscopic organization of proteins at the $\mathrm{AZ}$ gives rise to neurotransmission. Arguably, this is due to the diffraction-limited resolution of conventional light microscopy, which has hindered access to the spatial nanodomain in a physiologically relevant experimental setting.

Several SRM techniques now exist that have the capacity to localize proteins on the nanometer scale and to resolve components of macromolecular assemblies in their native environment. In this context, we believe that localization microscopy is of particular value, as it can be used to provide direct access to molecular coordinates and to count endogenous protein epitopes (Specht et al., 2013; Andreska et al., 2014; Ehmann et al., 2014). We expect that combining such quantitative information on protein organization with results from electrophysiology will contribute to a better understanding of the molecular mechanisms controlling AZ function. In addition, other correlative approaches, such as pairing SRM with biochemistry (Wilhelm et al., 2014), EM (Watanabe et al., 2011; Löschberger et al., 2014) and array tomography (Nanguneri et al., 2012) hold great promise for uncovering multiprotein architectures.

Harnessing the full potential of SRM will require expanding the repertoire of robust test samples and introducing optimized analytical tools (Bar-On et al., 2012). Likewise, small fluorescent probes with both efficient and specific binding properties will have to be developed to allow for simultaneous visualization of multiple targets in their native settings (Sauer, 2013). As already common practice in EM, users of SRM have to accept that fluorophores, labeling protocols and sample preparations need to be optimized for each new target molecule under investigation.

Dynamic, live-cell SRM remains challenging. As a rule of thumb, spatial resolution always comes at the cost of temporal resolution. Therefore, imaging complex structures, such as the cytoskeleton of a whole cell, requires several minutes acquisition time at a lateral resolution of about $20 \mathrm{~nm}$. This clearly limits the obtainable dynamic information. In contrast, modified SIM can easily resolve the movement of microtubules in entire living cells, albeit at lower spatial resolution (Chen et al., 2014). Hence, future efforts will have to optimize the trade-off between imaging area, temporal information and spatial resolution in order to monitor dynamic protein re-arrangements at the AZ directly. In principle, fluorescent protein-based SRM techniques offer the possibility of in vivo imaging in fully intact organisms. However, the feasibility of such applications must take into account light scattering and aberration in biological tissue, less amenable photophysical properties of fluorescent proteins compared with organic fluorophores and possible physiological alterations induced by recombinant protein expression (Sauer, 2013).

Despite its capacity to resolve multiprotein structures, so far relatively few studies have engaged SRM to study synaptic AZs. We anticipate that this situation will change as SRM techniques become increasingly available and affordable (Holm et al., 2014). Progress in efficient and stoichiometric labeling of endogenous proteins, together with the development of sample preparations that accurately preserve the molecular details of interest, will further advance SRM to shed light on the AZ.

\section{ACKNOWLEDGMENTS}

We thank M. Heckmann and T. Langenhan for discussions, C. Wichmann and S. J. Sigrist for providing the electron micrograph in figure panel D and L. Pließ for technical support. This work was supported by grants from the German Research foundation (KI 1460/1-1 and SFB 1047/A05 to Robert J. Kittel), a fellowship from the GSLS, University of Würzburg (Nadine Ehmann), the Biophotonics Initiative of the German Ministry of Research and Education (BMBF/Grants \#13N11019 and \#13N12507 to Markus Sauer), and the German-Israeli Foundation (GIF/Grant \#1125-145.1/2010 to Markus Sauer).

\section{REFERENCES}

Abbe, E. (1873). Beiträge zur Theorie des Mikroskops und der mikroskopischen Wahrnehmung. Arch. Mikroskopische Anat. 9, 456-468. doi: 10.1007/bf02956176 Alabi, A. A., and Tsien, R. W. (2012). Synaptic vesicle pools and dynamics. Cold Spring Harb. Perspect. Biol. 4:a013680. doi: 10.1101/cshperspect.a013680

Andreska, T., Aufmkolk, S., Sauer, M., and Blum, R. (2014). High abundance of BDNF within glutamatergic presynapses of cultured hippocampal neurons. Front. Cell. Neurosci. 8:107. doi: 10.3389/fncel.2014.00107

Atwood, H. L., and Karunanithi, S. (2002). Diversification of synaptic strength: presynaptic elements. Nat. Rev. Neurosci. 3, 497-516. doi: 10.1038/nrn876

Auger, C., Kondo, S., and Marty, A. (1998). Multivesicular release at single functional synaptic sites in cerebellar stellate and basket cells. J. Neurosci. 18, 4532-4547.

Augustine, G. J., Adler, E. M., and Charlton, M. P. (1991). The calcium signal for transmitter secretion from presynaptic nerve terminals. Ann. N Y Acad. Sci. 635, 365-381. doi: 10.1111/j.1749-6632.1991.tb36505.x 
Bar-On, D., Wolter, S., van de Linde, S., Heilemann, M., Nudelman, G., Nachliel, E., et al. (2012). Super-resolution imaging reveals the internal architecture of nano-sized syntaxin clusters. J. Biol. Chem. 287, 27158-27167. doi: 10.1074/jbc. M112.353250

Betzig, E., Patterson, G. H., Sougrat, R., Lindwasser, O. W., Olenych, S., Bonifacino, J. S., et al. (2006). Imaging intracellular fluorescent proteins at nanometer resolution. Science 313, 1642-1645. doi: 10.1126/science.1127344

Bollmann, J. H., Sakmann, B., and Borst, J. G. (2000). Calcium sensitivity of glutamate release in a calyx-type terminal. Science 289, 953-957. doi: 10. 1126/science.289.5481.953

Borst, J. G., and Sakmann, B. (1996). Calcium influx and transmitter release in a fast CNS synapse. Nature 383, 431-434. doi: 10.1038/383431a0

Branco, T., and Staras, K. (2009). The probability of neurotransmitter release: variability and feedback control at single synapses. Nat. Rev. Neurosci. 10, 373383. doi: $10.1038 / \mathrm{nrn} 2634$

Bucurenciu, I., Kulik, A., Schwaller, B., Frotscher, M., and Jonas, P. (2008). Nanodomain coupling between $\mathrm{Ca}^{2+}$ channels and $\mathrm{Ca}^{2+}$ sensors promotes fast and efficient transmitter release at a cortical GABAergic synapse. Neuron 57 , 536-545. doi: 10.1016/j.neuron.2007.12.026

Castillo, P. E., Schoch, S., Schmitz, F., Südhof, T. C., and Malenka, R. C. (2002). RIM $1 \alpha$ is required for presynaptic long-term potentiation. Nature 415, 327-330. doi: $10.1038 / 415327$ a

Chad, J. E., and Eckert, R. (1984). Calcium domains associated with individual channels can account for anomalous voltage relations of CA-dependent responses. Biophys. J. 45, 993-999. doi: 10.1016/s0006-3495(84)84244-7

Chen, B.-C., Legant, W. R., Wang, K., Shao, L., Milkie, D. E., Davidson, M. W., et al. (2014). Lattice light-sheet microscopy: imaging molecules to embryos at high spatiotemporal resolution. Science 346:1257998. doi: 10.1126/science. 1257998

Clements, J. D., and Silver, R. A. (2000). Unveiling synaptic plasticity: a new graphical and analytical approach. Trends Neurosci. 23, 105-113. doi: 10. 1016/s0166-2236(99)01520-9

Dani, A., Huang, B., Bergan, J., Dulac, C., and Zhuang, X. (2010). Superresolution imaging of chemical synapses in the brain. Neuron 68, 843-856. doi: 10.1016/j. neuron.2010.11.021

Del Castillo, J., and Katz, B. (1954). Quantal components of the end-plate potential. J. Physiol. 124, 560-573. doi: 10.1113/jphysiol.1954.sp005129

Eggermann, E., Bucurenciu, I., Goswami, S. P., and Jonas, P. (2012). Nanodomain coupling between $\mathrm{Ca}^{2+}$ channels and sensors of exocytosis at fast mammalian synapses. Nat. Rev. Neurosci. 13, 7-21. doi: 10.1038/nrn3125

Ehmann, N., van de Linde, S., Alon, A., Ljaschenko, D., Keung, X. Z., Holm, T., et al. (2014). Quantitative super-resolution imaging of Bruchpilot distinguishes active zone states. Nat. Commun. 5:4650. doi: 10.1038/ncomms5650

Erazo-Fischer, E., Striessnig, J., and Taschenberger, H. (2007). The role of physiological afferent nerve activity during in vivo maturation of the calyx of held synapse. J. Neurosci. 27, 1725-1737. doi: 10.1523/jneurosci.4116-06.2007

Fatt, P., and Katz, B. (1952). Spontaneous subthreshold activity at motor nerve endings. J. Physiol. 117, 109-128.

Fedchyshyn, M. J., and Wang, L.-Y. (2005). Developmental transformation of the release modality at the calyx of held synapse. J. Neurosci. 25, 4131-4140. doi: 10. 1523/jneurosci.0350-05.2005

Feeney, C. J., Karunanithi, S., Pearce, J., Govind, C. K., and Atwood, H. L. (1998). Motor nerve terminals on abdominal muscles in larval flesh flies, Sarcophaga bullata: comparisons with Drosophila. J. Comp. Neurol. 402, 197-209. doi: 10. 1002/(sici)1096-9861(19981214)402:2<197::aid-cne5>3.3.co;2-h

Fernández-Busnadiego, R., Asano, S., Oprisoreanu, A.-M., Sakata, E., Doengi, M., Kochovski, Z., et al. (2013). Cryo-electron tomography reveals a critical role of RIM1 $\alpha$ in synaptic vesicle tethering. J. Cell Biol. 201, 725-740. doi: 10.1083/jcb. 201206063

Forsythe, I. D. (1994). Direct patch recording from identified presynaptic terminals mediating glutamatergic EPSCs in the rat CNS, in vitro. J. Physiol. 479, 381-387. doi: 10.1113/jphysiol.1994.sp020303

Fouquet, W., Owald, D., Wichmann, C., Mertel, S., Depner, H., Dyba, M., et al. (2009). Maturation of active zone assembly by Drosophila Bruchpilot. J. Cell Biol. 186, 129-145. doi: 10.1083/jcb.200812150

Frank, T., Rutherford, M. A., Strenzke, N., Neef, A., Pangršič, T., Khimich, D., et al. (2010). Bassoon and the synaptic ribbon organize $\mathrm{Ca}^{2+}$ channels and vesicles to add release sites and promote refilling. Neuron 68, 724-738. doi: 10.1016/j. neuron.2010.10.027
Galbraith, C. G., and Galbraith, J. A. (2011). Super-resolution microscopy at a glance. J. Cell Sci. 124, 1607-1611. doi: 10.1242/jcs.080085

Gustafsson, M. G. L. (2000). Surpassing the lateral resolution limit by a factor of two using structured illumination microscopy. J. Microsc. 198, 82-87. doi: 10. 1046/j.1365-2818.2000.00710.x

Gustafsson, M. G. L. (2005). Nonlinear structured-illumination microscopy: widefield fluorescence imaging with theoretically unlimited resolution. Proc. Natl. Acad. Sci. U S A 102, 13081-13086. doi: 10.1073/pnas.0406877102

Hallermann, S., Heckmann, M., and Kittel, R. J. (2010a). Mechanisms of short-term plasticity at neuromuscular active zones of Drosophila. HFSP J. 4, 72-84. doi: 10. 2976/1.3338710

Hallermann, S., Kittel, R. J., Wichmann, C., Weyhersmüller, A., Fouquet, W., Mertel, S., et al. (2010b). Naked dense bodies provoke depression. J. Neurosci. 30, 14340-14345. doi: 10.1523/JNEUROSCI.2495-10.2010

Hallermann, S., Pawlu, C., Jonas, P., and Heckmann, M. (2003). A large pool of releasable vesicles in a cortical glutamatergic synapse. Proc. Natl. Acad. Sci. US A 100, 8975-8980. doi: 10.1073/pnas.1432836100

Hallermann, S., and Silver, R. A. (2013). Sustaining rapid vesicular release at active zones: potential roles for vesicle tethering. Trends Neurosci. 36, 185-194. doi: 10. 1016/j.tins.2012.10.001

Harlow, M. L., Ress, D., Stoschek, A., Marshall, R. M., and McMahan, U. J. (2001). The architecture of active zone material at the frog's neuromuscular junction. Nature 409, 479-484. doi: 10.1038/35054000

Haydon, P. G., Henderson, E., and Stanley, E. F. (1994). Localization of individual calcium channels at the release face of a presynaptic nerve terminal. Neuron 13 , 1275-1280. doi: 10.1016/0896-6273(94)90414-6

Heilemann, M., van de Linde, S., Schüttpelz, M., Kasper, R., Seefeldt, B., Mukherjee, A., et al. (2008). Subdiffraction-resolution fluorescence imaging with conventional fluorescent probes. Angew. Chem. Int. Ed Engl. 47, 6172-6176. doi: 10.1002/anie.200802376

Heintzmann, R., and Cremer, C. G. (1999). Laterally modulated excitation microscopy: improvement of resolution by using a diffraction grating. Proc. SPIE 3568, 185-196. doi: 10.1117/12.336833

Hell, S. W. (2007). Far-field optical nanoscopy. Science 316, 1153-1158. doi: 10. $1126 /$ science. 1137395

Hell, S. W. (2009). Microscopy and its focal switch. Nat. Methods 6, 24-32. doi: 10. 1038/nmeth.1291

Hell, S. W., and Wichmann, J. (1994). Breaking the diffraction resolution limit by stimulated emission: stimulated-emission-depletion fluorescence microscopy. Opt. Lett. 19, 780-782. doi: 10.1364/ol.19.000780

Hess, S. T., Girirajan, T. P. K., and Mason, M. D. (2006). Ultra-high resolution imaging by fluorescence photoactivation localization microscopy. Biophys. J. 91, 4258-4272. doi: 10.1529/biophysj.106.091116

Holderith, N., Lorincz, A., Katona, G., Rózsa, B., Kulik, A., Watanabe, M., et al. (2012). Release probability of hippocampal glutamatergic terminals scales with the size of the active zone. Nat. Neurosci. 15, 988-997. doi: 10.1038/ nn. 3137

Holm, T., Klein, T., Löschberger, A., Klamp, T., Wiebusch, G., van de Linde, S., et al. (2014). A blueprint for cost-efficient localization microscopy. Chemphyschem 15, 651-654. doi: 10.1002/cphc.201300739

Hosoi, N., Sakaba, T., and Neher, E. (2007). Quantitative analysis of calciumdependent vesicle recruitment and its functional role at the calyx of held synapse. J. Neurosci. 27, 14286-14298. doi: 10.1523/jneurosci.4122-07. 2007

Indriati, D. W., Kamasawa, N., Matsui, K., Meredith, A. L., Watanabe, M., and Shigemoto, R. (2013). Quantitative localization of $\mathrm{Ca}_{\mathrm{v}} 2.1$ (P/Q-type) voltagedependent calcium channels in Purkinje cells: somatodendritic gradient and distinct somatic coclustering with calcium-activated potassium channels. $J$. Neurosci. 33, 3668-3678. doi: 10.1523/JNEUROSCI.2921-12.2013

Jahn, R., and Fasshauer, D. (2012). Molecular machines governing exocytosis of synaptic vesicles. Nature 490, 201-207. doi: 10.1038/nature11320

Jiao, W., Masich, S., Franzén, O., and Shupliakov, O. (2010). Two pools of vesicles associated with the presynaptic cytosolic projection in Drosophila neuromuscular junctions. J. Struct. Biol. 172, 389-394. doi: 10.1016/j.jsb.2010. 07.007

Jones, S. A., Shim, S.-H., He, J., and Zhuang, X. (2011). Fast, three-dimensional super-resolution imaging of live cells. Nat. Methods 8, 499-508. doi: 10. 1038/nmeth.1605 
Kawasaki, F., Zou, B., Xu, X., and Ordway, R. W. (2004). Active zone localization of presynaptic calcium channels encoded by the cacophony locus of Drosophila. J. Neurosci. 24, 282-285. doi: 10.1523/jneurosci.3553-03.2004

Kempf, C., Staudt, T., Bingen, P., Horstmann, H., Engelhardt, J., Hell, S. W., et al. (2013). Tissue multicolor STED nanoscopy of presynaptic proteins in the calyx of held. PLoS One 8:e62893. doi: 10.1371/journal.pone.0062893

Khuong, T. M., Habets, R. L. P., Kuenen, S., Witkowska, A., Kasprowicz, J., Swerts, J., et al. (2013). Synaptic PI(3,4,5)P3 is required for Syntaxin1A clustering and neurotransmitter release. Neuron 77, 1097-1108. doi: 10.1016/j.neuron.2013. 01.025

Kittel, R. J., Wichmann, C., Rasse, T. M., Fouquet, W., Schmidt, M., Schmid, A., et al. (2006). Bruchpilot promotes active zone assembly, $\mathrm{Ca}^{2+}$ channel clustering and vesicle release. Science 312, 1051-1054. doi: 10.1126/science.1126308

Landis, D. M., Hall, A. K., Weinstein, L. A., and Reese, T. S. (1988). The organization of cytoplasm at the presynaptic active zone of a central nervous system synapse. Neuron 1, 201-209. doi: 10.1016/0896-6273(88)90140-7

Lichtman, J. W., and Conchello, J.-A. (2005). Fluorescence microscopy. Nat. Methods 2, 910-919. doi: 10.1038/nmeth817

Limbach, C., Laue, M. M., Wang, X., Hu, B., Thiede, N., Hultqvist, G., et al. (2011). Molecular in situ topology of Aczonin/Piccolo and associated proteins at the mammalian neurotransmitter release site. Proc. Natl. Acad. Sci. US A 108, E392E401. doi: 10.1073/pnas.1101707108

Liu, K. S. Y., Siebert, M., Mertel, S., Knoche, E., Wegener, S., Wichmann, C., et al. (2011). RIM-binding protein, a central part of the active zone, is essential for neurotransmitter release. Science 334, 1565-1569. doi: 10.1126/science.1212991

Llinás, R., Sugimori, M., and Silver, R. B. (1992). Microdomains of high calcium concentration in a presynaptic terminal. Science 256, 677-679. doi: 10 . 1126/science. 1350109

Löschberger, A., Franke, C., Krohne, G., van de Linde, S., and Sauer, M. (2014). Correlative super-resolution fluorescence and electron microscopy of the nuclear pore complex with molecular resolution. J. Cell Sci. 127, 4351-4355. doi: $10.1242 /$ jcs. 156620

Maglione, M., and Sigrist, S. J. (2013). Seeing the forest tree by tree: superresolution light microscopy meets the neurosciences. Nat. Neurosci. 16, 790-797. doi: 10.1038/nn.3403

Marvin, J. S., Borghuis, B. G., Tian, L., Cichon, J., Harnett, M. T., Akerboom, J., et al. (2013). An optimized fluorescent probe for visualizing glutamate neurotransmission. Nat. Methods 10, 162-170. doi: 10.1038/nmeth.2333

Matz, J., Gilyan, A., Kolar, A., McCarvill, T., and Krueger, S. R. (2010). Rapid structural alterations of the active zone lead to sustained changes in neurotransmitter release. Proc. Natl. Acad. Sci. U S A 107, 8836-8841. doi: 10. 1073/pnas.0906087107

Meinrenken, C. J., Borst, J. G. G., and Sakmann, B. (2002). Calcium secretion coupling at calyx of held governed by nonuniform channel-vesicle topography. J. Neurosci. 22, 1648-1667.

Meyer, L., Wildanger, D., Medda, R., Punge, A., Rizzoli, S. O., Donnert, G., et al. (2008). Dual-color STED microscopy at 30-nm focal-plane resolution. Small 4, 1095-1100. doi: 10.1002/smll.200800055

Moulder, K. L., and Mennerick, S. (2005). Reluctant vesicles contribute to the total readily releasable pool in glutamatergic hippocampal neurons. J. Neurosci. 25, 3842-3850. doi: 10.1523/jneurosci.5231-04.2005

Nägerl, U. V., Willig, K. I., Hein, B., Hell, S. W., and Bonhoeffer, T. (2008). Live-cell imaging of dendritic spines by STED microscopy. Proc. Natl. Acad. Sci. U S A 105, 18982-18987. doi: 10.1073/pnas.0810028105

Nanguneri, S., Flottmann, B., Horstmann, H., Heilemann, M., and Kuner, T. (2012). Three-dimensional, tomographic super-resolution fluorescence imaging of serially sectioned thick samples. PLoS One 7:e38098. doi: 10.1371/journal. pone. 0038098

Neher, E. (1998). Vesicle pools and $\mathrm{Ca}^{2+}$ microdomains: new tools for understanding their roles in neurotransmitter release. Neuron 20, 389-399. doi: 10.1016/s0896-6273(00)80983-6

Neher, E., and Sakaba, T. (2008). Multiple roles of calcium ions in the regulation of neurotransmitter release. Neuron 59, 861-872. doi: 10.1016/j.neuron.2008. 08.019

Owald, D., Fouquet, W., Schmidt, M., Wichmann, C., Mertel, S., Depner, H., et al. (2010). A Syd-1 homologue regulates pre- and postsynaptic maturation in Drosophila. J. Cell Biol. 188, 565-579. doi: 10.1083/jcb.200908055

Owald, D., and Sigrist, S. J. (2009). Assembling the presynaptic active zone. Curr. Opin. Neurobiol. 19, 311-318. doi: 10.1016/j.conb.2009.03.003
Patterson, G., Davidson, M., Manley, S., and Lippincott-Schwartz, J. (2010) Superresolution imaging using single-molecule localization. Annu. Rev. Phys. Chem. 61, 345-367. doi: 10.1146/annurev.physchem.012809. 103444

Peled, E. S., and Isacoff, E. Y. (2011). Optical quantal analysis of synaptic transmission in wild-type and rab3-mutant Drosophila motor axons. Nat. Neurosci. 14, 519-526. doi: 10.1038/nn.2767

Pfenninger, K., Akert, K., Moor, H., and Sandri, C. (1972). The fine structure of freeze-fractured presynaptic membranes. J. Neurocytol. 1, 129-149. doi: 10. 1007/bf01099180

Phillips, G. R., Huang, J. K., Wang, Y., Tanaka, H., Shapiro, L., Zhang, W., et al. (2001). The presynaptic particle web: ultrastructure, composition, dissolution and reconstitution. Neuron 32, 63-77. doi: 10.1016/S0896-6273(01) 00450-0

Rancz, E. A., Ishikawa, T., Duguid, I., Chadderton, P., Mahon, S., and Häusser, M. (2007). High-fidelity transmission of sensory information by single cerebellar mossy fibre boutons. Nature 450, 1245-1248. doi: 10.1038/nature 05995

Rosenmund, C., and Stevens, C. F. (1996). Definition of the readily releasable pool of vesicles at hippocampal synapses. Neuron 16, 1197-1207. doi: 10.1016/s08966273(00)80146-4

Rozov, A., Burnashev, N., Sakmann, B., and Neher, E. (2001). Transmitter release modulation by intracellular $\mathrm{Ca}^{2+}$ buffers in facilitating and depressing nerve terminals of pyramidal cells in layer $2 / 3$ of the rat neocortex indicates a target cell-specific difference in presynaptic calcium dynamics. J. Physiol. 531, 807826. doi: 10.1111/j.1469-7793.2001.0807h.x

Rust, M. J., Bates, M., and Zhuang, X. (2006). Sub-diffraction-limit imaging by stochastic optical reconstruction microscopy (STORM). Nat. Methods 3, 793 795. doi: $10.1038 /$ nmeth929

Sakaba, T., Schneggenburger, R., and Neher, E. (2002). Estimation of quantal parameters at the calyx of held synapse. Neurosci. Res. 44, 343-356. doi: 10. 1016/s0168-0102(02)00174-8

Sätzler, K., Söhl, L. F., Bollmann, J. H., Borst, J. G. G., Frotscher, M., Sakmann, B., et al. (2002). Three-dimensional reconstruction of a calyx of held and its postsynaptic principal neuron in the medial nucleus of the trapezoid body. $J$. Neurosci. 22, 10567-10579.

Sauer, M. (2013). Localization microscopy coming of age: from concepts to biological impact. J. Cell Sci. 126, 3505-3513. doi: 10.1242/jcs.123612

Schermelleh, L., Heintzmann, R., and Leonhardt, H. (2010). A guide to superresolution fluorescence microscopy. J. Cell Biol. 190, 165-175. doi: 10.1083/jcb. 201002018

Scheuss, V., Schneggenburger, R., and Neher, E. (2002). Separation of presynaptic and postsynaptic contributions to depression by covariance analysis of successive EPSCs at the calyx of held synapse. J. Neurosci. 22, 728-739.

Schmidt, H., Brachtendorf, S., Arendt, O., Hallermann, S., Ishiyama, S., Bornschein, G., et al. (2013). Nanodomain coupling at an excitatory cortical synapse. Curr. Biol. 23, 244-249. doi: 10.1016/j.cub.2012.12.007

Schneggenburger, R., and Neher, E. (2005). Presynaptic calcium and control of vesicle fusion. Curr. Opin. Neurobiol. 15, 266-274. doi: 10.1016/j.conb.2005.05. 006

Schneggenburger, R., Sakaba, T., and Neher, E. (2002). Vesicle pools and short-term synaptic depression: lessons from a large synapse. Trends Neurosci. 25, 206-212. doi: 10.1016/s0166-2236(02)02139-2

Sheng, J., He, L., Zheng, H., Xue, L., Luo, F., Shin, W., et al. (2012). Calcium-channel number critically influences synaptic strength and plasticity at the active zone. Nat. Neurosci. 15, 998-1006. doi: 10.1038/nn.3129

Sieber, J. J., Willig, K. I., Kutzner, C., Gerding-Reimers, C., Harke, B., Donnert, G., et al. (2007). Anatomy and dynamics of a supramolecular membrane protein cluster. Science 317, 1072-1076. doi: 10.1126/science.1141727

Siksou, L., Rostaing, P., Lechaire, J.-P., Boudier, T., Ohtsuka, T., Fejtová, A., et al. (2007). Three-dimensional architecture of presynaptic terminal cytomatrix. J. Neurosci. 27, 6868-6877. doi: 10.1523/jneurosci.1773-07.2007

Silver, R. A., Lubke, J., Sakmann, B., and Feldmeyer, D. (2003). High-probability uniquantal transmission at excitatory synapses in barrel cortex. Science 302, 1981-1984. doi: 10.1126/science. 1087160

Sochacki, K. A., Larson, B. T., Sengupta, D. C., Daniels, M. P., Shtengel, G., Hess, H. F., et al. (2012). Imaging the post-fusion release and capture of a vesicle membrane protein. Nat. Commun. 3:1154. doi: 10.1038/ncomms 2158 
Specht, C. G., Izeddin, I., Rodriguez, P. C., El Beheiry, M., Rostaing, P., Darzacq, X., et al. (2013). Quantitative nanoscopy of inhibitory synapses: counting gephyrin molecules and receptor binding sites. Neuron 79, 308-321. doi: 10. 1016/j.neuron.2013.05.013

Stanley, E. F. (1993). Single calcium channels and acetylcholine release at a presynaptic nerve terminal. Neuron 11, 1007-1011. doi: 10.1016/08966273(93)90214-c

Südhof, T. C. (2012). The presynaptic active zone. Neuron 75, 11-25. doi: 10.1016/j. neuron.2012.06.012

Sun, J., Pang, Z. P., Qin, D., Fahim, A. T., Adachi, R., and Südhof, T. C. (2007). A dual- $\mathrm{Ca}^{2+}$-sensor model for neurotransmitter release in a central synapse. Nature 450, 676-682. doi: 10.1038/nature06308

Szule, J. A., Harlow, M. L., Jung, J. H., De-Miguel, F. F., Marshall, R. M., and McMahan, U. J. (2012). Regulation of synaptic vesicle docking by different classes of macromolecules in active zone material. PLoS One 7:e33333. doi: 10. 1371/journal.pone.0033333

Tong, G., and Jahr, C. E. (1994). Multivesicular release from excitatory synapses of cultered hippocampal neurons. Neuron 12, 51-59. doi: 10.1016/08966273(94)90151-1

Tønnesen, J., Katona, G., Rózsa, B., and Nägerl, U. V. (2014). Spine neck plasticity regulates compartmentalization of synapses. Nat. Neurosci. 17, 678-685. doi: 10. 1038/nn.3682

Ulbrich, M. H., and Isacoff, E. Y. (2007). Subunit counting in membrane-bound proteins. Nat. Methods 4, 319-321. doi: 10.1038/nmeth1024

van de Linde, S., Löschberger, A., Klein, T., Heidbreder, M., Wolter, S., Heilemann, M., et al. (2011). Direct stochastic optical reconstruction microscopy with standard fluorescent probes. Nat. Protoc. 6, 991-1009. doi: 10.1038/nprot. 2011.336

Vyleta, N. P., and Jonas, P. (2014). Loose coupling between $\mathrm{Ca}^{2+}$ channels and release sensors at a plastic hippocampal synapse. Science 343, 665-670. doi: 10. 1126/science. 1244811

Wagh, D. A., Rasse, T. M., Asan, E., Hofbauer, A., Schwenkert, I., Dürrbeck, H., et al. (2006). Bruchpilot, a protein with homology to ELKS/CAST, is required for structural integrity and function of synaptic active zones in Drosophila. Neuron 49, 833-844. doi: 10.1016/j.neuron.2006.02.008

Watanabe, S., Punge, A., Hollopeter, G., Willig, K. I., Hobson, R. J., Davis, M. W., et al. (2011). Protein localization in electron micrographs using fluorescence nanoscopy. Nat. Methods 8, 80-84. doi: 10.1038/nmeth.1537

Westphal, V., Rizzoli, S. O., Lauterbach, M. A., Kamin, D., Jahn, R., and Hell, S. W. (2008). Video-rate far-field optical nanoscopy dissects synaptic vesicle movement. Science 320, 246-249. doi: 10.1126/science.11 54228

Weyhersmüller, A., Hallermann, S., Wagner, N., and Eilers, J. (2011). Rapid active zone remodeling during synaptic plasticity. J. Neurosci. 31, 6041-6052. doi: 10. 1523/JNEUROSCI.6698-10.2011

Wichmann, C., and Sigrist, S. J. (2010). The active zone T-bar-a plasticity module? J. Neurogenet. 24, 133-145. doi: 10.3109/01677063.2010.489626
Wilhelm, B. G., Mandad, S., Truckenbrodt, S., Kröhnert, K., Schäfer, C., Rammner, B., et al. (2014). Composition of isolated synaptic boutons reveals the amounts of vesicle trafficking proteins. Science 344, 1023-1028. doi: 10.1126/science. 1252884

Willig, K. I., Rizzoli, S. O., Westphal, V., Jahn, R., and Hell, S. W. (2006). STED microscopy reveals that synaptotagmin remains clustered after synaptic vesicle exocytosis. Nature 440, 935-939. doi: 10.1038/nature04592

Wojcik, S. M., and Brose, N. (2007). Regulation of membrane fusion in synaptic excitation-secretion coupling: speed and accuracy matter. Neuron 55, 11-24. doi: 10.1016/j.neuron.2007.06.013

Wojtowicz, J. M., Marin, L., and Atwood, H. L. (1994). Activity-induced changes in synaptic release sites at the crayfish neuromuscular junction. J. Neurosci. 14, 3688-3703.

Wong, A. B., Jing, Z., Rutherford, M. A., Frank, T., Strenzke, N., and Moser, T. (2013). Concurrent maturation of inner hair cell synaptic $\mathrm{Ca}^{2+}$ influx and auditory nerve spontaneous activity around hearing onset in mice. J. Neurosci. 33, 10661-10666. doi: 10.1523/JNEUROSCI.1215-13.2013

Xu-Friedman, M. A., Harris, K. M., and Regehr, W. G. (2001). Three-dimensional comparison of ultrastructural characteristics at depressing and facilitating synapses onto cerebellar Purkinje cells. J. Neurosci. 21, 6666-6672.

Yang, X., Kaeser-Woo, Y. J., Pang, Z. P., Xu, W., and Südhof, T. C. (2010). Complexin clamps asynchronous release by blocking a secondary $\mathrm{Ca}^{2+}$ sensor via its accessory $\alpha$ helix. Neuron 68, 907-920. doi: 10.1016/j.neuron.2010.11.001

Zhai, R. G., and Bellen, H. J. (2004). The architecture of the active zone in the presynaptic nerve terminal. Physiology (Bethesda) 19, 262-270. doi: 10. 1152/physiol.00014.2004

Zhang, Q., Li, Y., and Tsien, R. (2009). The dynamic control of kiss-and-run and vesicular reuse probed with single nanoparticles. Science 323, 1448-1453. doi: $10.1126 /$ science. 1167373

Zucker, R. S., and Regehr, W. G. (2002). Short-term synaptic plasticity. Annu. Rev. Physiol. 64, 355-405. doi: 10.1146/annurev.physiol.64.092501.114547

Conflict of Interest Statement: The authors declare that the research was conducted in the absence of any commercial or financial relationships that could be construed as a potential conflict of interest.

Received: 30 September 2014; accepted: 07 January 2015; published online: 30 January 2015.

Citation: Ehmann N, Sauer M and Kittel RJ (2015) Super-resolution microscopy of the synaptic active zone. Front. Cell. Neurosci. 9:7. doi: 10.3389/fncel.2015.00007

This article was submitted to the journal Frontiers in Cellular Neuroscience.

Copyright (C) 2015 Ehmann, Sauer and Kittel. This is an open-access article distributed under the terms of the Creative Commons Attribution License (CC BY). The use, distribution and reproduction in other forums is permitted, provided the original author(s) or licensor are credited and that the original publication in this journal is cited, in accordance with accepted academic practice. No use, distribution or reproduction is permitted which does not comply with these terms. 


\title{
Spontaneous vesicle recycling in the synaptic bouton
}

\author{
Sven Truckenbrodt ${ }^{1,2 *}$ and Silvio O. Rizzoli ${ }^{1 *}$ \\ Department of Neuro- and Sensory Physiology, University of Göttingen Medical Center, European Neuroscience Institute, Cluster of Excellence Nanoscale \\ Microscopy and Molecular Physiology of the Brain, Göttingen, Germany \\ 2 International Max Planck Research School for Molecular Biology, Göttingen, Germany
}

\author{
Edited by: \\ Hartmut Schmidt, University of \\ Leipzig, Germany \\ Reviewed by: \\ Annalisa Scimemi, SUNY Albany, \\ USA \\ Pascal Kaeser, Harvard Medical \\ School, USA

\section{*Correspondence:} \\ Sven Truckenbrodt and Silvio 0. \\ Rizzoli, Department of Neuro- and \\ Sensory Physiology, University of \\ Göttingen Medical Center, \\ Humboldtallee 23, 37073 Göttingen, \\ Germany \\ e-mail: strucke@gwdg.de; \\ srizzol@gwdg.de
}

The trigger for synaptic vesicle exocytosis is $\mathrm{Ca}^{2+}$, which enters the synaptic bouton following action potential stimulation. However, spontaneous release of neurotransmitter also occurs in the absence of stimulation in virtually all synaptic boutons. It has long been thought that this represents exocytosis driven by fluctuations in local $\mathrm{Ca}^{2+}$ levels. The vesicles responding to these fluctuations are thought to be the same ones that release upon stimulation, albeit potentially triggered by different $\mathrm{Ca}^{2+}$ sensors. This view has been challenged by several recent works, which have suggested that spontaneous release is driven by a separate pool of synaptic vesicles. Numerous articles appeared during the last few years in support of each of these hypotheses, and it has been challenging to bring them into accord. We speculate here on the origins of this controversy, and propose a solution that is related to developmental effects. Constitutive membrane traffic, needed for the biogenesis of vesicles and synapses, is responsible for high levels of spontaneous membrane fusion in young neurons, probably independent of $\mathrm{Ca}^{2+}$. The vesicles releasing spontaneously in such neurons are not related to other synaptic vesicle pools and may represent constitutively releasing vesicles (CRVs) rather than bona fide synaptic vesicles. In mature neurons, constitutive traffic is much dampened, and the few remaining spontaneous release events probably represent bona fide spontaneously releasing synaptic vesicles (SRSVs) responding to $\mathrm{Ca}^{2+}$ fluctuations, along with a handful of $C R V s$ that participate in synaptic vesicle turnover.

Keywords: spontaneous release, synaptic vesicle recycling, synaptic vesicle pools, synaptic vesicle biogenesis, synapse development

\section{INTRODUCTION: THE PHENOMENON OF SPONTANEOUS RELEASE AT THE SYNAPTIC BOUTON}

Neuronal communication relies on precisely timed synaptic vesicle exocytosis, which is typically triggered by a brief intracellular $\mathrm{Ca}^{2+}$ spike that follows action potentials (Südhof, 2004; Rizzoli, 2014). However, in parallel to stimulated release, all synapses also display spontaneous release of neurotransmitter. This phenomenon has been observed very early on in the history of research in synaptic communication, starting with several landmark papers quantifying synaptic release under physiological conditions, and typically occurs at very low rates of less than $0.1 \mathrm{~Hz}$ (Fatt and Katz, 1950, 1952; Del Castillo and Katz, 1954). This type of release did not receive significant further attention until recently (see reviews by Ramirez and Kavalali, 2011; Andreae and Burrone, 2014; Kaeser and Regehr, 2014; Rizzoli, 2014). The main difference between these two modes of release is that stimulated release is directly coupled to action potentials, while spontaneous release can occur seemingly without any trigger, at any time. Spontaneous release can be increased experimentally, by $\mathrm{Ca}^{2+}$-dependent mechanisms, such as caffeine triggered $\mathrm{Ca}^{2+}$ release from internal stores (Zefirov et al., 2006), as well as by $\mathrm{Ca}^{2+}$-independent mechanisms, including application of lanthanum ions (Heuser and Miledi, 1971), of black widow spider venom, whose main active component is alpha-latrotoxin (Ceccarelli et al., 1972, 1973; Betz and Henkel, 1994), or hyperosmotic sucrose (Rosenmund and Stevens, 1996).

We give here an overview of the phenomenon of spontaneous release, briefly reviewing some of the available evidence on its $\mathrm{Ca}^{2+}$ dependency. We then focus on the intense recent debate on whether active and spontaneous release originate from the same pool of vesicles. We suggest a simple solution to this problem: two completely different pools of vesicles share the synaptic bouton-synaptic vesicles, which can respond to stimulation but also act as spontaneously releasing synaptic vesicles (SRSVs) and constitutively releasing vesicles (CRVs), which are involved in constitutive membrane traffic and which are not responsive to stimulation. The prevailing view that we are following in this review is that exocytosis of synaptic vesicles is mainly $\mathrm{Ca}^{2+}$. dependent, either in response to action potentials or following spontaneous $\mathrm{Ca}^{2+}$ fluctuations. The CRVs only exocytose spontaneously, and may be completely independent of $\mathrm{Ca}^{2+}$. Finally, we show that this hypothesis is in agreement with the potential roles for spontaneously releasing vesicles throughout neuronal development.

\section{THE CALCIUM SENSORS FOR SPONTANEOUS RELEASE}

As indicated above, there are many ways in which spontaneous release can be elicited from synaptic terminals. The machinery 
for synaptic vesicle release is clearly prone to release even in the absence of action potentials, and even in the absence of $\mathrm{Ca}^{2+}$, both external and internal (Rizzoli and Betz, 2002). Whether physiological spontaneous release is $\mathrm{Ca}^{2+}$-dependent has been a matter of debate, and has triggered the search for a specific $\mathrm{Ca}^{2+}$ sensor. Synaptotagmin 1 has been recognized as the major $\mathrm{Ca}^{2+}$ sensor for mediating synaptic release, but mainly in the context of stimulation (Geppert et al., 1994; Südhof, 2004). However, synaptotagmin 1 has also been proposed to facilitate spontaneous release: knock-ins with enhanced apparent $\mathrm{Ca}^{2+}$ affinity increased spontaneous release, while knock-ins with reduced apparent $\mathrm{Ca}^{2+}$ affinity decreased spontaneous release (Xu et al., 2009; but also see Littleton et al., 1993). This is in agreement with the hypothesis that physiological spontaneous release is caused by spontaneous $\mathrm{Ca}^{2+}$ fluctuations. Paradoxically, however, the knock-out of synaptotagmin 1 does lead to a severe increase in spontaneous release (Xu et al., 2009), suggesting that alternative $\mathrm{Ca}^{2+}$ sensors must also play a role in this process. The cytoplasmic high-affinity $\mathrm{Ca}^{2+}$ binding protein Doc2b has instead been proposed as a specialized sensor for spontaneous release (Groffen et al., 2006, 2010). The knock-out of Doc2b leads to a severe decrease in spontaneous release, but leaves stimulated release unaltered (Groffen et al., 2010). Mechanistically, the higher affinity for $\mathrm{Ca}^{2+}$ of Doc2b could make it responsive to small fluctuations in local $\mathrm{Ca}^{2+}$ levels that would be subthreshold for the facilitation of release via synaptotagmin 1, an effect that is mimicked by synaptotagmin 1 mutants with high affinity for $\mathrm{Ca}^{2+}$ (Xu et al., 2009). Such local $\mathrm{Ca}^{2+}$ fluctuations might, however, also be high enough to trigger release via activation of synapotagmin 1 , and Doc $2 b$ might play a different role in spontaneous release, since a $\mathrm{Ca}^{2+}$ binding deficient Doc2b mutant restored spontaneous release in knock-downs (Pang et al., 2011).

\section{DIFFERENT VIEWS ON THE SPONTANEOUS POOL OF SYNAPTIC VESICLES}

The association of the soluble protein Doc2b with the vesicles containing synaptotagmin 1 would render them prone to both spontaneous and stimulated release (see also Walter et al., 2011). Alternatively, vesicles containing only one of these molecules would release only spontaneously, or only during stimulation. Whether the sensor molecules are present on all vesicles is currently unknown. Their large copy numbers, however (about 15 synaptotagmin 1 , and about 10 Doc2 molecules per vesicle, on average; Takamori et al., 2006; Wilhelm et al., 2014), make it probable that most of the bona fide synaptic vesicles are associated with at least a few copies of both.

The debate of whether spontaneous release occurs from the same pool of synaptic vesicles as stimulated release has been complicated by many conflicting data published in recent years (for example, Sara et al., 2005; Groemer and Klingauf, 2007; Mathew et al., 2008; Fredj and Burrone, 2009; Wilhelm et al., 2010). Spontaneous recycling has been recently investigated in neuronal cultures by silencing stimulated activity with tetrodotoxin (TTX), which abolishes action potentials. Under these conditions, synaptic vesicles participating in spontaneous release and recycling can be selectively loaded with membrane dyes, such as the FM dyes.
After washing out the dye, the exocytosis of the FM-loaded vesicles can be monitored by measuring the loss of fluorescence, as the dye is released from the vesicles into the bathing fluid (see Figure 1).

This type of experiment has initially suggested that vesicles loaded with dye under spontaneous conditions could not be induced to unload by stimulation, and, conversely, that synaptic vesicles loaded with dye during stimulation were not released spontaneously, in TTX (Sara et al., 2005). These observations were later verified by further FM dye experiments (Mathew et al., 2008; Chung et al., 2010). Surprisingly, other groups performed the same experiments, under comparable conditions, and found the opposite: namely that the same vesicles could recycle both under spontaneous and stimulated conditions (Groemer and Klingauf, 2007; Wilhelm et al., 2010).

One possible explanation for this controversy was that the FM dye experiments were difficult to analyse, and that the conclusions depended strongly on the methods used for quantification and normalization (Groemer and Klingauf, 2007). Several other types of labeling were therefore employed, ranging from the enzymatic coupling of biotin to synaptic vesicle proteins, to be later detected by fluorophore-coupled streptavidin (Fredj and Burrone, 2009), to synaptotagmin 1 labeling by antibodies recognizing its intravesicular tail (Hua et al., 2010; Wilhelm et al., 2010). These experiments served to deepen the controversy, by providing evidence both for a separate vesicle pool driving spontaneous release (Fredj and Burrone, 2009), and for the opposite, two independent pools (Hua et al., 2010; Wilhelm et al., 2010).

\section{THE ROLE OF SPONTANEOUS RELEASE DURING DEVELOPMENT SUGGESTS A SIMPLE SOLUTION FOR THE PROBLEM OF THE SPONTANEOUS POOL}

How can these conflicting results be reconciled? The key to understanding spontaneous release may lie in its likely role in synaptic biology. Spontaneous release has often been dismissed as a purely stochastic phenomenon, an accidental fusion of synaptic vesicles which should normally only fuse in response to a stimulus. This view is probably incorrect. The machinery of synaptic release has evolved to restrict accidental release of neurotransmitter, with various levels of fail-safe mechanisms to prevent accidental fusion (Jahn and Fasshauer, 2012; Südhof, 2013). At the same time, there is mounting evidence that spontaneous release is far from useless to the neuron, and that it actually has a crucial role in synapse biogenesis, maturation, and maintenance (McKinney et al., 1999; Saitoe et al., 2001; Sutton et al., 2006; Choi et al., 2014; Kaeser and Regehr, 2014).

First of all, we need to consider how synapses and synaptic vesicles are formed. The establishment of synapses entails the transport of various building blocks (for the active zone, the cytoskeleton, synaptic vesicles, and cell-cell adhesion complexes) from the cell soma to the site of the newly forming synapse. These building blocks are transported via various carriers, such as the so called piccolo-bassoon transport vesicles (PTVs) (Zhai et al., 2001; Shapira et al., 2003), or synaptic vesicle protein transport vesicles (STVs) (Sabo et al., 2006). Synaptic vesicles themselves are probably composed of proteins coming from different transport organelles and are later fully assembled in the synaptic bouton 


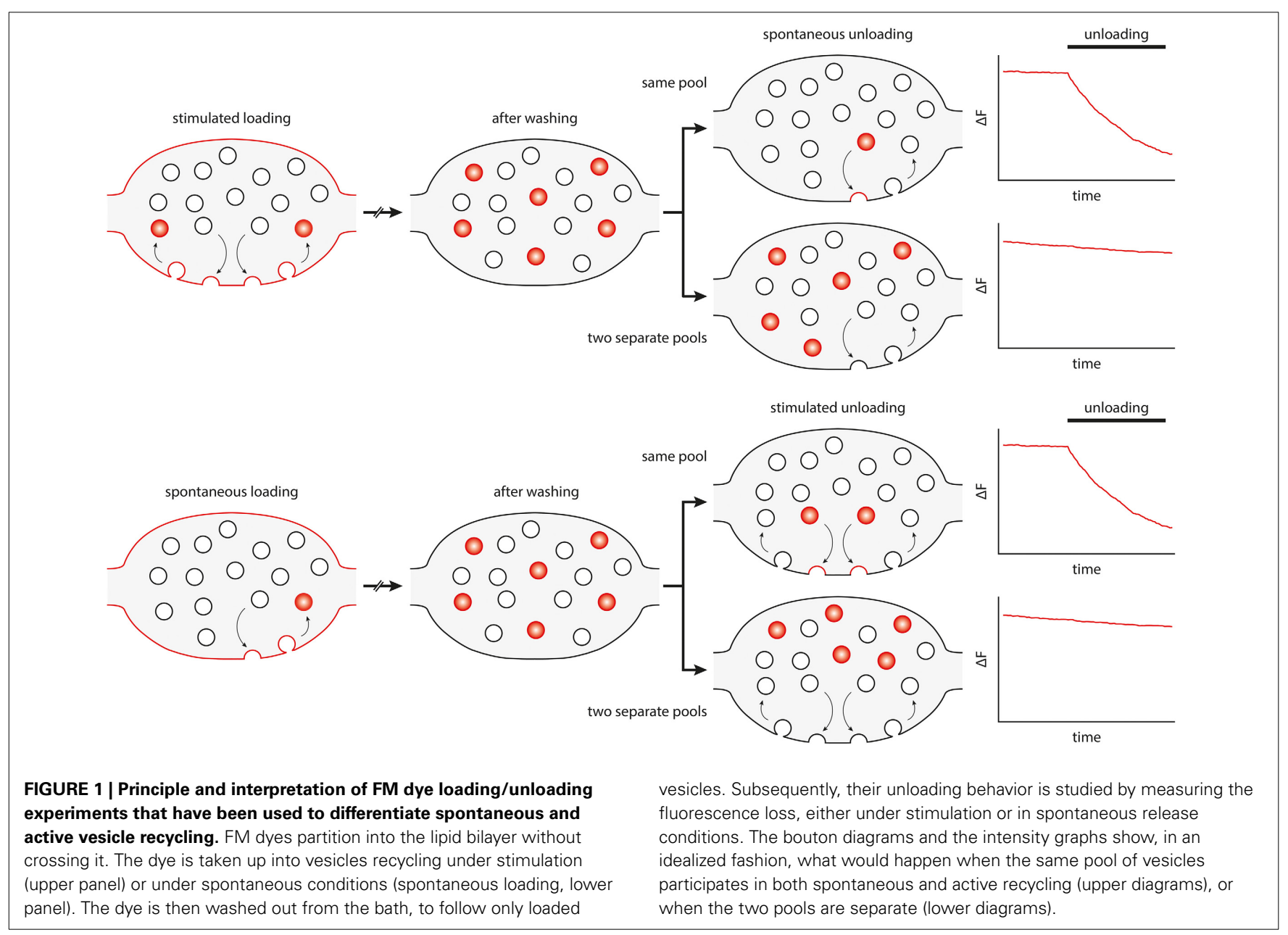

itself (see, for example, Rizzoli, 2014). Although various theories for synapse formation exist (see, for example, Scheiffele, 2003; McAllister, 2007), presynaptic STV clustering and release of neurotransmitter are essential, and may even predate the assembly of most other elements of the synapse (Sabo et al., 2006; see also Kaeser and Regehr, 2014).

Since the highly specific machinery of the active zone and bona fide synaptic vesicles are still missing from the to-be synapse, both neurotransmitter release and building block delivery must be ensured by CRVs. Such organelles are known from all cell types, where they typically participate in the early (sorting) endosome pathway. They have been described in nascent synapses as a heterogeneous population of dense-core, tubulovesicular, and pleomorphic vesicles, ranging in size up to $\sim 80 \mathrm{~nm}$ (Ahmari et al., 2000; Zhai et al., 2001; Ziv and Garner, 2004). This view is consistent with the observation that spontaneous neurotransmitter release is especially prevalent at developing synapses, where it is largely independent of stimulation (Kraszewski et al., 1995; Coco et al., 1998) and accounts for up to $80 \%$ of all release (Andreae et al., 2012). It should be noted in this context that constitutive fusion of vesicles outside active zones, and even outside synapses, where it cannot be stimulated by $\mathrm{Ca}^{2+}$, is a common observation, especially in developing neurons (Hume et al., 1983; Young and Poo, 1983; Matteoli et al., 1992; Zakharenko et al., 1999; Sabo and
McAllister, 2003). Furthermore, spontaneous release and synapse formation are not impaired in knock-outs of synaptotagmin 1, the main $\mathrm{Ca}^{2+}$ sensor of synaptic vesicles responsive to stimulation (Geppert et al., 1994). Additionally, synapse formation is not impaired in Doc2a/b double knock-outs (Groffen et al., 2010). Thus, it is tempting to hypothesize that in developing synapses spontaneously fusing vesicles are not bona fide SRSVs, but CRVs. As the synapses become established, stimulated release becomes predominant, and spontaneous release is dampened, consistent with the reduced need to deliver fresh building blocks to the synapse (Andreae et al., 2012).

The nature of the CRVs is not yet fully clear. A recent study suggested that they contain some synaptic vesicle markers, such as synaptophysin and neurotransmitter transporters, but have significantly lower amounts of some other proteins, most significantly the calcium sensor synaptotagmin 1 , when compared to bona fide synaptic vesicles. At the same time, CRVs were enriched in proteins of endosomal and constitutive trafficking pathways, such as the SNAREs syntaxin 13 and VAMP4 (Revelo et al., 2014). There are at least three different possible explanations for this observation. First, these vesicles might represent an organelle that is, in terms of biogenesis, completely separate from bona fide synaptic vesicles, and is involved in, for example, delivery of membrane to the synaptic bouton (unlikely, since these vesicles do 
contain synaptic vesicle markers). Second, CRVs might represent "rejects" from the synaptic vesicle biogenesis pathway. It is conceivable that biogenesis will not be successful for each and every synaptic vesicle, especially considering the complexity of the trafficking and final assembly steps involved in synaptic vesicle biogenesis (Bonanomi et al., 2006; Rizzoli, 2014). The CRVs might be faulty synaptic vesicles that have the ability to accumulate neurotransmitter (since otherwise there would not be any postsynaptic response to be observed), but without sufficient amounts of $\mathrm{Ca}^{2+}$ sensors for stimulated release, or inhibitors of spontaneous release (synaptotagmin 1, see above). Conversely, the insufficient clearing of constitutive and endosomal trafficking proteins from CRVs could thus enable them to release neurotransmitter independent of any stimulation, in a constitutive manner. Third, CRVs may not be "rejects" of the biogenesis pathway, but rather intermediates in the same pathway. The ability to release spontaneously might represent a transitory stage in the life cycle of a synaptic vesicle, during which it sheds unnecessary proteins, such as those of the constitutive pathway, and enriches synaptic vesicle proteins, via repeated fusion with the plasma membrane and subsequent recycling (Rizzoli, 2014). Thus, synaptic vesicles may fuse spontaneously early during their biogenesis, and stop doing so later, as they lose molecules of the constitutively trafficking pathway, and enrich bona fide synaptic vesicle molecules (Figure 2).

This third model also provides an explanation for the conflicting results obtained by several groups in the FM dye experiments relating to spontaneous release. If the CRV is an intermediate in the synaptic vesicle biogenesis pathway, it is probable that it still lacks some of the proteins necessary for stimulated release. Specifically, CRVs seem to contain lower levels of VAMP2 and synaptotagmin 1 (Revelo et al., 2014). This would render them less competent to respond to stimulation than bona fide synaptic vesicles, or even completely incompetent (see, for example, Rizzoli and Betz, 2002). Since a comparison of seven key studies on spontaneous release makes it evident that virtually identical experimental paradigms often yielded opposing results (Supplementary Table 1), we suggest the following explanation. It is well known that neuronal cultures of the same age can nevertheless have vastly different maturation rates, strongly dependent on, among other details, plating density (Fletcher et al., 1994; Biffi et al., 2013). This varies between laboratories, so despite the similar ages of the cultures, the different studies may have used cultures with different maturation states, and therefore with different rates of constitutive trafficking.

In line with this, studies suggesting one single pool are often based on tools specifically targeting synaptic vesicle proteins, such as VAMP2 or synaptotatmin 1 (see Supplementary Table 1) (Hua et al., 2010; Wilhelm et al., 2010). CRVs seem to contain less of these markers (Revelo et al., 2014), and therefore cannot be efficiently labeled this way, while FM dyes are non-specific and can label CRVs and SRSVs. The SRSVs would be releasable via stimulation, while the CRVs would not. Most studies discerning a separate spontaneous pool are based on FM dyes and find at least some unloading from this pool under stimulation (Sara et al., 2005; Mathew et al., 2008; Chung et al., 2010). The "spontaneous pool" labeled in FM-experiments was thus probably heterogeneously composed of CRVs and SRSVs. CRV prevalence decreases with neuron maturity (Andreae et al., 2012), preventing the detection of a separate spontaneous pool.

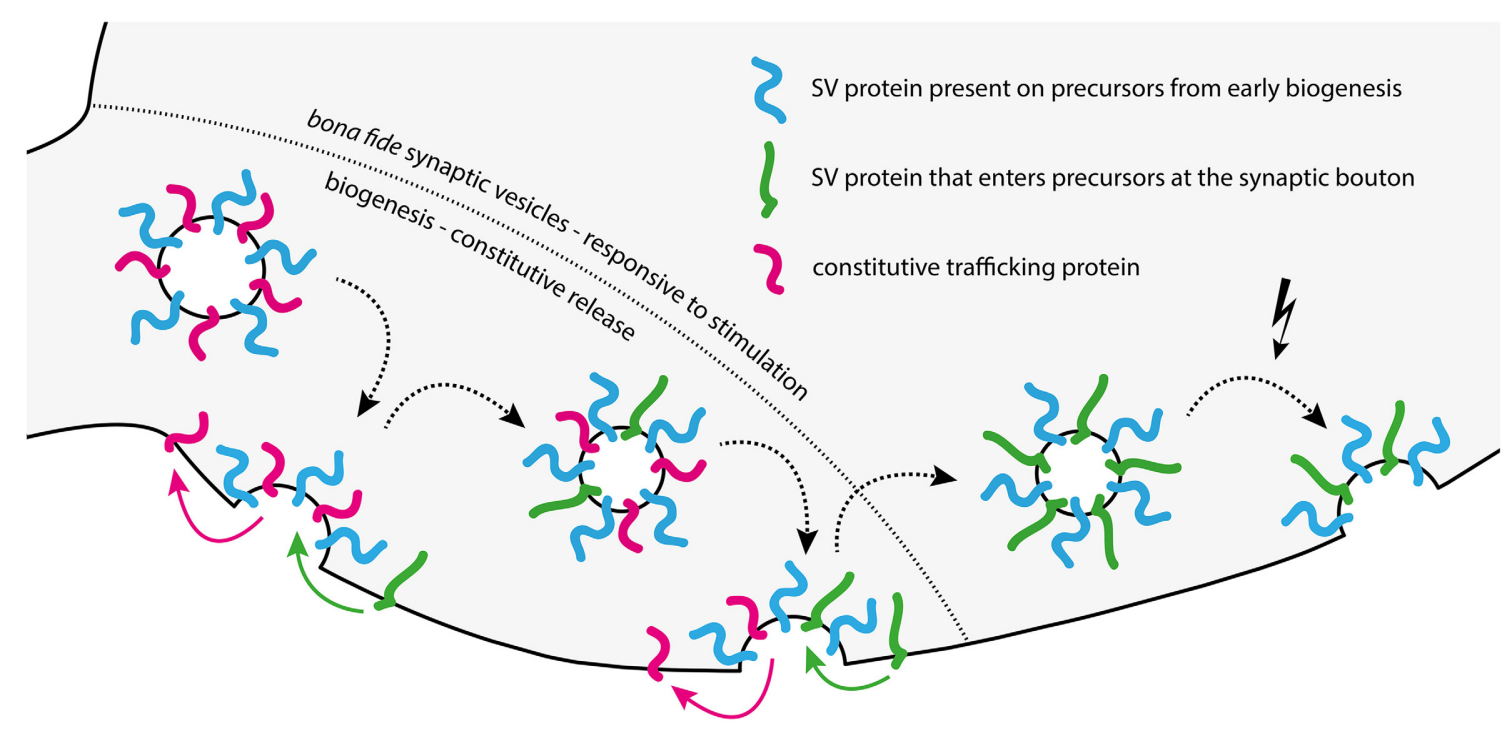

FIGURE 2 | Model of changes in synaptic vesicle composition and release behavior during biogenesis. Biogenesis from left to right: CRVs arriving at the synaptic bouton are molecularly "messy" synaptic vesicle precursors, having a different protein composition than bona fide synaptic vesicles. These precursors release spontaneously using constitutive trafficking proteins left over from biogenesis in the ER/Golgi system. They may lack the machinery necessary to sense and respond to stimulation. After several rounds of spontaneous release and recycling in the synapse, the emerging vesicle is cleared of most constitutive release proteins left over from biogenesis, and takes up additional synaptic vesicle (SV) proteins. With each recycling step during this phase, the vesicle becomes a bit less competent for spontaneous release, but a bit more competent for stimulated release. The end result is a fully formed, bona fide synaptic vesicle with little left over contamination (right of the dashed line). 


\section{MOLECULAR MARKERS OF THE SPONTANEOUSLY RECYCLING VESICLES}

Several recent studies found that vesicles containing the overexpressed SNAREs VAMP7 (Hua et al., 2011; Bal et al., 2013) or Vtila (Ramirez et al., 2012) behaved like CRVs, and were not responsive to stimulation. Since these molecules are classical constitutive trafficking markers, it is not surprising that they reach a type of vesicles that is not equivalent to synaptic vesicles. VAMP7 has been implicated in growth cone development and late endosome/lysosome trafficking (Wang and Tang, 2006; Burgo et al., 2012), while Vtila is known to participate in cis- and transGolgi trafficking (Fischer von Mollard and Stevens, 1998; Ganley et al., 2008). Additionally, a VAMP7/Vtila SNARE complex has been implicated in constitutive exocytosis and potassium channel trafficking to the plasma membrane, both in neuronal and non-neuronal cells (Flowerdew and Burgoyne, 2009).

The experiments in which overexpressed VAMP7 and Vtila reached spontaneously-, but not actively-releasing vesicles, have been interpreted as proof for their being the molecular mechanism that controls the reserve pool of synaptic vesicles (see Rizzoli and Betz, 2005, for a review on synaptic vesicle pools). This largely inert pool of vesicles, which does not recycle under physiological stimulation, was seen as the source of spontaneous release, via SNAREs such as VAMP7 (Fredj and Burrone, 2009; Hua et al., 2011; Bal et al., 2013) or Vtila (Ramirez et al., 2012). In view of more recent evidence, this is highly unlikely. Such molecules are present in mature synapses in very low copy numbers, two to three orders of magnitude lower than the exocytotic SNAREs (Takamori et al., 2006; Wilhelm et al., 2014). Such copy numbers would not allow them to be present in all reserve vesicles (Vtila averages at only $\sim 1$ copy per four vesicles in mature synapses; Wilhelm et al., 2014). Another argument against the hypothesis that VAMP7 and Vtila drive spontaneous release from the reserve pool is that there is very little spontaneous release in mature synaptic boutons (Andreae et al., 2012), although the reserve pool consists of at least $\sim 50 \%$ of all vesicles in mature synapses (Rizzoli, 2014). In contrast, the low copy numbers of SNAREs of the constitutive pathway agree with the hypothesis that spontaneous release is, at least in developing synapses, performed by constitutively recycling vesicles. Since such vesicles are not present in large numbers in mature synapses, neither should be their characteristic SNAREs.

\section{CONCLUSIONS: WHAT IS THE PURPOSE OF SPONTANEOUS RELEASE?}

We suggest that CRVs are, during the early development of the neuron, a pool of constitutively recycling membranes, which participate in the formation of synapses and synaptic vesicles. Putting this in perspective, a recent study determined that synapse development is perturbed by the abolishment of spontaneous, but not stimulated release (Choi et al., 2014). But even the established synapse is critically dependent on spontaneous neurotransmitter release (as recently reviewed by Andreae and Burrone, 2014). Abolishing it will first induce a compensatory receptor synthesis in the postsynapse (Sutton et al., 2006), later a loss of postsynaptic glutamate receptors (Saitoe et al., 2001), and will ultimately result in dissolving the synapse, evidenced by the loss of the dendritic spine (McKinney et al., 1999). Whether these spontaneously releasing vesicles are still members of the CRV pathway, or whether they are mature SRSVs responding to spontaneous $\mathrm{Ca}^{2+}$ fluctuations, perhaps via Doc $2 \mathrm{~b}$, still remains to be determined.

\section{ACKNOWLEDGMENTS}

Sven Truckenbrodt is supported by an Excellence Stipend of the Göttingen Graduate School for Neurosciences, Biophysics, and Molecular Biosciences (GGNB).

\section{SUPPLEMENTARY MATERIAL}

The Supplementary Material for this article can be found online at: http://www.frontiersin.org/journal/10.3389/fncel.2014. 00409/abstract

\section{REFERENCES}

Ahmari, S. E., Buchanan, J., and Smith, S. J. (2000). Assembly of presynaptic active zones from cytoplasmic transport packets. Nat. Neurosci. 3, 445-451. doi: $10.1038 / 74814$

Andreae, L. C., and Burrone, J. (2014). The role of neuronal activity and transmitter release on synapse formation. Curr. Opin. Neurobiol. 27C, 47-52. doi: 10.1016/j.conb.2014.02.008

Andreae, L. C., Fredj, N. B., and Burrone, J. (2012). Independent vesicle pools underlie different modes of release during neuronal development. J. Neurosci. 32, 1867-1874. doi: 10.1523/JNEUROSCI.5181-11.2012

Bal, M., Leitz, J., Reese, A. L., Ramirez, D. M. O., Durakoglugil, M., Herz, J., et al. (2013). Reelin mobilizes a VAMP7-dependent synaptic vesicle pool and selectively augments spontaneous neurotransmission. Neuron $80,934-946$. doi: 10.1016/j.neuron.2013.08.024

Betz, W. J., and Henkel, A. W. (1994). Okadaic acid disrupts clusters of synaptic vesicles in frog motor nerve terminals. J. Cell Biol. 124, 843-854. doi: 10.1083/jcb.124.5.843

Biffi, E., Regalia, G., Menegon, A., Ferrigno, G., and Pedrocchi, A. (2013). The influence of neuronal density and maturation on network activity of hippocampal cell cultures: a methodological study. PLOS ONE 8:e83899. doi: 10.1371/journal.pone.0083899

Bonanomi, D., Benfenati, F., and Valtorta, F. (2006). Protein sorting in the synaptic vesicle life cycle. Prog. Neurobiol. 80, 177-217. doi: 10.1016/j.pneurobio.2006.09.002

Burgo, A., Proux-Gillardeaux, V., Sotirakis, E., Bun, P., Casano, A., Verraes, A., et al. (2012). A molecular network for the transport of the TIVAMP/VAMP7 vesicles from cell center to periphery. Dev. Cell 23, 166-180. doi: 10.1016/j.devcel.2012.04.019

Ceccarelli, B., Hurlbut, W. P., and Mauro, A. (1973). Turnover of transmitter and synaptic vesicles at the frog neuromuscular junction. J. Cell Biol. 57, 499-524. doi: $10.1083 /$ jcb.57.2.499

Ceccarelli, B., Hurlbut, W. P., and Mauro, A. (1972). Depletion of vesicles from frog neuromuscular junctions by prolonged tetanic stimulation. J. Cell Biol. 54, 30-38. doi: 10.1083/jcb.54.1.30

Choi, B. J., Imlach, W. L., Jiao, W., Wolfram, V., Wu, Y., Grbic, M., et al. (2014). Miniature neurotransmission regulates Drosophila synaptic structural maturation. Neuron 82, 618-634. doi: 10.1016/j.neuron.2014.03.012

Chung, C., Barylko, B., Leitz, J., Liu, X., and Kavalali, E. T. (2010). Acute dynamin inhibition dissects synaptic vesicle recycling pathways that drive spontaneous and evoked neurotransmission. J. Neurosci. 30, 1363-1376. doi: 10.1523/JNEUROSCI.3427-09.2010

Coco, S., Verderio, C., De Camilli, P., and Matteoli, M. (1998). Calcium dependence of synaptic vesicle recycling before and after synaptogenesis. J. Neurochem. 71, 1987-1992. doi: 10.1046/j.1471-4159.1998.71051987.x

Del Castillo, J., and Katz, B. (1954). Quantal components of the end-plate potential. J. Physiol. 124, 560-573.

Fatt, B. Y. P., and Katz, B. (1950). Some observations on biological noise. Nature 166, 597-598. doi: 10.1038/166597a0

Fatt, B. Y. P., and Katz, B. (1952). Spontaneous subthreshold activity at motor nerve endings. J. Physiol. 117, 109-128. 
Fischer von Mollard, G., and Stevens, T. H. (1998). A human homolog can functionally replace the yeast vesicle-associated SNARE Vtilp in two vesicle transport pathways. J. Biol. Chem. 273, 2624-2630. doi: 10.1074/jbc.273.5.2624

Fletcher, L., De Camilli, P., and Banker, G. (1994). Synaptogenesis in hippocampal cultures: evidence indicating that axons and dendrites become competent to form synapses at different stages of neuronal development. J. Neurosci. 14, 6695-6706.

Flowerdew, S. E., and Burgoyne, R. D. (2009). A VAMP7/Vtila SNARE complex distinguishes a non-conventional traffic route to the cell surface used by KChIP1 and Kv4 potassium channels. Biochem. J. 418, 529-540. doi: 10.1042/BJ20081736

Fredj, N. B., and Burrone, J. (2009). A resting pool of vesicles is responsible for spontaneous vesicle fusion at the synapse. Nat. Neurosci. 12, 751-758. doi: $10.1038 / \mathrm{nn} .2317$

Ganley, I. G., Espinosa, E., and Pfeffer, S. R. (2008). A syntaxin 10-SNARE complex distinguishes two distinct transport routes from endosomes to the trans-Golgi in human cells. J. Cell Biol. 180, 159-172. doi: 10.1083/jcb.200707136

Geppert, M., Goda, Y., Hammer, R. E., Li, C., Rosahl, T. W., Stevens, C. F., et al. (1994). Synaptotagmin I: a major Ca2+ sensor for transmitter release at a central synapse. Cell 79, 717-727. doi: 10.1016/0092-8674(94)90556-8

Groemer, T. W., and Klingauf, J. (2007). Synaptic vesicles recycling spontaneously and during activity belong to the same vesicle pool. Nat. Neurosci. 10, 145-147. doi: $10.1038 / \mathrm{nn} 1831$

Groffen, A. J., Friedrich, R., Brian, E. C., Ashery, U., and Verhage, M. (2006). DOC2A and DOC2B are sensors for neuronal activity with unique calcium-dependent and kinetic properties. J. Neurochem. 97, 818-833. doi: 10.1111/j.1471-4159.2006.03755.x

Groffen, A. J., Martens, S., Díez Arazola, R., Cornelisse, L. N., Lozovaya, N., de Jong, A. P. H., et al. (2010). Doc2b is a high-affinity Ca2+ sensor for spontaneous neurotransmitter release. Science 327, 1614-1618. doi: 10.1126/science. 1183765

Heuser, J., and Miledi, R. (1971). Effect of lanthanum ions on function and structure of frog neuromuscular junctions. Proc. R. Soc. Lond. B Biol. Sci. 179, 247-260. doi: 10.1098/rspb.1971.0096

Hua, Y., Sinha, R., Martineau, M., Kahms, M., and Klingauf, J. (2010). A common origin of synaptic vesicles undergoing evoked and spontaneous fusion. Nat. Neurosci. 13, 1451-1453. doi: 10.1038/nn.2695

Hua, Z., Leal-Ortiz, S., Foss, S. M., Waites, C. L., Garner, C. C., Voglmaier, S. M., et al. (2011). v-SNARE composition distinguishes synaptic vesicle pools. Neuron 71, 474-487. doi: 10.1016/j.neuron.2011.06.010

Hume, R. I., Role, L. W., and Fischbach, G. D. (1983). Acetylcholine release from growth cones detected with patches of acetylcholine receptor-rich membranes. Nature 305, 632-634. doi: 10.1038/305632a0

Jahn, R., and Fasshauer, D. (2012). Molecular machines governing exocytosis of synaptic vesicles. Nature 490, 201-207. doi: 10.1038/nature11320

Kaeser, P. S., and Regehr, W. G. (2014). Molecular mechanisms for synchronous, asynchronous, and spontaneous neurotransmitter release. Annu. Rev. Physiol. 76, 333-363. doi: 10.1146/annurev-physiol-021113-170338

Kraszewski, K., Mundigl, O., Daniell, L., Verderio, C., Matteoli, M., and De Camilli, P. (1995). Synaptic vesicle dynamics in living cultured hippocampal neurons visualized with CY3-conjugated antibodies directed against the lumenal domain of synaptotagmin. J. Neurosci. 15, 4328-4342.

Littleton, J. T., Stern, M., Schuize, K., Perin, M., and Bellen, H. J. (1993). Mutational analysis of Drosophila synaptotagmin demonstrates its essential role in Ca2+activated neurotransmitter release. Cell 74, 1125-1134.

Mathew, S. S., Pozzo-Miller, L., and Hablitz, J. J. (2008). Kainate modulates presynaptic GABA release from two vesicle pools. J. Neurosci. 28, 725-731. doi: 10.1523/JNEUROSCI.3625-07.2008

Matteoli, M., Takei, K., Perin, M. S., Südhof, T. C., and De Camilli, P. (1992). Exoendocytotic recycling of synaptic vesicles in developing processes of cultured hippocampal neurons. J. Cell Biol. 117, 849-861. doi: 10.1083/jcb.117.4.849

McAllister, A. K. (2007). Dynamic aspects of synapse formation. Annu. Rev. Neurosci. 30, 425-450. doi: 10.1146/annurev.neuro.29.051605.112830

McKinney, R. A., Capogna, M., Dürr, R., Gähwiler, B. H., and Thompson, S. M. (1999). Miniature synaptic events maintain dendritic spines via AMPA receptor activation. Nat. Neurosci. 2, 44-49. doi: 10.1038/4548

Pang, Z. P., Bacaj, T., Yang, X., Zhou, P., Xu, W., and Südhof, T. C. (2011). Doc2 supports spontaneous synaptic transmission by a $\mathrm{Ca}(2+)$-independent mechanism. Neuron 70, 244-251. doi: 10.1016/j.neuron.2011.03.011
Ramirez, D. M. O., and Kavalali, E. T. (2011). Differential regulation of spontaneous and evoked neurotransmitter release at central synapses. Curr. Opin. Neurobiol. 21, 275-282. doi: 10.1016/j.conb.2011.01.007

Ramirez, D. M. O., Khvotchev, M., Trauterman, B., and Kavalali, E. T. (2012). Vtila identifies a vesicle pool that preferentially recycles at rest and maintains spontaneous neurotransmission. Neuron 73, 121-134. doi: 10.1016/j.neuron.2011.10.034

Revelo, N. H., Kamin, D., Truckenbrodt, S., Wong, A. B., Reuter-Jessen, K., Reisinger, E., et al. (2014). A new probe for super-resolution imaging of membranes elucidates trafficking pathways. J. Cell Biol. 205, 591-606. doi: $10.1083 /$ jcb. 201402066

Rizzoli, S. O. (2014). Synaptic vesicle recycling: steps and principles. EMBO J. 33, 788-822. doi: 10.1002/embj.201386357

Rizzoli, S. O., and Betz, W. J. (2002). Effects of 2-(4-morpholinyl)-8-phenyl-4H1-benzopyran-4-one on synaptic vesicle cycling at the frog neuromuscular junction. J. Neurosci. 22, 10680-10689.

Rizzoli, S. O., and Betz, W. J. (2005). Synaptic vesicle pools. Nat. Rev. Neurosci. 6, 57-69. doi: 10.1038/nrn1583

Rosenmund, C., and Stevens, C. F. (1996). Definition of the readily releasable pool of vesicles at hippocampal synapses. Neuron 16, 1197-1207. doi: 10.1016/S08966273(00)80146-4

Sabo, S. L., and McAllister, A. K. (2003). Mobility and cycling of synaptic protein-containing vesicles in axonal growth cone filopodia. Nat. Neurosci. 6, 1264-1269. doi: 10.1038/nn1149

Sabo, S. L., Gomes, R. A., and McAllister, A. K. (2006). Formation of presynaptic terminals at predefined sites along axons. J. Neurosci. 26, 10813-10825. doi: 10.1523/JNEUROSCI.2052-06.2006

Saitoe, M., Schwarz, T. L., Umbach, J. A., Gundersen, C. B., and Kidokoro, Y. (2001). Absence of junctional glutamate receptor clusters in Drosophila mutants lacking spontaneous transmitter release. Science 293, 514-517. doi: 10.1126/science. 1061270

Sara, Y., Virmani, T., Deák, F., Liu, X., and Kavalali, E. T. (2005). An isolated pool of vesicles recycles at rest and drives spontaneous neurotransmission. Neuron 45, 563-573. doi: 10.1016/j.neuron.2004.12.056

Scheiffele, P. (2003). Cell-cell signaling during synapse formation in the CNS. Annu. Rev. Neurosci. 26, 485-508. doi: 10.1146/annurev.neuro.26.043002. 094940

Shapira, M., Zhai, R. G., Dresbach, T., Bresler, T., Torres, V. I., Gundelfinger, E. D., et al. (2003). Unitary assembly of presynaptic active zones from Piccolo-Bassoon transport vesicles. Neuron 38, 237-252. doi: 10.1016/S0896-6273(03)00207-1

Südhof, T. C. (2004). The synaptic vesicle cycle. Annu. Rev. Neurosci. 27, 509-547. doi: 10.1146/annurev.neuro.26.041002.131412

Südhof, T. C. (2013). Neurotransmitter release: the last millisecond in the life of a synaptic vesicle. Neuron 80, 675-690. doi: 10.1016/j.neuron.2013.10.022

Sutton, M. A., Ito, H. T., Cressy, P., Kempf, C., Woo, J. C., and Schuman, E. M. (2006). Miniature neurotransmission stabilizes synaptic function via tonic suppression of local dendritic protein synthesis. Cell 125, 785-799. doi: 10.1016/j.cell.2006.03.040

Takamori, S., Holt, M., Stenius, K., Lemke, E. A., Grønborg, M., Riedel, D., et al. (2006). Molecular anatomy of a trafficking organelle. Cell 127, 831-846. doi: 10.1016/j.cell.2006.10.030

Walter, A. M., Groffen, A. J., Sørensen, J. B., and Verhage, M. (2011). Multiple Ca2+ sensors in secretion: teammates, competitors or autocrats? Trends Neurosci. 34, 487-497. doi: 10.1016/j.tins.2011.07.003

Wang, Y., and Tang, B. L. (2006). SNAREs in neurons-beyond synaptic vesicle exocytosis (Review). Mol. Membr. Biol. 23, 377-384. doi: 10.1080/0968786060 0776734

Wilhelm, B. G., Groemer, T. W., and Rizzoli, S. O. (2010). The same synaptic vesicles drive active and spontaneous release. Nat. Neurosci. 13, 1454-1456. doi: 10.1038/nn.2690

Wilhelm, B. G., Mandad, S., Truckenbrodt, S., Krohnert, K., Schafer, C., Rammner, B., et al. (2014). Composition of isolated synaptic boutons reveals the amounts of vesicle trafficking proteins. Science 344, 1023-1028. doi: 10.1126/science. 1252884

Xu, J., Pang, Z. P., Shin, O.-H., and Südhof, T. C. (2009). Synaptotagmin-1 functions as a Ca2+ sensor for spontaneous release. Nat. Neurosci. 12, 759-766. doi: $10.1038 / \mathrm{nn} .2320$

Young, S. H., and Poo, M. (1983). Spontaneous release of transmitter from growth cones of embryonic neurones. Nature 305, 634-637. doi: 10.1038/305634a0 
Zakharenko, S., Chang, S., O'Donoghue, M., and Popo, S. V. (1999). Neurotransmitter sectretion along growing nerve processes: comparison with synaptic vesicle exocytosis. J. Cell Biol. 144, 507-518.

Zefirov, A. L., Abdrakhmanov, M. M., Mukhamedyarov, M. A., and Grigoryev, P. N. (2006). The role of extracellular calcium in exo- and endocytosis of synaptic vesicles at the frog motor nerve terminals. Neuroscience 143, 905-910. doi: 10.1016/j.neuroscience.2006.08.025

Zhai, R. G., Vardinon-Friedman, H., Cases-Langhoff, C., Becker, B., Gundelfinger, E. D., Ziv, N. E., et al. (2001). Assembling the presynaptic active zone: a characterization of an active one precursor vesicle. Neuron 29, 131-143. doi: 10.1016/S0896-6273(01)00185-4

Ziv, N. E., and Garner, C. C. (2004). Cellular and molecular mechanisms of presynaptic assembly. Nat. Rev. Neurosci. 5, 385-399. doi: 10.1038/ nrn1370
Conflict of Interest Statement: The authors declare that the research was conducted in the absence of any commercial or financial relationships that could be construed as a potential conflict of interest.

Received: 24 September 2014; accepted: 11 November 2014; published online: 08 December 2014.

Citation: Truckenbrodt S and Rizzoli SO (2014) Spontaneous vesicle recycling in the synaptic bouton. Front. Cell. Neurosci. 8:409. doi: 10.3389/fncel.2014.00409

This article was submitted to the journal Frontiers in Cellular Neuroscience.

Copyright $\odot 2014$ Truckenbrodt and Rizzoli. This is an open-access article distributed under the terms of the Creative Commons Attribution License (CC BY). The use, distribution or reproduction in other forums is permitted, provided the original author(s) or licensor are credited and that the original publication in this journal is cited, in accordance with accepted academic practice. No use, distribution or reproduction is permitted which does not comply with these terms. 


\section{OPEN ACCESS}

Edited by:

Christian D. Wilms,

University College London, UK

Reviewed by:

Khursheed Wani,

University of Michigan, USA

Cheng-Chang Lien,

National Yang-Ming University, Taiwan

*Correspondence:

Ákos Kulik,

Institute of Physiology, University of Freiburg, Hermann Herder Straße

7, D-79104 Freiburg, Germany akos.kulik@physiologie.uni-freiburg.de

Received: 15 May 2015 Accepted: 28 July 2015

Published: 11 August 2015

Citation

Althof D, Baehrens D, Watanabe M, Suzuki N, Fakler B and Kulik Á (2015)

Inhibitory and excitatory axon terminals share a common nano-architecture of their Cav2.1 (P/Q-type) $\mathrm{Ca}^{2+}$ channels.

Front. Cell. Neurosci. 9:315 doi: 10.3389/fncel.2015.00315

\section{Inhibitory and excitatory axon terminals share a common nano-architecture of their $\mathrm{Ca}_{\mathrm{v}} 2.1$ (P/Q-type) $\mathrm{Ca}^{2+}$ channels}

\author{
Daniel Althof', David Baehrens ${ }^{1}$, Masahiko Watanabe ${ }^{2}$, Noboru Suzuki $^{3}$, Bernd Fakler $^{1,4}$ \\ and Ákos Kulik ${ }^{1,4 *}$
}

${ }^{1}$ Institute of Physiology, University of Freiburg, Freiburg, Germany, ${ }^{2}$ Department of Anatomy, Graduate School of Medicine, Hokkaido University, Sapporo, Japan, ${ }^{3}$ Department of Animal Genomics, Functional Genomics Institute, Mie University, Mie, Japan, ${ }^{4}$ Centre for Biological Signalling Studies, University of Freiburg, Freiburg, Germany

Tuning of the time course and strength of inhibitory and excitatory neurotransmitter release is fundamental for the precise operation of cortical network activity and is controlled by $\mathrm{Ca}^{2+}$ influx into presynaptic terminals through the high voltageactivated P/Q-type $\mathrm{Ca}^{2+}\left(\mathrm{Ca}_{v} 2.1\right)$ channels. Proper channel-mediated $\mathrm{Ca}^{2+}$-signaling critically depends on the topographical arrangement of the channels in the presynaptic membrane. Here, we used high-resolution SDS-digested freeze-fracture replica immunoelectron microscopy together with automatized computational analysis of Cav2.1 immunogold labeling to determine the precise subcellular organization of Cav2.1 channels in both inhibitory and excitatory terminals. Immunoparticles labeling the pore-forming $\alpha 1$ subunit of $\mathrm{Ca}_{v} 2.1$ channels were enriched over the active zone of the boutons with the number of channels (3-62) correlated with the area of the synaptic membrane. Detailed analysis showed that $\mathrm{Ca}_{v} 2.1$ channels are non-uniformly distributed over the presynaptic membrane specialization where they are arranged in clusters of an average five channels per cluster covering a mean area with a diameter of about $70 \mathrm{~nm}$. Importantly, clustered arrangement and cluster properties did not show any significant difference between GABAergic and glutamatergic terminals. Our data demonstrate a common nano-architecture of $\mathrm{Ca}_{v} 2.1$ channels in inhibitory and excitatory boutons in stratum radiatum of the hippocampal CA1 area suggesting that the cluster arrangement is crucial for the precise release of transmitters from the axonal boutons.

Keywords: $\mathrm{Ca}^{2+}$ channels, quantitative immunoelectron microscopy, cluster analysis, rat, hippocampus

\section{Introduction}

A balance between inhibitory and excitatory synaptic transmission is essential for the normal functioning of cortical neuronal circuits. The net effect of synaptic inhibition and excitation is determined by the firing properties of inhibitory GABAergic and excitatory glutamatergic cells as well as by the release dynamics of GABA- and glutamate-filled vesicles. The transmitter release is primarily triggered by $\mathrm{Ca}^{2+}$ influx through voltage-gated $\mathrm{Ca}^{2+}\left(\mathrm{Ca}_{\mathrm{v}}\right)$ channels $($ Clapham, 2007) that are 
activated by action potentials and/or sub-threshold depolarizing signals (Schneggenburger and Neher, 2005; Nadkarni et al., 2010). Consequently, number, density and spatial relationship of $\mathrm{Ca}_{\mathrm{V}}$ channels relative to the active zone of the presynaptic boutons, the actual locus of vesicle fusion, are assumed to be crucial factors in fine-tuning the temporal precision of transmitter release (Eggermann et al., 2012; Scimemi and Diamond, 2012; Sheng et al., 2012). At fast mammalian central synapses the subfamily two $\mathrm{Ca}_{\mathrm{v}}$ channels, $\mathrm{Ca}_{\mathrm{v}} 2.1$ (P/Q-type) and $\mathrm{Ca}_{\mathrm{v}} 2.2$ (N-type), are essential for coupling the presynaptic action potential to transmitter release (Wu and Saggau, 1994; Stevens, 2004; Cao and Tsien, 2010; Ariel et al., 2013) thus controlling the efficacy of transmission (Poncer et al., 1997; Catterall and Few, 2008; Lipscombe et al., 2013).

In the CA1 area of the hippocampus, pyramidal cells are under the control of inhibitory GABAergic and excitatory glutamatergic cells. GABAergic inputs originating mainly from local interneurons, controlling the firing rate of pyramidal cells and modulate their spike timing as well as synchronize their activity (Klausberger, 2009). In contrast, glutamatergic inputs arriving predominantly from pyramidal cells in CA3 and entorhinal cortex carry predictions based on memory recall and sensory information, respectively (Lisman, 1999; Otmakhova and Lisman, 2004). Thus, inhibitory and excitatory projections, targeting different subcellular domains of the CA1 principal cells, exert distinct effects on concerted and synchronous activities of hippocampal neurons and overall on rhythmic brain activities by released GABA and glutamate. The amount and kinetics of neurotransmitter release related to the intracellular $\mathrm{Ca}^{2+}$ concentration $\left(\left[\mathrm{Ca}^{2+}\right]_{\mathrm{i}}\right)$ needs to be tightly regulated in terminals by $\mathrm{Ca}^{2+}$ entry through $\mathrm{Ca}_{\mathrm{v}}$ channels, as even small changes in presynaptic $\mathrm{Ca}^{2+}$ influx lead to large changes in vesicle release and neurotransmission (Frank, 2014). This raises the question of whether hippocampal inhibitory and excitatory synapses are similar or fundamentally different regarding the subcellular organization of the $\mathrm{Ca}_{\mathrm{v}}$ channels. The $\mathrm{Ca}^{2+}$ dependent synchronous release of neurotransmitters require the concerted compliance of various functionally interacting proteins forming the $\mathrm{Ca}_{\mathrm{v}} 2$ channel-associated networks, termed nanoenvironment (Müller et al., 2010), in presynaptic compartments. The spatial arrangements of these specific proteins may determine the two-dimensional distribution pattern of $\mathrm{Ca}_{\mathrm{v}} 2$ channels in membrane segments of axonal boutons thereby placing the channel at a position optimal for triggering the release machinery. Although, recent functional studies achieved substantial progress in localizing $\mathrm{Ca}_{\mathrm{v}} 2$ channels in cortical inhibitory (Bucurenciu et al., 2008; Kisfali et al., 2013) and excitatory (Kulik et al., 2004; Holderith et al., 2012; Parajuli et al., 2012; Indriati et al., 2013; Baur et al., 2015) synapses as well as at the calyx of Held (Nakamura et al., 2015), qualitative and quantitative comparison of $\mathrm{Ca}_{\mathrm{v}} 2.1$ channel topographical arrangement in small presynaptic boutons in the CA1 area of the hippocampus remained unresolved.

Here, we combined the high-resolution sodium dodecyl sulfate-digested freeze-fracture replica labeling (SDS-FRL) immunoelectron microscopy with automatized computational cluster analysis of immunoreactivity to determine the number and the spatial distribution profile of $\mathrm{Ca}_{\mathrm{v}} 2.1$ channels in terminals of both GABAergic and glutamatergic cells in the stratum radiatum of the hippocampal CA1 region.

\section{Materials and Methods}

\section{Sodium Dodecyl Sulfate-Digested Freeze-Fracture Replica Immunolabeling (SDS-FRL) and Electron Microscopy \\ Immunolabeling}

For the current study 6-week-old male Wistar rats $(n=6)$, one adult male $\mathrm{Ca}_{\mathrm{v}} 2.1$ knock-out (ko) mouse, and one adult male wild type (wt) mouse were used. The perfusion of the animals and preparation of tissues and replicas for SDS-FRL were performed as described previously (Kulik et al., 2006; Masugi-Tokita and Shigemoto, 2007). Care and handling of the animals prior to and during the experimental procedures followed European Union regulations and was approved by the Animal Care and Use Committees of the authors' institutions. Animals were anesthetized with sodium pentobarbital $(50 \mathrm{mg} / \mathrm{kg}$, i.p.), and the hearts were surgically exposed for perfusion fixation. First, the vascular system was flushed by $25 \mathrm{mM}$ phosphatebuffered saline (PBS) followed by transcardial perfusion with a fixative containing $2 \%$ paraformaldehyde (Merck, Germany) and $15 \%$ saturated picric acid made up in $0.1 \mathrm{M}$ phosphate buffer (PB). Sagittal sections from the CA1 area were cut on a microslicer at a thickness of $110 \mu \mathrm{m}$. The slices were cryoprotected in a solution containing 30\% glycerol made up in $0.1 \mathrm{M} \mathrm{PB}$ and then frozen by a high-pressure freezing machine (HPM 100, Leica, Austria). Frozen samples were inserted into a double replica table and then fractured into two pieces at $-130^{\circ} \mathrm{C}$. Fractured faces were replicated by deposition of carbon (5 $\mathrm{nm}$ thickness), platinum (2 nm), and carbon $(18 \mathrm{~nm})$ in a freeze-fracture replica machine (BAF 060, BAL-TEC, Lichtenstein). They were digested in a solution containing $2.5 \%$ SDS and $20 \%$ sucrose made up in $15 \mathrm{mM}$ Tris buffer (TB), $\mathrm{pH} 8.3$, at $80^{\circ} \mathrm{C}$ for $18 \mathrm{~h}$. Replicas were washed in $50 \mathrm{mM}$ Tris-buffered saline (TBS) containing $0.05 \%$ BSA (Roth, Germany) and $0.1 \%$ Tween 20 (Tw20, Roth) and then incubated in a blocking solution (5\% BSA) and then in mixtures of primary antibodies: (i) $\mathrm{Ca}_{\mathrm{v}} 2.1$ [Guinea pig (Gp), $5 \mu \mathrm{g} / \mathrm{ml}$ ] and RIM1/2 [Rabbit (Rb), $1 \mu \mathrm{g} / \mathrm{ml}$; Synaptic System, Göttingen, Germany], (ii) vesicular GABA transporter (VGAT, Gp, $4.5 \mu \mathrm{g} / \mathrm{ml}$ ) and $\mathrm{Ca}_{\mathrm{v}} 2.1$ (Rb, $1 \mu \mathrm{g} / \mathrm{ml}$; Synaptic System, Göttingen), (iii) vesicular glutamate transporter-1 (VGLUT-1, $\mathrm{Rb}, 6 \mu \mathrm{g} / \mathrm{ml}$ ) and $\mathrm{Ca}_{\mathrm{v}} 2.1$ (Gp, $5 \mu \mathrm{g} / \mathrm{ml}$ ), (iv) VGAT (Gp, $4.5 \mu \mathrm{g} / \mathrm{ml}$ ) and VGLUT$1(\mathrm{Rb}, 6 \mu \mathrm{g} / \mathrm{ml})$ in $50 \mathrm{mM}$ TBS containing $1 \% \mathrm{BSA}$ and $0.1 \%$ Tw20 overnight $(\mathrm{O} / \mathrm{N})$ at room temperature. Replicas were reacted with a mixture of gold-coupled (10 and $15 \mathrm{~nm}$ or 5 and $10 \mathrm{~nm}$ ) goat anti-guinea pig and goat anti-rabbit IgGs secondary antibodies (1:30; BioCell Research Laboratories, Cardiff, UK) made up in $50 \mathrm{mM}$ TBS containing $5 \% \mathrm{BSA} \mathrm{O} / \mathrm{N}$ at $15^{\circ} \mathrm{C}$. 


\section{Electron Microscopy}

The labeled replicas were examined using a transmission electron microscope (Philips CM100).

\section{Control Experiments}

The specificity of immunolabeling for $\mathrm{Ca}_{\mathrm{v}} 2.1$ was controlled by staining of sections obtained from wt and ko mice. In wt animals [VGAT-Ca 2.1 ( $n=76$ terminals; VGLUT-1-Ca $2.1(n=40)$ ] the pattern of immunostaining was identical to that of rat, whereas in ko mouse [VGAT-Ca $2.1(n=68)$; VGLUT-1-Ca $2.1(n=53)$ ] no immunolabeling for the channel subunit was detected further confirming the specificity of the antibodies.

\section{Quantification of Immunogold Distribution}

The distribution of immunogold labeling for $\mathrm{Ca}_{\mathrm{v}} 2.1$ was evaluated using an in-house developed automatized computational procedure. As an input, the underlying algorithm used $\mathrm{x}^{-}$and $\mathrm{y}$-coordinates (in pixels) of the particles that were extracted from electron micrographs with the ImageJ software package (Schneider et al., 2012). The plasma membrane area covered with immunoparticles was calculated using the convex hull, the smallest area containing all particles as well as every line segment between all pairs of particles that was determined with the QuickHull algorithm (Barber et al., 1996). The cluster-assignment was obtained from the single-link method (Sibson, 1973). Accordingly, particles are assigned to the same cluster if their distances fall below a given threshold length that was set to $21 \mathrm{~nm}$ around the center of a gold particle. This distance is equivalent to the combined length of the radius of the $10 \mathrm{~nm}$ gold particle and the lengths of primary and secondary antibodies $(2 \times 8 \mathrm{~nm}=$ length of two IgGs, Figure 4A) (Amiry-Moghaddam and Ottersen, 2013).

To validate the clustering of $\mathrm{Ca}_{\mathrm{v}} 2.1$ immunoparticles our computational procedure was applied to random samples that were generated as follows: for each putative active zone, an equal number of control particles was randomly placed within a frame defined by the coordinates of the outer most particles (Figure 4D). When the size of the putative active zone was large enough while maintaining an equal number of particles they were placed not closer than $10 \mathrm{~nm}$, which corresponds to the diameter of a gold particle and avoids overlap of two neighboring particles. The final random control for any active zone was the average of ten individually generated random distributions.

\section{Control Experiments}

To assure that clustering of $\mathrm{Ca}_{\mathrm{v}} 2.1$ channels is not due to an artifact by the secondary antibody we investigated the subcellular distribution of VGAT and VGLUT-1 as well as the GluR $\delta 2$ receptor by using the same $10 \mathrm{~nm}$ gold-coupled secondary antibodies. These proteins showed a distribution pattern different from $\mathrm{Ca}_{\mathrm{v}} 2.1$ and did not form clusters. The automatized computational cluster analysis of immunoreactivity for GluR $\delta 2$ showed no significant difference compared to a random uniform sampling regarding both cohesion $(p=0.069)$ and separation $(p=0.86)$.

\section{Statistical Analysis}

Immunoreactivity for $\mathrm{Ca}_{\mathrm{v}} 2.1$ was quantitatively analyzed in putative active zones of GABAergic, putative glutamatergic and glutamatergic boutons $(n=54$ for VGAT,$+ n=67$ for VGAT,$- n=90$ for VGLUT-1+) obtained from two animals. Absolute numbers of $\mathrm{Ca}_{\mathrm{v}} 2.1$ immunoparticles per active zone were compared using the Mann-Whitney test. Correlation of the number of $\mathrm{Ca}_{\mathrm{v}} 2.1$ and the respective convex hull area was determined by the Spearman coefficient of correlation $\left(\mathrm{r}_{s}\right)$. Clusters of immunogold particles in VGAT + and VGAT - boutons as well as in VGAT + and VGLUT-1+ boutons as regards cohesion and separation were compared using the cumulative probability distributions and by performing the two-sample Kolmogorov-Smirnov test. The number of clusters per active zone, particles per clusters, and diameter of clusters in VGAT+ and VGAT- boutons as well as in VGAT+ and VGLUT-1+ boutons were statistically compared using the two-sample Kolmogorov-Smirnov test. Statistical significance was assessed by a $p$-value threshold of 0.05 .
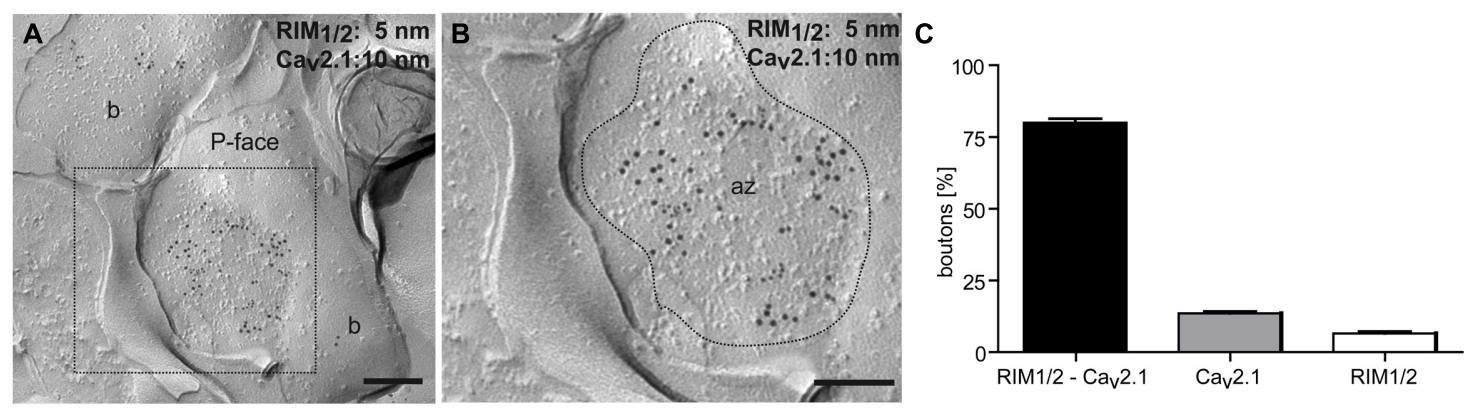

FIGURE 1 | $\mathrm{Ca}_{\mathrm{v}} 2.1$ localized to the active zone of axon terminals of hippocampal cells. (A,B) Colocalization of $\mathrm{Ca}_{\mathrm{v}} 2.1$ (10 nm gold particles) and the presynaptic marker proteins RIM1/2 (5 nm gold particles) in the active zone (az) of a bouton (b; inset, B) as assessed by the SDS-FRL method. The presynaptic active zone is indicated by the high density of intramembrane particles (IMPs) on the concave shape of the protoplasmic face (P-face) of the membrane (delineated by broken line). (C) Bar graph summarizing labeling and co-labeling of $\mathrm{Ca}_{\mathrm{v}} 2.1$ and RIM1/2 in 303 axon terminals. Note co-labeling in the majority of terminals (81\%). Scale bars, $0.2 \mu \mathrm{m}$. 


\section{Results}

\section{Cav2.1 Protein is Localized to the Active Zones of Axon Terminals}

All antibodies used target intracellular epitopes and, therefore, result in labeling of the protoplasmic face (P-face) of the replicas. First, we determined the distribution of the $\mathrm{Ca}_{\mathrm{v}} 2.1$ channels at presynaptic sites. Immunogold labeling for the channel's pore-forming $\alpha 1$ subunit was observed in the active zone of axon terminals that were recognized by their high density of intramembrane particles (IMPs) on the P-face of the invaginated plasma membrane and were identified by immunolabeling for the presynaptic marker proteins RIM1/2 (Figures 1A,B). Quantitative analysis further revealed a high degree of colocalization of $\mathrm{Ca}_{\mathrm{v}} 2.1$ and RIM1/2 in the majority (81\%) of the investigated terminals $(n=303$; Figure $1 \mathrm{C})$, indicating that
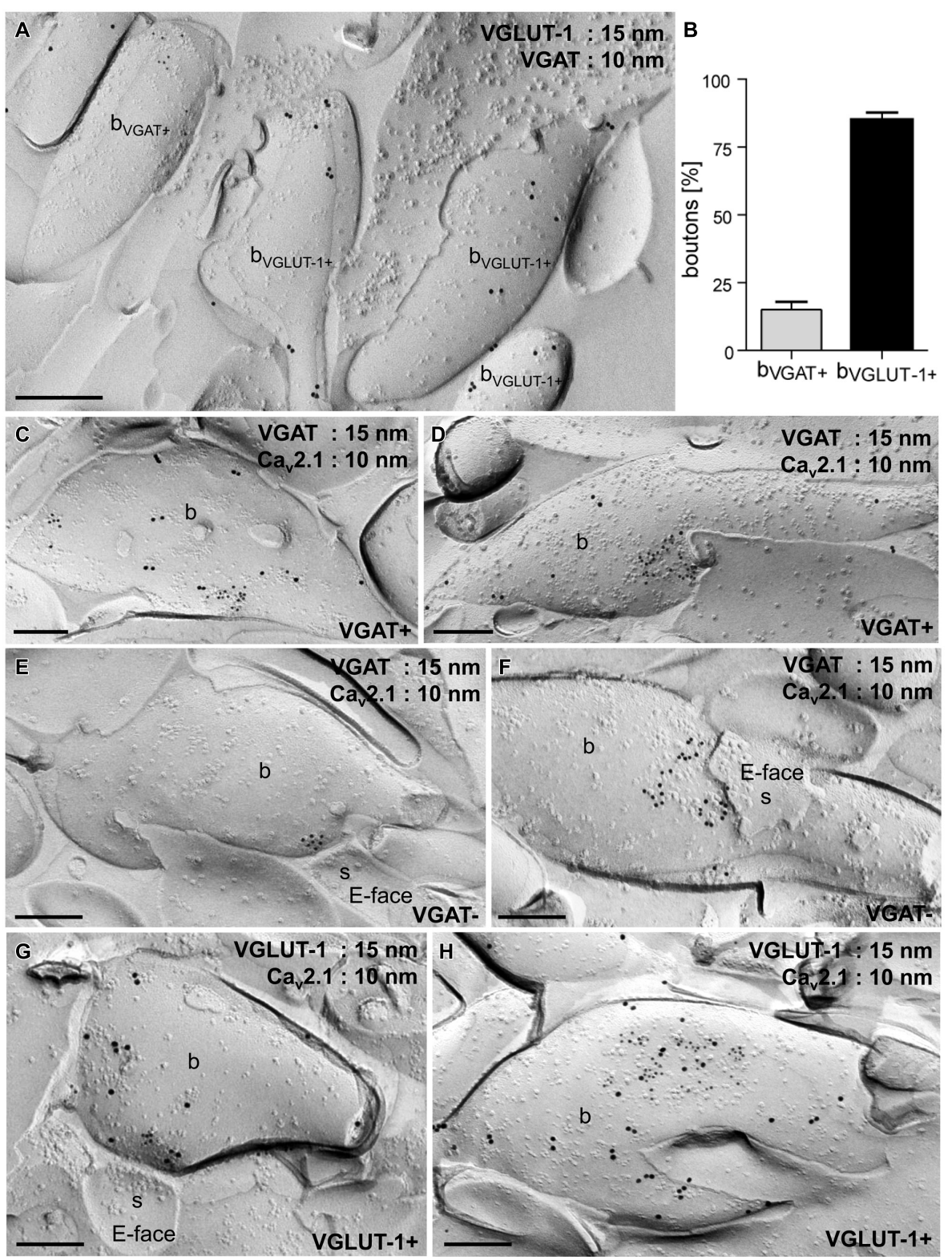

FIGURE 2 | $\mathrm{Ca}_{\mathrm{v}} 2.1$ channels are organized in discrete groups in the presynaptic active zone of boutons in inhibitory GABAergic and excitatory glutamatergic cells in the stratum radiatum of the hippocampal CA1 area. (A) Electron micrograph of a replica double-labeled for vesicular GABA transporter (VGAT; $10 \mathrm{~nm}$ gold particles) and VGLUT-1. $(15 \mathrm{~nm})$ showing no overlap between the two subpopulations. (B) Quantification of gold particles further demonstrated that $15 \%$ of the examined axon terminals $(n=328)$ were VGAT+ $($ bVGAT + ) and $85 \%$ were immunoreactive for VGLUT-1 (bVGLUT-1+). (C-H) Replica images showing aggregation of immunogold particles labeling Cav2.1 (10 nm) in small (C,E, G) and large (D,F,H) active zones of VGAT+ (15 nm; C,D), VGAT - (E,F) and VGLUT-1+ (15 nm; G,H) boutons (b). Note that VGAT - and VGLUT-1+ terminals make asmmetrical synapses with dendritic spines (s in $\mathbf{E}, \mathbf{F}, \mathbf{G}$ ) that can be recognized by the high density of IMPs on the E-face of the plasma membrane. Scale bars, $0.2 \mu \mathrm{m}$. 
$\mathrm{Ca}_{\mathrm{v}} 2.1$ channels are mainly confined to the active zone of boutons in the stratum radiatum of CA1.

\section{Inhibitory and Excitatory Boutons Show Similar Arrangement of $\mathrm{Ca}_{\mathbf{v}} \mathbf{2 . 1}$}

Next we compared the distribution of immunoparticles labeling $\mathrm{Ca}_{\mathrm{v}} 2.1$ in axon terminals of inhibitory GABAergic and excitatory glutamatergic neurons. For this purpose, three series of double immunolabeling experiments were performed: (i) labeling for vesicular GABA transporter (VGAT) and vesicular glutamate transporter-1 (VGLUT-1), (ii) labeling for VGAT and $\mathrm{Ca}_{\mathrm{v}} 2.1$, and (iii) labeling for VGLUT-1 and $\mathrm{Ca}_{\mathrm{v}}$ 2.1. Immunoreactivity for VGAT and VGLUT-1 appeared in two non-overlapping subpopulations of boutons (Figure 2A): $15 \%$ of the terminals $(n=328)$ showed immunoreactivity for VGAT, while $85 \%$ of them were labeled for VGLUT-1 (Figure 2B). To directly compare the localization of $\mathrm{Ca}_{\mathrm{v}} 2.1$ in GABAergic and glutamatergic terminals we then analyzed replicas double labeled for VGAT and the channel subunit. Inhibitory terminals were recognized from immunoreactivity for VGAT (VGAT+; Figures 2C,D), whereas VGAT- putative excitatory boutons were adjacent to postsynaptic dendritic spines that were characterized by a high density of IMPs on the exoplasmic face (E-face) of the membrane (Figures 2E,F) that

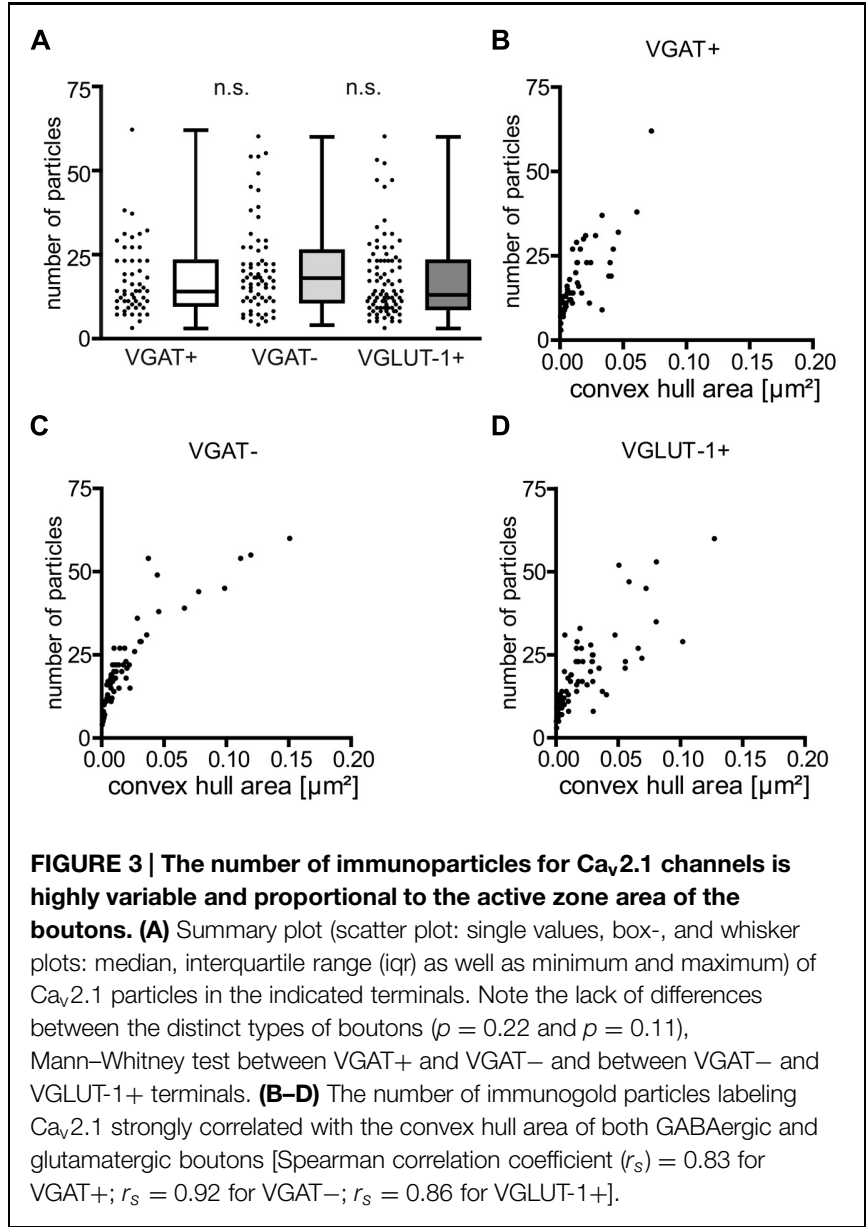

represent AMPA-type glutamate receptors in the postsynaptic membrane of asymmetrical synapses (Holderith et al., 2012). Immunoparticles for $\mathrm{Ca}_{\mathrm{v}} 2.1$ were highly concentrated in the synaptic membrane and were distributed non-homogeneously over small (Figures 2C,E) and large (Figures 2D,F) active zones of the terminals of both populations of neurons. Similar to VGAT - terminals, excitatory boutons, visualized by immunoreactivity for VGLUT-1 (VGLUT-1+), showed a nonhomogeneous pattern for $\mathrm{Ca}_{\mathrm{v}} 2.1$ distribution: gold particles labeling the channel protein were confined to the presynaptic membrane specialization where they formed discrete groups throughout the active zones (Figures 2G,H).

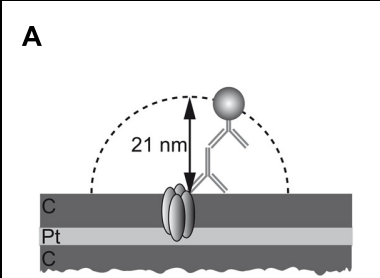

B

C

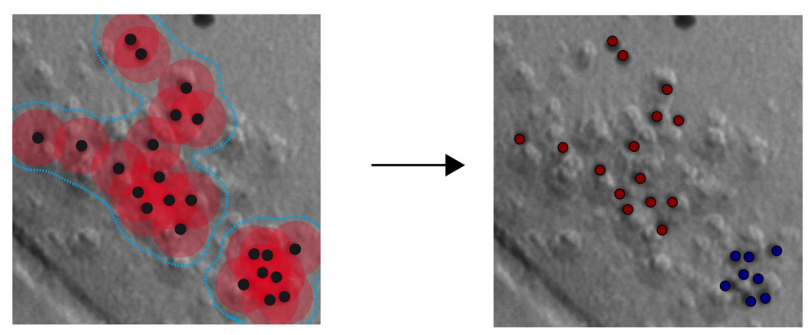

D

Cav2.1 distribution
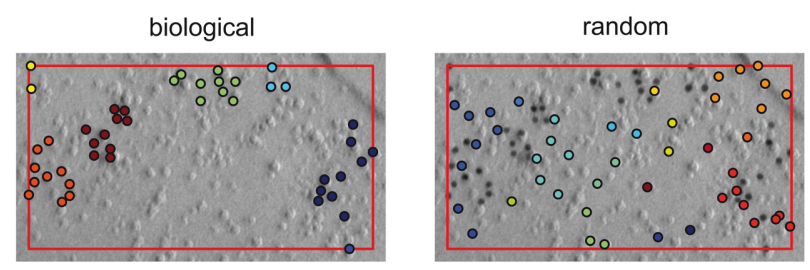

FIGURE 4 | Rational and operation of the automatized computational procedure used for quantitative assessment of immunoparticle distribution. (A) Spatial constraints arising from the $\mathrm{Ca}_{\mathrm{v}} 2.1$ (embedded into the carbon (C) and platinum (Pt) layers of the replica) labeling by primary and secondary antibodies ( $8 \mathrm{~nm}$ each) and the gold particle $(10 \mathrm{~nm})$.

(B) Agglomerative clustering of immunoparticles (black dots) using a maximal inter-particle distance of $42 \mathrm{~nm}$ (overlapping circles in red); blue broken lines frame individual clusters of immunoparticles derived by this distance constraint (overlapping vs non-overlapping circles). (C,D) Operation of the computational procedure: all immunoparticles (black dots) detected in an electron micrograph are evaluated for inter-particle distances based on their 2D-coordinates and grouped into clusters as shown in (B). (C) Application to a set of $\mathrm{Ca}_{\mathrm{v}} 2.1$ particles (left image) resulting in the assignment of two distinct clusters (right image). (D) Comparison of a clustered distribution ('biological') determined by the algorithm for a set of $\mathrm{Ca}_{\mathrm{v}} 2.1$ particles in an axon terminal (area given by box framed in red) and a random sample ('random') generated by randomly distributing the same number of particles on an area identical to that determined in the terminal. 
These results indicate that $\mathrm{Ca}_{\mathrm{v}} 2.1$ channels display similar distribution patterns with clustered appearance in the synaptic membrane of axon terminals of both GABAergic and glutamatergic neurons in the stratum radiatum of CA1.

Next we determined the absolute number of $\mathrm{Ca}_{\mathrm{v}} 2.1$ immunogold particles in the presynaptic active zones and correlated them with the convex hull area of either type of bouton. These analyses showed that the number of $\mathrm{Ca}_{\mathrm{v}} 2.1$ immunogold particles was highly variable ranging from 3 to 62 per active zone in all the three subpopulations of axon terminals [median $(\mathrm{mdn})=14$ and (interquartile range $($ iqr $)=10-23$ ] determined in 54 active zones of VGAT+, mdn $=18(\mathrm{iqr}=11-26)$ in 67 active zones of VGAT - and mdn $=13($ iqr $=9-23)$ in 90 active zones of VGLUT-1+ neurons from two animals, Figure 3A. These quantifications revealed no significant difference between VGAT+ and VGAT - groups of neurons ( $p=0.22$, Mann-Whitney test), neither between VGAT - and VGLUT-1+ terminals $(p=0.11$; Figure 3A). In addition, plotting the number of immunoparticles labeling $\mathrm{Ca}_{\mathrm{v}} 2.1$ against the convex hull area indicated a strong correlation between the number of $\mathrm{Ca}_{\mathrm{v}}$ channels and the synaptic area in both inhibitory and excitatory boutons
[Figures 3B-D; Spearman correlation coefficient $\left(\mathrm{r}_{S}\right)$ 0.83, 0.92, and 0.86 for VGAT+, VGAT - and VGLUT-1+ terminals, respectively].

Together, these analyses showed that the number of $\mathrm{Ca}_{\mathrm{v}} 2.1$ channels, despite clear synapse-to-synapse variation, is proportional to the area of active zones suggesting that their overall density in axon terminals of GABAergic and glutamatergic neurons in the stratum radiatum of CA1 is rather constant.

\section{$\mathrm{Ca}_{\mathrm{v}}$ 2.1 Proteins are Organized in Clusters within the Active Zone of Boutons}

For unbiased and quantitative assessment of the distribution of $\mathrm{Ca}_{\mathrm{v}} 2.1$ channels in the active zone, we set up a computational procedure performing automatized distribution analysis based on distances between neighboring immunoparticles (see Materials and Methods). Moreover, the underlying algorithm uses agglomerative clustering of particles when their distances fall below a threshold value that is given by the combined length of the primary and secondary antibodies as well as the radius of the gold particles ( $21 \mathrm{~nm}$; Figures $4 \mathbf{A}, \mathbf{B}$ ). Accordingly, particles located within distances of $\leq 42 \mathrm{~nm}$ from each other are assigned to a common cluster (Figure 4C).
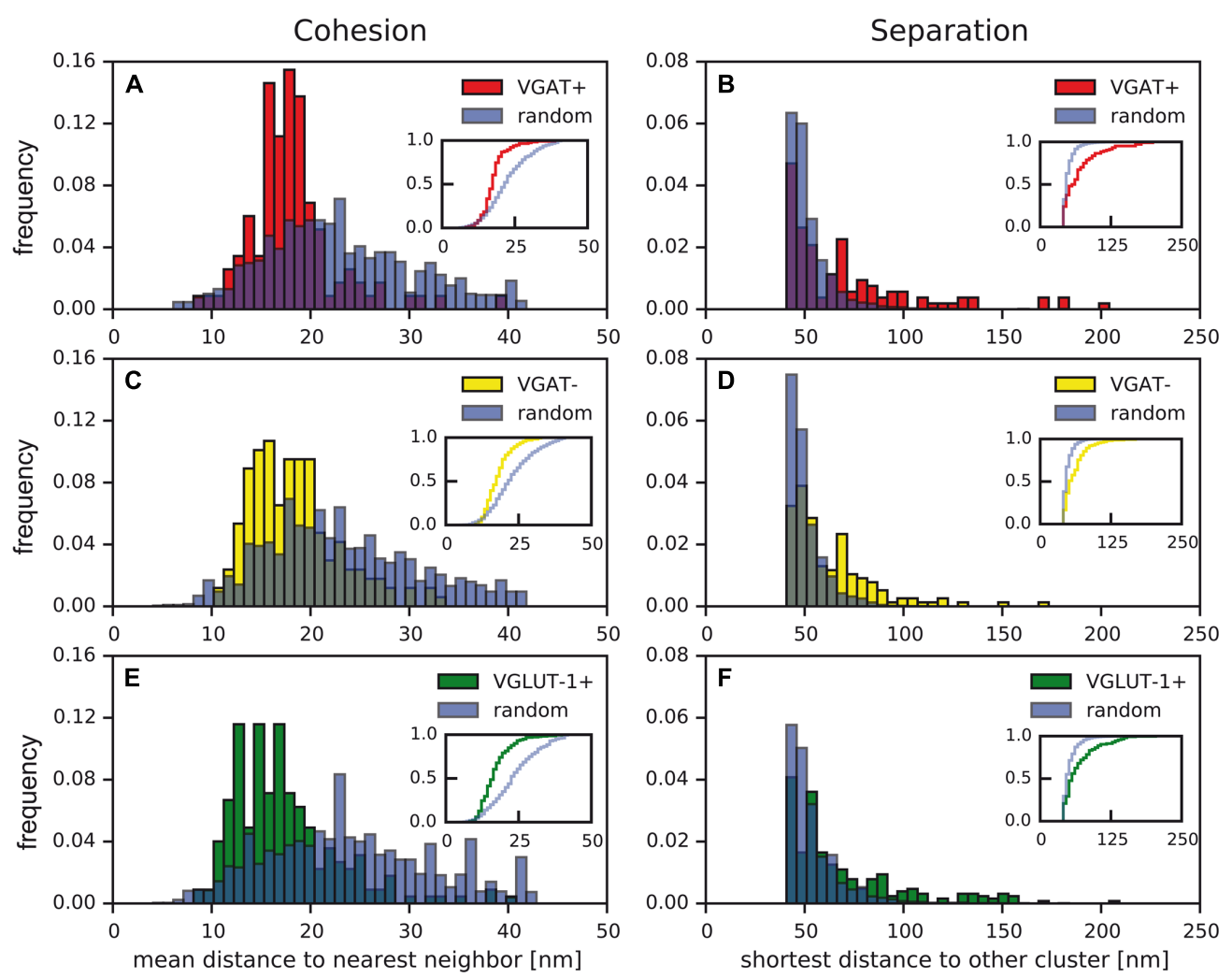

FIGURE 5 | Distinct distribution of $\mathrm{Ca}_{\mathrm{v}} 2.1$ channels in active zones of presynaptic boutons and in random controls. (A-F) Distances between nearest $\mathrm{Ca}_{\mathrm{v}} 2.1$ particles ('cohesion,' $\mathbf{A}, \mathbf{C}, \mathbf{E}$ ) and shortest distances between distinct clusters ('separation,' $\mathbf{B}, \mathbf{D}, \mathbf{F}$ ) determined in active zones of VGAT+ (A,B; red), VGAT- (C,D; yellow), VGLUT-1+

terminals (E,F; green) and in random control samples (blue). Insets: cumulative frequency distributions indicating significant differences between biological data (VGAT+, red; VGAT-, yellow; VGLUT-1+, green) and random controls (blue; two-sample Kolmogorov-Smirnov test; $p<0.05$, exact $p$-values given in Table 1). 
Using this computer-assisted analysis, we first probed the significance of clustered organization of $\mathrm{Ca}_{\mathrm{v}} 2.1$ channels illustrated above (Figures 1 and 2) over random distribution. For this purpose, we determined the distributions of (i) distances between nearest neighboring particles ('cohesion,' Figures $\mathbf{5 A}, \mathbf{C}, \mathbf{E}$ ) and of (ii) shortest distances between two clusters ('separation' Figures 5B,D,F), in active zones of VGAT+, VGAT-, and VGLUT-1+ terminals (biological distribution, Figure 4D, left panel) and in 'random controls' (Figure 4D, right panel). For the latter, the same number of particles was positioned randomly within the same area as determined for the respective active zones (red framed box, Figure 4D, and see also Materials and Methods).

As illustrated in Figure $\mathbf{5}$ and summarized in Table 1, the cohesion determined in the various types of boutons was almost identical (values for the median of 18 and $16 \mathrm{~nm}$ for VGAT+, VGAT-, and VGLUT-1+ terminals, respectively), but in either case was significantly stronger than the cohesion obtained in random control samples (respective medians of 25, 23, and $23 \mathrm{~nm}$, respectively; $p<0.05$ twosample Kolmogorov-Smirnov test, Figures 5A,C,E; Table 1). Conversely, the separation between clusters was significantly larger in all actual terminals than in random controls (values for the median of 61 and $48 \mathrm{~nm}$ (VGAT+), 56 and $48 \mathrm{~nm}$ (VGAT-), 58 and $50 \mathrm{~nm}$ (VGLUT-1+); $p<0.05$ twosample Kolmogorov-Smirnov test, Figures 5B,D,F; Table 1). These results were independent of the distance constraints, as biological distributions were still significantly different from the respective random controls upon variation of the maximal inter-particle distance between 35 and $55 \mathrm{~nm}$ (Table 2).

Together, these computational analyses indicated that $\mathrm{Ca}_{\mathrm{v}} 2.1$ channels are in fact organized in clusters over the active zones of both inhibitory and excitatory axon terminals.

\section{The Nano-Architecture of $\mathrm{Ca}_{\mathrm{v}} 2.1$ Channels is Shared between the Active Zones of Inhibitory and Excitatory Boutons}

Direct comparison of cohesion and separation of the $\mathrm{Ca}_{\mathrm{v}} 2.1$ clustering did not reveal statistically significant differences between inhibitory and excitatory terminals (Figures 6A-D), strongly suggesting a more general architecture that is shared among presynaptic compartments of different types of neurons. The slight difference observed for the cohesion between VGAT+ and VGLUT-1+ terminals is

TABLE 1 | Analysis of $\mathrm{Ca}_{\mathrm{v}} 2.1$ immunogold distribution in inhibitory and excitatory boutons.

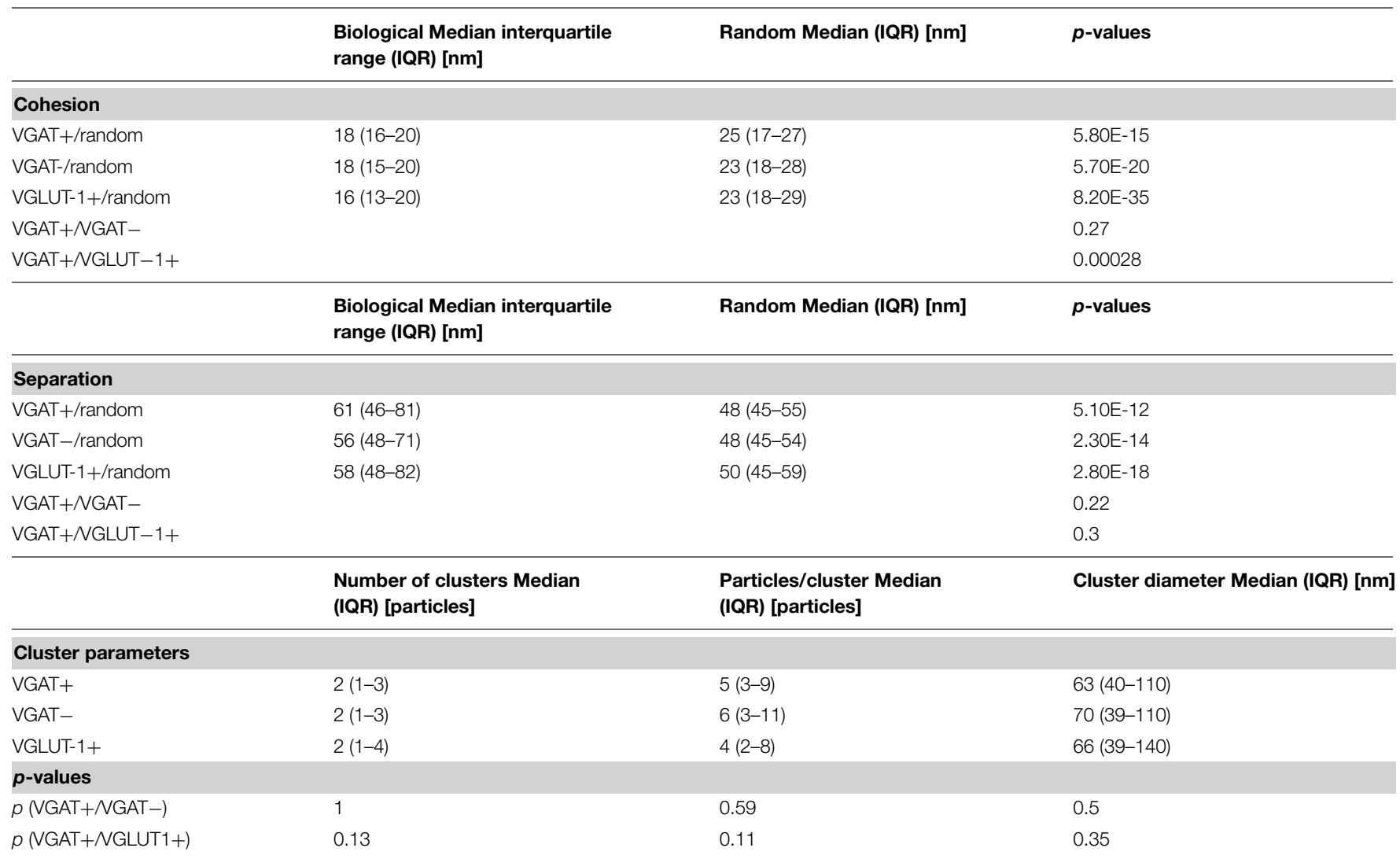

The cohesion, distances between nearest neighboring particles, in vesicular GABA transporter (VGAT+), VGAT-, and VGLUT-1+ terminals was significantly stronger than in random control samples, whereas the separation between clusters was significantly larger in all terminals than in random controls. Parameters such as number of clusters, number of particles for $\mathrm{Ca}_{\mathrm{v}} 2.1$ in individual cluster and diameter of clusters indicate a strikingly similar subcellular arrangement of Cav2.1 channels in inhibitory and excitatory terminals. 


\begin{tabular}{|c|c|c|c|c|c|c|}
\hline \multirow[b]{2}{*}{ Distance (nm) } & \multicolumn{2}{|c|}{ VGAT+ } & \multicolumn{2}{|c|}{ VGAT- } & \multicolumn{2}{|c|}{ VGLUT-1+ } \\
\hline & Cohesion & Separation & Cohesion & Separation & Cohesion & Separation \\
\hline 35 & $1.50 \mathrm{E}-11$ & $1.50 \mathrm{E}-07$ & $7.80 \mathrm{E}-14$ & $1.40 \mathrm{E}-10$ & 4.10E-25 & $5.70 \mathrm{E}-21$ \\
\hline 36 & $3.80 \mathrm{E}-12$ & 1.70E-08 & 1.10E-14 & $1.30 \mathrm{E}-10$ & $2.90 \mathrm{E}-26$ & $3.30 E-21$ \\
\hline 37 & $9.50 \mathrm{E}-15$ & 1.60E-08 & $7.20 \mathrm{E}-17$ & $4.00 E-12$ & $2.70 \mathrm{E}-31$ & $8.70 \mathrm{E}-19$ \\
\hline 38 & $2.60 E-15$ & 8.70E-10 & $8.50 \mathrm{E}-17$ & $9.30 \mathrm{E}-13$ & 4.40E-31 & 5.80E-20 \\
\hline 39 & $9.10 \mathrm{E}-15$ & $2.00 E-10$ & $4.50 \mathrm{E}-17$ & $5.50 E-14$ & $1.40 \mathrm{E}-30$ & $3.80 E-24$ \\
\hline 40 & $4.00 \mathrm{E}-16$ & $9.10 \mathrm{E}-12$ & 4.10E-19 & $6.00 E-14$ & 4.00E-32 & 4.10E-24 \\
\hline 41 & $1.40 \mathrm{E}-16$ & $7.90 \mathrm{E}-12$ & $1.60 \mathrm{E}-19$ & $9.30 \mathrm{E}-14$ & 3.80E-32 & 1.40E-23 \\
\hline 42 & $5.80 \mathrm{E}-15$ & $5.10 \mathrm{E}-12$ & $5.70 \mathrm{E}-20$ & $2.30 \mathrm{E}-14$ & $8.20 E-35$ & $2.80 \mathrm{E}-18$ \\
\hline 43 & $1.30 \mathrm{E}-16$ & $3.90 \mathrm{E}-12$ & 1.10E-20 & $1.00 \mathrm{E}-16$ & $9.10 \mathrm{E}-34$ & 1.60E-20 \\
\hline 44 & $3.70 \mathrm{E}-16$ & $5.50 E-14$ & $1.10 \mathrm{E}-21$ & $5.90 E-16$ & $5.50 \mathrm{E}-35$ & $1.10 \mathrm{E}-19$ \\
\hline 45 & $4.50 \mathrm{E}-16$ & $1.70 E-16$ & 5.30E-22 & $3.90 \mathrm{E}-17$ & $1.50 \mathrm{E}-34$ & $3.20 E-24$ \\
\hline 46 & $4.20 \mathrm{E}-17$ & $1.70 E-16$ & $3.90 E-22$ & $4.10 \mathrm{E}-17$ & $1.30 E-35$ & $2.60 E-19$ \\
\hline 47 & $4.30 \mathrm{E}-17$ & $1.10 \mathrm{E}-15$ & $3.40 E-23$ & $2.40 \mathrm{E}-17$ & $1.70 \mathrm{E}-35$ & $1.90 \mathrm{E}-18$ \\
\hline 48 & $6.90 \mathrm{E}-17$ & $5.80 E-16$ & $3.40 E-23$ & $3.10 E-19$ & $1.80 E-35$ & $4.80 \mathrm{E}-18$ \\
\hline 49 & $1.60 \mathrm{E}-17$ & 8.30E-17 & $1.40 E-23$ & $2.10 E-20$ & $6.60 E-36$ & $3.10 \mathrm{E}-17$ \\
\hline 50 & $5.90 \mathrm{E}-18$ & 3.30E-17 & $3.60 E-24$ & $4.40 E-20$ & $4.40 E-36$ & $2.30 \mathrm{E}-18$ \\
\hline 51 & $1.10 \mathrm{E}-17$ & $3.70 E-16$ & $2.90 E-24$ & $1.00 \mathrm{E}-18$ & 3.30E-36 & $5.00 \mathrm{E}-15$ \\
\hline 52 & $2.00 \mathrm{E}-17$ & $2.50 E-16$ & 3.10E-24 & 4.10E-19 & 6.30E-36 & $2.00 E-13$ \\
\hline 53 & $1.30 \mathrm{E}-16$ & $3.10 E-16$ & $1.80 E-24$ & 7.70E-20 & 5.30E-35 & $3.60 E-14$ \\
\hline 54 & $3.40 \mathrm{E}-16$ & 4.30E-16 & 3.30E-24 & 8.30E-19 & 3.00E-34 & $1.00 \mathrm{E}-15$ \\
\hline 55 & $1.40 \mathrm{E}-15$ & $4.80 E-15$ & 8.20E-24 & $6.60 E-21$ & $9.60 \mathrm{E}-33$ & $7.90 \mathrm{E}-15$ \\
\hline
\end{tabular}

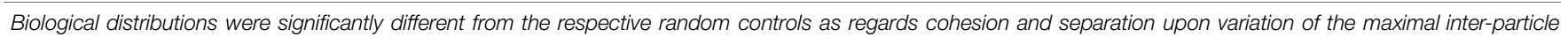
distance between 35 and $55 \mathrm{~nm}$.

most likely due to the distinct primary antibodies that target different epitopes on the channel protein and may distinctly impact the spatial arrangements of the gold grains and, therefore, the distances between nearest neighboring particles.

In subsequent analyses, the computational procedure was, therefore, used for a more detailed investigation of the parameters characterizing the architecture of the $\mathrm{Ca}_{\mathrm{v}} 2.1$ clusters in inhibitory and excitatory boutons. At this end, we determined (i) the number of clusters in presynaptic terminals, (ii) the number of immunogold particles for $\mathrm{Ca}_{\mathrm{v}} 2.1$ forming an individual cluster as well as, (iii) the diameter of the area covered by a cluster as accessible to our SDS-FRL configuration. The number of clusters varied roughly between 1 and 10 and averaged to two clusters per terminal in both inhibitory and excitatory boutons (Figure 6E; Table 1). Similarly, the number of $\mathrm{Ca}_{\mathrm{v}} 2.1$ immunogold particles integrated in the same cluster varies over a wide range (3-40) averaging to a value of 5 in all the three types of presynaptic terminals (Figure 6F; Table 1). Finally, the diameter of the individual clusters ranged from 10 to $250 \mathrm{~nm}$ and exhibit mean values of 63,70 , and $66 \mathrm{~nm}$ in VGAT+, VGAT-, and VGLUT-1+ terminals, respectively (Figure 6G; Table 1).

Together, these quantitative data unequivocally indicate a strikingly similar subcellular arrangement of presynaptic $\mathrm{Ca}_{\mathrm{v}} 2.1$ channels in inhibitory and excitatory axon terminals in the stratum radiatum of hippocampal CA1 region.

\section{Discussion}

In the present study we investigated and compared the ultrastructural organization of $\mathrm{Ca}_{\mathrm{v}} 2.1$ channels in axon terminals of inhibitory (VGAT+) and excitatory (VGAT - and VGLUT$1+$ ) neurons in the stratum radiatum of the CA1 hippocampal area using high-resolution SDS-FRL electron microscopy. Furthermore, we used an automatized computational analysis to compare the precise spatial arrangement of $\mathrm{Ca}_{\mathrm{v}} 2.1$ channels in the two subpopulations of axon terminals and suggest a common nano-architecture of the P/Q-type $\mathrm{Ca}^{2+}$ channel.

Immunoelectron microscopy unequivocally revealed enrichment of $\mathrm{Ca}_{\mathrm{v}} 2.1$ channels in the active zone of boutons as well as a close spatial relationship of the channel subunit to the presynaptic proteins RIM1/2, established components of the $\mathrm{Ca}_{\mathrm{v}} 2$ channel networks and major regulators of the coupling between $\mathrm{Ca}^{2+}$ channels and $\mathrm{Ca}^{2+}$ sensors of exocytosis (Müller et al., 2010; Han et al., 2011; Kaeser et al., 2011; Gundelfinger and Fejtova, 2012; Südhof, 2013). Quantitative morphological as well as detailed computational analysis further demonstrated a high degree of structural similarity between inhibitory and excitatory terminals with respect to clustering and average number of $\mathrm{Ca}_{\mathrm{v}} 2.1$ channels in the presynaptic membrane. These findings are consistent with the clustered distribution and estimates of the number of $\mathrm{Ca}_{\mathrm{v}} 2.1$ channels in the active zone of calyx of Held (Nakamura et al., 2015), cerebellar parallel 

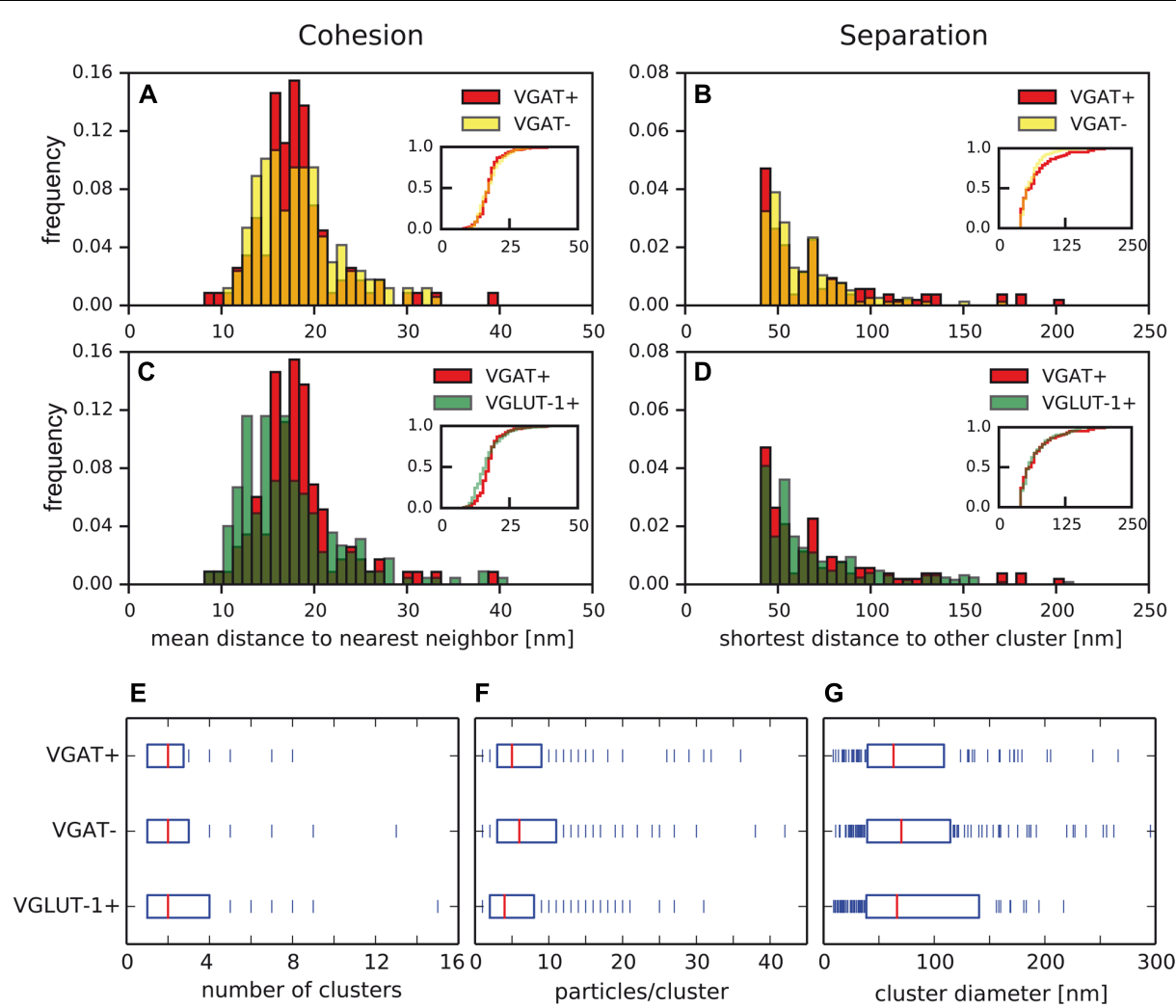

FIGURE 6 | Properties of $\mathrm{Ca}_{\mathrm{v}} \mathbf{2 . 1}$ clusters are similar in inhibitory and excitatory axon terminals. (A-D) Cohesion and separation of $\mathrm{Ca}_{\mathrm{v}} 2.1$ clusters as determined in VGAT+ (red), VGAT- (yellow), and VGLUT-1+ (green) terminals. No significant differences were detected in (A,B,D), $(p<0.05$, two-sample Kolmogorov-Smirnov test), while cohesion in (C) was significantly different. (E-G) Number of clusters per terminal $(\mathbf{E})$, number of $\mathrm{Ca}_{\mathrm{v}} 2.1$ particles per cluster $(\mathbf{F})$, and cluster diameter $(\mathbf{G})$ are determined in the boutons. Boxes indicate median (red) and iqr of the data (blue bars). No significant differences were detected between the data sets from distinct types of boutons (two-sample Kolmogorov-Smirnov test, exact $p$-values given in Table 1). fibers (Indriati et al., 2013; Schmidt et al., 2013; Baur et al., 2015), and hippocampal CA3 principal cells synapsing on either other CA3 (Holderith et al., 2012) or CA1 pyramidal (Ermolyuk et al., 2013) neurons. Interestingly, the size of the clusters and the number of $\mathrm{Ca}_{\mathrm{v}} 2.1$ proteins per cluster (4-6) correlate well with estimates derived from electrophysiological studies (Bucurenciu et al., 2010) and biochemical/proteomic analysis (Müller et al., 2010) that found a small number of $\mathrm{Ca}_{\mathrm{v}}$ channels located within a distance of less than $100 \mathrm{~nm}$ from the release machinery at central synapses. Therefore, our data provide qualitative and quantitative proof of principle that GABAergic and glutamatergic synapses in the stratum radiatum of the CA1 area share a common nano-architecture of $\mathrm{Ca}_{\mathrm{v}} 2.1$ channels making extensive use of tight coupling between the $\mathrm{Ca}_{\mathrm{v}} 2.1$ channels and $\mathrm{Ca}^{2+}$ sensors for fast transmitter release.

In that respect, the $\mathrm{Ca}_{\mathrm{v}} 2.1$ clusters reflect the molecular basis for local $\mathrm{Ca}^{2+}$ signaling in ' $\mathrm{Ca}^{2+}$ nano-domains' (Neher, 1998; Fakler and Adelman, 2008). The tight coupling of $\mathrm{Ca}_{\mathrm{v}} 2.1$ channels and $\mathrm{Ca}^{2+}$ sensors of exocytosis ensures high reliability in vesicle release (Scimemi and Diamond, 2012), reduced synaptic delay and duration of the release period as well as increased ratio of synchronous and 'entopic' (active zone) release resulting in similar high temporal precision of both GABAergic and glutamatergic transmission (Bucurenciu et al., 2008, 2010; Eggermann et al., 2012; Nadkarni et al., 2012).

In summary, our results demonstrate a large morphological homogeneity in the two non-overlapping populations of synapses (Figure 2A) suggesting that the processes and mechanisms underlying the formation of the $\mathrm{Ca}_{\mathrm{v}} 2.1$ nanoarchitecture and the evoked release of neurotransmitters are similar between inhibitory and excitatory central synapses (Xu et al., 2009; Eggermann et al., 2012). Regarding that the protein nano-environment of $\mathrm{Ca}_{\mathrm{v}} 2$ channels is highly complex (Berkefeld et al., 2006; Müller et al., 2010) consisting of quite a variety of auxiliary proteins and regulators (Arikkath and Campbell, 2003; Dolphin, 2012) that together form the channel-associated networks regulating the local $\mathrm{Ca}^{2+}$ signaling (Evans and Zamponi, 2006; Han et al., 2011; Hoppa et al., 2012; Davydova et al., 2014), it is conceivable that the assembly and operation of $\mathrm{Ca}_{\mathrm{v}} 2.1$ channel clusters can be dynamically regulated. In this respect, further extensive quantitative research is required to identify and localize additional components of the $\mathrm{Ca}_{\mathrm{v}} 2.1$ channel-associated networks and to unravel the 
synapse- and/or state-specific properties of the nanoenvironments of $\mathrm{P} / \mathrm{Q}$-type $\mathrm{Ca}^{2+}$ channels as well as their concerted (Wheeler et al., 1994; Spafford and Zamponi, 2003; Williams et al., 2012) implication in the homeostatic control of cortical synapses function.

\section{Author Contributions}

$\mathrm{DA}, \mathrm{BF}$, and $\mathrm{AK}$ designed the project; $\mathrm{DA}$ and $\mathrm{DB}$ performed computational cluster analysis; DA and AK performed SDSFRL immunoelectron microscopy; MW provided reagents; NS

\section{References}

Amiry-Moghaddam, M., and Ottersen, O. P. (2013). Immunogold cytochemistry in neuroscience. Nat. Neurosci. 16, 798-804. doi: 10.1038/nn.3418

Ariel, P., Hoppa, M. B., and Ryan, T. A. (2013). Intrinsic variability in Pv, RRP size, $\mathrm{Ca}^{2+}$ channel repertoire, and presynaptic potentiation in individual synaptic boutons. Front. Syn. Neurosci. 4:9. doi: 10.3389/fnsyn.2012.00009

Arikkath, J., and Campbell, K. P. (2003). Auxiliary subunits: essential components of the voltage-gated calcium channel complex. Curr. Opin. Neurobiol. 13, 298-307. doi: 10.1016/S0959-4388(03)00066-7

Barber, C. B., Dobkin, D. P., and Huhdanpaa, H. (1996). The quickhull algorithm for convex hulls. ACM Trans. Math. Softw. 22, 469-483. doi: $10.1145 / 235815.235821$

Baur, D., Bornschein, G., Althof, D., Watanabe, M., Kulik, A., Eilers, J., et al. (2015). Developmental tightening of cerebellar cortical synaptic influx-release coupling. J. Neurosci. 35, 1858-1871. doi: 10.1523/JNEUROSCI.2900-14.2015

Berkefeld, H., Sailer, C. A., Bildl, W., Rohde, V., Thumfart, J.-O., Eble, S., et al. (2006). BKCa-Ca $a_{v}$ channel complexes mediate rapid and localized $\mathrm{Ca}^{2+}$. activated $\mathrm{K}^{+}$signaling. Science 314, 615-620. doi: 10.1126/science.1132915

Bucurenciu, I., Bischofberger, J., and Jonas, P. (2010). A small number of open $\mathrm{Ca}^{2+}$ channels trigger transmitter release at a central GABAergic synapse. Nat. Neurosci. 13, 19-21. doi: 10.1038/nn.2461

Bucurenciu, I., Kulik, A., Schwaller, B., Frotscher, M., and Jonas, P. (2008). Nanodomain coupling between $\mathrm{Ca}^{2+}$ channels and $\mathrm{Ca}^{2+}$ sensors promotes fast and efficient transmitter release at a cortical GABAergic synapse. Neuron 57, 536-545. doi: 10.1016/j.neuron.2007.12.026

Cao, Y.-Q., and Tsien, R. W. (2010). Different relationship of N- and P/Q-type $\mathrm{Ca}^{2+}$ channels to channel-interacting slots in controlling neurotransmission at cultured hippocampal synapses. J. Neurosci. 30, 4536-4546. doi: 10.1523/JNEUROSCI.5161-09.2010

Catterall, W. A., and Few, A. P. (2008). Calcium channel regulation and presynaptic plasticity. Neuron 59, 882-898. doi: 10.1016/j.neuron.2008.09.005

Clapham, D. E. (2007). Calcium signaling. Cell 131, 1047-1058. doi: 10.1016/j.cell.2007.11.028

Davydova, D., Marini, C., King, C., Klueva, J., Bischof, F., Romorini, S., et al. (2014). Bassoon specifically controls presynaptic P/Q-type $\mathrm{Ca}^{2+}$ channels via RIM-binding protein. Neuron 82, 181-194. doi: 10.1016/j.neuron.2014.02.012

Dolphin, A. C. (2012). $\mathrm{Ca}^{2+}$ channel auxiliary a2d and b subunits: trafficking and one step beyond. Nat. Rev. Neurosci. 13, 542-555. doi: 10.1038/nrn3311

Eggermann, E., Bucurenciu, I., Goswami, S. P., and Jonas, P. (2012). Nanodomain coupling betwen $\mathrm{Ca}^{2+}$ channels and sensors of exocytosis at fast mammalian synapses. Nat. Rev. Neurosci. 13, 7-21. doi: 10.1038/nrn3125

Ermolyuk, Y. S., Alder, F. G., Surges, R., Pavlov, I. Y., Timofeeva, Y., Kullmann, D. M., et al. (2013). Differential triggering of spontaneous glutamate release by P/Q-, N- and R- type $\mathrm{Ca}^{2+}$ channels. Nat. Neurosci. 16, 1754-1763. doi: $10.1038 / \mathrm{nn} .3563$

Evans, R. M., and Zamponi, G. W. (2006). Presynaptic $\mathrm{Ca}^{2+}$ channels - integration centers for neuronal signaling pathways. Trends Neurosci. 29, 617-624. doi: 10.1016/j.tins.2006.08.006

Fakler, B., and Adelman, J. P. (2008). Control of KCa channels by $\mathrm{Ca}^{2+}$ nano/microdomains. Neuron 59, 873-881. doi: 10.1016/j.neuron.2008.09.001 provided knock-out tissues; $\mathrm{DA}, \mathrm{DB}, \mathrm{BF}$, and $\mathrm{AK}$ analyzed data; $\mathrm{DA}, \mathrm{BF}$, and $\mathrm{AK}$ wrote the paper.

\section{Acknowledgments}

We thank N. Wernet for technical support; A. Haupt for help with figures; Drs. Y. Fukazawa and H. Schmidt for reading the manuscript. This work was funded by BIOSS-2 A6 (AK) and grants of the Deutsche Forschungsgemeinschaft (SFB 746, TP16, Fa 332/9-1 to $\mathrm{BF})$.

Frank, C. A. (2014). How voltage-gated calcium channels gate forms of homeostatic synaptic plasticity. Front. Cell. Neurosci. 8:40. doi: 10.3389/fncel.2014.00040

Gundelfinger, E. D., and Fejtova, A. (2012). Molecular organization and plasticity of the cytomatrix at the active zone. Curr. Opin. Neurobiol. 22, 423-430. doi: 10.1016/j.conb.2011.10.005

Han, Y., Kaeser, P. S., Südhof, T. C., and Schneggenburger, R. (2011). RIM determines $\mathrm{Ca}^{2+}$ channel density and vesicle docking at the presynaptic active zone. Neuron 69, 304-316. doi: 10.1016/j.neuron.2010.12.014

Holderith, N., Lorincz, A., Katona, G., Rozsa, B., Kulik, A., Watanabe, M., et al. (2012). Release probability of hippocampal glutamatergic terminals scales with the size of the active zone. Nat. Neurosci. 15, 988-997. doi: 10.1038/nn.3137

Hoppa, M. B., Lana, B., Margas, W., Dolphin, A. C., and Ryan, T. A. (2012). Alpha2delta expression sets presynaptic calcium channel abundance and release probability. Nature 486, 122-125. doi: 10.1038/nature11033

Indriati, D. W., Kamasawa, N., Matsui, K., Meredith, A. L., Watanabe, M., and Shigemoto, R. (2013). Quantitative localization of $\mathrm{Ca}_{\mathrm{v}} 2.1$ (P/Q-type) voltage-dependent calcium channels in Purkinje cells: somatodendritic gradient and distinct somatic coclustering with $\mathrm{Ca}^{2+}$-activated potassium channels. J. Neurosci. 33, 3668-3678. doi: 10.1523/JNEUROSCI.2921-12.2013

Kaeser, P. S., Deng, L., Wang, Y., Dulubova, I., Liu, X., Rizo, J., et al. (2011). RIM proteins tether $\mathrm{Ca}^{2+}$ channels to presynaptic active zones via direct PDZdomain interaction. Cell 144, 282-295. doi: 10.1016/j.cell.2010.12.029

Kisfali, M., Lorincz, T., and Vizi, S. (2013). Comparision of $\mathrm{Ca}^{2+}$ transients and $\left[\mathrm{Ca}^{2+}\right]_{i}$ in the dendrites and boutons of non-fast-spiking GABAergic hippocampal interneurons using two-photon laser microscopy and highand low-affinity dyes. J. Physiol. 591, 5541-5553. doi: 10.1113/jphysiol.2013. 258863

Klausberger, T. (2009). GABAergic interneurons targeting dendrites of pyramidal cells in the CA1 area of the hippocampus. Eur. J. Neurosci. 30, 947-957. doi: 10.1111/j.1460- 9568.2009.06913.x

Kulik, A., Nakadate, K., Hagiwara, A., Fukazawa, Y., Lujan, R., Saito, H., et al. (2004). Immunocytochemical localization of the $\alpha_{1 \mathrm{~A}}$ subunit of the P/Q-type calcium channel in the rat cerebellum. Eur. J. Neurosci. 19, 2169-2178. doi: 10.1111/j.0953-816X.2004.03319.x

Kulik, A., Vida, I., Fukazawa, Y., Guetg, N., Kasugai, Y., Marker, C. L., et al. (2006). Compartment-dependent colocalization of Kir3.2-containing $\mathrm{K}^{+}$channels and GABA $_{B}$ receptors in hippocampal pyramidal cells. J. Neurosci. 26, 4289-4297. doi: 10.1523/JNEUROSCI.4178-05.2006

Lipscombe, D., Allen, S. E., and Toro, C. P. (2013). Control of neuronal voltagegated calcium ion channels from RNA to protein. Trends Neurosci. 36, 598-609. doi: 10.1016/j.tins.2013.06.008

Lisman, J. E. (1999). Relating hippocampal circuitry to function: recall of memory sequences by reciprocal dentate-CA3 interactions. Neuron 22, 233-242. doi: 10.1016/S0896-6273(00)81085-5

Masugi-Tokita, M., and Shigemoto, R. (2007). High-resolution quantitative visualization of glutamate and GABA receptors at central synapses. Curr. Opin. Neurobiol. 17, 387-393. doi: 10.1016/j.conb.2007.04.012

Müller, C. S., Haupt, A., Bildl, W., Schindler, J., Knaus, H.-G., Meissner, M., et al. (2010). Quantitative proteomics of the $\mathrm{Ca}_{\mathrm{v}} 2$ channel nano-environments in the mammalian brain. Proc. Natl. Acad. Sci. U.S.A. 107, 14950-14957. doi: $10.1073 /$ pnas. 1005940107 
Nadkarni, S., Bartol, T. M., Sejnowski, T. J., and Levine, H. (2010). Modelling vesicular release at hippocampal synapses. PLoS Comput. Biol. 6:e1000983. doi: 10.1371/journal.pcbi.1000983

Nadkarni, S., Bartol, T. M., Stevens, C. F., Sejnowski, T. J., and Levine, H. (2012). Short-term plasticity constrains spatial organization of a hippocampal presynaptic terminal. Proc. Natl. Acad. Sci. U.S.A. 109, 14657-14662. doi: 10.1073/pnas.1211971109

Nakamura, Y., Harada, H., Kamasawa, N., Matsui, K., Rothman, J. S., Shigemoto, R., et al. (2015). Nanoscale distribution of presynaptic $\mathrm{Ca}^{2+}$ channels and its impact on vesicular release during development. Neuron 85, 1-14. doi: 10.1016/j.neuron.2014.11.019

Neher, E. (1998). Vesicle pools and $\mathrm{Ca}^{2+}$ microdomains: new tools for understanding their roles in neurotransmitter release. Neuron 20, 389-399. doi: 10.1016/S0896-6273(00)80983-6

Otmakhova, N. A., and Lisman, J. E. (2004). Contribution of $\mathrm{I}_{\mathrm{h}}$ and $\mathrm{GABA}_{\mathrm{B}}$ to synaptically induced afterhyperpolarizations in CA1: a brake on the NMDA response. J. Neurophysiol. 92, 2027-2039. doi: 10.1152/jn.00427.2004

Parajuli, L. K., Nakajima, C., Kulik, A., Matsui, K., Schneider, T., Shigemoto, R., et al. (2012). Quantitative regional and ultrastructural localization of the $\mathrm{Ca}_{\mathrm{v}} 2.3$ subunit of R-type calcium channels in mouse brain. J. Neurosci. 32, 13555 13566. doi: 10.1523/JNEUROSCI.1142-12.2012

Poncer, J.-C., McKinney, R. E., Gähwiler, B. H., and Thompson, S. M. (1997). Either $\mathrm{N}$ - or P- type calcium channels mediate GABA release at distinct hippocampal inhibitory synapses. Neuron 18, 463-472. doi: 10.1016/S0896-6273(00)81246-5

Schmidt, H., Brachtendorf, S., Arendt, O., Hallermann, S., Ishiyama, S., Bornschein, G., et al. (2013). Nanodomain coupling at an excitatory cortical synapse. Curr. Biol. 23, 244-249. doi: 10.1016/j.cub.2012.12.007

Schneggenburger, R., and Neher, E. (2005). Presynaptic calcium and control of vesicle fusion. Curr. Opin. Neurobiol. 15, 266-274. doi: 10.1016/j.conb.2005.05.006

Schneider, C. A., Rasband, W. S., and Eliceiri, K. W. (2012). NIH Image to ImageJ: 25 years of image analysis. Nat. Meth. 9, 671-675. doi: 10.1038/nmeth.2089

Scimemi, A., and Diamond, J. S. (2012). The number and organization of $\mathrm{Ca}^{2+}$ channels in the active zone shapes neurotransmitter release from Schaffer collateral synapses. J. Neurosci. 32, 18157-18176. doi: 10.1523/JNEUROSCI.3827-12.2012
Sheng, J., He, L., Zheng, H., Xue, L., Luo, F., Shin, W., et al. (2012). Calciumchannel number critically influences synaptic strength and plasticity at the active zone. Nat. Neurosci. 15, 998-1006. doi: 10.1038/nn.3129

Sibson, R. (1973). SLINK: An optimally efficient algorithm for the single-link cluster method. Comput. J. 16, 30-34. doi: 10.1093/comjnl/16.1.30

Spafford, J. D., and Zamponi, G. W. (2003). Functional interactions between presynaptic calcium channels and the neurotransmitter release machinery. Curr. Opin. Neurobiol. 13, 308-314. doi: 10.1016/S0959-4388(03)00061-8

Stevens, C. F. (2004). Presynaptic function. Curr. Opin. Neurobiol. 14, 341-345. doi: 10.1016/j.conb.2004.04.004

Südhof, T. C. (2013). Neurotransmitter release: the last millisecond in the life of a synaptic vesicle. Neuron 80, 675-690. doi: 10.1016/j.neuron.2013.10.022

Wheeler, D. B., Randall, A., and Tsien, R. W. (1994). Roles of N-type and Q-type $\mathrm{Ca}^{2+}$ channels in supporting hippocampal synaptic transmission. Science 264, 107-111. doi: 10.1126/science.7832825

Williams, C., Chen, W., Lee, C-H., Yaeger, D., Vyleta, N. P., and Smith, S. M. (2012). Coactivation of multiple tightly coupled $\mathrm{Ca}^{2+}$ channels triggers spontaneous release of GABA. Nat. Neurosci. 15, 1195-1197. doi: 10.1038/nn.3162

Wu, L-G., and Saggau, P. (1994). Pharmacological identification of two types of presynaptic voltage-dependent calcium channels at CA3-CA1 synapses of the hippocampus. J. Neurosci. 14, 5613-5622.

Xu, J., Pang, Z. P., Shin, O. H., and Südhof, T. C. (2009). Synamptotagmin-1 functions as a $\mathrm{Ca}^{2+}$ sensor for spontaneous release. Nat. Neurosci. 12, 759-766. doi: $10.1038 / \mathrm{nn} .2320$

Conflict of Interest Statement: The authors declare that the research was conducted in the absence of any commercial or financial relationships that could be construed as a potential conflict of interest.

Copyright (c) 2015 Althof, Baehrens, Watanabe, Suzuki, Fakler and Kulik. This is an open-access article distributed under the terms of the Creative Commons Attribution License (CC BY). The use, distribution or reproduction in other forums is permitted, provided the original author(s) or licensor are credited and that the original publication in this journal is cited, in accordance with accepted academic practice. No use, distribution or reproduction is permitted which does not comply with these terms. 


\title{
Dendritic diameters affect the spatial variability of intracellular calcium dynamics in computer models
}

\author{
Haroon Anwar ${ }^{1,2 *}$, Christopher J. Roome ${ }^{3}$, Hermina Nedelescu ${ }^{1,2+}$, Weiliang Chen ${ }^{2}$, Bernd Kuhn ${ }^{3}$ and \\ Erik De Schutter ${ }^{1,2}$
}

1 Theoretical Neurobiology and Neuroengineering, University of Antwerp, Wirrijk, Belgium

${ }^{2}$ Computational Neuroscience Unit, Okinawa Institute of Science and Technology, Onna-Son, Okinawa, Japan

${ }^{3}$ Optical Neuroimaging Unit, Okinawa Institute of Science and Technology, Onna-Son, Okinawa, Japan

Edited by:

Hartmut Schmidt, University of Leipzig, Germany

Reviewed by:

Arnd Roth, University College

London, UK

Knut Holthoff, Universitätsklinikum

Jena, Germany

\section{*Correspondence:}

Haroon Anwar, Department of

Biological Sciences, New Jersey

Institute of Technology, University

Heights, Newark, NJ 07102-1982,

USA

e-mail: hanwar@njit.edu

${ }^{\boldsymbol{t}}$ Present address:

Hermina Nedelescu, Brain

Mechanisms for Behavior Unit,

Kunigami-gun, Okinawa, Japan
There is growing interest in understanding calcium dynamics in dendrites, both experimentally and computationally. Many processes influence these dynamics, but in dendrites there is a strong contribution of morphology because the peak calcium levels are strongly determined by the surface to volume ratio (SVR) of each branch, which is inversely related to branch diameter. In this study we explore the predicted variance of dendritic calcium concentrations due to local changes in dendrite diameter and how this is affected by the modeling approach used. We investigate this in a model of dendritic calcium spiking in different reconstructions of cerebellar Purkinje cells and in morphological analysis of neocortical and hippocampal pyramidal neurons. We report that many published models neglect diameter-dependent effects on calcium concentration and show how to implement this correctly in the NEURON simulator, both for phenomenological pool based models and for implementations using radial 1D diffusion. More detailed modeling requires simulation of 3D diffusion and we demonstrate that this does not dissipate the local concentration variance due to changes of dendritic diameter. In many cases 1D diffusion of models of calcium buffering give a good approximation provided an increased morphological resolution is implemented.

Keywords: intracellular calcium, calcium concentration, calcium buffering, diffusion, dendritic diameter, compartmentalization, active dendrite, morphology

\section{INTRODUCTION}

Intracellular $\mathrm{Ca}^{2+}$ has a central role in the information processing capabilities of neuronal dendrites. $\mathrm{Ca}^{2+}$ entering through voltagegated $\mathrm{Ca}^{2+}$ channels (VGCC) and ligand-gated channels gives rise to cytosolic $\mathrm{Ca}^{2+}$, which in turn controls $\mathrm{Ca}^{2+}$-activated $\mathrm{K}^{+}$ $\left(\mathrm{K}_{\mathrm{Ca}}\right)$ channels during dendritic $\mathrm{Ca}^{2+}$ spikes (Goldberg et al., 2004; Womack and Khodakhah, 2004; Kampa and Stuart, 2006). Free cytosolic $\mathrm{Ca}^{2+}$ can also activate complex molecular signaling pathways involved in different forms of synaptic and dendritic plasticity (Konnerth et al., 1992; Kampa et al., 2006; Rancz and Hausser, 2006; Canepari and Vogt, 2008; Antunes and De Schutter, 2012). The cytosolic spread and dynamics of $\mathrm{Ca}^{2+}$ in dendritic morphologies are controlled by intracellular $\mathrm{Ca}^{2+}$ mechanisms like diffusion, endogenous buffers, internal stores, exchangers and pumps (Berridge, 1998; Augustine et al., 2003; Hartmann and Konnerth, 2005). Therefore, correct representation of $\mathrm{Ca}^{2+}$ related mechanisms in complex dendritic structures is crucial in construction of biophysically faithful multi-scale models of dendrites.

In addition to intracellular $\mathrm{Ca}^{2+}$ mechanisms and ion channel distributions, dendritic geometry has been shown to greatly affect the spatial variability of $\mathrm{Ca}^{2+}$ dynamics (Lev-Ram et al., 1992; Regehr and Tank, 1994; Schiller et al., 1995; Holthoff et al., 2002; Rozsa et al., 2004). The effects of dendritic geometry on $\mathrm{Ca}^{2+}$ transients are often quantified in terms of the surface to volume ratio (SVR). This is because $\mathrm{Ca}^{2+}$ influx scales with membrane surface while the change in $\mathrm{Ca}^{2+}$ concentration due to diffusion and buffering strongly depends on the volume. This results in larger amplitude transients expected in small diameter dendrites because they have a large SVR. Considering each dendritic segment as a cylinder, SVR is inversely proportional to the diameter of the cylinder. Therefore, even in the absence of intracellular $\mathrm{Ca}^{2+}$ mechanisms (endogenous buffers, internal $\mathrm{Ca}^{2+}$ stores) and diffusion, changes in dendritic diameter across the dendrite will result in spatially variable $\mathrm{Ca}^{2+}$ levels. Moreover, because $\mathrm{Ca}^{2+}$ buffering and diffusion are also affected by geometry, dendrite diameters can also affect the decay time constants of $\mathrm{Ca}^{2+}$ transients (Holthoff et al., 2002). In this paper we characterize this spatial variability in Purkinje cell models and explore implementation issues that affect how well a biophysically detailed dendrite model can capture the spatio-temporal variability of $\mathrm{Ca}^{2+}$ dynamics caused by local variation of dendrite diameters.

Traditionally, a $\mathrm{Ca}^{2+}$ pool with a single relaxation time constant is used to model intracellular $\mathrm{Ca}^{2+}$ dynamics (Destexhe et al., 1994). Such models compute the effects of $\mathrm{Ca}^{2+}$ influx accurately but combine all removal systems, including diffusion, into one process with a fixed time constant. They usually represent the $\mathrm{Ca}^{2+}$ concentration in a submembrane shell with a 
fixed depth. Previously, we have shown that these pool based models can not capture the complex dynamics of intracellular $\mathrm{Ca}^{2+}$ because they fail to simulate the multiple time scales at which interactions between VGCC and $\mathrm{K}_{\mathrm{Ca}}$ channels occur (Anwar et al., 2012). Here we extend the comparison of $\mathrm{Ca}^{2+}$ pool to complex $\mathrm{Ca}^{2+}$ dynamics models to the spatial domain. We will show that many model implementations in the literature do not compute correct volumes for the submembrane shell and that accuracy of morphological reconstruction is a more important concern than the limitations of only modeling $1 \mathrm{D}$ radial diffusion. In addition we provide detailed instructions on how to model biophysically realistic $\mathrm{Ca}^{2+}$ dynamics in compartmental models of dendrites.

\section{MATERIALS AND METHODS MORPHOLOGY RECONSTRUCTIONS Purkinje cell morphologies}

Dendritic morphologies of 10 Purkinje cells (PC 3-12) used in this study were obtained from the NeuroMorpho database (http:// neuromorpho.org). An additional Purkinje cell morphology (PC 2) used in this study was provided by Ede Rancz and Michael Häusser, UCL, London, UK. Considering the small sample size of available PC neurons (11 cells) and their large variability in dendritic diameters, we decided to obtain an additional morphology (PC 1) with carefully reconstructed diameters.

\section{PC morphology with carefully reconstructed diameters}

All procedures for the care of animals were according to the Science Council of Japan Guidelines for Proper Conduct of Animal Experiments, and also the guideline approved by OIST Graduate University Animal Resources Section. A 4-week old mouse was anesthetized with isofluorane and decapitated. The cerebellum was removed from the skull and immediately collected into a vial containing ice cold carbogenated ACSF: $\mathrm{NaCl} 125 \mathrm{mM}, \mathrm{KCl} 2.5 \mathrm{mM}, \mathrm{NaH}_{2} \mathrm{PO}_{4} 1.2 \mathrm{mM}, \mathrm{MgSO}_{4}$ $1.9 \mathrm{mM}$, Glucose $10 \mathrm{mM}, \mathrm{NaHCO}_{3} 25 \mathrm{mM}, \mathrm{CaCl}_{2} 2 \mathrm{mM}$ at 300$305 \mathrm{mOsm}$. Sagittal slices of $250 \mu \mathrm{m}$ thickness were cut and placed in a recording chamber with carbogenated ACSF. The glass electrode $(4 \mathrm{MOhm})$ was filled with intracellular solution containing potassium gluconate $140 \mathrm{mM}, \mathrm{NaCl} 10 \mathrm{mM}$, HEPES 10 mM, EGTA $0.2 \mathrm{mM}$, MgATP $4 \mathrm{mM}$, NaGTP $0.4 \mathrm{mM}$, Phosphocreatine $10 \mathrm{mM}$ and $50 \mu \mathrm{M}$ Alexa 594 (Invitrogen) with pH 7.3 and 300 mOsm.

A custom-built two-photon microscope (MOM, Sutter) with a Ti:sapphire laser (Vision II, Coherent), GaAsP photomultiplier tubes, and a 25x water (NA 1.05, Olympus) objective lens was used to acquire a 3D image stack of the Alexafilled Purkinje cell with a z-step size of $0.25 \mu \mathrm{m}$ and an $x y$ field of view of $1024 \times 1024$ pixels. Next, the acquired 3D image stack was deconvoluted using AutoQuantX2 software (Media Cybernetics) using a theoretical point spread function (1-5 iterations) based on specifications from the image acquisition parameters. Later, the dendrite of Purkinje cell was reconstructed with Neurolucida, MBF Bioscience, (http:// www.mbfbioscience.com/neurolucida). A different reconstruction of the same Purkinje cell has previously been used in Anwar et al. (2013).

\section{$\mathrm{Ca}^{2+}$ SPIKING MODEL}

The detailed model of spontaneous $\mathrm{Ca}^{2+}$ spike generation was derived from the original biophysical model (Schmidt et al., 2003; Anwar et al., 2012) developed in the NEURON simulator (Hines and Carnevale, 1997). The model contained four types of ion channels: P-type $\mathrm{Ca}^{2+}$ channel $\left(\mathrm{P}_{\max }=2 \times 10^{-4} \mathrm{~cm} / \mathrm{s}\right.$, GHK equation) (Swensen and Bean, 2005), T-type $\mathrm{Ca}^{2+}$ channel $\left(\mathrm{P}_{\max }=8 \times 10^{-6} \mathrm{~cm} / \mathrm{s}\right.$, GHK equation) (Iftinca et al., 2006), BK-type $\mathrm{Ca}^{2+}$-activated $\mathrm{K}^{+}$channel $\left(\mathrm{G}_{\max }=7 \times 10^{-2} \mathrm{~S} / \mathrm{cm}^{2}\right)$ (Cox et al., 1997) and SK-type $\mathrm{Ca}^{2+}$-activated $\mathrm{K}^{+}$channel $\left(\mathrm{G}_{\max }=3.1 \times 10^{-4} \mathrm{~S} / \mathrm{cm}^{2}\right)$ (Hirschberg et al., 1998; Solinas et al., 2007), plus a leak channel $\left(\mathrm{G}_{\max }=1 \times 10^{-6} \mathrm{~S} / \mathrm{cm}^{2}\right.$ and $\left.\mathrm{E}_{\mathrm{rev}}=-61 \mathrm{mV}\right)$.

\section{$\mathrm{Ca}^{2+}$ BUFFERING MODELS}

Intracellular $\mathrm{Ca}^{2+}$ was modeled using the following $\mathrm{Ca}^{2+}$ buffering mechanisms.

\section{$\mathrm{Ca}^{2+}$ pool}

The exponential decaying $\mathrm{Ca}^{2+}$ pool was modeled as

$$
\frac{\mathrm{d}\left[\mathrm{Ca}^{2+}\right]_{\mathrm{i}}}{\mathrm{dt}}=-\frac{\mathrm{I}_{\mathrm{Ca}^{2+}}(\mathrm{t})}{2 \mathrm{Fd}_{\mathrm{eq}}}-\beta\left(\left[\mathrm{Ca}^{2+}\right]_{\mathrm{i}}-\left[\mathrm{Ca}^{2+}\right]_{0}\right)
$$

where $\left[\mathrm{Ca}^{2+}\right]_{i}$ is intracellular $\mathrm{Ca}^{2+}$ concentration, $\left[\mathrm{Ca}^{2+}\right]_{0}$ is $\mathrm{Ca}^{2+}$ concentration at rest and is $45 \mathrm{nM}, \mathrm{I}_{\mathrm{Ca}}(\mathrm{t})$ is total $\mathrm{Ca}^{2+}$ current per unit area through VGCC, $\mathrm{F}$ is the Faraday's constant, $\mathrm{d}_{\mathrm{eq}}$ is the equivalent depth of a submembrane shell to define the volume for effective $\mathrm{Ca}^{2+}$ concentration, and $\beta$ is the decay time constant. The values for depth (d) and $\beta, 0.169 \mu \mathrm{m}$ and $6.86 \mathrm{~ms}^{-1}$ respectively, were obtained from a past study (Anwar et al., 2012), where these values were fitted to generate dendritic $\mathrm{Ca}^{2+}$ spikes.

Two different definitions for $\mathrm{d}_{\mathrm{eq}}$ were used. The first definition $\left(\mathrm{SP}_{\mathrm{old}}\right)$, uses a mechanism widely used in multicompartment modeling studies using NEURON (e.g., Miyasho et al., 2001; Poirazi et al., 2003; Hemond et al., 2008; Hay et al., 2011) that takes the volume of the submembrane shell to be directly proportional to its depth $\mathrm{d}$ and therefore $\mathrm{d}_{\mathrm{eq}}=\mathrm{d}$. This results in an incorrect volume of submembrane shell (see details in Results). The second definition $\left(\mathrm{SP}_{\text {new }}\right)$ used in this study computed an equivalent depth $\left(\mathrm{d}_{\mathrm{eq}}\right)$ for each submembrane shell, which gives the correct volume (see details in Results) when used in the mechanism described by (1):

$$
\mathrm{d}_{\mathrm{eq}}=\mathrm{d}-\frac{\mathrm{d}^{2}}{\operatorname{diam}}
$$

where diam is the diameter of each compartment.

\section{Detailed $\mathrm{Ca}^{2+}$ dynamics}

The detailed $\mathrm{Ca}^{2+}$ dynamics model used in this study was obtained from our previous study (Anwar et al., 2012). It included calbindin (CB) and parvalbumin (PV) as buffers. In addition to $\mathrm{Ca}^{2+}$, both $\mathrm{PV}$ and $80 \%$ of $\mathrm{CB}$ were diffusible (Schmidt et al., 2005; Anwar et al., 2012). A single surface-based $\mathrm{Ca}^{2+}$ 
pump was modeled using Michaelis-Menten kinetics (Sala and Hernandez-Cruz, 1990) as follows:

$$
\text { pump }+\mathrm{Ca}^{2+} \underset{\mathrm{k}_{\mathrm{f}}}{\stackrel{\mathrm{k}_{\mathrm{b}}}{\rightleftharpoons}} \text { pump }-\mathrm{Ca}^{2+} \stackrel{\mathrm{k}_{\mathrm{ext}}}{\longrightarrow} \text { pump }
$$

where pump density was $1 \times 10^{-15}$ mol.cm ${ }^{-2}$, $\mathrm{k}_{\mathrm{f}}$ was $3 \times$ $10^{3} \mathrm{mM}^{-1} \cdot \mathrm{ms}^{-1}$, $\mathrm{k}_{\mathrm{b}}$ was $17.5 \mathrm{~ms}^{-1}$ and $\mathrm{k}_{\mathrm{ext}}$ was $72.55 \mathrm{~ms}^{-1}$.

\section{Diffusion in NEURON simulator}

In NEURON (Hines and Carnevale, 1997) simulations, diffusion of $\mathrm{Ca}^{2+}$, free and bound buffers was allowed only in the radial dimension, i.e., from membrane toward the center of the compartment and vice versa. Two different ways of discretizing space into concentric cylindrical shells were used. The first one, the variable depth scheme, is described as the standard example in the NEURON book (Carnevale and Hines, 2006). Each compartment is subdivided into radial shells (Figure 1) and the number of shells is computed using:

$$
\text { Shells }=\left\lfloor\frac{\text { diam }}{4 \mathrm{~d}}+1.5\right\rfloor
$$

where Shells is the number of radial shells, diam is diameter of the compartment and $d$ is depth of the outer radial shell, which was $0.1 \mu \mathrm{m}$. The discretization of the compartment volume into radial shells, where the depth of inner radial shells is twice the depth of outer radial shell, resulted in a varying depth of all shells, depending on the diameter of the compartment. The depth $\left(\mathrm{d}_{1}\right)$ of the outer shell and the inner most shell is then:

$$
\mathrm{d}_{1}=\frac{\text { diam }}{4(\text { Shells }-1)}
$$

and the other shells have a depth of $2 \times \mathrm{d}_{1}$ (see Figure $\mathbf{1}$ ).

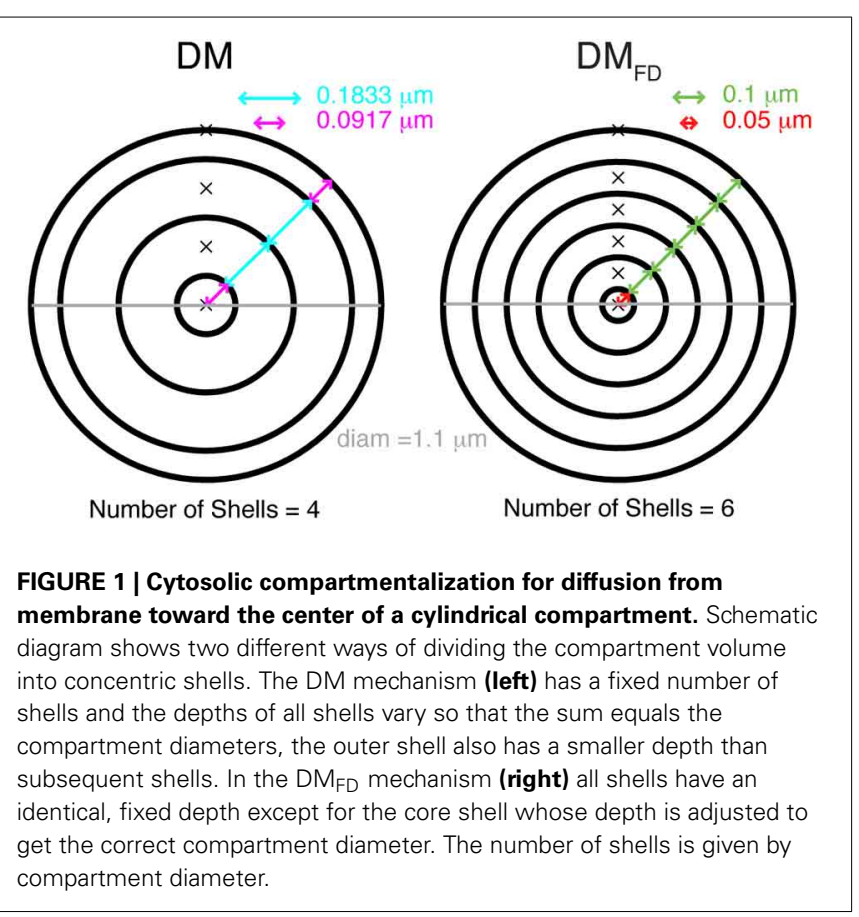

We also implemented a fixed depth scheme, where all the radial shells except the inner most core shell had a constant depth (Figure 1). The number of shells was computed using:

$$
\text { Shells }_{\mathrm{FD}}=\left\lceil\frac{\text { diam }}{2 \mathrm{~d}}\right\rceil
$$

Here $d_{1}$ as well as the depth of other shells was always $0.1 \mu \mathrm{m}$, and the core shell had a variable depth $(\leq 0.1 \mu \mathrm{m})$. Note that to model radial diffusion with a variable number of shells, a separate mechanism with a unique configuration of shells for every compartment with a different diameter needs to be created in NEURON.

\section{Diffusion in STEPS simulator}

To allow 3D diffusion in the stochastic reaction-diffusion simulator STEPS (Hepburn et al., 2012), the dendritic morphology (part of PC 1) was discretized into tetrahedral mesh using CUBIT (http://cubit.sandia.gov).

\section{COMPUTER SIMULATIONS}

All the simulations were run using a time step of $0.02 \mathrm{~ms}$. Model scripts for all models used in this work are available at http://senselab.med.yale.edu/modeldb/ShowModel.asp? model $=155731$.

\section{$\mathrm{Ca}^{2+}$ spike generation in realistic morphologies}

Spontaneous $\mathrm{Ca}^{2+}$ spikes were generated using realistic morphologies of PCs with ion channels uniformly distributed over the dendrites. The $\mathrm{Ca}^{2+}$ spike generation model was simulated with the following conditions: temperature of 34 Celsius, initial voltage of $-60 \mathrm{mV}$, membrane capacitance of $1.12 \mu \mathrm{F} . \mathrm{cm}^{-2}$ and axial resistance of $250 \mathrm{Ohm} . \mathrm{cm}$.

\section{$\mathrm{Ca}^{2+}$ transients in single compartments}

$\mathrm{Ca}^{2+}$ transients were simulated using different $\mathrm{Ca}^{2+}$ buffering models in single compartments with diameter varying from 0.1 to $6 \mu \mathrm{m}$ in steps of $0.1 \mu \mathrm{m}$. The P-type $\mathrm{Ca}^{2+}$ channel with $\mathrm{P}_{\max }$ of $5.2 \times 10^{-5} \mathrm{~cm} / \mathrm{s}$ was included in the model for $\mathrm{Ca}^{2+}$ influx. A "ramp-like" voltage step protocol (same as in Anwar et al., 2012) was used to depolarize the compartment to the voltage at which physiological dendritic $\mathrm{Ca}^{2+}$ spikes are generated.

\section{$\mathrm{Ca}^{2+}$ transients in part of dendritic morphology}

$\mathrm{Ca}^{2+}$ transients in a part of PC 1 dendritic morphology were simulated using the detailed $\mathrm{Ca}^{2+}$ dynamics model with $1 \mathrm{D}$ diffusion in NEURON and with 3D diffusion in STEPS. Because of the long runtime for $3 \mathrm{D}$ diffusion simulations it was not possible to simulate a complete PC in STEPS.

A uniform current (in $\mathrm{mA} / \mathrm{cm}^{2}$ ) recorded during a $\mathrm{Ca}^{2+}$ spike was applied to each compartment in the NEURON simulations to evoke a constant shape of the spike. Two types of compartmentalization approaches were used in these simulations. Firstly, using a single compartment per dendritic section (Total sections $=$ $45)$. Secondly, each of the dendritic section was split into multiple $(1-22)$ sections (Total sections $=300)$, where each section consisted of adjacent traced points on the dendrite. 
$\mathrm{Ca}^{2+}$ influx in STEPS was implemented using first-order surface based $\mathrm{Ca}^{2+}$ influx reaction $\left(\mathrm{X} \longrightarrow \mathrm{X}+\mathrm{Ca}^{2+}\right)$, where " $\mathrm{X}$ " channels $(100,000)$ were distributed uniformly over the surface triangles $(\sim 92,000)$ of the mesh. At each time point, the influx rate per channel was updated based on the $\mathrm{Ca}^{2+}$ influx profile (obtained using the total current applied in the NEURON simulation). Due to high rate and uniformity of influx, effects of stochasticity were negligible. The results of STEPS simulations in this study are reported as the mean computed over 10 trials.

\section{RESULTS}

In this study we explore the effect of dendrite diameter on $\mathrm{Ca}^{2+}$ dynamics in models of different complexity. Figure 2 shows that simulation results are strongly influenced by how one implements the model by comparing the integrated $\mathrm{Ca}^{2+}$ concentrations (for all time points in a time window, the sum of $\mathrm{Ca}^{2+}$ concentrations multiplied by the time step) in three different models of a spontaneous burst of $\mathrm{Ca}^{2+}$ spikes (Figure 2D; see the corresponding currents in Figure 2E) computed using the NEURON simulator. Figure $2 \mathrm{~A}$ shows the result when using a simple pool model to compute $\mathrm{Ca}^{2+}$ concentrations based on an approach used in most NEURON simulations ( $\mathrm{SP}_{\text {old }}$, e.g., Miyasho et al., 2001;
Poirazi et al., 2003; Hemond et al., 2008; Hay et al., 2011). Using this approach no gradients of $\mathrm{Ca}^{2+}$ concentration are predicted within the dendrite; this result is unlikely to be physiological considering the large variation in SVR across the dendrite. The model in Figure 2B ( $\left.\mathrm{SP}_{\text {new }}\right)$ also uses a simple pool but implemented differently; it results in strong $\mathrm{Ca}^{2+}$ gradients with higher concentrations in thin dendritic branches as expected from the SVR. Finally we simulated a detailed $\mathrm{Ca}^{2+}$ dynamics models with buffers and radial 1D diffusion (Figure 2C, DM). This shows similar gradients as $\mathrm{SP}_{\text {new }}$, but with higher $\mathrm{Ca}^{2+}$ peak values as expected from previous work comparing DM to simple pool models (Anwar et al., 2012).

Next we will describe in detail the differences between $\mathrm{SP}_{\text {new }}$ and $\mathrm{SP}_{\text {old }}$ and then analyze the diameter dependence in $\mathrm{SP}_{\text {new }}$ and DM.

\section{INACCURACY OF Ca ${ }^{2+}$ VOLUMES IN SIMPLE POOL MODELS AND THEIR EFFECTS ON Ca ${ }^{2+}$ LEVELS}

Many multi-compartment dendritic models use a single pool model of $\mathrm{Ca}^{2+}$ buffering, which simulates only the submembrane $\mathrm{Ca}^{2+}$ concentration to control $\mathrm{K}_{\mathrm{Ca}}$ channels. These phenomenological models convert $\mathrm{Ca}^{2+}$ current passing through VGCC to
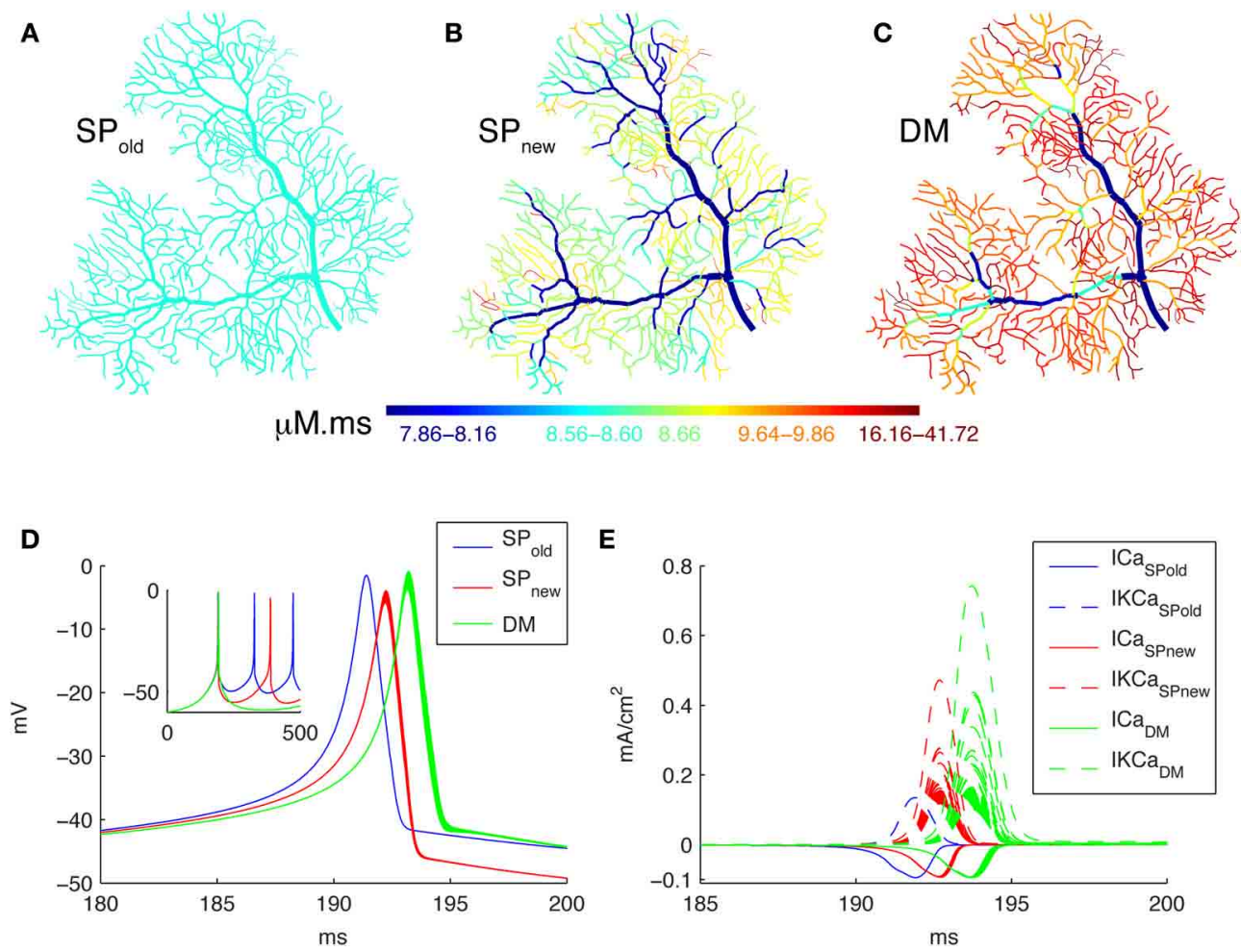

FIGURE 2 | Spatial $\mathrm{Ca}^{2+}$ gradients strongly depend on type of model implementation. Panels (A-C) show maps of the integrated calcium levels in the dendrite during a spontaneous burst of $\mathrm{Ca}^{2+}$ spikes (panel D). The dendritic branches are color coded to show the integrated calcium levels using a $20 \mathrm{~ms}$ window around the peak $\mathrm{Ca}^{2+}$ concentration of the first dendritic $\mathrm{Ca}^{2+}$ spike. The color scales used in these maps are nonlinear (using histogram equalization) to enhance the contrast. (A) Single $\mathrm{Ca}^{2+}$ pool model using $\mathrm{SP}_{\text {old }}$ mechanism results in homogenous $\mathrm{Ca}^{2+}$ levels. (B) Single

$\mathrm{Ca}^{2+}$ pool model using $\mathrm{SP}_{\text {new }}$ mechanism results in variable $\mathrm{Ca}^{2+}$ levels. (C) Detailed $\mathrm{Ca}^{2+}$ dynamics model with buffering and $1 \mathrm{D}$ diffusion results in variable $\mathrm{Ca}^{2+}$ levels with larger $\mathrm{Ca}^{2+}$ gradients. (D) Voltage traces show the first spike of the $\mathrm{Ca}^{2+}$ burst for each model in all dendritic compartments for the 3 different models (see color code in Figure). The inset shows complete traces. (E) The underlying $\mathrm{Ca}^{2+}$ and $\mathrm{K}_{\mathrm{Ca}}$ currents (recorded from all dendritic compartments) for the $\mathrm{Ca}^{2+}$ spike of the three different models (see color code in Figure). 
A

$1.414 \mu \mathrm{m}^{3}, 36 \%$ of total volume $\left(3.927 \mu \mathrm{m}^{3}\right)$ Surface to Volume ratio $=11.11$

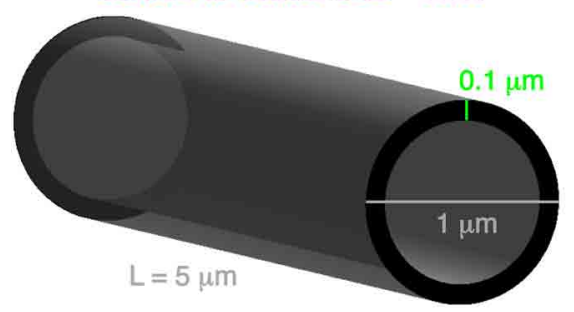

B

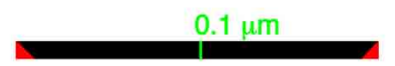

Surface to Volume $\left(1.571 \mu \mathrm{m}^{3}\right)$ ratio $=10$

C

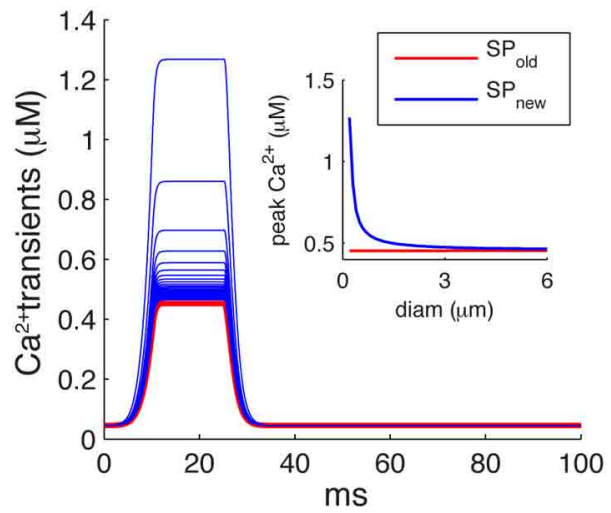

FIGURE 3 | Errors introduced by incorrect submembrane volumes of single pool models. (A) Comparison between cylindrical dendritic compartments with diameters of $1 \mu \mathrm{m}$ (left) and $0.5 \mu \mathrm{m}$ (right) with submembrane shells with a depth of $0.1 \mu \mathrm{m}$. A correct implementation of the volume of the submembrane shell representing the single $\mathrm{Ca}^{2+}$ pool (SPnew mechanism) results in a SVR that depends on the compartment diameter. (B) For the same compartments using the $\mathrm{SP}_{\text {old }}$ mechanism results in volumes that are too large and have a constant SVR. The cross-sectional area of each compartment (black disks shown in $\mathbf{A}$ ) is unfolded and drawn to show that the actual volume of the submembrane shell ( $\mathrm{SP}_{\text {new }}$ ) is smaller than the volume used in the $\mathrm{SP}_{\text {old }}$ mechanism. The red triangles represent extra cross-sectional area included in the volume of $\mathrm{SP}_{\text {old. }}$ (C) $\mathrm{Ca}^{2+}$ transients generated using a "ramp-like" voltage command in single compartments with diameters ranging from 0.2 to $6 \mu \mathrm{m}$ in steps
$S P$

new

$0.628 \mu \mathrm{m}^{3}, 64 \%$ of total volume $\left(0.982 \mu \mathrm{m}^{3}\right)$

Surface to Volume ratio $=12.5$

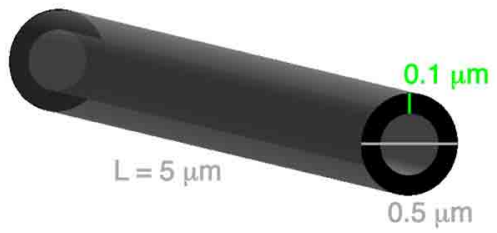

$\mathrm{SP}_{\text {old }}$

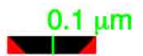

Surface to Volume $\left(0.785 \mu \mathrm{m}^{3}\right)$ ratio $=10$

D

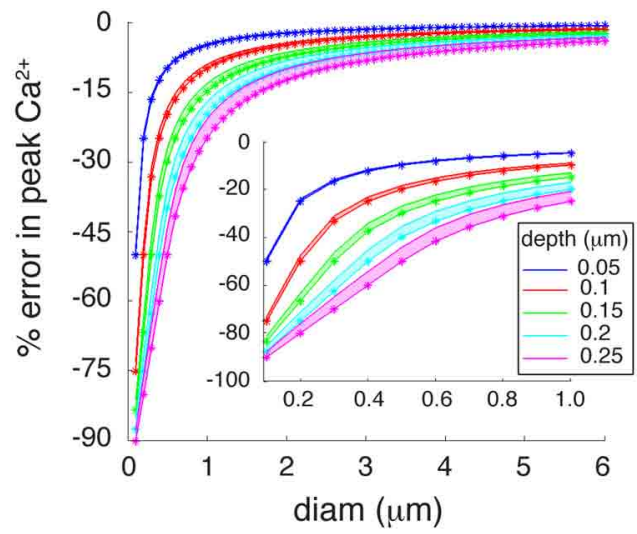

of $0.1 \mu \mathrm{m}$. P-type $\mathrm{Ca}^{2+}$ channel with $\mathrm{P}_{\max }$ of $5.2 \times 10^{-5} \mathrm{~cm} / \mathrm{s}$ was used for $\mathrm{Ca}^{2+}$ influx. Inset: comparison of peak amplitudes of $\mathrm{Ca}^{2+}$ transients using $\mathrm{SP}_{\text {old }}$ and $\mathrm{SP}_{\text {new }}$ show that the first mechanism causes exactly the same transient in all compartments, whereas, $\mathrm{SP}_{\text {new }}$ causes transients with varying peak $\mathrm{Ca}^{2+}$ amplitudes. (D) Error in peak $\mathrm{Ca}^{2+}$ levels caused by using the $\mathrm{SP}_{\text {old }}$ mechanism [error $=\left(\max \left(\left[\mathrm{Ca}^{2+}\right]_{\mathrm{SP}}\right.\right.$ old $)-$ $\left.\left.\max \left(\left[\mathrm{Ca}^{2+}\right]_{S_{\text {P_new }}}\right) / \max \left(\left[\mathrm{Ca}^{2+}\right]_{\text {SP_new }}\right)\right)\right]$. Pool models used $\beta$-values of 0.02 , 6.86 , and $10 \mathrm{~ms}^{-1}$; and depth (d) values of $0.05,0.1,0.15,0.2$, and $0.25 \mu \mathrm{m}$. The lower edge of shaded areas of each color shows error in peak calcium for $\beta$-value of $10 \mathrm{~ms}^{-1}$, whereas, the upper edge of shaded areas of each color show error for $\beta$-value of $0.02 \mathrm{~ms}^{-1}$. The colored asterisks show corresponding error for $\beta$-value (used to model PC dendrites) of $6.86 \mathrm{~ms}^{-1}$. Inset highlights large errors for branches with small diameters (diam $\leq 1 \mu \mathrm{m})$.
$\mathrm{Ca}^{2+}$ concentration using a submembrane shell of fixed depth, $\mathrm{d}$ [Equation (1); Figure 3A]. The volume of a submembrane shell (as for $\mathrm{SP}_{\text {new }}$ ) is defined as:

$$
\mathrm{Vol}_{s_{-} \text {new }}=\mathrm{Vol}_{\mathrm{f}}-\mathrm{Vol}_{\mathrm{c}}=\pi \mathrm{d}(\operatorname{diam}-\mathrm{d}) \mathrm{L}
$$

where $V_{0} l_{f}$ denotes the volume of a full compartment, $\mathrm{Vol}_{\mathrm{c}}$ denotes the volume of the core, $\mathrm{d}$ is the depth of submembrane shell, diam is the diameter of compartment and $\mathrm{L}$ is its length. Using such a representation of submembrane shell, $S R_{\text {new }}$ equals

$$
\mathrm{SVR}_{\text {new }}=\frac{\mathrm{SA}}{\mathrm{Vol}_{\text {Snew }_{-}}}=\frac{\text { diam }}{\mathrm{d}(\mathrm{diam}-\mathrm{d})}
$$

where SA is the surface area of the compartment. Note that $\mathrm{SVR}_{\text {new }}$ is less dependent on diameter than the SVR for the complete volume (1/diam), but, as shown in Figure $\mathbf{3 A}, \mathrm{SVR}_{\text {new }}$ still increases for smaller diameters.

However, we noticed that most of the $\mathrm{Ca}^{2+}$ shell models implemented in NEURON use an incorrect volume for the 
submembrane shell (Figure $3 \mathrm{~B})$. In those models $\left(\mathrm{SP}_{\mathrm{old}}\right)$, the volume of a submembrane shell is defined as:

$$
\mathrm{Vol}_{\text {s_old }}=\mathrm{SA} \times \mathrm{d}=\pi \operatorname{diamLd}
$$

Using such a representation of submembrane shell, gives $S_{V} R_{\text {old }}$

$$
\mathrm{SVR}_{\mathrm{old}}=\frac{\mathrm{SA}}{\text { Vol }_{\text {s_old }}}=\frac{1}{\mathrm{~d}}
$$

Because the depth (d) of submembrane shells is usually taken constant for variable diameter compartments, SVR old is constant and independent of the dendrite diameter. As a result, we observe changes in peak amplitudes of the simulated $\mathrm{Ca}^{2+}$ transients using $\mathrm{SP}_{\text {new }}$ in compartments with different diameters (Figure 3C) while the same compartments with $\mathrm{SP}_{\text {old }}$ always show exactly the same $\mathrm{Ca}^{2+}$ transient.

Since pool based models are phenomenological models, the values of depth (d) and decay time constants $(\beta)$ can be tuned to approximate the desired behavior of intracellular $\left[\mathrm{Ca}^{2+}\right]$. In Figure 3D we show how the error of using $\mathrm{SP}_{\text {old }}$ (compared to $\mathrm{SP}_{\text {new }}$ ) depends on the values of $\mathrm{d}$ and $\beta$ used. The errors in peak $\mathrm{Ca}^{2+}$ were computed using $\beta$-values of $0.02 \mathrm{~ms}^{-1}$ (Traub and Llinas, 1977), $6.86 \mathrm{~ms}^{-1}$ (Anwar et al., 2012), and $10 \mathrm{~ms}^{-1}$ (De Schutter and Bower, 1994) and using submembrane shells with depths ranging from 0.05 to $0.25 \mu \mathrm{m}$. The error increases with the size of depth used, as expected from $S_{V R}$ new. More importantly, these errors become significantly larger for smaller diameters (diam $<1 \mu \mathrm{m}$ ) and may reach up to $80 \%$ for $0.1 \mu \mathrm{m}$ diameter compartments (inset of Figure 3D). Typically, distal dendrites have large numbers of dendritic branches with diameters less than $1 \mu \mathrm{m}$.

In the rest of our study we will only focus on $\mathrm{SP}_{\text {new }}$ and DM to investigate how well they can capture $\mathrm{Ca}^{2+}$ gradients in dendrites.

\section{DETAILED $\mathrm{Ca}^{2+}$ DYNAMICS MODEL CAUSE LARGE SPATIAL VARIABILITY OF Ca ${ }^{2+}$ LEVELS IN REALISTIC DENDRITIC MORPHOLOGIES}

Figure 2 demonstrates in one dendritic morphology that SVR differences cause sharper $\mathrm{Ca}^{2+}$ gradients when it was modeled using $\mathrm{DM}$ compared to $\mathrm{SP}_{\text {new }}$. We next investigated whether this is a systematic observation by simulating the dendritic $\mathrm{Ca}^{2+}$ spike model in 11 additional dendritic reconstructions of PCs using both methods and comparing the results (Figure 4). For each PC, the ion channels were distributed uniformly on its dendrite and each unbranched segment had a constant diameter.

Because this study mostly focuses on local differences in $\mathrm{Ca}^{2+}$ concentration, we summarized the data on spatial gradients by computing the ratio of integrated $\left[\mathrm{Ca}^{2+}\right]$ in adjacent dendritic segments and plotting the distributions of these ratios in Figure 4. We observe a wide range of distributions of spatial fluctuations of $\mathrm{Ca}^{2+}$ levels in different neuron reconstructions, with the histograms of some neurons (e.g., PC 2 and 5) showing very large tails and other ones only small fluctuations (ratio $<2$ ). This observation may be related to differences in the quality of the reconstructions (see Discussion). But in all cases, the DM model
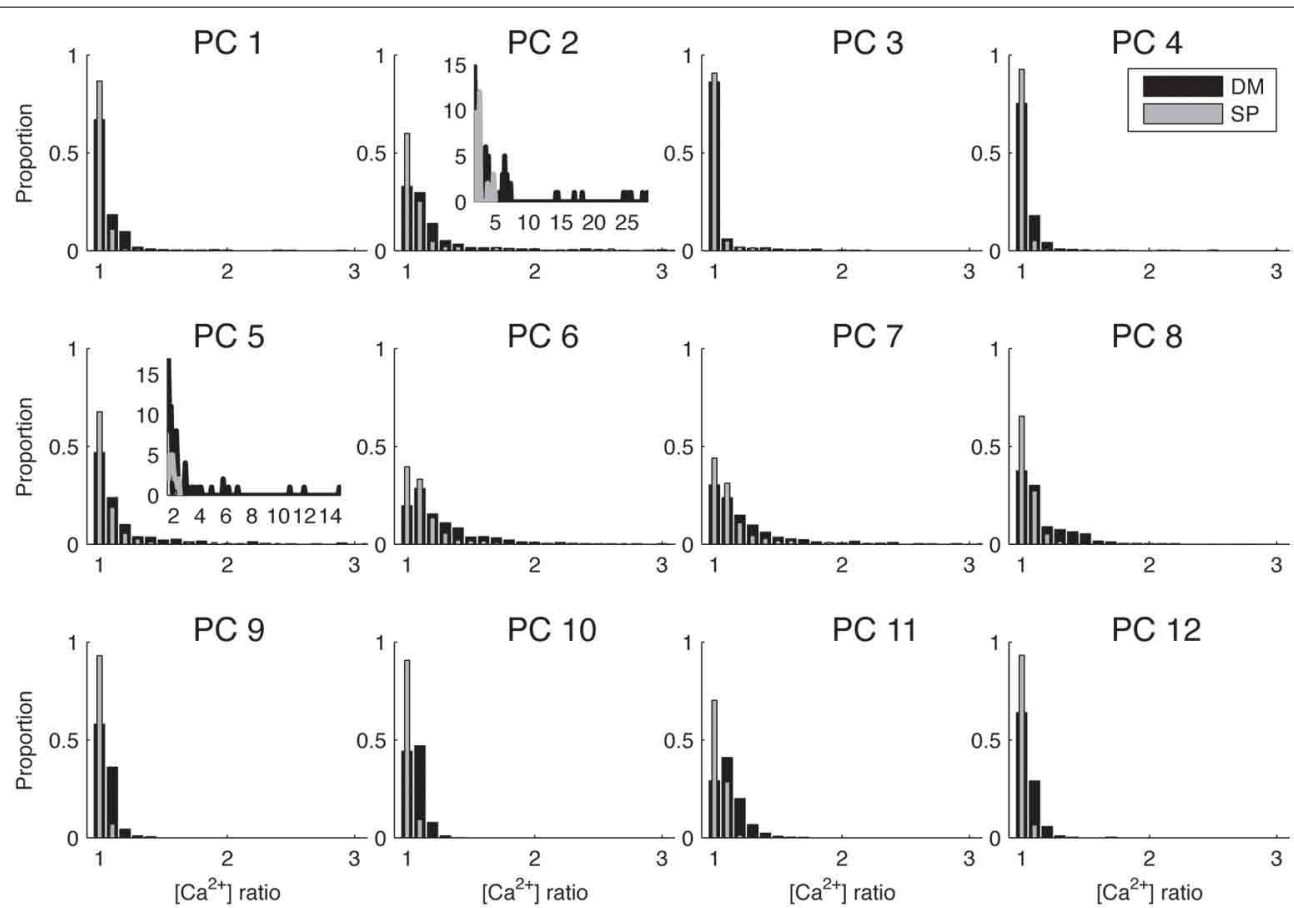

FIGURE 4 | Biophysically detailed $\mathrm{Ca}^{2+}$ dynamics model causes larger differences in calcium levels in adjacent dendritic branches than single pool models. Histograms of ratios between integrated calcium from adjacent dendritic branches for 12 different PCs using $\mathrm{SP}_{\text {new }}$ and DM. To make the differences between cells more visible only the range of ratios 1-3 is shown, for the two cells that have significantly larger ratios the full distribution is shown in the inset. $\mathrm{PC} 1$ is shown in Figure 2. Integrated $\mathrm{Ca}^{2+}$ was computed for $20 \mathrm{~ms}$ around the first peak of $\mathrm{Ca}^{2+}$ transients for all PCs. 
always results in a wider range of $\mathrm{Ca}^{2+}$ fluctuations as compared to $\mathrm{SP}_{\text {new. }}$.

\section{VARIABILITY OF DIAMETERS IN MORPHOLOGICAL RECONSTRUCTIONS OF NEURONS}

In Figure 4 we used a common approach in compartmental modeling: we ignored small variations in diameter by taking only a single compartment for each unbranched segment. However, due to the large jumps in $\mathrm{Ca}^{2+}$ concentrations between neighboring compartments observed in some parts of the model (Figures 2, 4) we wondered about the realism of this assumption of uniform diameter. We investigated this issue both in the 12 PCs modeled previously as well as in 284 neocortical and 38 hippocampal pyramidal neuron reconstructions, because larger changes in diameter may be present in morphological classes where the level of branching is not as extensive as in PCs. For both neuron types we computed the coefficient of variation $(\mathrm{CV})$ of diameters for every dendritic segment (between two branch points) based on all the measurements available in the morphological reconstruction (Figure 5). We observed a large variability in $\mathrm{CV}$ of reconstructed morphologies of neurons obtained from different laboratories for both cell classes (see Discussion), but overall the variability of diameter was much larger in pyramidal neurons where in many cells more than a quarter of the unbranched segments had CVs of 0.4 or more. In PCs more than half of the reconstructions had CVs of 0.2 or more in at least a quarter of their unbranched segments.

Our analysis suggests that for pyramidal neurons even more care should be taken when converting dendritic segments into cylindrical compartments. A good representation of dendritic segments with rapidly varying diameters is essential to model intracellular $\mathrm{Ca}^{2+}$ concentrations correctly (see also Figure 8).

\section{EFFECT OF VOLUME DISCRETIZATION ON DETAILED $\mathrm{Ca}^{2+}$ DYNAMICS MODELS}

Though the implementation of 1D diffusion in concentric cylindrical shells may seem straightforward, the NMODL language used in NEURON actually makes it difficult to do this in a flexible way and we discovered that many existing models do not implement it correctly. The standard example in the NEURON book (Carnevale and Hines, 2006) is a variable depth scheme where the volume is divided over a fixed number of concentric shells (4 in the standard example) with variable depth Equations (3) and (4) and Figure 1: DM, note that the submembrane and core shells have a smaller depth than the others). Many models using NEURON implement exactly this mechanism: 4 shells and all with variable depth. Because the volume of the submembrane shell is used to convert inward $\mathrm{Ca}^{2+}$ currents into a $\mathrm{Ca}^{2+}$ concentration that directly activates $\mathrm{K}_{\mathrm{Ca}}$ channels, varying its depth will affect the computed value of this $\mathrm{Ca}^{2+}$ concentration. From a biophysical perspective there is no reason why the depth of a submembrane shell that is assumed to simulate the effective volume affecting the $\mathrm{Ca}^{2+}$ sensors of $\mathrm{K}_{\mathrm{Ca}}$ channels (Fakler and Adelman, 2008; De Schutter, 2010) should vary greatly with dendrite diameter. We will therefore consider two issues: the number of shells to be modeled and a submembrane shell with variable (DM) or fixed depth $\left(\mathrm{DM}_{\mathrm{FD}}\right)$.

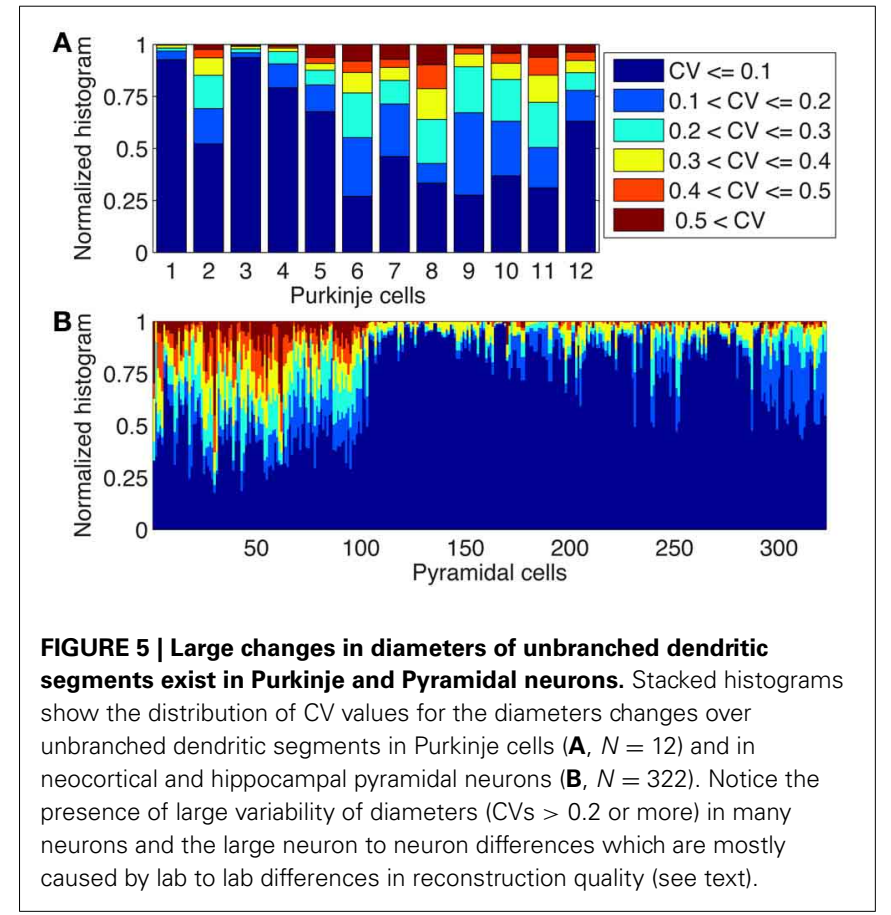

If one wants to vary the number of shells modeled depending on compartment diameter, which is the correct solution, a separate NEURON mechanism has to be created for each specific number of shells that is required. Some authors have therefore decided to use a fixed number of shells with variable depth of each shell (Migliore et al., 1995; Lazarewicz et al., 2002; Gold et al., 2007; Lavzin et al., 2012) (Figure 1: DM), but this can lead to significant errors in simulated submembrane $\mathrm{Ca}^{2+}$ concentration in large diameter dendrites if the number of shells is taken to be small (Figure 6A: circles). These errors show both a positive and negative component depending on compartment diameter, suggesting that two types of error contribute. Indeed, when we repeated these simulations with a fixed submembrane shell depth $\mathrm{d}_{1}$ of $0.1 \mu \mathrm{m}$ and the rest of the volume divided over the remaining shells with equal, variable depths (FD: Figure 6B: triangles) only a positive error, increasing with diameter, remains. Because this error is quite small for a large number of shells, a model with the same large number of shells in every compartment will give accurate results in NEURON, but this may cause unacceptably slow runtimes (Anwar et al., 2012) so it is better to vary the number of shells [DMFD mechanism, Equation (5)].

The next question is then how to compute shell depth as the depth of at least one shell has to vary to fit the total exactly to a variable compartment diameter. As already mentioned, in the standard NEURON implementation (Carnevale and Hines, 2006) the depths of all shells vary with compartment diameter [variable depth scheme; Equations (3) and (4) and Figure 1: DM], including that of the submembrane shell. In effect, the depth of the submembrane shell $\left(\mathrm{d}_{1}\right)$ may vary between $d-0.25 \mathrm{~d}$ and $d+0.25 d$. In Figure 6C, the broken line shows the theoretical error of $\mathrm{Ca}^{2+}$ influx conversion to $\mathrm{Ca}^{2+}$ concentration using the variable depth scheme (range of $\mathrm{d}_{1}$ due to discretization: 


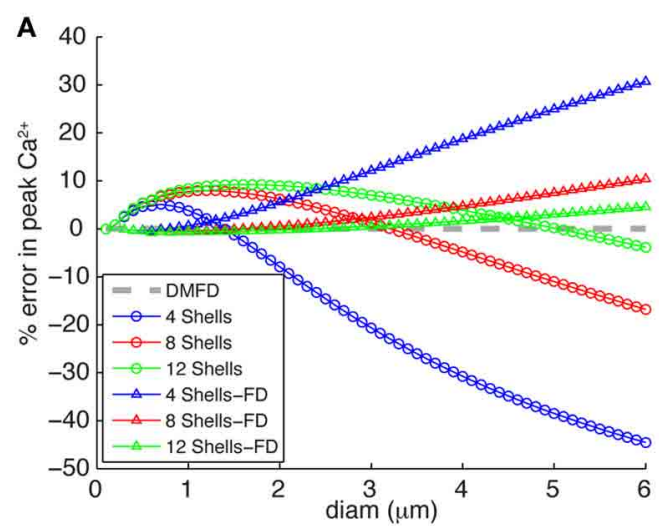

B

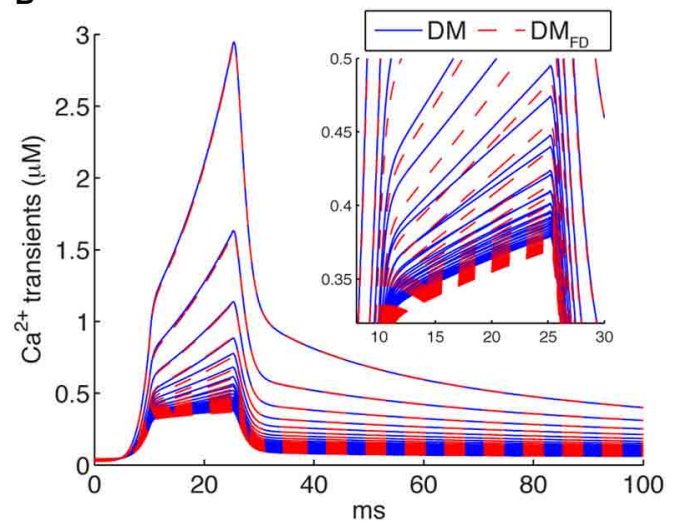

c

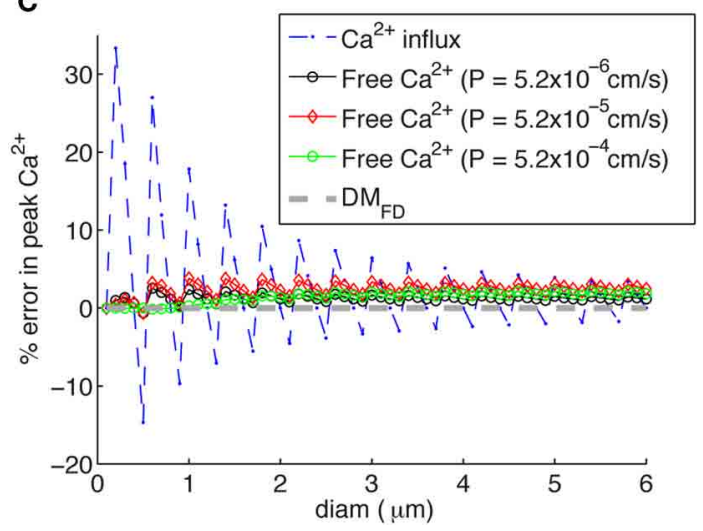

FIGURE 6 | Inaccuracies of different calcium 1D diffusion models result in erroneous calcium levels. (A) Errors introduced by making the number of concentric shells independent of compartment diameter, for 4,8 , or 12 shells respectively. Two mechanisms are implemented: the standard NEURON scheme with variable depths for all shells (circles) and an FD scheme where the submembrane shell has a constant depth $d_{1}=0.1 \mu \mathrm{m}$ and the rest of the shells has variable depth (triangles). The DMFD mechanism is used as reference. Note that for both mechanisms the errors become large for diameters beyond $2 \mu \mathrm{m}$ if only four shells are used (as is the case in some NEURON models). (B) $\mathrm{Ca}^{2+}$ transients generated using a "ramp-like" voltage command in single compartments (see Figure $\mathbf{3 C}$ for details) comparing the responses of the DM and DMFD models. Both models show very similar behavior with only small numerical differences. (C) Errors due to discretization of radial shells in DM, which may result in variable $d_{1}$ resulting in rapid changes of submembrane shell volume for

(Continued)

\section{FIGURE 6 | Continued}

increasing compartment diameter. The broken line with asterisks shows errors related to conversion of $\mathrm{Ca}^{2+}$ influx to $\mathrm{Ca}^{2+}$ concentration with variable depth $d_{1}$ of the submembrane shell (it varies between 0.075 and $0.125 \mu \mathrm{m}$ due to discretization) as compared to fixed $\mathrm{d}_{1}$ of $0.1 \mu \mathrm{m}$ (DM $\mathrm{DPD}_{\mathrm{F}}$ ). The solid lines with diamonds shows the actual error in free $\mathrm{Ca}^{2+}$ in the submembrane shell for DM models for different sizes of $\mathrm{Ca}^{2+}$ influx as indicated. Note that these errors are much smaller than predicted by the $\mathrm{Ca}^{2+}$ influx conversion.

$0.075-0.125 \mu \mathrm{m})$. The larger predicted errors in these cases are associated with small diameters, where small changes of diam will result in bigger changes in $d_{1}$ Equation (4) and submembrane shell volume.

These errors are large and should not be ignored. But what is the effect of these geometrical errors on actual computed $\mathrm{Ca}^{2+}$ concentrations? To quantify this we simulated $\mathrm{Ca}^{2+}$ transients using a mechanism with a variable number of shells, all with the same depth of $0.1 \mu \mathrm{m}$ except for the core shell which has a variable diameter $\left[\mathrm{DM}_{\mathrm{FD}}\right.$, Equation (5) and Figure 1], which is assumed to give the most accurate solution. We found that $\mathrm{DM}$ and $\mathrm{DM}_{\mathrm{FD}}$ show very similar peak amplitudes and decay time constants for different diameter compartments (Figure 6B), resulting in much smaller errors in peak amplitudes of $\mathrm{Ca}^{2+}$ using DM compared to $\mathrm{DM}_{\mathrm{FD}}$ than theoretically predicted (Figure 6C). The error depends on the size of $\mathrm{Ca}^{2+}$ influx in a nontrivial way, but for all levels of $\mathrm{Ca}^{2+}$ influx it was small with the largest error only about $4 \%$. This significant difference with the theoretical prediction is due to strong buffering (especially in PCs; (Hartmann and Konnerth, 2005) and diffusion of $\mathrm{Ca}^{2+}$, which removes most of $\mathrm{Ca}^{2+}$ entering into the submembrane shell.

How do the different $\mathrm{Ca}^{2+}$ buffering models respond to local fluctuations of dendrite diameter in terms of $\mathrm{Ca}^{2+}$ levels? To estimate the effect of dendritic diameter changes on $\mathrm{Ca}^{2+}$ dynamics using each model, we computed the ratio of integrated $\mathrm{Ca}^{2+}$ transients measured in each of the pair of simulated compartments using $\mathrm{SP}_{\mathrm{new}}, \mathrm{DM}$ and $\mathrm{DM}_{\mathrm{FD}}$ for many possible pairs of dendritic diameters (range: $0.1-6.0 \mu \mathrm{m}$ with increments of $0.1 \mu \mathrm{m}$ ) (Figure 7). As explained previously, the $\mathrm{SP}_{\text {old }}$ model does not show any sensitivity to changes in diameters. For $\mathrm{SP}_{\text {new }}$, large ratios $(>2)$ are limited to combinations where a compartment with an extremely small diameter $(\leq 0.3 \mu \mathrm{m})$ is connected to one with large diameters. For $\mathrm{DM}$ and $\mathrm{DM}_{\mathrm{FD}}$, this region expands to all compartments with diameter less than or equal to $1 \mu \mathrm{m}$ that are connected to ones with larger diameters. Therefore, the detailed $\mathrm{Ca}^{2+}$ dynamics models are more sensitive to changes in dendritic diameter compared to pool based models, which explains the differences observed in Figure 3. But, although the sensitivity maps of $\mathrm{DM}$ are noisier than those of $\mathrm{DM}_{\mathrm{FD}}$, due to the use of variable depth submembrane shells in DM, overall these maps are quite similar to each other.

We conclude from Figures 6, 7 that correct simulation of radial $1 \mathrm{D} \mathrm{Ca}^{2+}$ diffusion requires a variable number of concentric shells that scales with compartment diameter, but that the $\mathrm{Ca}^{2+}$ dynamics are less sensitive to the actual scheme used to compute the depth of these shells. 


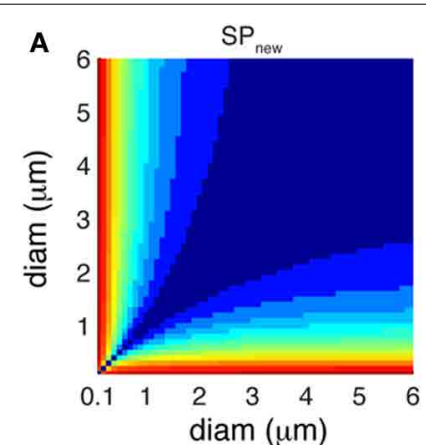

diam $(\mu \mathrm{m})$

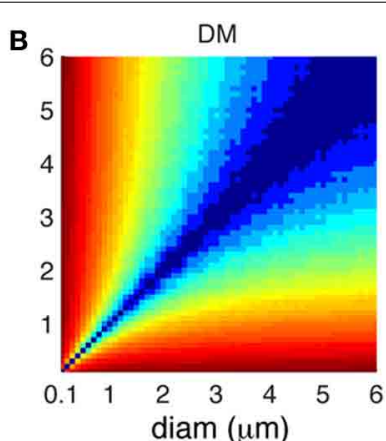

diam ( $\mu \mathrm{m})$

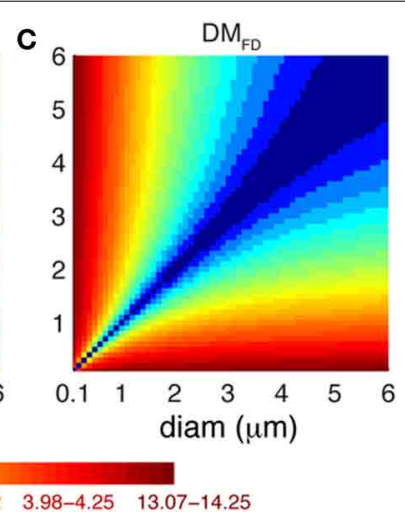

$1.29 \quad 1.66-1.72 \quad 3.98-4.25 \quad 13.07-14.25$
FIGURE 7 | Different $\mathrm{Ca}^{2+}$ buffering model respond variably to changes in dendrite diameters. Predicted ratio of integrated $\mathrm{Ca}^{2+}$ concentration ( $100 \mathrm{~ms}$ window) for different combinations of diameters of pairs of dendritic compartments using (A) $\mathrm{SP}_{\text {new }}$,
(B) DM, and (C) DMFD. The maps are derived from the data shown in Figure $\mathbf{3 C}(\mathbf{A})$ and Figure $\mathbf{6 B}(\mathbf{B}, \mathbf{C})$. The color scales used in these maps are nonlinear (using histogram equalization) to enhance the contrast.

\section{SPATIAL DIFFERENCES IN Ca ${ }^{2+}$ LEVELS PERSIST WITH 3D DIFFUSION}

The predictions of Figure 7 are based on no $\left(\mathrm{SP}_{\text {new }}\right)$ or only radial $1 \mathrm{D}$ diffusion $\left(\mathrm{DM}\right.$ and $\left.\mathrm{DM}_{\mathrm{FD}}\right)$. In this case, would the predicted large $\mathrm{Ca}^{2+}$ transients disappear in the presence of $3 \mathrm{D}$ diffusion? Also, what happens if dendritic diameter varies more smoothly than possible in a model using electrical compartmentalization? To address both issues, we used the STEPS simulator (Hepburn et al., 2012), which uses tetrahedral meshes to accurately represent detailed morphologies and 3D diffusion of molecules to simulate $\mathrm{Ca}^{2+}$ dynamics.

When we simulated $\mathrm{Ca}^{2+}$ transients with the detailed $\mathrm{Ca}^{2+}$ dynamics model in part of a PC dendritic arbor using STEPS, we still observed large fluctuations in $\mathrm{Ca}^{2+}$ levels along the different branches. Figure 8A shows the integrated $\mathrm{Ca}^{2+}$ levels for each tetrahedron located within $0.1 \mu \mathrm{m}$ from the membrane. The large fluctuations of $\mathrm{Ca}^{2+}$ levels appear to be related to dendrite diameter. High $\mathrm{Ca}^{2+}$ levels are observed in dendritic regions with small diameter (Figure 8B) and at the tips of terminating branches. Higher levels at the tips are due to the higher SVR as a result of their small diameters and the reduced effective diffusion because of the closed end condition (the latter is not predicted by radial diffusion models). Overall we conclude that neither $3 \mathrm{D}$ diffusion nor smooth changes in dendrite diameter reduce the pronounced $\mathrm{Ca}^{2+}$ gradients caused by variable dendrite diameter, raising the question what level of detail is necessary to model this effect correctly?

To address this question, we compare NEURON and STEPS simulations using two different compartmentalization schemes in NEURON. First, we simulated $\mathrm{Ca}^{2+}$ transients in NEURON using multiple compartments per unbranched segment to capture all changes in dendritic diameters (Figure 8D; right panel). For comparison Figure 8D (left panel) shows the STEPS simulation with mean integrated $\mathrm{Ca}^{2+}$ concentration computed for all tetrahedrons corresponding to every NEURON compartment. Next, we made a similar comparison with NEURON simulations where every unbranched dendritic segment is considered as a single compartment (Figure $\mathbf{8 F}$ ), which is the approach used in many compartmental models. Comparing these spatial maps
(Figures 8D,F), we observe only small differences between simulations with $1 \mathrm{D}$ diffusion (NEURON) or with 3D diffusion (STEPS). However, the actual $\mathrm{Ca}^{2+}$ levels are different in the respective simulations. To quantify the difference in $\mathrm{Ca}^{2+}$ levels between the two approaches and how they relate to fluctuations in dendritic diameters we computed the ratios of $\mathrm{Ca}^{2+}$ levels and diameters for all adjacent segments. Figure 8E shows $\mathrm{Ca}^{2+}$ ratios in adjacent compartments for small compartment sizes (data shown in Figure 8D) and Figure 8G shows $\mathrm{Ca}^{2+}$ ratios in adjacent compartments with one compartment per unbranched dendritic segment (data shown in Figure 8F). The comparison of Figure 8E with Figure 8G clearly shows that the use of large compartments will result in larger jumps in $\mathrm{Ca}^{2+}$ levels between adjacent compartments. Using many small compartments to capture the continuous change of dendritic diameters results in much smoother and smaller changes in $\mathrm{Ca}^{2+}$ levels. The overall behavior of these changes in $\mathrm{Ca}^{2+}$ levels (Figures 8E,G) is similar, respectively, to the ratios of diameters in the original morphological reconstruction and to the ratios for adjacent compartments diameters for one compartment per unbranched segment (Figure 8C). This confirms that the simulated $\mathrm{Ca}^{2+}$ gradients are largely caused by the SVR effect. Finally, notice that the effect of 3D diffusion is more prominent when using small compartments (Figure 8D, bigger difference between NEURON and STEPS simulation).

\section{DISCUSSION}

For a long time (until early 1960s), dendrites were thought to be passive structures, whose main function was to transfer and sum information from presynaptic to postsynaptic neurons (for review see Johnston et al., 1996). During the past couple of decades, it has been shown that dendrites contain a variety of voltage-gated channels (Llinas et al., 1992; Markram and Sakmann, 1994; Stuart and Sakmann, 1994; Magee and Johnston, 1995; Magee and Carruth, 1999; Lorincz and Nusser, 2010), voltage-dependent NMDA channels (Losonczy et al., 2008; Polsky et al., 2009; Major et al., 2013) and $\mathrm{K}_{\mathrm{Ca}}$ channels (Golding et al., 1999; Womack and Khodakhah, 2002, 2003), 

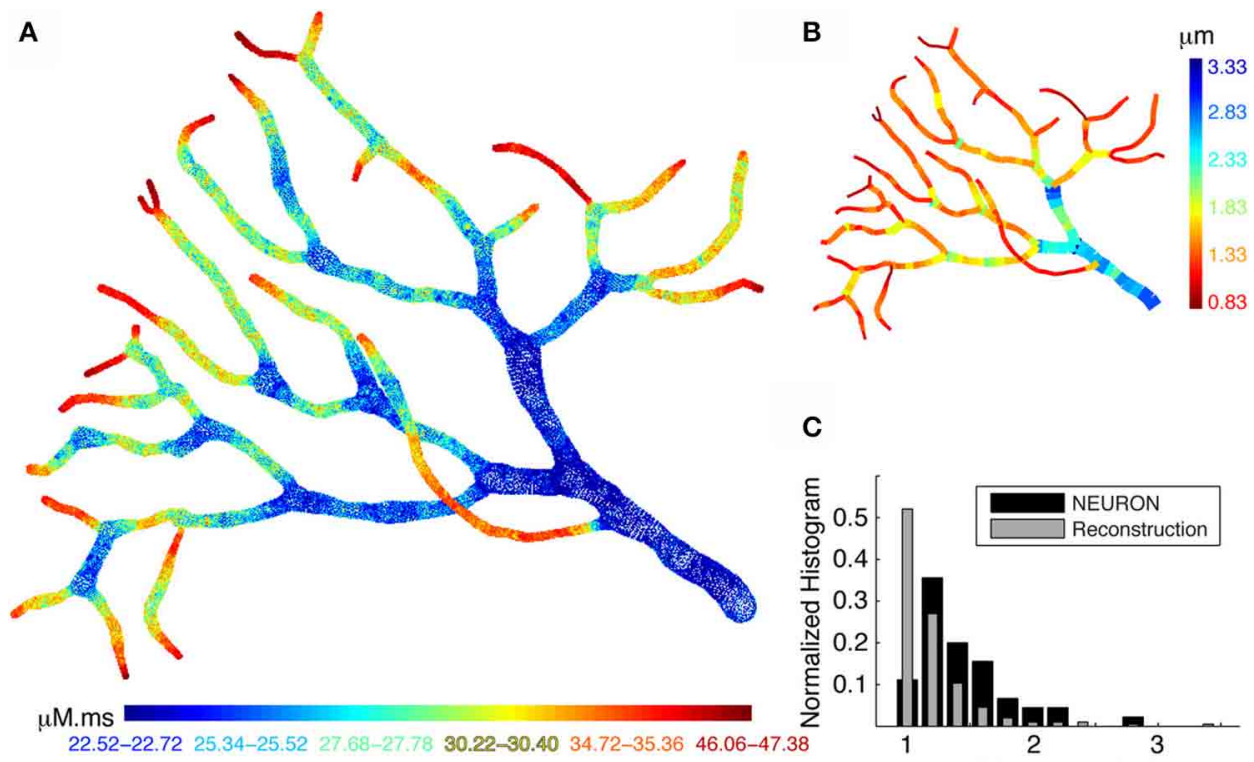

C

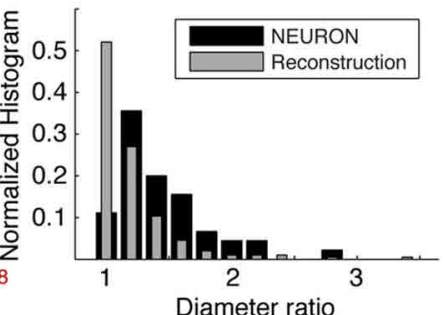

D

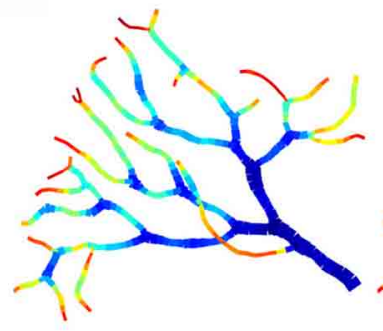

$\mathbf{F}$

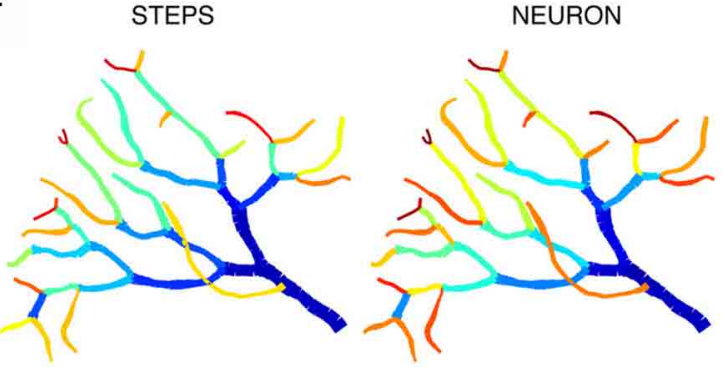

NEURON

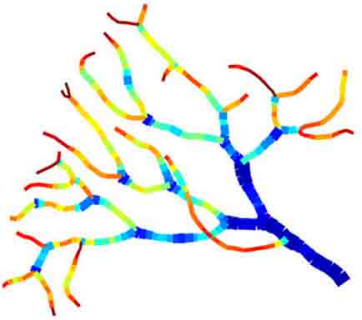

G

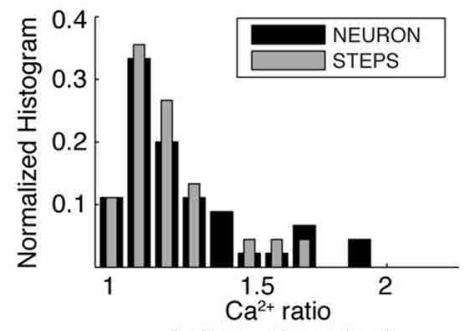

(adjacent branches)
FIGURE 8 | Large differences in calcium levels in adjacent dendritic branches persist in presence of 3D diffusion. (A) STEPS model using 3D buffered diffusion to compute the $\mathrm{Ca}^{2+}$ concentration resulting from the burst of $\mathrm{Ca}^{2+}$ spikes. Spatial map of integrated calcium ( $140 \mathrm{~ms}$ window) in a piece of carefully reconstructed PC dendritic arbor (part of PC 1). Every colored dot drawn at the center coordinates of each tetrahedron belonging to the mesh in which $3 \mathrm{D}$ diffusion was simulated shows the integrated $\mathrm{Ca}^{2+}$ in that particular tetrahedron. Only tetrahedrons representing the submembrane region are plotted. The color scales used in these maps are nonlinear (using histogram equalization) to enhance the contrast. (B) Spatial map of dendritic diameters in the dendrite shown in (A,D,F). (C) Normalized histograms compare the ratios of adjacent diameters in the original morphological reconstruction with similar ratios of diameters of adjacent compartments in the NEURON model 1 segment per unbranched section). (D,E) NEURON simulation with many compartments for each unbranched segment, carefully reflecting the variability of dendrite diameter. Data for the STEPS simulation are averaged over all tetrahedrons representing the corresponding NEURON

compartment. $(\mathbf{F}, \mathbf{G})$ NEURON simulation with a single compartment for each unbranched segment, data for the STEPS simulation averaged for corresponding NEURON compartments. (D,F) Spatial maps of integrated submembrane $\mathrm{Ca}^{2+}$ concentration using the detailed calcium dynamics model with 3D diffusion (STEPS) and 1D radial diffusion (NEURON) are shown for the different compartmentalization schemes. (D) and (F) Use same color as in $\mathbf{A}$. (E,G) Normalized histograms show the ratios of integrated $\mathrm{Ca}^{2+}$ concentration between every adjacent compartment using simulations with 3D diffusion (STEPS) and 1D diffusion (NEURON) for the results shown in (D,F) respectively. 
which make these structures active. In addition to their role in neuronal excitability and dendritic integration, dendrites with thousands of synapses also serve as a venue of memory storage through induction of synaptic plasticity. Intracellular $\mathrm{Ca}^{2+}$ is involved in many processing capabilities of dendrites. $\mathrm{Ca}^{2+}$ entering through VGCC and NMDA channels gives rise to cytosolic $\mathrm{Ca}^{2+}$, which in turn activates various $\mathrm{K}^{+}$channels and several molecular signaling pathways underlying synaptic plasticity. Therefore, it is important to correctly understand the dynamics of intracellular $\mathrm{Ca}^{2+}$ in dendrites with complex morphological structures.

\section{PREVIOUS MODELING OF DETAILED CALCIUM DYNAMICS}

The complexity of dendritic geometry and structure has been studied extensively to investigate its effects on propagation of action potentials, its role in synaptic efficacy and its effects on limiting interaction across different active dendritic regions (Mainen and Sejnowski, 1996; Vetter et al., 2001). Although variable levels of $\mathrm{Ca}^{2+}$ in different dendritic regions have been reported previously (Tank et al., 1988; Lev-Ram et al., 1992; Schiller et al., 1995), only a few studies specifically investigated the effect of dendritic diameters on $\mathrm{Ca}^{2+}$ dynamics (Holthoff et al., 2002; Rozsa et al., 2004). Therefore, those effects are often omitted while constructing biophysical models of dendrites. Due to limited quantitative information about the mechanisms controlling $\mathrm{Ca}^{2+}$ levels in many neurons, phenomenological models of $\mathrm{Ca}^{2+}$ buffering, such as the single exponential decaying pool, are commonly used for biophysical neuronal modeling. Such models when used correctly capture only some aspects of the highly complex behavior of intracellular $\mathrm{Ca}^{2+}$ buffering dynamics. In our previous work (Anwar et al., 2012), we showed that pool based models of $\mathrm{Ca}^{2+}$ buffering fail to correctly predict peak $\mathrm{Ca}^{2+}$ concentrations and decay time constants important for the interaction between VGCC and $\mathrm{K}_{\mathrm{Ca}}$ channels. In this study, we investigated the effect of dendritic diameters on $\mathrm{Ca}^{2+}$ dynamics using a modeling approach. Our results (Figures 2, 4) show that pool based models have limited ability to capture the spatial variability of $\mathrm{Ca}^{2+}$ dynamics in morphologically complex dendrites as compared to a detailed $\mathrm{Ca}^{2+}$ dynamics model with radial 1D diffusion. The detailed $\mathrm{Ca}^{2+}$ dynamics model shows different peak amplitudes of $\mathrm{Ca}^{2+}$ levels as well as different (and multiple) decay time constants (Figure 6B). In contrast, pool based models with correct submembrane volume only show different peak amplitudes of $\mathrm{Ca}^{2+}$ levels (Figure 3C).

In general, many studies, including this one, ignore additional properties of real neurons that will affect $\mathrm{Ca}^{2+}$ dynamics. The most important of these simplifications are the assumption of constant density of $\mathrm{Ca}^{2+}$ channels, which is known to be not true for many neurons (for review see Johnston et al., 1996; Migliore and Shepherd, 2002), and the omission of the effect of organelles in the cytoplasm that block diffusion and have additional membrane $\mathrm{Ca}^{2+}$ pumps (mainly endoplasmic reticulum and mitochondria). Another important determinant of $\mathrm{Ca}^{2+}$ dynamics is inhomogeneous distribution of $\mathrm{Ca}^{2+}$ buffers in dendrites of a given neuron, as well as their properties, causing competitive binding of $\mathrm{Ca}^{2+}$ to available
$\mathrm{Ca}^{2+}$ buffers and $\mathrm{Ca}^{2+}$ pumps (Markram et al., 1998). While the density of channels can easily be changed in compartmental models, accurate representation of intracellular organelles is possible in mesh based models only. We do not expect that inclusion of these properties would significantly change our conclusions.

\section{IMPORTANCE OF ACCURATE MORPHOLOGICAL RECONSTRUCTION}

Dendrites have variable diameters. Typically, the diameter of dendrites taper with increasing distance from the soma. It is generally assumed that the change in diameter of an unbranched dendrite is relatively small as compared to the change in diameter at branching, which allows representation of an unbranched dendrite segment as a single uniform diameter compartment. However, as shown in our morphological analysis in Figure 5, many reconstructions of both PCs and pyramidal neurons show great diameter variability within their unbranched segments, with $\mathrm{CV}$ values sometimes reaching above 0.5 . This implies that a correct $\mathrm{Ca}^{2+}$ dynamics model should represent this diameter variability by having several compartments for each unbranched segment (Figures 8D-E), but also that the quality of the morphological reconstruction is of utmost importance. We observed great differences of the diameter variability between different neural reconstructions which often could be related to the laboratory where the reconstructions have been done, as was reported previously for pyramidal neurons (Scorcioni et al., 2004; Szilagyi and De Schutter, 2004; Holmes et al., 2006). Because it seems more likely that human error causes an undersampling of diameter changes than an exaggeration, we assume that the reconstruction with high diameter $\mathrm{CV}$ tend to be more reliable. Finally, one should be aware that software like CVapp (Cannon et al., 1998), which converts morphology files into formats suitable for NEURON simulation, uses a specific discretization scheme that changes diameters at branch points (Figure S1).

Although morphological reconstructions obtained using electron microscopy (EM) capture dendrites much more precisely, because of rapid fluctuations in dendritic surface those reconstructions are not suitable for compartment based models. The proper use of EM reconstructions in modeling $\mathrm{Ca}^{2+}$ dynamics will require more advanced simulators with support for surface or tetrahedral meshes (e.g., M-Cell, STEPS). Also, this will require more detailed description of $\mathrm{Ca}^{2+}$ related mechanisms (e.g., spatial distribution of VGCa channels, $\mathrm{K}_{\mathrm{Ca}}$ channels, buffers, pumps and internal calcium stores).

\section{SIMULATOR IMPLEMENTATION ISSUES}

Almost all biophysically detailed models have been constructed using either the GENESIS or NEURON simulators. These software packages are based on compartmentalization of dendritic structures into multiple iso-potential cylinders, where voltage, currents and concentrations are computed for each of those compartments independently. Since these compartments are based on electrical properties of dendrites, biochemical representation of intracellular mechanisms in these compartments is always an approximation of the related biophysical process. Such a simplified molecular representation may result in unrealistic behavior of the model, depending on the 
rationale behind the assumption and its accuracy. One such example is the commonly used single pool model to simulate intracellular $\mathrm{Ca}^{2+}$ in the NEURON simulator Equations (8) and (9). The conversion of influx to intracellular $\mathrm{Ca}^{2+}$ concentration in these models is incorrect, which is due to the use of an inaccurate volume of the submembrane shell (Figure 3B). Although this inaccuracy does not influence the results of single compartment models dramatically because it can be easily tuned by adapting the shell depth, it becomes critically important in multi-compartment models. This incorrect single pool model will always underestimate the influx (Figure 3C), which may require unrealistic distribution of dendritic VGCC and $\mathrm{K}_{\mathrm{Ca}}$ channels during model construction and cause a mismatch in input resistance between model and actual cell, and it will not predict any spatial gradients of $\mathrm{Ca}^{2+}$ concentration due to fluctuations of dendrite diameter (Figure 2A).

More detailed $\mathrm{Ca}^{2+}$ dynamics models using radial 1D diffusion are thought to be more accurate, but again the compartmentalization of the dendrite may result in either an inaccurate or incomplete representation of model. It is a major challenge to model diffusion in the NEURON simulator correctly. NEURON allows radial (toward the center of each dendritic compartment) and longitudinal (from one compartment to neighboring compartment) diffusion. Radial diffusion requires virtual submembrane shells (Figure 1), where shells typically have a variable depth, depending on the diameter of each compartment. Furthermore, longitudinal diffusion is only allowed if the adjacent compartments have the same number of shells which will introduce a larger error (Figure 6A) unless a very large number of shells is used everywhere. Conversely, though theoretically the variable submembrane depths of the standard NEURON scheme (DM) should result in large errors, this effect was strongly filtered by the diffusion and buffering mechanisms, resulting in only small differences (Figure 6C) with a method $\left(\mathrm{DM}_{\mathrm{FD}}\right)$ that ensures a fixed depth of the submembrane shell. It should be noted, however, that these differences may be larger in models of other neurons because the buffering capacity of PCs is exceptionally high (Hartmann and Konnerth, 2005). Control simulations showed that although the extent of the changes in $\mathrm{Ca}^{2+}$ levels varied in models with lower buffers concentrations, the dependence of $\mathrm{Ca}^{2+}$ levels on changes in diameters persisted (results not shown).

Neither of the issues just mentioned are relevant for the GENESIS simulator. Both submembrane pools (as the concpool object, De Schutter and Bower, 1994) and radial diffusion (as the difshell object, De Schutter and Smolen, 1999) are implemented correctly and are easy to set up. Conversely, it is time consuming to create multiple calcium dynamics mechanisms with radial diffusion in NEURON because a separate mechanism has to be written for each set of diameters (see Materials and Methods) and this requires a lot of extra care. And then, for every different morphology, one will have to repeat the process. We expect that multilevel declarative model description languages (Raikov and De Schutter, 2012) may allow transparent and correct compartment based assignment of molecular mechanisms in NEURON in the future.

\section{RECOMMENDATIONS FOR CORRECT MODELING OF DENDRITIC $\mathrm{Ca}^{2+}$ DYNAMICS}

Even in PCs, where the estimated $\mathrm{Ca}^{2+}$ diffusion range is only about $5 \mu \mathrm{m}$ (Santamaria et al., 2006), we observe effects of 3D diffusion on $\mathrm{Ca}^{2+}$ transients compared to when only radial 1D diffusion is used, especially when $\mathrm{Ca}^{2+}$ concentration is averaged over short distances only (Figures 8D,E). Nevertheless, the error introduced by the $1 \mathrm{D}$ approach is much smaller than the errors caused by inaccurate morphologies (Figure 8C) and simulating $3 \mathrm{D}$ diffusion in tetrahedral meshes is quite slow. However, $3 \mathrm{D}$ diffusion must be included in biophysically accurate models of synaptic plasticity or models involving $\mathrm{Ca}^{2+}$ based signaling pathways.

For most of modeling projects with the goal of capturing excitability and integrative properties of dendrites, a correct implementation of 1D radial diffusion and buffering in NEURON (or any other compartment based simulator) will be an adequate approximation. It is then important to implement a variable number of submembrane shells, with larger number of shells in larger diameter compartments, and best using a fixed depth of the submembrane shell [DM $\mathrm{FD}$ model: Figure 1, Equation (5)]. The model should be based on a high quality morphological reconstruction (Jacobs et al., 2010) and the variability of diameter along dendritic segments should be retained by having as many compartments as required to capture diameter changes (Figures 8D,E).

Finally, we do not recommend the use of simple pool models, unless good data on the properties of $\mathrm{Ca}^{2+}$ buffering (e.g., Schmidt et al., 2003 for PCs) in the neuron type to be modeled is completely absent. If one is forced to use a simple pool model, make sure it is implemented correctly $\left[\mathrm{SP}_{\text {new }}\right.$, Figure 3A and Equations (2) and (6)].

\section{ACKNOWLEDGMENTS}

The authors wish to thank Sungho Hong and Iain Hepburn for comments on early drafts. This work was supported by OIST Graduate University. Hermina Nedelescu was supported by the European Union (CEREBNET FP7-ITN238686). We thank MBF Bioscience for providing Neurolucida tracing software and Professor Alanna Watt (McGill University) for providing the computer system and laboratory space to reconstruct the detailed morphology.

\section{SUPPLEMENTARY MATERIAL}

The Supplementary Material for this article can be found online at: http://www.frontiersin.org/journal/10.3389/fncel.2014. 00168/abstract

\section{REFERENCES}

Antunes, G., and De Schutter, E. (2012). A stochastic signaling network mediates the probabilistic induction of cerebellar long-term depression. J. Neurosci. 32, 9288-9300. doi: 10.1523/JNEUROSCI.5976-11.2012

Anwar, H., Hepburn, I., Nedelescu, H., Chen, W., and De Schutter, E. (2013). Stochastic calcium mechanisms cause dendritic calcium spike variability. J. Neurosci. 33, 15848-15867. doi: 10.1523/JNEUROSCI.172213.2013

Anwar, H., Hong, S., and De Schutter, E. (2012). Controlling $\mathrm{Ca}^{2+}$-activated $\mathrm{K}^{+}$ channels with models of $\mathrm{Ca}^{2+}$ buffering in purkinje Cells. Cerebellum 11, 681-693. doi: 10.1007/s12311-010-0224-3 
Augustine, G. J., Santamaria, F., and Tanaka, K. (2003). Local calcium signaling in neurons. Neuron 40, 331-346. doi: 10.1016/S0896-6273(03)00639-1

Berridge, M. (1998). Neuronal calcium signaling review. Neuron 21, 13-26. doi: 10.1016/S0896-6273(00)80510-3

Canepari, M., and Vogt, K. E. (2008). Dendritic spike saturation of endogenous calcium buffer and induction of postsynaptic cerebellar LTP. PLOS ONE 3:e4011. doi: 10.1371/journal.pone.0004011

Cannon, R. C., Turner, D. A., Pyapali, G. K., and Wheal, H. V. (1998). An on-line archive of reconstructed hippocampal neurons. J. Neurosci. Methods 84, 49-54. doi: 10.1016/S0165-0270(98)00091-0

Carnevale, N. T., and Hines, M. L. (2006). The NEURON Book. New York, NY: Cambridge University Press. doi: 10.1017/CBO9780511541612

Cox, D. H., Cui, J., and Aldrich, R. W. (1997). Allosteric gating of a large conductance Ca-activated K+ channel. J. Gen. Physiol. 110, 257-281. doi: 10.1085/jgp.110.3.257

De Schutter, E. (2010). "Modeling intracellular calcium dynamics," in Computational Modeling Methods for Neuroscientists, ed E. De Schutter (Cambridge, MA: The MIT Press), 93-106.

De Schutter, E., and Bower, J. M. (1994). An active membrane model of the cerebellar Purkinje cell. I. Simulation of current clamps in slice. J. Neurophysiol. 71, 375-400.

De Schutter, E., and Smolen, P. (1999). "Calcium dynamics in large neuronal models," in Methods Neuronal Modeling: From Ions to Networks, Vol. 1, eds C. Koch and I. Segev (Cambridge, MA: The MIT Press), 1-44.

Destexhe, A., Mainen, Z. F., and Sejnowski, T. J. (1994). Synthesis of models for excitable membranes, synaptic transmission and neuromodulation using a common kinetic formalism. J. Comput. Neurosci. 1, 195-230. doi: 10.1007/BF00961734

Fakler, B., and Adelman, J. (2008). Control of KCa channels by calcium nano/microdomains. Neuron 59, 873-881. doi: 10.1016/j.neuron.2008. 09.001

Gold, C., Henze, D., and Koch, C. (2007). Using extracellular action potential recordings to constrain compartmental models. J. Comput. Neurosci. 23, 39-58. doi: 10.1007/s10827-006-0018-2

Goldberg, J. H., Lacefield, C. O., and Yuste, R. (2004). Global dendritic calcium spikes in mouse layer 5 low threshold spiking interneurones: implications for control of pyramidal cell bursting. J. Physiol. 558, 465-478. doi: 10.1113/jphysiol.2004.064519

Golding, N. L., Jung, H.-Y., Mickus, T., and Spruston, N. (1999). Dendritic calcium spike initiation and repolarization are controlled by distinct potassium channel subtypes in CA1 pyramidal neurons. J. Neurosci. 19, 8789-8798.

Hartmann, J., and Konnerth, A. (2005). Determinants of postsynaptic Ca2+ signaling in Purkinje neurons. Cell Calcium 37, 459-466. doi: 10.1016/j.ceca.2005.01.014

Hay, E., Hill, S., Schürmann, F., Markram, H., and Segev, I. (2011). Models of neocortical layer $5 \mathrm{~b}$ pyramidal cells capturing a wide range of dendritic and perisomatic active properties. PLoS Comput. Biol. 7:e1002107. doi: 10.1371/journal.pcbi.1002107

Hemond, P., Epstein, D., Boley, A., Migliore, M., Ascoli, G. A., and Jaffe, D. B. (2008). Distinct classes of pyramidal cells exhibit mutually exclusive firing patterns in hippocampal area CA3b. Hippocampus 18, 411-424. doi: 10.1002/hipo.20404

Hepburn, I., Chen, W., Wils, S., and De Schutter, E. (2012). STEPS: efficient simulation of stochastic reaction-diffusion models in realistic morphologies. BMC Syst. Biol. 6:36. doi: 10.1186/1752-0509-6-36

Hines, M. L., and Carnevale, N. T. (1997). The NEURON simulation environment. Neural Comput. 9, 1179-1209. doi: 10.1162/neco.1997.9.6.1179

Hirschberg, B., Maylie, J., Adelman, J., and Marrion, N. V. (1998). Gating of recombinant small-conductance $\mathrm{Ca}$-activated $\mathrm{K}+$ channels by calcium. J. Gen. Physiol. 111, 565-581. doi: 10.1085/jgp.111.4.565

Holmes, W. R., Ambros-Ingerson, J., and Grover, L. M. (2006). Fitting experimental data to models that use morphological data from public databases. J. Comput. Neurosci. 20, 349-365. doi: 10.1007/s10827-006-7189-8

Holthoff, K., Tsay, D., and Yuste, R. (2002). Calcium dynamics of spines depend on their dendritic location. Neuron 33, 425-437. doi: 10.1016/S08966273(02)00576-7

Iftinca, M., McKay, B. E., Snutch, T. P., McRory, J. E., Turner, R. W., and Zamponi, G. W. (2006). Temperature dependence of T-type calcium channel gating. Neuroscience 142, 1031-1042. doi: 10.1016/j.neuroscience.2006.07.010
Jacobs, G., Claiborne, B., and Harris, K. M. (2010). "Reconstruction of neuronal morphology," in Computational Modeling Methods for Neuroscientists, ed E. De Schutter (Cambridge, MA: The MIT Press), 187-210.

Johnston, D., Magee, J. C., Colbert, C. M., and Christie, B. R. (1996). Active properties of neuronal dendrites. Annu. Rev. Neurosci. 19, 165-186. doi: 10.1146/annurev.ne.19.030196.001121

Kampa, B. M., Letzkus, J. J., and Stuart, G. (2006). Requirement of dendritic calcium spikes for induction of spike-timing-dependent synaptic plasticity. J. Physiol. 574, 283-290. doi: 10.1113/jphysiol.2006.111062

Kampa, B. M., and Stuart, G. J. (2006). Calcium spikes in basal dendrites of layer 5 pyramidal neurons during action potential bursts. J. Neurosci. 26, 7424-7432. doi: 10.1523/JNEUROSCI.3062-05.2006

Konnerth, A., Dreessen, J., and Augustine, G. J. (1992). Brief dendritic calcium signals initiate long-lasting synaptic depression in cerebellar Purkinje cells. Proc. Natl. Acad. Sci. U.S.A. 89, 7051-7055. doi: 10.1073/pnas.89.15.7051

Lavzin, M., Rapoport, S., Polsky, A., Garion, L., and Schiller, J. (2012). Nonlinear dendritic processing determines angular tuning of barrel cortex neurons in vivo. Nature 490, 397-401. doi: 10.1038/nature11451

Lazarewicz, M. T., Migliore, M., and Ascoli, G. A. (2002). A new bursting model of CA3 pyramidal cell physiology suggests multiple locations for spike initiation. Biosystems 67, 129-137. doi: 10.1016/S0303-2647(02)00071-0

Lev-Ram, V., Miyakawa, H., Lasser-Ross, N., and Ross, W. N. (1992). Calcium transients in cerebellar Purkinje neurons evoked by intracellular stimulation. J. Neurophysiol. 68, 1167-1177.

Llinas, R., Sugimori, M., Hillman, D. E., and Cherksey, B. (1992). Distribution and functional significance of the P-type, voltage-dependent $\mathrm{Ca} 2+$ channels in the mammalian central nervous system. Trends Neurosci. 15, 351-355. doi: 10.1016/0166-2236(92)90053-B

Lorincz, A., and Nusser, Z. (2010). Molecular identity of dendritic voltage-gated sodium channels. Science 328, 906-909. doi: 10.1126/science.1187958

Losonczy, A., Makara, J. K., and Magee, J. C. (2008). Compartmentalized dendritic plasticity and input feature storage in neurons. Nature 452, 436-441. doi: 10.1038 /nature06725

Magee, J. C., and Carruth, M. (1999). Dendritic voltage-gated ion channels regulate the action potential firing mode of hippocampal CA1 pyramidal neurons. J. Neurophysiol. 82, 1895-1901.

Magee, J. C., and Johnston, D. (1995). Characterization of single voltage-gated $\mathrm{Na}+$ and $\mathrm{Ca} 2+$ channels in apical dendrites of rat CA1 pyramidal neurons. J. Physiol. 487, 67-90.

Mainen, Z., and Sejnowski, T. (1996). Influence of dendritic structure on firing pattern in model neocortical neurons. Nature 382, 363-366. doi: 10.1038/382363a0

Major, G., Larkum, M. E., and Schiller, J. (2013). Active properties of neocortical pyramidal neuron dendrites. Annu. Rev. Neurosci. 36, 1-24. doi: 10.1146/annurev-neuro-062111-150343

Markram, H., Roth, A., and Helmchen, F. (1998). Competitive calcium binding: implications for dendritic calcium signaling. J. Comput. Neurosci. 5, 331-348. doi: 10.1023/A:1008891229546

Markram, H., and Sakmann, B. (1994). Calcium transients in dendrites of neocortical neurons evoked by single subthreshold excitatory postsynaptic potentials via low-voltage-activated calcium channels. Proc. Natl. Acad. Sci. U.S.A. 91, 5207-5211. doi: 10.1073/pnas.91.11.5207

Migliore, M., Cook, E. P., Jaffe, D. B., Turner, D. A., and Johnston, D. (1995). Computer simulations of morphologically reconstructed CA3 hippocampal neurons. J. Neurophysiol. 73, 1157-1168.

Migliore, M., and Shepherd, G. M. (2002). Emerging rules for the distributions of active dendritic conductances. Nat. Rev. Neurosci. 3, 362-370. doi: $10.1038 / \mathrm{nrn} 810$

Miyasho, T., Takagi, H., Suzuki, H., Watanabe, S., Inoue, M., Kudo, Y., et al. (2001). Low-threshold potassium channels and a low-threshold calcium channel regulate $\mathrm{Ca}^{2+}$ spike firing in the dendrites of cerebellar Purkinje neurons: a modeling study. Brain Res. 891, 106-115. doi: 10.1016/S0006-8993(00)03206-6

Poirazi, P., Brannon, T., and Mel, B. W. (2003). Pyramidal neuron as two-layer neural network. Neuron 37, 989-999. doi: 10.1016/S0896-6273(03)00149-1

Polsky, A., Mel, B., and Schiller, J. (2009). Encoding and decoding bursts by NMDA spikes in basal dendrites of layer 5 pyramidal neurons. J. Neurosci. 29, 11891-11903. doi: 10.1523/JNEUROSCI.5250-08.2009

Raikov, I., and De Schutter, E. (2012). The layer-oriented approach to declarative languages for biological modeling. PLoS Comput. Biol. 8:e1002521. doi: 10.1371/journal.pcbi.1002521 
Rancz, E. A., and Hausser, M. (2006). Dendritic calcium spikes are tunable triggers of cannabinoid release and short-term synaptic plasticity in cerebellar purkinje neurons. J. Neurosci. 26, 5428-5437. doi: 10.1523/JNEUROSCI.528405.2006

Regehr, W. G., and Tank, D. W. (1994). Dendritic calcium dynamics. Curr. Opin. Neurobiol. 4, 373-382. doi: 10.1016/0959-4388(94)90099-X

Rozsa, B., Zelles, T., Vizi, E. S., and Lendvai, B. (2004). Distance-dependent scaling of calcium transients evoked by backpropagating spikes and synaptic activity in dendrites of hippocampal interneurons. J. Neurosci. 24, 661-670. doi: 10.1523/JNEUROSCI.3906-03.2004

Sala, F., and Hernandez-Cruz, A. (1990). Calcium diffusion modeling in a spherical neuron. Relevance of buffering properties. Biophys. J. 57, 313-324. doi: 10.1016/S0006-3495(90)82533-9

Santamaria, F., Wils, S., De Schutter, E., and Augustine, G. J. (2006). Anomalous diffusion in purkinje cell dendrites caused by spines. Neuron $52,635-648$. doi: 10.1016/j.neuron.2006.10.025

Schiller, J., Helmchen, F., and Sakmann, B. (1995). Spatial profile of dendritic calcium transients evoked by action potentials in rat neocortical pyramidal neurones. J. Physiol. 487, 583-600.

Schmidt, H., Schwaller, B., and Eilers, J. (2005). Calbindin D28k targets myo-inositol monophosphatase in spines and dendrites of cerebellar Purkinje neurons. Proc. Natl. Acad. Sci. U.S.A. 102, 5850-5855. doi: 10.1073/pnas.0407855102

Schmidt, H., Stiefel, K. M., Racay, P., Schwaller, B., and Eilers, J. (2003). Mutational analysis of dendritic $\mathrm{Ca} 2+$ kinetics in rodent Purkinje cells: role of parvalbumin and calbindin D28k. J. Physiol. 551, 13-32. doi: 10.1113/jphysiol.2002. 035824

Scorcioni, R., Lazarewicz, M. T., and Ascoli, G. A. (2004). Quantitative morphometry of hippocampal pyramidal cells: differences between anatomical classes and reconstructing laboratories. J. Comp. Neurol. 473, 177-193. doi: $10.1002 / \mathrm{cne} .20067$

Solinas, S., Forti, L., Cesana, E., Mapelli, J., De Schutter, E., and D’Angelo, E. (2007). Computational reconstruction of pacemaking and intrinsic electroresponsiveness in cerebellar golgi cells. Front. Cell. Neurosci. 1:2. doi: 10.3389/neuro.03.002.2007

Stuart, G. J., and Sakmann, B. (1994). Active propagation of somatic action potentials into neocortical pyramidal cell dendrites. Nature 367, 69-72. doi: $10.1038 / 367069 \mathrm{a} 0$

Swensen, A. M., and Bean, B. P. (2005). Robustness of burst firing in dissociated purkinje neurons with acute or long-term reductions in sodium conductance. J. Neurosci. 25, 3509-3520. doi: 10.1523/JNEUROSCI.392904.2005

Szilagyi, T., and De Schutter, E. (2004). Effects of variability in anatomical reconstruction techniques on models of synaptic integration by dendrites: a comparison of three internet archives. Eur. J. Neurosci. 19, 1257-1266. doi: 10.1111/j.1460-9568.2004.03222.x

Tank, D., Sugimori, M., Connor, J., and Llinas, R. (1988). Spatially resolved calcium dynamics of mammalian Purkinje cells in cerebellar slice. Science 242, 773-777. doi: $10.1126 /$ science. 2847315

Traub, R. D., and Llinas, R. (1977). The spatial distribution of ionic conductances in normal and axotomized motorneurons. Neuroscience 2, 829-849. doi: 10.1016/0306-4522(77)90110-5

Vetter, P., Roth, A., and Hausser, M. (2001). Propagation of action potentials in dendrites depends on dendritic morphology. J. Neurophysiol. 85, 926-937.

Womack, M. D., and Khodakhah, K. (2002). Characterization of large conductance $\mathrm{Ca} 2+$-activated $\mathrm{K}+$ channels in cerebellar Purkinje neurons. Eur. J. Neurosci. 16, 1214-1222. doi: 10.1046/j.1460-9568.2002.02171.x

Womack, M. D., and Khodakhah, K. (2003). Somatic and dendritic smallconductance calcium-activated potassium channels regulate the output of cerebellar Purkinje neurons. J. Neurosci. 23, 2600-2607.

Womack, M. D., and Khodakhah, K. (2004). Dendritic control of spontaneous bursting in cerebellar Purkinje cells. J. Neurosci. 24, 3511-3521. doi: 10.1523/JNEUROSCI.0290-04.2004

Conflict of Interest Statement: The authors declare that the research was conducted in the absence of any commercial or financial relationships that could be construed as a potential conflict of interest.

Received: 21 March 2014; accepted: 02 June 2014; published online: 23 July 2014 Citation: Anwar H, Roome CJ, Nedelescu H, Chen W, Kuhn B and De Schutter E (2014) Dendritic diameters affect the spatial variability of intracellular calcium dynamics in computer models. Front. Cell. Neurosci. 8:168. doi: 10.3389/fncel. 2014.00168

This article was submitted to the journal Frontiers in Cellular Neuroscience. Copyright (c) 2014 Anwar, Roome, Nedelescu, Chen, Kuhn and De Schutter. This is an open-access article distributed under the terms of the Creative Commons Attribution License (CC BY). The use, distribution or reproduction in other forums is permitted, provided the original author(s) or licensor are credited and that the original publication in this journal is cited, in accordance with accepted academic practice. No use, distribution or reproduction is permitted which does not comply with these terms. 


\section{OPEN ACCESS}

Edited by:

Christian D. Wilms,

University College London, UK

Reviewed by:

Arnd Roth,

University College London, UK

Lisa Mapelli,

University of Pavia, Italy

${ }^{*}$ Correspondence: Igor Delvendahl and Stefan

Hallermann, Medical Faculty, Carl-Ludwig-Institute for Physiology,

University of Leipzig, Liebigstr. 27 04103 Leipzig, Germany igor.delvendahl@ medizin.uni-leipzig.de; hallermann@medizin.uni-leipzig.de

Received: 13 October 2014 Accepted: 28 February 2015

Published: 19 March 2015

Citation:

Delvendahl I,

Straub I and Hallermann S (2015) Dendritic patch-clamp recordings from cerebellar granule cells demonstrate electrotonic compactness.

Front. Cell. Neurosci. 9:93 doi: 10.3389/fncel.2015.00093

\section{Dendritic patch-clamp recordings from cerebellar granule cells demonstrate electrotonic compactness}

\author{
Igor Delvendahl *, Isabelle Straub and Stefan Hallermann * \\ Medical Faculty, Carl-Ludwig Institute for Physiology, University of Leipzig, Leipzig, Germany
}

Cerebellar granule cells (GCs), the smallest neurons in the brain, have on average four short dendrites that receive high-frequency mossy fiber inputs conveying sensory information. The short length of the dendrites suggests that GCs are electrotonically compact allowing unfiltered integration of dendritic inputs. The small average diameter of the dendrites $(\sim 0.7 \mu \mathrm{m})$, however, argues for dendritic filtering. Previous studies based on somatic recordings and modeling indicated that GCs are electrotonically extremely compact. Here, we performed patch-clamp recordings from GC dendrites in acute brain slices of mice to directly analyze the electrotonic properties of GCs. Strikingly, the input resistance did not differ significantly between dendrites and somata of GCs. Furthermore, spontaneous excitatory postsynaptic potentials (EPSP) were similar in amplitude at dendritic and somatic recording sites. From the dendritic and somatic input resistances we determined parameters characterizing the electrotonic compactness of GCs. These data directly demonstrate that cerebellar GCs are electrotonically compact and thus ideally suited for efficient high-frequency information transfer.

Keywords: granule cell, dendrites, cerebellum, patch-clamp techniques, electrophysiology

\section{Introduction}

Synaptic information transfer is strongly determined by the electrotonic properties of the postsynaptic neuron and the location of the synapse within the neuron. Dendrites receiving synaptic input provide the backbone for the computation performed by neurons (Magee, 2000; Abbott and Regehr, 2004; Gulledge et al., 2005; London and Häusser, 2005; Spruston, 2008). The morphology and passive properties of dendrites critically influence the processing of synaptic inputs (Jack et al., 1983; Mainen and Sejnowski, 1996; Segev and London, 2000; Schaefer et al., 2003; Abrahamsson et al., 2012). Thus, knowledge about the electrical properties of dendrites is crucial for our understanding of information transfer and computation in the central nervous system.

Cerebellar granule cells (GCs) are the most numerous neurons in the brain (Williams and Herrup, 1988) and compose the majority of the input layer of the cerebellar cortex (Billings et al., 2014). GCs have small somata and, on average, four short dendrites (Palkovits et al., 1972; Palay and Chan-Palay, 1974). The dendrites end with claw-like shaped digits (DiGregorio et al., 2007), which receive excitatory mossy fiber input in cerebellar glomeruli (D’Angelo et al., 1990; Silver et al., 1992). A glomerulus is formed 
by a single presynaptic mossy fiber bouton, Golgi cell axons, and dendrites of more than 10 GCs (Jakab and Hámori, 1988; Billings et al., 2014; Ritzau-Jost et al., 2014). GCs integrate the broadbandwidth sensory information conveyed by mossy fiber inputs, transforming it into higher dimensional, sparser code (Marr, 1969; Billings et al., 2014). Thus, the anatomical structure of the GC layer is optimal for pattern separation (Olshausen and Field, 2004), which is important for network functions such as adaptive filtering (Fujita, 1982; Dean et al., 2010) and associative learning (D'Angelo and De Zeeuw, 2009).

Regarding the electrical properties of cerebellar GCs, previous studies based on somatic recordings and modeling indicated that these small neurons are electrotonically compact (Silver et al., 1992; D’Angelo et al., 1993; Gabbiani et al., 1994), thus affording good somatic voltage-clamp. Consequently, GC soma and dendrites are generally assumed to form a single electrical compartment, thereby acting as a point neuron (Billings et al., 2014). In recent years, direct patch-clamp recordings from dendrites have significantly advanced our understanding of many neurons' electrical properties and their signaling (see e.g., Stuart and Sakmann, 1994; Nevian et al., 2007; $\mathrm{Hu}$ et al., 2010). The electrotonic properties of the small GC dendrites, however, have not been directly determined. In particular, passive membrane properties of GC dendrites such as the input resistance and their relation to somatic values remain unclear. Furthermore, model predictions critically depend on the diameter of dendrites, which is difficult to measure. Here, we establish whole-cell patch-clamp recordings from GC dendrites to directly determine their electrotonic properties. We compare the input resistance and measure spontaneous excitatory postsynaptic potentials (EPSP) at dendritic and somatic recording sites. Our experimental findings provide direct evidence for the electrotonic compactness of GCs.

\section{Materials and Methods}

\section{Electrophysiology}

Cerebellar slices were prepared from mature $(\mathrm{P} 37 \pm 3$, range P22-P98) CD-1 or C57BL/6 mice of either sex. Animals were bred in the animal facility of the Medical Faculty of the University of Leipzig, and treated in accordance with the German Protection of Animals Act (TierSchG $\$ 4$ Abs. 3) and with the guidelines for the welfare of experimental animals issued by the European Communities Council Directive of 24 . November 1986 (86/609/EEC). The local authorities approved the experiments (Landesdirektion Leipzig, registration number T86/13). Mice were housed in a $12 \mathrm{~h}$ light/dark cycle with food and water ad libitum. Animals were lightly anesthetized with isoflurane (Baxter, Deerfield, IL) before being killed by rapid decapitation. The cerebellar vermis was quickly removed and mounted in a chamber filled with chilled extracellular solution. Parasagittal $300-\mu \mathrm{m}$ slices were cut using a Leica VT1200 microtome (Leica Microsystems, Wetzlar, Germany), transferred to an incubation chamber at $\sim 35^{\circ} \mathrm{C}$ for $30 \mathrm{~min}$ and subsequently stored at room temperature. Artificial cerebrospinal fluid (ACSF) was used for slice cutting, storage, and experiments.
ACSF contained (in mM): $125 \mathrm{NaCl}, 25 \mathrm{NaHCO}_{3}, 2.5 \mathrm{KCl}$, $1.25 \mathrm{NaH}_{2} \mathrm{PO}_{4}, 2 \mathrm{CaCl}_{2}, 1 \mathrm{MgCl}_{2}, 20$ Glucose $(\sim 310 \mathrm{mOsm}$, $\mathrm{pH} 7.3$ when bubbled with Carbogen $\left.\left(5 \% \mathrm{O}_{2} / 95 \% \mathrm{CO}_{2}\right)\right)$. Patch pipettes were pulled from borosilicate glass (Science Products, Hofheim, Germany) using a DMZ Puller (Zeitz-Instruments, Martinsried, Germany). Patch pipettes had open-tip resistances of 9-14 $\mathrm{M} \Omega$ or 14-18 $\mathrm{M} \Omega$ for somatic and dendritic recordings, respectively. The intracellular solution contained (in $\mathrm{mM}$ ): $150 \mathrm{~K}$-gluconate, $10 \mathrm{NaCl}, 10 \mathrm{~K}$-HEPES, $3 \mathrm{Mg}$ ATP, $0.3 \mathrm{Na}-\mathrm{GTP}$ (300-305 mOsm, pH adjusted to 7.3 with $\mathrm{KOH})$. In addition, the intracellular solution contained 10$-20 \mu \mathrm{M}$ of the fluorescence dye Atto594. Experiments were performed at $35-37^{\circ} \mathrm{C}$ and slices were continuously superfused with ACSF. Atto594 was obtained from Atto-Tec (Atto-Tec, Siegen, Germany); all other chemicals were purchased from Sigma-Aldrich (St. Louis, MO).

Cerebellar GCs were visualized with oblique infrared illumination and were identified as previously described (Silver et al., 1996). For dendritic recordings, putative cerebellar glomeruli were approached with patch-pipettes. In 12 out of $>700$ attempts, a whole-cell recording could be established at a GC dendrite. Dendritic recording sites were confirmed by twophoton imaging of the GC filled with Atto594 via the dendrite (Figure 1B). Patch-clamp recordings were made using a HEKA EPC10/2 USB amplifier (HEKA Elektronik, Lambrecht/Pfalz, Germany). Data were sampled at $200 \mathrm{kHz}$. Measurements were corrected for a liquid junction potential of $+13 \mathrm{mV}$. Series resistance ranged from $20-57 \mathrm{M} \Omega$ for somatic recordings (mean $36.6 \pm 2.4 \mathrm{M} \Omega$ ), and from $42-155 \mathrm{M} \Omega$ for recordings from GC dendrites (mean $88.7 \pm 16.9 \mathrm{M} \Omega$ ).

\section{Two-photon Imaging}

We used a Femto2D laser-scanning microscope (Femtonics, Budapest, Hungary) for imaging. Two-photon excitation was performed with a MaiTai femtosecond pulsed Ti:Sapphire laser (SpectraPhysics, Santa Clara, CA) tuned to $810 \mathrm{~nm}$. Both reflected and transmitted fluorescence were collected by the imaging setup with a $60 \times$ water-immersion objective (Olympus, NA 1.0) and an oil-immersion condenser (Olympus, NA 1.4), respectively. Imaging data were acquired and processed using MES software (Femtonics). Stacks of two-photon images covering $20-50 \mu \mathrm{m}$ in $z$-dimension were obtained. Diameters of GC dendrites and somata were measured as full-width at half-maximum of intensity line profiles made perpendicular to the dendrite or soma, respectively, in maximum $z$-projections of image stacks. Dendrite length was measured as $x y z$-distance in image stacks using MES software.

\section{Data Analysis}

Input resistance $\left(R_{\text {in }}\right)$ was calculated from voltage deflections in response to tonic current injection $(-20 \mathrm{pA}$, duration $300 \mathrm{~ms})$. Voltage was calculated as mean over $60 \mathrm{~ms}$ at steady-state. In a separate set of experiments, the subthreshold current-voltage relationship was determined with small current steps $( \pm 2 \mathrm{pA})$ in order to characterize the dependence of $R_{\text {in }}$ on the amplitude of current injection. As previously reported (D'Angelo et al., 


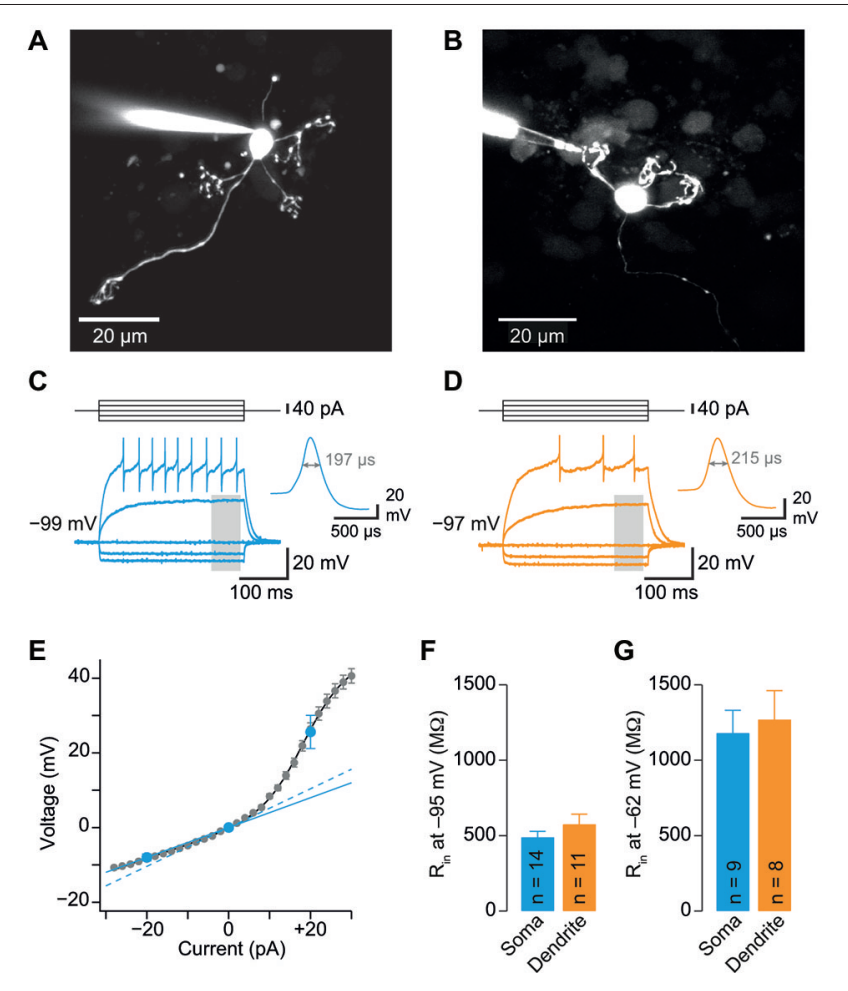

FIGURE 1 | Somatic and dendritic input resistances are similar.

(A) Cerebellar granule cell (GC) filled with a fluorescent dye via the patch-pipette during a somatic recording. Maximum intensity projection of a two-photon stack of 11 images, z-step $2 \mu \mathrm{m}$. (B) GC filled with a fluorescent dye via the patch-pipette during a dendritic recording. Maximum intensity projection of a two-photon stack of 8 images, z-step $3 \mu \mathrm{m}$. (C) Voltage transients obtained in response to tonic current injections (steps of $\pm 20 \mathrm{pA}$ ) in a somatic GC recording. Action potentials are truncated for clarity. Inset shows action potential on enlarged time scale, the duration at the half-maximal amplitude is indicated. Same cell as in (A). The gray area indicates time window used for analysis of input resistance. (D) Corresponding data from a dendritic recording. Same cell as in (B). (E) Voltage-current relation determined with small current steps in $n=35$ GC somata in a different set of experiments (gray markers). Blue markers represent data obtained with \pm 20 pA steps. Input resistance determined using small (2 pA, dashed line) and larger steps (-20 pA, continuous line) differed by a factor of 1.3. Input resistance measured using $-20 \mathrm{pA}$ steps was corrected by this factor (see Material and Methods). (F) Average input resistance $\left(R_{\text {in }}\right)$ at GC somata and dendrites was not significantly different. $R_{\text {in }}$ was determined at a membrane potential of $-95.4 \pm 1.1 \mathrm{mV}$ (corresponding to $0 \mathrm{pA}$ in panel E). Bars represent means \pm SEM (number of somatic and dendritic GC recordings is indicated). (G) $R_{\text {in }}$ recorded from somata and dendrites at a membrane potential of $-62.4 \pm 3.2 \mathrm{mV}$ (corresponding to $+20 \mathrm{pA}$ in panel E).

1995; Cathala et al., 2003), GCs exhibited outward and inward rectification (Figure 1E). Consequently, the data obtained with $-20 \mathrm{pA}$ current steps were corrected for by using the slope at $0 \mathrm{pA}$ of a sum of a sigmoid and a linear function fit to the data, resulting in a correction factor of 1.3 (Figure 1E). Spontaneous EPSP were detected with a template matching routine implemented in NeuroMatic software. ${ }^{1}$ For analysis of $20-80 \%$ rise times and decay time constants of EPSPs, data were filtered to avoid distortions of the kinetics measurements

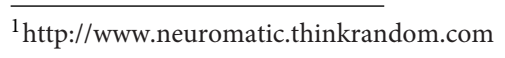

by noise. Statistical analysis was performed using unpaired or paired $t$-tests. Level of statistical significance was set at $p<0.05$. Data are expressed as mean \pm SEM except where stated.

\section{Modeling}

To determine the electrotonic properties of GCs from the somatic and dendritic input resistance, the following approach was used: GCs were represented by a spherical soma with radius, $a_{\text {soma }}$, an axon with radius, $a_{\mathrm{axon}}$, and four cylindrical dendrites with radius, $a_{\text {dend }}$ (Figure 3A). Dendritic claws were not included as additional compartments, because their diameter does not exceed the diameter of the parent dendrite (Jakab and Hámori, 1988; DiGregorio et al., 2002). The resulting somatic input conductance, $g_{\text {somatic }}$, is:

$$
g_{\text {somatic }}=g_{\text {soma }}+4 g_{\text {dendrite }}+g_{\text {axon }}
$$

where $g_{\text {soma }}, g_{\text {axon }}$, and $g_{\text {dendrite }}$ are the input conductance of an isolated soma, isolated axon, and a single isolated dendrite, respectively. $g_{\text {soma }}$ is calculated as:

$$
g_{\text {soma }}=\frac{1}{R_{m}} 4 \pi a_{\mathrm{soma}}^{2}
$$

where $R_{m}$ is the specific membrane resistance. $g_{\text {dendrite }}$ is calculated as the input conductance of a finite cable (Rall, 1969; Jack et al., 1983):

$$
g_{\text {dendrite }}=\frac{\tanh L}{r_{a} \lambda}
$$

where $L$ is the electrotonic length of the dendrites, $r_{a}$ is the intracellular resistance to axial flow of current along the cylinder, and $\lambda$ is the membrane length constant defined as:

$$
L=\frac{l}{\lambda}
$$

where $l$ is the length of the dendrite,

$$
r_{a}=\frac{R_{i}}{\pi a_{\mathrm{dend}}^{2}}
$$

where $R_{i}$ is the intracellular resistivity, and

$$
\lambda=\sqrt{R_{m} a_{\mathrm{dend}} / 2 R_{i}}
$$

$g_{\text {axon }}$ is calculated as the input conductance of a finite cable, accordingly.

First, $R_{m}$ was calculated to obtain the measured somatic input resistance as a function of dendrite diameter $\left(=2 a_{\text {dend }}\right.$; Figure $3 \mathbf{B}$ ) by numerically solving equation (1) for $R_{m}$ (using the FindRoot function of Mathematica). Note, that the three remaining parameters were measured $\left(a_{\text {soma }}\right.$ and $l$ ) or taken from the literature $\left(R_{i}\right.$, Silver et al., 1992; Gabbiani et al., 1994; Cathala et al., 2003). In addition, two parameters describing the electrotonic compactness of neurons were plotted as a function of dendritic diameter: The above defined electrotonic length of the dendrites, $L$, and the dendrite-to-soma conductance ratio, $\rho$ (also referred to as 
dendritic dominance), defined as (Rall, 1969; Jack et al., 1983):

$$
\rho=\frac{4 g_{\text {dend }}}{g_{\text {soma }}}
$$

Finally, the predicted dendritic input resistance was calculated using the NEURON simulation environment (Carnevale and Hines, 2006). A multi-compartment cylinder (nseg $=20)$ with radius $a_{\text {soma }}$ and length $2 a_{\text {soma }}$ represented the soma; the axon was represented by a multi-compartment cylinder $(n s e g=20)$ with radius $a_{\text {axon }}=0.09 \mu \mathrm{m}$ (Sultan, 2000) and length $300 \mu \mathrm{m}$. Increasing the length of the axon had marginal impact on the results. Four cylinders with radius $a_{\mathrm{dend}}$ and length $l$ represented the dendrites (see Table 1; nseg $=20$ ). Membrane capacitance $\left(C_{m}\right)$ was $0.9 \mu \mathrm{F} \mathrm{cm}^{-2}$. For each dendritic diameter, $R_{m}$ of the NEURON model was set to a value ensuring the correct somatic input resistance. Current injection at the soma resulted in voltage deflections at the soma consistent with the calculated somatic input resistance. Current injection at the tip of one dendrite resulted in voltage deflections at the tip of the dendrite from which the dendritic input resistance was calculated.

\section{Results}

\section{Somatic and Dendritic Input Resistances are Similar}

To investigate the electrotonic properties of cerebellar GCs, we performed direct patch-clamp recordings from GC somata and dendrites (Figures 1A,B). For dendritic recordings, putative cerebellar glomeruli containing mossy fiber boutons and dendrites of GCs were approached with patch pipettes. After establishing the whole-cell configuration, GCs were unequivocally identified by the following two criteria: (1) In contrast to presynaptic mossy fiber terminals, which fire a single action potential upon current injection (Rancz et al., 2007;

\section{TABLE 1 | Parameters of GCs.}

\begin{tabular}{lcl}
\hline Parameter & Value & \multicolumn{1}{c}{ Method } \\
\hline Somatic $R_{\text {in }}$ at $-95 \mathrm{mV}(\mathrm{M} \Omega)$ & $492 \pm 37(n=14)$ & patch-clamp recording \\
Dendritic $R_{\text {in }}$ at $-95 \mathrm{mV}(\mathrm{M} \Omega)$ & $578 \pm 65(n=11)$ & patch-clamp recording \\
Somatic $R_{\text {in }}$ at $-62 \mathrm{mV}(\mathrm{M} \Omega)$ & $1182 \pm 150(n=9)$ & patch-clamp recording \\
Dendritic $R_{\text {in }}$ at $-62 \mathrm{mV}(\mathrm{M} \Omega)$ & $1273 \pm 189(n=8)$ & patch-clamp recording \\
Soma diameter $(\mu \mathrm{m})$ & $5.9 \pm 0.3(n=11)$ & two-photon imaging \\
Dendrite length $(\mu \mathrm{m})$ & $20.7 \pm 2.9(n=11)$ & two-photon imaging \\
Dendrite diameter $(\mu \mathrm{m})$ & $0.69 \pm 0.3(n=10)$ & two-photon imaging \\
Dendrite diameter $(\mu \mathrm{m})$ & $0.52(0.40-1.02)$ & Figure 3C \\
$R_{m}\left(\mathrm{k} \Omega \mathrm{cm}^{2}\right)$ & $1.42(1.25-2.12)$ & Figure 3C \\
$\rho$ & $1.23(0.93-2.43)$ & Figure 3C \\
$L$ & $0.15(0.09-0.19)$ & Figure 3C
\end{tabular}

Summary of determined GC parameters (mean \pm SEM, or mean with 16-84\% confidence interval in brackets). The input resistance $\left(R_{\text {in }}\right)$ was obtained with somatic or direct dendritic patch-clamp recordings. The diameter of the soma and the length of the dendrites were measured from stacks of two-photon images. The diameter of the dendrites, the specific membrane resistance $\left(R_{m}\right)$, the dendrite-tosoma conductance ratio $(\rho)$ and the electrotonic length of the dendrites $(L)$ were determined in Figure $\mathbf{3} \mathbf{C}$.
Ritzau-Jost et al., 2014), GCs display distinctive repetitive firing (Cathala et al., 2003); and (2) The dendritic recording site was verified by including a fluorescence dye (Atto594) in the patch pipette and using two-photon imaging (Figure 1B). Interestingly, in 5 out of 12 GCs the axon originated from the dendrite (Thome et al., 2014). In all our experiments, the dendritic recording site was located at the distal part of the dendrites with an average distance from the soma of $20.7 \pm 2.9 \mu \mathrm{m}(n=11$; range: 11-42 $\mu \mathrm{m}$; Table 1). Thus, our data show that direct patchclamp recordings from the small dendrites of cerebellar GCs are feasible.

We compared the input resistance $\left(R_{i n}\right)$ of somatic and dendritic recordings to investigate the electrotonic compactness of GCs. Analysis of the spatial distribution of $R_{i n}$ alone is necessary, but not sufficient to make conclusions on electrical compactness of neuronal structures. In our case, however, the length of dendrites is known, which allows investigating the electrical compactness of GCs with additional knowledge of $R_{i n}$. We determined $R_{\text {in }}$ in current-clamp mode using 300-ms long hyperpolarizing current steps of $-20 \mathrm{pA}$ (Figures 1C,D). Because $R_{\text {in }}$ depends on the amount of current injection (D'Angelo et al., 1995; Cathala et al., 2003), we also determined the voltagecurrent relation in a separate set of GC somatic recordings using smaller $( \pm 2 \mathrm{pA})$ current steps. These data were fit with the sum of a sigmoid and a linear function. From this fit, $R_{\text {in }}$ was determined as the slope at $0 \mathrm{pA}$, which was 1.3 -fold higher than $R_{\text {in }}$ calculated from $-20 \mathrm{pA}$ step current injections (Figure 1E, blue lines). Therefore, $R_{\text {in }}$ values measured in somatic and dendritic recordings using $-20 \mathrm{pA}$ current injection were corrected accordingly (cf. Material and Methods). Interestingly, $R_{\text {in }}$ was not significantly different at dendritic and somatic recording sites (soma: $0.49 \pm 0.04 \mathrm{G} \Omega$; dendrite: $0.58 \pm 0.07$ $\mathrm{G} \Omega ; p=0.24$, unpaired $t$-test; Figure 1F; Table 1). Also, $R_{\text {in }}$ in our somatic measurements was comparable to values previously reported for P39 mice (Cathala et al., 2003) and adult cats (Jörntell and Ekerot, 2006), but lower than previously determined in young rats (D'Angelo et al., 1993, 1995; Silver et al., 1996; Prestori et al., 2013). Cerebellar GCs show pronounced inward rectification ((D'Angelo et al., 1995; Cathala et al., 2003), cf. Figures 1C,D,F), which could impact the $R_{\text {in }}$ measurements with hyperpolarizing current steps. When analyzing $R_{i n}$ with depolarizing current steps of $+20 \mathrm{pA}$, we obtained higher values, which were again similar in somatic and dendritic recordings (soma: $1.18 \pm 0.15 \mathrm{G} \Omega$; dendrite: $1.27 \pm 0.19 \mathrm{G} \Omega$, Figure 1G; Table 1). These data directly demonstrate that the distal part of the dendrites of GCs has similar $R_{\text {in }}$ compared to the soma. Furthermore, the membrane time constant $\left(\tau_{m}\right)$ determined with hyperpolarizing current injections was comparable for somatic and dendritic recordings (soma: $1.4 \pm 0.12 \mathrm{~ms}$, dendrite: $1.63 \pm$ $0.17 \mathrm{~ms} ; p=0.25$, unpaired $t$-test).

\section{GC Dendrites do not Significantly Filter Spontaneous EPSPs}

The similar $R_{i n}$ of soma and dendrites suggests that GCs are electrotonically compact. To further investigate this hypothesis, we measured spontaneous EPSPs in GC somata and dendrites. 


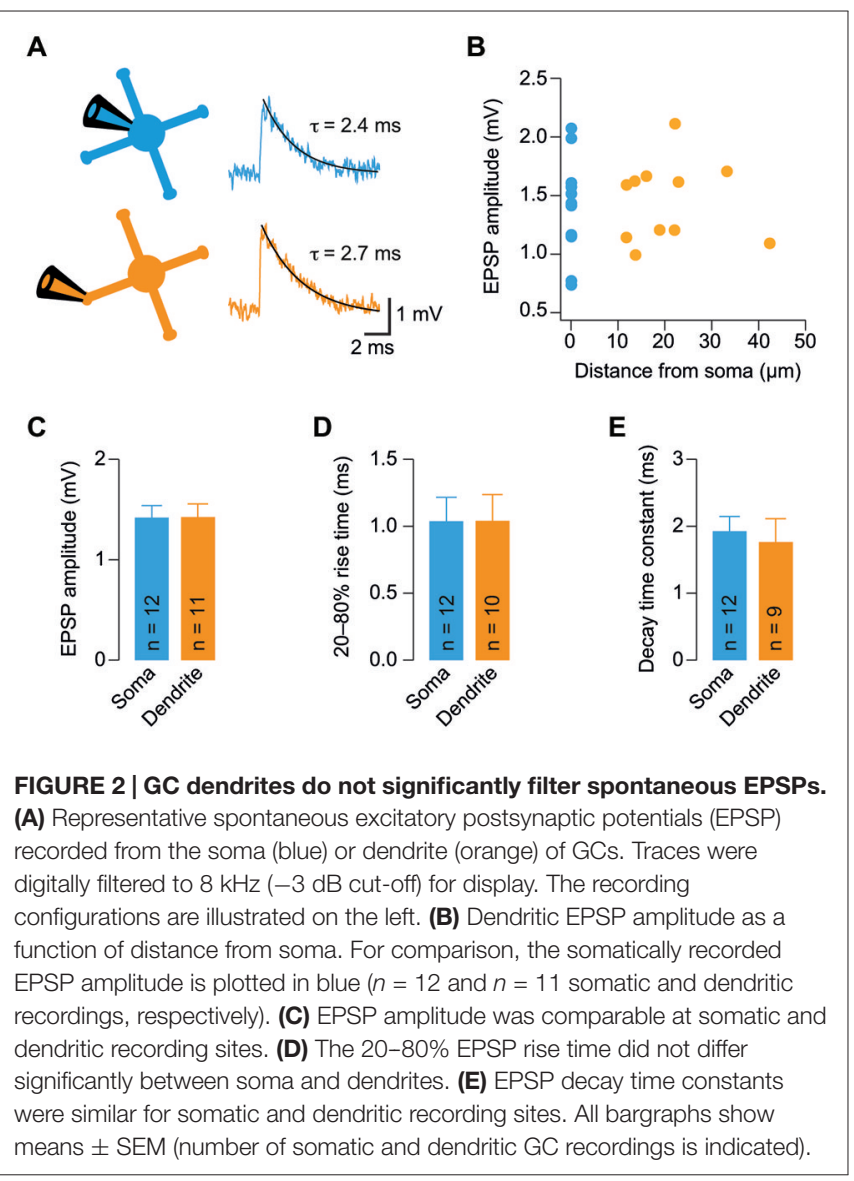

We observed spontaneous EPSPs (Figure 2A) at a mean frequency of $0.85 \pm 0.16 \mathrm{~Hz}$ and $1.19 \pm 0.22 \mathrm{~Hz}$ in somatic and dendritic recordings, respectively, consistent with previous reports (Cathala et al., 2003; Hallermann et al., 2010). The amplitude of spontaneous EPSPs of GCs did not display a strong dependence on distance of dendritic recording sites from the soma (Figure 2B). Accordingly, the mean amplitude of spontaneous EPSPs was not significantly different between somatic and dendritic recording sites $(p=0.98$, unpaired $t$-test; Figure 2C). In addition, the rise times and decay time constants of spontaneous EPSPs were comparable at the two distinct recording sites $(20-80 \%$ rise time: $1.04 \pm 0.18 \mathrm{~ms}$ vs. $1.04 \pm 0.19 \mathrm{~ms}, n=12$ and $10, p=0.99$; decay time constant: $1.9 \pm 0.2 \mathrm{~ms}$ vs. $1.8 \pm 0.4 \mathrm{~ms}, n=12$ and $9, p=0.68$; for somatic and dendritic recordings, respectively, unpaired $t$-tests; Figures 2D,E). When recording from a dendrite, some EPSPs will be locally generated and the rest originate from the remaining three dendrites. However, we did not observe an increased heterogeneity of the EPSP amplitude during the dendritic recordings (dendritic vs. somatic coefficient of variation, $\mathrm{CV}=$ mean/SD: $50.4 \%$ vs. $61.4 \%$ ) and the variability of kinetic parameters was comparable $(20-80 \%$ rise time dendritic vs. somatic CV: $111.4 \%$ vs. $91.8 \%$; decay time constant dendritic vs. somatic CV: $93.4 \%$ vs. $76.8 \%$ ). These results indicate that dendrites do not filter EPSPs in GCs to a large extent.

\section{Analysis of Electrotonic Properties Demonstrates Electrical Compactness}

We next determined the electrotonic properties of GCs by analyzing the measured somatic and dendritic input resistance using analytical calculations and numerical modeling implemented in NEURON (Carnevale and Hines, 2006). GCs were modeled by a spherical soma with a cylindrical axon and four cylindrical dendrites (Figure 3A). The somatic input resistance of this simplified GC can be calculated analytically (Equation 1, Material and Methods) and depends on the diameter of the soma, the length and diameter of the axon and dendrites, the intracellular resistivity, and the specific membrane resistance. These values were determined as described in the following: The soma diameter and dendrite length were measured from image stacks of GCs filled with Atto594 during dendritic recordings. In these experiments, the mean GC soma diameter was $5.9 \mu \mathrm{m}$ and the mean length of GC dendrites, which were recorded from, was $20.7 \mu \mathrm{m}$ (Table 1). The intracellular resistivity is similar across cell types and was set at previously estimated values from cerebellar GCs (100 $\Omega \mathrm{cm}$; Silver et al., 1992; Gabbiani et al., 1994; Cathala et al., 2003). The axon diameter was taken from the literature $(0.18 \mu \mathrm{m}$, Sultan, 2000) and its length was set at $300 \mu \mathrm{m}$. The two remaining parameters-the diameter of the dendrites and the membrane resistance-are more difficult to measure. Since the diameter of GC dendrites is not exactly known and has a strong influence on the input resistance, we systematically varied the diameter of the dendrites in our GC model. We set the specific membrane resistance $\left(R_{m}\right)$ at a value that ensured that the model predicted our measured somatic $R_{\text {in }}$ of $492 \mathrm{M} \Omega$ for each dendrite diameter (Figure 3B, upper two graphs; cf. Material and Methods). We then calculated two parameters describing the electrotonic properties of GCs: the dendrite-to-soma conductance ratio, $\rho$ (also referred to as dendritic dominance), and the electrotonic length of the dendrites, $L$ (Rall, 1969). $\rho$ increased with dendrite diameter (Figure 3B), corresponding to a larger contribution of the dendrites to $R_{\text {in }}$. Also, $L$ decreased with increasing dendrite diameter (Figure 3B), corresponding to increased electrotonic compactness and thus a convergence to a single compartment (which would have $L=0$ ).

Based on these results, the measured dendritic $R_{\text {in }}$ was used to determine the average properties of our GCs. Therefore, we first determined the dendritic $R_{i n}$ in our model as a function of dendrite diameter (Figure 3C, top graph; see Material and Methods). Comparison with the measured dendritic $R_{\text {in }}$ of $578.1 \pm 64.9 \mathrm{M} \Omega$ revealed that our GCs are best characterized by a dendritic diameter of $0.52 \mu \mathrm{m}$. In our two-photon images, the patched GC dendrites had an average diameter of $0.69 \pm 0.03 \mu \mathrm{m}(n=11$, range $0.5-0.8 \mu \mathrm{m})$. Taking into account the limited spatial resolution of twophoton microscopy, these values seem consistent. According to the relations shown in Figure $\mathbf{3 B}$, the comparison of the model prediction and the measured dendritic $R_{\text {in }}$ revealed an $R_{m}$ of $1.4 \mathrm{k} \Omega \mathrm{cm}^{2}, \rho$ of 1.23 , and $L$ of 0.15 (Figure $3 \mathrm{C}$; see Table 1). These results and in particular the small electrotonic length of the dendrites demonstrate that GCs are electrotonically very compact. 
A

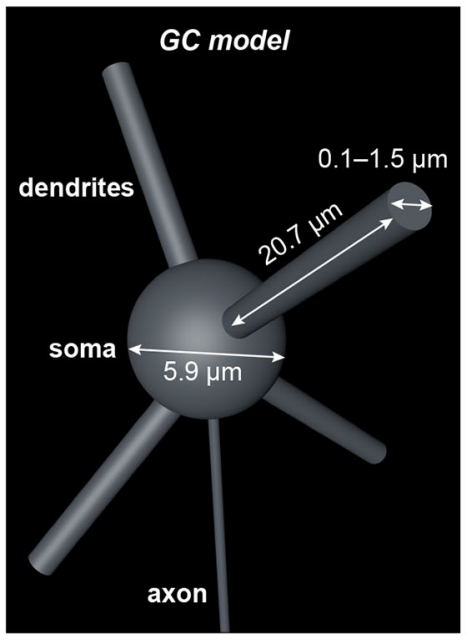

B

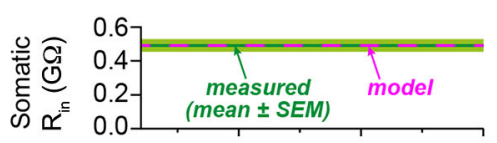

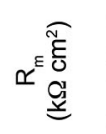

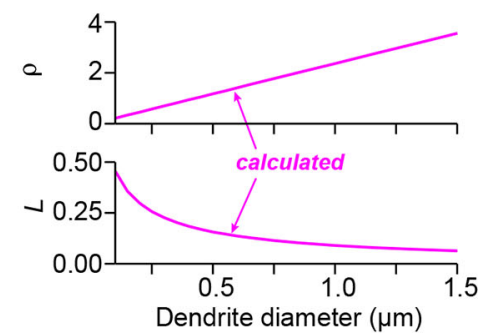

C

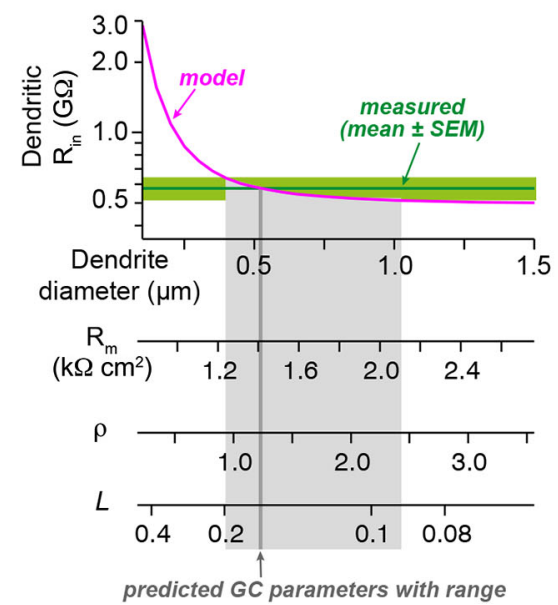

FIGURE 3 | Analysis of electrotonic properties demonstrates electrical compactness. (A) Illustration of our GC model. The model consisted of a spherical soma and four dendrites. The indicated diameter of the soma and the length of dendrites were measured from stacks of two-photon microscopic images obtained during dendritic recordings. Axon diameter was taken from the literature. The diameter of the dendrites was systematically varied between 0.1 and $1.5 \mu \mathrm{m}$. (B) Superposition of the measured somatic input resistance $\left(R_{\text {in }}\right)$ with the prediction of the model. For each diameter of the dendrites, the specific membrane resistance $\left(R_{m}\right)$ was adjusted to ensure the correct somatic $R_{\text {in }}$. The dendrite-to-soma conductance ratio $(\rho)$ and the electrotonic length of the dendrites $(L)$ were calculated as a function of dendrite diameter. (C) Superposition of the measured dendritic $R_{\text {in }}$ with the prediction of the model as a function of dendrite diameter. Comparison of the model prediction with the mean and the SEM of the dendritic $R_{\text {in }}$ revealed estimates with confidence ranges for the dendrite diameter, $R_{m}, \rho$, and $L$ (see Table 1).
We also used the measured membrane time constant as independent constraint in our simulations. To this end, we compared the measured membrane time constant with the one predicted by the model resulting in a graph comparable to Figure 3C (data not shown). This analysis yielded a dendrite diameter estimate of $0.69 \mu \mathrm{m}, R_{m}$ of $1.7 \mathrm{k} \Omega \mathrm{cm}^{2}, \rho$ of 1.64 , and $L$ of 0.12 . These values are very similar to the approach based on $R_{i n}$, providing independent support for our conclusion of electronics compactness of GCs.

As described above, our measurements of $R_{\text {in }}$ might be influenced by the inward rectification present in GCs (cf. Figure 1). We therefore repeated the simulations as in Figures 3B,C with the higher $R_{\text {in }}$ values obtained from $+20 \mathrm{pA}$ current steps (cf. Figure 1G). The resulting estimates were dendrite diameter $0.52 \mu \mathrm{m}, R_{m}=3.7 \mathrm{k} \Omega \mathrm{cm}^{2}, \rho=1.23$, and $L=0.10$, again indicating that GCs can be considered as electrotonically very compact.

\section{Discussion}

In this study, we established dendritic patch-clamp recordings from GC dendrites, which have a thin diameter of $\sim 0.7 \mu \mathrm{m}$ (Eccles et al., 1967). To the best of our knowledge, these are the thinnest dendrites recorded from. Dendritic recordings have been performed at other thin dendrites, such as the basal dendrites of layer 5 pyramidal neurons with a diameter of $\sim 1.9 \mu \mathrm{m}$ (Nevian et al., 2007), or dendrites of hippocampal basket cells with a diameter of $\sim 1.4 \mu \mathrm{m}$
(Hu et al., 2010; Nörenberg et al., 2010). We exploited this technique to directly investigate the passive electrical properties of GCs.

\section{Electrical Compactness of Cerebellar CGs}

Previous studies using somatic recordings in rats indicated that GCs are electrotonically very compact (Silver et al., 1992; D'Angelo et al., 1993). In these two studies, the values for the dendrite-to-soma conductance ratio $(\rho)$ were 0.98 and $\leq 0.5$ (upper boundary), respectively. The electrotonic length of the dendrites $(L)$ was determined as 0.05 and 0.04 . These figures are slightly smaller than our estimates in mice of 1.23 for $\rho$ and 0.15 for $L$ (Figure 3; Table 1). Furthermore, the specific membrane resistance $R_{m}$ was previously estimated as $16 \mathrm{k} \Omega \mathrm{cm}^{2}$ (Silver et al., 1992), whereas our estimate was $1.4 \mathrm{k} \Omega \mathrm{cm}^{2}$. Species and recording temperature differences could contribute to these discrepancies and may also explain why in our experiments at physiological temperature $\left(35-37^{\circ} \mathrm{C}\right), R_{\text {in }}$ was lower than previous estimates (D’Angelo et al., 1993, 1995; Brickley et al., 2001). In addition, the developmental state of the animals could be a reason, because pronounced changes in the morphological properties of GC and their membrane properties during development have previously been described (Cathala et al., 2003). For example, $R_{m}$ was decreased from $9.2 \mathrm{k} \Omega \mathrm{cm}^{2}$ in P8 to $2.6 \mathrm{k} \Omega \mathrm{cm}^{2}$ in P39 mice (Cathala et al., 2003). Note, that our mice had an average age of P37, but previous studies used rats of age P10-P22 (Silver et al., 1992; D'Angelo et al., 1993). Nevertheless, the compactness of GCs was confirmed when using higher $R_{\text {in }}$ values for our analyses (see Results). Thus, 
our dendritic recordings strongly support the previous studies analyzing the electrotonic compactness of GCs. Furthermore, the low $R_{m}$ of cerebellar GCs will contribute to a fast time course of EPSPs and facilitate rapid action potential initiation (Nörenberg et al., 2010). Consistent with the spatially uniform $R_{\text {in }}$ and electrotonic compactness, spontaneous EPSPs recorded at the soma and the dendrites were similar (Figure 2). Thus, our data indicate that cerebellar GCs are electrotonically extremely compact.

\section{Functional Implications}

The electrotonic compactness allows GCs to rapidly and precisely integrate the fast EPSCs originating from mossy fiber activation (Silver et al., 1992; Cathala et al., 2005; Sargent et al., 2005) and to process high-frequency inputs (Saviane and Silver, 2006; Rancz et al., 2007; Ritzau-Jost et al., 2014). Furthermore, their compactness enables GCs to compare mossy fiber inputs independent of the distance of the synaptic site from the soma. Some less compact neurons with longer dendrites receive stronger inputs at distal parts of the dendrites (Magee and Cook,

\section{References}

Abbott, L. F., and Regehr, W. G. (2004). Synaptic computation. Nature 431, 796-803. doi: 10.1038/nature03010

Abrahamsson, T., Cathala, L., Matsui, K., Shigemoto, R., and DiGregorio, D. A. (2012). Thin dendrites of cerebellar interneurons confer sublinear synaptic integration and a gradient of short-term plasticity. Neuron 73, 1159-1172. doi: 10.1016/j.neuron.2012.01.027

Billings, G., Piasini, E., Lorincz, A., Nusser, Z., and Silver, R. A. (2014). Network structure within the cerebellar input layer enables lossless sparse encoding. Neuron 83, 960-974. doi: 10.1016/j.neuron.2014.07.020

Blot, A., and Barbour, B. (2014). Ultra-rapid axon-axon ephaptic inhibition of cerebellar Purkinje cells by the pinceau. Nat. Neurosci. 17, 289-295. doi: 10. 1038/nn.3624

Brickley, S. G., Revilla, V., Cull-Candy, S. G., Wisden, W., and Farrant, M. (2001). Adaptive regulation of neuronal excitability by a voltage-independent potassium conductance. Nature 409, 88-92. doi: 10.1038/35051086

Carnevale, N. T., and Hines, M. L. (2006). The NEURON Book. Cambridge: Cambridge University Press.

Cathala, L., Brickley, S., Cull-Candy, S., and Farrant, M. (2003). Maturation of EPSCs and intrinsic membrane properties enhances precision at a cerebellar synapse. J. Neurosci. 23, 6074-6085.

Cathala, L., Holderith, N. B., Nusser, Z., DiGregorio, D. A., and Cull-Candy, S. G. (2005). Changes in synaptic structure underlie the developmental speeding of AMPA receptor-mediated EPSCs. Nat. Neurosci. 8, 1310-1318. doi: 10. 1038/nn 1534

Chadderton, P., Margrie, T. W., and Häusser, M. (2004). Integration of quanta in cerebellar granule cells during sensory processing. Nature 428, 856-860. doi: 10.1038/nature02442

Chen, C. H., Fremont, R., Arteaga-Bracho, E. E., and Khodakhah, K. (2014). Short latency cerebellar modulation of the basal ganglia. Nat. Neurosci. 17, 1767-1775. doi: 10.1038/nn.3868

Crowley, J. J., Carter, A. G., and Regehr, W. G. (2007). Fast vesicle replenishment and rapid recovery from desensitization at a single synaptic release site. J. Neurosci. 27, 5448-5460. doi: 10.1523/jneurosci.1186-07.2007

D’Angelo, E., De Filippi, G., Rossi, P., and Taglietti, V. (1995). Synaptic excitation of individual rat cerebellar granule cells in situ: evidence for the role of NMDA receptors. J. Physiol. 484, 397-413. doi: 10.1113/jphysiol.1995.sp020673

D'Angelo, E., and De Zeeuw, C. I. (2009). Timing and plasticity in the cerebellum: focus on the granular layer. Trends Neurosci. 32, 30-40. doi: 10.1016/j.tins.2008. 09.007
2000) or express dendritic hyperpolarization-activated currents (Williams and Stuart, 2000) to counterbalance dendritic filtering of EPSPs. On the other hand, dendritic filtering might have the advantage to encode the spatial information of synaptic inputs (Rall, 1964). For GCs, however, this would not be of any benefit, because these neurons receive excitatory inputs only at the end of their dendrites. Furthermore, electrotonic compactness likely represents an important factor for the relay function of cerebellar GCs (Chadderton et al., 2004), which efficiently signal to postsynaptic stellate and Purkinje cells (Crowley et al., 2007; Valera et al., 2012), and thereby contribute to rapid cerebellar signaling (Blot and Barbour, 2014; Chen et al., 2014).

\section{Conclusion}

In summary, our dendritic patch-clamp recordings demonstrate that dendrites of cerebellar GCs have a low dendritic dominance and short electrotonic length. Thus, GCs are electrotonically very compact, which seems ideally suited to rapidly process the highfrequency inputs arriving in the cerebellar cortex.

D’Angelo, E., Rossi, P., and Garthwaite, J. (1990). Dual-component NMDA receptor currents at a single central synapse. Nature 346, 467-470. doi: 10. $1038 / 346467 \mathrm{a} 0$

D’Angelo, E., Rossi, P., and Taglietti, V. (1993). Different proportions of Nmethyl-D-aspartate and non-N-methyl-D-aspartate receptor currents at the mossy fibre-granule cell synapse of developing rat cerebellum. Neuroscience 53, 121-130. doi: 10.1016/0306-4522(93)90290-v

Dean, P., Porrill, J., Ekerot, C. F., and Jörntell, H. (2010). The cerebellar microcircuit as an adaptive filter: experimental and computational evidence. Nat. Rev. Neurosci. 11, 30-43. doi: 10.1038/nrn2756

DiGregorio, D. A., Nusser, Z., and Silver, R. A. (2002). Spillover of glutamate onto synaptic AMPA receptors enhances fast transmission at a cerebellar synapse. Neuron 35, 521-533. doi: 10.1016/s0896-6273(02)00787-0

DiGregorio, D. A., Rothman, J. S., Nielsen, T. A., and Silver, R. A. (2007). Desensitization properties of AMPA receptors at the cerebellar mossy fiber granule cell synapse. J. Neurosci. 27, 8344-8357. doi: 10.1523/jneurosci.239907.2007

Eccles, J. C., Ito, M., and Szentagothai, J. (1967). The Cerebellum as a Neuronal Machine. New York: Springer Verlag.

Fujita, M. (1982). Adaptive filter model of the cerebellum. Biol. Cybern. 45, 195-206. doi: 10.1007/bf00336192

Gabbiani, F., Midtgaard, J., and Knöpfel, T. (1994). Synaptic integration in a model of cerebellar granule cells. J. Neurophysiol. 72, 999-1009.

Gulledge, A. T., Kampa, B. M., and Stuart, G. J. (2005). Synaptic integration in dendritic trees. J. Neurobiol. 64, 75-90. doi: 10.1002/neu.20144

Hallermann, S., Fejtova, A., Schmidt, H., Weyhersmüller, A., Silver, R. A., Gundelfinger, E. D., et al. (2010). Bassoon speeds vesicle reloading at a central excitatory synapse. Neuron 68, 710-723. doi: 10.1016/j.neuron.2010.10.026

Hu, H., Martina, M., and Jonas, P. (2010). Dendritic mechanisms underlying rapid synaptic activation of fast-spiking hippocampal interneurons. Science 327, 52-58. doi: 10.1126/science.1177876

Jack, J. J. B., Noble, D., and Tsien, R. W. (1983). Electric Current Flow in Excitable Cells. Oxford: Clarendon Press.

Jakab, R. L., and Hámori, J. (1988). Quantitative morphology and synaptology of cerebellar glomeruli in the rat. Anat. Embryol. (Berl) 179, 81-88. doi: 10 1007/bf00305102

Jörntell, H., and Ekerot, C. F. (2006). Properties of somatosensory synaptic integration in cerebellar granule cells in vivo. J. Neurosci. 26, 11786-11797. doi: 10.1523/jneurosci.2939-06.2006

London, M., and Häusser, M. (2005). Dendritic computation. Annu. Rev. Neurosci. 28, 503-532. doi: 10.1146/annurev.neuro.28.061604.135703 
Magee, J. C. (2000). Dendritic integration of excitatory synaptic input. Nat. Rev. Neurosci. 1, 181-190. doi: 10.1038/35044552

Magee, J. C., and Cook, E. P. (2000). Somatic EPSP amplitude is independent of synapse location in hippocampal pyramidal neurons. Nat. Neurosci. 3, 895-903. doi: $10.1038 / 78800$

Mainen, Z. F., and Sejnowski, T. J. (1996). Influence of dendritic structure on firing pattern in model neocortical neurons. Nature 382, 363-366. doi: 10. 1038/382363a0

Marr, D. (1969). A theory of cerebellar cortex. J. Physiol. 202, 437-470. doi: 10. 1113/jphysiol.1969.sp008820

Nevian, T., Larkum, M. E., Polsky, A., and Schiller, J. (2007). Properties of basal dendrites of layer 5 pyramidal neurons: a direct patch-clamp recording study. Nat. Neurosci. 10, 206-214. doi: 10.1038/nn1826

Nörenberg, A., Hu, H., Vida, I., Bartos, M., and Jonas, P. (2010). Distinct nonuniform cable properties optimize rapid and efficient activation of fastspiking GABAergic interneurons. Proc. Natl. Acad. Sci. U S A 107, 894-899. doi: 10.1073/pnas.0910716107

Olshausen, B. A., and Field, D. J. (2004). Sparse coding of sensory inputs. Curr. Opin. Neurobiol. 14, 481-487. doi: 10.1016/j.conb.2004.07.007

Palay, S. L., and Chan-Palay, V. (1974). Cerebellar Cortex: Cytology and Organization. Berlin; Heidelberg: Springer.

Palkovits, M., Magyar, P., and Szentágothai, J. (1972). Quantitative histological analysis of the cerebellar cortex in the cat. IV. Mossy fiber-Purkinje cell numerical transfer. Brain Res. 45, 15-29. doi: 10.1016/0006-8993(72) 90213-2

Prestori, F., Bonardi, C., Mapelli, L., Lombardo, P., Goselink, R., De Stefano, M. E., et al. (2013). Gating of long-term potentiation by nicotinic acetylcholine receptors at the cerebellum input stage. PLoS One 8:e64828. doi: 10. 1371/journal.pone.0064828

Rall, W. (1964). "Theoretical significance of dendritic trees for neuronal inputoutput relations," in Neural Theory and Modeling, ed E. Reiss (Stanford, CA: Stanford University Press), 73-97.

Rall, W. (1969). Time constants and electrotonic length of membrane cylinders and neurons. Biophys. J. 9, 1483-1508. doi: 10.1016/s0006-3495(69) 86467-2

Rancz, E. A., Ishikawa, T., Duguid, I., Chadderton, P., Mahon, S., and Häusser, M. (2007). High-fidelity transmission of sensory information by single cerebellar mossy fibre boutons. Nature 450, 1245-1248. doi: 10.1038/nature 05995

Ritzau-Jost, A., Delvendahl, I., Rings, A., Byczkowicz, N., Harada, H., Shigemoto, R., et al. (2014). Ultrafast action potentials mediate kilohertz signaling at a central synapse. Neuron 84, 152-163. doi: 10.1016/j.neuron.2014. 08.036

Sargent, P. B., Saviane, C., Nielsen, T. A., DiGregorio, D. A., and Silver, R. A. (2005). Rapid vesicular release, quantal variability and spillover contribute to the precision and reliability of transmission at a glomerular synapse. J. Neurosci. 25, 8173-8187. doi: 10.1523/jneurosci.2051-05.2005
Saviane, C., and Silver, R. A. (2006). Fast vesicle reloading and a large pool sustain high bandwidth transmission at a central synapse. Nature 439, 983-987. doi: 10. 1038/nature04509

Schaefer, A. T., Larkum, M. E., Sakmann, B., and Roth, A. (2003). Coincidence detection in pyramidal neurons is tuned by their dendritic branching pattern. J. Neurophysiol. 89, 3143-3154. doi: 10.1152/jn.00046.2003

Segev, I., and London, M. (2000). Untangling dendrites with quantitative models. Science 290, 744-750. doi: 10.1126/science.290.5492.744

Silver, R. A., Cull-Candy, S. G., and Takahashi, T. (1996). Non-NMDA glutamate receptor occupancy and open probability at a rat cerebellar synapse with single and multiple release sites. J. Physiol. 494, 231-250. doi: 10.1113/jphysiol.1996. sp021487

Silver, R. A., Traynelis, S. F., and Cull-Candy, S. G. (1992). Rapid-time-course miniature and evoked excitatory currents at cerebellar synapses in situ. Nature 355, 163-166. doi: 10.1038/355163a0

Spruston, N. (2008). Pyramidal neurons: dendritic structure and synaptic integration. Nat. Rev. Neurosci. 9, 206-221. doi: 10.1038/nrn2286

Stuart, G. J., and Sakmann, B. (1994). Active propagation of somatic action potentials into neocortical pyramidal cell dendrites. Nature 367, 69-72. doi: 10. $1038 / 367069 \mathrm{a} 0$

Sultan, F. (2000). Exploring a critical parameter of timing in the mouse cerebellar microcircuitry: the parallel fiber diameter. Neurosci. Lett. 280, 41-44. doi: 10. 1016/s0304-3940(99)00984-2

Thome, C., Kelly, T., Yanez, A., Schultz, C., Engelhardt, M., Cambridge, S. B., et al. (2014). Axon-carrying dendrites convey privileged synaptic input in hippocampal neurons. Neuron 83, 1418-1430. doi: 10.1016/j.neuron.2014. 08.013

Valera, A. M., Doussau, F., Poulain, B., Barbour, B., and Isope, P. (2012). Adaptation of granule cell to Purkinje cell synapses to high-frequency transmission. J. Neurosci. 32, 3267-3280. doi: 10.1523/JNEUROSCI.317511.2012

Williams, R. W., and Herrup, K. (1988). The control of neuron number. Annu. Rev. Neurosci. 11, 423-453. doi: 10.1146/annurev.neuro.11.1.423

Williams, S. R., and Stuart, G. J. (2000). Site independence of EPSP time course is mediated by dendritic $I_{h}$ in neocortical pyramidal neurons. J. Neurophysiol. 83, $3177-3182$

Conflict of Interest Statement: The authors declare that the research was conducted in the absence of any commercial or financial relationships that could be construed as a potential conflict of interest.

Copyright $\odot 2015$ Delvendahl, Straub and Hallermann. This is an open-access article distributed under the terms of the Creative Commons Attribution License (CC BY). The use, distribution and reproduction in other forums is permitted, provided the original author(s) or licensor are credited and that the original publication in this journal is cited, in accordance with accepted academic practice. No use, distribution or reproduction is permitted which does not comply with these terms. 


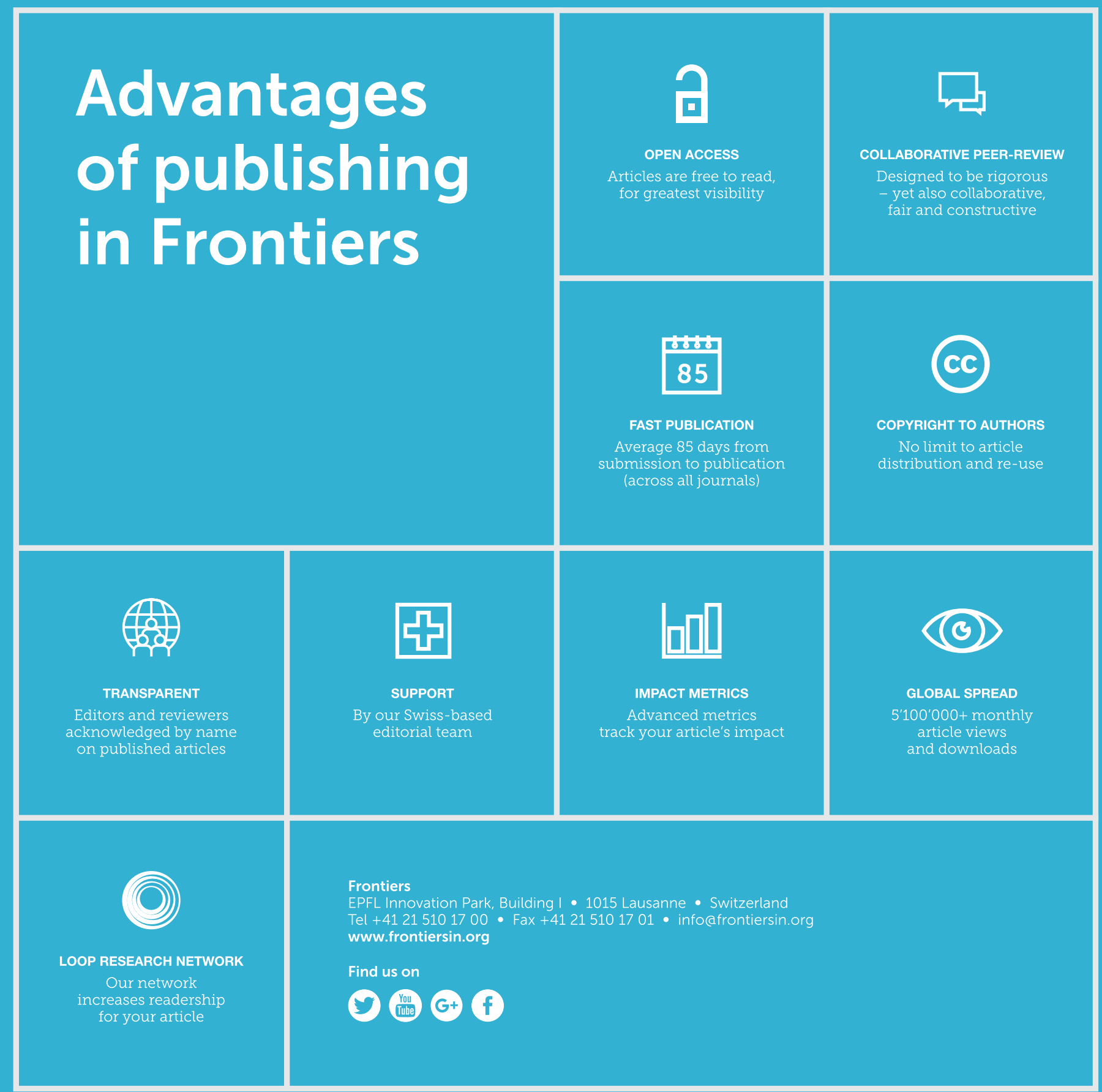

